\title{
WestVirginiaUniversity
}

THE RESEARCH REPOSITORY @ WVU

Graduate Theses, Dissertations, and Problem Reports

2005

\section{Towards large eddy simulation of dispersed gas -liquid two-phase turbulent flows}

Gusheng Hu

West Virginia University

Follow this and additional works at: https://researchrepository.wvu.edu/etd

\section{Recommended Citation}

$\mathrm{Hu}$, Gusheng, "Towards large eddy simulation of dispersed gas -liquid two-phase turbulent flows" (2005). Graduate Theses, Dissertations, and Problem Reports. 2681.

https://researchrepository.wvu.edu/etd/2681

This Dissertation is protected by copyright and/or related rights. It has been brought to you by the The Research Repository @ WVU with permission from the rights-holder(s). You are free to use this Dissertation in any way that is permitted by the copyright and related rights legislation that applies to your use. For other uses you must obtain permission from the rights-holder(s) directly, unless additional rights are indicated by a Creative Commons license in the record and/ or on the work itself. This Dissertation has been accepted for inclusion in WVU Graduate Theses, Dissertations, and Problem Reports collection by an authorized administrator of The Research Repository @ WVU.

For more information, please contact researchrepository@mail.wvu.edu. 


\title{
TOWARDS LARGE EDDY SIMULATION OF DISPERSED GAS-LIQUID TWO-PHASE TURBULENT FLOWS
}

\author{
Gusheng Hu \\ Dissertation submitted to the \\ College of Engineering and Mineral Resources \\ at West Virginia University \\ in partial fulfillment of the requirements \\ for the degree of \\ Doctor of Philosophy \\ in \\ Mechanical Engineering \\ Ismail Celik, Ph.D., Chair \\ John Kuhlman, Ph.D. \\ Thomas O'Brien, Ph.D. \\ Andrei Smirnov, Ph.D. \\ Ray Yang, Ph.D. \\ Department of Mechanical and Aerospace Engineering \\ Morgantown, West Virginia \\ 2005
}

Key words: Large eddy simulation (LES); Multiphase flow; Dispersed two-phase flow; Bubbly flow; Bubble column; Computational fluid dynamics (CFD); Navier-Stokes solver; Turbulence modeling; Lagrangian particle tracking; Two-way coupling

Copyright 2005 Gusheng Hu 


\section{ABSTRACT \\ Towards Large Eddy Simulation of Gas-Liquid Dispersed Two-Phase Turbulent Flows}

\section{Gusheng Hu}

This study presents a detailed investigation of all essential components of computational and modeling issues necessary for a successful large-eddy simulation (LES) of dispersed twophase turbulent flows. In particular, a two-layer concept is proposed to enable the LES capability in two-phase flows involving dispersed bubbles that are relatively large compared to the mesh size. The work comprises three major parts.

Part I focuses on the development and verification of a transient, three-dimensional, finite-volume-method (FVM) based accurate Navier-Stokes solver, named DREAM II (second generation of the DREAM code). Several high-order schemes are implemented for both the spatial and temporal discretization. Solution of the coupled partial differential equations is attacked with a fractional step (projection) method. The developed solver is verified against various benchmarks including Taylor's vortex, free-shear layer, backward-facing step flow and square cavity. A second-order overall accuracy is achieved in both space and time.

Part II concerns the modeling and LES of single-phase turbulent flows. A review of the LES theory and subgrid-scale (SGS) models is presented. Three SGS models, namely, Smagorinsky model, dynamic model and implicit model, are implemented and investigated. Then turbulent channel flow, plane mixing layer, and flow past a square cylinder are simulated, and comparisons of the first-, second-order statistics, and characteristic flow structures are made with direct numerical simulation (DNS) and/or benchmark experiments. The test results show superior quality of the present LES.

Part III delves into the theory, modeling and simulation of dispersed two-phase flow systems. A conceptual review of the characteristics and description of such system is made, considering both Eulerian-Eulerian (E-E) and Eulerian-Lagrangian (E-L) approaches, but with an emphasis on the latter. Various hydrodynamic forces acting on particles or bubbles are summarized and interpreted. Formulations regarding interphase coupling is discussed in depth. Typical computational treatments of modeled two-way couplings in an E-L DNS/LES are reviewed. Issues related to the interpolation are addressed. A general Lagrangian particletracking (LPT) program, named PART, is developed and verified using analytical solutions.

A critical issue in the E-L approach is that, the particle size is conventionally required to be much smaller than the characteristic flow length scale and the computational grid size. This presents a considerable restriction on the E-L's applicability to practical engineering flows such as bubble column reactors. A two-layer concept, aimed at decoupling the geometric feature of the particles from that of the grid, is proposed. In this approach, the carrier phase and the dispersed phase are viewed as two independent computational layers, and the reverse coupling takes place at those discrete particle locations through modeled momentum exchange forces with the help of a predefined influence sphere. The proposed realization of the backward coupling, given the name PSI-Ball (particle-source-in ball), can be regarded as a generalization of the PSI-Cell method, and it ensures a "fair" redistribution of the interphase coupling force to its neighboring Eulerian grid nodes. A significant advantage is also that, the Eulerian grid can be constructed to a desired fineness (e.g., in the wall layer) without concern for the particle size. Finally, this idea is proven to work well in an application of two-way coupled E-L LES to a locally aerated turbulent bubble column, with surprisingly good success. 
My wife:

Daughter:

Dad:

Mom:

and younger sister:
Mingying Lu

Deanna Hu

Xihua Hu

Jinlan Zhao

Guqing $\mathrm{Hu}$ 


\section{ACKNOWLEDGEMENT}

I would like to express my deepest sense of gratitude to my advisor, Dr. Ismail Celik for his great academic and financial support. The research freedom and the personal trust he offered to me have been very important elements in encouraging my independent thinking and creative work. I am especially grateful for his "famous" CFD and turbulence courses at WVU, which were the initial triggering of my strong interest in this fascinating research area.

I will be forever indebted to my parents, Xihua $\mathrm{Hu}$ and Jinlan Zhao. Their everlasting love has been the powerful source of my spiritual strength; their mind and encouragement have made a profound impact on my academic life; their imperceptible illustration has shaped my solid appreciation for the value of higher education.

This work would have never come to a completion without the enormous dedication and sacrifice my wife, Mingying Lu, has made for me. I genuinely appreciate her support, patience, and understanding during this long, long endeavor. On August 13th, 2004 our little Deanna announced her arrival with her big cries.

I would like to sincerely thank my committee members, Drs. John Kuhlman, Thomas O'Brien, Andrei Smirnov and Ray Yang, for their valuable time reading this awfully dense manuscript. Their careful corrections and critical comments are highly appreciated.

A wide variety of other individuals made my academic life at WVU fulfilling. To Dr. Gary Morris, who welcomed me to Morgantown with a generous TA offer; to Dr. Jacky Prucz, who facilitated my ambition of pursuing another advanced degree in applied

mathematics; to Mr. Don McLaughlin, who diligently maintains the university Beowulf Cluster on which many of my computations were performed; and to all of my CFD labmates who extended their generous hands whenever needed. 


\section{TABLE OF CONTENTS}

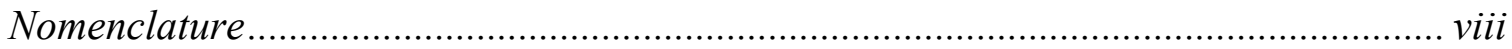

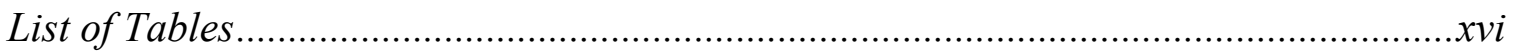

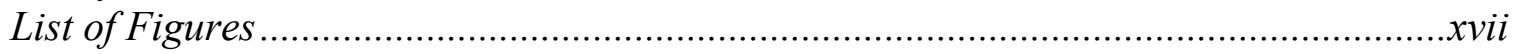

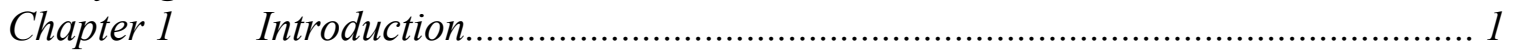

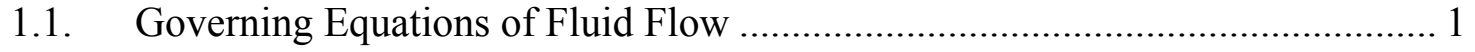

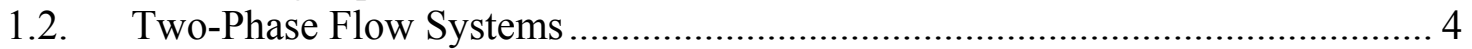

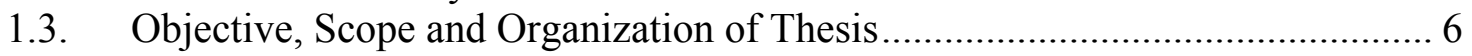

Part I Numerical Solution of Navier-Stokes Equations ............................................. 8

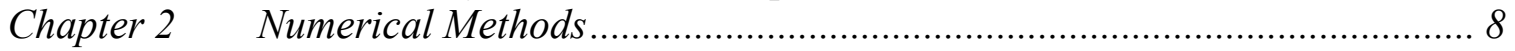

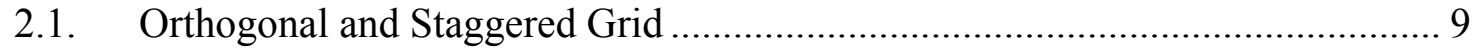

2.2. Discretization of a General Scalar Transport Equation ................................ 11

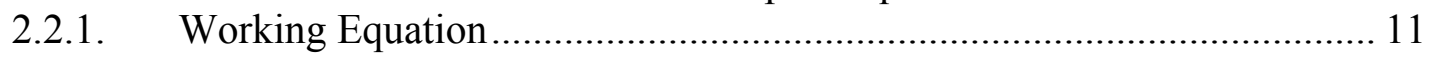

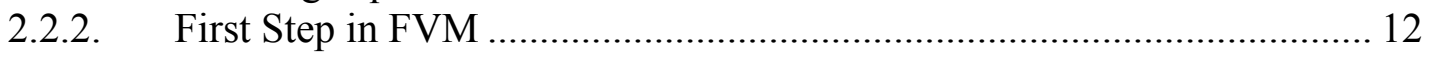

2.2.3. Approximation under Assumptions ................................................ 13

2.2.4. Patankar's Formulae ....................................................................... 14

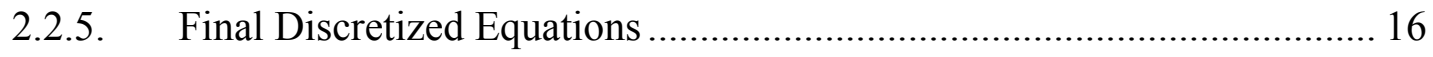

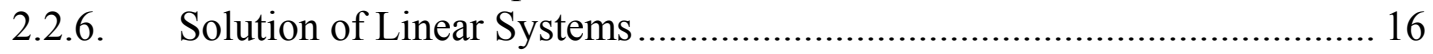

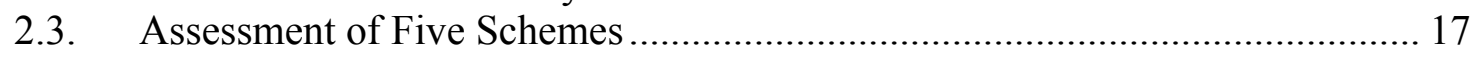

2.4. Implicitness Factors ................................................................................ 19

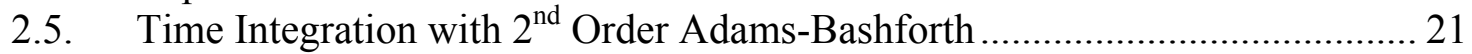

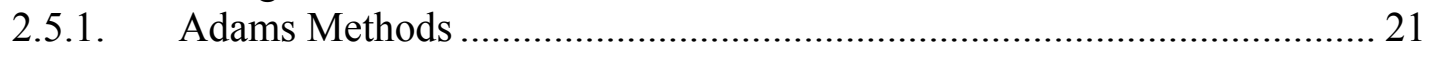

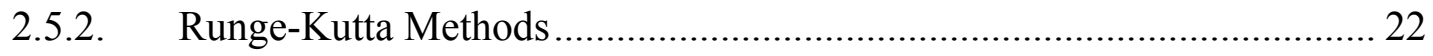

2.5.3. Adams Methods vs. Runge-Kutta Methods ............................................ 24

2.6. Higher Order Schemes with $3^{\text {rd }}$ order QUICK and $4^{\text {th }}$ order CD..................... 25

2.7. Fractional Step (Projection) Method........................................................... 27

2.8. Discretization of Pressure Equation and $4^{\text {th }}$ Order Deferred Correction .......... 30

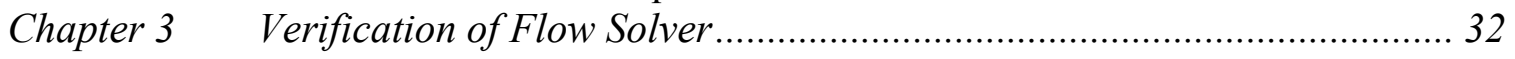

3.1. Couette Flow Between a Fixed and a Moving Plate..................................... 32

3.2. Couette-Poiseuille Flow Between a Fixed and a Moving Plate...................... 34

3.3. Poiseuille Flow Between Fixed Parallel Plates................................................. 36

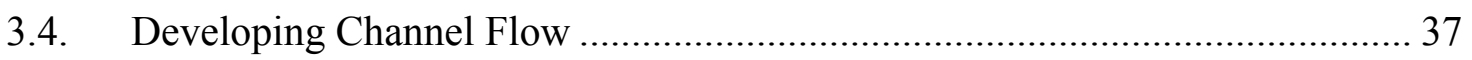

3.5. Poiseuille Flow Between Plates with Bottom Injection and Top Suction ........ 52

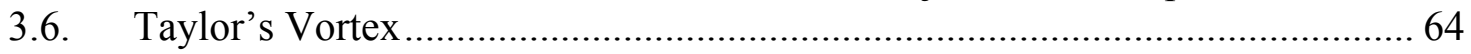

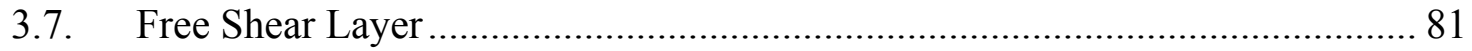

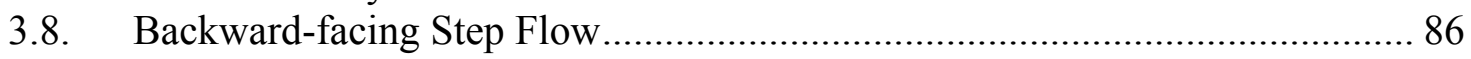

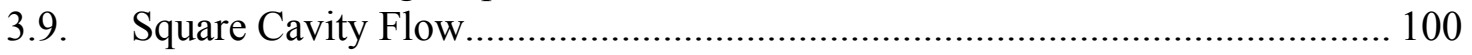

Part II Modeling and Simulation of Turbulent Flows ............................................ 113

Chapter $4 \quad$ Modeling Strategies for Turbulent Flows ......................................... 113

4.1. Scales in Turbulent Flows and Direct Numerical Simulation ...................... 113

4.2. Reynolds Averaged Navier-Stokes and Its Closure Models ........................ 115

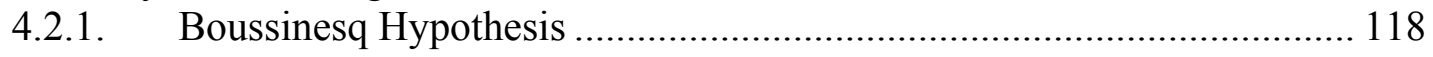

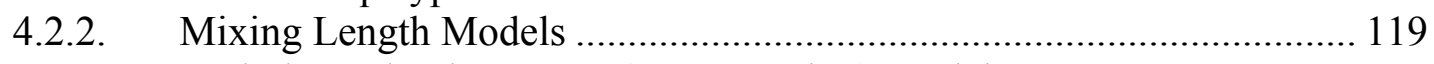

4.2.3. Turbulent Kinetic Energy (One-equation) Model................................. 119 
4.2.4. k- $\quad$ (Two-equation) Model .................................................................. 120

4.2.5. Second Moment Models ...................................................................... 121

4.3. Large Eddy Simulation ………………….......................................... 122

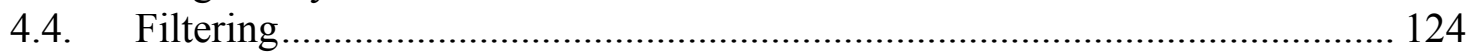

4.5. Filtered Navier-Stokes Equations ............................................................... 130

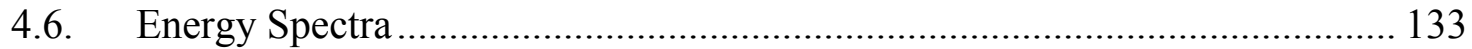

4.7. Grid Resolution Requirement in LES ..................................................... 137

4.8. Subgrid Scale Modeling........................................................................... 142

4.8.1. Kinetic Energy Equations ……………………................................. 143

4.8.2. Smagorinsky Model ............................................................................ 144

4.8.3. Dynamic Smagorinsky Model ............................................................. 145

4.8.4. One-equation SGS Model ............................................................... 148

4.8.5. Implicit Turbulence Modeling ............................................................ 150

Chapter 5 Large Eddy Simulation of Building-Block Turbulent Flows.................. 155

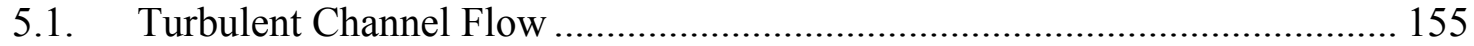

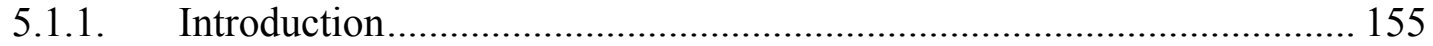

5.1.2. Computational Details ..................................................................... 156

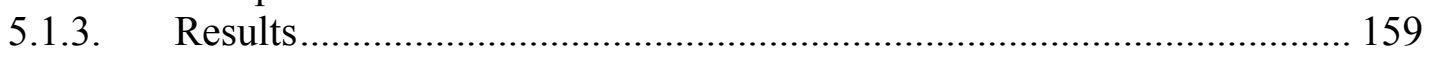

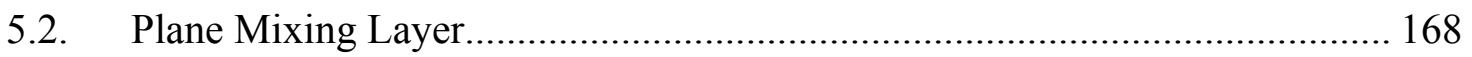

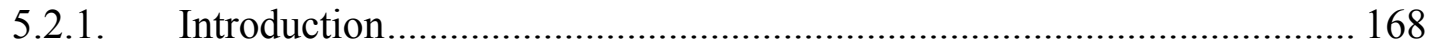

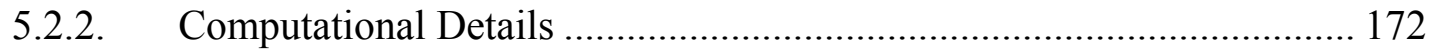

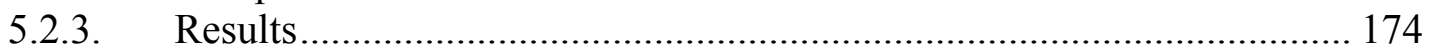

5.3. Flow Past a Square Cylinder.................................................................... 190

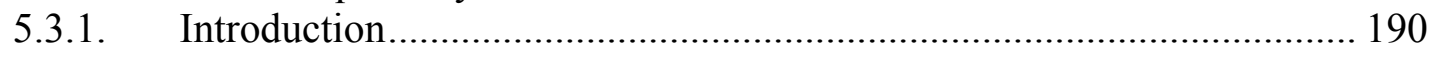

5.3.2. Computational Details .................................................................... 190

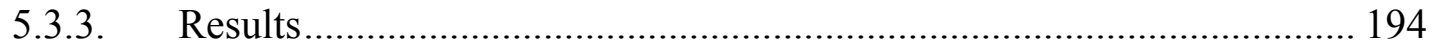

Part III Modeling and Simulation of Gas-Liquid Dispersed Two-Phase Flows ........... 209 Chapter $6 \quad$ Verification of Lagrangian Tracking with a Single Rigid Particle in Imposed Flow Fields..................................................................................... 209

6.1. Steady-state Drag and Momentum Relaxation Time.................................... 209

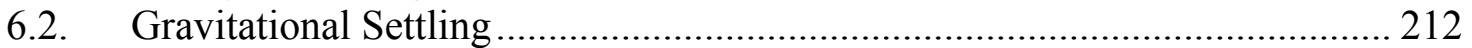

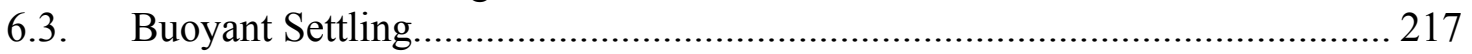

6.4. Particle Injected across a Uniform Flow......................................................... 218

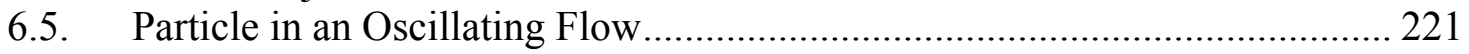

6.6. Particle in a Rotating Flow …………................................................... 225

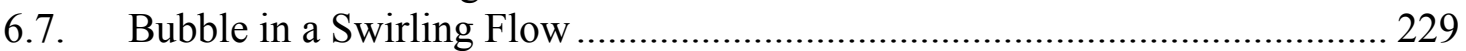

Chapter 7 Formulations of Turbulent Gas-Liquid Dispersed Two-Phase Flows and

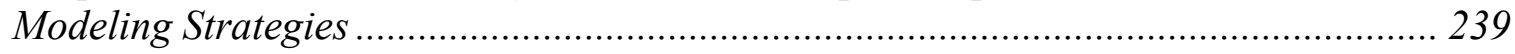

7.1. Parameters and Criteria for Dispersed Two-phase Flow ………………......... 239

7.1.1. Five Parameters........................................................................... 240

7.1.2. Number Density and Volume Fraction ................................................... 242

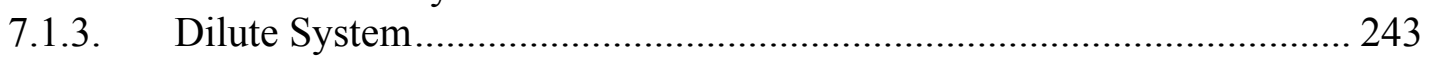

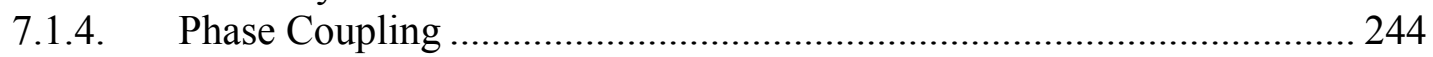

7.1.5. Turbulent Dispersion and Diffusion .................................................... 245

7.1.6. Turbulence Modulation........................................................................ 247

7.2. General Formulations of Two-phase Flow System ……………………...... 248 
7.3. Volume-averaged Conservation Equations.................................................... 250

7.4. Eulerian and Lagrangian Description of Dispersed Phase.............................. 254

7.4.1. Mixed-fluid Eulerian Approach........................................................ 255

7.4.2. Two-fluid Eulerian Approach ............................................................. 256

7.4.3. Resolved-volume Lagrangian Approach …………………………....... 258

7.4.4. Point-volume Lagrangian Approach..................................................... 259

7.5. Hydrodynamic Forces for a Single Particle ................................................. 263

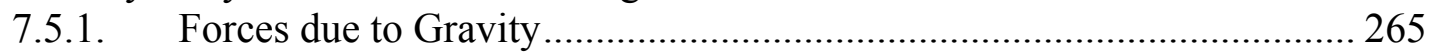

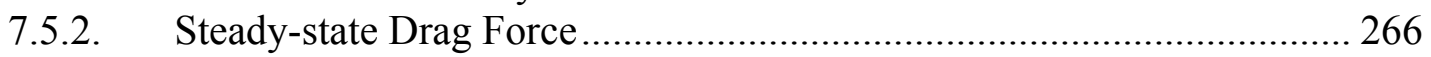

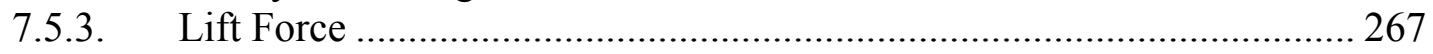

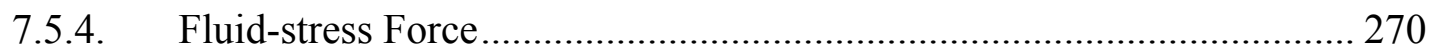

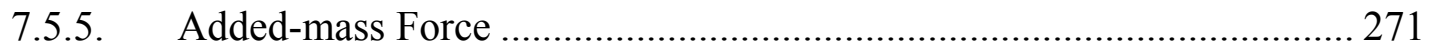

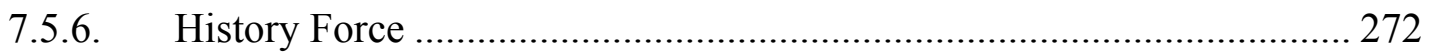

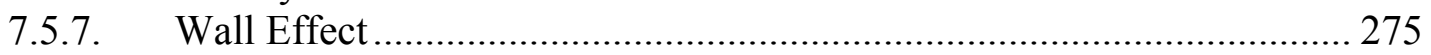

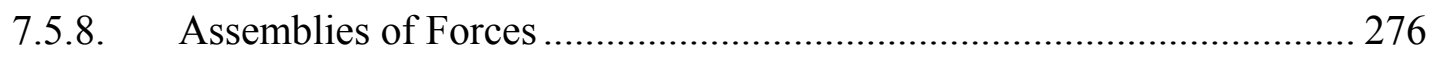

7.5.9. Bubble Terminal Rise Velocity and Relaxation Time............................ 279

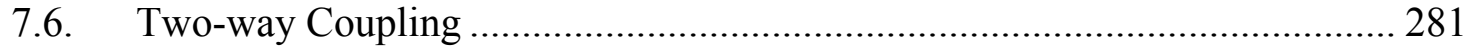

7.6.1. A Simplified Two-way Model from Two-fluid Approach ...................... 281

7.6.2. Forward Coupling in E-L Approach ....................................................... 284

7.6.3. Backward Coupling in E-L Approach: Force Coupling .......................... 286

7.6.4. Backward Coupling in E-L Approach: Velocity Coupling...................... 292

7.6.5. Backward Coupling in E-L Approach: PHYSALIS ................................ 294

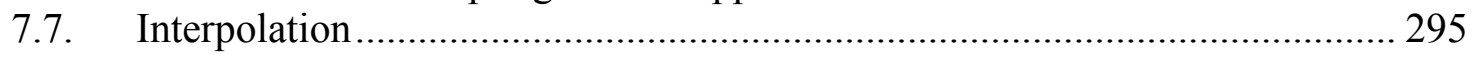

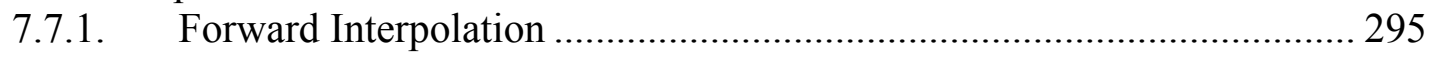

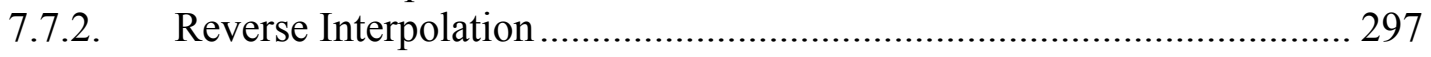

7.8. Formulation of Two-way Coupled LES ………............................................ 299

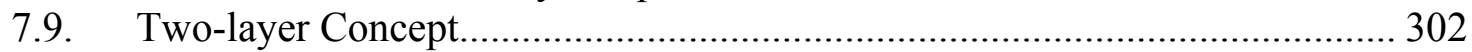

7.9.1. Particle Size vs. Grid Size....................................................................... 302

7.9.2. Point Volume or PHYSALIS? ..................................................................... 304

7.9.3. An Assumption: Point Volume and Relatively Dilute............................. 304

7.9.4. An Assertion: Geometric Decoupling..................................................... 305

7.9.5. An Approach: PSI-Ball (Particle-Source-in Ball).................................... 305

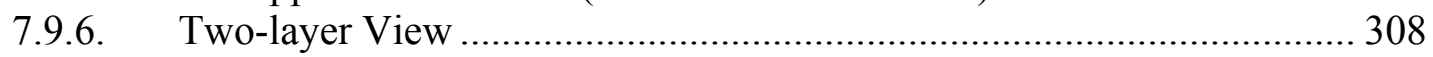

Chapter $8 \quad$ Large Eddy Simulation of Bubble Column .......................................... 309

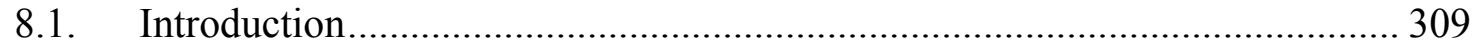

8.2. Experimental Setup and Flow Feature ....................................................... 312

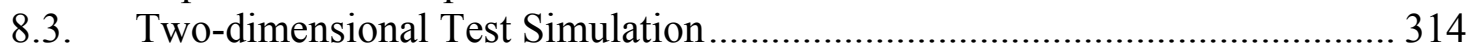

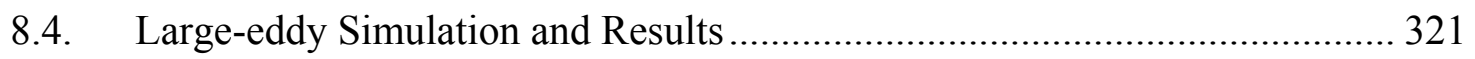

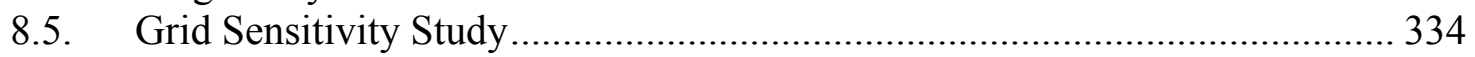

Chapter $9 \quad$ Conclusions and Future Work ………............................................ 340

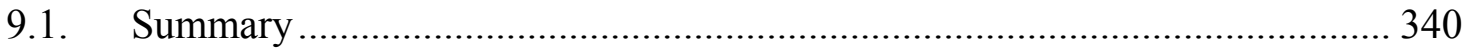

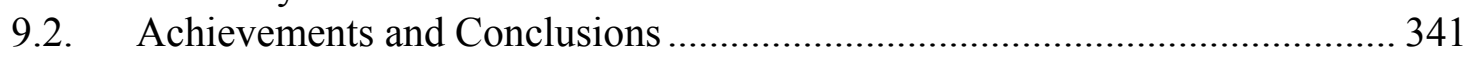

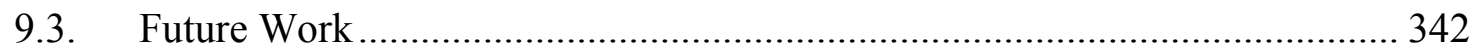

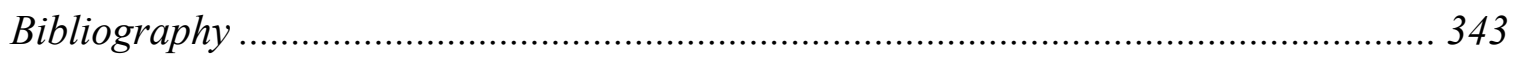

Appendix A About the Navier-Stokes Solver "DREAM II"..................................... 368

Appendix B About the Lagrangian Particle Tracking Program "PART"................. 370 


\section{Nomenclature}

\section{English Symbols}

$a$

A

$A_{f r}$

A

b, $b_{i}$

$B$

$c_{n}$

$\bar{c}_{n}$

$c_{n 1, n 2, n 3}$

C

$C_{A}$

$C_{D}$

$C_{L}$

$C_{1}, C_{2}, C_{\mu}, C_{\varepsilon 1}, C_{\varepsilon 2}$

$C_{v}, C_{\varepsilon}$

$C_{i j}$

$d_{p}$

D

$D_{h}$

$D_{p t}$

$D_{t}$

$\bar{D} / \bar{D} t$

$e$

erf

e

E

f

$f_{0}$

$f_{\text {rhs }}$

$f_{\text {conv }}$

$f_{\text {diff }}$

fothers

$f_{p p}$

F

F

$\mathbf{F} p$

$\mathbf{F}_{A}, \mathbf{F}_{B}, \mathbf{F}_{D}, \mathbf{F}_{G}, \mathbf{F}_{L}, \mathbf{F}_{M}$,
Coefficient in a discretized equation; particle radius; speed

of sound

Area; Generic constant expression

Frontal area

Function for calculating coefficients

Body force

Generic constant expression

Fourier coefficients for 1D variable

Fourier coefficients for 1D filtered variable

Fourier coefficients for 3D variable

Generic constant expression; Integration constant

Added mass coefficient

Drag coefficient

Lift coefficient

Model constants in RANS models

Model constants in one-equation SGS model

Cross stress tensor

Particle diameter

Diffusion conductance; total derivative operator; ball

diameter in PSI-Ball approach

Hydraulic diameter

Particle turbulent diffusion coefficient

Scalar (mass) turbulent diffusion coefficient

Substantial derivative based on mean or filtered velocity

Total energy per unit mass

Error function

Unit vector

Energy spectrum

A generic function or variable; frequency; drag factor

(Stokes correction)

Fundamental frequency in a mixing layer

Symbol including all spatial discretization terms

Symbol including all discretized convection terms

Symbol including all discretized diffusion terms

Symbol including the rest discretized terms other than $f_{\text {conv }}$ and $f_{\text {diff }}$

Average particle-particle collision frequency

Mass flow rate

Force

Total forces acting on a point-mass particle

Forces acting on a bubble (added mass, Basset (history), 


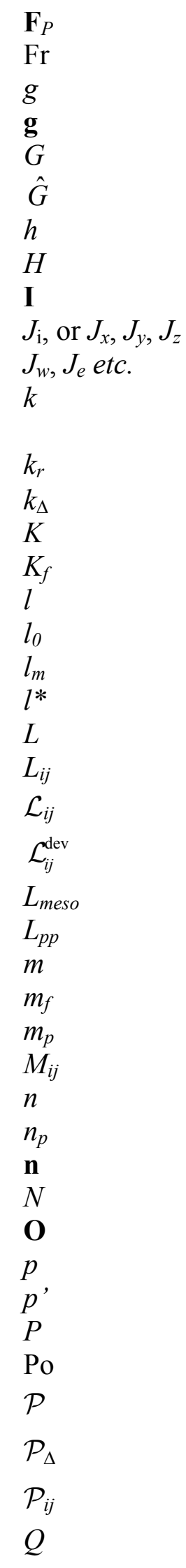

drag, buoyancy, lift, Magnus, pressure gradient)

Froude number

A generic function or variable; distribution function

Gravitational acceleration

Filter function or filter kernel

Fourier transfer function of $G$

Half channel height; grid size; step height

Channel height

Unitary tensor (Identity matrix)

Total (convection and diffusion) fluxes

Integrated total fluxes

Conductivity; slope; proportionality constant; turbulent kinetic energy;

Resolved turbulent kinetic energy

Residual (SGS) kinetic energy

Kinetic energy of the mean flow

Kinetic energy of the filtered flow field

Characteristic length scale

Length scale of largest eddies

Mixing length

Turbulent length scale in eddy-viscosity models

Characteristic length scale of the flow

Leonard stress tensor

Germano identity (stress tensor)

Deviatoric part of $\mathcal{L}_{i j}$

Mesoscale

Average inter-particle spacing

Mass

Mass of fluid particle

Particle mass

Scaled composite strain rate tensor

Order of a method

Number density of dispersed phase

Unit vector

Number of grid nodes

Oseen tensor

Pressure or pressure-like variable (pseudo-pressure)

Fluctuating pressure or pseudo-pressure

Channel or duct perimeter; continuous function in an ODE

Dimensionless pressure gradient

Production of turbulent kinetic energy

Production of residual kinetic energy

Production of turbulent kinetic energy tensor

Volume flow rate; heat; continuous function in an ODE 


\begin{tabular}{|c|c|}
\hline$r$ & Spreading rate of a mixing layer \\
\hline$(r, \theta, z)$ & Cylindrical (polar) coordinates \\
\hline$R$ & Radius; two-point correlation function (auto-covariance) \\
\hline $\bar{R}$ & $\begin{array}{l}\text { two-point correlation function (auto-covariance) of a } \\
\text { filtered quantity }\end{array}$ \\
\hline $\mathbf{R}^{3}$ & Three-dimensional space \\
\hline$R_{i j}$ & Two-point correlation tensor; SGS Reynolds stress tensor \\
\hline$\hat{R}_{i j}$ & Spectrum tensor (Fourier transform of $R_{i j}$ ) \\
\hline $\mathcal{R}_{i j}$ & Pressure rate of strain tensor \\
\hline $\operatorname{Re}$ & Reynolds number \\
\hline $\operatorname{Re}_{L}, \operatorname{Re}_{\delta}$ & Reynolds numbers based on corresponding length scales \\
\hline $\mathrm{Re}_{p}$ & Particle Reynolds number \\
\hline $\operatorname{Re}_{w}$ & Wall Reynolds number \\
\hline$s_{i j}$ & Fluctuating strain rate tensor \\
\hline$S_{*}$ & Coefficient polynomial of a interpolation scheme \\
\hline$S^{*}$ & Coefficient polynomial of an inverse interpolation scheme \\
\hline $\mathbf{S}$ or $S_{i j}$ & Strain rate tensor \\
\hline St & Stokes number \\
\hline $\bar{S}_{i j}$ & Mean strain rate tensor; filtered strain rate tensor \\
\hline $\bar{S}$ & $\begin{array}{l}\text { Characteristic mean strain rate; characteristic filtered } \\
\text { strain rate }\end{array}$ \\
\hline$\tilde{\bar{S}}_{i j}$ & Double-filtered strain rate tensor \\
\hline$\tilde{\bar{S}}$ & Characteristic double-filtered strain rate \\
\hline$S_{\phi}$ & Source term in a generic transport equation \\
\hline$t$ & Time \\
\hline$T$ & Time interval \\
\hline $\mathbf{T}$ & Total stress tensor \\
\hline $\mathbf{T}_{c}$ & Total stress tensor of continuous phase \\
\hline $\mathbf{T}_{d}$ & Total stress tensor of dispersed phase \\
\hline$T_{i j}^{R}$ & Residual stress from double filtering operation \\
\hline$T_{i j}^{r}$ & Deviatoric part of $T_{i j}^{R}$ \\
\hline $\mathcal{T}, \mathcal{T}_{i}$ & Fluxes responsible for diffusive transport \\
\hline $\mathcal{T}_{i j}$ & Stress tensor responsible for diffusive transport \\
\hline $\mathcal{T}_{k i j}$ & Reynolds stress flux \\
\hline $\mathbf{u}, u_{i}$, or $u, v, w$ & Flow velocity field \\
\hline$u(x)$ & 1D flow field \\
\hline $\bar{u}(x)$ & 1D filtered flow field \\
\hline$\hat{u}_{i}$ & Intermediate velocity field in the projection method \\
\hline$\overline{\mathbf{u}}, \bar{u}_{i}$, or $\bar{u}, \bar{v}, \bar{w}$ & $\begin{array}{l}\text { Mean flow velocity in RANS; filtered flow velocity in } \\
\text { LES }\end{array}$ \\
\hline $\mathbf{u}^{\prime}, u_{i}^{\prime}$, or $u^{\prime}, v^{\prime}, w^{\prime}$ & Fluctuating flow velocity in RANS; subgrid scale flow \\
\hline
\end{tabular}




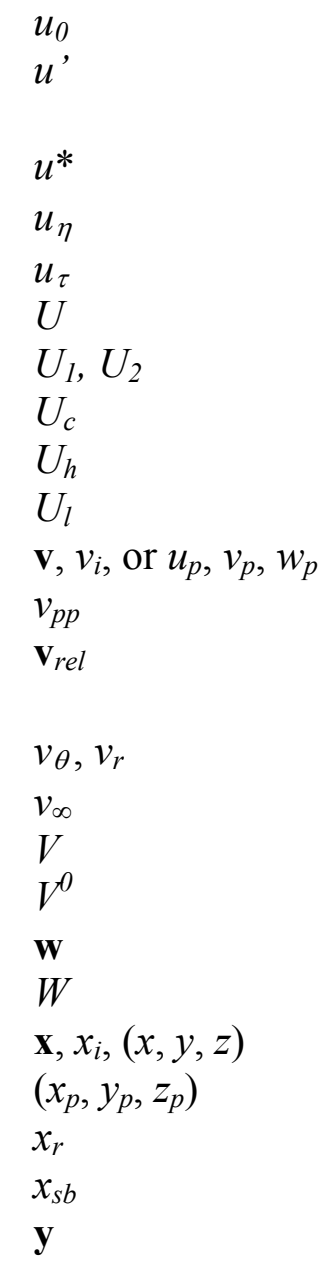

\section{Greek Symbols}

$\alpha$

$\alpha_{c}$
$\alpha_{d}$
$\alpha_{c o n v}$
$\alpha_{\text {diff }}$
$\alpha_{k}$
$\alpha_{p}$
$\beta$
$\gamma_{\rho}$
$\gamma_{D}$
$\gamma_{l}$
$\gamma_{v}$
$\delta$

velocity in LES

Velocity (fluctuation) scale of largest eddies

RMS velocity fluctuation; characteristic velocity

fluctuation

Turbulent velocity scale in eddy viscosity model

Kolmogorov velocity scale

Friction velocity

A reference velocity; free stream velocity

Reference velocities in a free shear layer

Mean convective velocity in a mixing layer

Velocity of high-speed stream in a mixing layer

Velocity of low-speed stream in a mixing layer

Dispersed phase velocity

Inter-particle relative velocity scale

Relative velocity between the continuous phase and the dispersed phase

Dispersed phase velocity in spherical coordinates

Particle terminal velocity scale

Volume

Limiting volume

Interface velocity; disturbance velocity

Width

Cartesian coordinates

Particle location

Reattachment length in backward-facing step flow

Secondary bubble length in backward-facing step flow

Particle location

Implicitness factor for terms in $f_{r h s}$; a generic coefficient; volume fraction

Volume fraction of continuous phase

Volume fraction of dispersed phase

Implicitness factor for terms in $f_{\text {conv }}$

Implicitness factor for terms in $f_{\text {diff }}$

Volume fraction of phase $k$

Volume fraction of dispersed phase

A generic coefficient

Density ratio between two phases

Particle diffusion ratio

Length scale ratio between two phases

Drift parameter (ratio of particle's terminal velocity to the

RMS velocity of the fluid)

Discretized approximation to a continuum derivative

expression; velocity boundary layer thickness; half

channel height; Dirac delta function 


\begin{tabular}{|c|c|}
\hline$\delta_{i j}$ & Kronecker delta \\
\hline$\delta_{L}$ & Level thickness in a mixing layer \\
\hline$\delta_{\theta}$ & Momentum thickness in a mixing layer \\
\hline$\delta_{\omega}$ & Vorticity thickness in a mixing layer \\
\hline$\Delta$ & Filter width; Characteristic filter width \\
\hline $\bar{\Delta}$ & Grid filter width \\
\hline$\tilde{\Delta}$ & Test filter width \\
\hline$\tilde{\bar{\Delta}}$ & Effective double-filter width \\
\hline$\Delta t$ & Time step size \\
\hline$\Delta x, \Delta y, \Delta z$ & Sizes a of computational cell \\
\hline$\Delta U$ & Free stream difference in a mixing layer \\
\hline$\Delta V$ & Volume of a computational cell \\
\hline$\Delta \rho$ & Density difference between two phases \\
\hline$\varepsilon$ & $\begin{array}{l}\text { Numerical error; viscous dissipation rate of turbulent } \\
\text { kinetic energy }\end{array}$ \\
\hline$\varepsilon_{f}$ & Dissipation rate of filtered flow field \\
\hline$\varepsilon_{m}$ & Dissipation rate of mean flow \\
\hline$\varepsilon_{\text {num }}$ & Numerical dissipation rate \\
\hline$\varepsilon_{i j}$ & Dissipation tensor \\
\hline$\kappa$ & Wave number; curvature \\
\hline$\kappa_{c}$ & Cut-off wave number \\
\hline$\kappa_{r}$ & nth wave number \\
\hline$\kappa_{r}$ & Resolved wave number \\
\hline$\kappa$ & Wave number vector \\
\hline$\theta$ & Angle \\
\hline$\eta$ & Similarity variable; Kolmogorov length scale \\
\hline$\phi$ & A generic intensive property \\
\hline $\bar{\phi}$ & A mean quantity in RANS; filtered quantity in LES \\
\hline$\tilde{\phi}$ & A filtered quantity using a test filter \\
\hline$\phi^{\prime}$ & $\begin{array}{l}\text { Fluctuating quantity in RANS; subgrid scale quantity in } \\
\text { LES }\end{array}$ \\
\hline$\hat{\phi}$ & Fourier transfer function of $\phi$ \\
\hline$\varphi$ & Angle; disturbed pressure field \\
\hline$\varphi_{0}$ & Phase angle \\
\hline$\Phi$ & A generic extensive property; velocity spectrum \\
\hline$\Gamma$ & Diffusion coefficient \\
\hline$\lambda$ & $\begin{array}{l}\text { Second viscosity coefficient; most unstable wavelength } \\
\text { (or billow-to-billow distance) }\end{array}$ \\
\hline$\lambda_{0}$ & Fundamental wavelength in a mixing layer \\
\hline$\Lambda$ & Turbulent integral length scale \\
\hline$\mu$ & Dynamic viscosity \\
\hline$\mu_{c}$ & Dynamic viscosity of continuous phase \\
\hline$\mu_{d}$ & Dynamic viscosity of dispersed phase \\
\hline
\end{tabular}




\begin{tabular}{ll}
$\mu_{t}$ & Turbulent (eddy) dynamic viscosity \\
$v$ & Kinematic viscosity \\
$v_{t}$ & Turbulent (eddy) kinematic viscosity \\
$\Pi$ & Momentum coupling parameter \\
$\rho$ & Density \\
$\rho_{c}$ & Density of continuous phase \\
$\rho_{d}$ & Density of dispersed phase \\
$\rho^{\prime}$ & Density fluctuations; SGS density fluctuations \\
$\sigma$ & Surface tension coefficient \\
$\sigma$ or $\sigma_{i j}$ & Total stress tensor \\
$\sigma_{k}$ & Turbulent Prandtl-Schmidt number for kinetic energy \\
$\sigma_{p}$ & Turbulent Prandtl-Schmidt number for particle diffusion \\
$\sigma_{\varepsilon}$ & Turbulent Prandtl number for dissipation \\
$\tau$ & Characteristic time scale; shear stress \\
$\tau$ or $\tau_{i j}$ & Viscous stress tensor \\
$\tau_{i j}^{R}$ & Residual stress tensor \\
$\tau_{i j}^{r}$ & Deviatoric (anisotropic) residual stress \\
$\tau_{p}$ & Particle momentum relaxation time \\
$\tau_{p e}$ & Particle-eddy interaction time scale \\
$\tau_{p p}$ & Average particle-particle collision time \\
$\tau_{t r a v}$ & Particle traversal time scale \\
$\tau_{0}$ & Time scale of largest eddies \\
$\tau_{\eta}$ & Kolmogorov time scale \\
$\tau_{\Lambda}$ & Turbulent integral time scale \\
$\omega$ & Angular velocity \\
$\omega$ & Vorticity \\
$\Omega$ & Volume \\
$\Omega_{i j}$ & Rate of rotation tensor \\
$\bar{\Omega}$ & Mean rotation rate tensor \\
$\bar{\Omega}$ & Characteristic mean rotation rate \\
$\chi$ & Phase indication function \\
$\psi$ & Stream function \\
$\Psi$ & Dissipation function \\
& \\
\hline &
\end{tabular}

\section{Other Symbols}

$\begin{array}{ll}\mathcal{F} & \text { Fourier transform operator } \\ \mathcal{F}^{-1} & \text { Inverse Fourier transform operator } \\ \mathcal{I} & \text { Interphase coupling term } \\ \mathcal{S} & \text { Surface; sphere } \\ \nabla & \text { Gradient operator } \\ \nabla^{2} & \text { Laplacian operator } \\ \Delta & \text { Laplacian operator }\end{array}$


\langle\rangle

\langle\rangle$^{i}$

$\equiv$

\section{Subscripts}

ave

$b$

$c$

$d$

D

$i, j, k$

$l$

$\max$

$n$

$p$

ref

$t$

$w, e, s, n, b, t$

$W, E, S, N, B, T, P$

$W W, E E$ etc.

0

$\infty$

\section{Superscripts}

\section{H}

$L$

$n$

o

$T$

$v$

\section{Abbreviations}

$\mathrm{AB}$

AM

ASM

BBO

CD

CFL

$\mathrm{CM}$

C-N

$\mathrm{CV}$

CS

DFT

DNS

E-E
Convolution

Mean (expectation); volume averaging

Intrinsic volume averaging

Defined as

Average quantity

Bubble

Primary continuous phase in a two-phase flow system

Secondary dispersed phase in a two-phase flow system

Drag

Indices for Cartesian coordinates or vector quantities

Dummy indices; liquid

Maximum quantity

$n$th particle

Particle

Reference value

Partial derivative with respect to time

West, east, south, north, bottom, top faces

West, east, south, north, bottom, top and center nodes

West of west, east of east etc.

Initial condition

Infinity; terminal velocity

Higher order approximation

Lower order approximation

Time level index; particle number index

Old time level

Transpose

Grid point (vertex) index

Adams-Bashforth

Adams-Moulton

Algebraic stress model

Basset-Boussinesq-Oseen

Central differencing

Courant-Friedrichs-Lewy

Control mass

Crank-Nicolson

Control volume

Control surface

Discrete Fourier transform

Direct numerical simulation

Eulerian-Eulerian 


\begin{tabular}{|c|c|}
\hline E-L & Eulerian-Lagrangian \\
\hline FCT & Flux-corrected transport \\
\hline FFT & Fast Fourier transform \\
\hline FLOP & Floating-point operation \\
\hline FVM & Finite volume method \\
\hline ITM & Implicit turbulence modeling \\
\hline LDV & Laser-Doppler velocimetry \\
\hline LES & Large-eddy simulation \\
\hline LHS & Left hand side \\
\hline LPT & Lagrangian particle tracking \\
\hline MILES & Monotone integrated LES \\
\hline $\mathrm{N}-\mathrm{S}$ & Navier-Stokes \\
\hline ODE & Ordinary differential equation \\
\hline $\mathrm{PDE}$ & Partial differential equation \\
\hline PHYSALIS & A numerical method for DNS with many solid spheres \\
\hline $\mathrm{PIC}$ & Particle in cell \\
\hline PPM & Piecewise parabolic method \\
\hline PSIB, PSI-Ball & Particle-source-in ball \\
\hline PSIC, PSI-Cell & Particle-source-in cell \\
\hline QSOU & Quasi second order upwind \\
\hline QUICK & Quadratic upwind interpolation for convective kinematics \\
\hline RANS & Reynolds-averaged Navier-Stokes \\
\hline RFG & Random flow generation \\
\hline RHS & Right hand side \\
\hline RK & Runge-Kutta \\
\hline RK2 & $2^{\text {nd }}$ order RK \\
\hline RK4 & $4^{\text {th }}$ order RK \\
\hline RMS, rms & Root mean square \\
\hline rpm & Rotations per minute \\
\hline RSM & Reynolds stress model \\
\hline SGS & Subgrid scale \\
\hline SIMPLE & Semi-implicit method for pressure-linked equations \\
\hline TKE & Turbulent kinetic energy \\
\hline TVD & Total variation diminishing \\
\hline
\end{tabular}




\section{List of Tables}

Table 1-1 Classification of two-phase systems and flow regimes................................... 5

Table 2-1 The "A" function A(|Pe|) for different schemes (Patankar 1980) ................... 15

Table 3-1 Order of method analysis for the calculations of Couette-Poiseuille flow between a fixed and a moving plate; schemes considered: semi-implicit power-law,

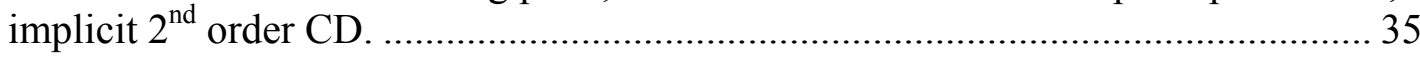

Table 3-2 Order of method analysis for the calculations of Couette-Poiseuille Flow with bottom injection and top suction; schemes considered: semi-implicit power-law, semi-implicit $2^{\text {nd }}$ order CD, explicit QUICK, explicit $4^{\text {th }}$ order CD...

Table 3-3 Order of method analysis based on the RMS error for the calculations of Couette-Poiseuille Flow with bottom injection and top suction, $4^{\text {th }}$ order CD is used

Table 3-4 Order of method analysis for the calculated u-velocity of a Taylor's vortex, $\Delta \mathrm{t}$ $=0.001 \mathrm{~s}$.

Table 3-5 Order of method analysis for the calculated v-velocity of a Taylor's vortex, $\Delta \mathrm{t}$ $=0.001 \mathrm{~s}$.

Table 3-6 Effective overall order of the QUICK scheme for the calculated u-velocity of a Taylor's vortex.

Table 3-7 Effective overall order of the QUICK scheme for the calculated v-velocity of a Taylor's vortex 68

Table 3-8 Calculated reattachment length and length of secondary bubble as a function of Reynolds numbers

Table 3-9 Center locations of the primary vortex of cavity flow at various Reynolds number

Table 4-1 Commonly used one-dimensional filter functions and their Fourier transfer functions

Table 5-1 Reynolds numbers of available DNS channel calculations............................ 156

Table 5-2 Spatial resolution of the channel flow simulation, $\operatorname{Re}_{\tau}=640 \ldots \ldots \ldots \ldots \ldots \ldots \ldots \ldots . . . . .157$

Table 5-3 Summary of grid information for flow past a square cylinder ....................... 192

Table 6-1 Commonly used drag correction factor $f$.

Table 6-2 Order of method analysis of RK2 for the calculation of gravitational settling.

Table 7-1 Drag correction factor $\mathrm{f}$ for air bubble in clean or contaminated water. ........ 267

Table 7-2 Drag coefficient for air bubble in tap water ................................................ 267

Table 7-3 Bubble terminal rise velocities, terminal Reynolds numbers and relaxation time

Table 8-1 Parameters used in the 2D simulation of a locally aerated bubble column.... 315

Table 8-2 Parameters used in 3D E-L large-eddy simulation of a locally aerated bubble column

Table 8-3 Grid information in coarser and finer-grid computation............................... 335

Table 8-4 Comparison of period and mean vertical velocity at point A and B............. 335 


\section{List of Figures}

Figure 1-1 Mathematical models with respect to levels of approximation........................ 1

Figure 2-1 A typical 2D computational cell used in finite volume discretization ............ 10

Figure 2-2 A typical 3D computational cell used in finite volume discretization............ 10

Figure 2-3 Quadratic interpolation used in QUICK scheme ...................................... 26

Figure 2-4 Flow chart of fractional step (projection) method for the solution of Navier-

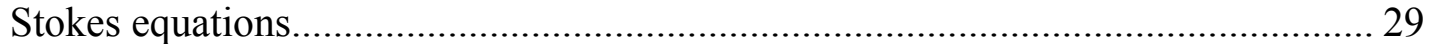

Figure 3-1 Couette flow between a fixed and a moving plate ..................................... 33

Figure 3-2 Contour plots of Couette flow between a fixed and a moving plate. (a) u-

velocity, (b) v-velocity, (c) pressure.................................................................. 40

Figure 3-3 u-velocity profiles (a) and its exact error (b) of Couette flow between a fixed

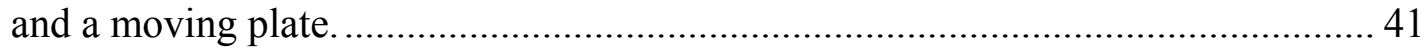

Figure 3-4 Contour plots of Couette-Poiseuille flow between a fixed and a moving plate,

$\mathrm{Po}_{\mathrm{o}}$ 1. (a) u-velocity, (b) v-velocity, (c) pressure-like variable.............................. 42

Figure 3-5 u-velocity profiles (a) and its exact error (b) of Couette-Poiseuille flow

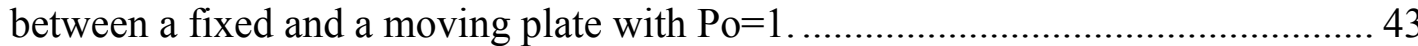

Figure 3-6 u-velocity profiles of Couette-Poiseuille flow between a fixed and a moving plate at different Po. Square symbols: present calculation; circle symbols: exact

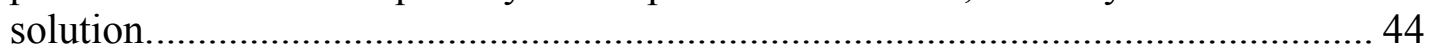

Figure 3-7 Flow separation with backflow of Couette-Poiseuille flow between a fixed and

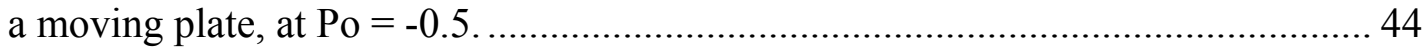

Figure 3-8 Maximum error as a function of mesh refinement for the Couette-Poiseuille flow between a fixed and a moving plate, (a) semi-implicit power-law, (b) implicit

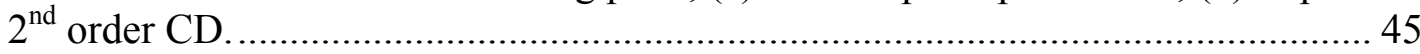

Figure 3-9 u-velocity profiles (a) and its exact error (b) of Poiseuille flow between fixed

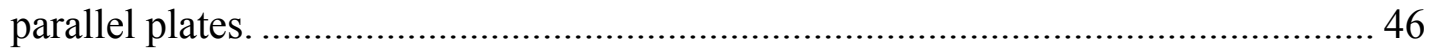

Figure 3-10 u-velocity contour of Poiseuille flow between fixed parallel plates............ 47

Figure 3-11 Exact error of the u-velocity of Poiseuille flow between fixed parallel plates,

$\mathrm{u}$-velocity calculated with $2^{\text {nd }}$ order central differencing. 47

Figure 3-12 u-velocity variation along the horizontal centerline of a developing channel

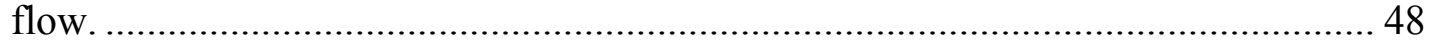

Figure 3-13 u-velocity profiles at different x-station of a developing channel flow....... 48

Figure 3-14 Entrance (developing) region of a developing channel flow, (a) u-velocity

contour, (b) v-velocity contour. ................................................................... 49

Figure 3-15 u-velocity profiles (a) and its exact error (b) in the fully developed region of

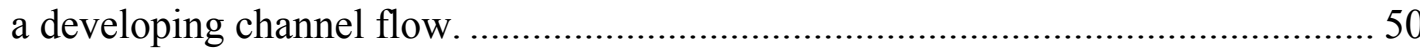

Figure 3-16 (a) Pressure contour of a developing channel flow, (b) pressure variation along horizontal centerline.......................................................................... 51

Figure 3-17 u-velocity profiles (a) and its exact error (b) of Poiseuille flow between plates with bottom injection and top suction; power-law scheme is used...............55

Figure 3-18 Comparison of u-velocity profiles calculated from different higher order schemes for Poiseuille flow between plates with bottom injection and top suction,

(e) $4^{\text {th }}$ order explicit CD. 
Figure 3-19 Comparison of exact error of u-velocities calculated with different higher order schemes for Poiseuille flow between plates with bottom injection and top suction, (e) $4^{\text {th }}$ order explicit CD.

Figure 3-20 Maximum error as a function of mesh refinement for the Couette-Poiseuille flow between plates with bottom injection and top suction, (b) semi-implicit $2^{\text {nd }}$ order CD, (c) explicit and QUICK, (d) explicit $4^{\text {th }}$ order CD.

Figure 3-21 RMS error as a function of mesh refinement for the Couette-Poiseuille flow between plates with bottom injection and top suction, $4^{\text {th }}$ order CD is used....

Figure 3-22 u-velocity profiles of Poiseuille flow between plates with bottom injection and top suction at different wall Reynolds number. Square symbols: present calculation; circle symbols: exact solution.

Figure 3-23 Streamline plot of Taylor's vortex at $t=50$ s for $\mathrm{Re}=100$; background contour plot is the magnitude of velocity.

Figure 3-24 Contour plots of Taylor's vortex at $t=50$ s for $\mathrm{Re}=100$, (b) v-velocity, (c) pressure.

Figure 3-25 u-velocity profile (a) and its exact error (b) of a Taylor's vortex at $t=50 \mathrm{~s}$ for $\mathrm{Re}=100, x=0.5 \mathrm{~m}$. 71

Figure 3-26 v-velocity profile (a) and its exact error (b) of a Taylor's vortex at $t=50 \mathrm{~s}$ for $\operatorname{Re}=100, y=0.5 \mathrm{~m}$ 72

Figure 3-27 u-velocity profile (a) and its exact error (b) of a Taylor's vortex at $t=50$ s for $\mathrm{Re}=100, x=0.5 \mathrm{~m}$; explicit QUICK scheme..... 73

Figure 3-28 v-velocity profile (a) and its exact error (b) of a Taylor's vortex at $t=50 \mathrm{~s}$ for $\mathrm{Re}=100, y=0.5 \mathrm{~m}$; explicit QUICK scheme.

Figure 3-29 Velocity profiles at different time of a Taylor's vortex with $\mathrm{Re}=100$. (a) uvelocity, (b) v-velocity. Square symbol: present calculation; circle symbol: exact solution.

Figure 3-30 u-velocity profile (a) and its exact error (b) of a Taylor's vortex at $t=50 \mathrm{~s}$ for $\mathrm{Re}=1000, x=0.5 \mathrm{~m}$; explicit QUICK scheme 76

Figure 3-31 v-velocity profile (a) and its exact error (b) of a Taylor's vortex at $t=50 \mathrm{~s}$ for $\operatorname{Re}=1000, y=0.5 \mathrm{~m}$; explicit QUICK scheme.

Figure 3-32 RMS error of u-velocity as a function of mesh refinement for Taylor's vortex flow, $\Delta \mathrm{t}=0.001 \mathrm{~s}$. (a) Implicit power-law, (b) QUICK. ..................................... 78

Figure 3-33 RMS error of v-velocity as a function of mesh refinement for Taylor's vortex flow, $\Delta \mathrm{t}=0.001 \mathrm{~s}$. (a) Implicit power-law, (b) QUICK. 79

Figure 3-34 Effective overall error of the QUICK scheme for Taylor's vortex problem.

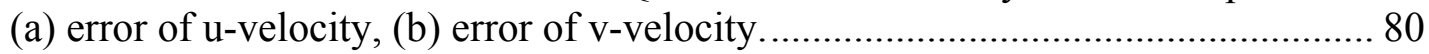

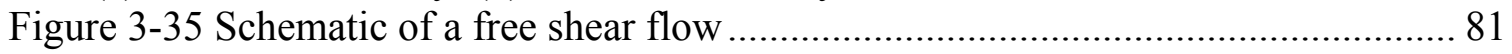

Figure 3-36 Contour plot of free shear layer, (a) u-velocity, (b) v-velocity................... 84

Figure 3-37 u-velocity profiles at three different $\mathrm{x}$-stations of a free shear layer, $\mathrm{U}_{2} / \mathrm{U}_{1}=$ 0.5 .

Figure 3-38 Comparison of normalized u-velocity profiles with similarity solution (Lock

1951) for a free shear layer, $U_{2} / U_{1}=0.5$. 85

Figure 3-39 Schematic of flow past a backward-facing step (1:2 expansion ratio)......... 86

Figure 3-40 Location of detachment and reattachment in a backward facing step flow.

(From (Armaly et al. 1983) with permission). 88 
Figure 3-41 u-velocity contours and streamlines of backward-facing step flow at various

Reynolds numbers $(\mathrm{Re} \leq 400)$.

Figure 3-42 v-velocity contours of backward-facing step flow at various Reynolds numbers $(\mathrm{Re} \leq 400)$. 94

Figure 3-43 u-velocity profiles at various $\mathrm{x}$-stations of a backward-facing step flow with different Reynolds number $(\operatorname{Re} \leq 400)$. Both $x$ - and y-locations are in step-height unit. (a) $\operatorname{Re}=100$, (b) $\operatorname{Re}=200$, (c) $\operatorname{Re}=300$, (d) $\operatorname{Re}=400$.

Figure 3-44 u-velocity contours and streamlines of backward-facing step flow at various

Reynolds numbers $(400<\operatorname{Re} \leq 1000)$.

Figure 3-45 v-velocity contours of backward-facing step flow at various Reynolds numbers $(400<\operatorname{Re} \leq 1000)$.

Figure 3-46 u-velocity profiles at various $\mathrm{x}$-stations of a backward-facing step flow with different Reynolds number $(400<\mathrm{Re} \leq 1000)$. Both $\mathrm{x}$ - and $\mathrm{y}$-locations are in stepheight unit. (a) $\operatorname{Re}=500$, (b) $\operatorname{Re}=600$, (c) $\operatorname{Re}=800$, (d) $\operatorname{Re}=1000$. 98

Figure 3-47 Comparison of reattachment length of a backward-facing step flow as a function of Reynolds number. Circle: experimental data from (Armaly et al. 1983); dash-dotted line: computation of (Kim and Moin 1985); square with solid line: present computation; dashed line: computation of (Armaly et al. 1983).

Figure 3-48 Schematic of a lid-driven cavity with possible vortices.

Figure 3-49 $\mathrm{u}$-velocity profile of a cavity flow at $\mathrm{x}=0.5 \mathrm{~L}$ for $\mathrm{Re}=400$. (a) Comparison of results using different scheme and grids, (b) comparison with the computation from (Ghia et al. 1982) and (Kim and Moin 1985).

Figure 3-50 u-velocity profiles of a cavity flow at $\mathrm{x}=0.5 \mathrm{~L}$ for various Reynolds numbers. (e) $\mathrm{Re}=5000$. Solid line: present results; circles: results from (Ghia et al. 1982) with $129 \times 129$ grid nodes. 106

Figure 3-51 v-velocity profiles of a cavity flow at $\mathrm{y}=0.5 \mathrm{~L}$ for various Reynolds numbers. (e) $\mathrm{Re}=5000$. Solid line: present results; circles: results from (Ghia et al. 1982) with $129 \times 129$ grid nodes.

Figure 3-52 Velocity vectors and streamlines of cavity flow at various Reynolds number.

(e) $\operatorname{Re}=3200,61 \times 61$, (f) $\mathrm{Re}=5000,61 \times 61$. 112

Figure 4-1 (a) One-dimensional filter functions, $G(\xi)$, (b) Filter transfer functions, $\hat{G}(\kappa)$

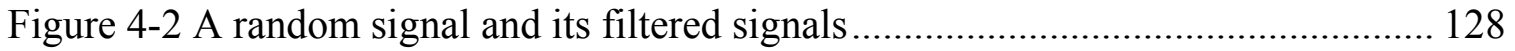

Figure 4-3 Attenuation factor of one-dimensional filters. ....................................... 135

Figure 4-4 Sample energy spectra. Solid line: three-dimensional spectrum; dashed line: one-dimensional spectrum; dotted line: filtered one-dimensional spectrum .......... 137

Figure 4-5 Energy spectrum of filtered velocity derivative..................................... 141

Figure 4-6 A summary of nonlinear stability conditions for scalar conservation laws .. 153

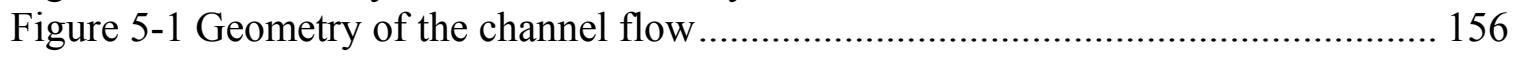

Figure 5-2 Mean streamwise velocity profile in a turbulent channel flow................... 162

Figure 5-3 Mean-velocity logarithmic profile in a turbulent channel flow. .................. 163

Figure 5-4 Resolved turbulence intensity in a turbulent channel flow......................... 163

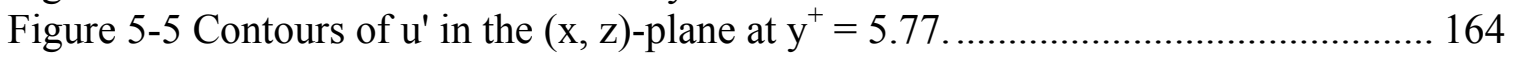

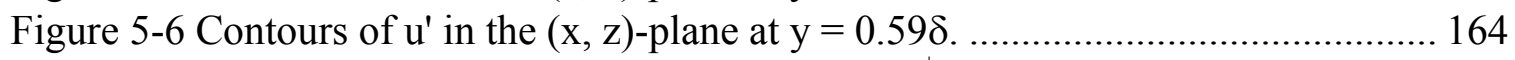

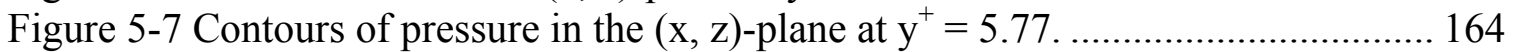

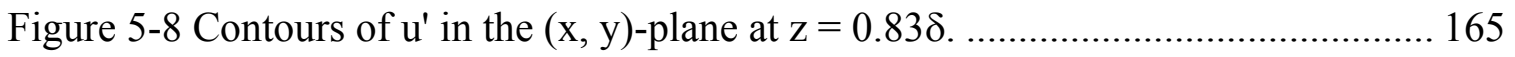




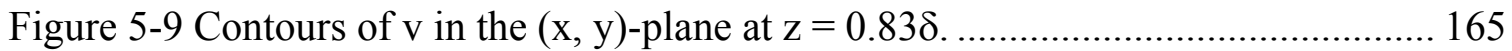

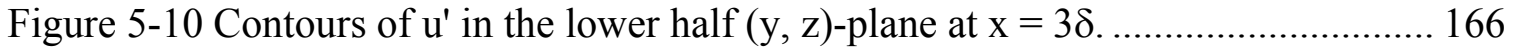

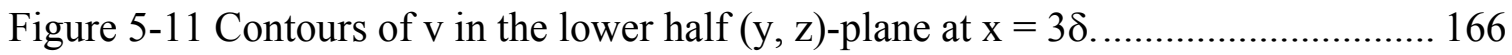

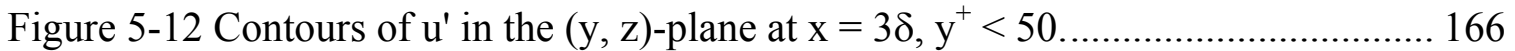

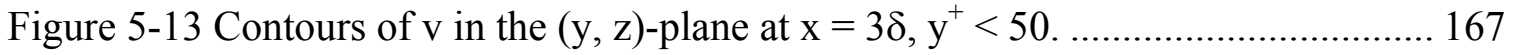

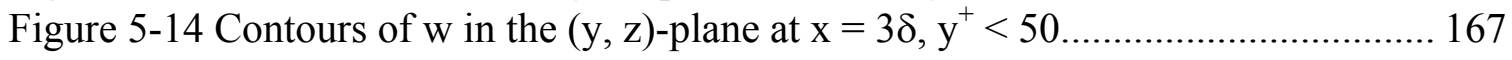

Figure 5-15 Contours of streamwise vorticity and streamlines in the $(\mathrm{y}, \mathrm{z})$-plane at $\mathrm{x}=3 \delta$.

Figure 5-16 Illustration of vortex "pairing" in a plane mixing layer............................ 168

Figure 5-17 Illustration of vortex "tearing" in a plane mixing layer. ........................... 168

Figure 5-18 Illustration of "rib-vortices" in a plane mixing layer (provided by (Lopez and

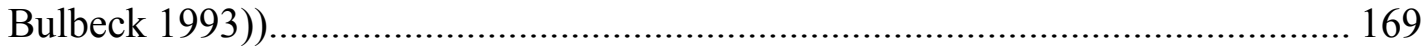

Figure 5-19 Schematic of a plane mixing layer ................................................... 173

Figure 5-20 Contours of mean streamwise velocity of a mixing layer: (a) entire domain,

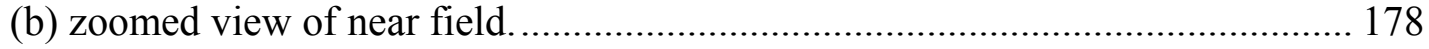

Figure 5-21 Mean flow visualization from Rightley \& Lasheras (2000). ..................... 178

Figure 5-22 Mean streamwise velocity profiles in a mixing layer. Dynamic model is used.

Figure 5-23 RMS streamwise velocity profiles in a mixing layer. Dynamic model is used.

Figure 5-24 RMS vertical velocity profiles in a mixing layer. Dynamic model is used. 180 Figure 5-25 Mean streamwise velocity profiles in a mixing layer. No SGS model is used.

Figure 5-26 RMS streamwise velocity profiles in a mixing layer. No SGS model is used.

Figure 5-27 RMS vertical velocity profiles in a mixing layer. No SGS model is used.. 181

Figure 5-28 Streamwise (a) mean and (b) RMS velocity profiles from Rightley \&

Lasheras (2000). Solid line, $x / \lambda=0.31$; dash, $x / \lambda=0.63$; dash-dot, $x / \lambda=1.25$; long-

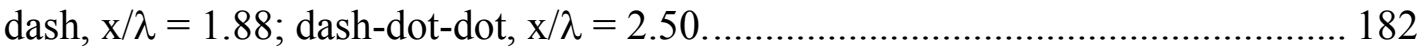

Figure 5-29 Comparison of mean streamwise velocity profiles at $x / \lambda=0.63 \ldots \ldots \ldots \ldots \ldots . .183$

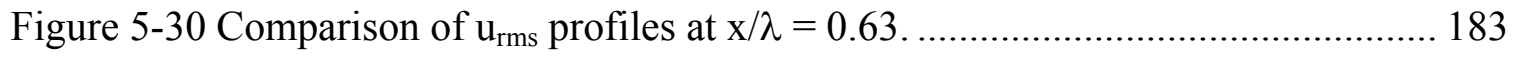

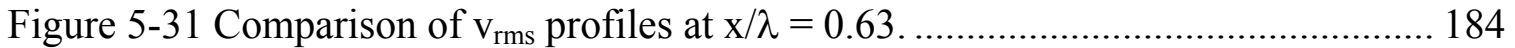

Figure 5-32 Comparison of mean streamwise velocity profiles at $x / \lambda=2.5 \ldots \ldots \ldots \ldots \ldots . . .184$

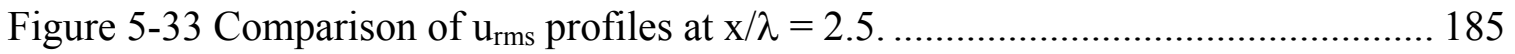

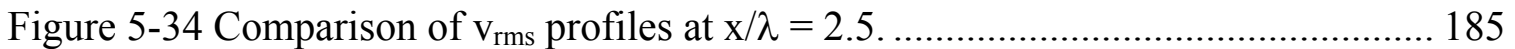

Figure 5-35 Comparison of $\mathrm{u}_{\mathrm{rms}}$ along centerline computed with dynamic model and

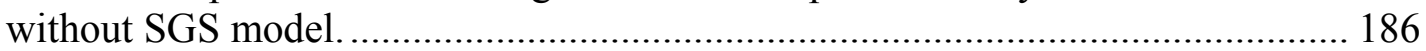

Figure 5-36 Comparison of level thickness between prediction and measurements..... 186

Figure 5-37 A snapshot of (a) spanwise vorticity contours, and (b) streamlines and velocity vectors, mean convective velocity is subtracted from the streamwise velocity component.

Figure 5-38 Phase-averaged velocity field at (a) $x / \lambda=1.25$ and (b) $x / \lambda=1.88$ from Rightley \& Lasheras (2000). Mean velocity is subtracted from the streamwise velocity component. 
Figure 5-39 Instantaneous velocity vector field (a) between $\mathrm{x} / \lambda=1.25$ and $\mathrm{x} / \lambda=3.88$, (b) between $\mathrm{x} / \lambda=1.88$ and $\mathrm{x} / \lambda=3.88$. Instant is taken at zero phase angle of the forcing function. Mean convective velocity is subtracted from the streamwise velocity component.

Figure 5-40 Schematic of flow past a square cylinder.

Figure 5-41 Grid distribution on the x-y plane for flow past a square cylinder ............. 191

Figure 5-42 Mean streamwise velocity along centerline of square cylinder.

Figure 5-43 Results from Rottach-Egern workshop: mean streamwise velocity along centerline of square cylinder. (Reprinted from (Rodi 1997) with permission)....... 197

Figure 5-44 Total $\mathrm{u}_{\mathrm{rms}}$ fluctuations (periodic + turbulent) along centerline of square cylinder.

Figure 5-45 Total $\mathrm{v}_{\mathrm{rms}}$ fluctuations (periodic + turbulent) along centerline of square cylinder.

Figure 5-46 Total kinetic energy of fluctuations (turbulent + periodic) along centerline of square cylinder.

Figure 5-47 Results from Rottach-Egern workshop: total kinetic energy of fluctuations along centerline of square cylinder. (Reprinted from (Rodi 1997) with permission)

Figure 5-48 Mean streamwise velocity profile along $y$-direction at cylinder center $(x=0)$.

Figure 5-49 Total $\mathrm{u}_{\mathrm{rms}}$ fluctuations (periodic + turbulent) along y-direction at cylinder center $(\mathrm{x}=0)$.

Figure 5-50 Contours of mean streamwise velocity in the center (x,y)-plane............... 201

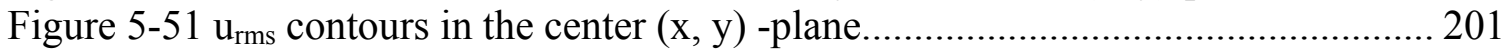

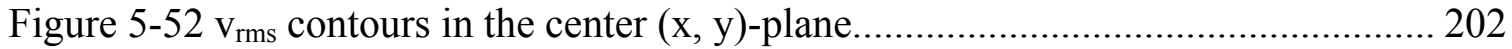

Figure 5-53 Contours of total kinetic energy of fluctuations in the center $(x, y)$ plane.. 202 Figure 5-54 Phase-averaged streamlines of flow past a square cylinder at three phases (phase 1, 9 and 17). Left column is from experiment of Lyn et al. (1995), right column is present simulation.

Figure 5-55 Instantaneous streamwise velocity in the center $(\mathrm{x}, \mathrm{y})$ plane...................... 204

Figure 5-56 Instantaneous vertical velocity in the center $(\mathrm{x}, \mathrm{y})$ plane............................ 204

Figure 5-57 Instantaneous spanwise velocity in the center (x, y) plane........................ 205

Figure 5-58 Instantaneous spanwise vorticity in the center (x, y) plane. ........................ 205

Figure 5-59 Time history of fluctuating signals sampled at (1D, 0D).......................... 206

Figure 5-60 Time history of fluctuating signals sampled at (1D, 0.5D)...................... 206

Figure 5-61 Time history of fluctuating signals sampled at (1D, 3D) ......................... 207

Figure 5-62 Power spectra of u'-signals sampled at, in order, (1D, 0D), (1D, 0.5D) and

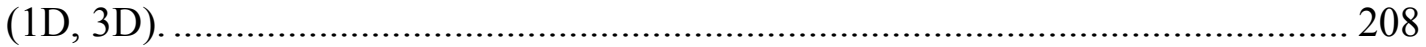

Figure 5-63 Power spectra of v'-signals sampled at, in order, (1D, 0D), (1D, 0.5D) and

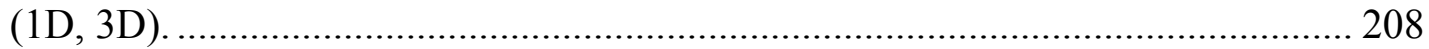

Figure 6-1 (a) Gravitational settling, (b) buoyant settling......................................... 213

Figure 6-2 Gravitational settling of water drop in quiescent air: (a) velocity evolution, (b)

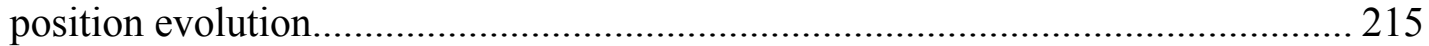

Figure 6-3 Comparison of exact error of the calculated water drop velocity with two different Runge-Kutta schemes; (a) $2^{\text {nd }}$ order RK, (b) $4^{\text {th }}$ order RK....................... 216

Figure 6-4 Order of method of RK2 for the calculation of gravitational settling........... 216 
Figure 6-5 Buoyant settling of air bubble rising in quiescent water: (a) velocity evolution,

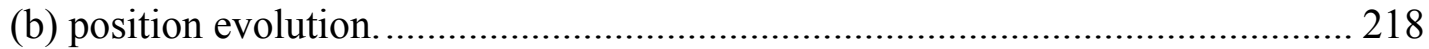

Figure 6-6 Particle injected across a uniform flow.................................................... 219

Figure 6-7 Water drop injected across a uniform flow. (a) u-velocity, and (b) v-velocity

evolution of the water drop...................................................................... 220

Figure 6-8 Parametric trajectory of a water drop injected across a uniform flow......... 221

Figure 6-9 Two-dimensional view of the trajectory of a water drop injected across a uniform flow. Circles represent locations passed by the drop............................. 221

Figure 6-10 Velocity evolution of a water-drop in an oscillating air flow.................... 224

Figure 6-11 Position evolution of a water-drop in an oscillating air flow. Dashed line is

the analytical location at sufficiently large time................................................ 224

Figure 6-12 Position evolution of a water-drop in an oscillating air flow. Dashed line is the analytical location at sufficiently large time. Total time of evolution is 50s... 225

Figure 6-13 Particle in a rotating flow................................................................. 225

Figure 6-14 x- and y-position evolution of a particle in a rotating air. (a) x-location, (b) y-location 228

Figure 6-15 Two-dimensional view of the trajectory of a particle in a rotating air. Circles represent locations passed by the particle................................................... 229

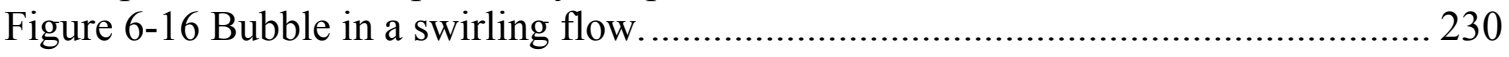

Figure 6-17 Decay of absolute r-coordinate of a bubble in a swirling flow. The angular velocity of the swirling flow is (a) $104 \mathrm{rpm}$, (b) $209 \mathrm{rpm}$, (c) $313 \mathrm{rpm}$, (d) $522 \mathrm{rpm}$.

Figure 6-18 Top view of bubble trajectory in a swirling flow. The angular velocity of the swirling flow is (c) $313 \mathrm{rpm}$ (d) $522 \mathrm{rpm}$. Circle: calculated, line: exact.............. 236

Figure 6-19 3D view of bubble trajectory in a swirling flow. The angular velocity of the swirling flow is (c) $313 \mathrm{rpm}$, (d) $522 \mathrm{rpm}$. Circle: calculated, line: exact.............. 238

Figure 7-1 Difference between particle diffusion and dispersion in a turbulent boundary

layer. (Reprinted from (Loth 2000) with permission) ........................................ 246

Figure 7-2 Illustration of mesoscale ................................................................. 251

Figure 7-3 Shape regimes for bubbles and drops rising or falling freely in quiesent liquids. (From (Clift et al. 1978) with permission, chapter numbers annotated on the figure refer to those of the original textbook, not of the present thesis) ................ 264

Figure 7-4 Bubble terminal rise velocities and terminal Reynolds numbers.................. 280

Figure 7-5 Schematic of Particle-Source-In Cell (PSI-Cell) method. ............................ 291

Figure 7-6 Schematic of PSI-Cell method with force distribution. ............................... 291

Figure 7-7 Schematic of area- or volume-weighted distribution of a quantity defined at the particle location. 292

Figure 7-8 Schematic of inverse-distance-weighted distribution of a quantity defined at the particle location. 292

Figure 7-9 Schematic of a micro particle vs. grid distribution in a wall-resolving LES or DNS 302

Figure 7-10 Schematic of particle size with respect to grid size. Eulerian grid is (a) coarse, (b) fine.

Figure 7-11 Schematic of force redistribution using a predefined influence circle or ball

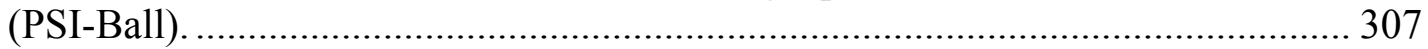

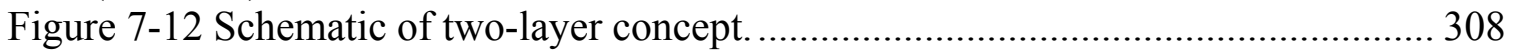


Figure 8-1 Sketch of flat bubble column used by (Becker et al. 1994). 313

Figure 8-2 (a) LDA-measurements of vertical velocity at two selected locations A and B in a locally aerated bubble column. (b) A typical instantaneous snapshot of rising bubble swarm. (Reprinted from (Sokolichin and Eigenberger 1999) with permission).

Figure 8-3 Instantaneous bubble dispersion in a locally aerated bubble column. From top to bottom: (a) Experiment (binary and inverted photograph) of (Sokolichin and Eigenberger 1999), (b) present 2D E-L simulation, (c) 2D E-L simulation of (Delnoij et al. 1997b), (d) 2D E-E simulation of (Sokolichin and Eigenberger 1999). Gas flow rate $=1.61 / \mathrm{min} . \Delta \mathrm{t}=10 \mathrm{~s}$. (Figures on $1^{\text {st }}, 3^{\text {rd }}$ and $4^{\text {th }}$ rows reprinted with permission).

Figure 8-4 Instantaneous liquid vector field in a locally aerated bubble column. From top to bottom: (a) present 2D E-L simulation, (b) 2D E-L simulation of (Delnoij et al. 1997b), (c) 3D E-E simulation of (Sokolichin and Eigenberger 1999). Gas flow rate $=1.6 \mathrm{l} / \mathrm{min} . \Delta \mathrm{t}=10 \mathrm{~s}$. (Figures on $2^{\text {nd }}$ and $3^{\text {rd }}$ rows reprinted with permission).... 318

Figure 8-5 Long-time averaged liquid velocity field (a) present 2D E-L simulation (0 300s), (b) (Becker et al. 1994)'s experiment, (c) (Becker et al. 1994)'s 2D E-E simulation, (d) (Sokolichin and Eigenberger 1999)'s experiment. (Figures (b), (c) and $(d)$ reprinted with permission).

Figure 8-6 Time history of liquid vertical velocity sampled at point A $(900 \mathrm{~mm}$ from bottom, $35 \mathrm{~mm}$ from left wall). From top to bottom: (a) present 2D simulation, (b) (Becker et al. 1994)'s experiment, (c) (Becker et al. 1994)'s 2D simulation, (d) (Sokolichin and Eigenberger 1999)'s experiment, (e) (Sokolichin and Eigenberger 1999)'s 3D simulation. (Last four figures reprinted with permission).

Figure 8-7 Instantaneous bubble dispersion in a locally aerated bubble column. From top to bottom: (a) present LES, (b) experiment of (Sokolichin and Eigenberger 1999), (c) E-E 3D simulation of (Sokolichin and Eigenberger 1999), (d) experiment of (Becker et al. 1994). Gas flow rate $=1.6 \mathrm{l} / \mathrm{min} . \Delta \mathrm{t}=5 \mathrm{~s}$. (Figures on last three rows reprinted with permission). 325

Figure 8-8 Instantaneous liquid vector field in a locally aerated bubble column. From top to bottom: (a) instantaneous bubble dispersion (from present LES), (b) present LES (grid index skip in both $\mathrm{x}$ - and y-directions is 2), (c) E-E 3D simulation of (Sokolichin and Eigenberger 1999). Gas flow rate $=1.61 / \mathrm{min} . \Delta \mathrm{t}=5 \mathrm{~s}$. (Figure on $3^{\text {rd }}$ rows reprinted with permission).

Figure 8-9 Time history of liquid vertical velocity sampled at point A $(900 \mathrm{~mm}$ from bottom, $35 \mathrm{~mm}$ from left wall). From top to bottom: (a) present LES, (b) (Sokolichin and Eigenberger 1999)'s experiment, (c) (Becker et al. 1994)'s experiment (0 581s), (d) (Sokolichin and Eigenberger 1999)'s 3D simulation. (Last three figures reprinted with permission).

Figure 8-10 Time history of liquid vertical velocity sampled at point B $(1.05 \mathrm{~m}$ from bottom, $50 \mathrm{~mm}$ from right wall). From top to bottom: (a) present LES, (b) (Sokolichin and Eigenberger 1999)'s experiment, (c) (Sokolichin and Eigenberger 1999)'s 3D simulation. (Second and third figures reprinted with permission)....... 328

Figure 8-11 Power spectra of u-velocity fluctuations at Point A (upper) and B (lower).329 Figure 8-12 Long-time averaged liquid velocity field (a) present LES (0 - 300s, grid index skip in both $\mathrm{x}$ - and y- directions is 2), (b) (Becker et al. 1994)'s experiment, 
(c) (Sokolichin and Eigenberger 1999)'s experiment, (d) (Sokolichin and Eigenberger 1999)'s 3D simulation. (Figures (b), (c), and (d) reprinted with permission).

Figure 8-13 Long-time averaged vertical velocity profiles at different heights of the middepth plane. Eight comparison stations are, from bottom to top, $\mathrm{y} / \mathrm{H}=0.065,0.2$, $0.32,0.45,0.58,0.7,0.83$, and 0.96. Solid lines are present LES (left) or (Sokolichin and Eigenberger 1999)'s 3D E-E simulation (right), dash-dot or dashed lines (Sokolichin and Eigenberger 1999)'s experiment. (Right figure reprinted with permission).

Figure 8-14 Turbulence intensities at different heights in the mid-depth plane, from top to bottom: (a) $u_{\mathrm{rms}}$, (b) $v_{\mathrm{rms}}$, (c) turbulent kinetic energy.

Figure 8-15 TKE profile at $\mathrm{y} / \mathrm{H}=0.5$ calculated from (Sokolichin and Eigenberger 1999)'s 2D E-E simulation with $k-\varepsilon$ model. (Figure reprinted with permission)... 333

Figure 8-16 Instantaneous volume fraction of dispersed phase (bubble). Upper row: present LES; lower row: E-E 3D simulation of (Sokolichin and Eigenberger 1999).

Figure 8-17 Mean volume fraction of dispersed phase (bubble). 334

Figure 8-18 Comparison of instantaneous bubble dispersion calculated with two different grids. Upper row: 96x64x8; lower row: 120x80x10.

Figure 8-19 Comparison of time history of vertical velocity at Point A calculated with two different grids. Upper row: 96x64x8; lower row: 120x80x10 ……………... 336

Figure 8-20 Comparison of time history of vertical velocity at Point B calculated with two different grids. Upper row: $96 \times 64 \times 8$; lower row: $120 \times 80 \times 10$.

Figure 8-21 Comparison of long-time $(0-300 \mathrm{~s})$ averaged liquid velocity field in the mid-

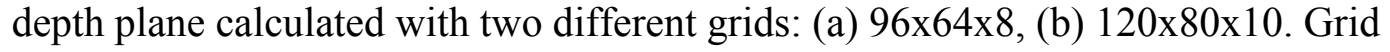
index skip is 2 in both $\mathrm{x}$ - and y-directions.

Figure 8-22 Comparison of turbulent kinetic energy profiles at different height in the

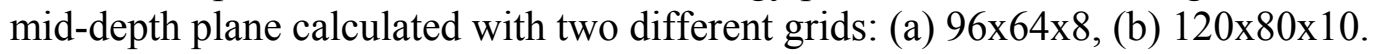

Figure 8-23 Comparison of turbulence intensity and turbulent kinetic energy profiles at two different height in the mid-depth plane calculated with two different grids. From top to bottom: (a) $\mathrm{u}_{\mathrm{rms}}$, (b) $\mathrm{v}_{\mathrm{rms}}$, (c) TKE. 


\section{Chapter 1 Introduction}

\subsection{Governing Equations of Fluid Flow}

"A mathematical model for the behavior of a physical system, and in particular the system of fluid flows, can only be defined after consideration of the level of the approximation required in order to achieve an acceptable accuracy on a defined set of dependent and independent variables." (Hirsch 1988)

Various levels of description of our physical world (a physical understanding to the real world) have been proposed and defined, ranging from subatomic, atomic or molecular, microscopic or macroscopic, up to the astronomical scale. At a certain approximation level a mathematic model can be derived aiming at describing the physical system at this level with a reasonable accuracy. Figure 1-1 shows a block diagram of this top-down hierarchy. The classical fluid mechanics is based on the continuum hypothesis, which is valid on a macroscopic scale and assumes individual fluid particles to be large compared with the distance between molecules.

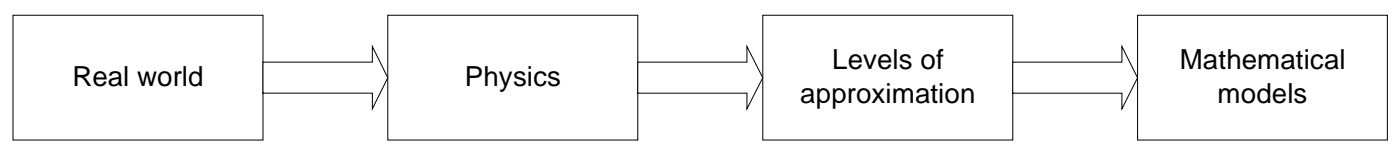

Figure 1-1 Mathematical models with respect to levels of approximation

The law of fluid dynamics is well established, whereby the key observation is that during the motion of a fluid certain number of properties, such as mass, generalized momentum and energy, are conserved. These conservation properties are then used to deduce a set of integral-differential equations (mathematical model) describing a dynamical system of fluid flow (real world problem). This well-known set of equations governing the fluid dynamics is commonly referred to as the Navier-Stokes (N-S) equations. In what follows the N-S equations will be briefly outlined. A detailed derivation of the N-S equations is however, abridged here, as they can be found in a great deal of classical texts. (Batchelor 1967; Daily and Harleman 1973; Schlichting 1979; White 1991; Munson et al. 1994; Bird et al. 2002; Fox et al. 2003)

Consider a given quantity of matter, called control mass (CM) or system. Let $\phi$ be a generic conserved intensive property that is not dependent on the amount of matter (mass or volume). Examples of $\phi$ are density (mass per unit volume), specific volume (volume per unit mass), velocity (momentum per unit mass), pressure and temperature. The corresponding extensive property $\Phi$, whose value varies directly with mass, can be expressed as

$$
\Phi=\int_{\Omega_{C M}} \rho \phi d \Omega
$$


where $\Omega_{C M}$ stands for the volume occupied by the CM, $\rho$ is the density. A control volume $(\mathrm{CV})$ is a certain spatial region that can be either fixed or moving. By Reynolds transport theorem, which links the control mass frame (or the Lagrangian frame) with the control volume frame (or the Eulerian or laboratory frame), the following integral-differential equations are obtained describing a conserved property:

$$
\frac{d \Phi}{d t}=\frac{d}{d t} \int_{C V} \rho \phi d \Omega+\int_{C S} \rho \phi\left(\mathbf{u}-\mathbf{u}_{C S}\right) \cdot \mathbf{n} d \mathcal{S},
$$

where CS (control surface) is the surface enclosing CV, $\mathbf{n}$ is the unit vector perpendicular to CS and directed outwards, $\mathbf{u}$ is the fluid velocity and $\mathbf{u}_{\mathrm{CS}}$ is the velocity with which the $\mathrm{CS}$ moves. If the $\mathrm{CV}$ is fixed, a situation to be considered here, $\mathbf{u}_{\mathrm{CS}}$ equals zero and the first derivative on the right hand side becomes a local (partial) derivative. Thus,

$$
\frac{d \Phi}{d t}=\frac{\partial}{\partial t} \int_{C V} \rho \phi d \Omega+\int_{C S} \rho \phi \mathbf{u} \cdot \mathbf{n} d \mathcal{S}
$$

If $\phi$ is taken to be unity ( $\phi=1)$, flow velocity $(\phi=\mathbf{u})$, and total energy per unit mass $(\phi=e)$ respectively, the integral form of the mass conservation (continuity), momentum conservation and energy conservation equation is obtained, respectively:

$$
\begin{gathered}
\frac{\partial}{\partial t} \int_{C V} \rho d \Omega+\int_{C S} \rho \mathbf{u} \cdot \mathbf{n} d \mathcal{S}=0, \\
\frac{\partial}{\partial t} \int_{C V} \rho \mathbf{u} d \Omega+\int_{C S} \rho \mathbf{u u} \cdot \mathbf{n} d \mathcal{S}=\sum_{l} \mathbf{f}_{l}, \\
\frac{\partial}{\partial t} \int_{C V} \rho e d \Omega+\int_{C S} \rho e \mathbf{u} \cdot \mathbf{n} d \mathcal{S}=S_{\phi} .
\end{gathered}
$$

In the above equations, $\sum_{l} \mathbf{f}_{l}$ represents all possible forces acting on a $\mathrm{CV}$, which may include surface forces (pressure, shear stresses, surface tension etc.) and body forces (gravity, Coriolis forces etc.); $S_{\phi}$ represents all the source or sink terms that contribute to the energy transfer with respect to a CV. Examples are the work done to the CV and heat flux through the CV surfaces. With Gauss' divergence theorem the surface integral can be transformed into volume integral. Further allowing the control volume to be infinitely small, at the limit, the differential coordinate-free form of the conservation equations is obtained. In particular, assuming the fluid to be viscous leads to the well-known set of Navier-Stokes equations (White 1991):

$$
\begin{gathered}
\frac{\partial \rho}{\partial t}+\operatorname{div}(\rho \mathbf{u})=0, \\
\frac{\partial \rho \mathbf{u}}{\partial t}+\operatorname{div}(\rho \mathbf{u u})=\operatorname{div}(\boldsymbol{\sigma})+\rho \mathbf{b}, \\
\frac{\partial \rho h}{\partial t}+\operatorname{div}(\rho h \mathbf{u})=\operatorname{div}(k \nabla T)+\frac{D p}{D t}+\Psi+S_{\phi},
\end{gathered}
$$

where $\sigma$ is the total stress tensor due to the surface forces, $\mathbf{b}$ is the body force per unit volume including gravitational force, $h$ is the fluid enthalpy, $T$ is the temperature, $p$ is the pressure, $\Psi$ is the viscous dissipation function representing the degradation of mechanical energy per unit volume into the thermal energy. The second term on the left in the above equation describes the convection effect, and is known as the convective or advective 
term. The first term on the right of Eq. (1.8) and (1.9) represents the diffusion effect and is called the diffusion or conduction term. By assuming Newtonian fluid and using Stokes' hypothesis, the momentum conservation equation (Eq. (1.8)) can be closed with the following relation for the total stress tensor $\sigma$ :

$$
\boldsymbol{\sigma}=-[p \mathbf{I}+\lambda \operatorname{div}(\mathbf{u})]+2 \mu \mathbf{S}, \quad \lambda=\frac{2}{3} \mu,
$$

where $\mu$ is the dynamics viscosity, $\lambda$ is the second viscosity coefficient, $\mathbf{I}$ is the unitary (or identity) tensor, and $\mathbf{S}$ is the rate of strain (deformation) tensor:

$$
\mathbf{S}=\frac{1}{2}\left[\nabla \mathbf{u}+(\nabla \mathbf{u})^{T}\right] .
$$

Very often, the viscous shear stress tensor $2 \mu \mathbf{S}$ is denoted by a single symbol $\tau$.

In a Cartesian coordinate system, which is considered throughout this study, the Navier-Stokes equations (Eq. (1.7) through (1.9)) can be written as:

$$
\begin{gathered}
\frac{\partial \rho}{\partial t}+\frac{\partial}{\partial x_{i}}\left(\rho u_{i}\right)=0, \\
\frac{\partial}{\partial t}\left(\rho u_{i}\right)+\frac{\partial}{\partial x_{j}}\left(\rho u_{j} u_{i}\right)=-\frac{\partial p}{\partial x_{i}}+\frac{\partial \tau_{i j}}{\partial x_{j}}+\rho b_{i}, \\
\frac{\partial}{\partial t}(\rho h)+\frac{\partial}{\partial x_{j}}\left(\rho u_{j} h\right)=\frac{D p}{D t}+\frac{\partial}{\partial x_{j}}\left(k \frac{\partial T}{\partial x_{j}}\right)+\tau_{i j}^{T} \frac{\partial u_{i}}{\partial x_{j}}+S_{\phi},
\end{gathered}
$$

with

$$
\begin{gathered}
\tau_{i j}=2 \mu S_{i j}-\left(\lambda \frac{\partial u_{j}}{\partial x_{j}}\right) \delta_{i j}, \lambda=\frac{2}{3} \mu, \\
S_{i j}=\frac{1}{2}\left[\frac{\partial u_{i}}{\partial x_{j}}+\frac{\partial u_{j}}{\partial x_{i}}\right] .
\end{gathered}
$$

Note that $\tau_{i j}$ is the viscous stress tensor excluding pressure; $S_{i j}$ is the rate of stain tensor on Cartesian coordinates; superscript $T$ denotes the transpose of a stress tensor; $i$ is used as free index and $j$ as dummy index, both may be valued at from one to three representing three directions in a Cartesian coordinate system.

If the flow is compressible and the assumption of local thermodynamic equilibrium applies, the governing equations must be supplemented by the thermal equations of state, i.e.,

$$
\begin{array}{ll}
\rho=\rho(p, T) & h=h(p, T) \\
\mu=\mu(p, T) & k=k(p, T)
\end{array}
$$

In this study only isothermal systems is considered, in which temperature is constant and the energy equation drops. An isothermal systems can be formally defined if in the system there are no externally imposed temperature gradients and no appreciable temperature change resulting from expansion, contraction, or viscous dissipation (Bird et al. 2002). Following this, it can be further assumed a constant viscosity and conductivity 
of the fluid. Only the density, in addition to the primary flow field variables (velocity and pressure), is allowed to change with both space and time. Variable density requirement could be important in the modeling of a gas-liquid two-phase flow system.

For an incompressible flow which satisfies

$$
\nabla \cdot \mathbf{u}=0
$$

the substantial (or material, particle) derivative of the fluid density is zero, i.e.,

$$
\frac{D \rho}{D t}=0
$$

This can be shown by rewriting the continuity equation (1.12) in an equivalent nonconservative form as

$$
\frac{D \rho}{D t}+\rho \nabla \cdot \mathbf{u}=0
$$

It should be stressed that a variable density flow, in which $\rho$ is a function of time and space, can still be incompressible as long as the material derivative of density is equal to zero or negligibly small. Further, with the incompressibility condition plus the constant viscosity assumption the shear stress term in the momentum equation (1.13) can be simplified to

$$
\frac{\partial \tau_{i j}}{\partial x_{j}}=\frac{\partial}{\partial x_{j}}\left(2 \mu S_{i j}\right)=\frac{\partial}{\partial x_{j}}\left(\mu \frac{\partial u_{i}}{\partial x_{j}}\right)
$$

since

$$
\frac{\partial}{\partial x_{j}}\left(\mu \frac{\partial u_{j}}{\partial x_{i}}\right)=\mu \frac{\partial}{\partial x_{i}}\left(\frac{\partial u_{j}}{\partial x_{j}}\right)=0 \text {. }
$$

This gives the following momentum equation for an incompressible flow expressed on the Cartesian coordinates:

$$
\frac{\partial}{\partial t}\left(\rho u_{i}\right)+\frac{\partial}{\partial x_{j}}\left(\rho u_{j} u_{i}\right)=-\frac{\partial p}{\partial x_{i}}+\frac{\partial}{\partial x_{j}}\left(2 \mu S_{i j}\right)+\rho b_{i}
$$

or

$$
\frac{\partial}{\partial t}\left(\rho u_{i}\right)+\frac{\partial}{\partial x_{j}}\left(\rho u_{j} u_{i}\right)=-\frac{\partial p}{\partial x_{i}}+\frac{\partial}{\partial x_{j}}\left(\mu \frac{\partial u_{i}}{\partial x_{j}}\right)+\rho b_{i} .
$$

Eq. (1.12) and (1.24) serves as the most fundamental equations throughout the entire study.

\subsection{Two-Phase Flow Systems}

A large number of flows encountered in nature and industrial application are a mixture of phases. A physical phase is defined as a state of the matter, i.e., solid, liquid gas or vapor. Dust storm, air pollution and smog, bubbly ship wake, ocean-atmosphere interactions, paint sprays, spray drying, coal or liquid fuel combustion, fluidized beds, bubble column reactors, cyclone separators are just a few examples of a multiphase flow system. Unlike the single-phase flow where the governing equations for the motion and thermal 
properties (N-S equations) are well accepted, a proper and efficient formulation of a multiphase flow system is still subject to debate (Crowe et al. 1996); many multiphase systems of physical and technological importance exhibit highly complex nature and are not yet well understood. It is for these reasons that the multiphase flow research is still considered to be primitive, and represents a challenging and potentially very fruitful area.

Within the broad science of multiphase flow, a subset is named two-phase flow where only two phases are present in the system. Depending on the combination of the physical phases, a two-phase system can be generally classified into three categories, namely, gas-liquid flows, gas-solid flows and liquid-solid flows. Within each class of flow there can be different regimes characterized by distinct flow patterns. These are summarized in Table 1-1.

Table 1-1 Classification of two-phase systems and flow regimes

\begin{tabular}{|l|l|l|}
\hline \multicolumn{1}{|c|}{ Category } & \multicolumn{1}{|c|}{ Regime } & \multicolumn{1}{c|}{ Description or examples } \\
\hline \multirow{4}{*}{ Gas-liquid flows } & Bubbly flow & Discrete gaseous bubbles in a liquid \\
\cline { 2 - 3 } & Droplet flow & Discrete fluid droplets in a gas \\
\cline { 2 - 3 } & Slug flow & Very large bubbles in a liquid \\
\cline { 2 - 3 } & $\begin{array}{l}\text { Stratified/free- } \\
\text { surface flow }\end{array}$ & $\begin{array}{l}\text { Immiscible fluids separated by clearly } \\
\text { defined interface }\end{array}$ \\
\hline \multirow{5}{*}{ Gas-solid flows } & Particle-laden flow & Discrete solid particles in gas \\
\cline { 2 - 3 } & Pneumatic transport & $\begin{array}{l}\text { Pattern varies depending on factors such as } \\
\text { the solid loading }\end{array}$ \\
\cline { 2 - 3 } & Fluidized bed & $\begin{array}{l}\text { Rising gas suspends densely-distributed solid } \\
\text { particles }\end{array}$ \\
\hline \multirow{3}{*}{ Liquid-solid flows } & Slurry flow & Transport of particles in a liquid \\
\cline { 2 - 3 } & Hydrotransport & Densely-distributed solid particles in a liquid \\
\cline { 2 - 3 } & Sedimentation & $\begin{array}{l}\text { Characterized by a sludge layer at the } \\
\text { bottom, a clear interphase at the top and a } \\
\text { settling zone in the middle }\end{array}$ \\
\hline
\end{tabular}

A special group of the two-phase flow that covers several flow regimes, namely, the bubbly flow, droplet flow and particle-laden flow as described in Table 1-1, is known as the dispersed two-phase flow. In a dispersed two-phase system a secondary discrete phase, present in form of "small" particles/bubbles/droplets, is dispersed in the primary continuous phase (liquid or gas). An important characteristic of such system is that the dispersed phase has a negligibly low volume fraction (not mass fraction!). Dispersed twophase flows can be found in a broad range of engineering and scientific disciplines including biological, chemical, mechanical, meteorological, petrochemical, nuclear, aerospace, civil and environmental applications, ranging from droplet sprays in highspeed combusting flow, pollutant dispersion, to bubbly pipe flows of nuclear reactors. The dispersed two-phase flows will be the primary interest of the current study. 


\subsection{Objective, Scope and Organization of Thesis}

In a broader sense, this dissertation is aimed at simulating dispersed two-phase flows using proper numerical techniques. Specifically, it presents a fundamental study of all essential components of computational and modeling issues for a successful large-eddy simulation (LES) of dispersed two-phase turbulent flows. Resent research activities have shown an intense trend of using LES to predict turbulent two-phase flows. It is generally believed that two-phase LES would show considerable promise when proper closure models become well established. The present work strives to advance the two-phase LES research by (i) formalizing various two-way coupling formulations and models, which are of fundamental importance and practical interest, and (ii) resolving the scale restrictions imposed to the particle size in a conventional two-phase simulation.

Overall, the study is heavily approach-oriented rather than results-oriented. It is focused more on the right way, not just on the seemingly right results. A very large portion of this study is devoted to a careful development and interpretation of the theories and methodologies. The work is organized into the following three major parts.

Part I focuses on the development, verification and validation of a transient, threedimensional, finite-volume-method (FVM) based accurate Navier-Stokes solver capable of tackling both laminar and turbulent flow problems. Chapter 2 supplies details of the implemented spatial and temporal discretizations. Schemes considered for the convective transport are Patankar's generalized formula (Patankar 1980), which incorporates the $1^{\text {st }}$ order upwind, hybrid, power-law, and $2^{\text {nd }}$ order central differencing (CD), the $3^{\text {rd }}$ order QUICK (quadratic upwind interpolation for convective kinematics) and $4^{\text {th }}$ order CD; and schemes considered for the time integration are the $2^{\text {nd }}$ order Adams-Bashforth. Solution of the coupled partial differential equations is attacked with a fractional step method proposed by (Kim and Moin 1985). In Chapter 3, the developed solver is verified in a systematic manner against a variety of benchmark laminar flows, including CouettePoiseuille flows, the developing channel flow, Taylor's vortex, free-shear layer, backward-facing step flow and the square cavity. It is shown that a second-order overall accuracy is achieved in both space and time.

Part II concerns the modeling and large-eddy simulation (LES) of single-phase turbulent flows. In Chapter 4, a review of the LES theory, methodology and subgridscale (SGS) models is presented. Resolution requirements in a LES are addressed. Three SGS models, namely, Smagorinsky model, dynamic model and implicit model, all with implicit filtering, are implemented and investigated. Large-eddy simulations are then carried out in Chapter 5 for three building-block turbulent flows, namely, turbulent channel flow, plane mixing layer, and flow past a square cylinder. Extensive comparisons of the first-, second-order statistics, and characteristic flow structures are made with direct numerical simulation (DNS) and/or benchmark experiments. The test results show superior quality of the present LES.

Part III delves into the theory, modeling and simulation of dispersed two-phase flow systems. In Chapter 7 a conceptual review of the characteristics and description of 
such systems is made, considering both Eulerian-Eulerian (E-E) and Eulerian-Lagrangian (E-L) approaches, but with an emphasis on the latter. Hydrodynamic forces acting on particles or bubbles are addressed in depth. Formulations regarding interphase coupling is reviewed. Typical computational treatments of modeled two-way coupling in E-L DNS/LES are summarized and evaluated. Issues related to interpolation are discussed. In Chapter 5 a general Lagrangian particle-tracking (LPT) program is developed and it is verified using analytical solutions and by considering the motion of a single rigid particle subject to various imposed flow fields, such as the oscillating flow, rotating flow and swirling flow. Chapter 5 is intentionally arranged before Chapter 6 because it can serve as a proper introduction to the topic of Lagrangian particle dynamics.

A critical issue in the E-L approach is that, the particle size is required to be much smaller than the characteristic flow length scale and the computational grid size. This presents a considerable restriction of the E-L's applicability to practical engineering flows such as bubble column reactors. A two-layer concept, aimed at decoupling the particle's dynamic feature from the geometric feature, is proposed at end of Chapter 7. In this approach, the carrier phase and the dispersed phase are viewed as two independent computational layers, and the reverse coupling takes place at those discrete particle locations through modeled momentum exchange forces with the help of a predefined influence circle. A significant advantage is that the Eulerian grid can be constructed to a desired fineness (e.g., in the wall layer) without concerning with the particle size. Finally, in Chapter 8, this idea is proven to work well in an application of two-way coupled E-L LES to a locally aerated turbulent bubble column, with surprisingly good success. 


\section{Part I Numerical Solution of Navier- Stokes Equations}

\section{Chapter 2 Numerical Methods}

Here sought is an efficient numerical solution procedure to solve the unsteady, threedimensional (3D) Navier-Stokes (N-S) equations,

$$
\begin{gathered}
\frac{\partial \rho}{\partial t}+\frac{\partial}{\partial x_{i}}\left(\rho u_{i}\right)=0 \\
\frac{\partial}{\partial t}\left(\rho u_{i}\right)+\frac{\partial}{\partial x_{j}}\left(\rho u_{j} u_{i}\right)=-\frac{\partial p}{\partial x_{i}}+\frac{\partial}{\partial x_{j}}\left(\mu \frac{\partial u_{i}}{\partial x_{j}}\right)+\rho b_{i} .
\end{gathered}
$$

In that, the four primary flow field variables, i.e., three velocity components and the pressure, are functions of both space and time, and they need to be coped with in coupled manner. Density is kept as an optional variable. A discretization for a fully compressible flow, which incorporates a stress term due to the second viscosity $\left(2^{\text {nd }}\right.$ term on the RHS of Eq. (1.10)), and possibly requires a coupling of the energy equation (1.14) and thermal dynamic properties, is avoided in this study. Discretization of these partial differential equations (PDE) follows the standard finite volume method (FVM). Some introductory material concerning FVM can be found for example, in (Patankar 1980; Hoffmann and Chiang 1993; Versteeg and Malalasekera 1995; Ferziger and Peric 1996; Wendt et al. 1996; Chung 2002). For the sake of simplicity and solution efficiency, the computational grid is restricted to be orthogonal, i.e., they are aligned with Cartesian coordinate system.

The text will start with a brief discussion on the computational grid or cell used in the present FVM. Discussed is a staggered grid arrangement used to accommodate the three velocity components and the pressure field. Next, a detailed derivation is presented for the discretization of a generalized transport equation. The generalized scheme of (Patankar 1980) is incorporated in this step. The transport equation under consideration can be regarded as a generalization of equation (2.2), so that the derived discretization can be equally applied to the three momentum equations. The five schemes contained in Patankar's formulation are assessed in the following section. The standard discretization is then further generalized by introducing implicitness factors. As an important addition, the order of the method is improved by higher order schemes. In particular, the spatial accuracy can be improved by the $3^{\text {rd }}$ order QUICK (quadratic upwind interpolation for convective kinematics) or the $4^{\text {th }}$ order central differencing (CD), and a higher order temporal accuracy can be achieved with the $2^{\text {nd }}$ order Adams-Bashforth scheme. It is then 
followed by a description of a procedure for solving the coupled Navier-Stokes equations known as the fractional step (or projection) method, due to (Kim and Moin 1985). This procedure employs the idea of operator splitting, which essentially decouples the pressure from the solution of the velocity field. The discretization of the pressure equation, which arises from the fractional step procedure, is explicated in the subsequent section. The Poisson-type pressure equation is solved using the standard $2^{\text {nd }}$ order central differencing, with an optional accuracy improvement using the $4^{\text {th }}$ order deferred correction.

\subsection{Orthogonal and Staggered Grid}

In finite volume approach the solution domain is subdivided into a finite number of small control volumes (CVs), or computational cells, which have no overlap and together fill up the whole solution domain. In this study only the orthogonal grid is considered. In Figure 2-1 a typical two-dimensional CV (shaded area) is shown along with the notation to be used in the later sections. A capital letter indicates a CV node while a lower case letter represents the face of a $\mathrm{CV}$. These letters suggest the relative orientation of a $\mathrm{CV}$ node or face with respect to the CV under consideration (shaded area with node "P" inside). For example, "W" denotes the node located west of the shaded CV, and "W" is the west face of it. A three-dimensional CV is also depicted in Figure 2-2 with additional bottom face (denoted by "b") and top face (denoted by "t") oriented in the z-direction.

The solution of the Navier-Stokes equation typically involves four unknowns, i.e., three velocity components and a pressure. The conservation properties can be readily achieved if the four variables are solved on a staggered grid arrangement (Patankar 1980). That is, the pressure is solved on a main grid points, the x-direction velocity $u$ is staggered in the $\mathrm{x}$-direction with respect to the main grid, $\mathrm{y}$-direction velocity $v$ staggered in the y-direction, and $w$ staggered in the z-direction. In Figure 2-1, the solid dots represent the main grid, the horizontal arrows indicate grid points of the $u$-velocity, and vertical arrows that of the v-velocity. It is noted that the staggered quantities $(u, v$ and $w$ velocities) are located at the face of the main grid. The N-S solver developed in the present study uses such a staggered grid system.

Usually, there are two approaches to arrange the node and face locations. Either control volumes are first constructed and then the nodes are placed in the center of the corresponding control volumes, or, the cell nodes are defined first and then let cell faces lie midway between nodes. The advantage of the first approach is that the nodal value represents the mean of a control volume more accurately than it does in the second approach; and the advantage of the second approach is that the derivatives evaluated at cell faces, especially when central differencing is used, are more accurate than in the first approach. In this study, the second approach is adopted. 


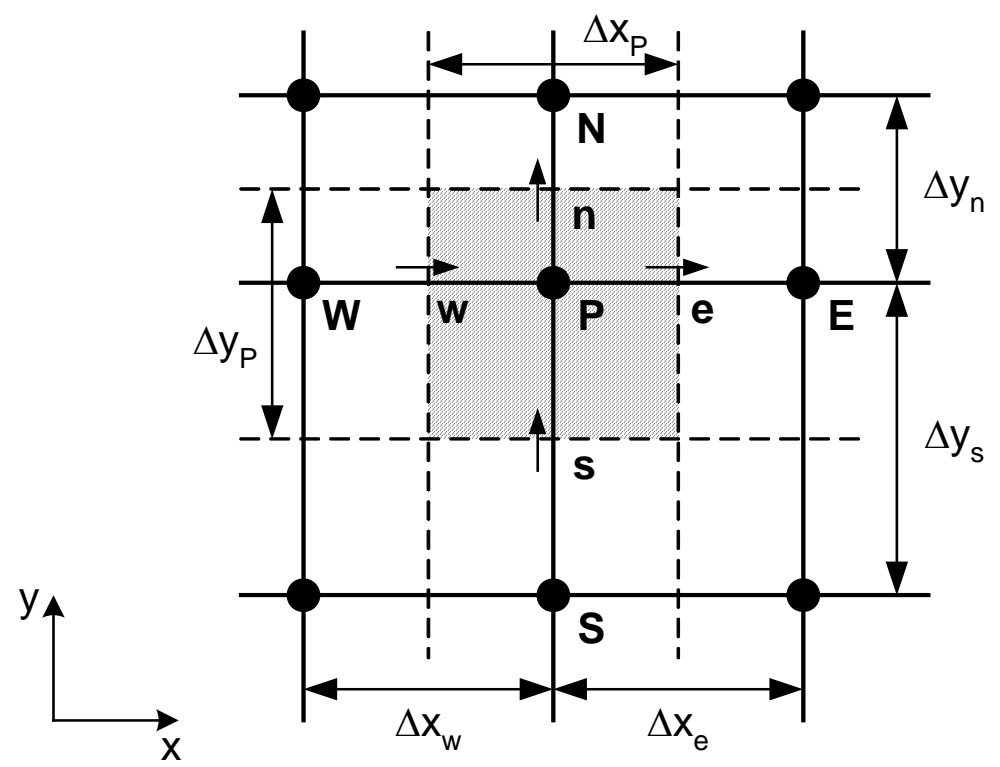

Figure 2-1 A typical 2D computational cell used in finite volume discretization

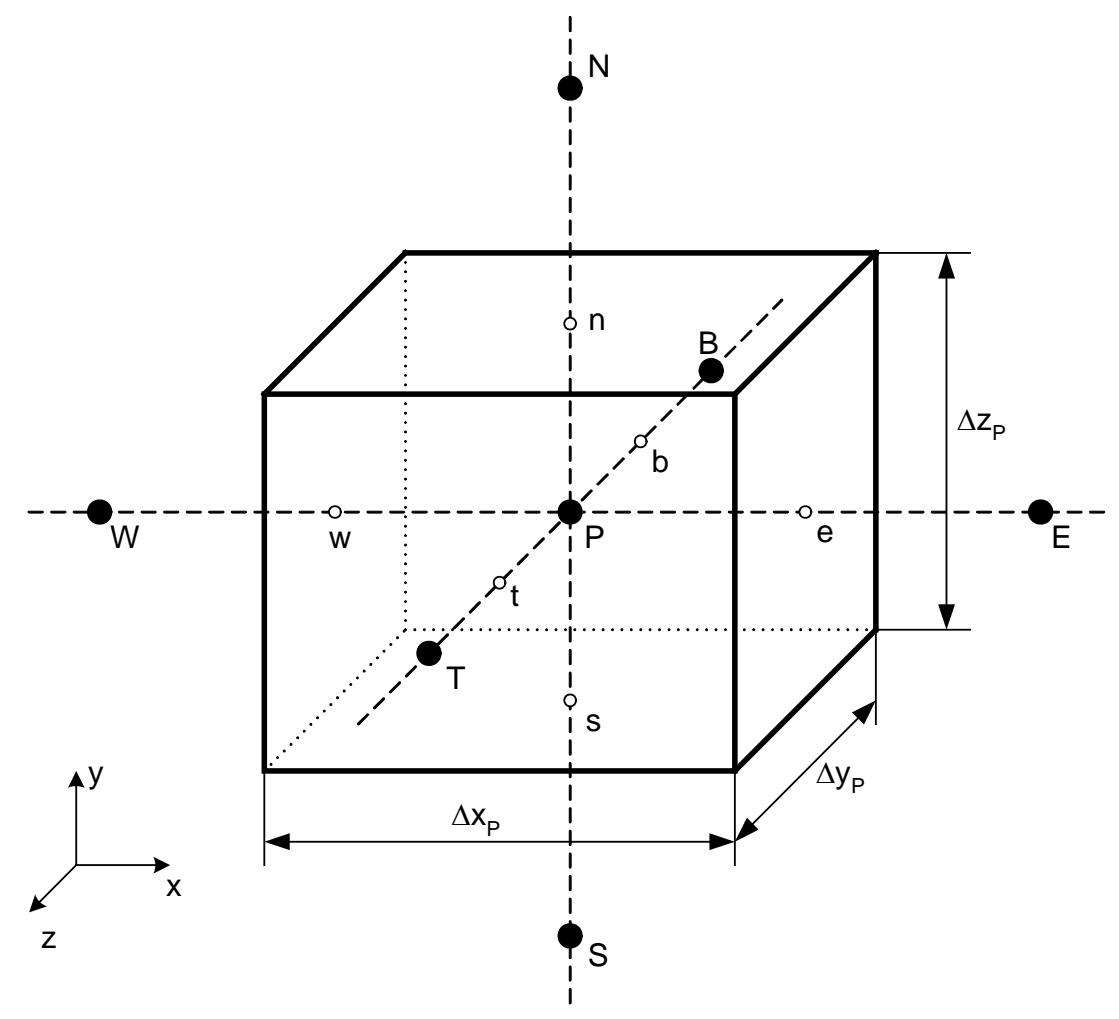

Figure 2-2 A typical 3D computational cell used in finite volume discretization 


\subsection{Discretization of a General Scalar Transport Equation}

\subsubsection{Working Equation}

Consider a general three-dimensional scalar transport equation expressed in the integral form as

$$
\frac{\partial}{\partial t} \int_{C V} \rho \phi d \Omega+\int_{C S} \rho \phi \mathbf{u} \cdot \mathbf{n} d \mathcal{S}=\int_{C S} \Gamma \nabla \phi \cdot \mathbf{n} d \mathcal{S}+\int_{C V} S_{\phi} d \Omega,
$$

and in the differential conservative form as

$$
\frac{\partial}{\partial t}(\rho \phi)+\frac{\partial}{\partial x_{j}}\left(\rho u_{j} \phi\right)=\frac{\partial}{\partial x_{j}}\left(\Gamma \frac{\partial \phi}{\partial x_{j}}\right)+S_{\phi},
$$

with the velocity field satisfying the continuity equation,

$$
\frac{\partial \rho}{\partial t}+\frac{\partial}{\partial x_{j}}\left(\rho u_{j}\right)=0 \text {. }
$$

Here $\Gamma$ represents a generic diffusion coefficient; $S_{\phi}$ includes all source terms as a function of $\phi ; j$ is the dummy index that runs from one to three (for a three-dimensional problem). The expression $\rho u_{j} \phi$ is usually called convection flux and $\Gamma \partial \phi / \partial x_{j}$ called diffusion flux. By using continuity relation (2.5), equation (2.4) can be written in an equivalent but non-conservative form as

$$
\rho \frac{\partial \phi}{\partial t}+\rho u_{j} \frac{\partial \phi}{\partial x_{j}}=\frac{\partial}{\partial x_{j}}\left(\Gamma \frac{\partial \phi}{\partial x_{j}}\right)+S_{\phi} .
$$

On the one hand, it is desired to isolate the temporal derivative of $\phi$ from the density, as in Eq. (2.6), so that the influence of a fluctuating density field on an accurate evaluation of the temporal variation can be minimized. On the other hand, the conservative form of the convection term, as in Eq. (2.4), is preferred due to its capability of being combined with the diffusion term. Thus, (Patankar 1980) has suggested a slightly modified version of Eq. (2.4) and (2.6), in which the spatial derivative is kept in conservative form while the time derivative term on the left hand side (LHS) is split into two, the resulting $\partial \rho / \partial t$ substituted by the spatial derivative from continuity equation. This yields a proper working (or starting) equation for discretization.

$$
\rho \frac{\partial \phi}{\partial t}+\frac{\partial}{\partial x_{j}}\left(\rho u_{j} \phi-\Gamma \frac{\partial \phi}{\partial x_{j}}\right)-\phi \frac{\partial}{\partial x_{j}}\left(\rho u_{j}\right)=S_{\phi} .
$$

Note that in the above equation the convective and diffusive fluxes have been grouped into one parenthesis. By introducing a mnemonic symbol $J_{i}$ to denote the total (convection plus diffusion) flux of the scalar in all three directions, i.e.,

$$
J_{i} \equiv \rho u_{i} \phi-\Gamma \frac{\partial \phi}{\partial x_{i}}
$$

Eq. (2.7) can be rewritten in a more compact way:

$$
\rho \frac{\partial \phi}{\partial t}+\frac{\partial J_{i}}{\partial x_{j}}-\phi \frac{\partial}{\partial x_{j}}\left(\rho u_{j}\right)=S_{\phi}
$$




\subsubsection{First Step in FVM}

The essence of the discretization in FVM is to apply the integral conservation equations (2.3) (or equivalently, the integrated form of Eq. (2.4), (2.7) or (2.9)) to each control volume in the solution domain, and to use certain approximation practice to yield a set of algebraic (discretized) equations for all CVs, so that they can be solved with the help of digital computer. Notice that summing up all the integral conservation equations on each $\mathrm{CV}$ yields a global conservation equation that has the same form as Eq. (2.3) and governs the entire solution domain (since surface integrals over all inner cell faces cancel out). A good discretization scheme should be able to preserve this global conservation property.

The goal of this section is to discretize Eq. (2.7) or (2.9) with a generalized scheme (Patankar 1980) in a fully implicit fashion. Five frequently used (especially in RANS calculations) schemes, i.e., the $1^{\text {st }}$ order upwind, the $2^{\text {nd }}$ order central differencing scheme, the hybrid scheme, the power law scheme and the exponential scheme are compactly expressed via a so-called "A" function. Higher order schemes are presented in the subsequent sections. In conformity with the formulae derived in (Patankar 1980) similar notations shall be used in the following presentation, but with slight modification and generalization suitable for a true three-dimensional situation. A similar derivation based on the same literature can also be found in (Celik and Badeau 2003).

Without loss of generality, let $x, y, z$ denote three axes in Cartesian coordinates corresponding to $x_{1}, x_{2}$ and $x_{3}$, and further let $u, v, w$ denote three velocity components aligned with positive $x, y$ and $z$, respectively. Thus,

$$
\begin{aligned}
& J_{1}=J_{x}=\rho u \phi-\Gamma \frac{\partial \phi}{\partial x}, \\
& J_{2}=J_{y}=\rho v \phi-\Gamma \frac{\partial \phi}{\partial y}, \\
& J_{3}=J_{z}=\rho w \phi-\Gamma \frac{\partial \phi}{\partial z} .
\end{aligned}
$$

Note that this orientation shall always be adopted in this study.

Performing integration on both sides of Eq. (2.9) over a Cartesian computational cell (see Figure 2-1 and Figure 2-2) yields

$$
\begin{aligned}
& \int_{C V} \rho \frac{\partial \phi}{\partial t} d V+\int_{A_{e}}\left(J_{x}\right)_{e} d A-\int_{A_{w}}\left(J_{x}\right)_{w} d A+\int_{A_{n}}\left(J_{y}\right)_{n} d A-\int_{A_{s}}\left(J_{y}\right)_{s} d A \\
& +\int_{A_{t}}\left(J_{z}\right)_{t} d A-\int_{A_{b}}\left(J_{z}\right)_{b} d A-\int_{C V} \phi \frac{\partial}{\partial x_{j}}\left(\rho u_{j}\right) d V=\int_{C V} S_{\phi} d V,
\end{aligned}
$$

where those $A$ 's appearing in the integral indicate a corresponding plane surface area of the control volume, on which the surface integral is calculated. Those surface integrals are the integrated total fluxes over the control volume faces; physically, they can be interpreted as certain amount of extensive property that is carried into or out of the 
control volume within unit time. Let $J_{w}, J_{e}, J_{s}, J_{n}, J_{b}, J_{t}$ denote such integrated total fluxes, i.e.,

$$
\begin{aligned}
& J_{w} \equiv \int_{A_{w}}\left(J_{x}\right)_{w} d A, J_{e} \equiv \int_{A_{e}}\left(J_{x}\right)_{e} d A, \\
& J_{s} \equiv \int_{A_{s}}\left(J_{y}\right)_{s} d A, J_{n} \equiv \int_{A_{n}}\left(J_{y}\right)_{n} d A, \\
& J_{b} \equiv \int_{A_{b}}\left(J_{z}\right)_{b} d A, J_{t} \equiv \int_{A_{t}}\left(J_{z}\right)_{t} d A .
\end{aligned}
$$

Rewriting Eq. (2.11) with the above notation gives

$$
\int_{C V} \rho \frac{\partial \phi}{\partial t} d V+J_{e}-J_{w}+J_{n}-J_{s}+J_{t}-J_{b}-\int_{C V} \phi \frac{\partial}{\partial x_{j}}\left(\rho u_{j}\right) d V=\int_{C V} S_{\phi} d V .
$$

\subsubsection{Approximation under Assumptions}

Note that up to this point all the equations presented above are exact; no approximation has been made yet. To achieve a discretized algebraic equation that gives reasonably accurate approximation to Eq. (2.13), certain assumptions are necessary. This will be worked term by term for Eq. (2.13) following major assumptions suggested by (Patankar $1980)$. First, $\phi_{P}$ and $\rho_{P}$ is assumed to prevail over the whole control volume. Thus, the unsteady term on the left hand side (LHS) of Eq. (2.13) can be approximated by

$$
\int_{C V} \rho \frac{\partial \phi}{\partial t} d V=\rho_{P} \Delta V \frac{\partial \phi_{P}}{\partial t}
$$

and the last term on the LHS by

$$
\int_{C V} \phi \frac{\partial}{\partial x_{j}}\left(\rho u_{j}\right) d V=\phi_{P} \int_{C V} \frac{\partial}{\partial x_{j}}\left(\rho u_{j}\right) d V .
$$

Second, the source term in general can be linearized as

$$
S_{\phi}=S_{C}+S_{P} \phi_{P}
$$

and similarly it is assumed to be constant within the control volume. This gives

$$
\int_{C V} S_{\phi} d V=\left(S_{C}+S_{P} \phi_{P}\right) \Delta V \text {. }
$$

Third, uniform distribution is assumed for quantities evaluated at cell faces, such as $\rho u_{j}$.

This implies that, e.g., if $\rho u$ is taken at point $w$ (cf. Figure 2-1 and Figure 2-2) it then prevails over the whole west face. This leads to a further simplification for the expression on the right hand side (RHS) of (2.15). Before doing this, the following set of symbols should be defined to represent the mass flow rate through the faces of the control volume:

$$
\begin{aligned}
& F_{w} \equiv \int_{A_{w}}(\rho u) d A, F_{e} \equiv \int_{A_{e}}(\rho u) d A, \\
& F_{s} \equiv \int_{A_{s}}(\rho v) d A, F_{n} \equiv \int_{A_{n}}(\rho v) d A, \\
& F_{b} \equiv \int_{A_{b}}(\rho w) d A, F_{t} \equiv \int_{A_{t}}(\rho w) d A .
\end{aligned}
$$

Hence, with the assumption made, one may set 


$$
\begin{aligned}
& F_{w}=(\rho u)_{w} A_{w}, F_{e}=(\rho u)_{e} A_{e}, \\
& F_{s}=(\rho v)_{s} A_{s}, F_{n}=(\rho v)_{n} A_{n}, \\
& F_{b}=(\rho w)_{b} A_{b}, F_{e}=(\rho w)_{t} A_{t},
\end{aligned}
$$

where

$$
\begin{aligned}
& A_{w}=A_{e}=\Delta y_{P} \Delta z_{P}, \\
& A_{s}=A_{n}=\Delta z_{P} \Delta x_{P}, \\
& A_{b}=A_{t}=\Delta x_{P} \Delta y_{P} .
\end{aligned}
$$

Using the symbols introduced in Eq. (2.18) and (2.19), the RHS of (2.15) becomes:

$$
\phi_{P} \int_{C V} \frac{\partial}{\partial x_{j}}\left(\rho u_{j}\right) d V=\phi_{P}\left(F_{e}-F_{w}+F_{n}-F_{s}+F_{t}-F_{b}\right) .
$$

In the same manner, the integral total flux may be simplified to

$$
\begin{aligned}
& J_{w}=A_{w}\left(J_{x}\right)_{w}, J_{e}=A_{e}\left(J_{x}\right)_{e}, \\
& J_{s}=A_{s}\left(J_{y}\right)_{s}, J_{n}=A_{n}\left(J_{y}\right)_{n}, \\
& J_{b}=A_{b}\left(J_{z}\right)_{b}, J_{t}=A_{t}\left(J_{z}\right)_{t} .
\end{aligned}
$$

Notice that the integral total fluxes and the mass flow rates are related, i.e.,

$$
\begin{aligned}
& J_{w}=F_{w} \phi_{w}-\left(\Gamma A \frac{\partial \phi}{\partial x}\right)_{w}, J_{e}=F_{e} \phi_{e}-\left(\Gamma A \frac{\partial \phi}{\partial x}\right)_{e}, \\
& J_{s}=F_{s} \phi_{s}-\left(\Gamma A \frac{\partial \phi}{\partial y}\right)_{s}, J_{n}=F_{n} \phi_{n}-\left(\Gamma A \frac{\partial \phi}{\partial y}\right)_{n}, \\
& J_{b}=F_{b} \phi_{b}-\left(\Gamma A \frac{\partial \phi}{\partial z}\right)_{b}, J_{t}=F_{t} \phi_{t}-\left(\Gamma A \frac{\partial \phi}{\partial z}\right)_{t} .
\end{aligned}
$$

Now, substituting Eq. (2.14), (2.17) and (2.21) into Eq. (2.13) yields:

$$
\begin{aligned}
& \rho_{P} \Delta V \frac{\partial \phi_{P}}{\partial t}+J_{e}-J_{w}+J_{n}-J_{s}+J_{t}-J_{b}-\phi_{P}\left(F_{e}-F_{w}+F_{n}-F_{s}+F_{t}-F_{b}\right) \\
& =\left(S_{C}+S_{P} \phi_{P}\right) \Delta V
\end{aligned}
$$

\subsubsection{Patankar's Formulae}

In the end, one would like to set up algebraic equations for each control volume cast in the following form:

$$
a_{P} \phi_{P}=\sum a_{n b} \phi_{n b}+b \text {. }
$$

Here $a$ 's are the coefficients of the corresponding nodal variables, " $n b$ " denotes neighboring nodes, $b$ usually is a constant, can also be a function of $\phi$ evaluated at an old time level.

If Eq. (2.24) is recast into the following, 


$$
\begin{aligned}
\rho_{P} \Delta V \frac{\partial \phi_{P}}{\partial t}= & f_{r h s}=\left(J_{w}-F_{w} \phi_{P}\right)-\left(J_{e}-F_{e} \phi_{P}\right)+\left(J_{s}-F_{s} \phi_{P}\right)-\left(J_{n}-F_{n} \phi_{P}\right) \\
& +\left(J_{b}-F_{b} \phi_{P}\right)-\left(J_{t}-F_{t} \phi_{P}\right)+\left(S_{C}+S_{P} \phi_{P}\right) \Delta V,
\end{aligned}
$$

one is then in a position to use the generalized scheme supplied by (Patankar 1980). This gives

$$
\begin{aligned}
& J_{w}-F_{w} \phi_{P}=a_{W}\left(\phi_{W}-\phi_{P}\right), J_{e}-F_{e} \phi_{P}=a_{E}\left(\phi_{P}-\phi_{E}\right), \\
& J_{s}-F_{s} \phi_{P}=a_{S}\left(\phi_{S}-\phi_{P}\right), J_{n}-F_{n} \phi_{P}=a_{N}\left(\phi_{P}-\phi_{N}\right), \\
& J_{b}-F_{b} \phi_{P}=a_{B}\left(\phi_{B}-\phi_{P}\right), J_{t}-F_{t} \phi_{P}=a_{T}\left(\phi_{P}-\phi_{T}\right) .
\end{aligned}
$$

where the coefficients $a$ 's are determined from the following relations:

$$
\begin{array}{ll}
a_{W}=D_{w} \mathbf{A}\left(\left|\mathrm{Pe}_{w}\right|\right)+\max \left(F_{w}, 0\right), & a_{E}=D_{e} \mathbf{A}\left(\left|\mathrm{Pe}_{e}\right|\right)+\max \left(-F_{e}, 0\right), \\
a_{S}=D_{s} \mathbf{A}\left(\left|\mathrm{Pe}_{s}\right|\right)+\max \left(F_{s}, 0\right), & a_{N}=D_{n} \mathbf{A}\left(\left|\mathrm{Pe}_{n}\right|\right)+\max \left(-F_{n}, 0\right), \\
a_{B}=D_{b} \mathbf{A}\left(\left|\mathrm{Pe}_{b}\right|\right)+\max \left(F_{b}, 0\right), & a_{T}=D_{t} \mathbf{A}\left(\left|\mathrm{Pe}_{t}\right|\right)+\max \left(-F_{t}, 0\right) .
\end{array}
$$

In the above equations, those $D$ 's represent diffusive conductance and have the same unit as the mass flow rate (those $F$ 's). They are defined as

$$
\begin{aligned}
& D_{w}=\frac{\Gamma_{w} A_{w}}{\Delta x_{w}}, D_{e}=\frac{\Gamma_{e} A_{e}}{\Delta x_{e}}, \\
& D_{s}=\frac{\Gamma_{s} A_{s}}{\Delta y_{s}}, D_{n}=\frac{\Gamma_{n} A_{n}}{\Delta y_{n}}, \\
& D_{b}=\frac{\Gamma_{b} A_{b}}{\Delta z_{b}}, D_{t}=\frac{\Gamma_{t} A_{t}}{\Delta z_{t}},
\end{aligned}
$$

$F$ 's are provided in Eq. (2.19), and Pe is the grid Peclet number defined by

$$
\begin{aligned}
& \mathrm{Pe}_{w}=F_{w} / D_{w}, \mathrm{Pe}_{e}=F_{e} / D_{e}, \\
& \mathrm{Pe}_{s}=F_{s} / D_{s}, \mathrm{Pe}_{n}=F_{n} / D_{n}, \\
& \mathrm{Pe}_{b}=F_{b} / D_{b}, \mathrm{Pe}_{t}=F_{t} / D_{t} .
\end{aligned}
$$

Finally, "A" (not symbol $A$ !) denotes a function whose selection will determine a certain discretization scheme. The expressions of the A-function have been summarized in (Patankar 1980), and repeated in following Table 2-1 merely for convenience in a future use.

Table 2-1 The “A” function $A(|P e|)$ for different schemes (Patankar 1980)

\begin{tabular}{|l|l|}
\hline Scheme & Formula for $\mathbf{A}(|\mathrm{Pe}|)$ \\
\hline $1^{\text {st }}$ order upwind & 1 \\
\hline Central differencing & $1-0.5|\mathrm{Pe}|$ \\
\hline Hybrid (Spalding 1972) & $\max (0,1-0.5|\mathrm{Pe}|)$ \\
\hline Power-law & $\max \left(0,(1-0.1|\mathrm{Pe}|)^{5}\right)$ \\
\hline Exponential & $|\mathrm{Pe}| /[\exp (|\mathrm{Pe}|)-1]$ \\
\hline
\end{tabular}




\subsubsection{Final Discretized Equations}

What remains now is the discretization for the unsteady term in Eq. (2.26). This is usually done with the $1^{\text {st }}$ order backward (or implicit) Euler scheme that gives

$$
\rho_{P} \Delta V \frac{\partial \phi_{P}}{\partial t}=\rho_{P} \Delta V \frac{\phi_{P}-\phi_{P}{ }^{o}}{\Delta t}=a_{P}^{o}\left(\phi_{P}-\phi_{P}^{o}\right),
$$

where $\phi_{P}{ }^{\circ}$ denotes the nodal value evaluated at an old time level, and

$$
a_{P}^{o}=\frac{\rho_{P} \Delta V}{\Delta t} \text {. }
$$

By using small time steps, the influence of the $1^{\text {st }}$ order time integration on the overall accuracy of the scheme is negligible (Ferziger and Peric 1996). A higher order scheme for time integration, namely, the $2^{\text {nd }}$ order Adams-Bashforth scheme, is presented in Section 2.5.

With all the manipulations made above, one finally arrives at a fully implicit discretization formula summarized as follows:

$$
a_{P} \phi_{P}=a_{W} \phi_{W}+a_{E} \phi_{E}+a_{S} \phi_{S}+a_{N} \phi_{N}+a_{B} \phi_{B}+a_{T} \phi_{T}+b
$$

where

$$
\begin{aligned}
& a_{P}=a_{C}+a_{P}{ }^{o}+S_{P} \Delta V, \\
& a_{C}=a_{W}+a_{E}+a_{S}+a_{N}+a_{B}+a_{T}, \\
& b=a_{P}^{o} \phi_{P}{ }^{o}+S_{C} \Delta V,
\end{aligned}
$$

all the neighboring node coefficients ( $a_{w}$ etc.) are provided in Eq. (2.28) to (2.30), and $a_{P}{ }^{o}$ is given in Eq. (2.32).

\subsubsection{Solution of Linear Systems}

Let $N$ denote the total number of control volumes, then, there are $N$ algebraic equations (usually linear or linearized) in form of Eq. (2.33), and they together need to be solved simultaneously. The resulting system of equations can be written in matrix form, with the coefficient (or system) matrix having a sparse tri-diagonal block structure.

The solution of the linear equation systems can be accomplished by employing classical iterative matrix solution methods, e.g., the Alternating Direction Implicit (ADI, a line-by-line method), the strongly implicit procedure (SIP, an incomplete LU decomposition method), the conjugate gradient (CG), the conjugate gradient square (CGS), the bi-conjugate gradient stabilized (CGSTAB), and the incomplete conjugate gradient (ICCG) method. For further details of these methods the readers are referred to the original papers (Peaceman and Rachfod 1955; Stone 1968; Sonneveld 1989; Golub and van Loan 1990; Van den Vorst and Sonneveld 1990; Van den Vorst 1992). 


\subsection{Assessment of Five Schemes}

A discretization should be a reasonable approximation to its parent equation, i.e., the continuum partial differential equation. One of the principle characteristics of a discretization scheme is how accurate the numerical solution associated with it can provide. The measure of the accuracy is termed as order of accuracy or order of the method/scheme or simply accuracy. The difference between the discretization equation and its continuum parent equation, called discretization error, is due to neglecting the truncated terms in Taylor series expansion of the discretized equation (e.g. Eq. (2.33)) about node $P$. The truncated terms usually contain factors $\Delta x^{n}$; the power $n$ of $\Delta x$ governs the rate at which the error tends to zero as the grid spacing approaches infinitely small, thus the name order of the scheme or order of accuracy. By default, this order of accuracy should be understood as a local quantity (i.e., confined in CVs) instead of a global one that covers the entire computation domain and is very difficult to estimate (Mitchell and Griffiths 1980).

One can always show by means of Taylor series expansion that the upwind is $1^{\text {st }}$ order accurate, central differencing is $2^{\text {nd }}$ order. Although hybrid scheme and power-law scheme is also $1^{\text {st }}$ order in terms of Taylor series truncation error, they proved in practice to be much more accurate than the $1^{\text {st }}$ order upwind. The exponential scheme is exact for a one-dimensional problem, but its extension to multi-dimensional problem is not justified.

A further study (Hirsch 1988) on the truncation error terms of a certain discretization shows that the error terms with even-order derivatives are responsible for artificially diffusive (or dissipative) results (error in magnitude), and that the error terms with odd-derivatives are associated with the error on the phase of the solution (error in phase). Therefore, the former error is called the diffusion or dissipation error and the latter is called the dispersion or phase error. Numerical dispersion may corrupt large regions of solution with unphysical oscillations, and may lead to divergence of the numerical method. Therefore, the $1^{\text {st }}$ order upwind, which contains a leading error term with a $2^{\text {nd }}$ order derivative, usually produces fairly dissipative results. On the other hand, it is mainly due to the $3^{\text {rd }}$ order derivative contained in the truncation error term that the $2^{\text {nd }}$ order central differencing sometimes generates oscillatory solutions.

Other than the order of accuracy, three mathematical concepts are crucial in assessing a numerical discretization scheme. They are the consistency, convergence, and stability. A method is said to be consistent if the resulting system of algebraic equations from certain discretization procedure is equivalent to the original differential equation as the grid spacing tends to zero. A method is said to be convergent if the numerical solution approaches exact solution as the grid size goes to zero. A method is said to be stable if there is no exponential growth of round-off error as the computation proceed in time. Usually, the convergence property, albeit difficult to establish theoretically, can still be determined with the help of Lax's equivalence theorem (Isaacson and Keller 1967; Richtmyer and Morton 1967; Mitchell and Griffiths 1980). The theorem states that given 
a properly posed linear initial value problem and a consistent discretization method the stability is the necessary and sufficient condition for convergence. In other words, if a method is consistent and stable, it is also convergent.

In a computational fluid dynamics (CFD) simulation that uses a finite volume scheme it has been commonly accepted that the conservativeness, boundedness and transportiveness can serve as alternatives for the more mathematically rigorous concepts of consistency, convergence and stability (Versteeg and Malalasekera 1995). The three "engineering-sensed" properties are designed into all finite volume schemes and have been widely shown to lead to successful CFD simulations. A scheme is said to be conservative if a local conservation of a fluid property $\phi$ is guaranteed for each control volume, thus satisfying a global conservation on the entire solution domain. For the property to be locally conservative, consistent expressions for fluxes through the cell faces between two adjacent control volumes have to be ensured. The boundedness property tells the solution is bounded in the absence of sources. (Scarborough 1958) has shown that a sufficient condition for a convergent iterative method can be expressed as

$$
\frac{\sum\left|a_{n b}\right|}{\left|a_{P}\right|}\left\{\begin{array}{ll}
\leq 1 & \text { for all equations } \\
<1 & \text { for at least one equations }
\end{array}\right. \text {. }
$$

A matrix is diagonally dominant if the above criterion is satisfied. In fact, the diagonal dominance is a desirable feature for satisfying the boundedness. As it is well known that all flow processes involve both convective and diffusive effects. The transportiveness property is thus used to take into account the relative strength of diffusion to convection.

Having defined the three important properties for a numerical scheme, the five schemes derived in the proceeding section is briefly assessed here. A good thing is that all the five schemes presented in this section preserves conservation, because they all use consistent expression to evaluate convective and diffusive fluxes at the $\mathrm{CV}$ faces. Among the five schemes, the $1^{\text {st }}$ order upwind always produces bounded solutions (robust), it also accounts for the direction of the flow (thus has transportiveness property), but its solution is highly diffusive. Although the central differencing is most accurate for multidimensional calculation among the five schemes, it may produce unphysical solution with spurious oscillation when $\mathrm{Pe}>2$, often leading to a divergent solution; also it does not recognize the direction of the flow (no transportiveness). The hybrid scheme and powerlaw are highly stable and also possess the transportiveness property. Reasonably accurate solutions are obtained for steady, quasi-one-dimensional flows (with one main flow direction). However, it is possible that the two schemes exhibit high artificial diffusivity, especially when the grid is not aligned with the main flow direction, e.g., the recirculation flow, leading to a seriously degraded solution (Leonard and Drummond 1995). Although the exponential scheme satisfies the same properties as the hybrid and power-law, but the evaluation of the exponential function is expensive, plus its extension to a $2 \mathrm{D}$ or $3 \mathrm{D}$ calculation is not justifiable.

From the above discussion it can be concluded that the hybrid and power law are preferred when the flow is steady and has one main convective direction. For simulating unsteady, complex flows the central differencing should be used and its grid Peclet number should be monitored. Since hybrid and power law are highly robust, it is also 
possible to use them at a certain percentage merely for the purpose of stabilizing and work on a more accurate explicit discretization, which does not involve a matrix solution. This point will become clear when the discussion develops in the following sections.

\subsection{Implicitness Factors}

In the previous section a fairly general discretization equation have been developed based on a fully implicit approach, meaning that all the unknowns are located at a new time level and should be solved simultaneously. Also recall that for the time integration the $1^{\text {st }}$ order backward Euler has been used, which involves two time levels. A method that involves only two time levels is usually called a two-level method. For a two-level method, the time discretization of the equation,

$$
\rho_{P} \Delta V \frac{\partial \phi_{P}}{\partial t}=f_{r h s},
$$

where $f_{\text {rhs }}$ contains all the spatial discretized terms, and can be evaluated with an arbitrary implicitness in regard with the weight taken from the current and previous time levels.

Introduce an implicitness factor, $\alpha$, whose value is between zero and one. Then, $\alpha$ portion of the RHS of Eq. (2.36) will be evaluated at the new time level while (1- $\alpha$, portion of the RHS at the old time level (denoted by superscript " $O$ "), i.e.,

$$
\rho_{P} \Delta V \frac{\partial \phi_{P}}{\partial t}=\alpha f_{r h s}+(1-\alpha) f_{r h s}^{o} .
$$

A value of $\alpha=1$ corresponds to a fully implicit scheme in which the coefficients derived in the previous section can apply, and $\alpha=0$ and 0.5 correspond to a fully explicit scheme and Crank-Nicolson (C-N) scheme, respectively. In case with the fully explicit scheme, the time integration becomes the $1^{\text {st }}$ order forward (or explicit) Euler method. Also, the $\mathrm{C}-\mathrm{N}$ can be viewed as an equal blending of the first order explicit and implicit Euler schemes. Only as such, a $2^{\text {nd }}$ order temporal accuracy can be achieved for the diffusion terms.

Very often, it is also desirable to have the option of making the implicitness of the convection terms and the diffusion terms separately. For this purpose, one should split $f_{r h s}$ into the following three parts:

$$
f_{\text {rhs }}=f_{\text {conv }}+f_{\text {diff }}+f_{\text {others }} .
$$

By further letting $\alpha_{c o n v}$ and $\alpha_{\text {diff }}$ stand for the implicitness factor of the convection and diffusion terms, respectively, a scheme involving two independent implicitness factors can then be formulated as

$$
\rho_{P} \Delta V \frac{\partial \phi_{P}}{\partial t}=\alpha_{\text {conv }} f_{\text {conv }}+\alpha_{\text {diff }} f_{\text {diff }}+\left(1-\alpha_{\text {conv }}\right) f_{\text {conv }}^{o}+\left(1-\alpha_{\text {diff }}\right) f_{\text {diff }}^{o}+f_{\text {others }} .
$$

It is important to recognize that $f_{\text {others }}$, for the case of a general scalar transport equation, contains the linearized source of the original equation (2.17), and should be treated in a usually way (Cf. previous section). With this modification, the new discretization equation for one $\mathrm{CV}$ can be reformulated by directly using the results obtained in the preceding section. This gives 


$$
\begin{aligned}
a_{P} \phi_{P}= & a_{W} \phi_{W}+a_{E} \phi_{E}+a_{S} \phi_{S}+a_{N} \phi_{N}+a_{B} \phi_{B}+a_{T} \phi_{T} \\
& +\left(1-\alpha_{c o n v}\right) f_{c o n v}^{o}+\left(1-\alpha_{\text {diff }}\right) f_{\text {diff }}^{o}+b
\end{aligned}
$$

where

$$
\begin{aligned}
& a_{P}=a_{C}+a_{P}^{o}+S_{P} \Delta V, \\
& a_{C}=a_{W}+a_{E}+a_{S}+a_{N}+a_{B}+a_{T}, \\
& b=a_{P}^{o} \phi_{P}^{o}+S_{C} \Delta V,
\end{aligned}
$$

and

$$
\begin{aligned}
& a_{W}=\alpha_{\text {diff }} D_{w} \mathbf{A}\left(\left|\mathrm{Pe}_{w}\right|\right)+\alpha_{c o n v} \max \left(F_{w}, 0\right), \\
& a_{E}=\alpha_{\text {diff }} D_{e} \mathbf{A}\left(\left|\mathrm{Pe}_{e}\right|\right)+\alpha_{c o n v} \max \left(-F_{e}, 0\right), \\
& a_{S}=\alpha_{\text {diff }} D_{s} \mathbf{A}\left(\left|\mathrm{Pe}_{s}\right|\right)+\alpha_{c o n v} \max \left(F_{s}, 0\right), \\
& a_{N}=\alpha_{\text {diff }} D_{n} \mathbf{A}\left(\left|\mathrm{Pe}_{n}\right|\right)+\alpha_{c o n v} \max \left(-F_{n}, 0\right), \\
& a_{B}=\alpha_{\text {diff }} D_{b} \mathbf{A}\left(\left|\mathrm{Pe}_{b}\right|\right)+\alpha_{c o n v} \max \left(F_{b}, 0\right), \\
& a_{T}=\alpha_{\text {diff }} D_{t} \mathbf{A}\left(\left|\mathrm{Pe}_{t}\right|\right)+\alpha_{\text {conv }} \max \left(-F_{t}, 0\right),
\end{aligned}
$$

with D's and Pe's given in Eq. (2.29) and (2.30) respectively. The expression for $f_{c o n v}^{o}$ and $f_{\text {diff }}^{o}$ can also be deduced from Eq. (2.23) and (2.24), i.e.,

$$
f_{\text {conv }}^{o}=\left(1-\alpha_{\text {conv }}\right)\left[\begin{array}{l}
\left(F_{w} \phi_{w}-F_{e} \phi_{e}+F_{s} \phi_{s}-F_{n} \phi_{n}+F_{b} \phi_{b}-F_{t} \phi_{t}\right) \\
+\phi_{P}\left(F_{e}-F_{w}+F_{n}-F_{s}+F_{t}-F_{b}\right)
\end{array}\right]^{o},
$$

and

$$
f_{\text {diff }}^{o}=\left(1-\alpha_{\text {diff }}\right)\left[\begin{array}{l}
\left(\Gamma A \frac{\delta \phi}{\delta x}\right)_{e}-\left(\Gamma A \frac{\delta \phi}{\delta x}\right)_{w}+\left(\Gamma A \frac{\delta \phi}{\delta y}\right)_{n} \\
-\left(\Gamma A \frac{\delta \phi}{\delta y}\right)_{s}+\left(\Gamma A \frac{\delta \phi}{\delta z}\right)_{t}-\left(\Gamma A \frac{\delta \phi}{\delta z}\right)_{b}
\end{array}\right]^{o} .
$$

In Eq. (2.44) the operator $\delta$ is used to denote certain discretized approximation to the continuum partial derivative. Those terms evaluated at the new time level may be called implicit discretization and those at the old time level(s) explicit discretization. It should be remarked that (i) the second term in the square bracket of Eq. (2.43) usually is zero for the incompressible flow, and (ii) those values or derivatives evaluated at cell faces (Eq. (2.43) and (2.44)) should be approximated using the same discretization scheme as being used for the implicit part in general; however, it is also possible to calculate these quantities by employing a higher order scheme, which is dealt with in Section 2.6. 


\subsection{Time Integration with $2^{\text {nd }}$ Order Adams- Bashforth}

As already been explained in the previous sections, the backward Euler is a $1^{\text {st }}$ order twolevel scheme for time discretization. To simulate unsteady flows, as is the case with unsteady RANS (Reynolds-averaged Navier-Stokes), large-eddy simulation (LES) and direct numerical simulation (DNS), a more accurate time discretization is desired if time step is not kept very small (Choi and Moin 1994; Manson et al. 1996; Manson and Wallis 1997; Smith and Celik 1999). The accuracy of the time advancement can be improved by either incorporating more time levels, called Adams-methods, or inserting more evaluation data points within one time step, called Runge-Kutta (RK) methods. Description of these two families of methods can be found in many textbooks (Conte and de Boor 1980; Ferziger 1981; Burden and Faires 1989; Press et al. 1992; Chapra and Canale 1998). Adams methods and RK methods are originally devised in the solution of ordinary differential equations (ODE), but their idea is also applicable to the timedependent solution of partial differential equations (PDE). In Adams method two variants exist, namely, the explicit Adams-Bashforth (AB) method and implicit Adams-Moulton (AM) method. In what follows the concept of those families of methods are briefly described; popular formulae in each category are summarized. For simulations performed in the later chapters the $2^{\text {nd }}$ order $A B$ method shall be used whenever possible; the reason will become clear when the discussion develops in this section.

Without loss of generality, an unsteady partial differential equation of the form

$$
\frac{\partial \phi}{\partial \mathrm{t}}=f(t, \phi),
$$

where $\phi$ is a function of time and space, is first simplified to an ordinary differential equation

$$
\frac{d \phi}{\mathrm{dt}}=f(t, \phi),
$$

with the assumption that $\phi$ is a function of $t$ only; however, the formulae derived later in this section should be justifiable for the application in an unsteady problem like Eq. (2.45).

\subsubsection{Adams Methods}

The basic idea of Adams methods is to fit a polynomial to the time derivative using a number of points at different time levels. Let superscript $n$ denote the time level. By using Maclaurin series of $\phi$ about $\phi^{n}$ and letting $\Delta t$ denote a constant time step size, variable $\phi$ at time level $n+1$ can be expressed as

$$
\phi^{n+1}=\phi\left(t_{n}+\Delta t\right)=\phi^{n}+\phi_{t}^{n} \Delta t+\frac{1}{2} \phi_{t t}^{n}(\Delta t)^{2}+\frac{1}{6} \phi_{t t t}^{n}(\Delta t)^{3}+\ldots,
$$

where subscript $t$ denote partial derivative with respect to time (not top face here!), and by Eq. (2.46)

$$
\phi_{t}^{n}=f\left(t_{n}, \phi^{n}\right)
$$


The higher order derivatives are then approximated by backward differences using Eq. (2.48):

$$
\begin{aligned}
& \phi_{t t}^{n}=\frac{\phi_{t}^{n}-\phi_{t}^{n-1}}{\Delta t}=\frac{f\left(t_{n}, \phi^{n}\right)-f\left(t_{n-1}, \phi^{n-1}\right)}{\Delta t}, \\
& \phi_{t t}^{n}=\frac{\phi_{t t}^{n}-\phi_{t t}^{n-1}}{\Delta t}=\frac{f\left(t_{n}, \phi^{n}\right)-2 f\left(t_{n-1}, \phi^{n-1}\right)+f\left(t_{n-2}, \phi^{n-2}\right)}{\Delta t}
\end{aligned}
$$

and so on. Substituting Eq. (2.48) and (2.49) into (2.47) and truncating the higher order terms accordingly, the $1^{\text {st }}$ (forward Euler), $2^{\text {nd }}$ and $3^{\text {rd }}$ order Adams-Bashforth formula are obtained, respectively:

$$
\begin{gathered}
\phi^{n+1}=\phi^{n}+\Delta t\left[f\left(t_{n}, \phi^{n}\right)\right], \\
\phi^{n+1}=\phi^{n}+\frac{\Delta t}{2}\left[3 f\left(t_{n}, \phi^{n}\right)-f\left(t_{n-1}, \phi^{n-1}\right)\right], \\
\phi^{n+1}=\phi^{n}+\frac{\Delta t}{12}\left[23 f\left(t_{n}, \phi^{n}\right)-16 f\left(t_{n-1}, \phi^{n-1}\right)+5 f\left(t_{n-2}, \phi^{n-2}\right)\right] .
\end{gathered}
$$

Note that Adams-Bashforth method is explicit in nature. Its implicit counterpart is called Adams-Moulton method, in which the approximation of derivatives involves points at time step $n+1$. Its $1^{\text {st }}$ order (backward Euler), $2^{\text {nd }}$ (also called trapezoid rule) and $3^{\text {rd }}$ order formulae are given as

$$
\begin{gathered}
\phi^{n+1}=\phi^{n}+\Delta t\left[f\left(t_{n+1}, \phi^{n+1}\right)\right], \\
\phi^{n+1}=\phi^{n}+\frac{\Delta t}{2}\left[f\left(t_{n+1}, \phi^{n+1}\right)+f\left(t_{n}, \phi^{n}\right)\right], \\
\phi^{n+1}=\phi^{n}+\frac{\Delta t}{12}\left[5 f\left(t_{n+1}, \phi^{n+1}\right)+8 f\left(t_{n}, \phi^{n}\right)-f\left(t_{n-1}, \phi^{n-1}\right)\right] .
\end{gathered}
$$

It is also a common practice that two families of methods are combined to produce some mixed methods. For example, one may use $2^{\text {nd }}$ order Adams-Bashforth method as a predictor, and apply $3^{\text {rd }}$ order Adams-Moulton method as a corrector.

\subsubsection{Runge-Kutta Methods}

Different from Adams methods, the Runge-Kutta methods do not need data points from less than time level $t_{n}$; instead, they use several intermediate points between $t_{n}$, and $t_{n+l}$, and special slope averaging schemes to determine $\phi^{n+1}$, which can be written in the general form of

$$
\phi^{n+1}=\phi^{n}+\Delta t \sum_{l} \alpha_{l} k_{l} .
$$

Here $k$ 's are the slopes evaluated at intermediate points and $\alpha$ 's are the relative weight of the corresponding slope; the summation of all $\alpha$ 's is unity.

A $2^{\text {nd }}$ order RK method will be of the form

$$
\phi^{n+1}=\phi^{n}+\Delta t\left(\alpha_{1} k_{1}+\alpha_{2} k_{2}\right),
$$

where $k_{1}=f\left(t, \phi^{n}\right)$ and

$$
k_{2}=f\left(t_{n}+\beta_{1} \Delta t, \phi^{n}+\beta_{2} \Delta t k_{1}\right) .
$$


To define the scheme it is necessary to determine the constants $\alpha_{1}, \alpha_{2}$ and $\beta_{1}, \beta_{2}$. Taylor series expansion of $k_{2}$ about $t_{n}$ and $\phi_{n}$ gives

$$
\begin{aligned}
k_{2} & =f\left(t_{n}, \phi^{n}\right)+\left.\left(\beta_{1} \Delta t\right) \frac{\partial f}{\partial t}\right|_{t_{n}, \phi^{n}}+\left.\left(\beta_{2} \Delta t k_{1}\right) \frac{\partial f}{\partial \phi}\right|_{t_{n}, \phi^{n}}+O\left(\Delta t^{2}\right), \\
& =k_{1}+\left(\beta_{1} \Delta t\right) f_{t}^{n}+\left(\beta_{2} \Delta t k_{1}\right) f_{\phi}^{n}+O\left(\Delta t^{2}\right)
\end{aligned}
$$

where $f_{\phi}^{n}$ denotes partial derivative of $f$ with respect to $\phi$ evaluated at $\left(t_{n}, \phi^{n}\right)$, and similarly for $f_{t}^{n}$. Substitute Eq. (2.59) into (2.57) yields

$$
\begin{aligned}
\phi^{n+1} & =\phi^{n}+\Delta t\left[\alpha_{1} k_{1}+\alpha_{2}\left(k_{1}+\left(\beta_{1} \Delta t\right) f_{t}^{n}+\left(\beta_{2} \Delta t k_{1}\right) f_{t}^{n}\right)\right]+O\left(\Delta t^{3}\right) \\
& =\phi^{n}+\Delta t\left(\alpha_{1}+\alpha_{2}\right) k_{1}+(\Delta t)^{2}\left(\alpha_{2} \beta_{1} f_{t}^{n}+\alpha_{2} \beta_{2} k_{1} f_{t}^{n}\right)+O\left(\Delta t^{3}\right)
\end{aligned} .
$$

At the same time, expanding $\phi^{\text {n+1 }}$ about $t_{n}$ using Taylor series (see Eq. (2.47)) and recognizing the following relation due to train rule

$$
\phi_{t t}=\frac{D f(t, \phi)}{D t}=\frac{\partial f}{\partial t}+\frac{\partial f}{\partial \phi} \frac{d \phi}{d t}=\frac{\partial f}{\partial t}+k_{1} \frac{\partial f}{\partial \phi}
$$

gives

$$
\phi^{n+1}=\phi\left(t_{n}+\Delta t\right)=\phi^{n}+\Delta t \phi_{t}^{n}+\frac{(\Delta t)^{2}}{2}\left(\frac{\partial f}{\partial t}+k_{1} \frac{\partial f}{\partial \phi}\right)+O\left(\Delta t^{3}\right) .
$$

A term-by-term comparison of Eq. (2.60) and (2.62) gives

$$
\begin{aligned}
& \alpha_{1}+\alpha_{2}=1, \\
& \alpha_{2} \beta_{1}=\frac{1}{2}, \\
& \alpha_{2} \beta_{2}=\frac{1}{2} .
\end{aligned}
$$

The system consists of four equations with four unknowns. If any one of the parameters is specified, the system can be determined. For example, letting $\beta_{1}=1$ leads to one of the popular version of $2^{\text {nd }}$ order RK method, i.e.,

$$
\begin{aligned}
& k_{1}=f\left(t_{n}, \phi^{n}\right), \\
& k_{2}=f\left(t_{n+1}, \phi^{n}+\Delta t k_{1}\right), \\
& \phi^{n+1}=\phi^{n}+\frac{\Delta t}{2}\left(k_{1}+k_{2}\right),
\end{aligned}
$$

or prescribing $\alpha_{1}=1$ gives an alternative $2^{\text {nd }}$ order RK, i.e.,

$$
\begin{aligned}
& k_{1}=f\left(t_{n}, \phi^{n}\right), \\
& k_{2}=f\left(t_{n+1 / 2}, \phi^{n}+\frac{\Delta t}{2} k_{1}\right), \\
& \phi^{n+1}=\phi^{n}+\Delta t k_{2} .
\end{aligned}
$$

Following similar procedure, any order of RK method can be derived. A $3^{\text {rd }}$ order RK formula is given by 


$$
\begin{aligned}
& k_{1}=f\left(t_{n}, \phi^{n}\right), \\
& k_{2}=f\left(t_{n+1 / 2}, \phi^{n}+\frac{\Delta t}{2} k_{1}\right), \\
& k_{3}=f\left(t_{n+1}, \phi^{n}-\Delta t k_{1}+2 \Delta t k_{2}\right), \\
& \phi^{n+1}=\phi^{n}+\frac{\Delta t}{6}\left(k_{1}+4 k_{2}+k_{3}\right),
\end{aligned}
$$

and a $4^{\text {th }}$ order $\mathrm{RK}$ is given by

$$
\begin{aligned}
& k_{1}=f\left(t_{n}, \phi^{n}\right), \\
& k_{2}=f\left(t_{n+1 / 2}, \phi^{n}+\frac{\Delta t}{2} k_{1}\right), \\
& k_{3}=f\left(t_{n+1 / 2}, \phi^{n}+\frac{\Delta t}{2} k_{2}\right), \\
& k_{4}=f\left(t_{n+1}, \phi^{n}+\Delta t k_{3}\right), \\
& \phi^{n+1}=\phi^{n}+\frac{\Delta t}{6}\left(k_{1}+2 k_{2}+2 k_{3}+k_{4}\right) .
\end{aligned}
$$

\subsubsection{Adams Methods vs. Runge-Kutta Methods}

In general, a method is called stable if it produces bounded solution. An unstable method is due to the fact that any errors (e.g. numerical errors, round-off errors etc.) introduced into the computation are amplified as computation progresses. It is known that for the forward Euler method, stability condition requires:

$$
\left|1+\Delta t \frac{\partial f(t, \phi)}{\partial \phi}\right|<1 \text {. }
$$

For a real valued function $f$, as in the present case, Eq. (2.68) reduces to

$$
\left|\Delta t \frac{\partial f(t, \phi)}{\partial \phi}\right|<2 \text {. }
$$

Therefore, for explicit Euler the time step $\Delta \mathrm{t}$ cannot be too large and its size should be monitored by Eq. (2.69). On the other hand, the backward Euler method is unconditionally stable, meaning that they produced bounded solution as long as the underlying exact solution is also bounded, i.e., $\partial f / \partial \phi<0$. Performing some mathematical analysis (Conte and de Boor 1980; Burden and Faires 1989) one can show that the Adams family of methods offers relatively good stability properties. However, in practice all multi-step methods will exhibit some instability for some range of step sizes; and it can be further shown that the AM implicit methods have regions of stability that are more than ten times larger than those for the $\mathrm{AB}$ methods of the same order (Conte and de Boor 1980). Also, it is well agreed that the Runge-Kutta method is more stable than the Adams method of the same order (Ferziger and Peric 1996).

The Adams methods mainly offer two advantages. Above all, they are easy to construct and implement. It is the case especially for the explicit Adams-Bashforth 
methods since the implicit coefficients in a discretized equation would not be changed, and only minimal modification for the explicit flux is necessary. Second, the Adams methods require only one evaluation per time step. This advantage becomes important when intensive computation is heavily involved, e.g., calculation for an unsteady threedimensional flow; the time gain makes it a worthwhile choice.

But, as it can be seen from Eq. (2.50) to (2.52) that $1^{\text {st }}, 2^{\text {nd }}$ and $3^{\text {rd }}$ order $A B$ schemes require data points from two, three and four time levels, respectively. This makes a higher order Adams scheme expensive because, for example, the $3^{\text {rd }}$ order one will need doubled data storage as compared to the $1^{\text {st }}$ order Euler. For this reason, for the solution of partial differential equation only the low order methods are considered. Another drawback is that the Adams methods are not self-starting, meaning that at the beginning of time advancement other methods have to be used, and they will start to kick in once the required number of time levels is available. But this shall not be a big problem in practical use because one usually starts with a stable scheme, say, the $1^{\text {st }}$ order implicit Euler, and with a small (even a big) time step to achieve some preliminary flow field, which serves as the new initial condition for a more accurate run, e.g., the $2^{\text {nd }}$ order Adams-Bashforth method.

On the other hand, the RK methods can start right from the initial stage (selfstarting), and a RK method is more stable and slightly accurate than an Adams method of the same order (Ferziger and Peric 1996). But at the same time, the advantage of the Adams methods also becomes the disadvantages in RK. In particular, at each time step, for a RK scheme of order $n$, the derivatives need to be evaluated $n$ times, making the scheme cumbersome and potentially expensive.

Clearly, a perfect scheme would be to use two-level RK in the initial stage, and then switching to a combined Adams method, say, the $2^{\text {nd }}$ order $A B$ as predictor and $3^{\text {rd }}$

order AM as corrector. For the research code developed in the present study the $2^{\text {nd }}$ order $\mathrm{AB}$ is preferred because it features simplicity, efficiency and a desired order of accuracy. Also note that all methods will produce good solutions so long as time step is small (Ferziger and Peric 1996).

\subsection{Higher Order Schemes with $3^{\text {rd }}$ order QUICK and $4^{\text {th }}$ order CD}

At the end of Section 2.4 it is mentioned that the explicit discretization could be either consistent with the implicit discretization, or use a more accurate discretization scheme. Note that the implicit discretization is directly linked to the resulting matrix structure. For example, the formula derived in Section 2.2 will yield a band-structured matrix that can be solved with some efficient routines such as ADI, SIP and CGSTAB. However, when one increases the order of the method for the implicit part, a larger computational molecular (or coefficients structure) will result in the discretized equation, and the produced system of equations would then be very expensive to solve. On the other hand, the relatively low-order implicit discretizations with a maximum order being two are 
fairly robust and they find wide applicability especially in the RANS (Reynolds-averaged Navier-Stokes) simulations. Further, the robustness of the implicit part can be useful in stabilizing a high-order explicit scheme by way of adjusting the implicitness factors (cf. Section 2.4). Given these, in the current study high-order spatial discretization schemes are implemented preferably for explicit part only. Also, it is well known that the $2^{\text {nd }}$ order central differencing is usually good enough for the diffusion terms, a high-order scheme therefore refers to the convection terms only. This default terminology shall be frequently used in this text. The methods to be considered herein are the $3^{\text {rd }}$ order QUICK (quadratic upwind interpolation for convective kinematics) due to (Leonard 1979), and the $4^{\text {th }}$ order central differencing $(\mathrm{CD})$.

Remark that the central distinction among all schemes is that how the cell face values are evaluated. The $1^{\text {st }}$ order upwind takes only a first upstream node and nothing from downstream, while the $2^{\text {nd }}$ order central takes one node on either side. Following this line of thinking, a natural improvement will be to take two upwind nodes and one downstream node, i.e., the QUICK scheme. In this way not only one more node is involved but also the direction of the flow is considered (transportiveness). The scheme can be best understood by considering a one-dimensional cell displayed in Figure 2-3.

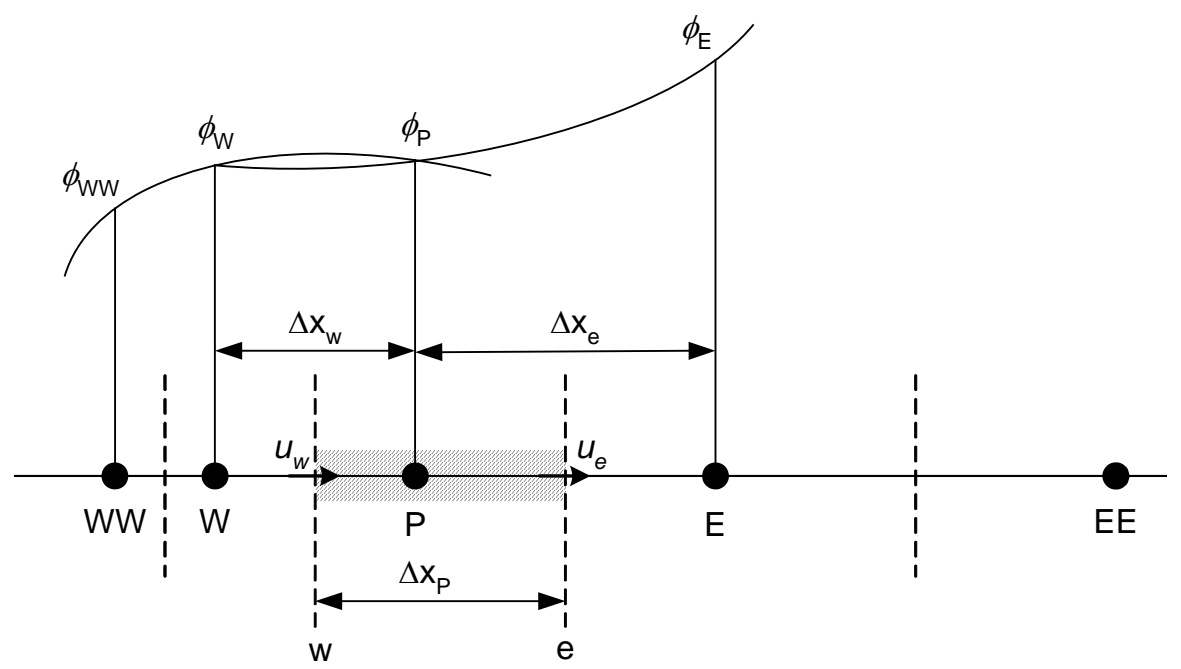

Figure 2-3 Quadratic interpolation used in QUICK scheme

Here the same notation as those in Section 2.2 is used. In addition, "WW" denotes a further node west of node "W", and "EE" denote a further node east of node "E". When $u_{w}>0$, one takes two upstream nodes "WW", "W" and one downstream node "P" to fit a quadratic polynomial which is used to evaluate the face value $\phi_{w}$; when $u_{w}<0$, one then picks nodes "W", "P" and "E" for the quadratic interpolation of $\phi_{w}$. It works similarly for $\phi_{e}$ and face values in other directions. On uniform grid this practice gives, for example for the west and east face,

$$
\phi_{w}= \begin{cases}\frac{1}{8}\left(-\phi_{W W}+6 \phi_{W}+3 \phi_{P}\right) & u_{w}>0, \\ \frac{1}{8}\left(3 \phi_{W}+6 \phi_{P}-\phi_{E}\right) & u_{w}<0,\end{cases}
$$


and

$$
\phi_{e}= \begin{cases}\frac{1}{8}\left(-\phi_{W}+6 \phi_{P}+3 \phi_{E}\right) & u_{e}>0, \\ \frac{1}{8}\left(3 \phi_{P}+6 \phi_{E}-\phi_{E E}\right) & u_{e}<0 .\end{cases}
$$

An extension of the formula to other directions is straightforward and abridged here. A formula base on non-uniform Cartesian grids can also be found in (Ferziger and Peric 1996). In an explicit discretization it should be understood that those nodal values used in the interpolation come from an old time level.

The $4^{\text {th }}$ order central differencing, on the contrary, does not take into account the flow direction. It simply uses two nodes on both upstream side and downstream side for a cubic polynomial interpolation. This gives the following formulae on a uniform grid:

$$
\phi_{w}=\frac{1}{16}\left(-\phi_{W W}+9 \phi_{W}+9 \phi_{P}-\phi_{E}\right),
$$

and

$$
\phi_{e}=\frac{1}{16}\left(-\phi_{W}+9 \phi_{P}+9 \phi_{E}-\phi_{E E}\right)
$$

For non-uniform grid, the complete expression is cumbersome and Lagrangian polynomial may be used for that purpose.

\subsection{Fractional Step (Projection) Method}

The fractional step (projection) method presented here follows closely the one proposed by (Kim and Moin 1985). A brief review of this method can also be found in the text of (Ferziger and Peric 1996). In essence, the method originates from the idea of operator splitting, i.e., splitting the solution procedure of Navier-Stokes equations into two steps: a prediction step followed by a projection step. Unlike the traditional SIMPLE (semiimplicit method for pressure-linked equations) class of N-S solution procedure (Patankar 1980), the fractional step approach uncouples the solution of velocity field from the solution of pressure, and does not ask for inner iterations within one time step. The continuity of the velocity field is ensured by the projection step.

Suppose the discretized equation has the following form

$$
\frac{(\rho \mathbf{u})^{n+1}-(\rho \mathbf{u})^{n}}{\Delta t}=\mathbf{c o n v}+\mathbf{d i f f}-\nabla p,
$$

where $\mathbf{u}$ is the velocity field, $p$ denotes pressure or a pressure-like variable (pseudopressure), conv and diff represent the convection and diffusion terms in the N-S equations, respectively, the superscripts indicate the time level. For a variable density flow, one wishes to uncouple density from the solution of velocity. One way is to assume the density change within the time step is negligible, and let $\rho^{n+1}=\rho^{n}$; once the velocity field is advanced to a new time level, density could be updated accordingly. Thus, one writes, 


$$
\rho^{n} \frac{\mathbf{u}^{n+1}-\mathbf{u}^{n}}{\Delta t}=\mathbf{c o n v}+\operatorname{diff}-\nabla p
$$

For an incompressible flow with constant density, as is in the present study, Eq. (2.74) and (2.75) are equivalent.

In the prediction step, pressure term is ignored, only a tentative velocity field, $\hat{\mathbf{u}}$, is solved:

$$
\rho^{n} \frac{\hat{\mathbf{u}}-\mathbf{u}^{n}}{\Delta t}=\mathbf{c o n v}+\operatorname{diff} .
$$

The action of the pressure is taken into account in the projection step, i.e.,

$$
\rho^{n} \frac{\mathbf{u}^{n+1}-\hat{\mathbf{u}}}{\Delta t}=-\nabla p
$$

Eq. (2.77) can be rewritten as

$$
\mathbf{u}^{n+1}=\hat{\mathbf{u}}-\frac{\Delta t}{\rho^{n}} \nabla p
$$

Note that the velocity field $\hat{\mathbf{u}}$ obtained after the prediction step generally does not preserve continuity. The very role of the pressure appearing in the projection step (2.77) is to correct $\hat{\mathbf{u}}$ in such a way that continuity of the final velocity field at the new time level is satisfied. That is, the tentative velocity field $\hat{\mathbf{u}}$ is projected onto a divergence-free field $\mathbf{u}^{n+1}$.

To find the pressure (or pseudo-pressure) that will turn $\hat{\mathbf{u}}$ into a divergence-free field $\mathbf{u}^{n+1}$, one takes divergence of both sides of Eq. (2.77). This yields, for the case of an incompressible flow,

$$
\nabla^{2} p=\frac{1}{\Delta t} \nabla \cdot(\rho \hat{\mathbf{u}}) \cong \frac{1}{\Delta t} \frac{\delta\left(\rho^{n} \hat{u}_{i}\right)}{\delta x_{i}},
$$

and the case of a variable density flow,

$$
\nabla^{2} p=\frac{1}{\Delta t}\left[\nabla \cdot(\rho \hat{\mathbf{u}})+\frac{\partial \rho}{\partial t}\right] \cong \frac{1}{\Delta t}\left[\frac{\delta\left(\rho^{n} \hat{u}_{i}\right)}{\delta x_{i}}+\frac{\partial \rho}{\partial t}\right],
$$

where $\delta$ here is the discrete representation of the gradient operator. Equations (2.79) and (2.80) are of Poisson type, and they are usually solved with either Neumann or periodic boundary conditions. It has been known that, if pressure boundary conditions are either periodic or homogeneous Neumann, the differencing equation system of the Poisson equation will be singular, and it will give either no solution, or multiple solutions. To ensure a unique solution, the sum of the RHS of the discrete equations over all cells should be zero, or have a numerical error less than $10^{-6}$ (Su et al. 2001).

To summarize the procedure, one first seeks a predicted velocity field, $\hat{\mathbf{u}}$, via Eq. (2.76), then solve the pressure field with Eq. (2.79) or (2.80), and finally correct $\hat{\mathbf{u}}$ by Eq. (2.78). These steps are also depicted in a flow chart (Figure 2-4). 
A variant of the projection method, known as projection-correction method (Hirsch 1990) exists, in which pressure term is not removed in the prediction step. In this case, a pressure correction field, instead of pressure or pseudo-pressure, is solved. Once the pressure correction is available, not only velocity but also pressure is then updated.

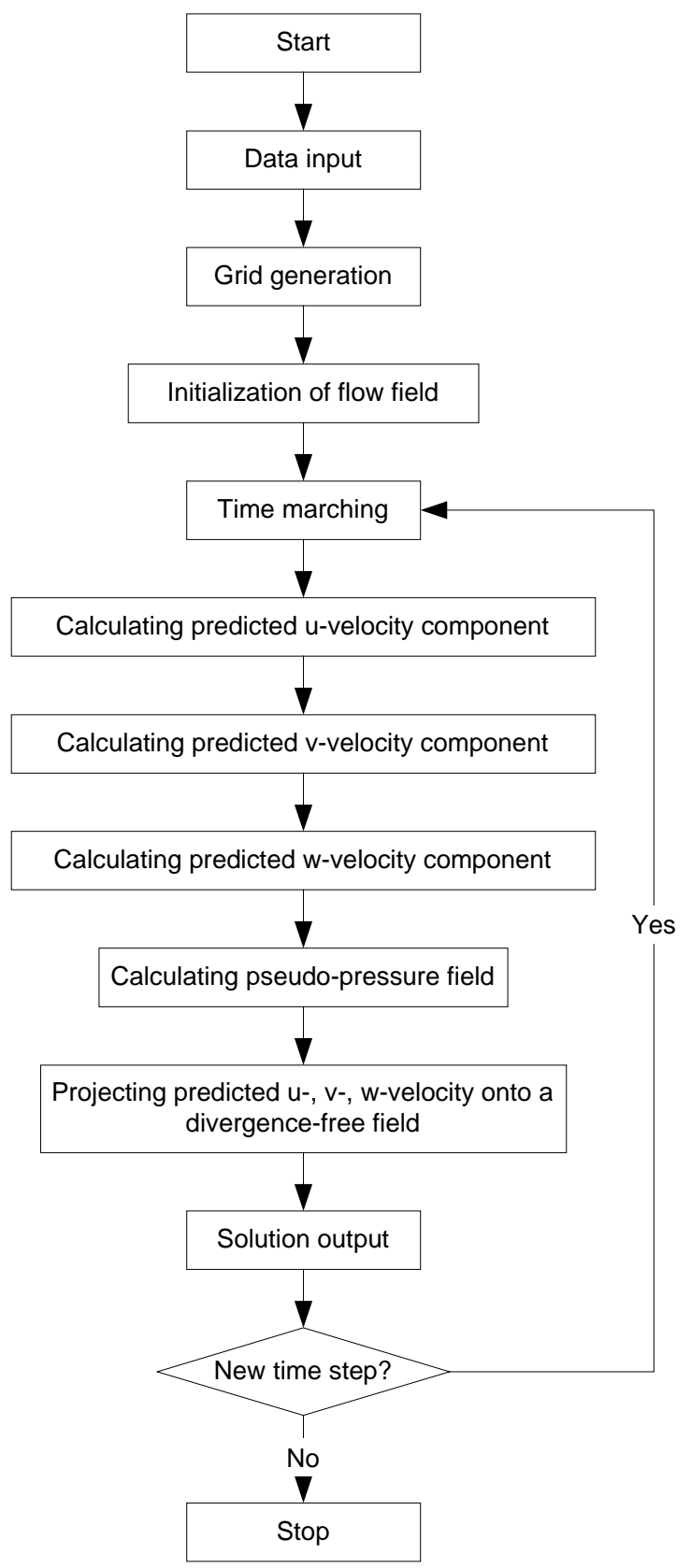

Figure 2-4 Flow chart of fractional step (projection) method for the solution of Navier-Stokes equations. 


\subsection{Discretization of Pressure Equation and $4^{\text {th }}$ Order Deferred Correction}

In this section a brief presentation of the discretization for the pressure equation (2.80) is made. Similar assumptions and simplifications as addressed in Section 2.2 should apply here as well.

As usual, Eq. (2.80) is first integrated over a Cartesian control volume to yield an integral form. The resulting first derivatives on the LHS are assumed to be constant over the cell face and can be discretized with the $2^{\text {nd }}$ order central differencing. This gives:

$$
\begin{aligned}
& \int_{A_{x}}\left(\left.\frac{\partial p}{\partial x}\right|_{e}-\left.\frac{\partial p}{\partial x}\right|_{w}\right) d A+\int_{A_{y}}\left(\left.\frac{\partial p}{\partial y}\right|_{n}-\left.\frac{\partial p}{\partial y}\right|_{s}\right) d A+\int_{A_{z}}\left(\left.\frac{\partial p}{\partial z}\right|_{t}-\left.\frac{\partial p}{\partial z}\right|_{b}\right) d A= \\
& A_{e} \frac{p_{E}-p_{P}}{\Delta x_{e}}-A_{w} \frac{p_{P}-p_{W}}{\Delta x_{w}}+A_{n} \frac{p_{N}-p_{P}}{\Delta y_{n}}-A_{s} \frac{p_{P}-p_{S}}{\Delta y_{s}}+A_{t} \frac{p_{T}-p_{P}}{\Delta z_{t}}-A_{b} \frac{p_{P}-p_{B}}{\Delta z_{b}},
\end{aligned}
$$

where $A_{x}, A_{y}$ and $A_{z}$ denote the areas of the cell faces aligned in $x, y$ and $z$ directions respectively. With proper assumptions, the integral form of the RHS of Eq. (2.80)turns into terms expressed at cell faces, i.e.,

$$
\begin{aligned}
& \frac{1}{\Delta t}\left\{\begin{array}{l}
\int_{A_{x}}\left[(\rho \hat{u})_{e}-(\rho \hat{u})_{w}\right] d A+\int_{A_{y}}\left[(\rho \hat{v})_{n}-(\rho \hat{v})_{s}\right] d A \\
+\int_{A_{z}}\left[(\rho \hat{w})_{t}-(\rho \hat{w})_{b}\right] d A+\int_{C V} \frac{\partial \rho}{\partial t} d V
\end{array}\right\} \\
& =\frac{1}{\Delta t}\left[\begin{array}{l}
(\rho \hat{u})_{e} A_{e}-(\rho \hat{u})_{w} A_{w}+(\rho \hat{v})_{n} A_{n}-(\rho \hat{v})_{s} A_{s} \\
+(\rho \hat{w})_{t} A_{t}-(\rho \hat{w})_{b} A_{b}+V \frac{\partial \rho}{\partial t}
\end{array}\right] .
\end{aligned}
$$

Rearranging terms and defining the following coefficients

$$
\begin{aligned}
& a_{W}=-\frac{A_{w}}{\Delta x_{w}}, a_{E}=-\frac{A_{e}}{\Delta y_{e}}, \\
& a_{S}=-\frac{A_{s}}{\Delta y_{s}}, a_{N}=-\frac{A_{n}}{\Delta y_{n}}, \\
& a_{B}=-\frac{A_{b}}{\Delta z_{b}}, a_{T}=-\frac{A_{t}}{\Delta z_{t}}, \\
& a_{P}=a_{W}+a_{E}+a_{S}+a_{N}+a_{B}+a_{T},
\end{aligned}
$$

one arrives at the discretized equation for the pressure

$$
a_{P} p_{P}=a_{W} p_{W}+a_{E} p_{E}+a_{S} p_{S}+a_{N} p_{N}+a_{B} p_{B}+a_{T} p_{T}+b,
$$

where $b$ is given as the negative of the RHS of Eq. (2.82) evaluated using the approximate velocity field $\hat{u}, \hat{v}$ and $\hat{w}$.

The order of the above CD discretization is $2^{\text {nd }}$ order if the grid is uniform. However, there will be accuracy loss if it is applied to a non-uniform grid. To remedy this deficiency one may employ the so-called deferred correction method (Khosla and Rubin 
1974). In that, one keeps a lower order implicit discretization, and adjusts it into a higher order scheme by explicitly eliminating the lower-order fluxes and then adding the explicit higher-order fluxes evaluated from the previous iteration or time step. For example, at the west cell face, one can write

$$
F_{w}=F_{w}^{L}+\left(F_{w}^{H}-F_{w}^{L}\right)^{o}
$$

where $F_{w}^{L}$ stands for the flux approximation by some lower order scheme and $F_{w}^{H}$ is the higher order approximation. Due to the elliptic nature of the Poison equation, one should use the $4^{\text {th }}$ order central differencing to approximate the explicit flux $F_{w}^{H}$. The formula presented in Section 2.6 for uniform grid is also applicable to this case. A key benefit of this method is the accuracy gain, while retaining the banded matrix structure represented by Eq. (2.84). One has to bear in mind that a higher order approximation does not necessarily guarantee a more accurate solution on any single grid; high accuracy is achieved only when the grid is sufficiently fine to capture the essential details of the solution (Ferziger and Peric 1996). 


\section{Chapter 3 Verification of Flow Solver}

In this chapter the aim is to verify a self-developed finite-volume-method based NavierStokes solver that solves transient, both two-dimensional (2D) and three-dimensional (3D) flow problems. The implementation of the solver follows the numerical methods presented in the preceding chapter. The solver will be tested on various benchmark laminar flow problems. Obtained numerical solutions will be compared with analytical, experimental or other widely accepted calculations. The laminar flow cases considered herein are Couette-type flows, injection and suction flow, laminar channel flow, Taylor's vortex, shear-layer, flow past a backward-facing step and lid-driven square cavity. In all situations the flow is assumed to be steady state, 2D or quasi-2D and with constant properties, i.e., constant density and viscosity. Note that although the flow problems can be simplified to a quasi $1 \mathrm{D}$ or $2 \mathrm{D}$ ones, the developed solver itself is $3 \mathrm{D}$. That is, all the three dimensions enter the calculation but with the solution exhibiting proper 1D or 2D behaviors. Pressure has to be solved in the numerical procedure to enforce continuity. In the case of an imposed pressure gradient (e.g. the Poiseuille flow) it is also possible to add this constant gradient into the momentum source and solve a pressure-like variable to adjust continuity. It is agreed in this chapter that the streamwise direction always refers to the $\mathrm{x}$-direction, and the cross-stream direction corresponds to the y-direction. Also note that $\mathrm{u}$-velocity is always aligned with the $\mathrm{x}$-direction and v-velocity with the $\mathrm{y}$-direction.

\subsection{Couette Flow Between a Fixed and a Moving Plate}

A schematic sketch of the flow configuration is shown in Figure 3-1. The upper plate is moving at a constant velocity while the lower plate is fixed. By assuming zero pressure gradients and no flow in the y-direction the Navier-Stokes equations can be simplified to a quasi one-dimensional problem:

$$
\begin{aligned}
& \frac{\partial u}{\partial x}=0, \\
& \mu \frac{d^{2} u}{d y^{2}}=0 .
\end{aligned}
$$

The analytical solution is a linear profile given by

$$
u=\frac{U}{2}\left(1+\frac{y-h}{h}\right),
$$

where $U$ is the upper plate velocity, $h$ is the half-channel height (White 1991). Note that the origin used herein is located at the bottom wall whereas it is placed in the middle in White's book, and this coordinate arrangement should be adopted as default in the following sections without further mention. 


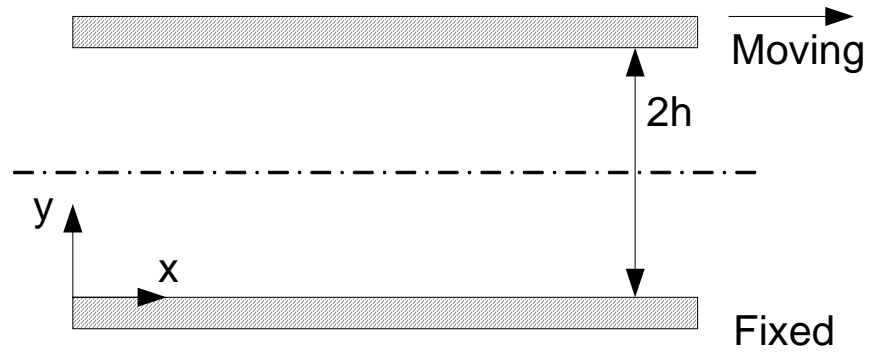

Figure 3-1 Couette flow between a fixed and a moving plate

Numerically a full set of Navier-Stokes equations should be solved. With properly posed boundary conditions the numerical solution should exhibit the quasi-1D behavior. Thus, periodic boundary condition is applied in the streamwise direction; in the vertical direction no-slip wall condition is applied at the lower bound while slip wall with a constant plate velocity $U$ is imposed at the upper bound. Calculation as well as the boundary conditions in the $z$-direction is neglected. In a fractional step method (see Section 2.7) the pressure or pseudo-pressure variable is uncoupled from the momentum equation, and solved via a Poisson type equation with Neumann boundary conditions. The resulting pressure or pseudo-pressure is used to correct a predicted velocity field, thus enhancing continuity.

The computational domain is sized as $1.0 \mathrm{~m} \times 0.2 \mathrm{~m}$ in $\mathrm{x}$ - and $\mathrm{y}$ - direction, respectively. A uniform grid of 50 cells in $\mathrm{x}$ and 20 cells in y direction is used within the computational domain. This corresponds to a grid spacing of $0.02 \mathrm{~m}$ in $\mathrm{x}$ and $0.01 \mathrm{~m}$ in $\mathrm{y}$ direction. A constant velocity of $1 \mathrm{~m} / \mathrm{s}$ is used for the moving upper plate and the Reynolds number based on the channel height and upper plate velocity is 100 .

The Crank-Nicolson $(\mathrm{C}-\mathrm{N})$ is used for time discretization, corresponding to a fifty percent of implicit discretization and fifty percent explicit discretization. The power-law is used for implicit spatial discretization and the $2^{\text {nd }}$ order central differencing is used for explicit spatial discretization. The pressure-related Poisson equation is solved using the $4^{\text {th }}$ order deferred correction. Note that the $4^{\text {th }}$ order deferred correction is always used for solving Poisson equation unless otherwise mentioned.

The computation is started with a stationary flow field. A time step of $0.1 \mathrm{~s}$ is used for time advancement. The time iteration is stopped when no appreciable change is observed in the flow field, i.e., the steady state is reached. A normalized global mass residual (normalized by mass flow rate at the inlet) of order $10^{-8}$ is achieved.

The obtained u-velocity contour is displayed in Figure 3-2(a), showing a linear increase in magnitude along the vertical direction and a uniform distribution in the streamwise direction. A vertical line is then extracted at an $\mathrm{x}$-station close to the outlet and compared with the analytical solution given by Eq. (3.2). This is shown in Figure 3-3(a) with vertical position normalized by the channel height and velocity normalized by the upper plate velocity. The two curves well coincide. The exact error, which is defined 
as the difference between the exact and calculated values, i.e., $u_{\text {exa }}-u_{\text {calc }}$, is shown in Figure 3-3(b) and it is of order $10^{-7}$.

Due to the nature of $2 \mathrm{D}$ calculation the contour plots for v- velocity and pressure are also displayed in Figure 3-2(b) and Figure 3-2(c) respectively. It is seen that in a 2D calculation these quantities may not be strictly zero or zero gradients; some noise may exist owing to the limitations in the iterative convergence as well as approximations in the boundary condition implementation. Overall, the results from the present computation with fairly coarse grid conform well to the analytical solution.

\subsection{Couette-Poiseuille Flow Between a Fixed and a Moving Plate}

A little different from the Couette flow in the preceding section is that a constant pressure gradient is imposed in the streamwise direction. This gives

$$
\begin{aligned}
& \frac{\partial u}{\partial x}=0, \\
& \mu \frac{d^{2} u}{d y^{2}}=\frac{d p}{d x} .
\end{aligned}
$$

The analytical solution (White 1991), given by

$$
\frac{u}{U}=\frac{y}{2 h}+\mathrm{Po}\left[1-\left(\frac{y-h}{h}\right)^{2}\right], \operatorname{Po}=\left(-\frac{d p}{d x}\right) \frac{h^{2}}{2 \mu U},
$$

is a superposition of Couette wall-driven flow (the first term on the RHS) and Poiseuille pressure-driven flow (the second term). Note that in Eq. (3.4) Po can be regarded as a dimensionless pressure gradient (with opposite sign of the actual pressure gradient).

The computation is performed using the same geometry, grid and scheme. The Reynolds number based on the channel height and upper plate velocity is again 100 . The constant pressure gradient is imposed as a constant source onto the RHS of the momentum equation. A pressure-like variable still needs to be solved to take care of the global continuity, but with the expectation of a nearly uniform distribution.

The results are first illustrated with Po $=1$. The contour plots of u-velocity, vvelocity and pressure are shown in Figure 3-4(a) through (c). Notice that the increase of the streamwise velocity along the vertical direction is no longer linear, resulting from the influence of constant pressure gradient. Also, as expected, the vertical velocities are near zero everywhere; the pressure-like variable varies insignificantly with an order of magnitude $10^{-6}$.

Next, the dependency of the u-velocity on the dimensionless pressure gradient Po is reproduced. For this purpose a series of computations is performed at different Po, and the u-velocity profiles are then collected and combined into a single Figure (Figure 3-6). In all cases the calculated profile is in complete compliance with the exact solution. It is 
worthwhile to mention that (i) at $\mathrm{Po}=0$, the flow turns to exactly the same Couette flow addressed in the preceding section, (ii) at Po $=-1 / 4$ the shear stress $\mu \partial u / \partial y$ at the lower wall is zero, indicating a critical number below which a flow separation will occur, and (iii) when Po $<-1 / 4$ the flow is separated due to a strong positive pressure gradient, resulting in the so-called backflow; this phenomenon is shown in Figure 3-7 for the flow calculated at $\mathrm{Po}=-1 / 2$.

To determine the order of accuracy of the current power-law scheme, three calculations are performed with three different mesh sizes at the same Reynolds number and Po value (equal unity). Since the numerical solution for u-velocity is constant along horizontal line and does not depend on the grid resolution in that direction, a systematic refinement of the grid is only necessary in y-direction. With a refinement factor of two, the three grids have 20,40 and 80 internal cells in the vertical direction, respectively. The data of relevance to error analysis from three different runs are listed in Table 3-1, in which $h$ denotes the grid size, $h_{\text {ref }}$ refers to the finest grid (here 80$), \varepsilon_{\max }$ is the maximum exact absolute error, and $u_{\max }$ is the maximum $\mathrm{u}$-velocity. Thus, the three nondimensional grid sizes normalized by $h_{\text {ref }}$ are 1,2 and 4, ordered from the finest to coarsest. Similarly, the exact absolute errors normalized by the maximum u-velocity gives a non-dimensional measure for the absolute error. Note that here the maximum error has been used as the error norm, and this norm is regarded as the one of the most stringent criteria. Other error measures also exist such as the RMS error, where average is taken on the square root of the summation of squares of the exact error at all computed grid nodes.

Table 3-1 Order of method analysis for the calculations of Couette-Poiseuille flow between a fixed and a moving plate; schemes considered: semi-implicit power-law, implicit $2^{\text {nd }}$ order CD.

\begin{tabular}{|c|c|c|c|c|c|c|c|}
\hline grid in $y$ & $\mathrm{~h} / \mathrm{h}_{\text {ref }}$ & $\varepsilon_{\max }$ & $\mathrm{u}_{\max }$ & $\varepsilon_{\max } / u_{\max }$ & $\ln \left(\mathrm{h} / \mathrm{h}_{\mathrm{ref}}\right)$ & $\ln \left(\varepsilon_{\max } / u_{\max }\right)$ & slope \\
\hline \multicolumn{8}{|c|}{ C-N, power-law } \\
\hline 80 & 1 & 1.5998E-04 & 1.5625 & 1.0239E-04 & 0.0000 & -9.1867 & \\
\hline 40 & 2 & $6.2575 \mathrm{E}-04$ & 1.5625 & 4.0048E-04 & 0.6931 & -7.8228 & \\
\hline 20 & 4 & 2.5098E-03 & 1.5650 & 1.6037E-03 & 1.3863 & -6.4354 & 1.9847 \\
\hline \multicolumn{8}{|c|}{ Implicit, 2nd CD } \\
\hline 80 & 1 & 1.5651E-04 & 1.5625 & 1.0017E-04 & 0.0000 & -9.2087 & \\
\hline 40 & 2 & 6.0284E-04 & 1.5625 & 3.8582E-04 & 0.6931 & -7.8601 & \\
\hline 20 & 4 & 2.5006E-03 & 1.5650 & 1.5978E-03 & 1.3863 & -6.4391 & 1.9978 \\
\hline
\end{tabular}

According to Richardson's theory (Richardson 1910; Richardson and Gaunt 1927), the absolute error is proportional to the grid size, i.e.

$$
\varepsilon=C h^{n}
$$

where $C$ is a constant and $n$ represents the order of a method. Eq. (3.5) has been accepted sort of as a defacto standard for verifying the order of a numerical scheme. In literature one usually use the logarithmic form of Eq. (3.5) to produce a linear relationship between the error and grid size, i.e.,

$$
\ln \varepsilon=\ln C+n \ln h \text {. }
$$

The advantage of Eq. (3.6) is that one can easily tell the order of a method by just examining the slope of the straight line from the graph. Thus, the natural logarithmic 
values of the normalized grid size and absolute error are also listed in Table 3-1; the two columns are then used to produce a line plot showed in Figure 3-8(a). The observed order of method follows from a simple calculation of the slope, which gives a value slightly less than 2. Although this result the power-law provides is encouraging, one should be cautious that the actual order of the power-law and the hybrid scheme behaves differently in different flow systems and different grids, depending on how much the higher order part $\left(2^{\text {nd }}\right.$ order CD) is being used.

For comparison, similar computations are performed with full implicitness and the $2^{\text {nd }}$ order central differencing scheme. A similar set of data is presented in the lower rows of Table 3-1, along with a linear fit in log-log scale shown in Figure 3-8(b). It is evident that the $2^{\text {nd }}$ order central differencing is indeed $2^{\text {nd }}$ order accurate.

A side note is that the Richardson's idea has led to tremendous research work in the area of the discretization error estimation, in which the so-called Richardson's extrapolation becomes the most popular and possibly significant tool. More information of that relevance can be found for example in ( $\mathrm{Hu}$ 2002) and is not quite pertinent to the present study.

\subsection{Poiseuille Flow Between Fixed Parallel Plates}

If the upper wall is held fixed the flow described in Section 3.2 reduces to pure Poiseuille, i.e., pressure-driven, flow between two fixed parallel plates. Since this type of flow is described by the same equation (3.3), the analytical solution can be either solved fresh from Eq. (3.3) with a modified boundary condition, or directly deduced from Eq. (3.4); this yields

$$
u=u_{\max }\left[1-\left(\frac{y-h}{h}\right)^{2}\right], u_{\max }=\left(-\frac{d p}{d x}\right) \frac{h^{2}}{2 \mu} .
$$

One may further deduce an average velocity from a preserved volume flow rate; this gives

$$
\begin{aligned}
& Q=\int_{0}^{2 h} u d y=\frac{4}{3} h u_{\max }, \\
& u_{\mathrm{ave}}=\frac{Q}{2 h}=\frac{2}{3} u_{\max } .
\end{aligned}
$$

Again, the same geometry, grid and scheme is used here. Similar to the approach presented in the last section a constant pressure gradient is imposed in the momentum equation and a pressure-like variable is solved in a subsequent step to ensure continuity. The Reynolds number used is 50, based on the half-channel height and the maximum velocity (equal to unity), or 66.67, based on the channel height and the average velocity. This corresponds to a constant pressure gradient equal to $-0.4 \mathrm{~N} / \mathrm{m}^{3}$. The results are collected when a steady state is reached. 
The calculated $\mathrm{u}$-velocity profile in vertical direction is displayed along with the exact solution in Figure 3-9(a), with their difference displayed in Figure 3-9(b). A contour plot of the u-velocity is also shown in Figure 3-10. The parabolic profile reminds us of the fully developed laminar pipe flow or channel flow. Indeed, the pure Poiseuille flow between parallel plates can be viewed as a simplified two-dimensional channel flow in which the pressure gradient is already known (imposed) and does not need to be calculated. For comparison, computation of a developing laminar channel flow is carried out in the next section (Section 3.4), where the pressure gradient is part of the unknowns. It will be good to see that the fully developed profile looks similar.

An interesting observation from Figure 3-9(b) is that for the fifty percent powerlaw scheme the numerical error is asymmetrically distributed in the vertical direction while the u-velocity itself exhibits a perfect symmetric parabola. This arouses our interest in a further investigation with a different scheme, say, the $2^{\text {nd }}$ order central differencing with full implicitness. The error curve with the latter scheme is shown in Figure 3-11. It can be seen that the numerical error tends to be distributed more symmetrically as compared with the fifty percent power-law. Further, in spite of the $2^{\text {nd }}$ order scheme, the order of magnitude of the absolute error is still about the same as the fifty percent powerlaw. This behavior is expected because the $2^{\text {nd }}$ order scheme is $2^{\text {nd }}$ order only in the context of the solution of a single equation without interference of the unsteady term. In other words, a $2^{\text {nd }}$ order accuracy may not be achieved when (i) a lower order scheme is used for the time discretization or the time step is large, (ii) coupled equations are solved sequentially, as in the fractional step method, and non-linearity of linearized terms is not strictly satisfied without performing time-consuming inner-iterations, (iii) pressure is not solved to a high accuracy, and (iv) there is accuracy loss in the implementation of periodic boundary condition. Aside from the discretization error, the limited accuracy may also due to the machine round-off error and insufficiently accurate grid information.

\subsection{Developing Channel Flow}

For the pressure-driven flow considered in the previous section the pressure gradient is given and does not require a stand-alone solution. Although a pressure-like variable was still solved, it is only to fine-tune the flow field to satisfy continuity. In this section a developing laminar channel is considered with the inlet fed by the uniform profile. The flow-driving pressure gradient plays an important role in this case and must be correctly solved.

Here a computational domain of $8 \mathrm{~m} \times 0.2 \mathrm{~m}$ is selected in the streamwise and vertical direction, respectively. The Reynolds number is 50, based on the half-channel height and the centerline velocity (equal unity) of a fully developed channel. Note that the Reynolds number chosen is same as the one considered in the previous section; the computational domain has the same height as well. But the domain length is largely extended to allow for a full development of the wall boundary layer. A scale analysis using boundary layer theory (Schlichting 1979) shows an estimate of laminar boundary layer thickness in dependence of the Reynolds number $\mathrm{Re}_{L}$ : 


$$
\frac{\delta}{L} \sim \operatorname{Re}_{L}^{-1 / 2}
$$

or equivalently,

$$
\delta \sim\left(\frac{v L}{U}\right)^{1 / 2}
$$

and

$$
L \sim \frac{U \delta^{2}}{v}=\operatorname{Re}_{\delta} \delta
$$

where $\delta$ is the velocity boundary layer thickness, $L$ is a proper length scale in streamwise direction, and $\operatorname{Re}_{L}$ and $\mathrm{Re}_{\delta}$ is the Reynolds number defined by using the characteristic length $L$ and $\delta$ respectively. This relation implies that for, say, a $\operatorname{Re}_{\delta}$ of 50 with a boundary layer thickness of $\delta=h$, a length of about $50 h$ is required for the boundary layer to be fully developed to $h$ thick. Thus, with an $8 \mathrm{~m}$ long it should be sufficient to get a fully developed flow of the $0.2 \mathrm{~m}$ high channel with $\mathrm{Re}_{\delta}=50$.

For the computation a mesh of $80 \times 20$ cells is used in $\mathrm{x}$ - and $\mathrm{y}$ - directions, respectively. As for the numerical scheme Crank-Nicolson, the implicit power-law and explicit central differencing are considered. A uniform inlet and an outlet condition is prescribed at the west and east boundaries, respectively. The flow field is initialized with the average u-velocity obtained from the flow rate at inlet. A large time step of 0.1 second is first used to establish some preliminary flow field; after that a smaller time step of $0.01 \mathrm{~s}$ is taken to achieve more accurate results. To measure the duration of simulation, the term, flow-through time or step-to-outlet time, is often used, representing the time required for a fluid particle to travel from the inlet to outlet in the main flow direction. For the developing channel a rule-of-thumb estimation is to divide the domain length $(8 \mathrm{~m})$ by the inlet average velocity, giving a time of around 12 seconds. Typically a simulation is required to last for at least several flow-through times to ensure fully steady state solution.

Figure 3-12 shows the u-velocity variation along the horizontal centerline of the channel at steady state. It is seen that after a developing region where the velocity continuously increases the streamwise centerline velocity is stabilized at around $1 \mathrm{~m} / \mathrm{s}$. The fully developed region can be roughly marked at from around $x=1.6 \mathrm{~m}$. In Figure 3-13 u-velocity profiles at various $\mathrm{x}$-station in the developing region are demonstrated. One notices that while the shape of the profiles tends to be parabolic the areas enclosed by the corresponding curves remain the same, thus continuity-preserving. A complete picture for the developing (or entrance) region can be gained in the contour plots of Figure 3-14, where two wall boundary layers are seen to gradually build up and merge at the center of the channel (Figure 3-14(a)). As previously mentioned, the v-velocity is not zero in the developing region, while it is the case when the flow is fully developed. This point is illustrated in Figure 3-14(b).

In the fully developed region the u-velocity profile is extracted at an x-station near the outlet and compared with the exact solution provided in the previous section (Eq. (3.7)). The results are shown in Figure 3-15(a) and (b) indicating a satisfactory 
agreement. An interesting phenomenon is that the exact error curve shows a similar parabola to the original u-velocity profile, but certainly with much less magnitude!

The pressure field is displayed in Figure 3-16(a) along with a horizontal centerline plot given in Figure 3-16(b). It is seen that the solved pressure gives a negative gradient, as desired, and it decreases linearly with the x-station with a constant slope in the fully developed region $(\mathrm{x}>1.6 \mathrm{~m})$. If one takes the pressure values at the first and last node on the center $x$-line $\left(0.9920 \mathrm{~N} / \mathrm{m}^{2}\right.$ and $\left.-1.5084 \mathrm{~N} / \mathrm{m}^{2}\right)$, divide their difference by the distance between the two nodes $(7.95 \mathrm{~m}-1.65 \mathrm{~m}=6.3 \mathrm{~m})$, an approximated pressure gradient equal to $-0.397 \mathrm{~N} / \mathrm{m}^{3}$ can be obtained. Compared to the analytical value of $-0.4 \mathrm{~N} / \mathrm{m}^{3}$ the solved pressure gradient gives about only $0.8 \%$ relative error. 


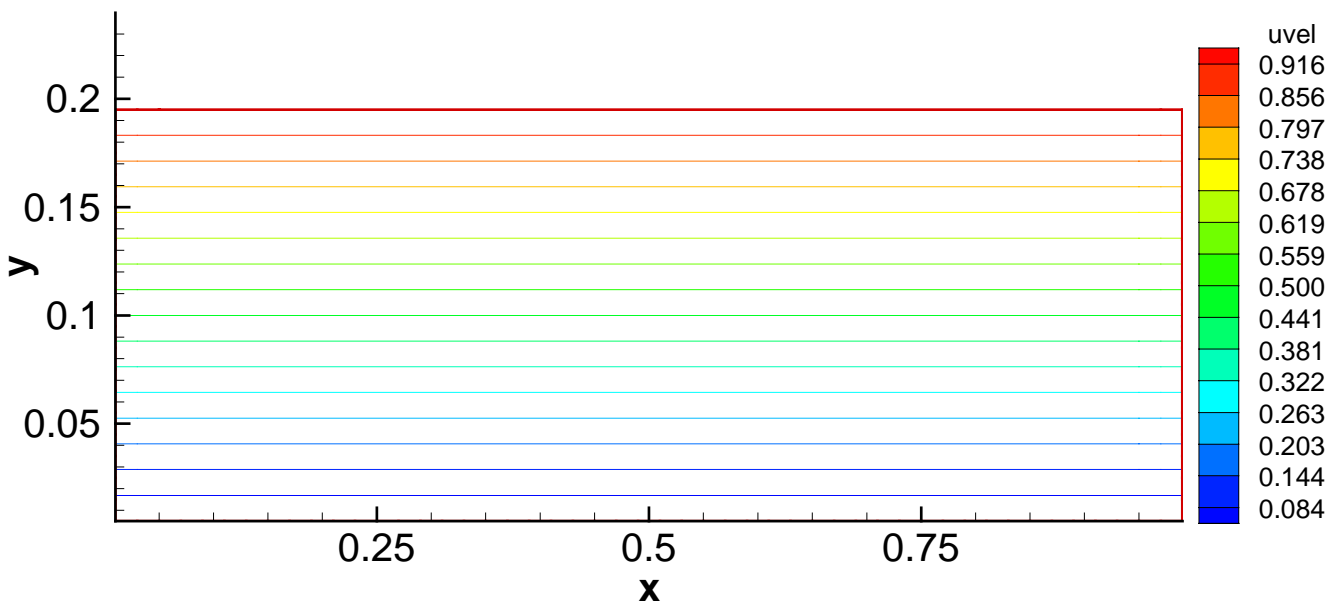

(a)

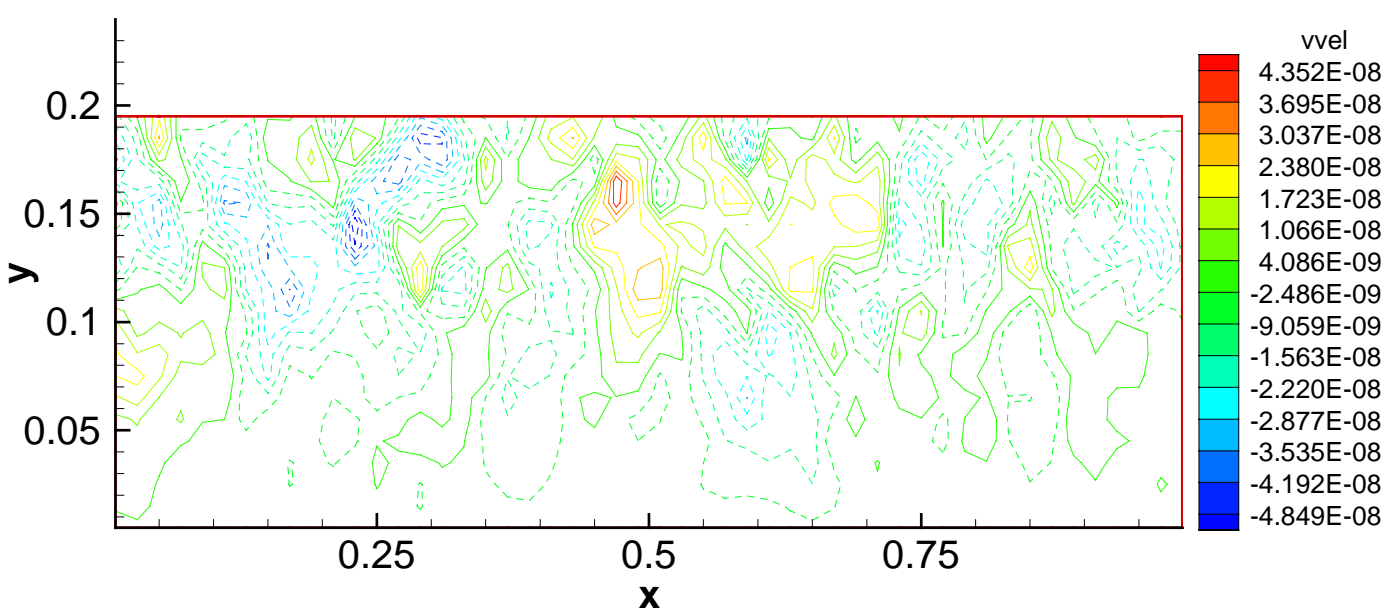

(b)

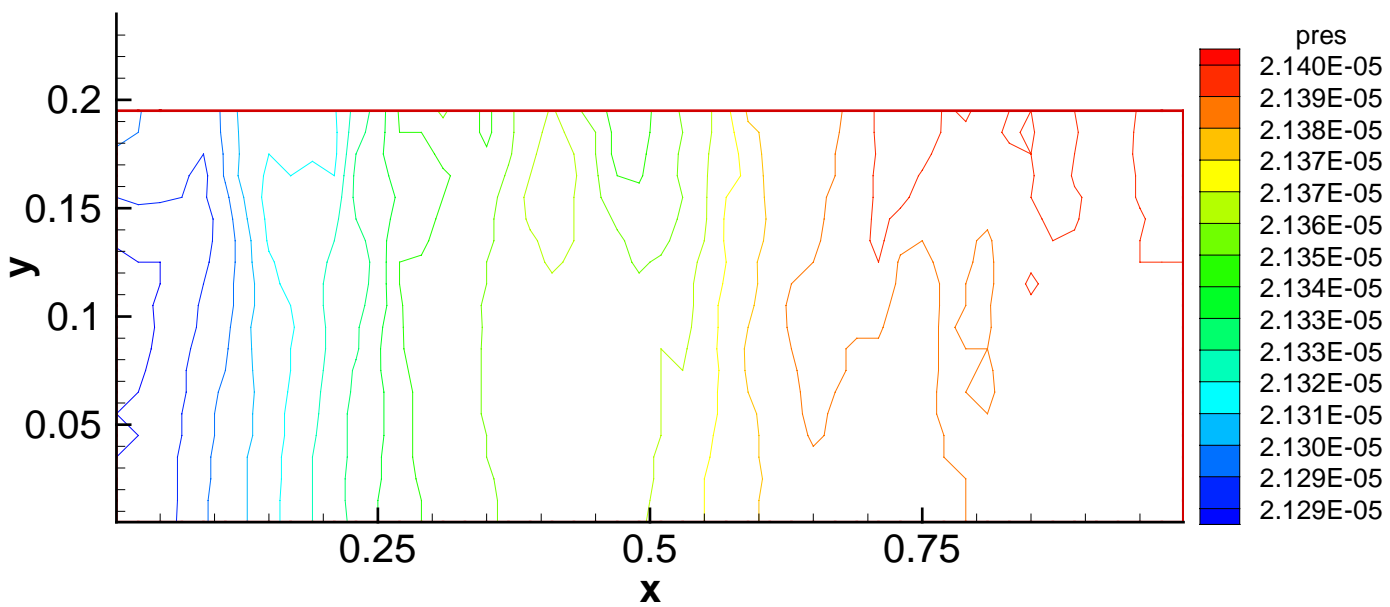

(c)

Figure 3-2 Contour plots of Couette flow between a fixed and a moving plate. (a) u-velocity, (b) vvelocity, (c) pressure. 


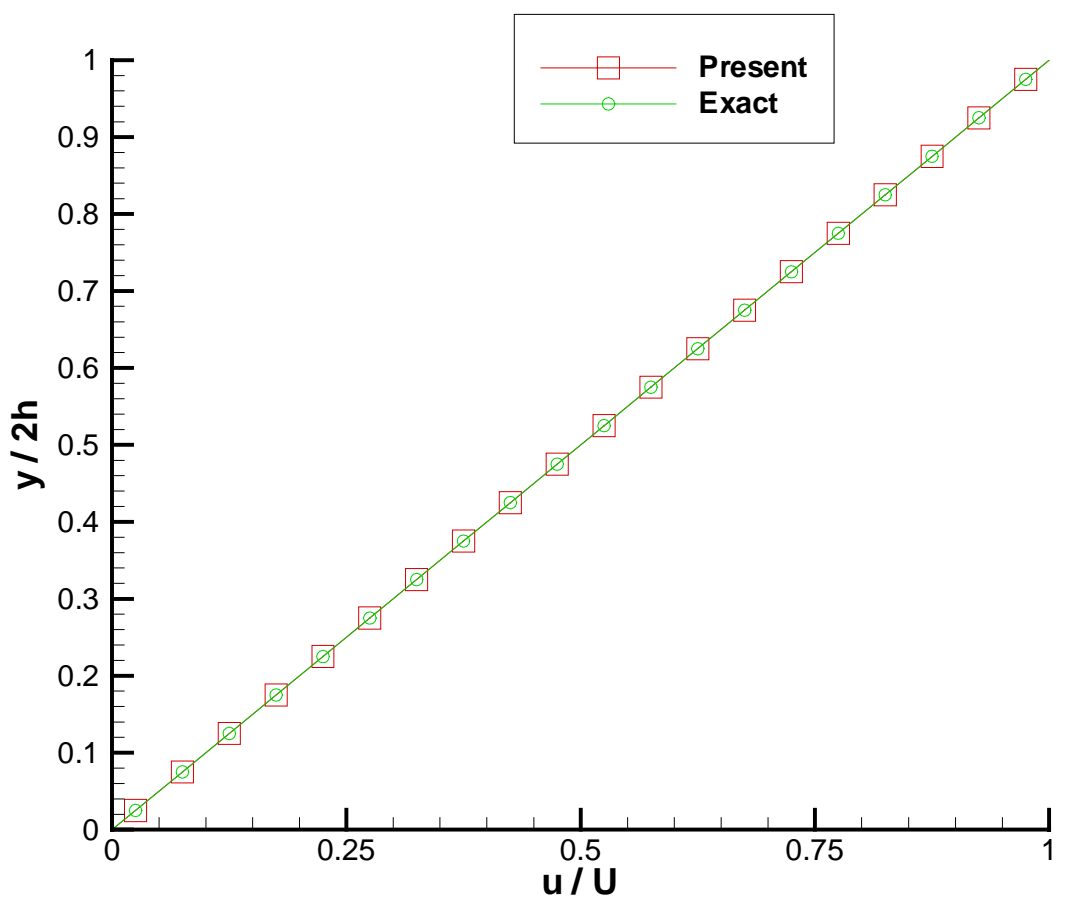

(a)

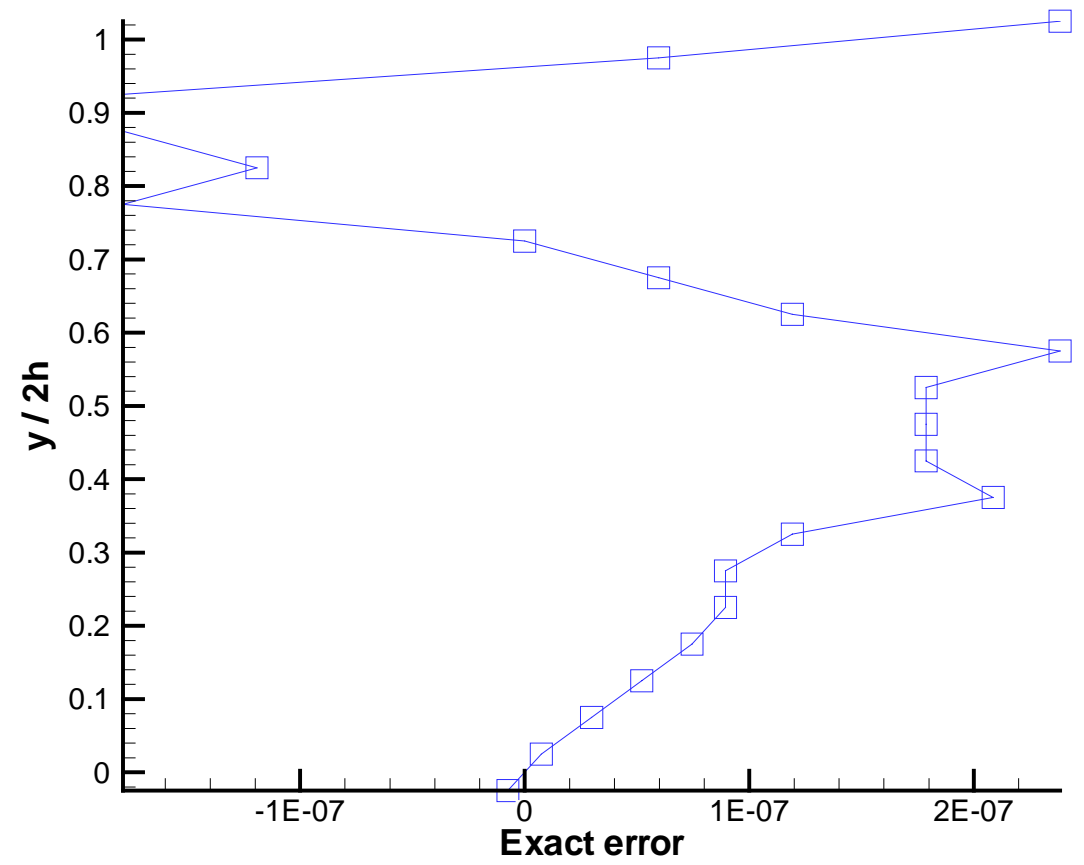

(b)

Figure 3-3 u-velocity profiles (a) and its exact error (b) of Couette flow between a fixed and a moving plate. 


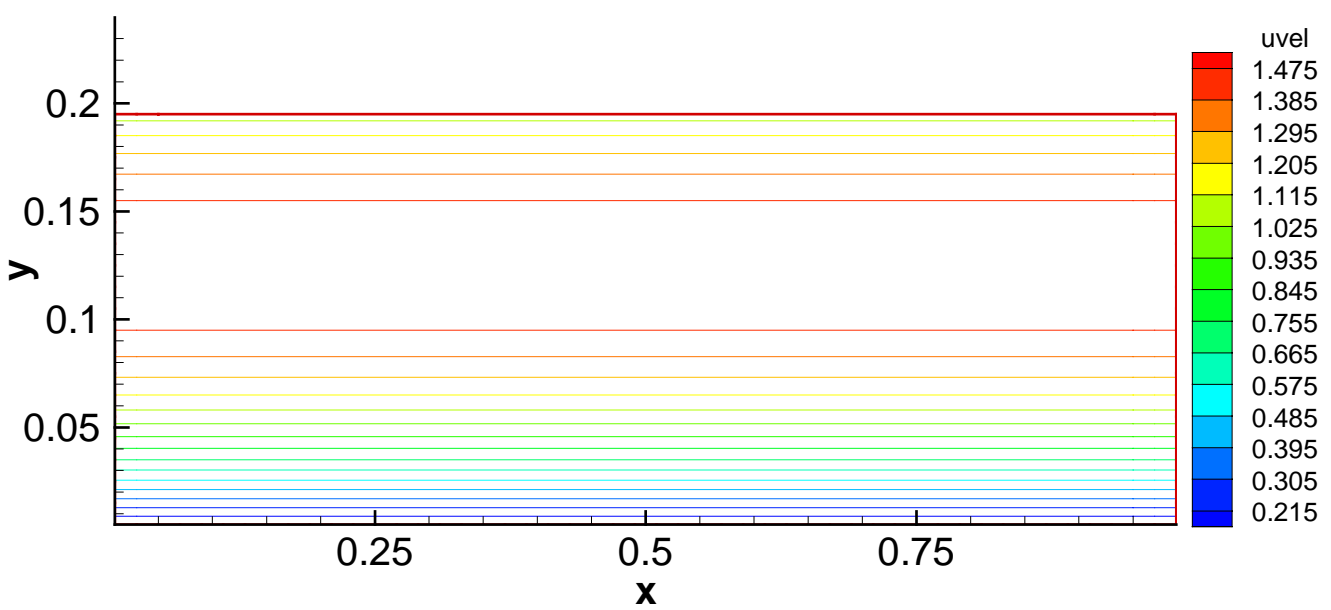

(a)

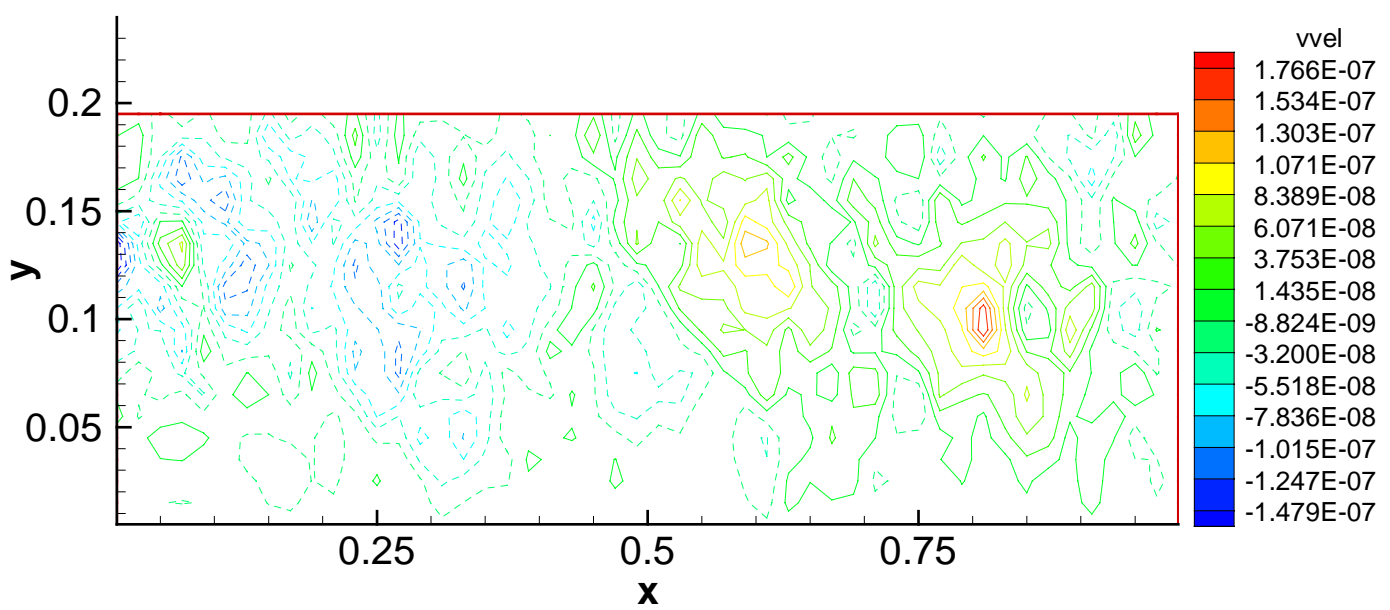

(b)

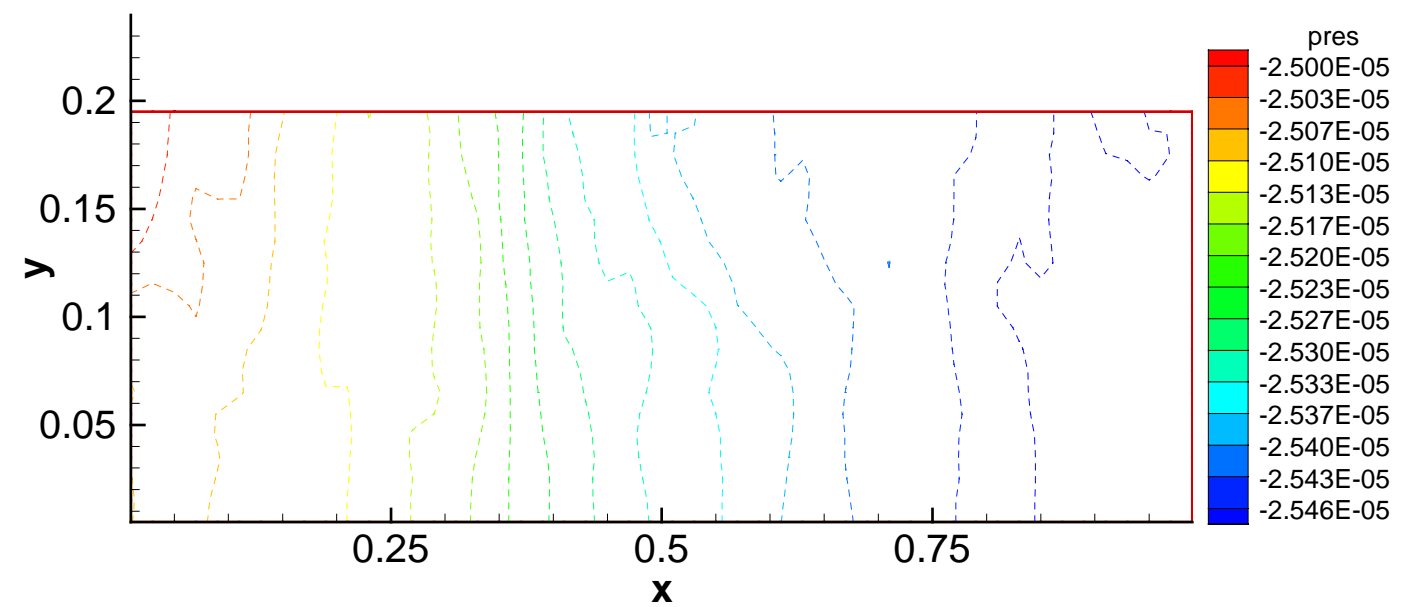

(c)

Figure 3-4 Contour plots of Couette-Poiseuille flow between a fixed and a moving plate, $P o=1$. (a) $u$ velocity, (b) v-velocity, (c) pressure-like variable. 


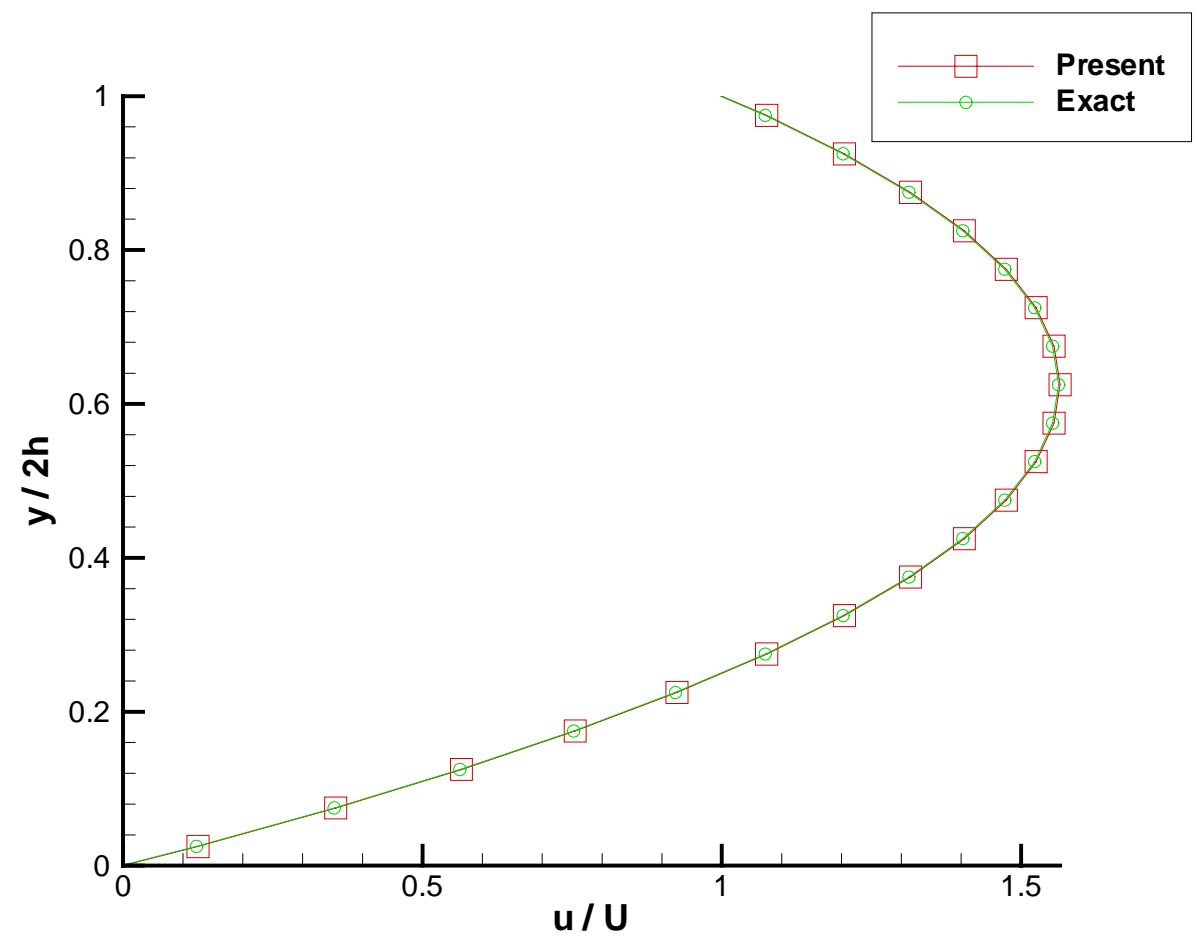

(a)

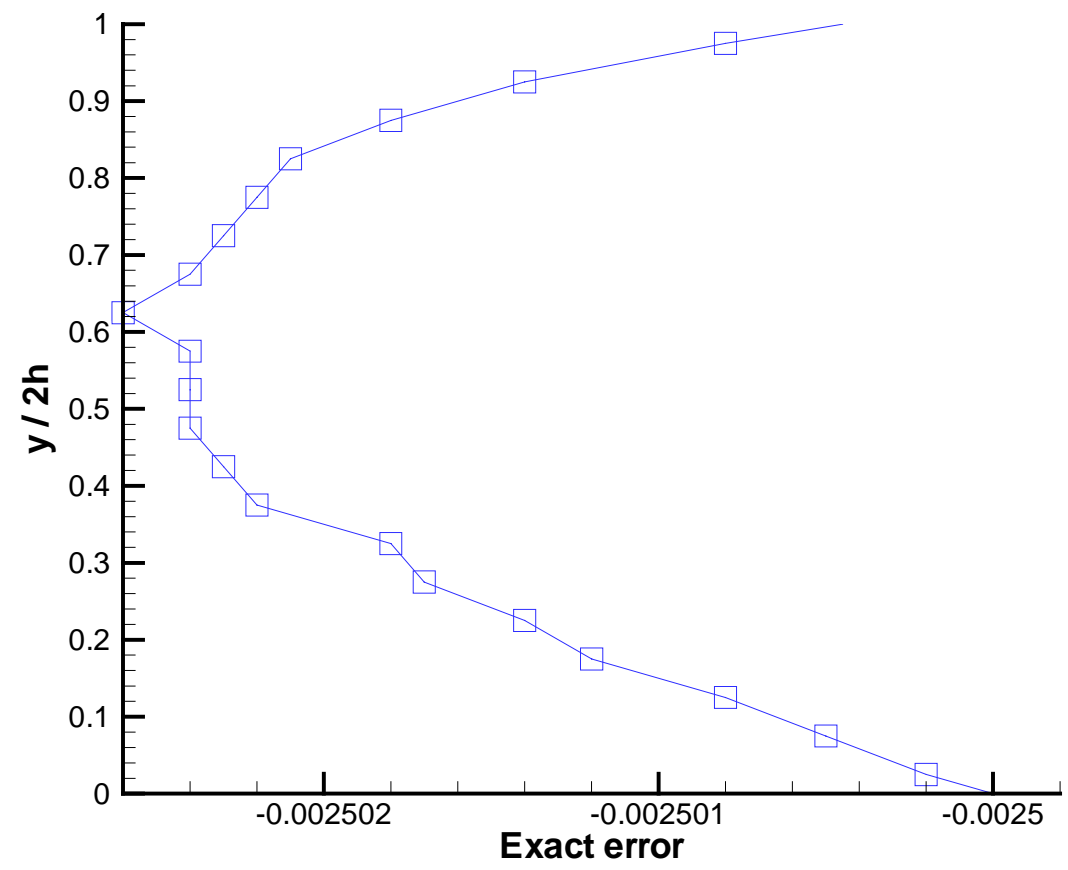

(b)

Figure 3-5 u-velocity profiles (a) and its exact error (b) of Couette-Poiseuille flow between a fixed and a moving plate with $P o=1$. 


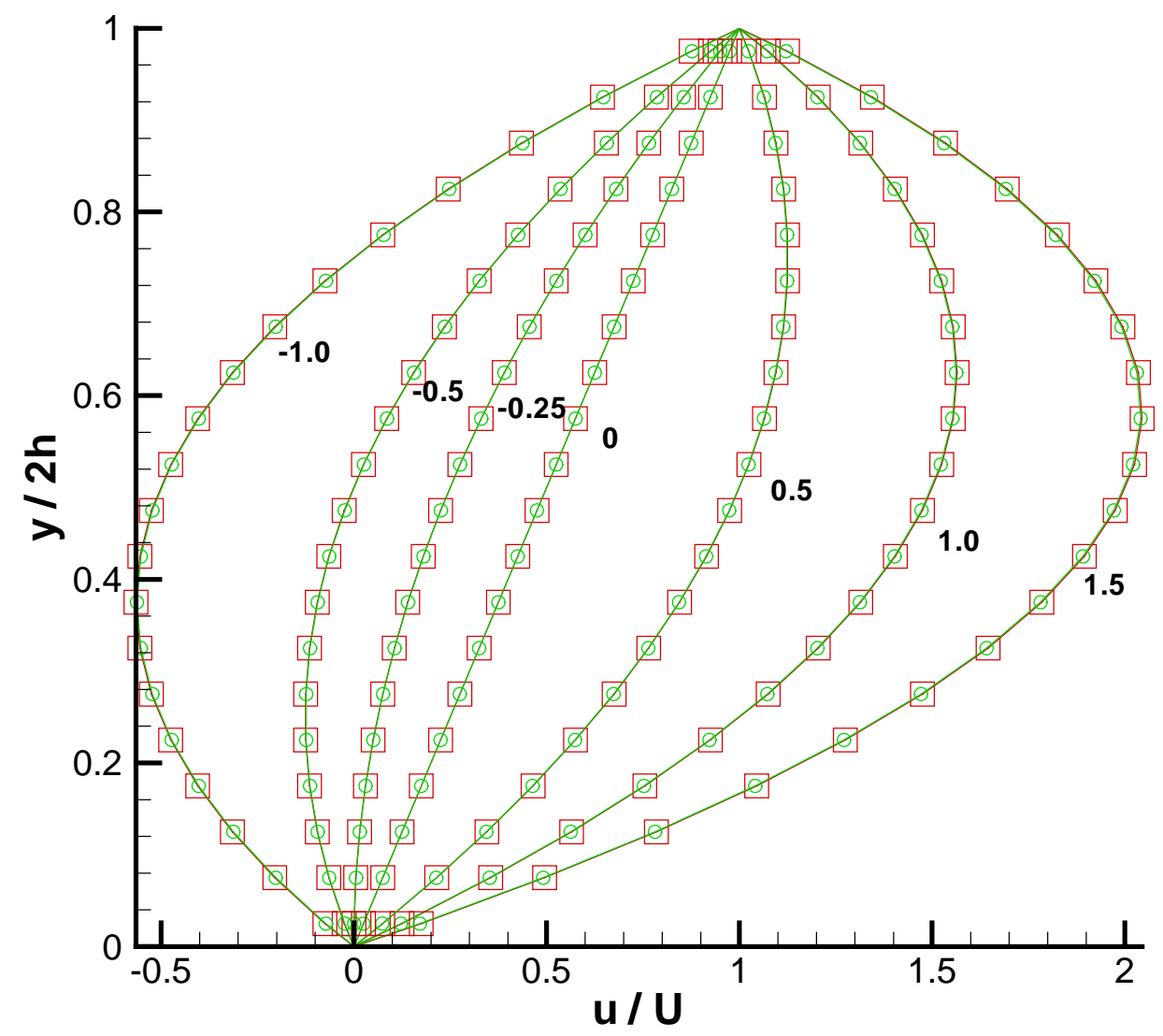

Figure 3-6 u-velocity profiles of Couette-Poiseuille flow between a fixed and a moving plate at different Po. Square symbols: present calculation; circle symbols: exact solution.

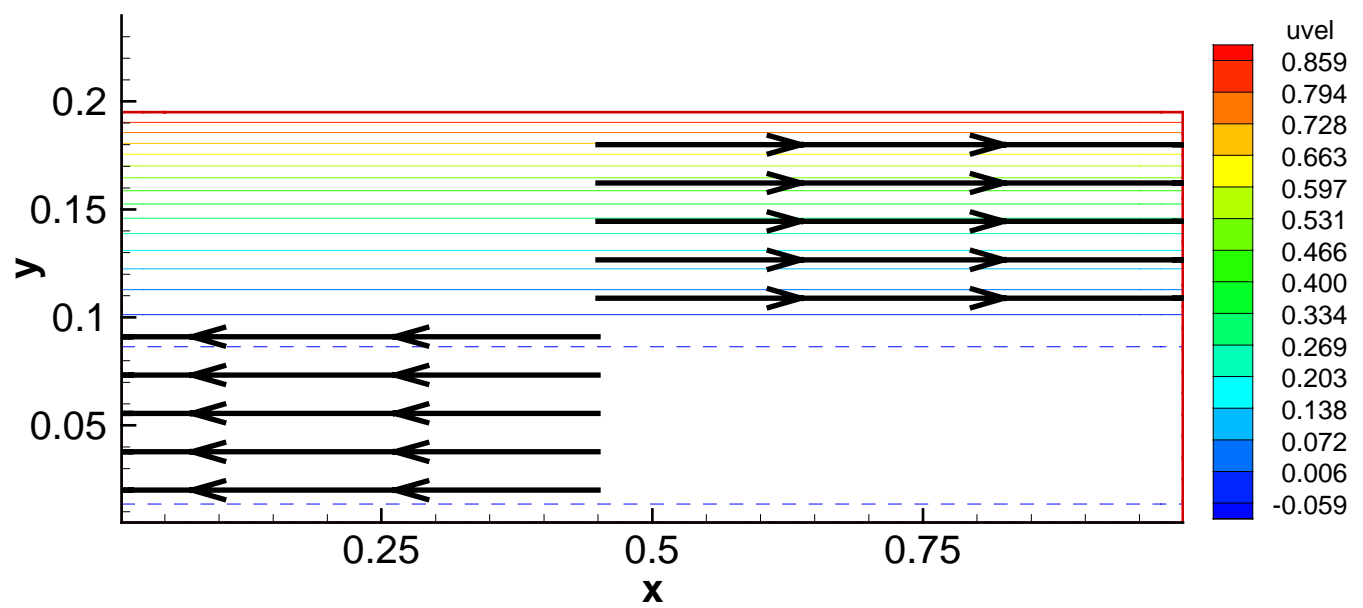

Figure 3-7 Flow separation with backflow of Couette-Poiseuille flow between a fixed and a moving plate, at $\mathrm{Po}=\mathbf{- 0 . 5}$. 


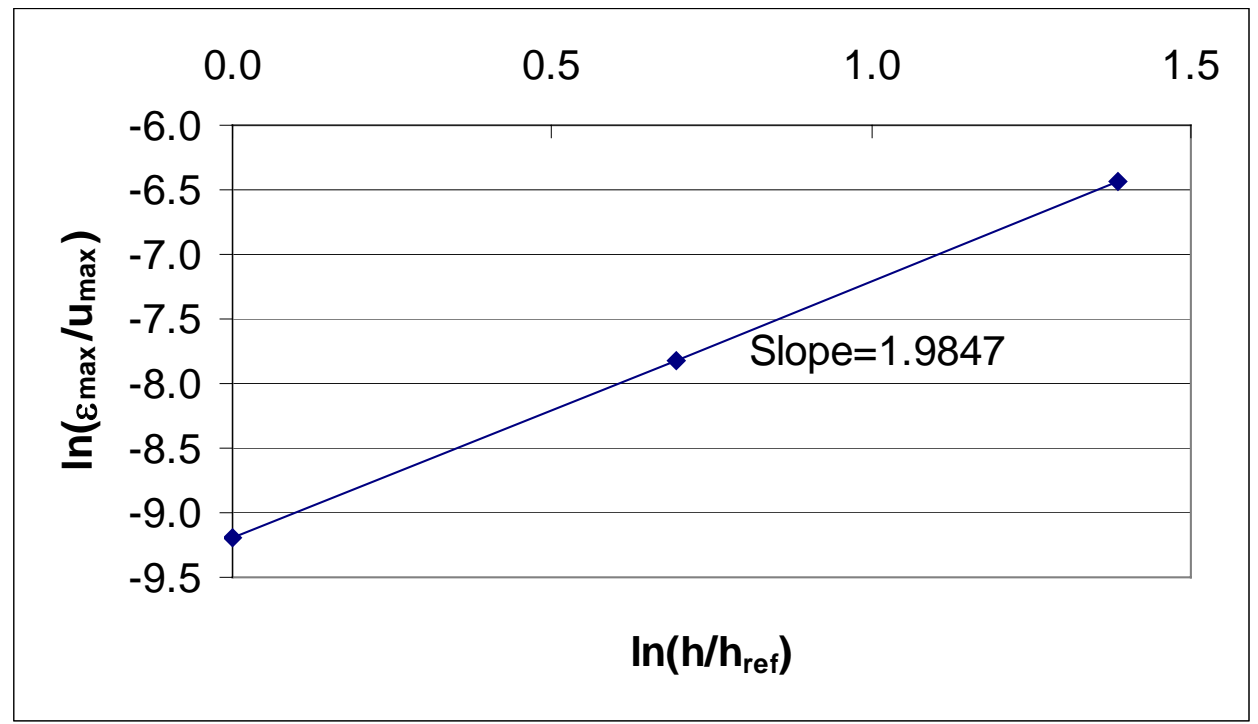

(a)

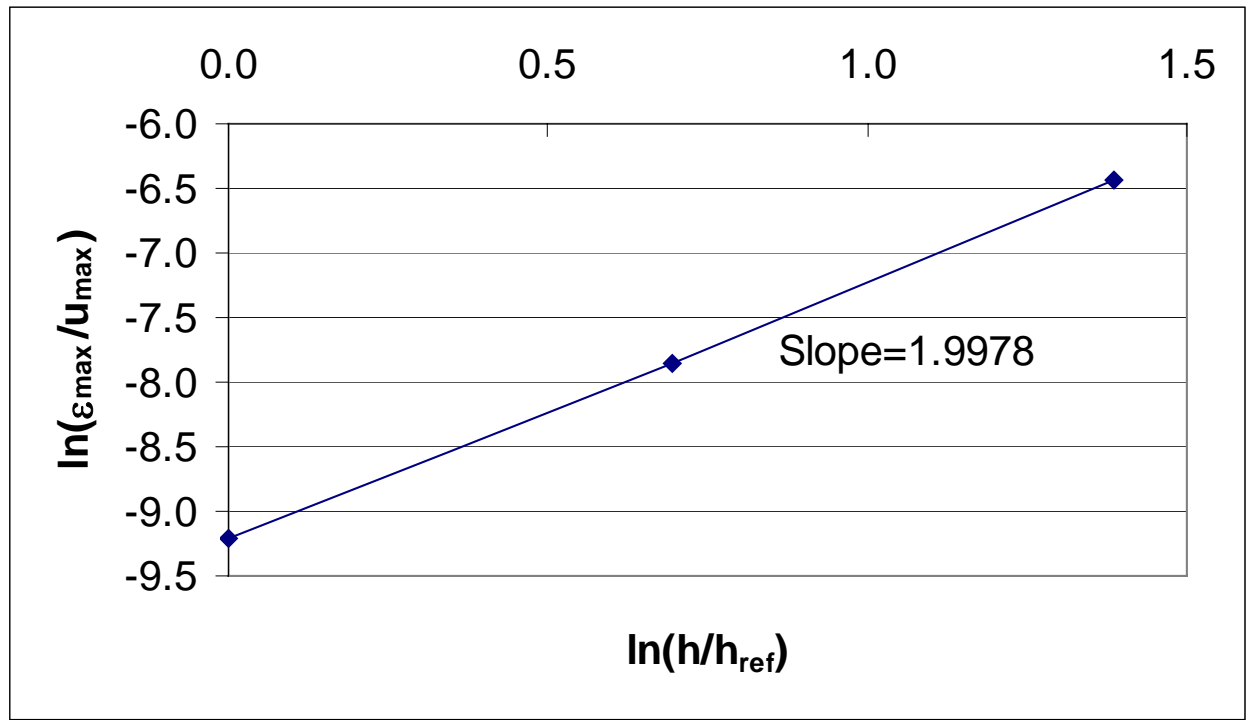

(b)

Figure 3-8 Maximum error as a function of mesh refinement for the Couette-Poiseuille flow between a fixed and a moving plate, (a) semi-implicit power-law, (b) implicit $2^{\text {nd }}$ order CD. 


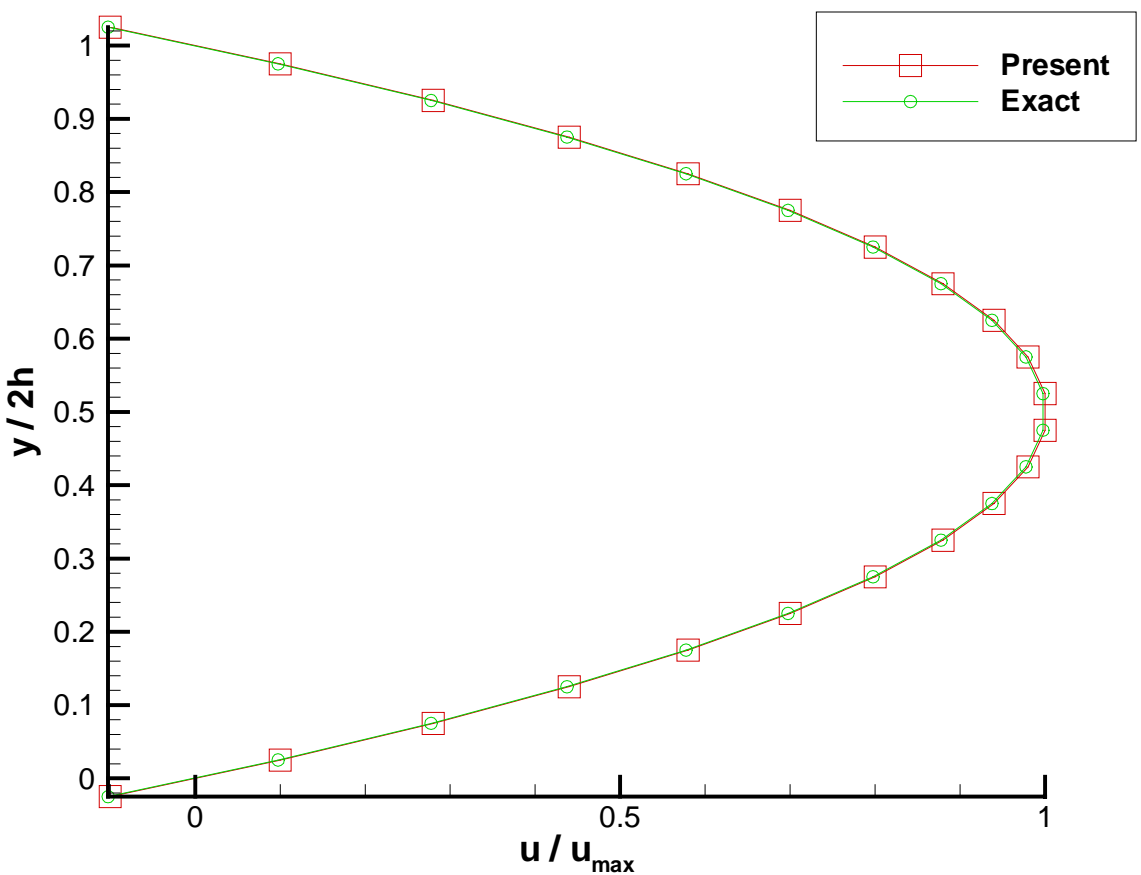

(a)

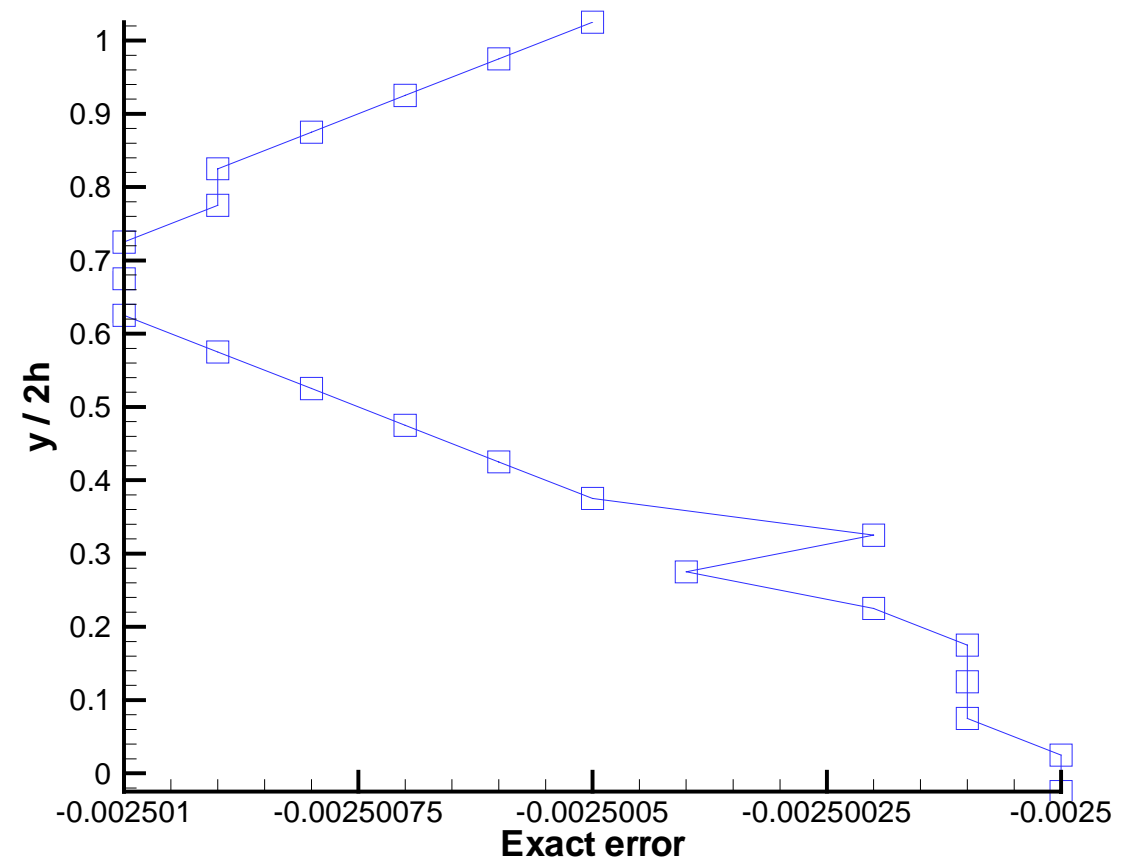

(b)

Figure 3-9 u-velocity profiles (a) and its exact error (b) of Poiseuille flow between fixed parallel plates. 


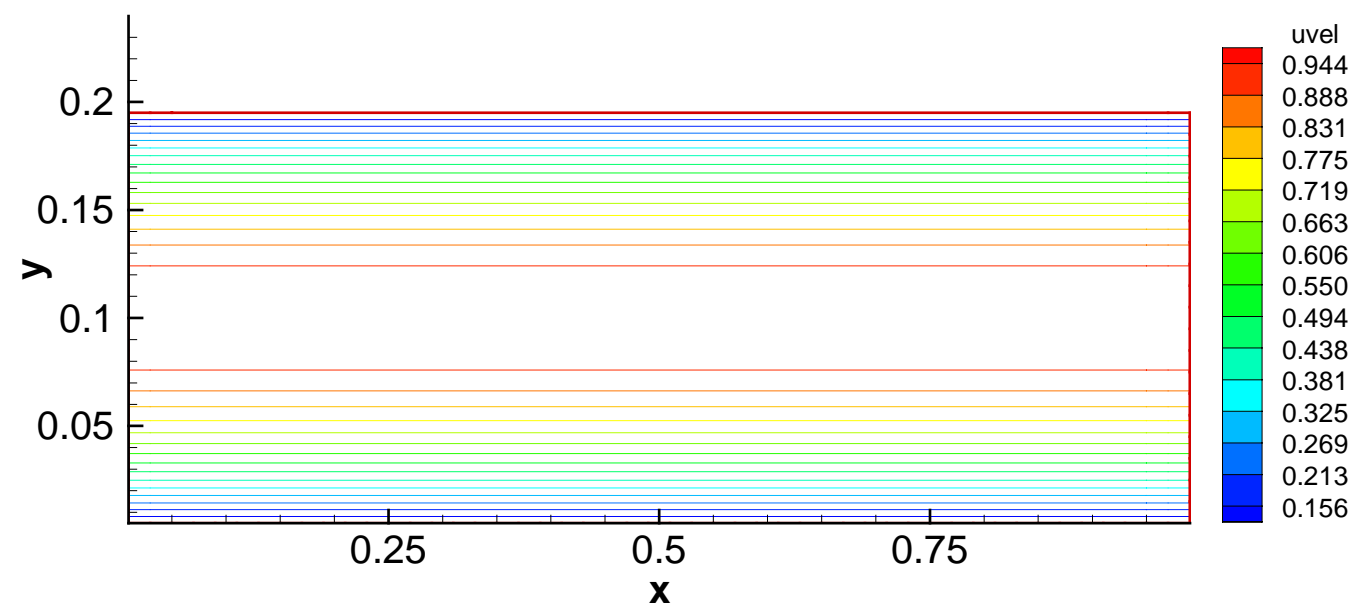

Figure 3-10 u-velocity contour of Poiseuille flow between fixed parallel plates.

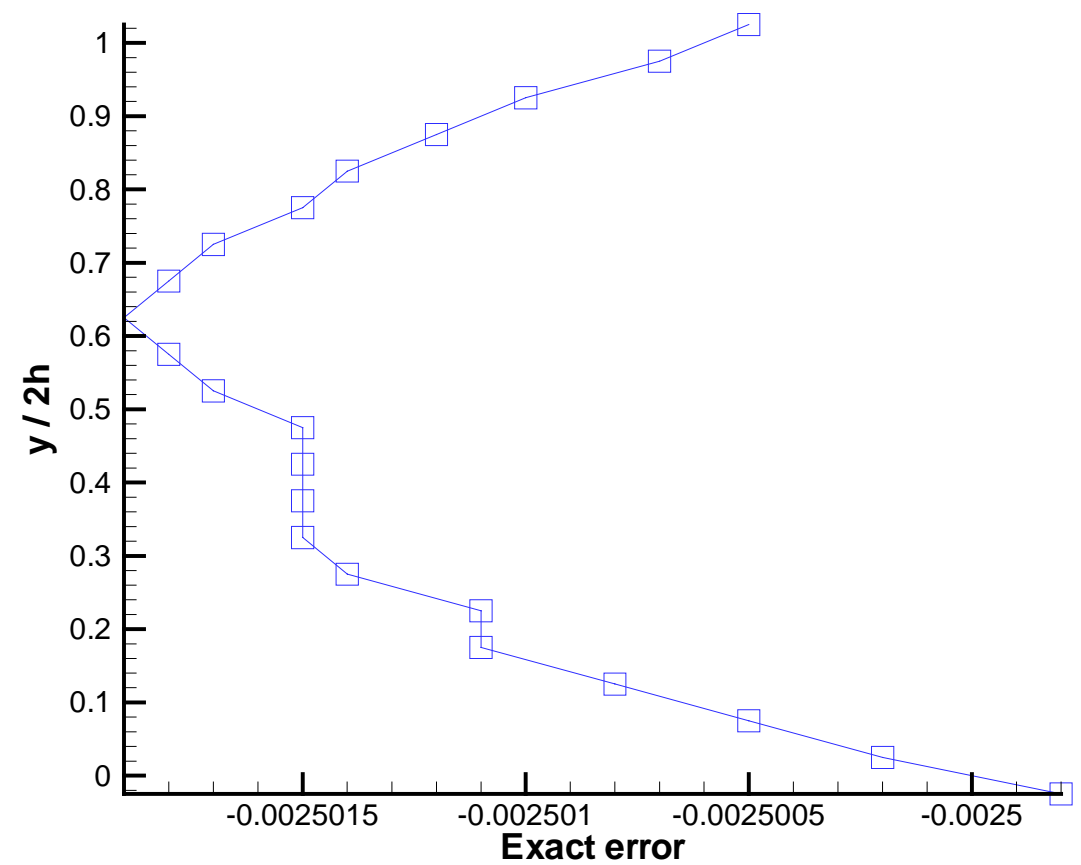

Figure 3-11 Exact error of the u-velocity of Poiseuille flow between fixed parallel plates, u-velocity calculated with $2^{\text {nd }}$ order central differencing. 


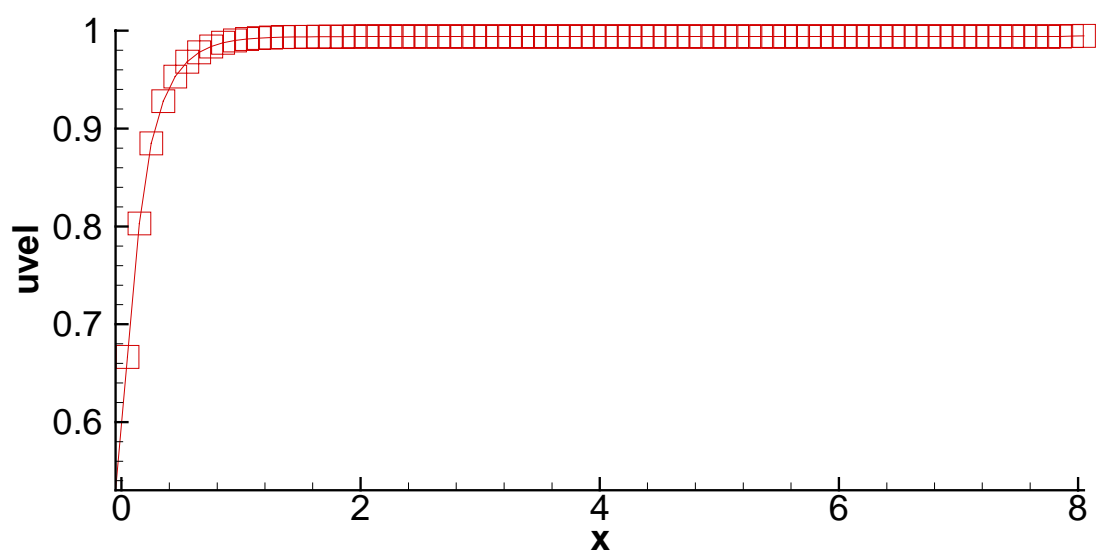

Figure 3-12 u-velocity variation along the horizontal centerline of a developing channel flow.

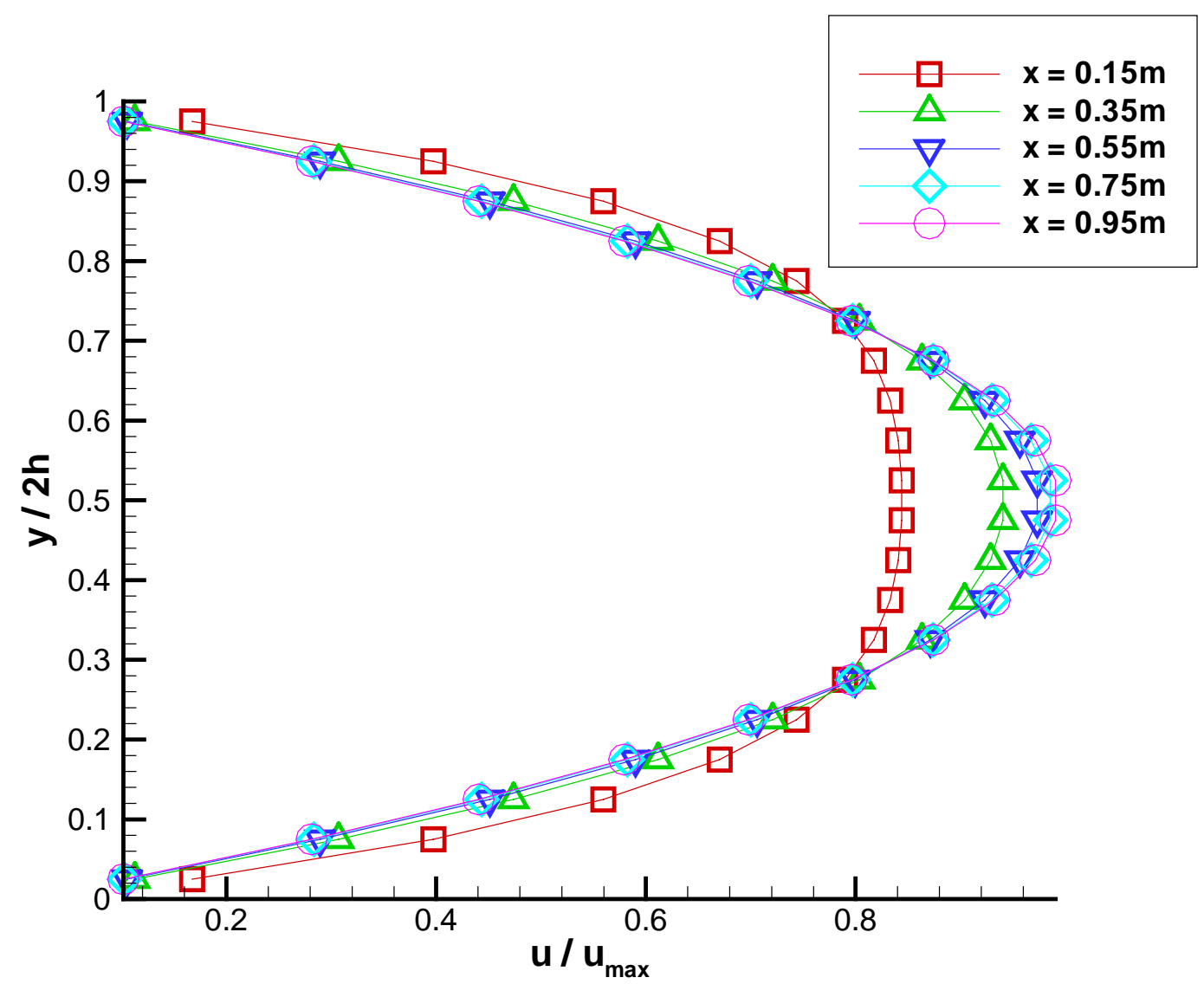

Figure 3-13 u-velocity profiles at different $x$-station of a developing channel flow. 


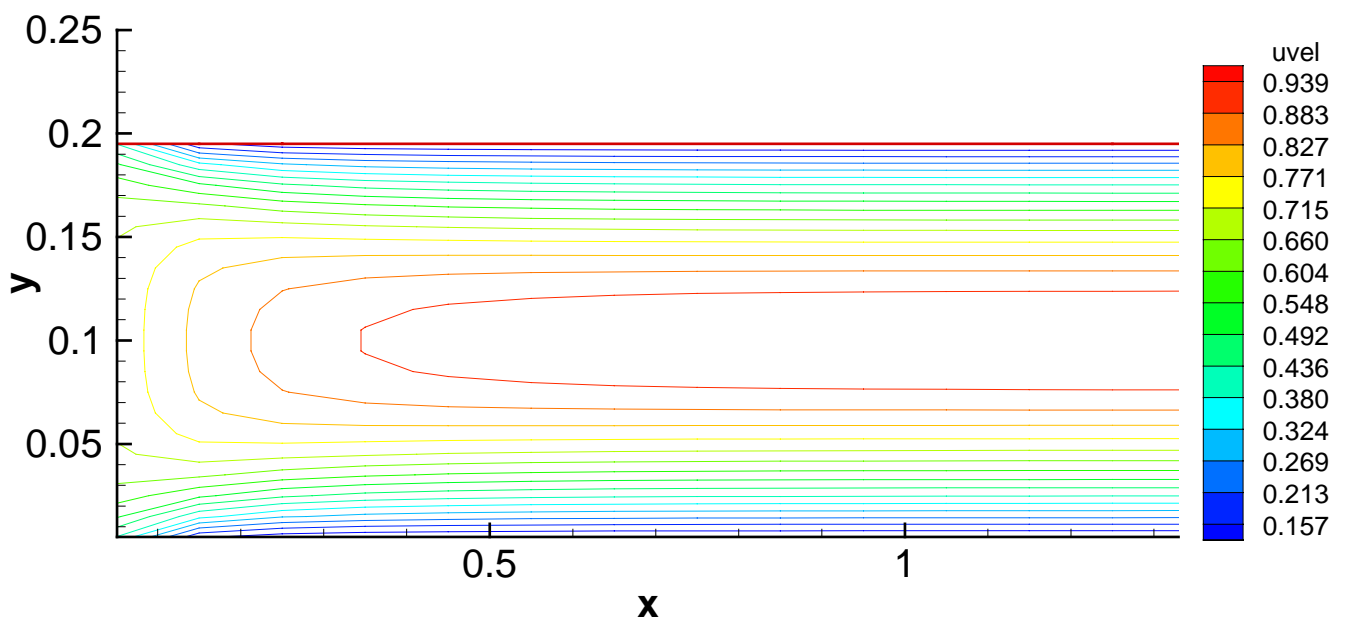

(a)

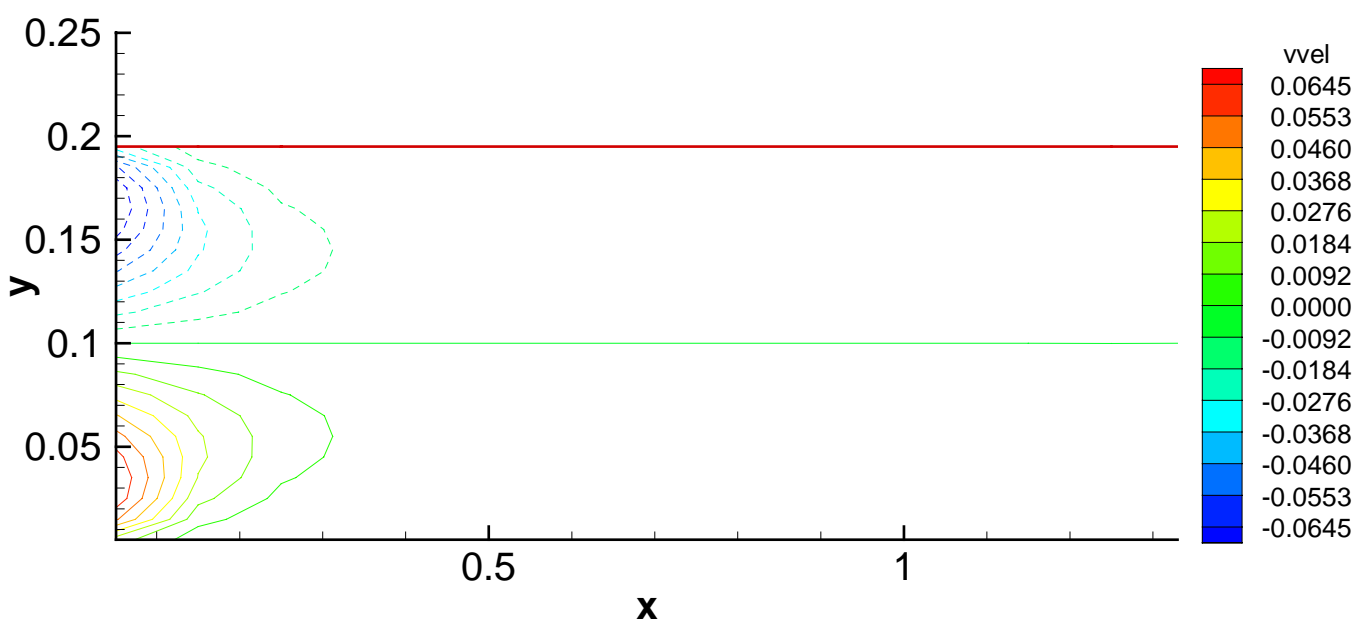

(b)

Figure 3-14 Entrance (developing) region of a developing channel flow, (a) u-velocity contour, (b) vvelocity contour. 


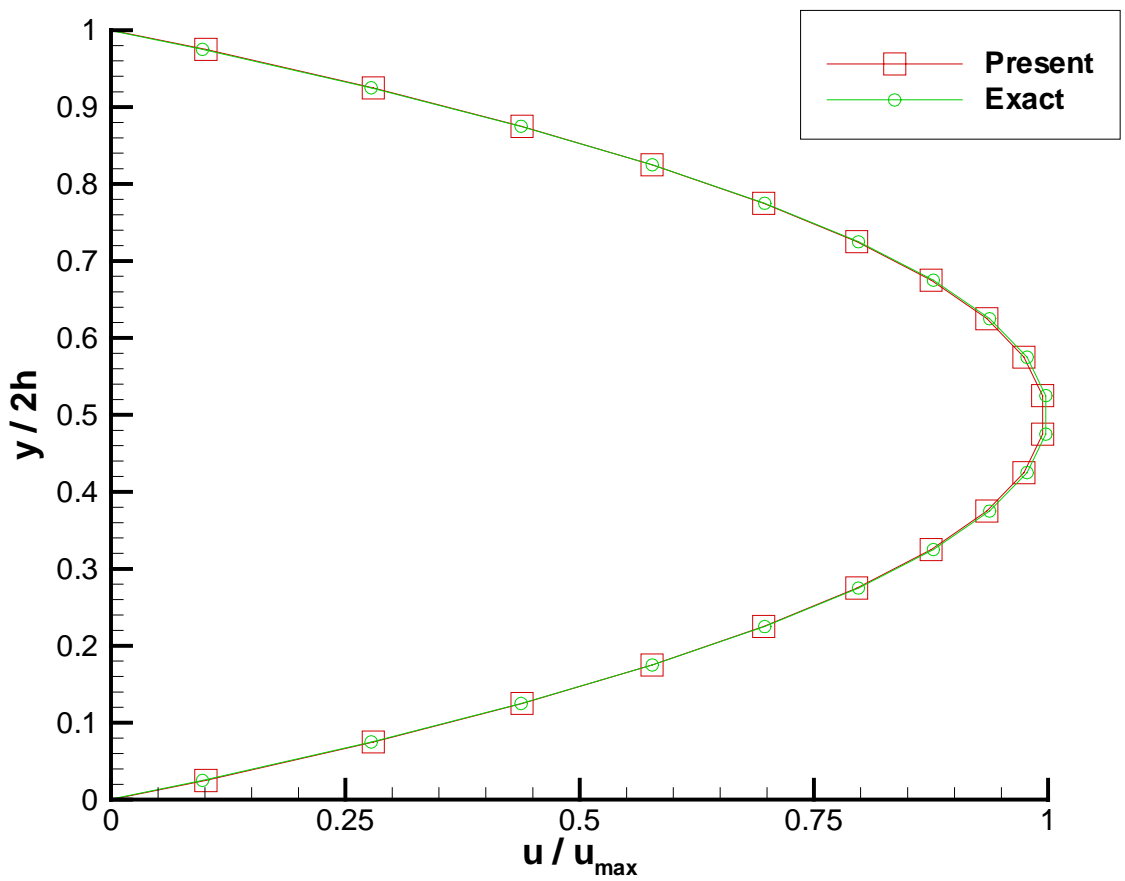

(a)

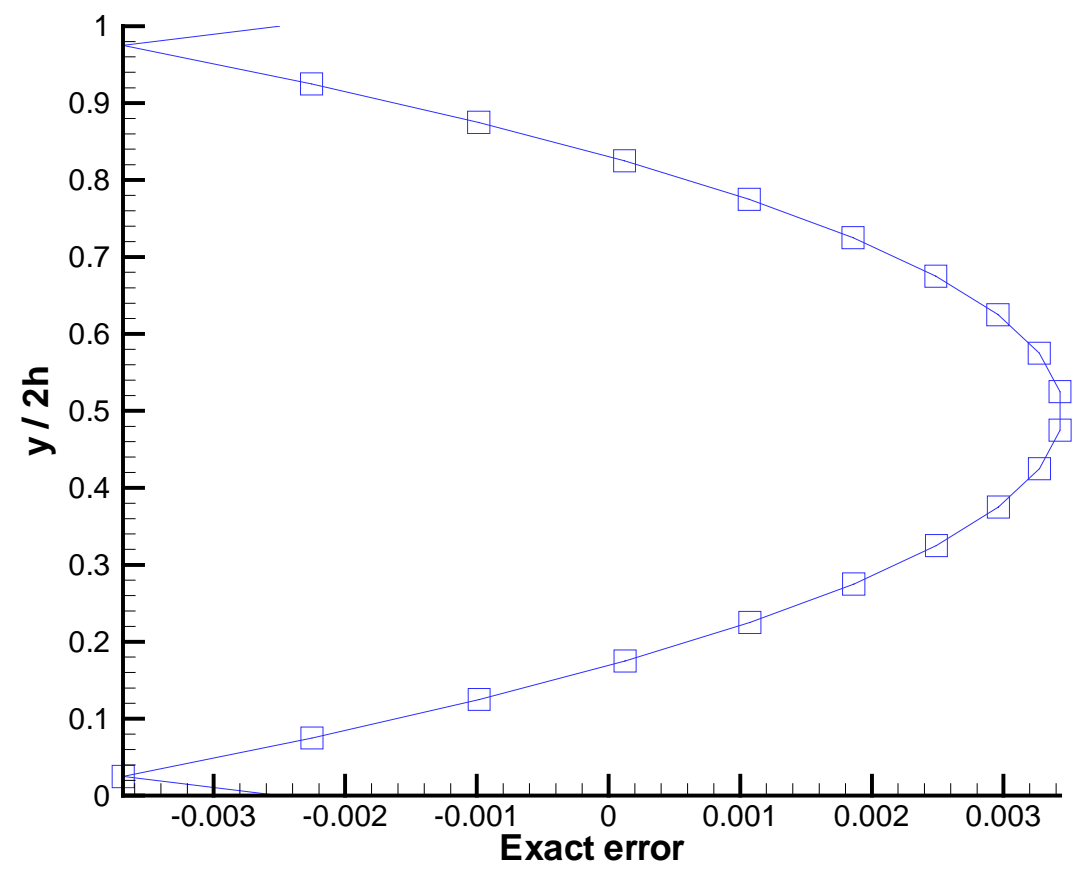

(b)

Figure 3-15 u-velocity profiles (a) and its exact error (b) in the fully developed region of a developing channel flow. 


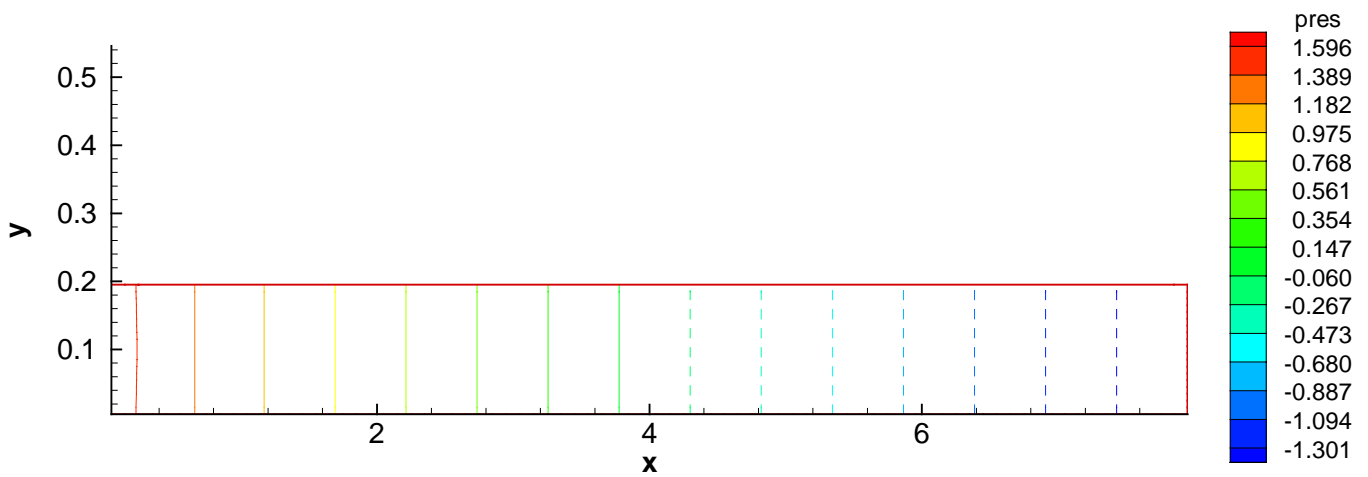

(a)

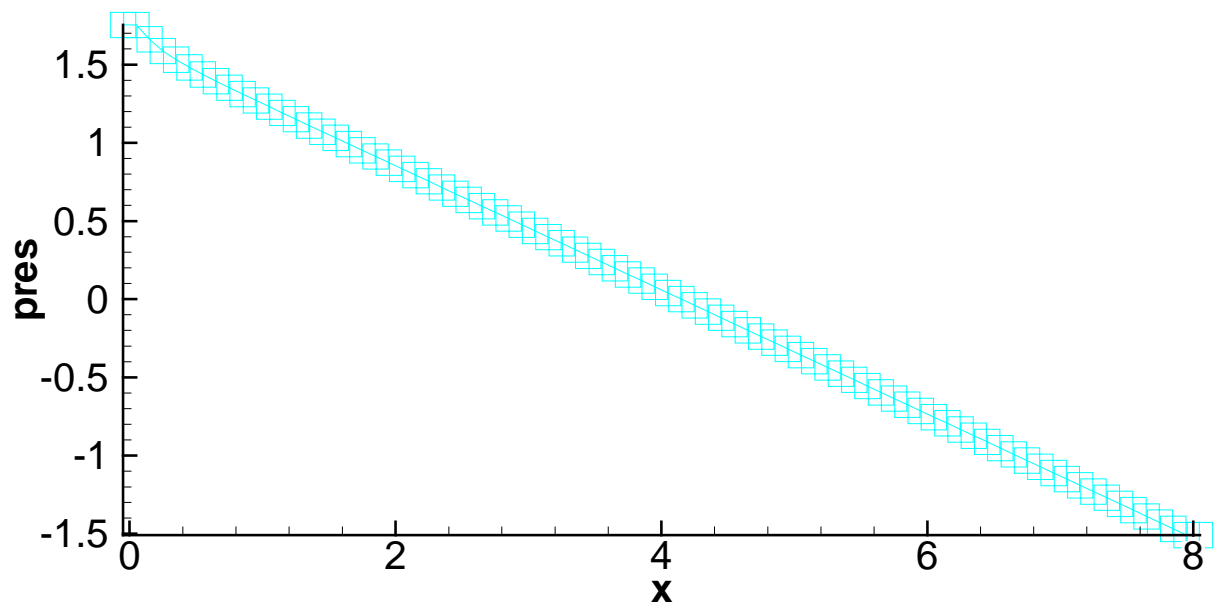

(b)

Figure 3-16 (a) Pressure contour of a developing channel flow, (b) pressure variation along horizontal centerline. 


\subsection{Poiseuille Flow Between Plates with Bottom Injection and Top Suction}

This is the last Poiseuille flow to be considered. The main flow is generated by a constant pressure gradient between two fixed parallel plates. Also let the upper and lower wall to be porous such that a vertical stream in and out of the wall is possible. Suppose the vertical steam is injected from the lower wall and fully drawn into the upper plate. If the vertical crossflow, denoted by $v_{\text {wall }}$, is assumed to be uniform, the flow field can then be described as

$$
\rho v_{\text {wall }} \frac{d u}{d y}=-\frac{d p}{d x}+\mu \frac{d^{2} u}{d y^{2}} .
$$

This second order linear inhomogeneous ordinary differential equation (ODE) can be readily solved with the no-slip boundary condition for $u$ at the upper and lower walls. The analytical solution, for example in (White 1991), is given in the following form:

$$
\frac{u}{u_{\max }}=\frac{2}{\operatorname{Re}_{\text {wall }}}\left(\frac{y}{h}-2+\frac{e^{\mathrm{Re}_{\text {wall }}}-e^{\operatorname{Re}_{\text {wall }} /(y-h)}}{\sinh \left(\operatorname{Re}_{\text {wall }}\right)}\right),
$$

where $u_{\max }$ is same as the one in Eq. (3.7), and the wall Reynolds number is defined as

$$
\operatorname{Re}_{\text {wall }}=\frac{v_{\text {wall }} h}{v} \text {. }
$$

As the wall Reynolds number approaches zero, the Poiseuille solution of the flow between two parallel plates (Eq. (3.7)) is reclaimed. This can be seen by a power series expansion for the last term in the parentheses, which cancels out the first order and constant terms and leaves the leading $2^{\text {nd }}$ order parabola exposed.

It should be noted that the analytical solution is derived based on the assumption of a uniform crossflow. In the numerical implementation, on the contrary, this simplification is not necessary. Both $\mathrm{u}$ - and v-velocity need to be solved; the injection and suction velocity appear as the boundary conditions to the v-component at lower and upper plate, respectively. On the other hand, similar to the approach described in Section 3.3, a constant pressure gradient is imposed as a source in the momentum equation with the pressure Poisson equation still being solved for a pressure-like variable to conserve the mass.

The same geometry and grid as the one in Section 3.1 through 3.3 shall be used here. Like before, Crank-Nicolson and power-law scheme is first used for the simulation. The time step used is 0.01 second. The calculated and exact u-velocity profiles at steady state are shown in Figure 3-17(a) for a wall Reynolds number of five. The corresponding exact error is depicted in Figure 3-17(b).

Unlike previous calculations where the numerical solution obtained by halfimplicitness and power-law gives close agreement to the exact one, the results computed using this scheme for the current case indicate a relatively high numerical diffusion, which can be seen in Figure 3-17 through a undershot of the numerical solution near the 
maximum velocity. There is reason to believe that for flows with complicated flow structures, such as the existence of re-circulation zones, the power-law or hybrid scheme may give rather diffusive results. The reader is referred to (Leonard and Drummond 1995) for further discussions. This example also presents a needed warning when one is about to employ a diffusive scheme to simulate a turbulent flow because turbulence may be "killed" or damped by the numerical dissipation.

Thus, to improve the accuracy, one should turn to a higher order scheme, such as the $2^{\text {nd }}$ order CD, $3^{\text {rd }}$ order QUICK or the $4^{\text {th }}$ order CD. As already mentioned in Section 2.6, a higher order scheme should be understood as the one applied to the convection terms only; for the diffusion terms the $2^{\text {nd }}$ order CD with Crank-Nicolson is always used in this study.

The flow is then computed with different high-order schemes (the $2^{\text {nd }}$ order explicit CD, $2^{\text {nd }}$ order implicit $C D$, semi-implicit $2^{\text {nd }}$ order CD, $3^{\text {rd }}$ order QUICK, and the $4^{\text {th }}$ order explicit CD), while keeping the same wall Reynolds number. In Figure 3-18(a) through (e) the u-velocity profiles calculated from the four schemes are displayed. Their corresponding exact errors are shown in Figure 3-19(a) through (e). A noticeable improvement with the four schemes is that around the peak velocity they all produce an overshot instead of the underestimation shown with the power-law scheme (Figure 3-17). That may indicate that a higher order scheme would rather make the signal too strong than too weak. From Figure 3-18(a) through (c) it is seen that the explicit and implicit version of the $2^{\text {nd }}$ order CD doesn't make much difference. Among the five schemes the $3^{\text {rd }}$ order QUICK and the $4^{\text {th }}$ order CD prevail over the other two $2^{\text {nd }}$ order methods; they generally predict the peak value more accurately (Figure 3-18(d) and (e)) and have more points whose errors are agglomerated around zero (see Figure 3-19(d) and (e)).

To see if one scheme is quantitatively superior over the other an error analysis is performed to determine the apparent order of the schemes. Under consideration are four schemes: Crank-Nicolson and power-law, Crank-Nicolson and $2^{\text {nd }}$ order CD, explicit QUICK, and explicit $4^{\text {th }}$ order CD. Again, the maximum exact absolute errors, maximum $\mathrm{u}$-velocity and other post-processed data are listed in Table 3-2 for respective schemes calculated at three different grids. For each scheme a log-log plot is then produced based on the normalized grid size and the normalized maximum error. A linear fit of the three points in each graph is followed, giving the desired order of a scheme. For the four different schemes in question, the observed orders are 2.0094, 2.0405, 2.0451 and 2.0469 respectively.

Table 3-2 Order of method analysis for the calculations of Couette-Poiseuille Flow with bottom injection and top suction; schemes considered: semi-implicit power-law, semi-implicit $2^{\text {nd }}$ order CD, explicit QUICK, explicit $4^{\text {th }}$ order CD.

\begin{tabular}{|c|c|c|c|c|c|c|c|}
\hline grid in $y$ & $\mathrm{~h} / \mathrm{h}_{\mathrm{ref}}$ & $\varepsilon_{\max }$ & $\mathrm{u}_{\max }$ & $\varepsilon_{\max } / u_{\max }$ & $\ln \left(h / h_{\text {ref }}\right)$ & $\ln \left(\varepsilon_{\max } / u_{\max }\right)$ & slope \\
\hline \multicolumn{8}{|c|}{ C-N, power-law } \\
\hline 80 & 1 & 3.1052E-03 & 0.5305 & 5.8537E-03 & 0.0000 & -5.1407 & \\
\hline 40 & 2 & 1.2391E-02 & 0.5260 & 2.3558E-02 & 0.6931 & -3.7483 & \\
\hline 20 & 4 & 4.9187E-02 & 0.5184 & 9.4891E-02 & 1.3863 & -2.3550 & 2.0094 \\
\hline
\end{tabular}




\begin{tabular}{|c|c|c|c|c|c|c|c|}
\hline \multicolumn{8}{|c|}{$\overline{C-N, \text { 2nd CD }}$} \\
\hline 80 & 1 & 1.5907E-03 & 0.5362 & 2.9666E-03 & 0.0000 & -5.8204 & \\
\hline 40 & 2 & 6.5160E-03 & 0.5371 & 1.2132E-02 & 0.6931 & -4.4119 & \\
\hline 20 & 4 & $2.7226 \mathrm{E}-02$ & 0.5423 & 5.0208E-02 & 1.3863 & -2.9916 & 2.0405 \\
\hline \multicolumn{8}{|c|}{ Explicit, QUICK } \\
\hline 80 & 1 & 1.5914E-03 & 0.5359 & 2.9697E-03 & 0.0000 & -5.8193 & \\
\hline 40 & 2 & $6.5156 \mathrm{E}-03$ & 0.5360 & 1.2157E-02 & 0.6931 & -4.4099 & \\
\hline 20 & 4 & 2.7223E-02 & 0.5382 & 5.0582E-02 & 1.3863 & -2.9842 & 2.0451 \\
\hline \multicolumn{8}{|c|}{ Explicit, 4th CD } \\
\hline 80 & 1 & 1.5914E-03 & 0.5359 & 2.9699E-03 & 0.0000 & -5.8192 & \\
\hline 40 & 2 & $6.5155 \mathrm{E}-03$ & 0.5358 & 1.2160E-02 & 0.6931 & -4.4096 & \\
\hline 20 & 4 & 2.7223E-02 & 0.5369 & 5.0709E-02 & 1.3863 & -2.9817 & 2.0469 \\
\hline
\end{tabular}

For this flow configuration, all the four schemes perform well. They all exhibit an order of at least two. Although the order of the power-law scheme is slightly lower than the other three, it is already very encouraging to achieve a $2^{\text {nd }}$ order accuracy with a theoretically only $1^{\text {st }}$ order scheme (see Section 2.3 ). The $2^{\text {nd }}$ order CD is doing what it supposed to be dong. Although the $3^{\text {rd }}$ order QUICK is theoretically more accurate than $2^{\text {nd }}$ order $C D$, but they converge asymptotically in a second order manner and the differences between them are rarely large (Ferziger and Peric 1996); this point is exactlly reflected in the current results. Discrepancy occurs with the theoretically $4^{\text {th }}$ order $\mathrm{CD}$ as the observed order does not meet its commensurate demand. Even though the exact cause is not quite clear yet, one can postulate that the accuracy loss may be related to several factors discussed at the end of Section 3.3; among which the inheritance of the splitting method may be an important one to restrict a further increase in accuracy. One also notices that although all the calculated orders are at about the same level, they are still sorted in an order it should be; in other words, the $3^{\text {rd }}$ order QUICK is slightly more accurate than $2^{\text {nd }}$ order $C D$, and the $4^{\text {th }}$ order CD is slightly more accurate than the QUICK etc.

As already mentioned in Section 3.2, the maximum error is one of the most stringent error criteria. A slightly looser one is the RMS (root mean square) error. A similar error calculation based on the RMS measure is provided in Table 3-3 and the corresponding linear fit is shown in Figure 3-21. It is noticed that with this less strict criteria the order of the method, positioned at 2.2347 , increases about $10 \%$, compared to the 2.0469 from the maximum error measure.

Table 3-3 Order of method analysis based on the RMS error for the calculations of Couette-Poiseuille Flow with bottom injection and top suction, $4^{\text {th }}$ order $\mathrm{CD}$ is used

\begin{tabular}{cccccccc} 
grid in $\mathrm{y}$ & $\mathrm{h} / \mathrm{h}_{\text {ref }}$ & $\varepsilon_{\text {rms }}$ & $\mathrm{u}_{\max }$ & $\varepsilon_{\max } / \mathrm{u}_{\max }$ & $\ln \left(\mathrm{h} / \mathrm{h}_{\text {ref }}\right)$ & $\ln \left(\varepsilon_{\max } / \mathrm{u}_{\max }\right)$ & slope \\
\hline 80 & 1 & $3.3476 \mathrm{E}-04$ & 0.5359 & $6.2472 \mathrm{E}-04$ & 0.0000 & -7.3782 & \\
40 & 2 & $1.5325 \mathrm{E}-03$ & 0.5358 & $2.8602 \mathrm{E}-03$ & 0.6931 & -5.8569 & \\
20 & 4 & $7.4298 \mathrm{E}-03$ & 0.5369 & $1.3840 \mathrm{E}-02$ & 1.3863 & -4.2802 & $\mathbf{2 . 2 3 4 7}$
\end{tabular}


Finally, the behavior of the injection-suction flow at different wall Reynolds numbers should be examined. To this end the $3^{\text {rd }}$ order QUICK is picked. The results at $\operatorname{Re}_{\text {wall }}=10,5,3,1$ and 0 are plotted in Figure 3-22. In all situations it is seen that the quality of the numerical solution is satisfying; the location and magnitude of u-velocity at those turning points are all accurately captured. In particular, when $\mathrm{Re}_{\text {wall }}=0$ the profile reproduces to a Poiseuille parabola that has been encountered many times in the previous sections.

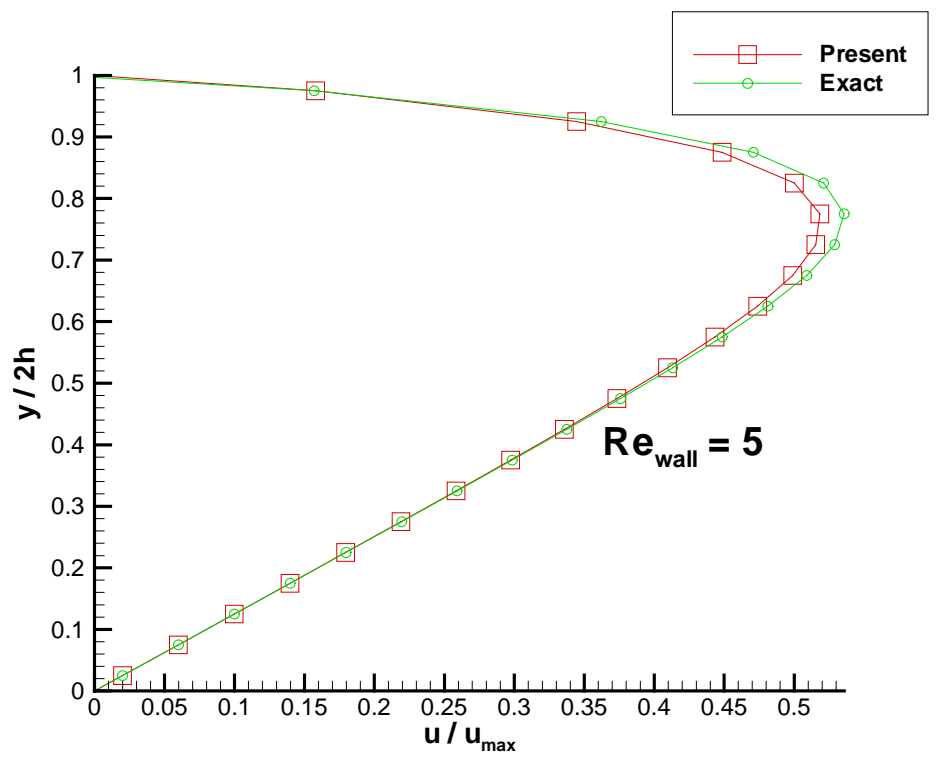

(a)

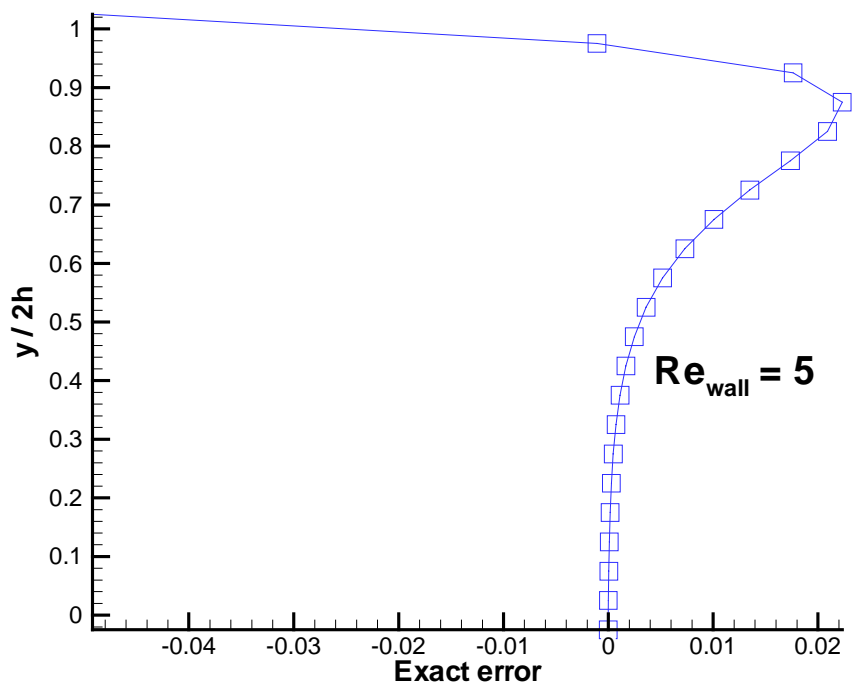

(b)

Figure 3-17 u-velocity profiles (a) and its exact error (b) of Poiseuille flow between plates with bottom injection and top suction; power-law scheme is used. 


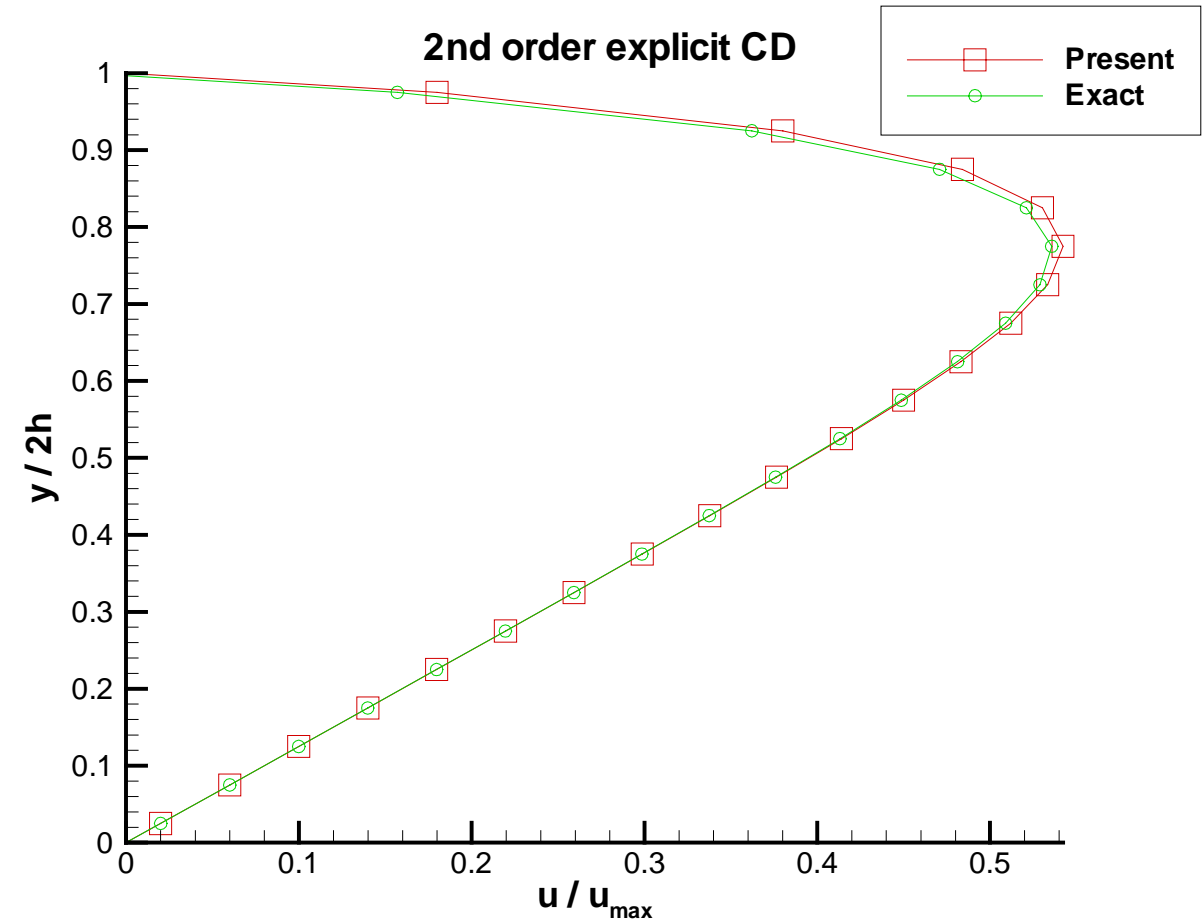

(a)

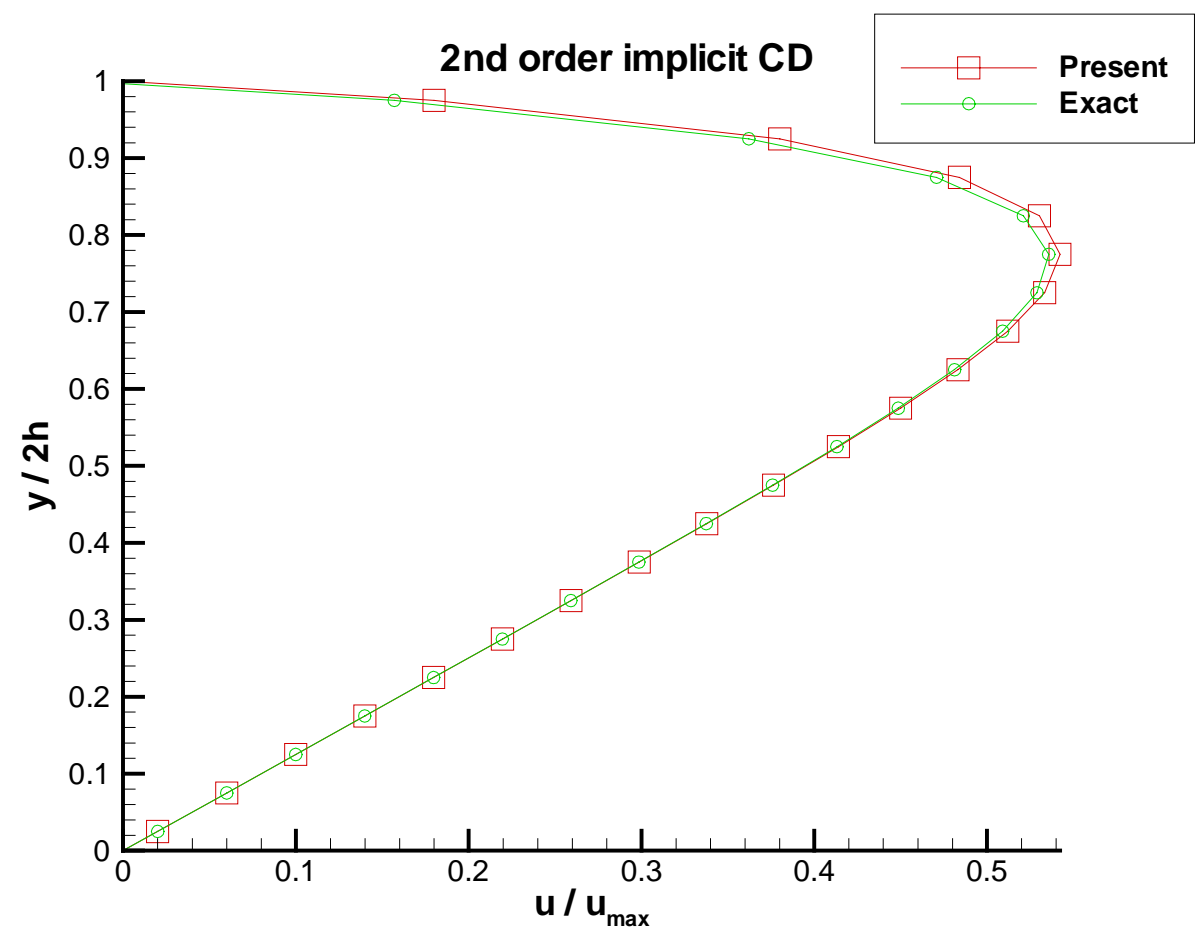

(b)

Figure 3-18 Comparison of u-velocity profiles calculated from different higher order schemes for Poiseuille flow between plates with bottom injection and top suction, (a) $2^{\text {nd }}$ order explicit central differencing (CD), (b) $2^{\text {nd }}$ order implicit CD. 


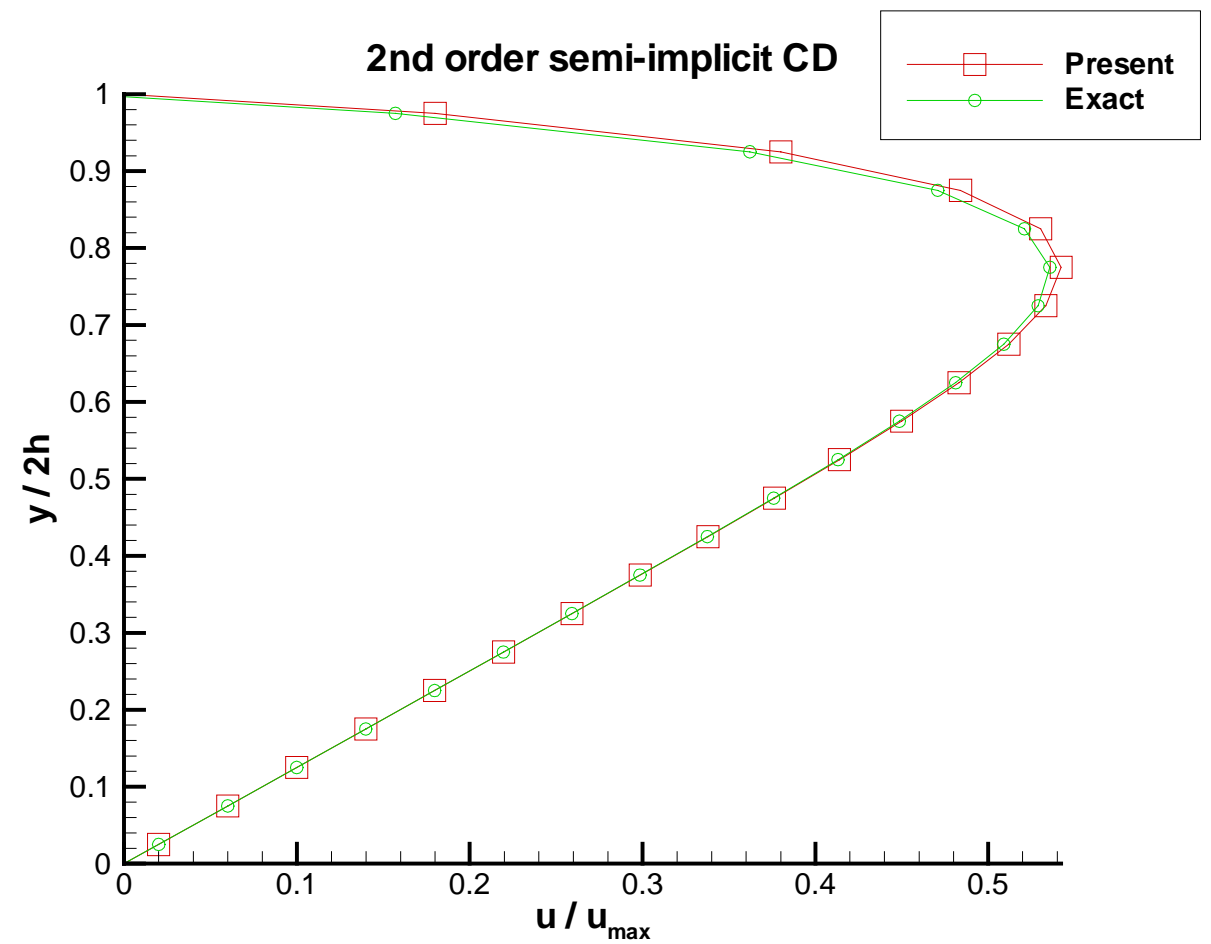

(c)

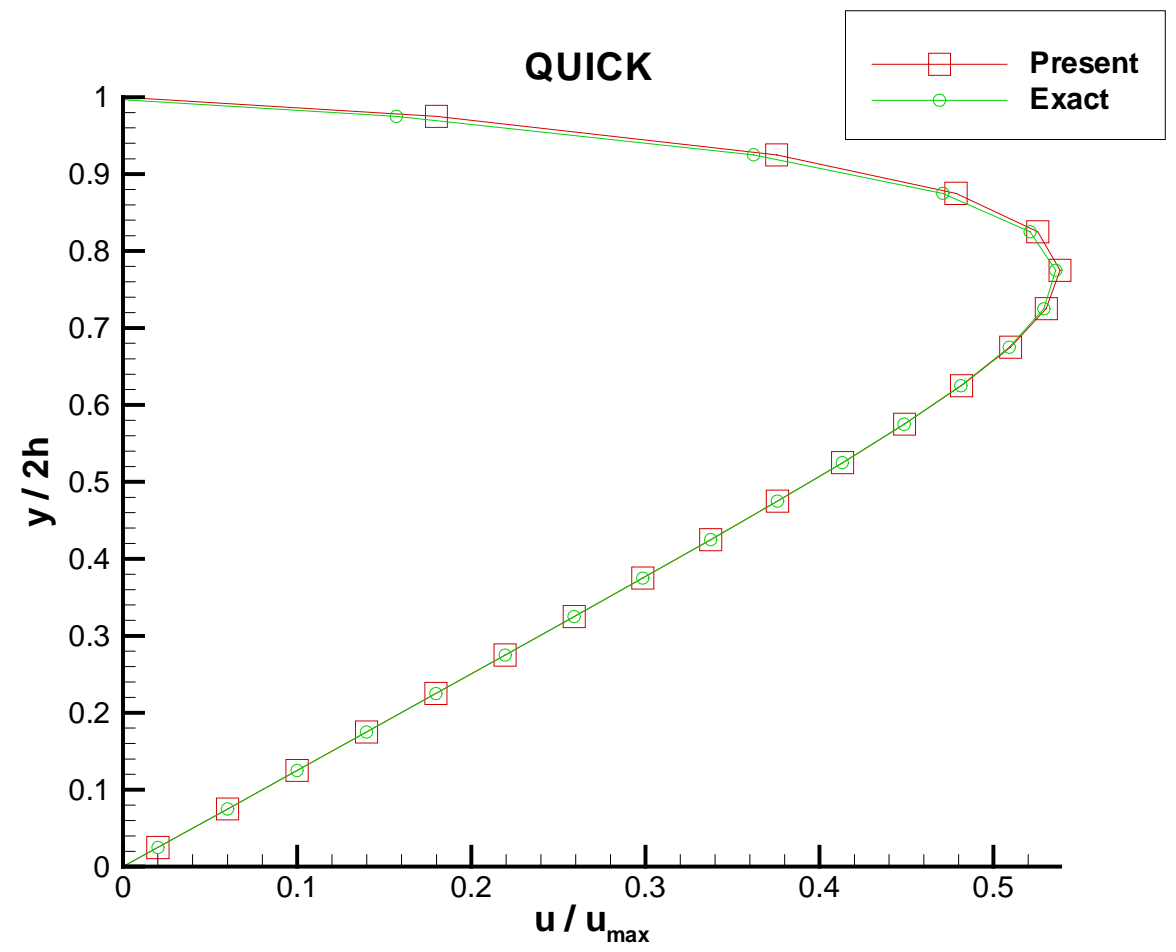

(d)

Figure 3-18 Comparison of u-velocity profiles calculated from different higher order schemes for Poiseuille flow between plates with bottom injection and top suction, (c) $2^{\text {nd }}$ order semi-implicit $C D$, (d) $3^{\text {rd }}$ order QUICK. 


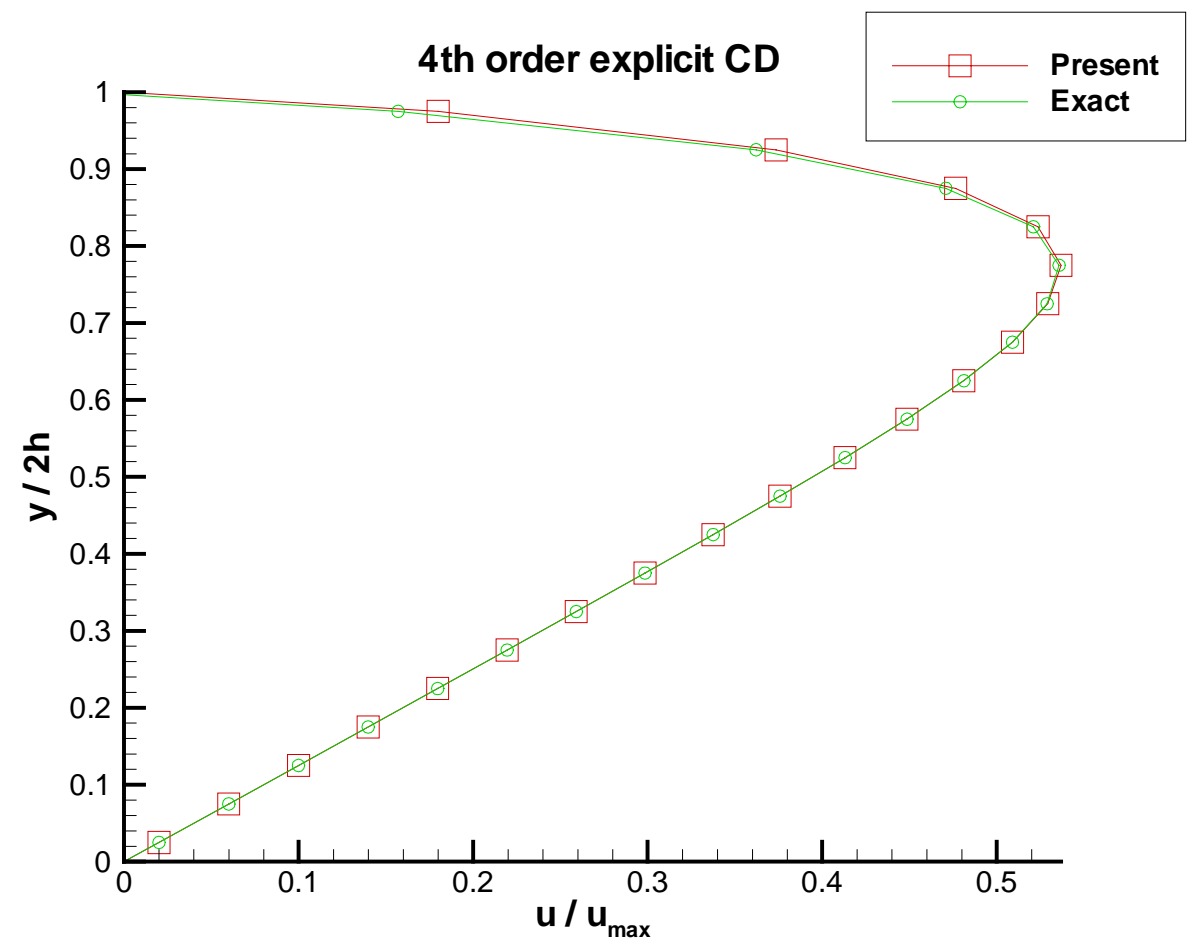

(e)

Figure 3-18 Comparison of u-velocity profiles calculated from different higher order schemes for Poiseuille flow between plates with bottom injection and top suction, (e) $4^{\text {th }}$ order explicit CD. 


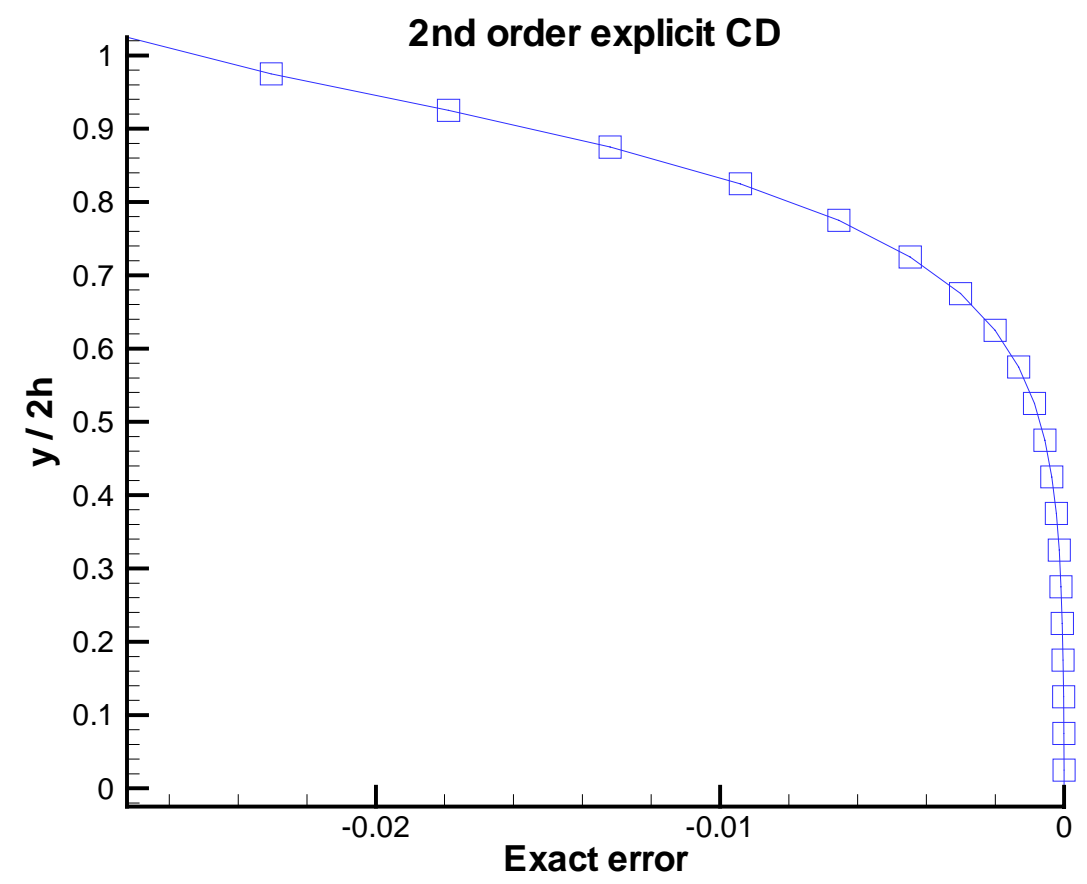

(a)

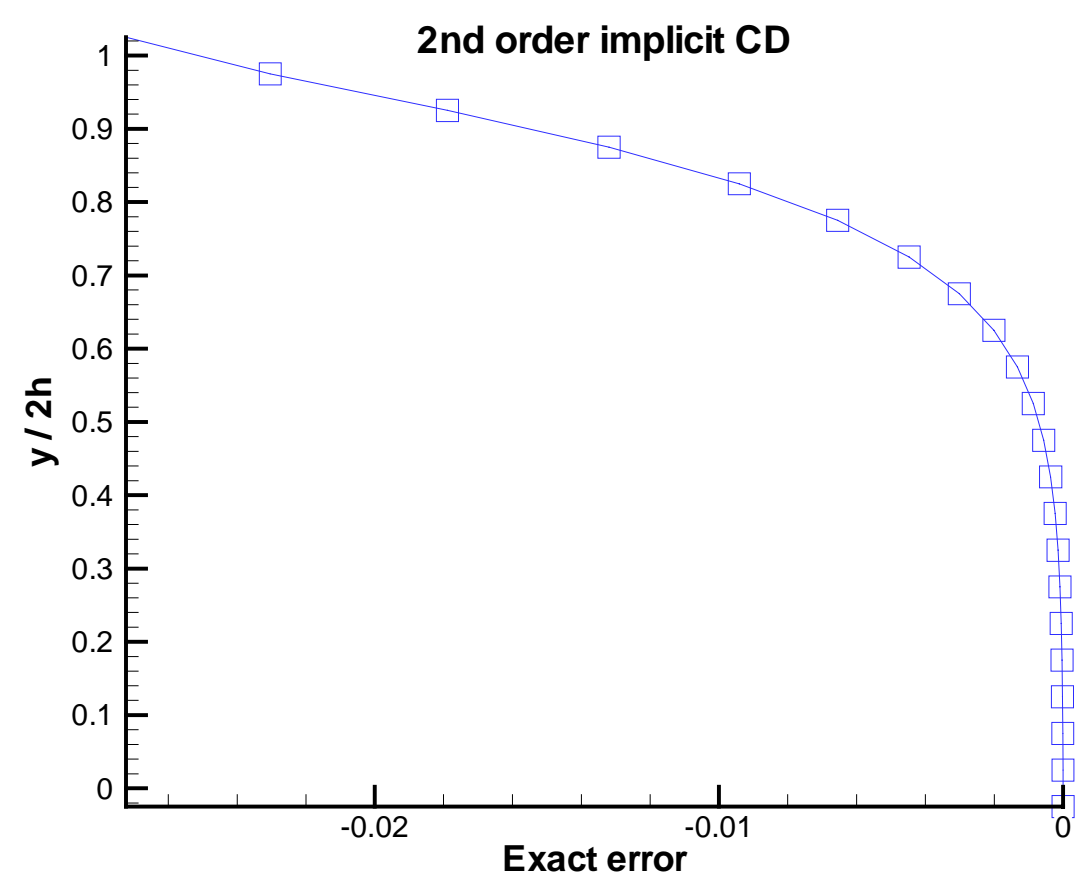

(b)

Figure 3-19 Comparison of exact error of u-velocities calculated with different higher order schemes for Poiseuille flow between plates with bottom injection and top suction, (a) $2^{\text {nd }}$ order explicit $C D$, (b) $2^{\text {nd }}$ order implicit CD. 


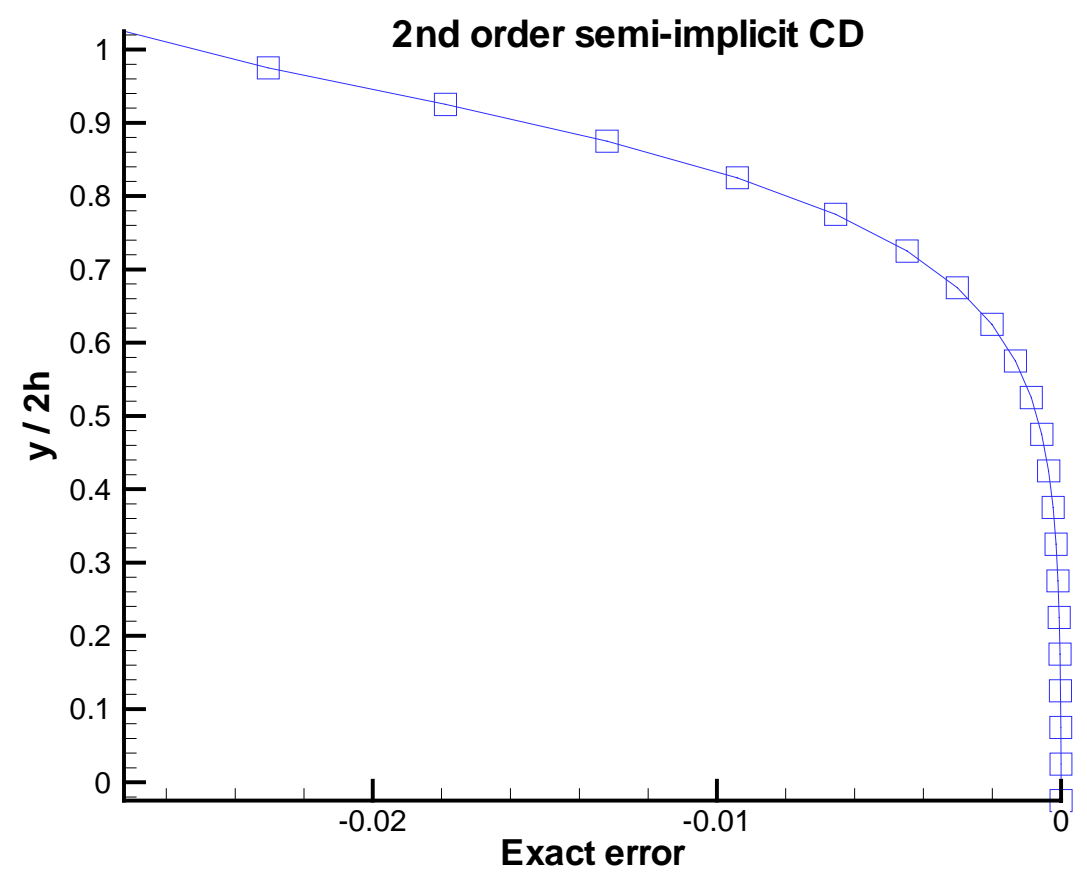

(c)

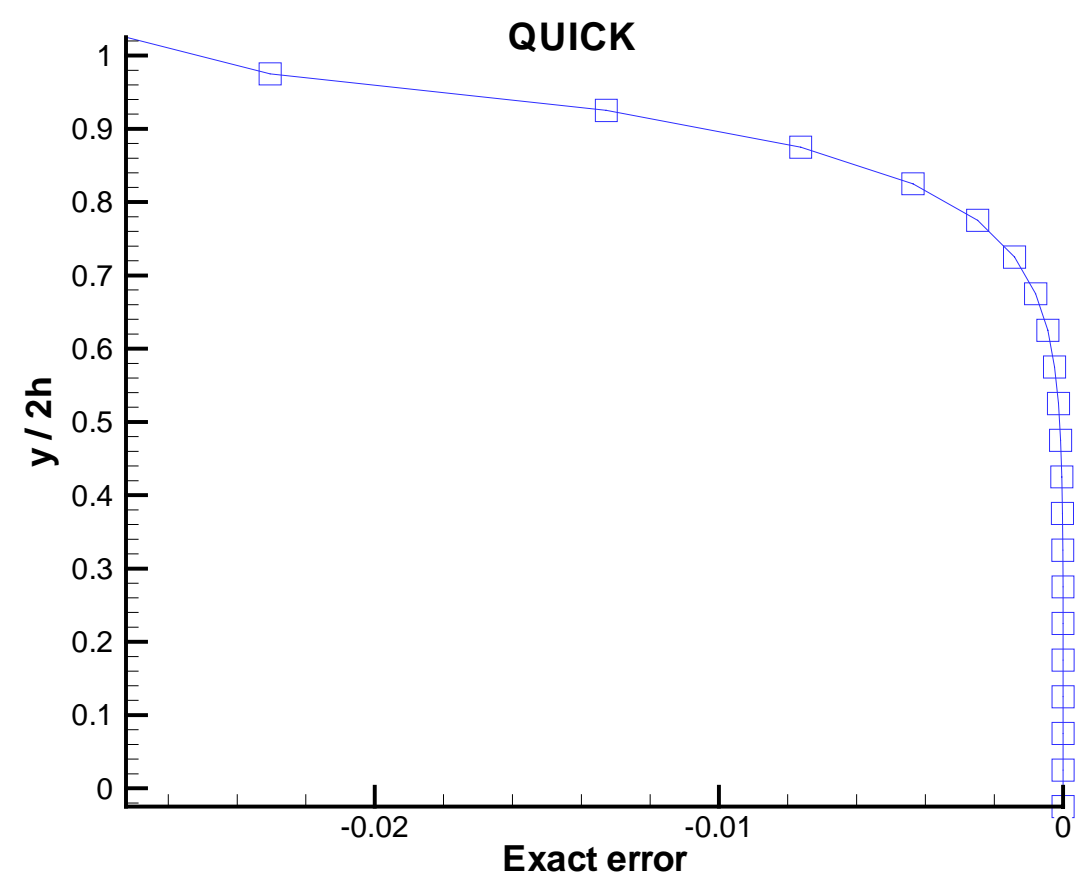

(d)

Figure 3-19 Comparison of exact error of u-velocities calculated with different higher order schemes for Poiseuille flow between plates with bottom injection and top suction, (c) $2^{\text {nd }}$ order semi-implicit CD, (d) $3^{\text {rd }}$ order QUICK. 


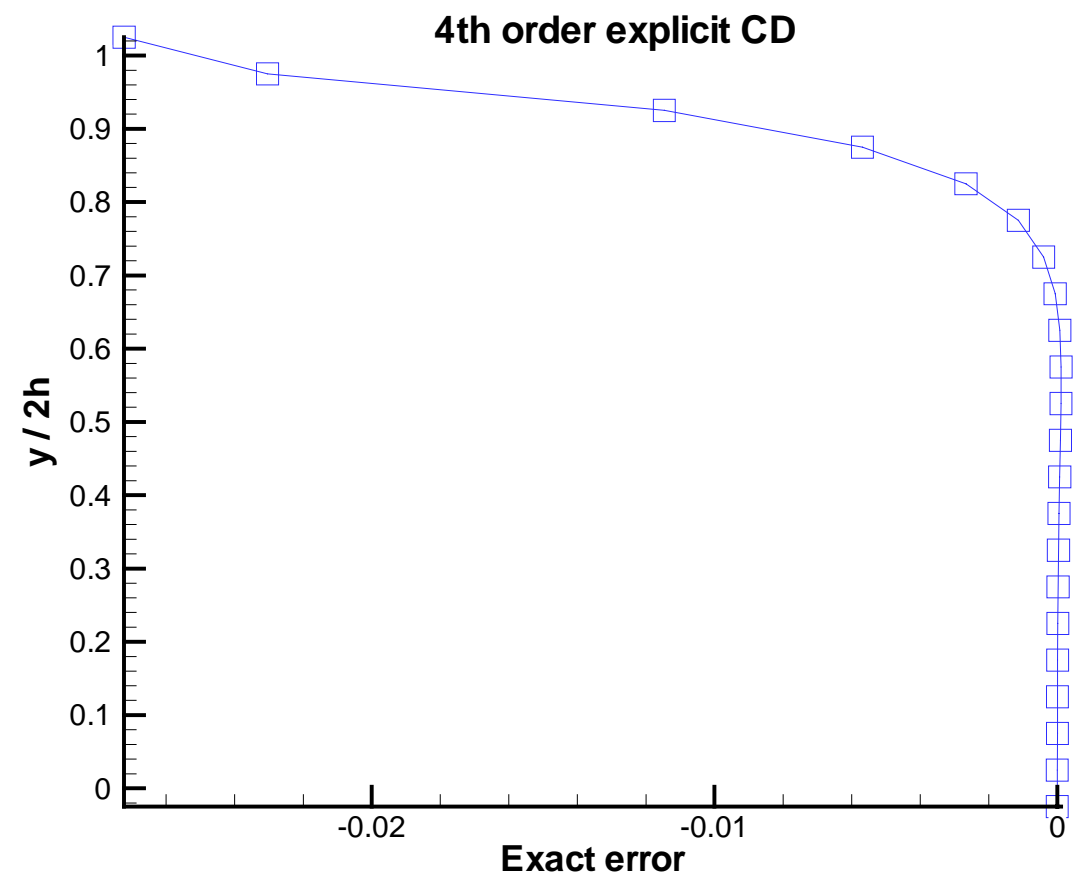

(e)

Figure 3-19 Comparison of exact error of u-velocities calculated with different higher order schemes for Poiseuille flow between plates with bottom injection and top suction, (e) $4^{\text {th }}$ order explicit CD.

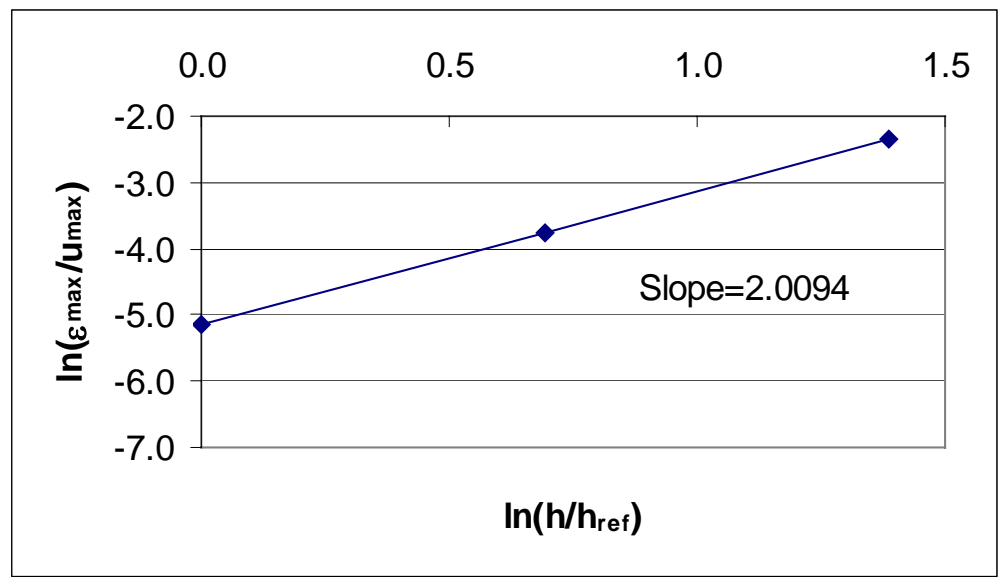

(a)

Figure 3-20 Maximum error as a function of mesh refinement for the Couette-Poiseuille flow between plates with bottom injection and top suction, (a) semi-implicit power-law. 


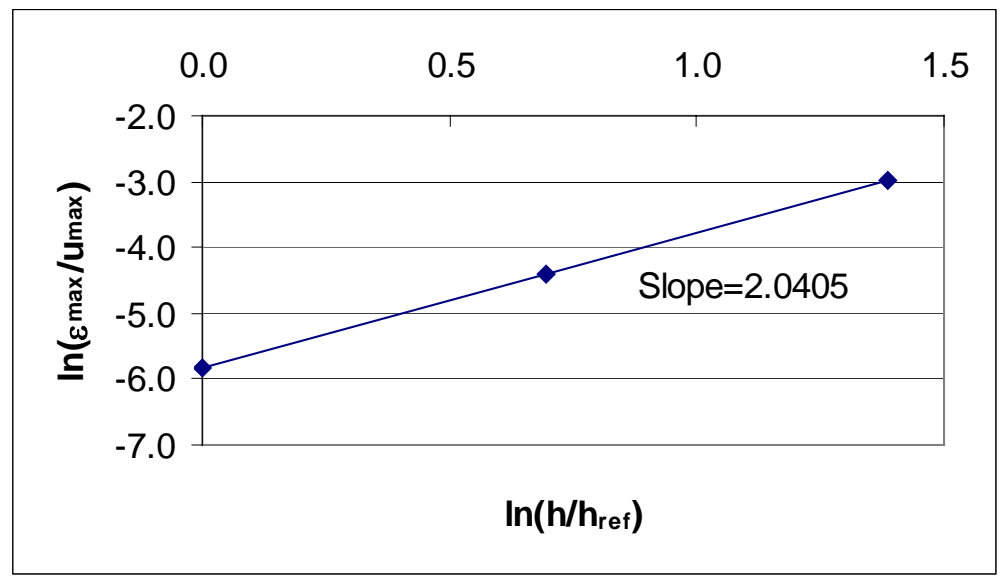

(b)

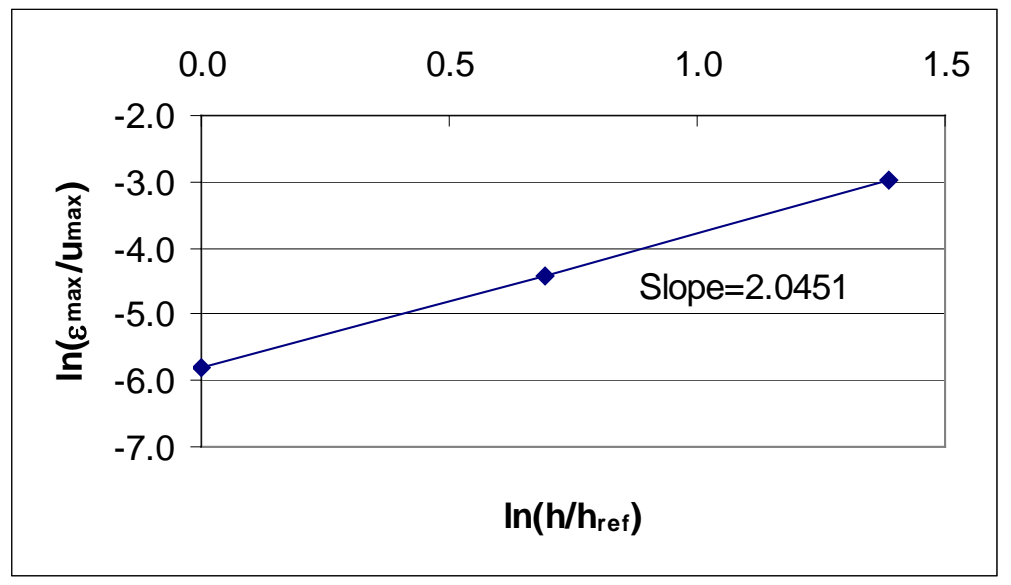

(c)

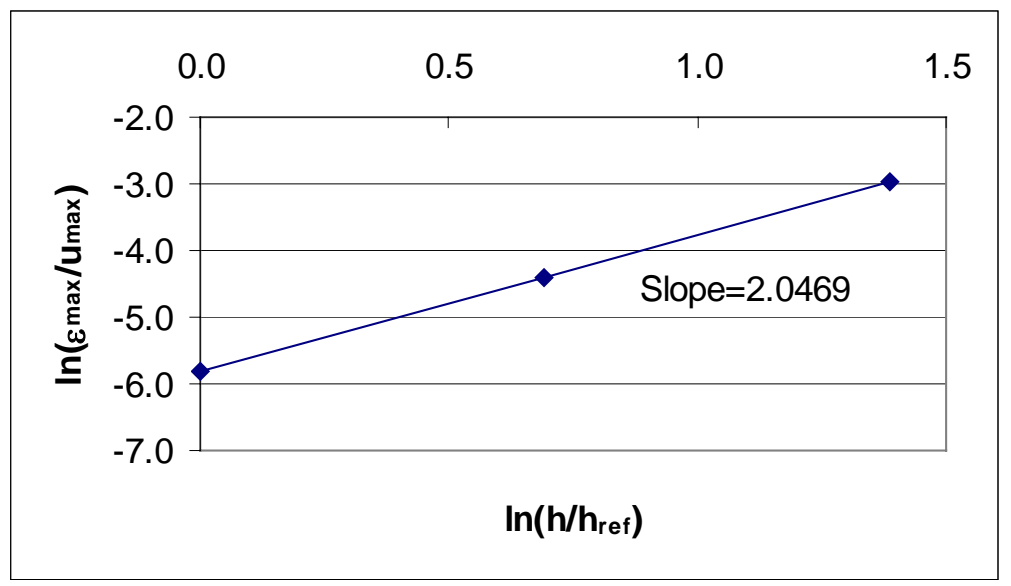

(d)

Figure 3-20 Maximum error as a function of mesh refinement for the Couette-Poiseuille flow between plates with bottom injection and top suction, (b) semi-implicit $2^{\text {nd }}$ order CD, (c) explicit and QUICK, (d) explicit $4^{\text {th }}$ order CD. 


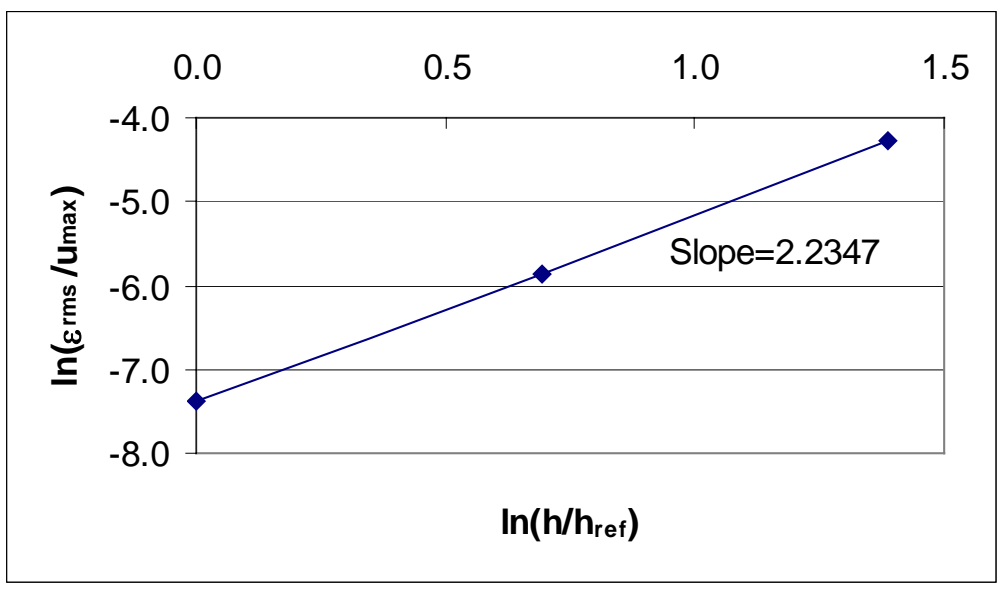

Figure 3-21 RMS error as a function of mesh refinement for the Couette-Poiseuille flow between plates with bottom injection and top suction, $4^{\text {th }}$ order CD is used

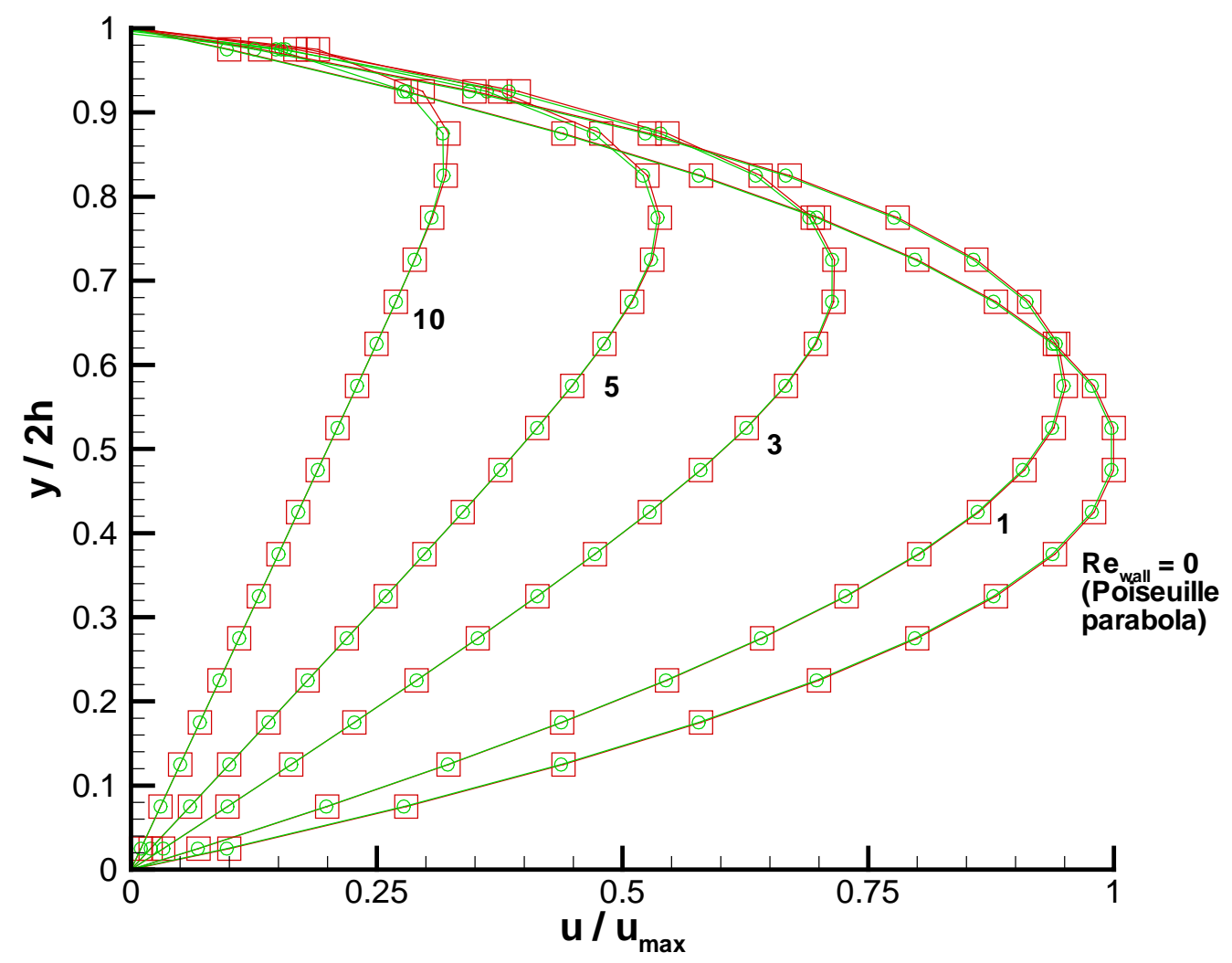

Figure 3-22 u-velocity profiles of Poiseuille flow between plates with bottom injection and top suction at different wall Reynolds number. Square symbols: present calculation; circle symbols: exact solution. 


\subsection{Taylor's Vortex}

Taylor's vortex is one of the very few benchmarking test cases that not only offers a full analytical solution to the two-dimensional Navier-Stokes equations but also includes unsteady, convective and diffusive effects. The analytical solution was originally obtained by (Taylor 1923a) which describes a system of counter-rotating decaying, and incompressible vortices in a infinitely large domain. The vortices can be formulated in terms of a steam function as

$$
\psi(x, y, t)=\frac{C}{\pi} \sin (\pi x) \sin (\pi y) \exp \left(-2 \pi^{2} v t\right),
$$

where $C$ is a constant coefficient related to the amplitude of velocity components, i.e., the maximum velocity. By the definition of stream function the velocity field can be obtained as

$$
\begin{aligned}
& u=\frac{\partial \psi}{\partial y}=C \sin (\pi x) \cos (\pi y) \exp \left(-2 \pi^{2} v t\right), \\
& v=-\frac{\partial \psi}{\partial x}=-C \cos (\pi x) \sin (\pi y) \exp \left(-2 \pi^{2} v t\right) .
\end{aligned}
$$

It can be readily shown that this velocity field satisfies continuity. The pressure, $p$, can be found from the two-dimensional momentum equations:

$$
p=\frac{C^{2}}{4}[\cos (2 \pi x)+\cos (2 \pi y)] \exp \left(-4 \pi^{2} v t\right) .
$$

Other configurations of Taylor's vortices also exist, given one example as

$$
\begin{aligned}
& \psi(x, y, t)=\frac{C}{\pi} \cos (\pi x) \cos (\pi y) \exp \left(-2 \pi^{2} v t\right), \\
& u=\frac{\partial \psi}{\partial y}=-C \cos (\pi x) \sin (\pi y) \exp \left(-2 \pi^{2} v t\right), \\
& v=-\frac{\partial \psi}{\partial x}=C \sin (\pi x) \cos (\pi y) \exp \left(-2 \pi^{2} v t\right), \\
& p=-\frac{C^{2}}{4}[\cos (2 \pi x)+\cos (2 \pi y)] \exp \left(-4 \pi^{2} v t\right) .
\end{aligned}
$$

It is worthwhile to mention that the Taylor's vortices described above is closely related to a classical experiment (Taylor 1923b) conducted by the same scientist, in which he used two concentric rotating cylinders to predict the onset of instability of the azimuthal Couette flow, and he further showed that when the inner-cylinder reaches a critical rotating speed a cellular pattern is formed and the fluid travels in helical paths around the cylinder in layers of vortices, called Taylor vortex flow.

Very often the analytical solution is used to study only a single vortex; that is why it is called Taylor's vortex instead of Taylor's vortices. For example, Eq. (3.15) through (3.17) can define a vortex centered at $(0.5,0.5)$ with a radius of unity. In the verification practice of a Navier-Stokes solver the Taylor's vortex has been widely used (e.g., (Kim 
and Moin 1985; Smith 1996; Arunajatesan and Shipman 2004)) to test both the space accuracy and temporal accuracy of the solver. In the present study Eq. (3.15) through (3.17) shall be used to confirm the results and the order of the method.

The computational domain considered here is a square with the side length, $L$, equal to $1.0 \mathrm{~m}$. The origin is placed at lower left corner of the square. The exact solution is used to specify the initial condition at $t=0$, as well as to impose the Dirichlet boundary conditions for $\mathrm{u}$ - and v-velocities. Note that the boundary conditions applied are timedependent and vary along the $\mathrm{x}$ - or $\mathrm{y}$-position. Pressure boundary condition is still Neumann, because when velocity is specified at boundary, pressure should not be prescribed at boundary, and vise versa (Ferziger and Peric 1996). The Reynolds number is based on the side length and the maximum velocity.

Simulation is first carried out on $41 \times 41$ grid using Crank-Nicolson and power-law scheme at a Reynolds number of 100 , which corresponds to a maximum velocity and kinematic viscosity equal to $0.1 \mathrm{~m} / \mathrm{s}$ and $1 \times 10^{-3} \mathrm{~m}^{2} / \mathrm{s}$, respectively. The time step used is $0.1 \mathrm{~s}$. At time $t=50 \mathrm{~s}$, at whose point the vortex has decayed to about $37.27 \%$ of its initial strength, results are collected and presented in Figure 3-23 through Figure 3-26.

Figure 3-23 shows streamlines of a Taylor's vortex with the background being the magnitude of the velocity. It is noticed that the vortex has a counter-clockwise flow direction, and a nice symmetry of the velocity magnitude is present. Three contour plots are also presented in Figure 3-24 for the u-, v- velocity and pressure. The maximum uvelocity and v-velocity is $0.03692 \mathrm{~m} / \mathrm{s}$ and $0.03691 \mathrm{~m} / \mathrm{s}$ respectively, which matches the approximate $37 \%\left(\mathrm{e}^{-1}\right)$ decay of the vortex. In Figure 3-24(c) it is observed that the pressure grows radially toward the outside, a low-pressure region is concentrated around the center, and four high-pressure regions are distributed at four corners. Quantitative comparisons between exact and numerical solution at this instant are presented in Figure 3-25 and Figure 3-26, where the two plots are created at center x-station and center ystation respectively. One can see the exact error for both u- and v-velocity exhibits an interesting vertical or horizontal "S" shape. Attention should be paid to the relatively large numerical error in both the horizontal and vertical directions. The deficiency of the essentially $1^{\text {st }}$ order power-law scheme is exposed in this unsteady flow calculation.

Next, the explicit QUICK is used to see if it would improve the results. As expected, a drastic improvement in the accuracy is clearly seen from Figure 3-27 and Figure 3-28; the exact error is reduced by nearly an order of magnitude! The excellent agreement encourages us to record line profiles at more time instants to explore its full performance. The results are plotted in Figure 3-29(a) and (b) at $t=0,20,50,100$ and 200 seconds.

The dependency of the flow on the Reynolds number should be checked as well. To this end the Reynolds number is increased to 1000 . This can be done by, for example, changing the maximum velocity to $1.0 \mathrm{~m} / \mathrm{s}$. Due to stability constraints at high Reynolds number the time step is reduced to $0.005 \mathrm{~s}$ correspondingly, and it takes ten thousand time iterations to reach $t=50 \mathrm{~s}$. The u-velocity profile and its error at the center of the $\mathrm{x}$-axis, 
the v-velocity profile and its error at the center of the y-axis are plotted in Figure 3-30(a), (b) and Figure 3-31(a), (b), respectively. In general, the magnitude of the numerical error not only depends on the scheme, but also the nature of the flow. Comparing Figure 3-30(b) with Figure 3-27(b), and Figure 3-31(b) with Figure 3-28(b), it is seen that with a ten times increase of Re, the numerical error is also increased by a factor of ten. Yet, this error produced by the explicit QUICK, even at such high Re value, is still smaller than the one given by semi-implicit power-law (see Figure 3-25(b) and Figure 3-26(b)).

This unsteady, fully 2D flow problem in fact presents an excellent test case since it has an exact solution, and it involves convective, viscous and unsteady effects. Here, the semi-implicit power-law scheme and explicit QUICK will be considered for error analysis. Three grids with a successive double refinement are $10 \times 10,20 \times 20$ and $40 \times 40$. The RMS (root mean square) value of the exact absolute errors will be used to estimate the order of the method. The Reynolds number under consideration is again 100.

To examine the spatial accuracy the time step size is taken to be constant. On the one hand, the time step should be kept reasonably small to eliminate as much as possible the influence of temporal discretization on the spatial error (Roache 1972; Gresho and Lee 1981; Smith and Celik 1999). On the other hand, because the numerical accuracy for convection-diffusion problem is strongly Courant number dependent (Manson et al. 1996; Manson and Wallis 1997), in order to compare the results from three different grids it is necessary to keep the Courant number constant. The Courant number is defined by

$$
\mathrm{Co}=\frac{u_{\mathrm{max}, 0} \Delta t}{h}
$$

where $h$ is a representative grid size; in the case of a uniform grid in both $\mathrm{x}$ - and $\mathrm{y}$ direction, $h=\Delta x=\Delta y . u_{\max , 0}$ is the maximum velocity at initial stage, i.e., equal to $C$. In practice it suffices if a constant time step is used, since the order of method analysis will yield an even better order estimate if the Co is kept constant (smaller $\Delta x$ requires smaller $\Delta t$, and thus more accurate results). Also, different choices of grid size $h$ is available, for example,

$$
h=\sqrt{\Delta t^{2}+\Delta x^{2}}
$$

or,

$$
h=\sqrt{\Delta t \Delta x} .
$$

In all cases, the dominant spatial error will prevail as long as the time step is relatively small. For simplicity, let $h=\Delta x=\Delta y$ and keep in mind that the time step should be negligibly small. Here $\Delta \mathrm{t}$ is taken to be $0.001 \mathrm{~s}$.

For each scheme, the RMS errors of both u- and v-velocity are recorded after 100 time steps for three different grids. The obtained errors are post-processed, and the results are summarized in Table 3-4 and Table 3-5, and shown in Figure 3-32 and Figure 3-33. From these calculations one notices that the schemes being used are truly $2^{\text {nd }}$ order accurate in space. The difference of the order of method in the u- and v-velocity is due to the unequal weight of influence of the temporal error, despite the small time step. A hand calculation shows that the power-law scheme is switched to central differencing in many 
local regions, which explains why it shows $2^{\text {nd }}$ order accurate. The advantage of QUICK over the power-law is again clearly seen.

Table 3-4 Order of method analysis for the calculated u-velocity of a Taylor's vortex, $\Delta t=0.001 \mathrm{~s}$.

\begin{tabular}{|c|c|c|c|c|c|c|c|}
\hline grid & $\mathrm{h} / \mathrm{h}_{\mathrm{ref}}$ & $\varepsilon_{\mathrm{rms}}$ & $\mathrm{u}_{\max }$ & $\varepsilon_{\mathrm{rms}} / \mathrm{u}_{\max }$ & $\ln \left(h / h_{\text {ref }}\right)$ & $\ln \left(\varepsilon_{\mathrm{rms}} / \mathrm{u}_{\max }\right)$ & slope \\
\hline \multicolumn{8}{|c|}{ Implicit power-law } \\
\hline 40 & 1 & 1.6490E-06 & 0.0997 & 1.6546E-05 & 0.0000 & -11.0093 & \\
\hline 20 & 2 & 8.6303E-06 & 0.0992 & 8.7010E-05 & 0.6931 & -9.3495 & \\
\hline 10 & 4 & 5.9692E-05 & 0.0976 & 6.1169E-04 & 1.3863 & -7.3993 & 2.6041 \\
\hline \multicolumn{8}{|c|}{ QUICK } \\
\hline 40 & 1 & 8.9624E-07 & 0.0997 & 8.9916E-06 & 0.0000 & -11.6192 & \\
\hline 20 & 2 & $6.8616 \mathrm{E}-06$ & 0.0993 & 6.9122E-05 & 0.6931 & -9.5796 & \\
\hline 10 & 4 & 5.0376E-05 & 0.0978 & 5.1508E-04 & 1.3863 & -7.5712 & 2.9200 \\
\hline
\end{tabular}

Table 3-5 Order of method analysis for the calculated $v$-velocity of a Taylor's vortex, $\Delta t=0.001 \mathrm{~s}$.

\begin{tabular}{cccccccc} 
grid & $\mathrm{h} / \mathrm{h}_{\text {ref }}$ & $\varepsilon_{\mathrm{rms}}$ & $\mathrm{u}_{\max }$ & $\varepsilon_{\mathrm{rms}} / \mathrm{u}_{\max }$ & $\ln \left(\mathrm{h} / \mathrm{h}_{\mathrm{ref}}\right)$ & $\ln \left(\varepsilon_{\mathrm{rms}} / \mathrm{u}_{\max }\right)$ & slope \\
\hline \multicolumn{2}{l}{ Implicit power-law } & & & & & & \\
40 & 1 & $1.6489 \mathrm{E}-06$ & 0.0997 & $1.6546 \mathrm{E}-05$ & 0.0000 & -11.0094 & \\
20 & 2 & $8.6307 \mathrm{E}-06$ & 0.0992 & $8.7014 \mathrm{E}-05$ & 0.6931 & -9.3494 & \\
10 & 4 & $5.9693 \mathrm{E}-05$ & 0.0976 & $6.1170 \mathrm{E}-04$ & 1.3863 & -7.3993 & $\mathbf{2 . 6 0 4 2}$ \\
& & & & & & & \\
QUICK & & & & & & & \\
40 & 1 & $8.9654 \mathrm{E}-07$ & 0.0997 & $8.9946 \mathrm{E}-06$ & 0.0000 & -11.6189 & \\
20 & 2 & $6.8620 \mathrm{E}-06$ & 0.0993 & $6.9125 \mathrm{E}-05$ & 0.6931 & -9.5796 & \\
10 & 4 & $5.0377 \mathrm{E}-05$ & 0.0978 & $5.1508 \mathrm{E}-04$ & 1.3863 & -7.5712 & $\mathbf{2 . 9 1 9 8}$
\end{tabular}

Temporal accuracy alone is difficult to determine in general, because the time discretization is hardly isolated from the spatial discretization. This is because the time step size cannot be arbitrarily small due to stability constraints. However, if one would still like to examine the time accuracy alone, very small grid spacing compared to the time step size has to be used. To avoid expensive computation, a simple alternative is to look at the overall accuracy in which both the spatial and temporal errors are evaluated as a whole. Again, three grids, namely, $10 \times 10,20 \times 20$ and $40 \times 40$, are considered. Further it is necessary in this approach to keep Courant number the same, giving three time steps as $0.08 \mathrm{~s}, 0.04 \mathrm{~s}$ and $0.02 \mathrm{~s}$, respectively. The QUICK is employed along with the $2^{\text {nd }}$ order Adams-Bashforth. The results for $\mathrm{u}$ - and v-velocity errors are presented in Table 3-6, Table 3-7 and Figure 3-34. The obtained slopes verify the scheme being used is indeed at least $2^{\text {nd }}$ order accurate in both space and time.

Table 3-6 Effective overall order of the QUICK scheme for the calculated u-velocity of a Taylor's vortex.

$\begin{array}{ccccccccr}\mathrm{dt} & \text { grid } & \mathrm{h} / \mathrm{h}_{\text {ref }} & \varepsilon_{\mathrm{rms}} & \mathrm{u}_{\max } & \varepsilon_{\mathrm{rms}} / \mathrm{u}_{\max } & \ln \left(\mathrm{h} / \mathrm{h}_{\mathrm{ref}}\right) & \ln \left(\varepsilon_{\mathrm{rms}} / \mathrm{u}_{\max }\right) & \text { slope } \\ 0.02 & 40 & 1 & 1.3270 \mathrm{E}-06 & 0.0969 & 1.3690 \mathrm{E}-05 & 0.0000 & -11.1988 & \\ 0.04 & 20 & 2 & 6.5774 \mathrm{E}-06 & 0.0969 & 6.7858 \mathrm{E}-05 & 0.6931 & -9.5981 & \end{array}$


$\begin{array}{lllllllll}0.08 & 10 & 4 & 4.2474 \mathrm{E}-05 & 0.0962 & 4.4160 \mathrm{E}-04 & 1.3863 & -7.7251 & \mathbf{2 . 5 0 5 8}\end{array}$

Table 3-7 Effective overall order of the QUICK scheme for the calculated v-velocity of a Taylor's vortex.

$\begin{array}{ccccccccr}\mathrm{dt} & \text { grid } & \mathrm{h} / \mathrm{h}_{\text {ref }} & \varepsilon_{\text {rms }} & \mathrm{u}_{\max } & \varepsilon_{\mathrm{rms}} / \mathrm{u}_{\max } & \ln \left(\mathrm{h} / \mathrm{h}_{\text {ref }}\right) & \ln \left(\varepsilon_{\mathrm{rms}} / \mathrm{u}_{\max }\right) & \text { slope } \\ 0.02 & 40 & 1 & 3.2099 \mathrm{E}-06 & 0.0969 & 3.3116 \mathrm{E}-05 & 0.0000 & -10.3155 & \\ 0.04 & 20 & 2 & 1.3751 \mathrm{E}-05 & 0.0969 & 1.4186 \mathrm{E}-04 & 0.6931 & -8.8606 & \\ 0.08 & 10 & 4 & 6.5683 \mathrm{E}-05 & 0.0962 & 6.8290 \mathrm{E}-04 & 1.3863 & -7.2892 & \mathbf{2 . 1 8 3 0}\end{array}$

As a further check, the above calculations are repeated for the vortex located in the $\mathrm{y}-\mathrm{z}$ plane. In this case, the u-velocity is replaced by the w-velocity and the $\mathrm{x}$ coordinate replaced by the z-coordinate. Exactly same results are obtained, including the order of the schemes. 


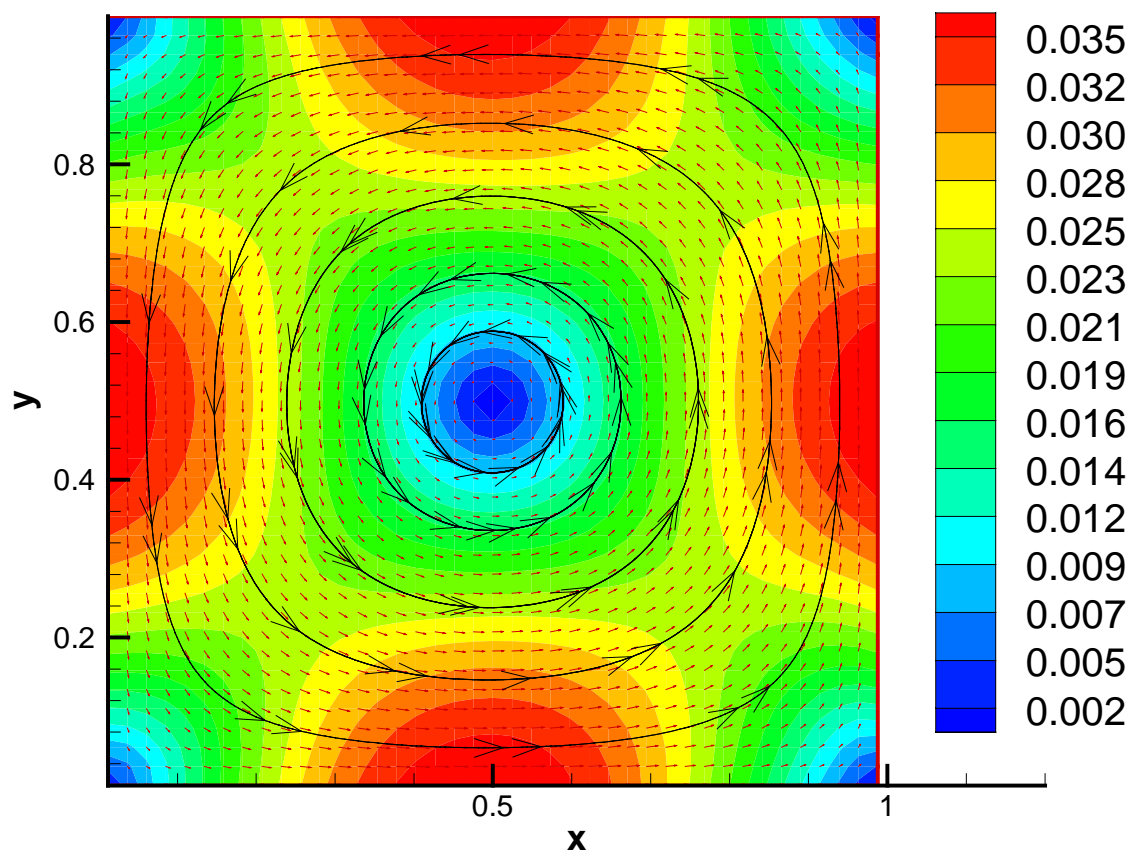

Figure 3-23 Streamline plot of Taylor's vortex at $t=50 \mathrm{~s}$ for $\mathrm{Re}=100$; background contour plot is the magnitude of velocity.

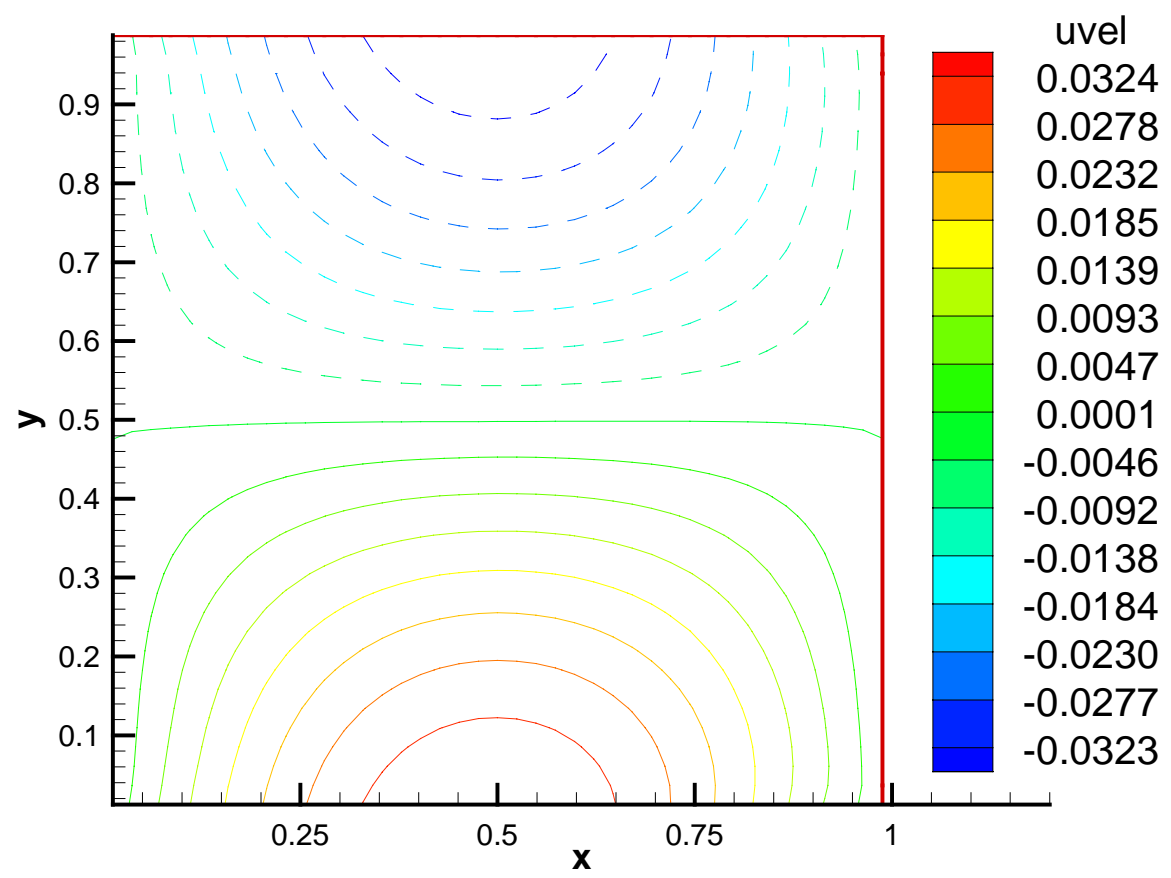

(a)

Figure 3-24 Contour plots of Taylor's vortex at $t=50 \mathrm{~s}$ for $\mathrm{Re}=100$, (a) u-velocity. 


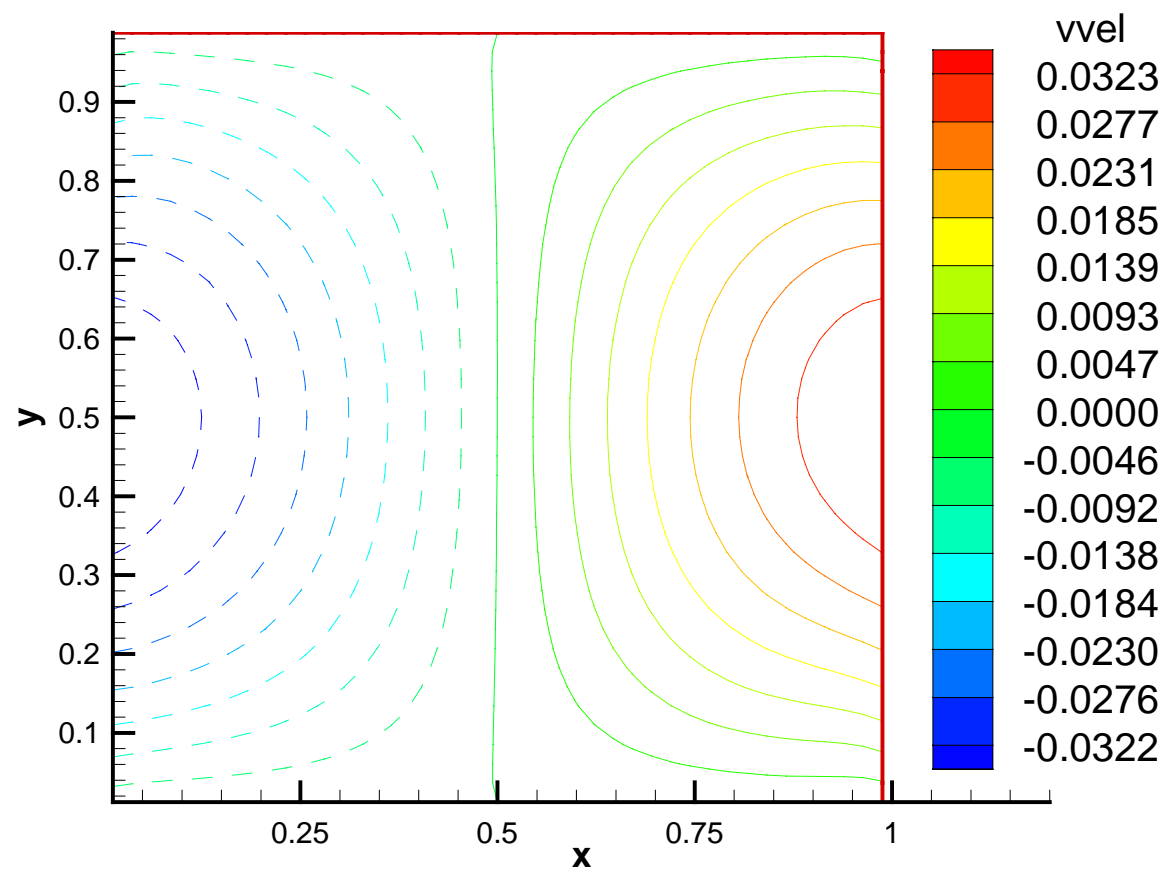

(b)

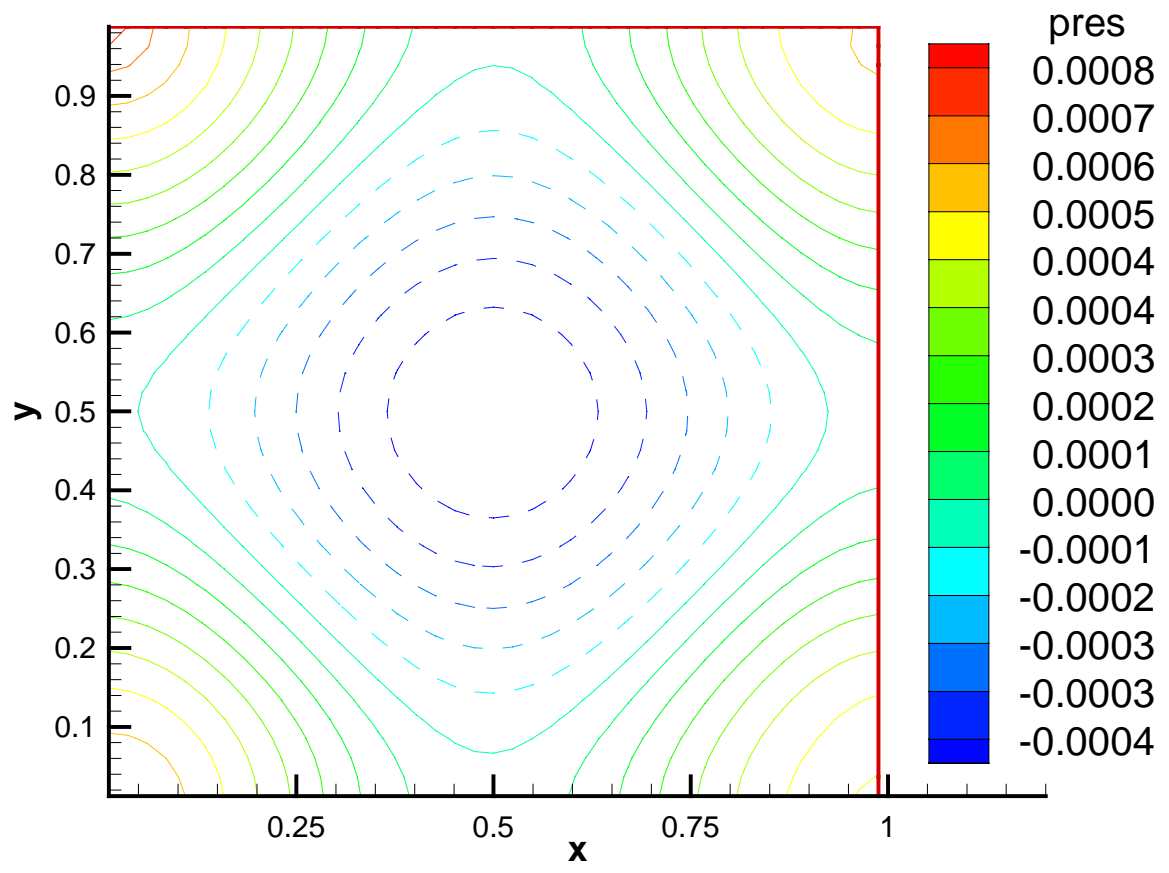

(c)

Figure 3-24 Contour plots of Taylor's vortex at $t=50$ s for $R e=100$, (b) v-velocity, (c) pressure. 


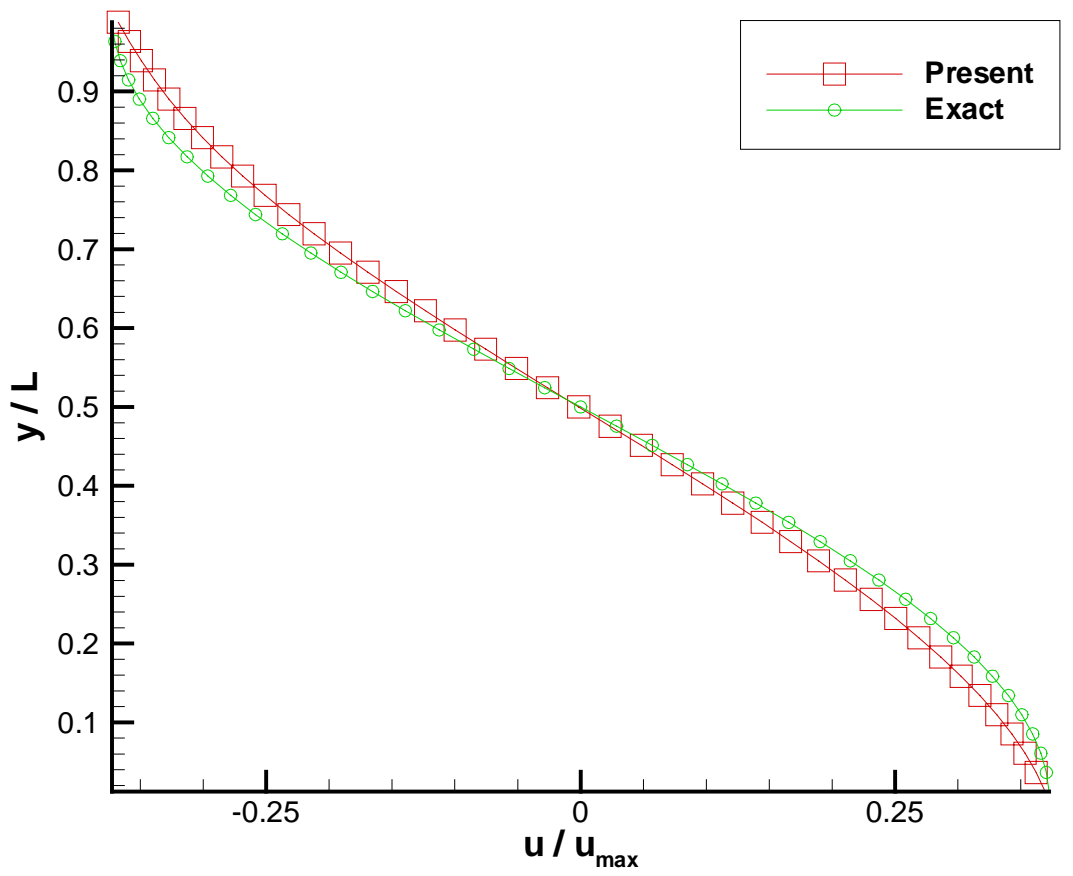

(a)

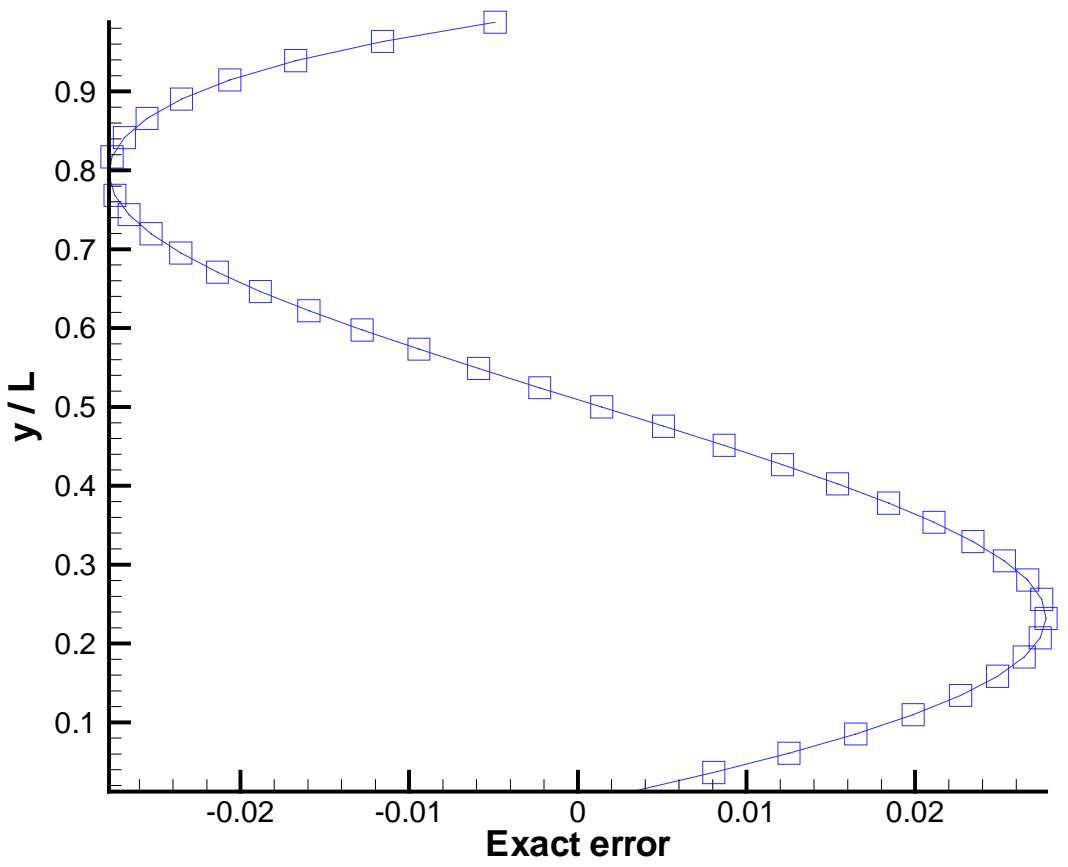

(b)

Figure 3-25 u-velocity profile (a) and its exact error (b) of a Taylor's vortex at $t=50$ s for $\operatorname{Re}=100, x$ $=0.5 \mathrm{~m}$. 


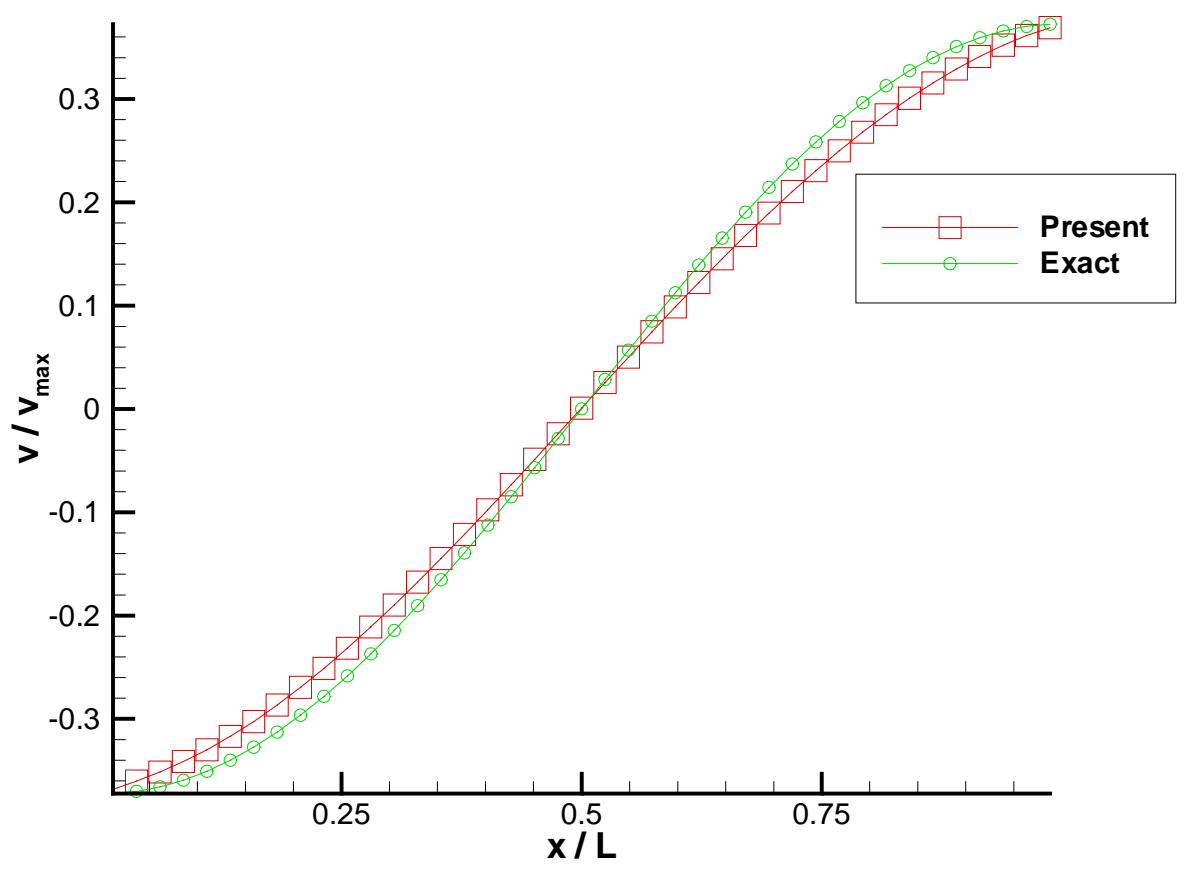

(a)

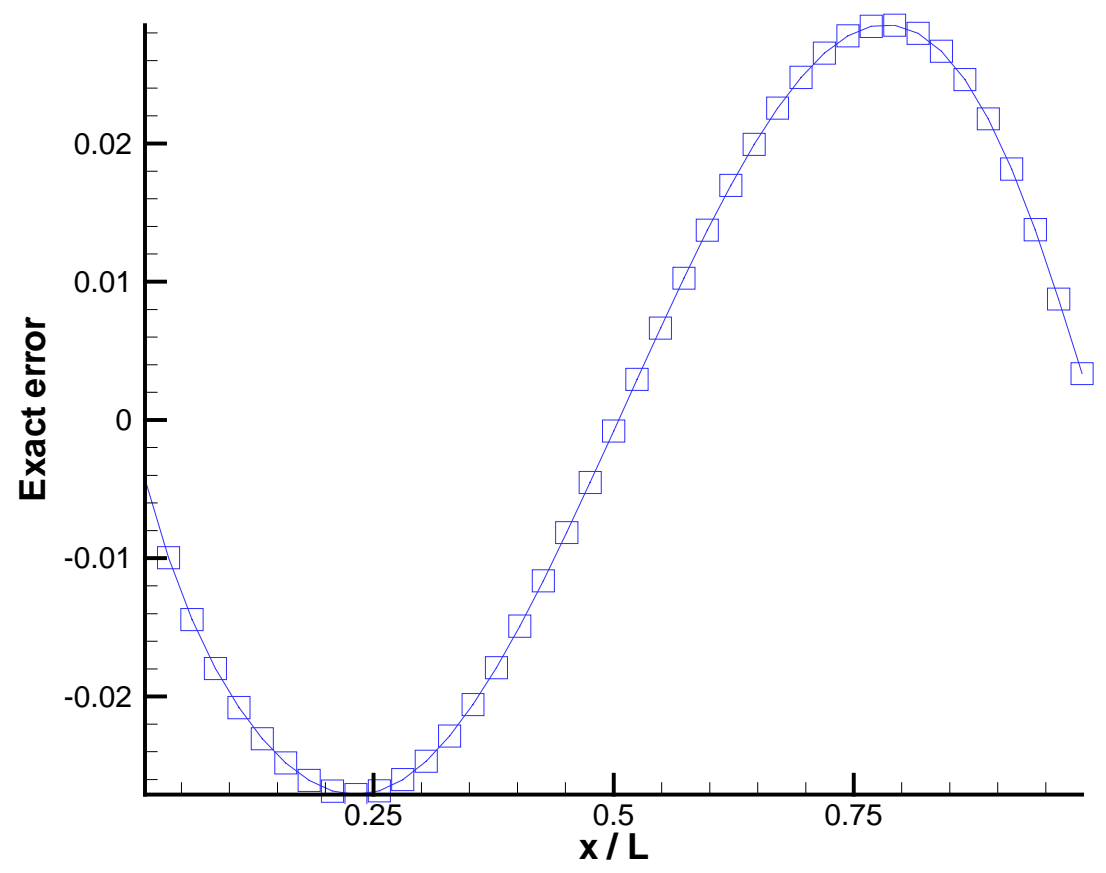

(b)

Figure 3-26 v-velocity profile (a) and its exact error (b) of a Taylor's vortex at $t=50$ s for $\operatorname{Re}=100, y$ $=0.5 \mathrm{~m}$. 


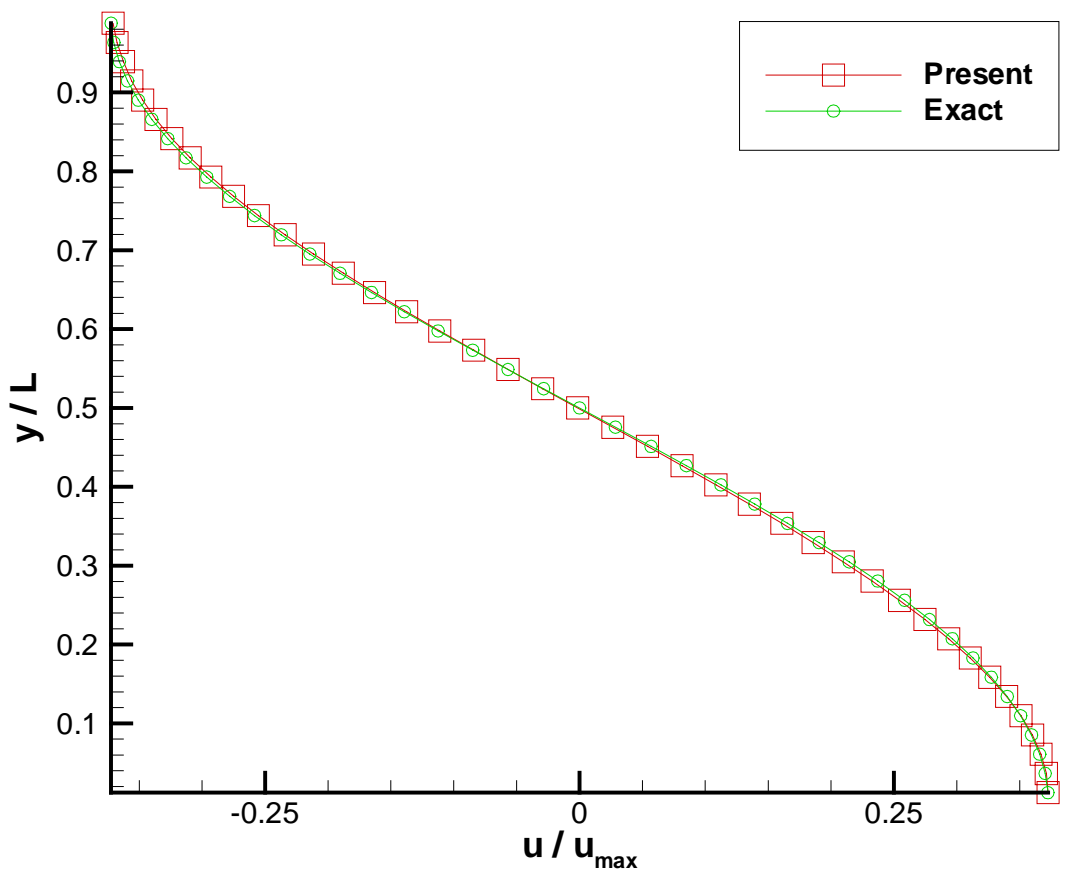

(a)

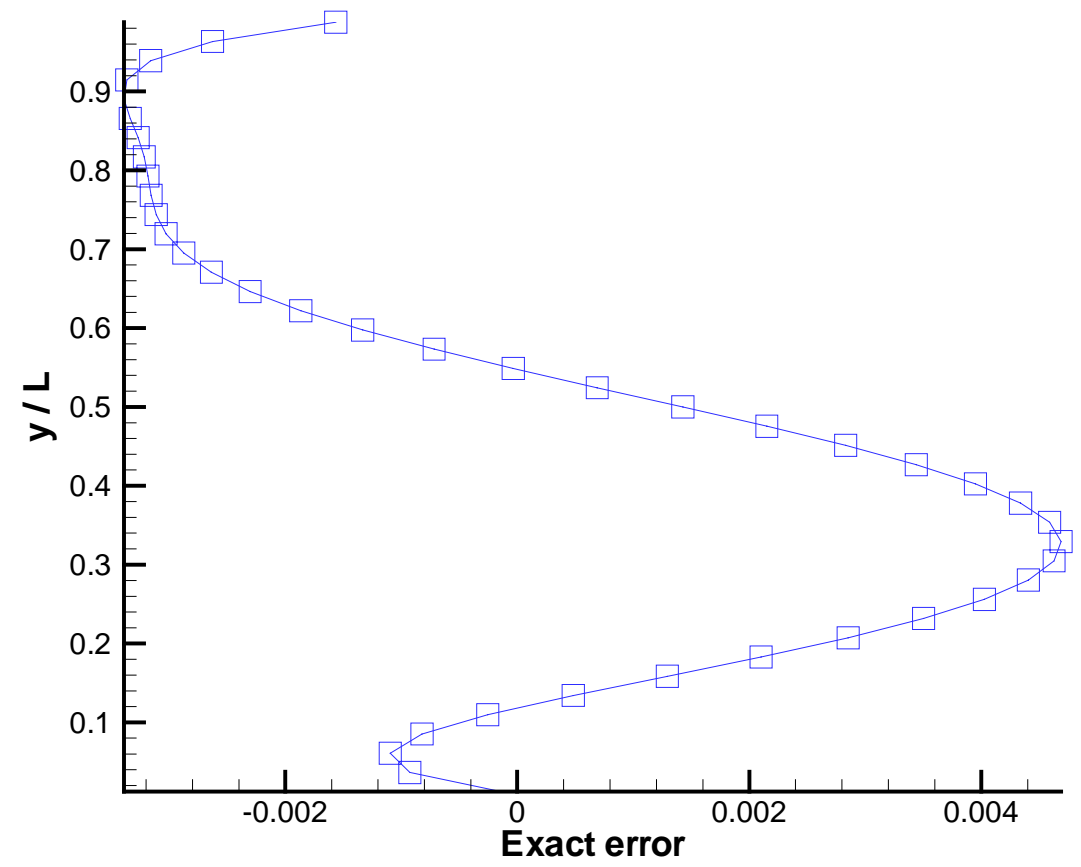

(b)

Figure 3-27 u-velocity profile (a) and its exact error (b) of a Taylor's vortex at $t=50$ s for $\operatorname{Re}=100, x$ $=0.5 \mathrm{~m}$; explicit QUICK scheme. 


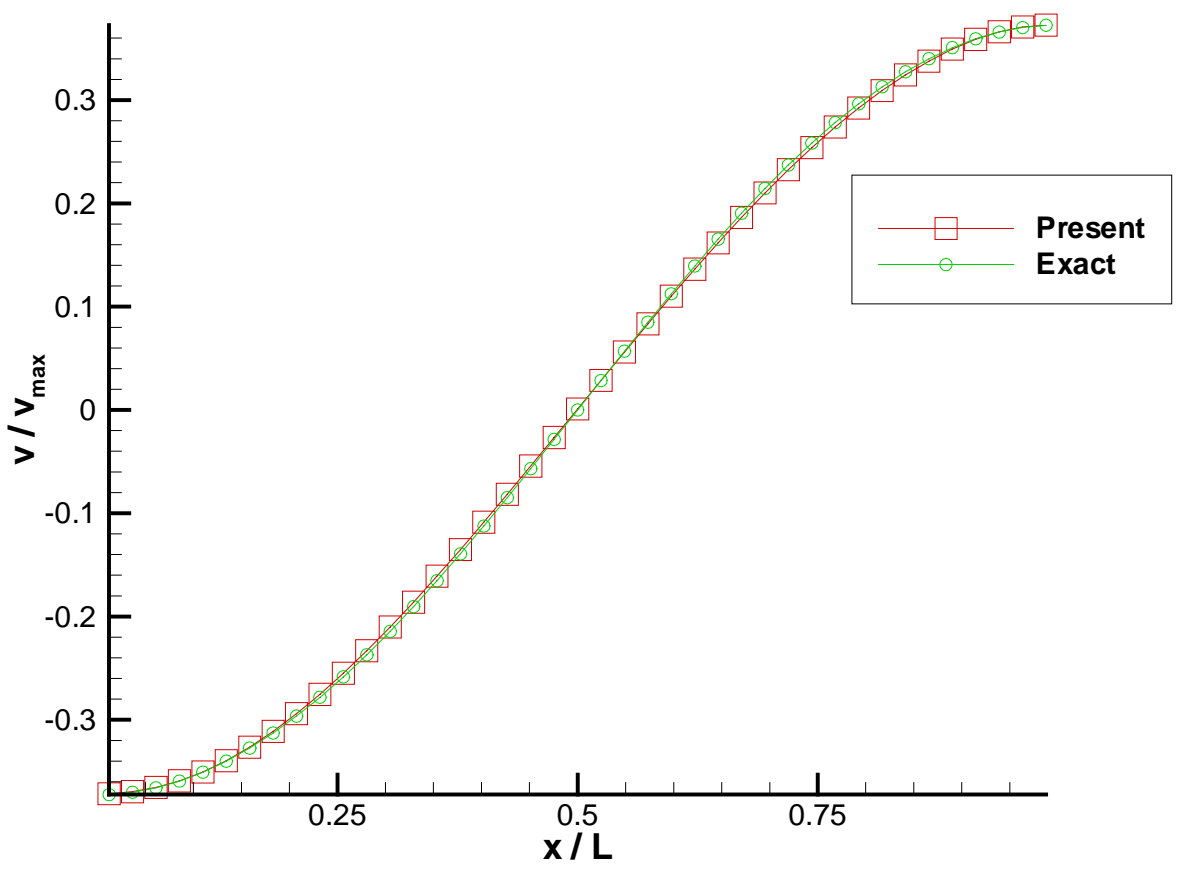

(a)

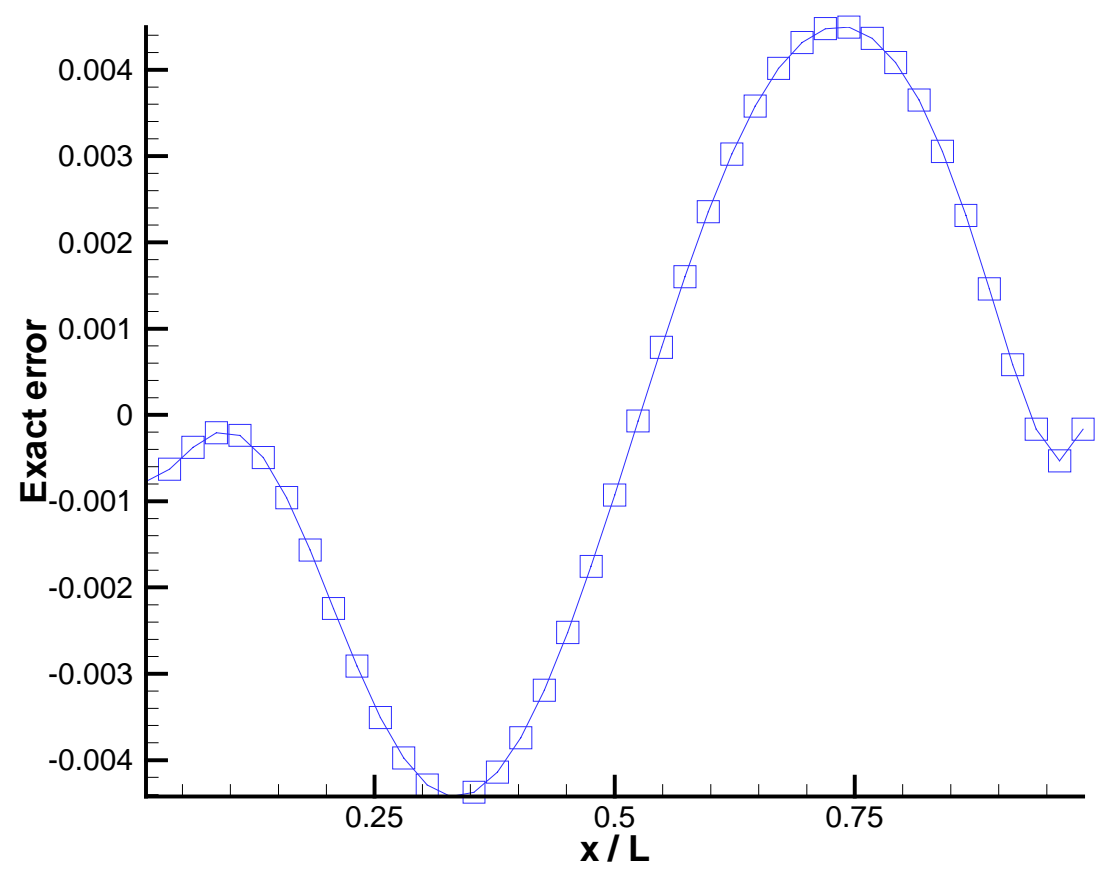

(b)

Figure 3-28 v-velocity profile (a) and its exact error (b) of a Taylor's vortex at $t=50 \mathrm{~s}$ for $\operatorname{Re}=100, y$ $=0.5 \mathrm{~m}$; explicit QUICK scheme. 


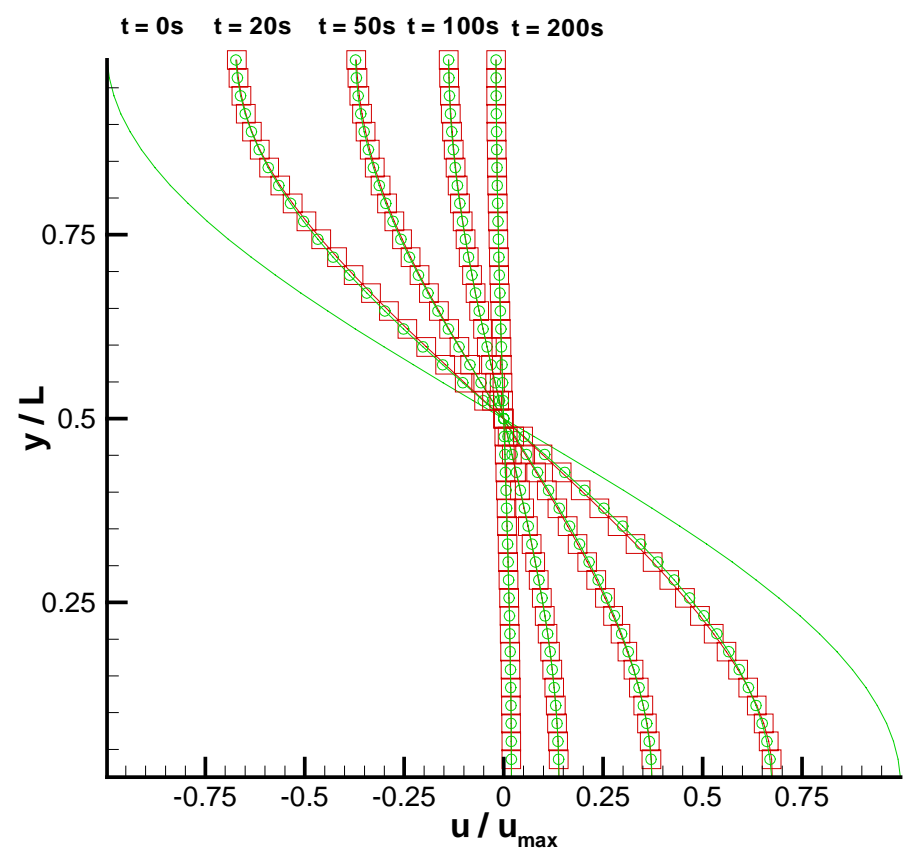

(a)

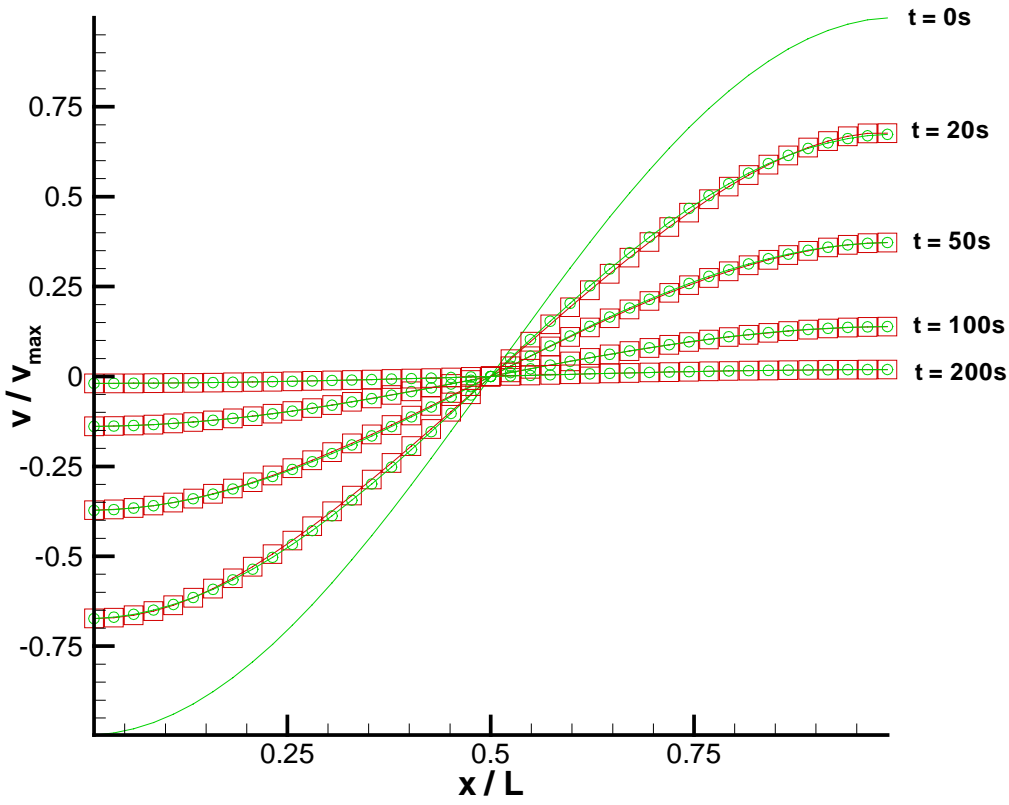

(b)

Figure 3-29 Velocity profiles at different time of a Taylor's vortex with $R e=100$. (a) u-velocity, (b) vvelocity. Square symbol: present calculation; circle symbol: exact solution 


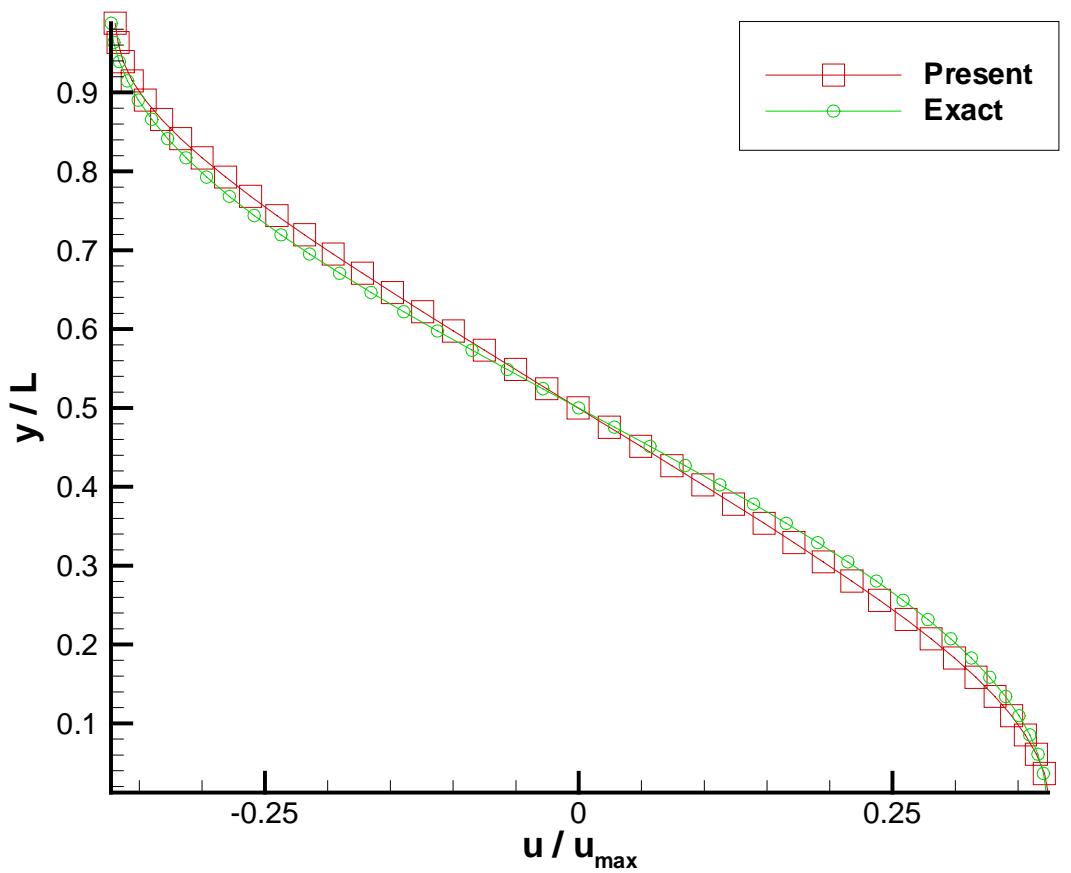

(a)

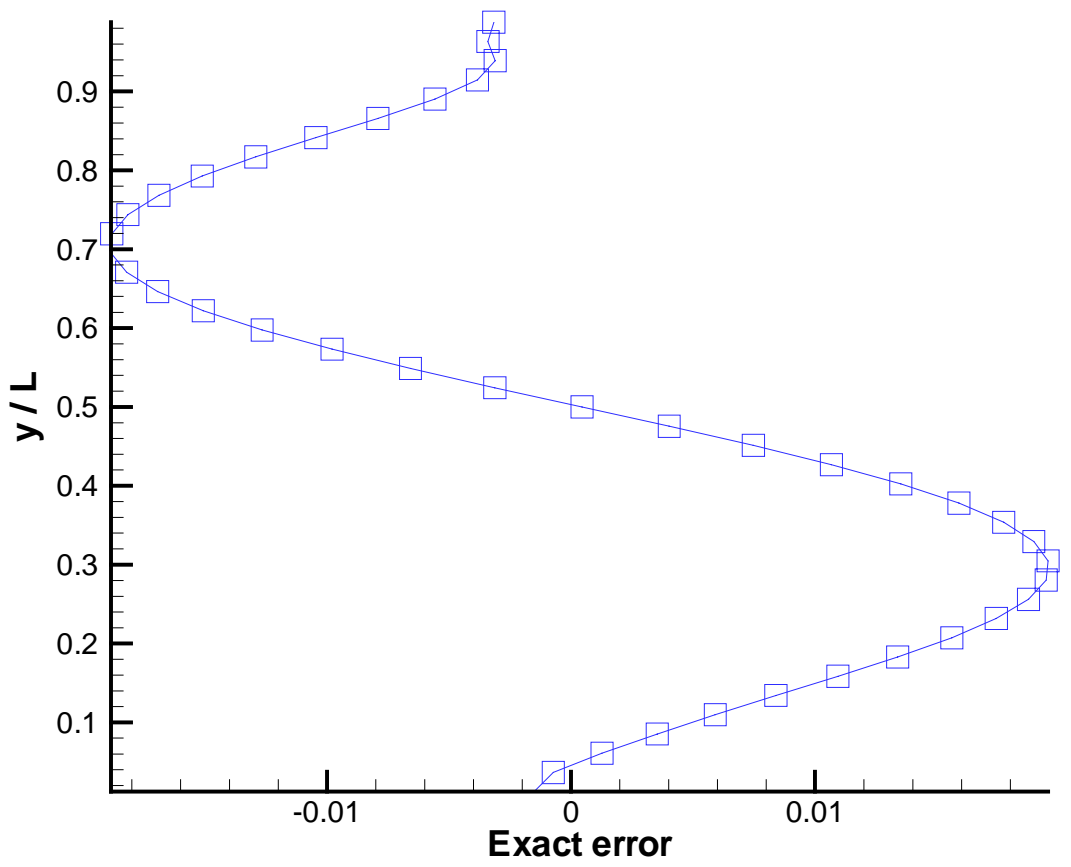

(b)

Figure 3-30 u-velocity profile (a) and its exact error (b) of a Taylor's vortex at $t=50$ s for $\operatorname{Re}=1000, x$ $=0.5 \mathrm{~m}$; explicit QUICK scheme. 


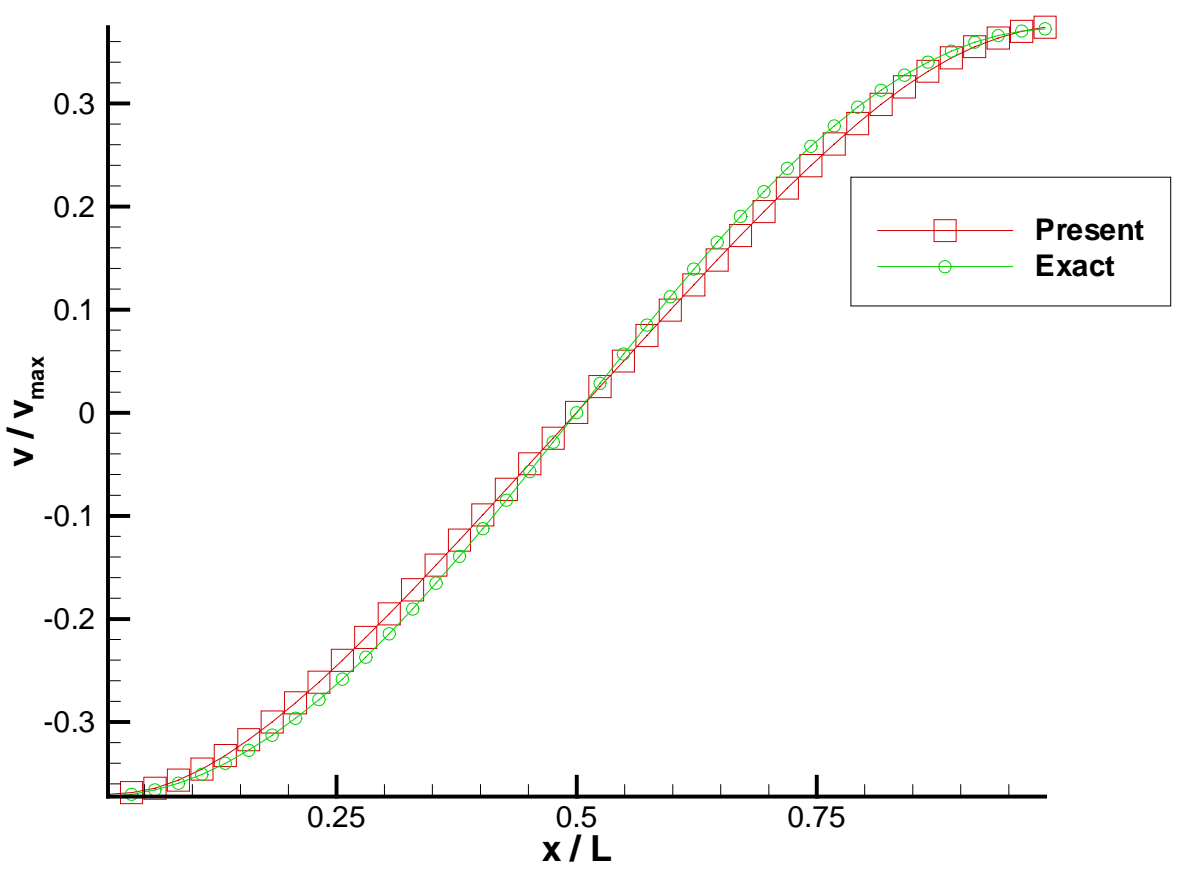

(a)

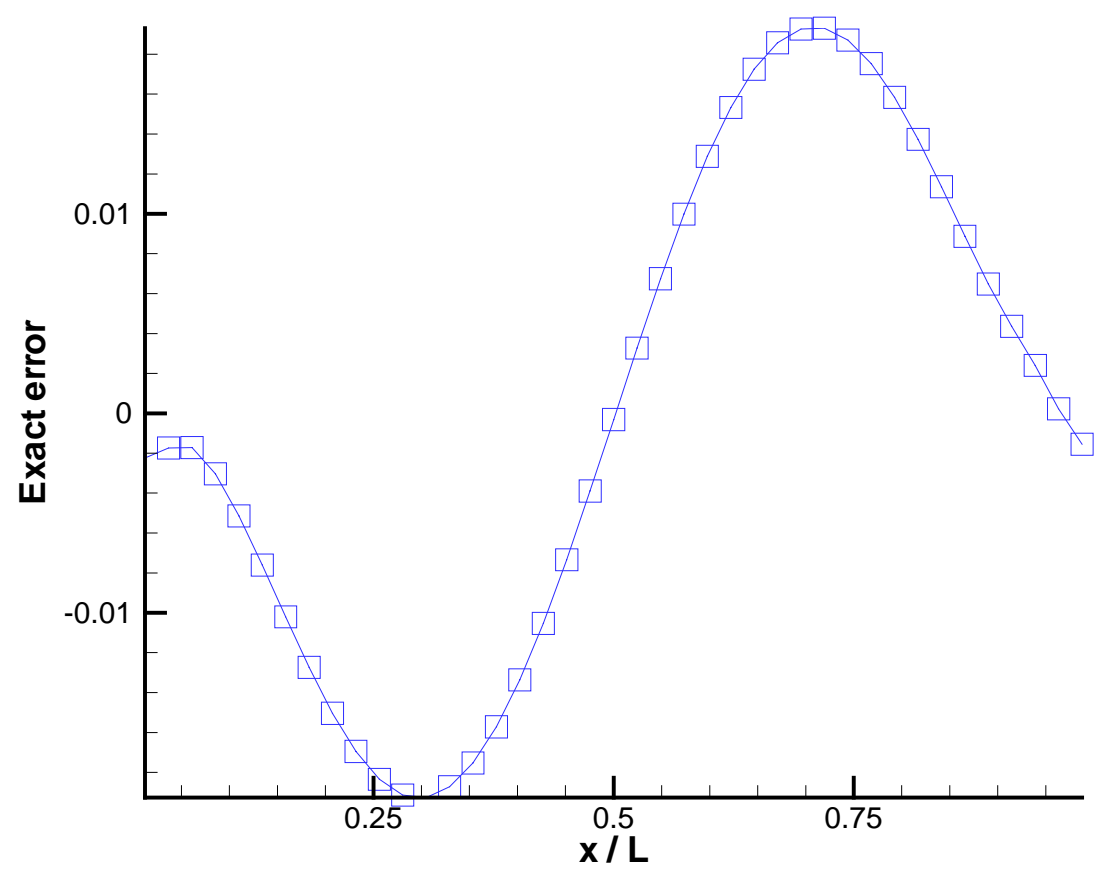

(a)

Figure 3-31 v-velocity profile (a) and its exact error (b) of a Taylor's vortex at $t=50$ s for $\operatorname{Re}=1000, y$ $=0.5 \mathrm{~m}$; explicit QUICK scheme. 


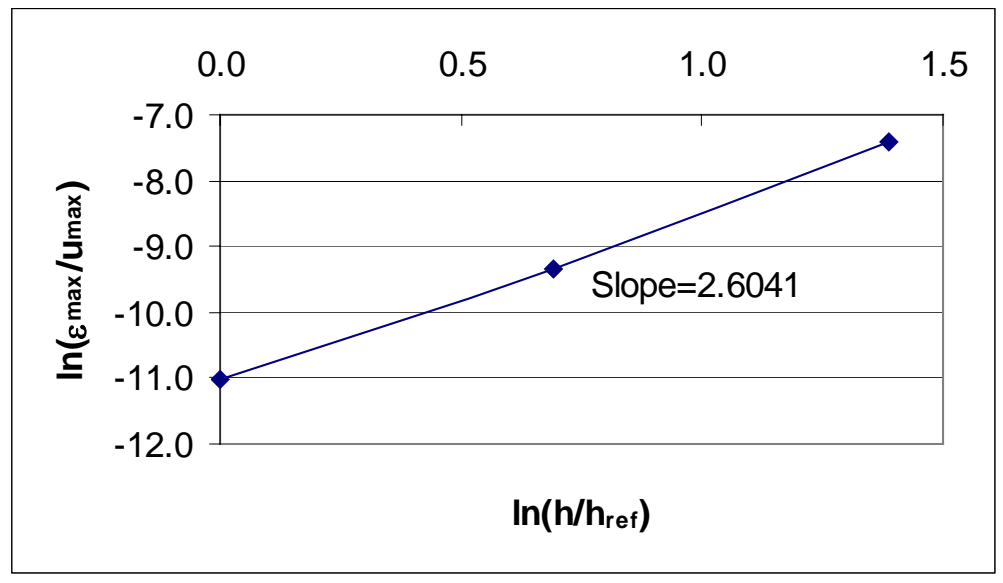

(a)

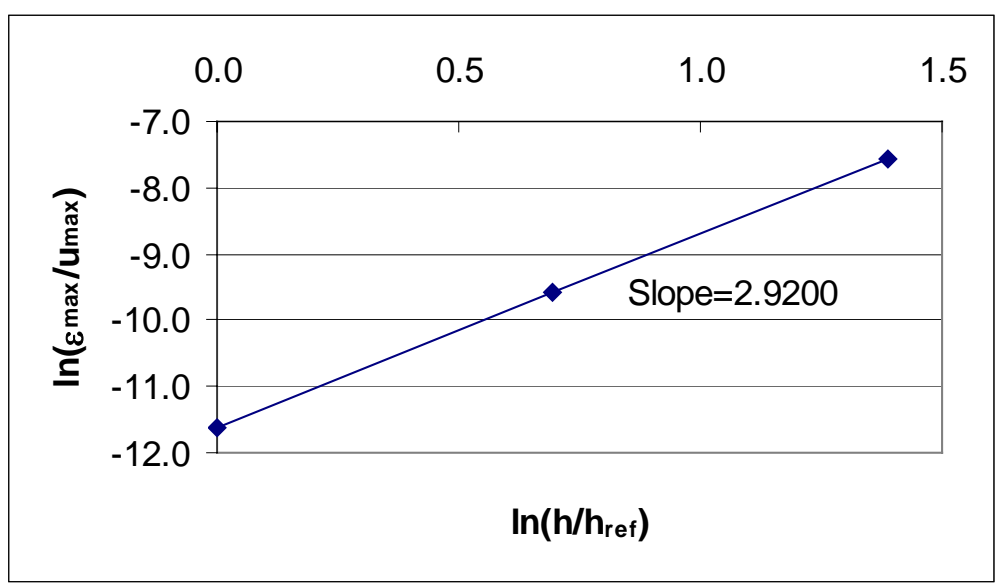

(b)

Figure 3-32 RMS error of u-velocity as a function of mesh refinement for Taylor's vortex flow, $\Delta t=$ 0.001s. (a) Implicit power-law, (b) QUICK. 


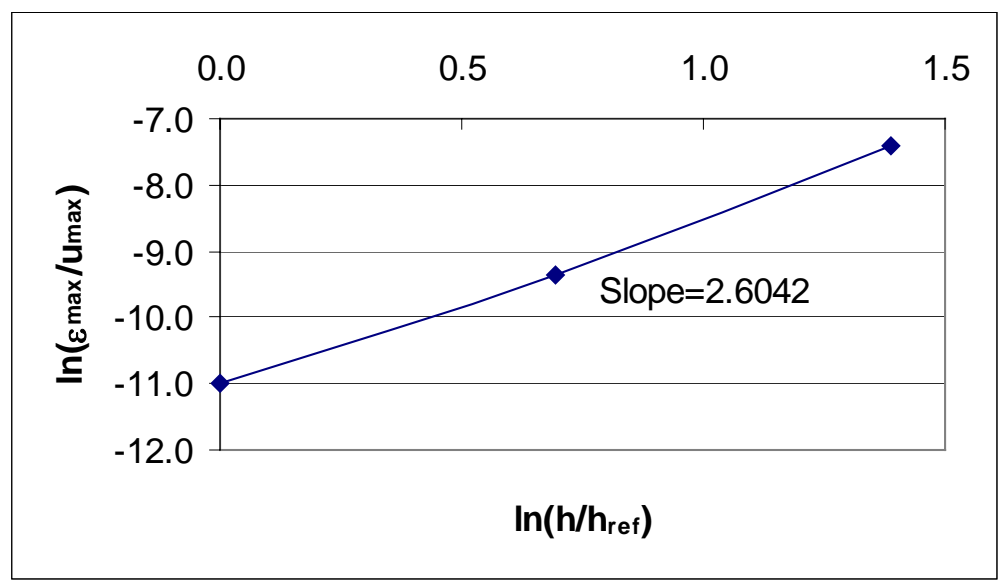

(a)

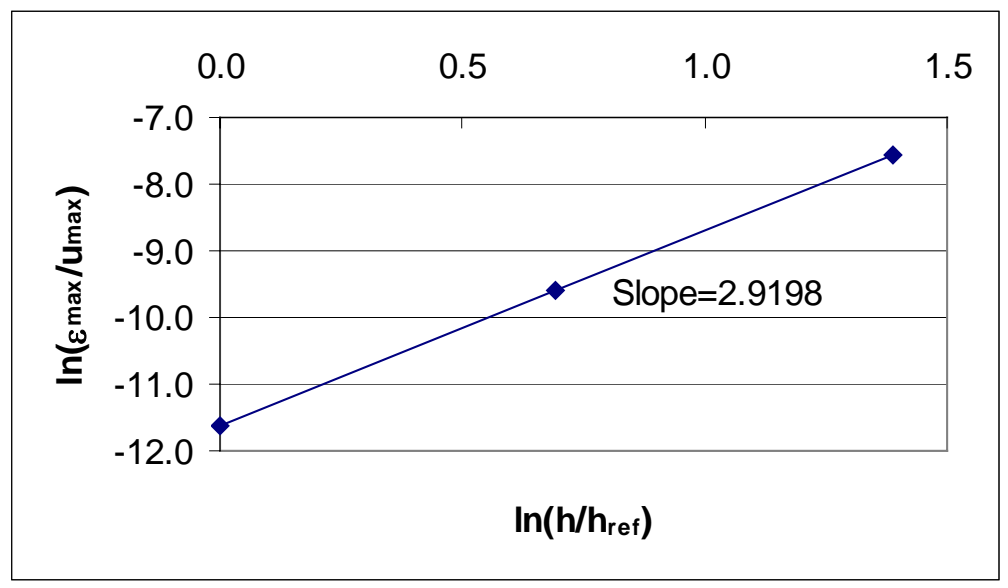

(b)

Figure 3-33 RMS error of v-velocity as a function of mesh refinement for Taylor's vortex flow, $\Delta t=$ 0.001s. (a) Implicit power-law, (b) QUICK. 


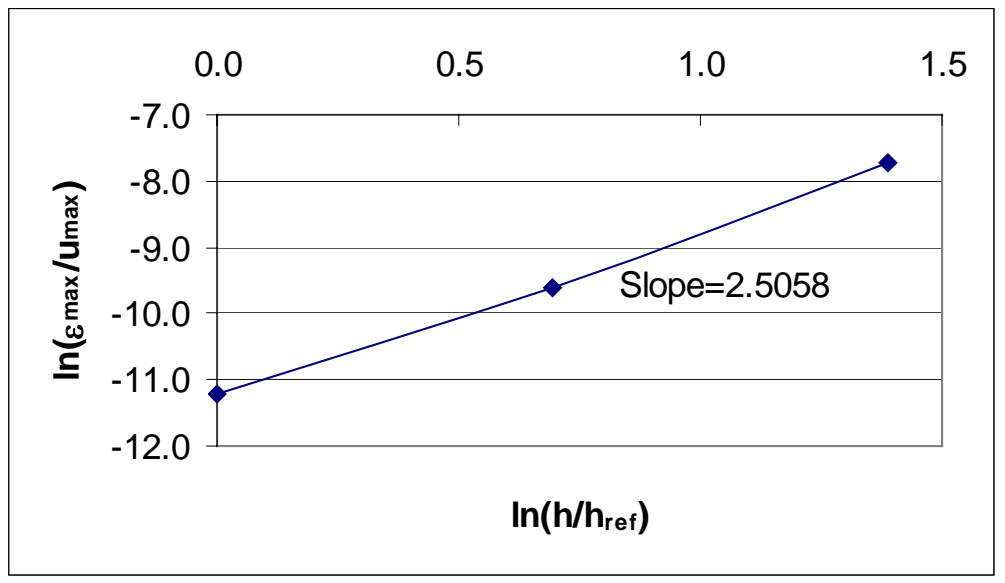

(a)

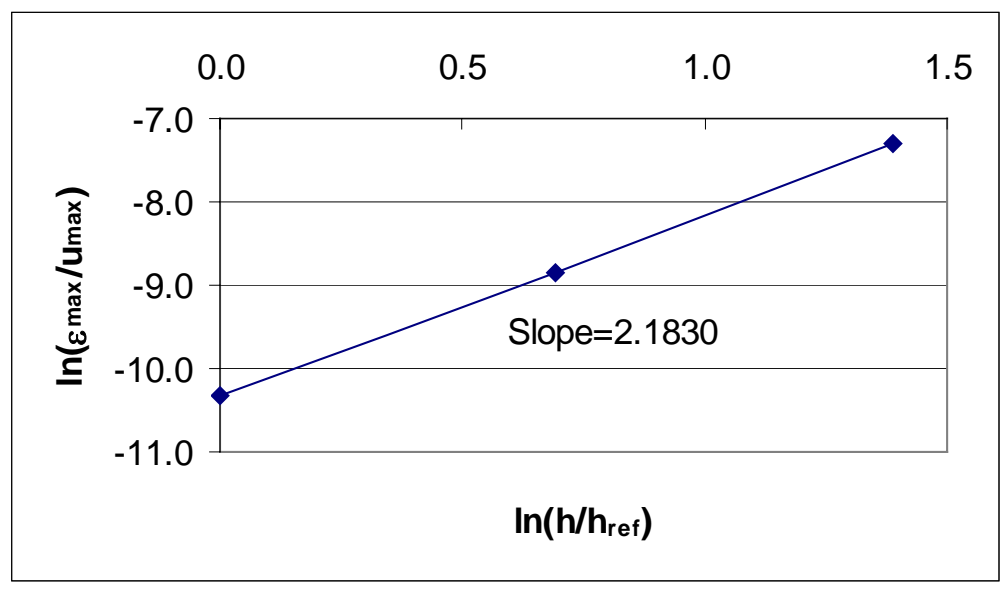

(b)

Figure 3-34 Effective overall error of the QUICK scheme for Taylor's vortex problem. (a) error of uvelocity, (b) error of $v$-velocity. 


\subsection{Free Shear Layer}

The free shear layer is another classical flow case, where two parallel uniform streams $U_{1}$ (upper) and $U_{2}$ (lower) meet at an inlet and the interface between the two gradually forms a S-shaped velocity profile as they develop downstream. A schematic sketch of the flow configuration is shown in Figure 3-35.

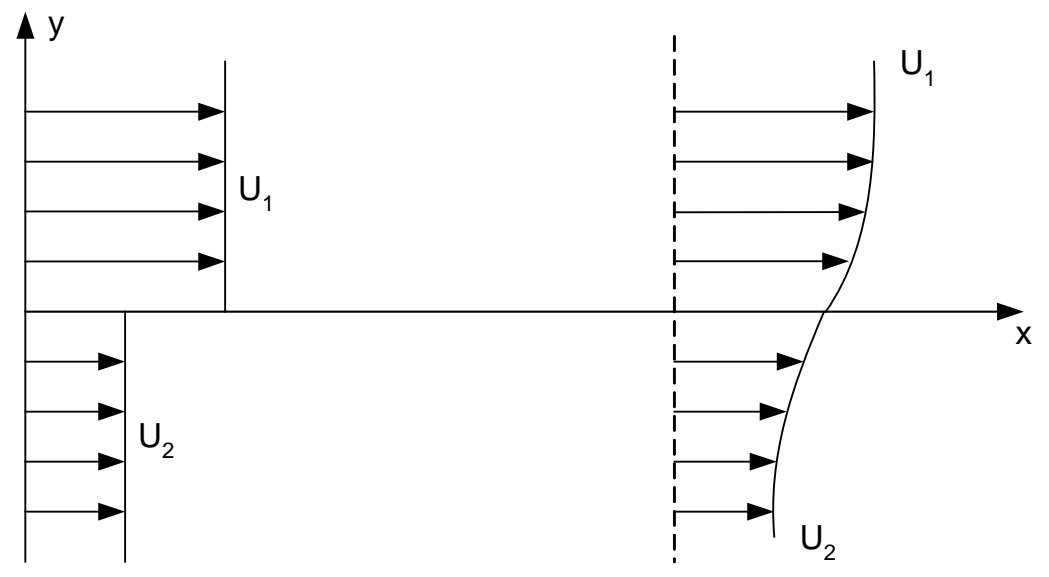

Figure 3-35 Schematic of a free shear flow

The steady two-dimensional incompressible laminar flow with negligible gravitational effects can be described by the simplified 2D Navier-Stokes equations

$$
\begin{aligned}
& u \frac{\partial u}{\partial x}+v \frac{\partial u}{\partial y}=-\frac{1}{\rho} \frac{\partial p}{\partial x}+v\left(\frac{\partial^{2} u}{\partial x^{2}}+\frac{\partial^{2} u}{\partial y^{2}}\right) \\
& u \frac{\partial v}{\partial x}+v \frac{\partial v}{\partial y}=-\frac{1}{\rho} \frac{\partial p}{\partial y}+v\left(\frac{\partial^{2} v}{\partial x^{2}}+\frac{\partial^{2} v}{\partial y^{2}}\right) \\
& \frac{\partial u}{\partial x}+\frac{\partial v}{\partial y}=0
\end{aligned}
$$

Although the mathematical problem is well posed, no one was able to obtain an analytical solution to these equations not only for the free shear flow but also for flows past any shaped body! Yes, researchers are poor when confronting with exact solutions; but, we are not destitute. While admitting obtaining analytical solutions is a formidable practice, one can at least try to acquire some type of approximate solution.

First recall the boundary layer equations simplified from Eq. (3.22) by certain assumptions (see (Schlichting 1979)):

$$
\begin{aligned}
& u \frac{\partial u}{\partial x}+v \frac{\partial u}{\partial y}=v \frac{\partial^{2} u}{\partial y^{2}} \\
& \frac{\partial p}{\partial y}=0, \frac{\partial u}{\partial x}+\frac{\partial v}{\partial y}=0
\end{aligned}
$$


Further recall the so-called Blasius (1883-1970) solution for the flow past a flat-plate, in which the boundary layer partial differential equations are turned into a third order ODE by (i) arguing that dimensionless velocity profiles are similar, and (ii) applying a clever coordinate transformation and changes of variables to collapse $x$ and $y$ into a single variable, called similarity variable $\eta$, and $u$ and $v$ into a single stream function $\psi$, whose respective definition is given by

$$
\begin{aligned}
& \eta=y\left(\frac{U}{2 v x}\right)^{1 / 2}, \\
& \psi=(v x U)^{1 / 2} f^{\prime}(\eta) .
\end{aligned}
$$

In the above definition, the velocity scale $U$ is taken from the free stream velocity and ( )' stands for $\mathrm{d} / \mathrm{d} \eta$, and $f$ is the function that satisfies $f^{\prime}=u / U$ and appears in the ODE to be solved. The third order ODE can be written as

$$
f^{\prime \prime \prime}+f f^{\prime \prime}=0
$$

with the boundary conditions

$$
\begin{aligned}
& f^{\prime}(0)=f(0)=1, \\
& f^{\prime}(\infty)=1 .
\end{aligned}
$$

This basic idea was then adopted by (Lock 1951) who by analogy introduced a Blasius-type similarity variables for each stream of the shear layer, i.e.,

$$
\begin{aligned}
& \eta_{j}=y\left(\frac{U_{1}}{2 v_{j} x}\right)^{1 / 2}, \\
& f_{j}^{\prime}=\frac{u_{j}}{U_{1}}, j=1,2 .
\end{aligned}
$$

Following a similar procedure as the Blasius', the governing equations (Eq. (3.22)) are then turned into two third-order ODEs for the two streams, coupled by their interface condition, i.e.,

$$
f_{j}^{\prime \prime \prime}+f_{j} f_{j}^{\prime \prime}=0, j=1,2,
$$

with the boundary and interface conditions:

$$
\begin{aligned}
& f_{1}^{\prime}(+\infty)=1, f_{2}^{\prime}(-\infty)=\frac{U_{2}}{U_{1}}, \\
& f_{1}(0)=f_{2}(0)=0 .
\end{aligned}
$$

In the numerical computation, if the upper and lower layer has the same density and viscosity, or no mixing of the two layers occurs, one may directly attack the governing equation (3.22) without simplification. Here the velocity ratio $U_{2} / U_{1}$ is chosen to be 0.5 , with the upper velocity being unity. Further let the kinematic viscosity equal to $1.0 \mathrm{E}-3 \mathrm{~m} / 2 / \mathrm{s}$ such that the Reynolds number based on the maximum inlet velocity and the maximum momentum thickness at outlet is approximately 100 . The computation domain should be long enough to grant the flow sufficient space to develop, and should be high enough to have the u-velocity to reach the theoretical velocities at plus and minus infinity 
$(1 \mathrm{~m} / \mathrm{s}$ and $0.5 \mathrm{~m} / \mathrm{s})$. Thus, a rectangular domain sized at $1 \mathrm{~m} \times 0.4 \mathrm{~m}$ is chosen. 50 and 40 equally spaced internal nodes are placed in the streamwise and transverse direction, resulting a grid spacing of $0.02 \mathrm{~m}$ and $0.01 \mathrm{~m}$, respectively.

At the inlet, a Dirichlet boundary condition is specified with the given inlet velocity profile, i.e., the upper stream enters at $1 \mathrm{~m} / \mathrm{s}$ and the lower stream at $0.5 \mathrm{~m} / \mathrm{s}$. At the outlet a Neumann boundary condition is employed. At the south and north boundaries a Neumann condition is again applied to both u- and v-component. Note that in order to compare with the similarity solutions one should not use the slip-wall condition. The reasons are: (i) With a finite domain one usually cannot ensure the u-velocity has reached the theoretical velocities at those boundaries, and moreover, the y-location of the edge velocities $(99 \%$ of the free stream velocity) varies itself along $\mathrm{x}$-station. (ii) In the similarity solution presented above the vertical velocity at south or north is not assumed to be zero; in fact one can simply show that they are non-zero by just examining the vvelocity expression from the stream function. Thus, if the slip-wall condition was used for the u-component, the Neumann boundary condition for the v-velocity cannot be justified.

The semi-implicit power-law is first applied with a relatively large time step (0.1s) to obtain some primary flow field. The scheme is then switched to the explicit QUICK accompanied with small a time step $(0.01 \mathrm{~s})$ to reach a steady state flow. The global normalized mass residual is stabilized at around an order of $10^{-6}$. The calculated uand v-velocity contour are shown in Figure 3-36(a) and (b), from which a developing shear layer is clearly seen, and in particular, the calculated v-velocity is not zero at the south and north boundaries.

To get a comparison with Lock's similarity solution (Lock 1951), three x-stations, namely, $x$ / (domain length $)=0.4,0.6$ and 0.8 , are selected such a way that they are in the developed similarity region, on the one hand, and away from the outlet (to eliminate the outlet influence), on the other hand. The collected three u-velocity profiles are shown in Figure 3-37. It is evident from Figure 3-37 that the shear layer thickness is getting thicker as the flow develops downstream. The non-dimensional similarity variable $\eta$ is then calculated and plotted versus the normalized u-velocity $\left(u / U_{1},\right)$, along with the data extracted from Lock's study (Figure 3-38). It is clearly seen from Figure 3-38 that the three velocity profiles calculated from the present study do very nicely exhibit close similitude, as they can be hardly differentiated from each other. Moreover, the agreement with Lock's solution is close with an acceptable error due to the fact that the similarity solution is obtained based on boundary conditions that are not exactly the same as the one being implemented into the $2 \mathrm{D}$ calculation. In this sense, it is unsafe to use the similarity solution as an exact solution for the error analysis. Nevertheless, it does offer important insight into the flow physics and a well-suited justification to the obtained numerical solutions. 


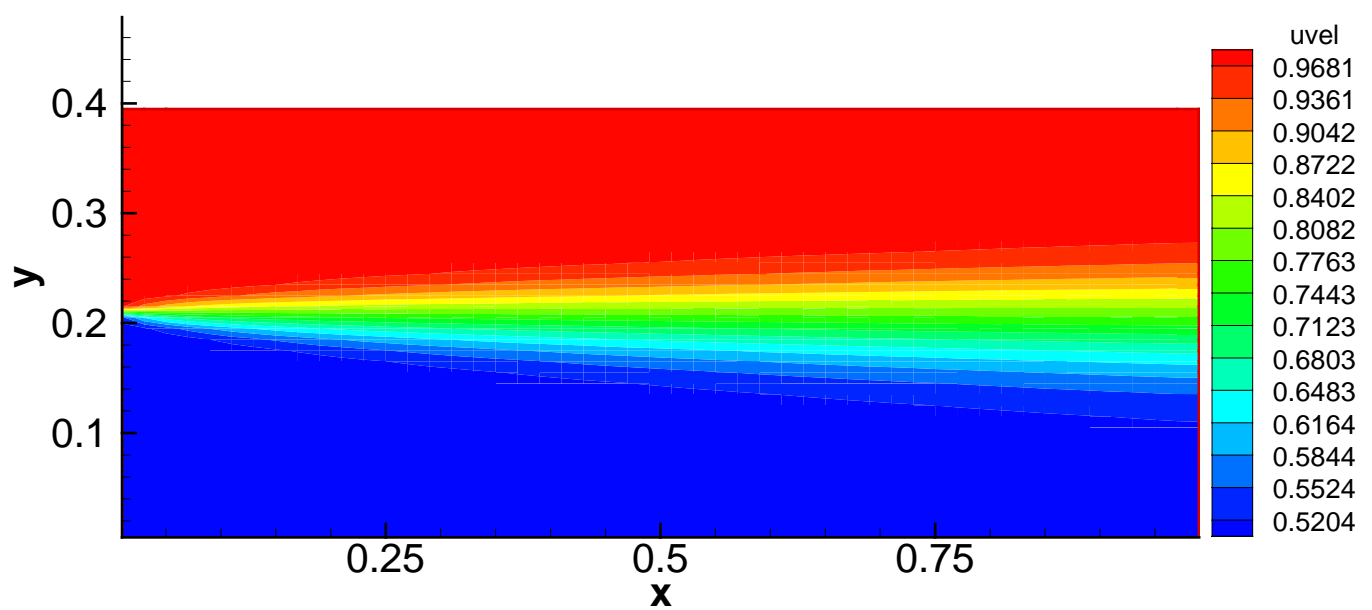

(a)

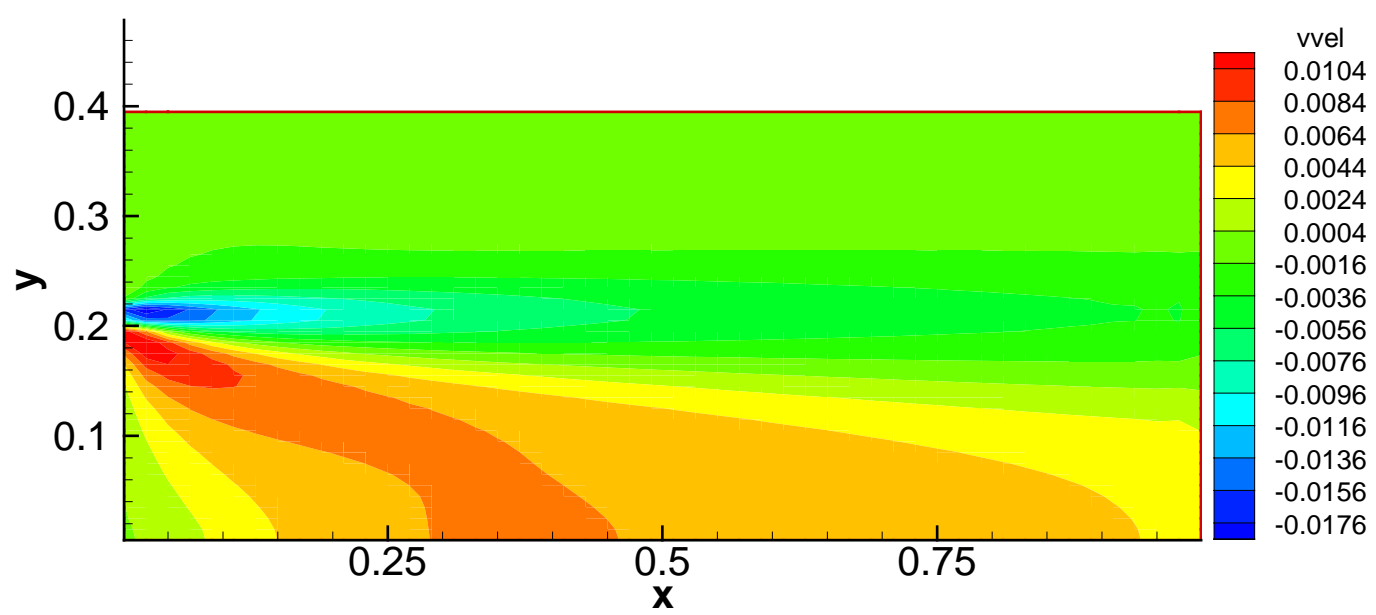

(b)

Figure 3-36 Contour plot of free shear layer, (a) u-velocity, (b) v-velocity. 


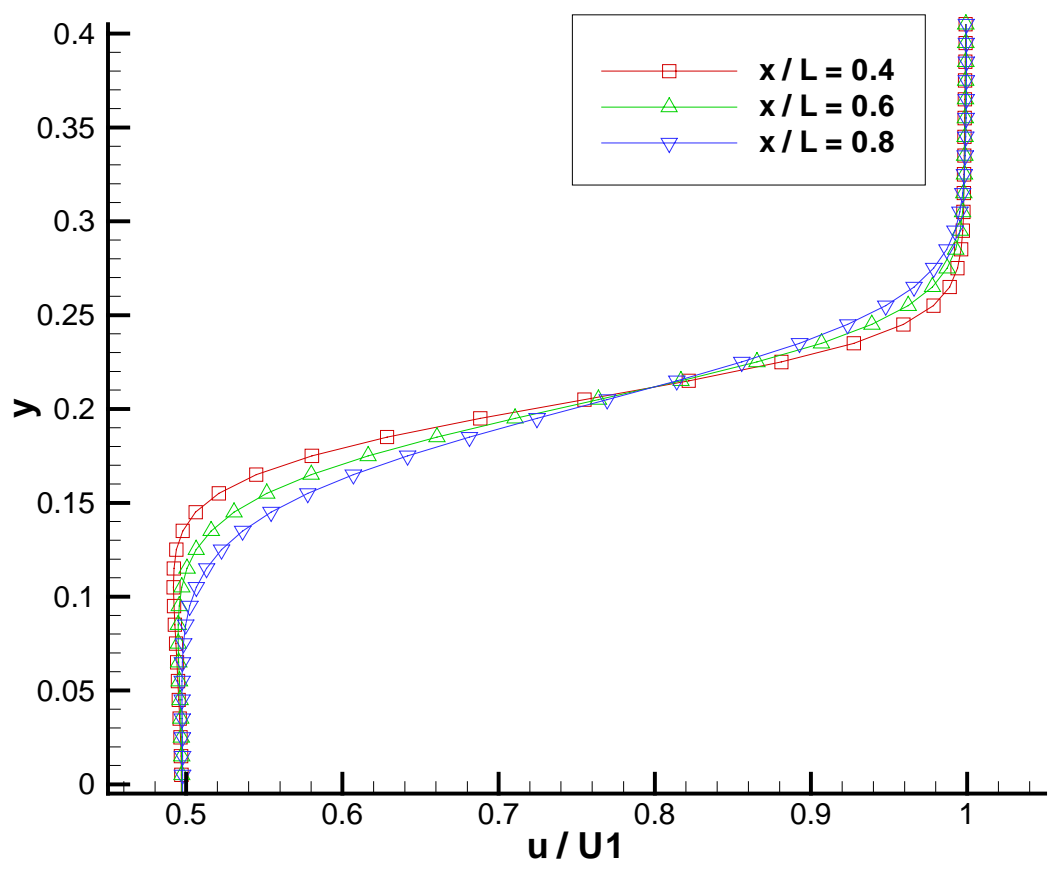

Figure 3-37 u-velocity profiles at three different $x$-stations of a free shear layer, $U_{2} / U_{1}=0.5$.

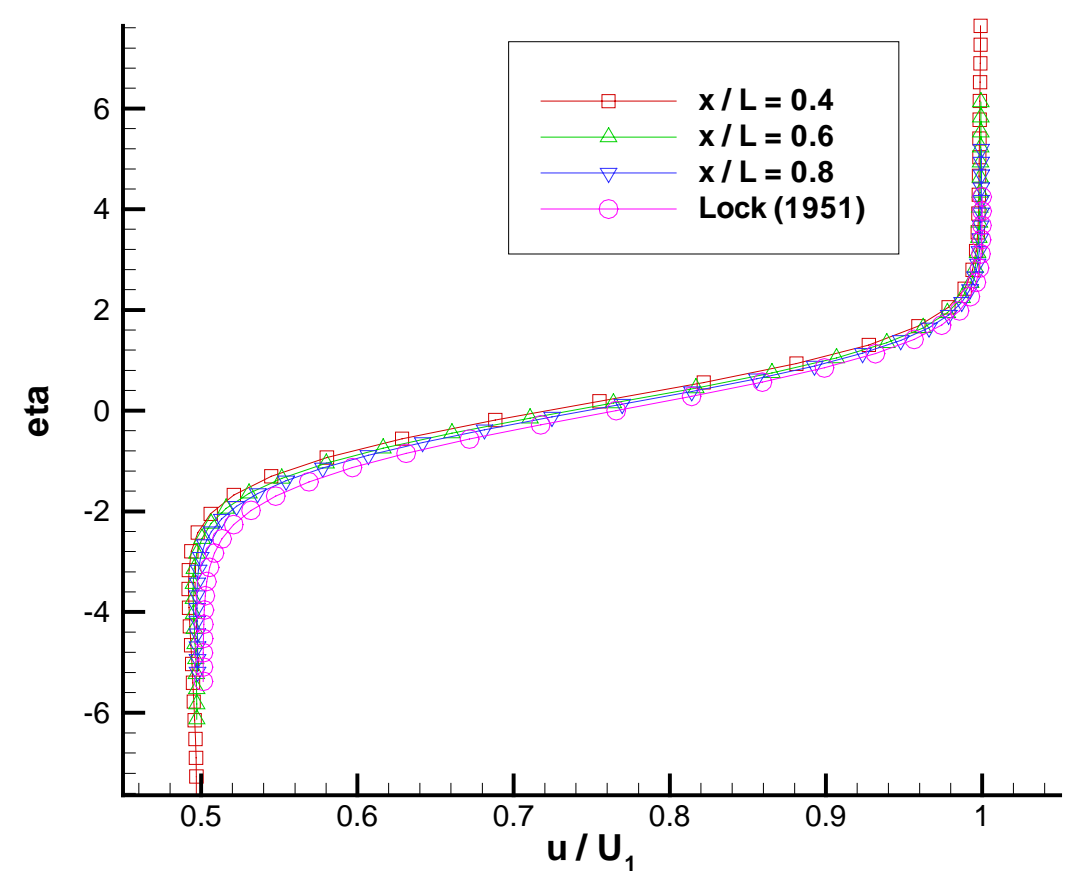

Figure 3-38 Comparison of normalized u-velocity profiles with similarity solution (Lock 1951) for a free shear layer, $U_{2} / U_{1}=0.5$. 


\subsection{Backward-facing Step Flow}

Up to the last section, flow problems being considered have available analytical solution. Starting from this section, judgment on the computation would have to be made based on either the experimental data or numerical solution obtained from an independent study.

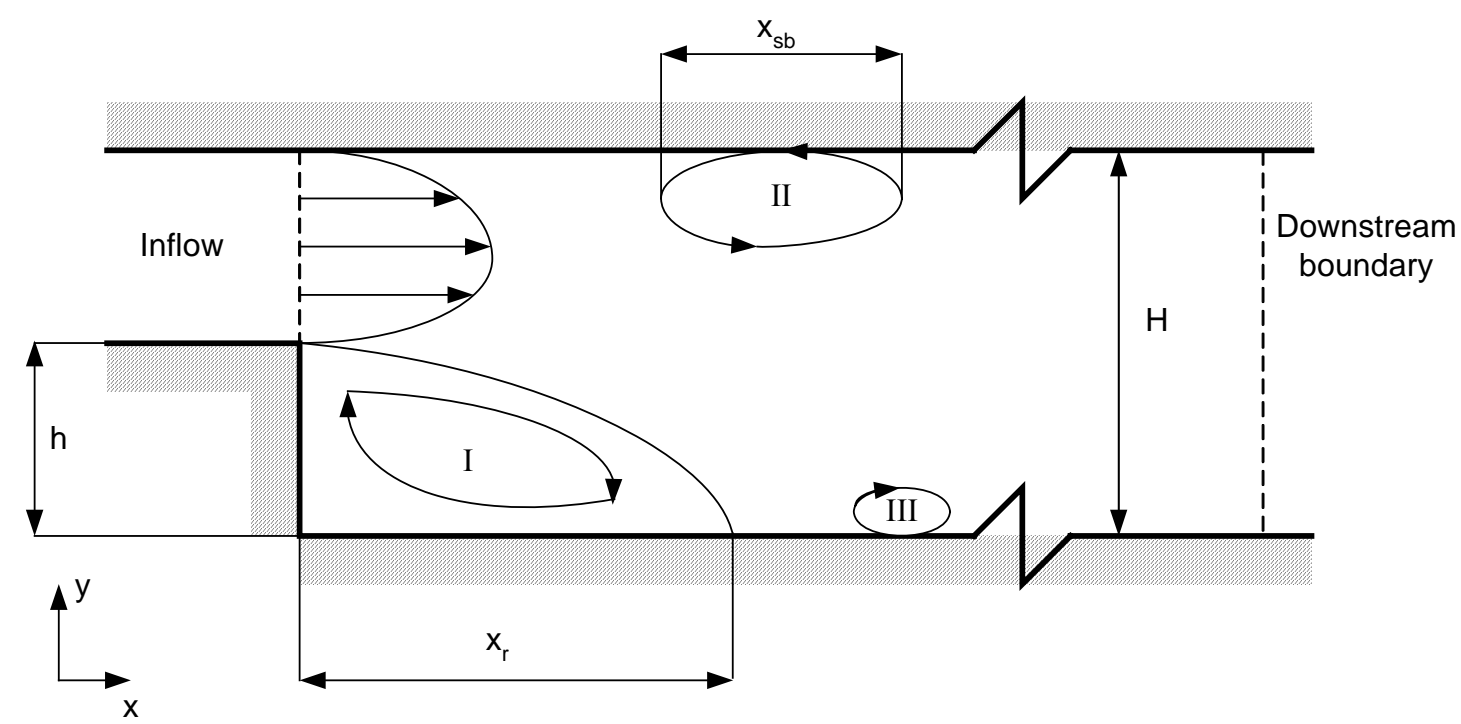

Figure 3-39 Schematic of flow past a backward-facing step (1:2 expansion ratio)

In this section considered is a steady, incompressible flow over a backward-facing step in a wall-bounded channel. Figure 3-39 shows a schematic of the flow configuration, where $h$ is the step height. The narrower channel is called the inlet channel or upstream channel; the other part of the channel is called the expanded channel or downstream channel. This type of flow has been widely investigated by numerous authors both experimentally and numerically (Goldstein et al. 1970; Denham and Patrick 1974; Etheridge and Kemp 1978; Armaly et al. 1983; Kim and Moin 1985; Zhu 1994; Hwang and Peng 1995; Le et al. 1997). Many of those studies used a channel with a relatively large width (size in the spanwise direction), and an expansion ratio $(h / H)$ of 1:2. The Reynolds number used for this flow is usually defined as

$$
\operatorname{Re}=\frac{U D_{h}}{v},
$$

where $U$ represents an average velocity in the inlet channel which is equal to two third of the maximum velocity, $D_{h}$ is the hydraulic diameter of the inlet channel. For a channel with relatively large width and an expansion ratio of two, $D_{h}$ is equal to twice as the step height, since

$$
D_{h}=\frac{4 A}{P}=\frac{4 W(H-h)}{2(W+H)} \cong \frac{4 W h}{2 W}=2 h .
$$

Alternative definitions of Reynolds numbers such the one based on the centerline velocity and step height can be easily based from the above relation. 
As observed in many experiments, a direct consequence of the channel expansion is a large re-circulating flow attached to the backward-facing step, called the primary recirculation zone (zone I in Figure 3-39). The primary recirculation zone usually is measured by its reattachment length $x_{r}$, before which the flow in the near-wall region goes in a reverse direction (see Figure 3-39), thus the phenomena of boundary layer separation.

In a representative work presented by (Armaly et al. 1983) which also used a 1:2 expansion channel, it is pointed out that the flow exhibits two-dimensionality only at Reynolds numbers $\operatorname{Re}<400$ and $\operatorname{Re}>6000$; in between these Reynolds numbers the flow was found to be strongly three-dimensional, and at the same time maintained its symmetry to the center x-y plane. (Armaly et al. 1983) further identified three flow regimes based on the relation between the measured reattachment length and the Reynolds number, i.e., laminar when $\operatorname{Re}<1200$, transitional when $1200<\operatorname{Re}<6600$, and turbulent when $\operatorname{Re}>6600$.

They also, as the first research group, reported two additional recirculation zones (zone II and III in Figure 3-39), one at the upper wall downstream of the expansion and the other, being very thin, at the lower wall a little distanced downstream from the primary recirculation zone. The secondary recirculation zone (zone II) is also called secondary bubble; its formation is due to the adverse pressure gradient created by the sudden expansion, and exists in the late part of the laminar range and throughout the transition phase. The third recirculation zone, zone III, occurs only in the early part of the transition region where reattachment length experiences a sharp drop in its magnitude (Armaly et al. 1983). The size of both the primary and secondary recirculation zone increases in the laminar flow regime with Reynolds number, and starts to decrease in the transitional region. In the turbulent regime the secondary and tertiary zone disappear while the primary recirculation zone is fixed at a constant size. To clarify the above explanation, a figure (Figure 3-40) from the original paper is included herein. 


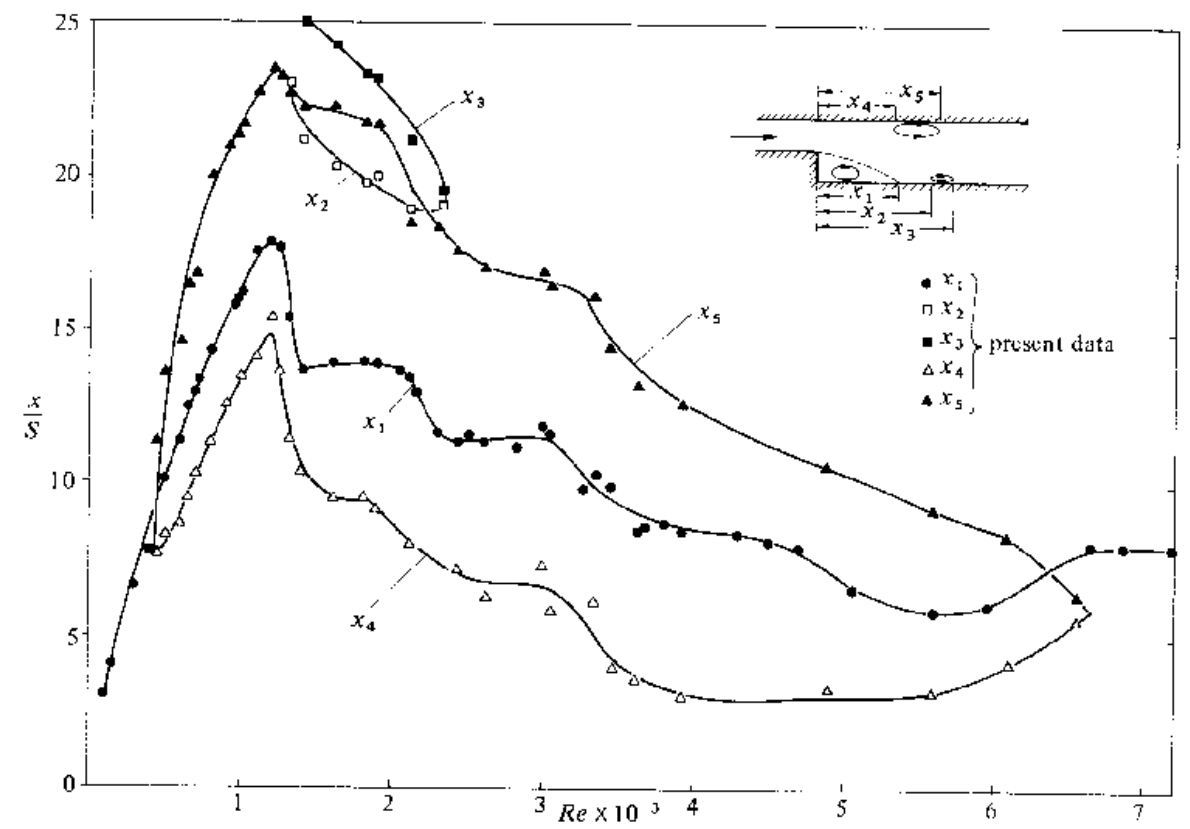

Figure 3-40 Location of detachment and reattachment in a backward facing step flow. (From (Armaly et al. 1983) with permission)

In general, the characteristic of the backward-facing step flow, in particular the reattachment length, is determined by the Reynolds number, channel expansion ratio, dimensionality (2D or 3D), inlet condition, and channel boundaries (i.e., close channel or open channel). In this section, focus is placed on the two-dimensional wall-bounded laminar flow with Reynolds number $<400$. Attempt will also be given to the prediction of 2D laminar flow but with higher Reynolds numbers $400<\operatorname{Re}<1000$, to see how the 2D computation performs. As has been done in many other computations, the standard step geometry was simplified by excluding the upstream channel, and accordingly a proper velocity profile, typically a parabolic profile is specified at the inlet of the downstream channel (see Figure 3-39). A study for the high Reynolds number turbulent flow will be presented in a later chapter.

Simulations are first performed for Reynolds numbers within 400, a range in which the flow can be considered two-dimensional. The flows with Reynolds number valued at $100,150,200,250,300,350$ and 400 is configured by adjusting the inlet velocity while keeping the kinematic viscosity constant $\left(1 \mathrm{E}-6 \mathrm{~m}^{2} / \mathrm{s}\right)$. The domain size used is 2.5 meter in length and $0.2 \mathrm{~m}$ in height, excluding the inlet channel. The step height is $0.1 \mathrm{~m}$, which gives a channel expansion ratio of the $1: 2$. A $75 \times 60$ grid is used which yields a grid resolution of $0.033 \mathrm{~m}$ and $0.0033 \mathrm{~m}$ in longitudinal and transverse direction, respectively. A relatively fine grid in the y-direction is necessary to capture the recirculation zone triggered by the wall boundary layer separation. At the inlet an assumed parabolic profile is prescribed, as the same was used by (Kim and Moin 1985). An outlet boundary condition is used at the outlet. Wall boundary conditions are applied at the south and north walls, as well as at the downstream side of the step. 
At each Reynolds number, the simulation starts with a relatively large time step, typically $0.1 \mathrm{~s}$, using fifty percent implicit power-law and fifty percent explicit QUICK. The established flow field is then further advanced using a smaller time step size $(0.01 \mathrm{~s}$ when $\operatorname{Re}<300,0.005$ s when $300 \leq \operatorname{Re}<300$ ) and the explicit QUICK to reach a final steady-state flow.

The steady-state u-velocity contour plots at each Reynolds number along with flow streamlines are shown in Figure 3-41(a) through (g). The steady-state v-velocity contours at those Reynolds numbers are also shown in Figure 3-42(a) through (g). It is clearly seen that the size of the recirculation zone gradually increases with the rise of the Reynolds number. This phenomenon is accompanied by a progressively elongated flow core region originating from an increased inlet velocity. As reported in the experiments, it is confirmed here that no secondary and tertiary recirculation zone is present in this Reynolds number range. However, with the increasing Reynolds number, a basin-like (or opposite bell-shaped) region is slowly formed at about 7 step-heights away from the inlet on the upper wall, where the deceleration of the u-velocity in that region is gradually intensified and spreads around the bell base center. This decelerated cone of the flow acts like an obstacle that forces other part of the flow to bypass it (Figure 3-41(g)), and prepares the flow a formation of the secondary recirculation zone at a further increase of Reynolds number. The streamwise velocity profiles at various $\mathrm{x}$-stations are also shown in Figure 3-43 for Reynolds numbers 100, 200, 300 and 400.

When Reynolds number goes beyond 400, as reported in (Armaly et al. 1983), the flow becomes three dimensional in nature. Nevertheless, attempt is also made to explore possible outcomes at high Re's with this inexpensive quasi two-dimensional calculation. Before doing so, notice from Figure 3-41(g) that the decelerating core of the stream has almost reached the domain end. In order to eliminate the possible influence of the outflow boundary, extend the domain length is extended to $4.0 \mathrm{~m}$, and correspondingly, the grid nodes in the $\mathrm{x}$-direction are increased to 120. Calculations are then carried out for $\operatorname{Re}=500,600,800$ and 1000. The obtained u- and v-velocity contours are shown in Figure 3-44(a) through (d) and Figure 3-45(a) through (d) respectively. The velocity profiles are displayed in Figure 3-46. It is observed that while the primary recirculation zone keeps increasing, a secondary recirculation zone (separation bubble) is formed from about $\mathrm{Re}=500$ at the upper wall. The size of the separation bubble grows with the increase of $\mathrm{Re}$ in the Re range considered herein. This result, in particular, the triggering Reynolds number and the growing trend of the bubble size, agrees with the experimental observation reported in (Armaly et al. 1983). Verification of the tertiary recirculation zone which occurs only in the transitional region is avoided in this study.

The reattachment lengths, as the most critical characteristic in the backwardfacing step flow, are also measured at all Reynolds numbers being considered here. This is done by plotting the $\mathrm{u}$-velocity variation along $\mathrm{x}$-axis at the first node near the lower wall. Excluding the origin point $(x=0)$, the first intersection of the curve with zero gives the desired reattachment length. Interpolation practice is usually necessary to determine the approximate location at which the u-curve crosses zero. The reattachment lengths calculated from the current flow simulation are included in Table 3-8, and are also further 
graphed in Figure 3-47 in comparison with the data given in (Armaly et al. 1983) and (Kim and Moin 1985). The dependence of the reattachment length on Reynolds numbers is in good agreement with both experimental data and Kim and Moin's simulation up to about $\operatorname{Re}=400$. From $\operatorname{Re}=500$ the computed results start to deviate from the experimental values. Similar behavior of the solution is reported by (Kim and Moin 1985). A mesh-refinement study as well as variation of the location of downstream boundary at $R e=600$ gives similar prediction. In fact, the downstream boundary used in (Kim and Moin 1985) is 30 step-heights while the current simulation has 40h. This tells that the difference between the experimental and computational results is not a result of numerical errors. Instead, this discrepancy confirms, from the numerical computation point of view, the possible deficiency of simulating an essentially 3D flow with a 2D calculation starting from $\mathrm{Re}=400$, just as (Armaly et al. 1983) have pointed out.

Table 3-8 Calculated reattachment length and length of secondary bubble as a function of Reynolds numbers

\begin{tabular}{|c|c|c|c|c|}
\hline $\mathbf{R e}$ & $\mathbf{x}_{\mathbf{r}}$ & $\mathbf{x}_{\mathbf{r}} / \mathbf{h}$ & $\mathbf{x}_{\mathbf{s b}}$ & $\mathbf{x}_{\mathbf{s b}} / \mathbf{h}$ \\
\hline 100 & 0.3261 & 3.261 & & \\
\hline 150 & 0.4379 & 4.379 & & \\
\hline 200 & 0.5376 & 5.376 & & \\
\hline 250 & 0.6230 & 6.230 & & \\
\hline 300 & 0.7135 & 7.135 & & \\
\hline 350 & 0.7791 & 7.791 & & \\
\hline 400 & 0.8448 & 8.448 & & \\
\hline 500 & 0.9475 & 9.475 & 0.6384 & 6.384 \\
\hline 600 & 1.0015 & 10.015 & 0.8713 & 8.713 \\
\hline 800 & 1.1338 & 11.338 & 1.1776 & 11.776 \\
\hline 1000 & 1.3179 & 13.179 & 1.3546 & 13.546 \\
\hline
\end{tabular}

The length of the secondary bubble is measured in a similar way as to the primary recirculation zone except that two intersections (not one) of the u-velocity curve (at the first nodes from the upper wall) with zero needs to be recorded; the distance between the two zero locations gives the bubble length. The obtained values are also listed in Table 3-8. (Kim and Moin 1985) reported their calculated lengths at $\mathrm{Re}=600$ and 800 , which is 7.8 and 11.5 step-heights respectively; the current study gives $8.7 \mathrm{~h}$ and $11.8 \mathrm{~h}$ at these two Reynolds numbers. At $\mathrm{Re}=1000$, (Armaly et al. 1983) reported a length of $10.4 \mathrm{~h}$ while the present study yields $13.5 \mathrm{~h}$. Again, the somewhat large discrepancy is very possibly due to the three-dimensionality at that Reynolds number. 


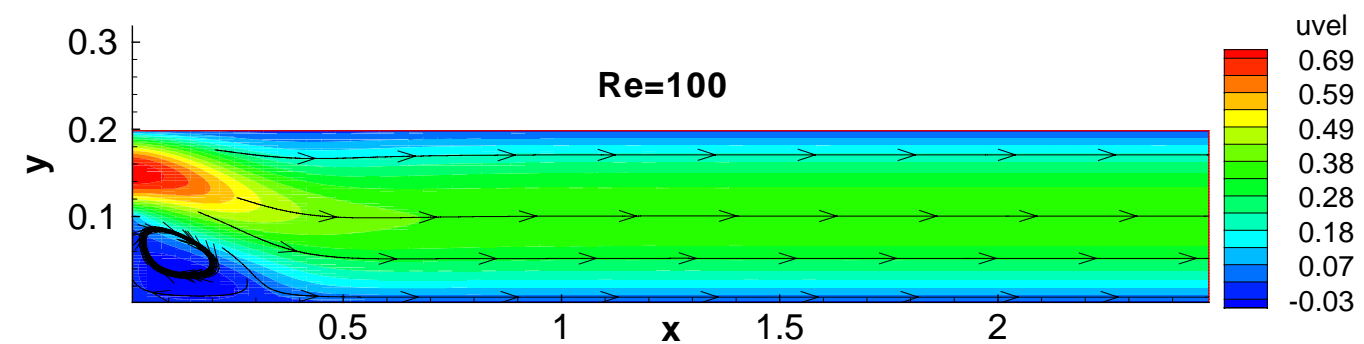

(a)

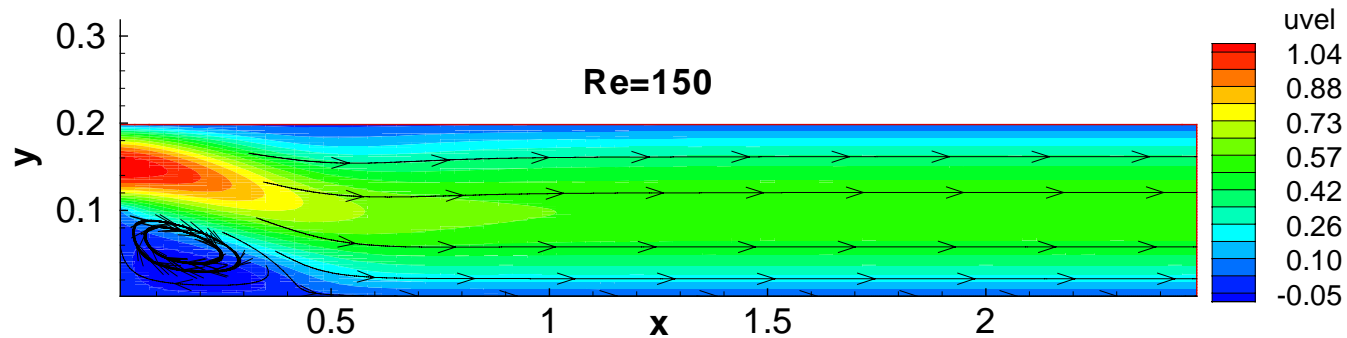

(b)

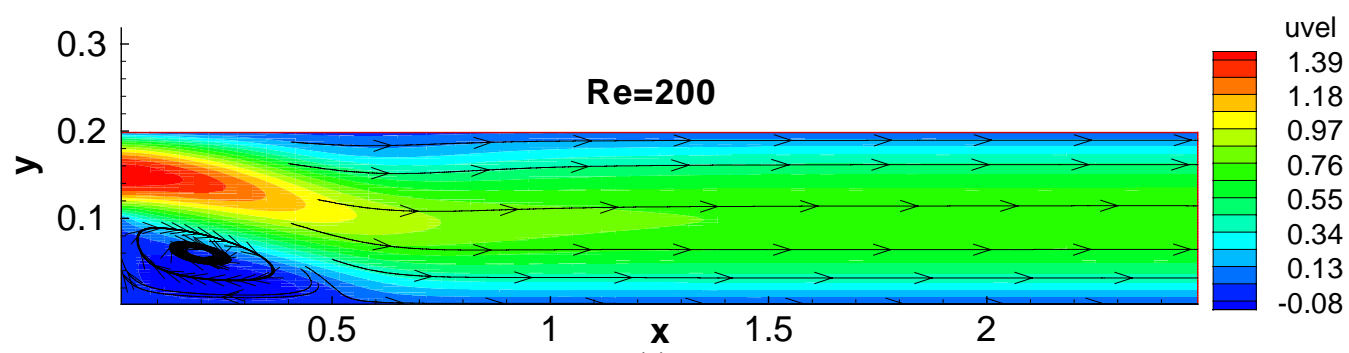

(c)

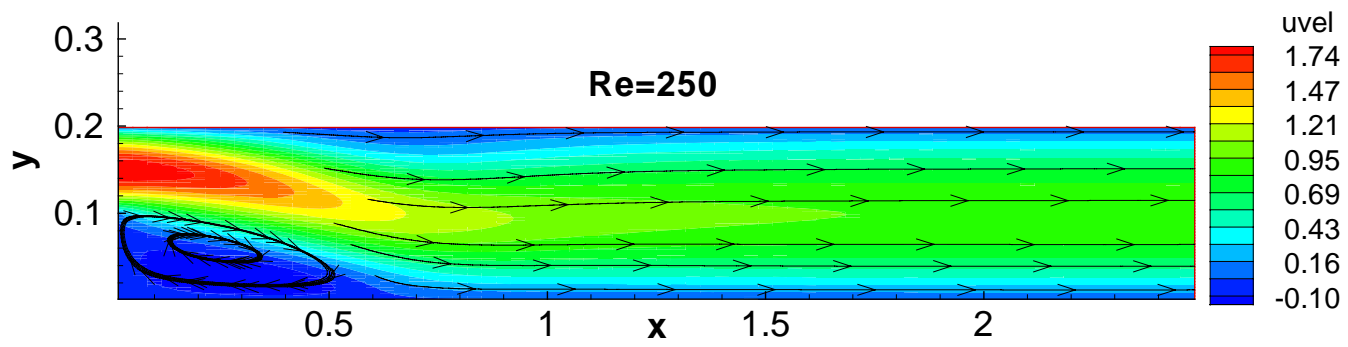

(d)

Figure 3-41 u-velocity contours and streamlines of backward-facing step flow at various Reynolds numbers $(\operatorname{Re} \leq 400)$. 


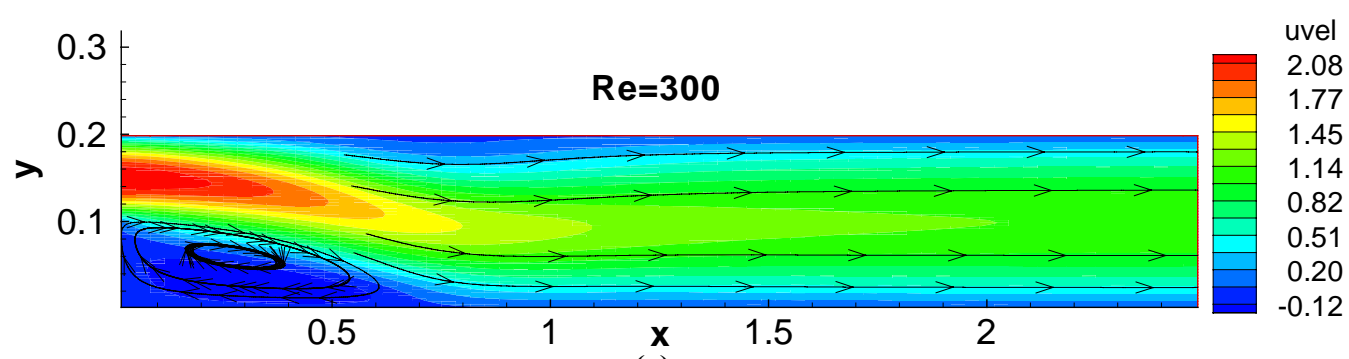

(e)

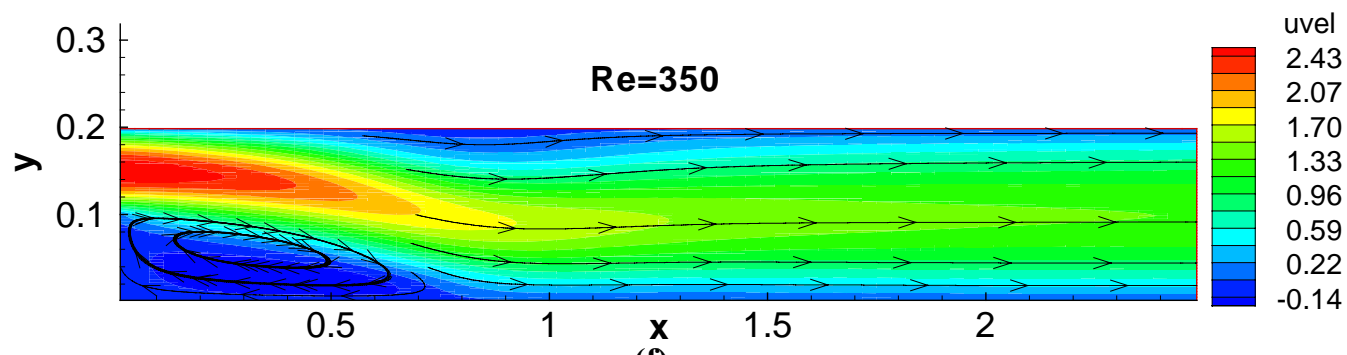

(f)

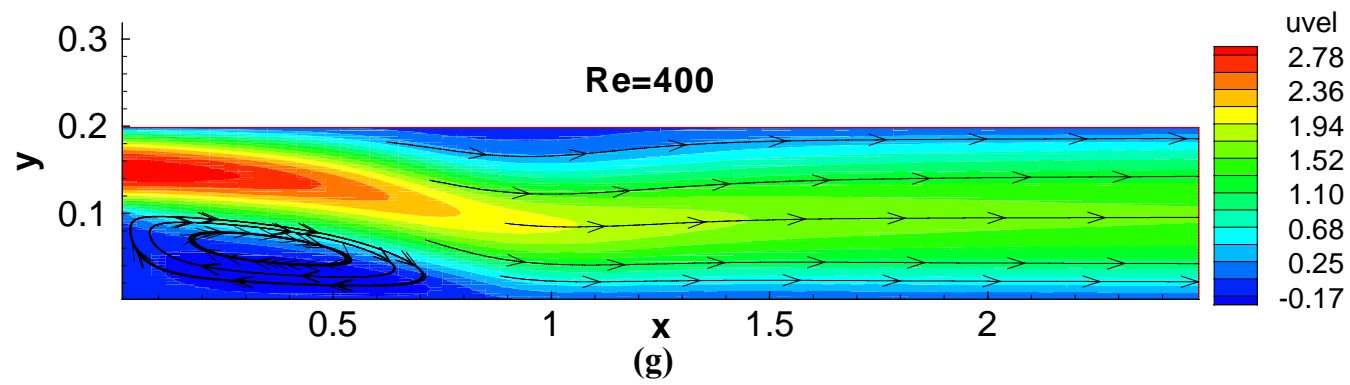

Figure 3-41 u-velocity contours and streamlines of backward-facing step flow at various Reynolds numbers $(\operatorname{Re} \leq 400)$. 


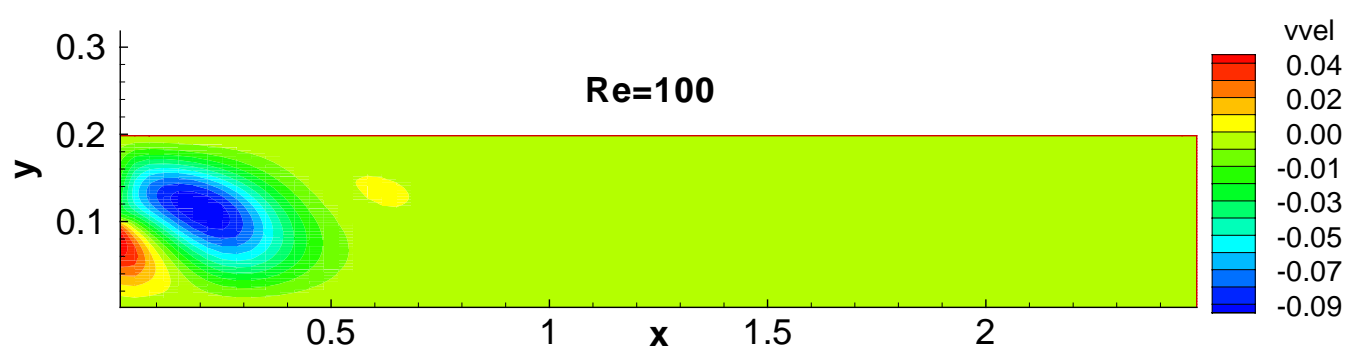

(a)

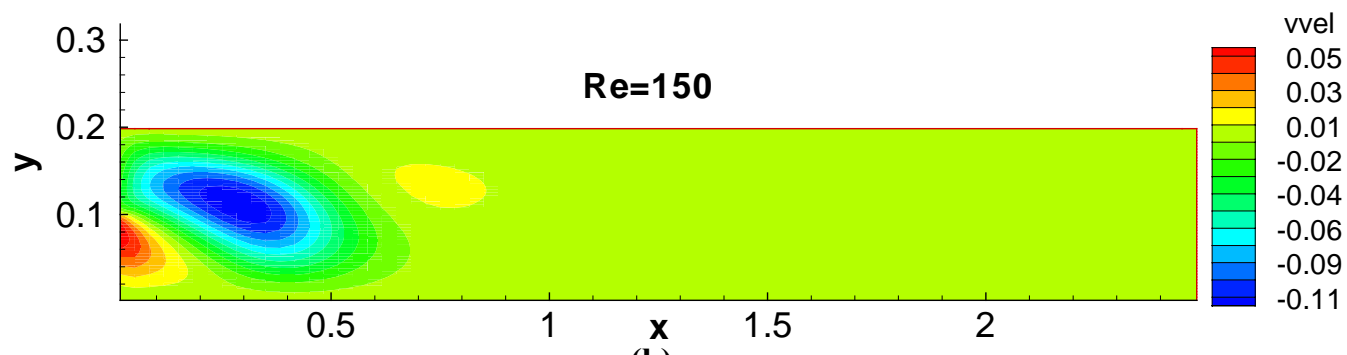

(b)

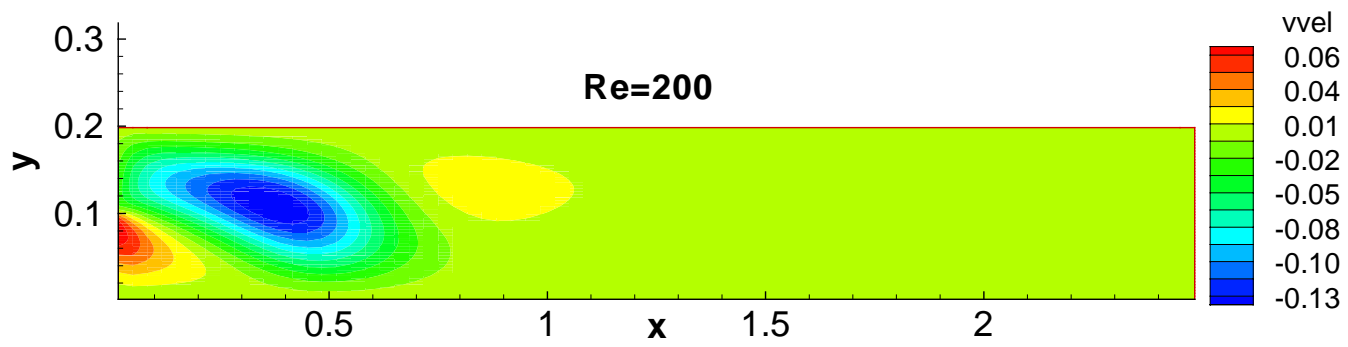

(c)

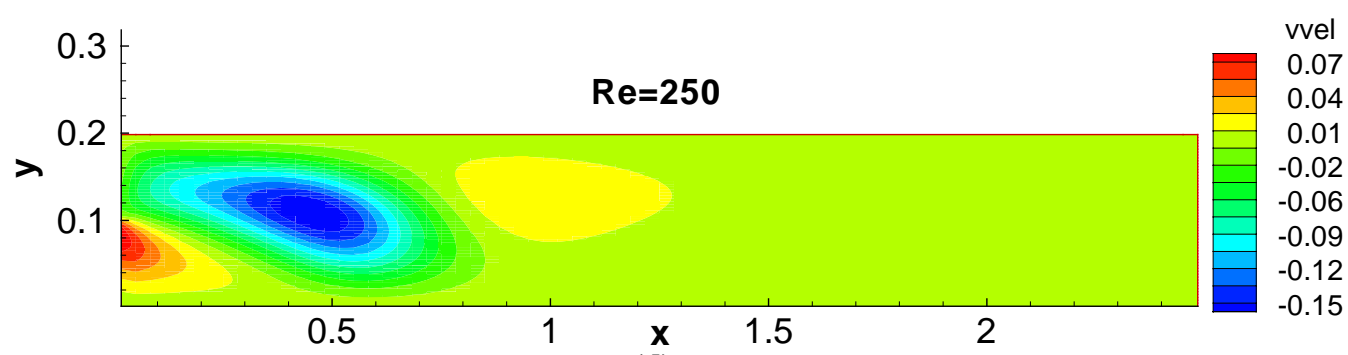

(d)

Figure 3-42 v-velocity contours of backward-facing step flow at various Reynolds numbers $(\operatorname{Re} \leq$ 400). 


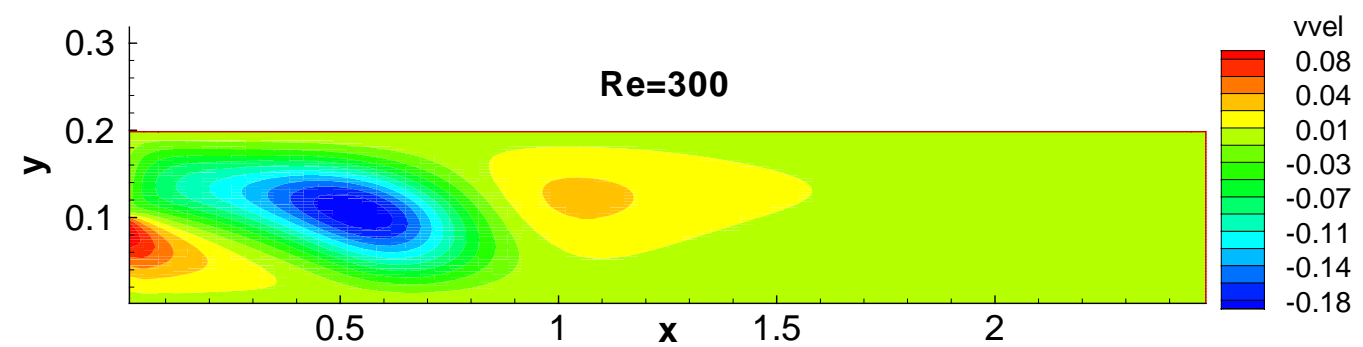

(e)
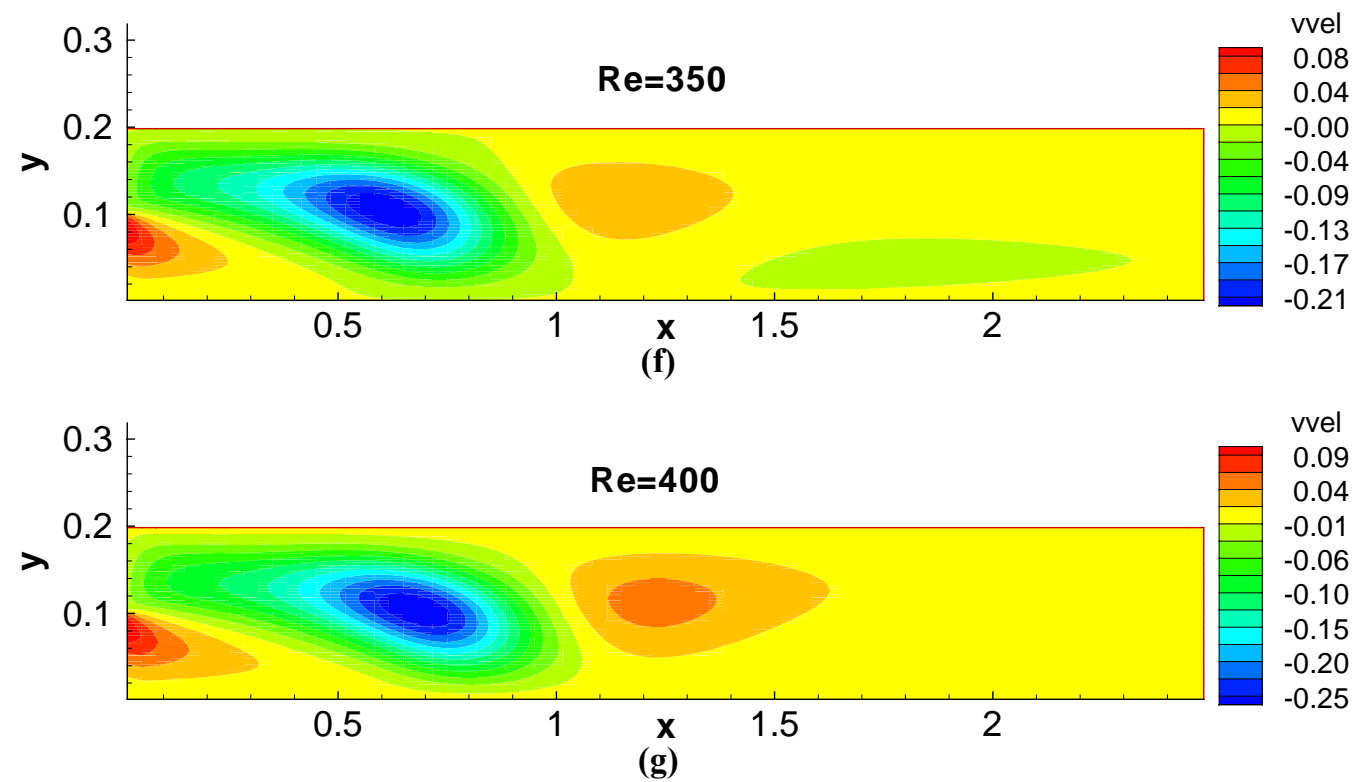

Figure 3-42 v-velocity contours of backward-facing step flow at various Reynolds numbers $(\operatorname{Re} \leq$ 400). 


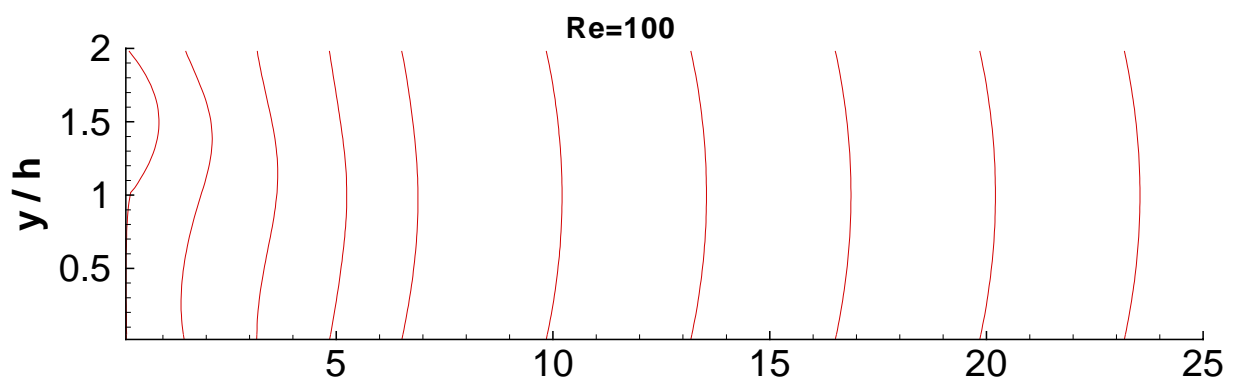

(a)

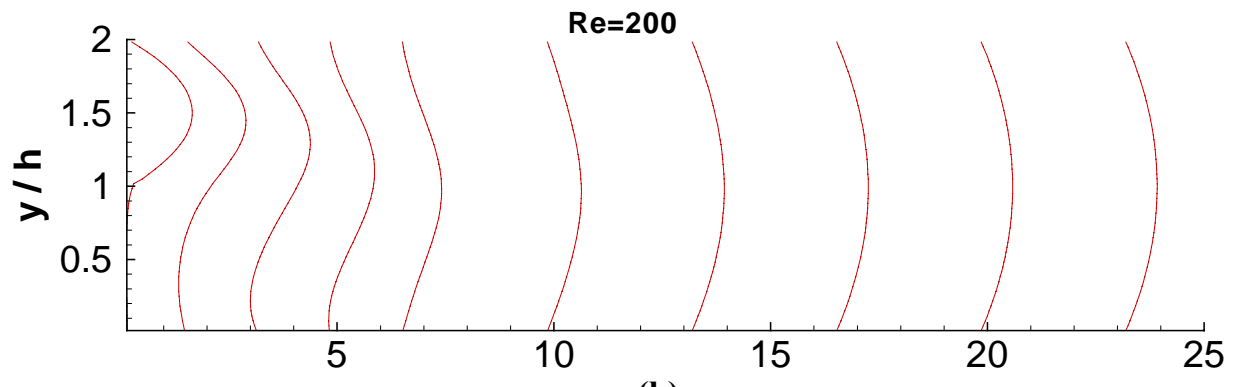

(b)

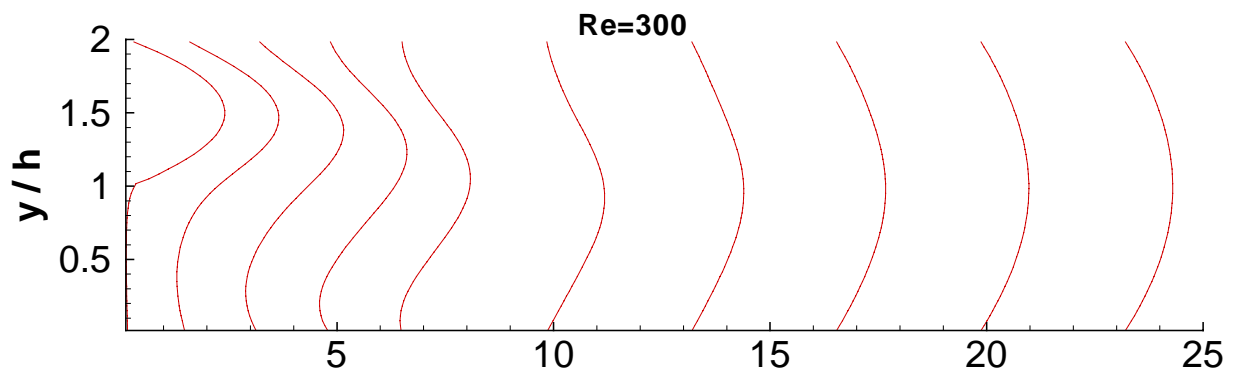

(c)

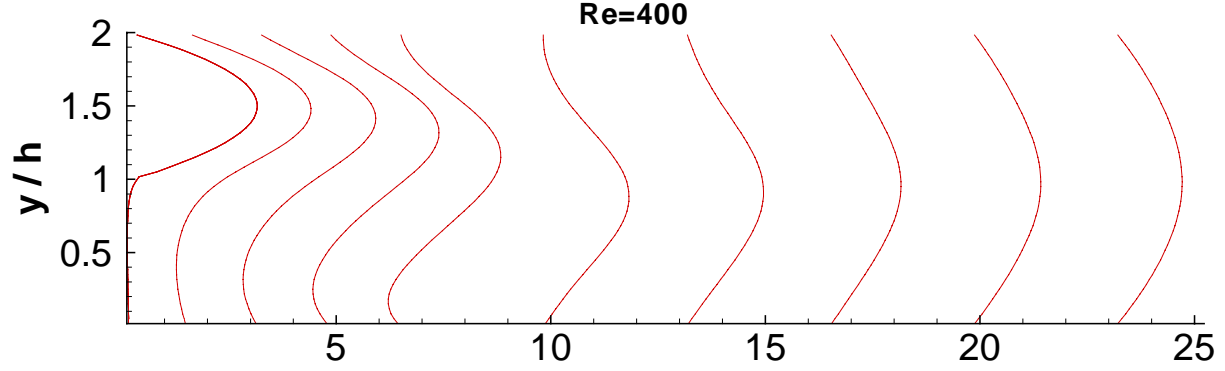

(d)

Figure 3-43 u-velocity profiles at various x-stations of a backward-facing step flow with different Reynolds number $(\operatorname{Re} \leq 400)$. Both $x$ - and y-locations are in step-height unit. (a) $\operatorname{Re}=100,(b) \operatorname{Re}=$ 200, (c) $\operatorname{Re}=300$, (d) $\operatorname{Re}=400$. 


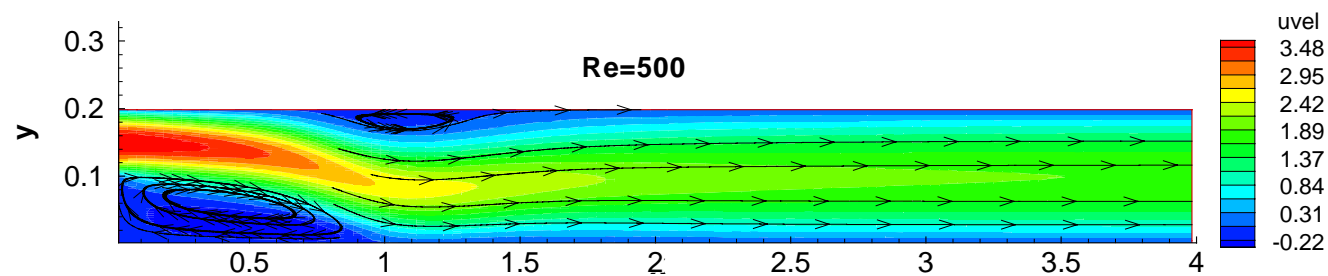

(a)

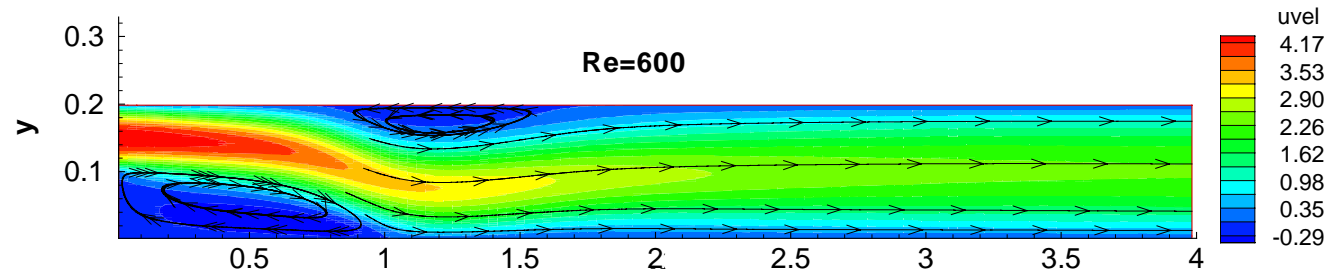

(b)

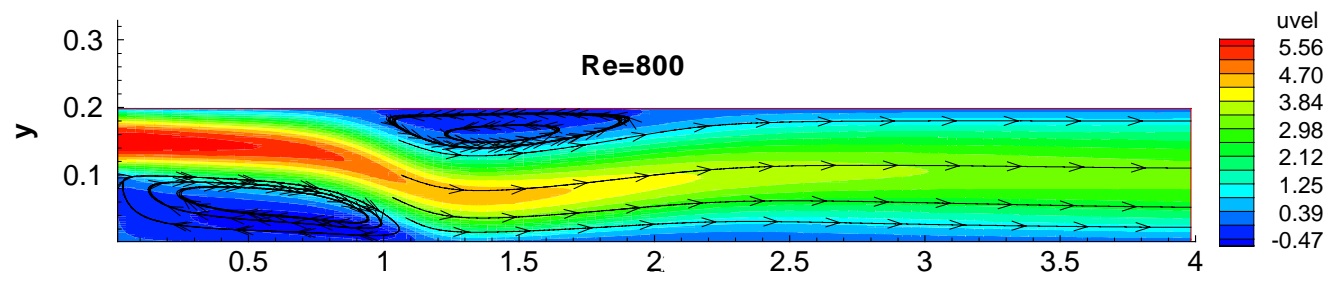

(c)

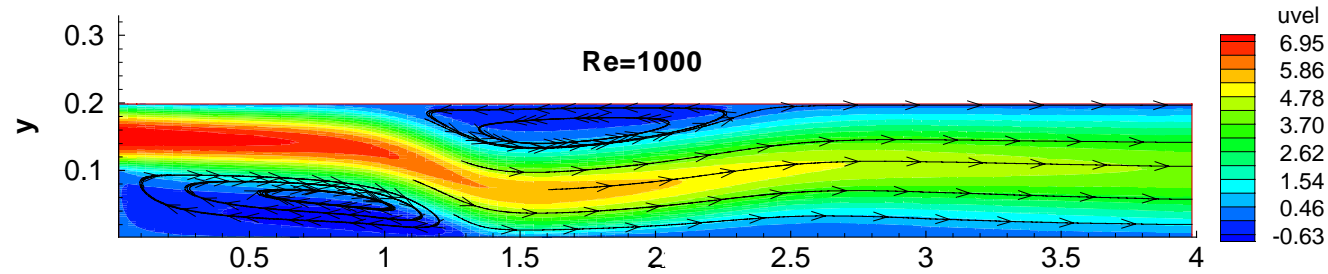

(d)

Figure 3-44 u-velocity contours and streamlines of backward-facing step flow at various Reynolds numbers $(400<\operatorname{Re} \leq 1000)$. 


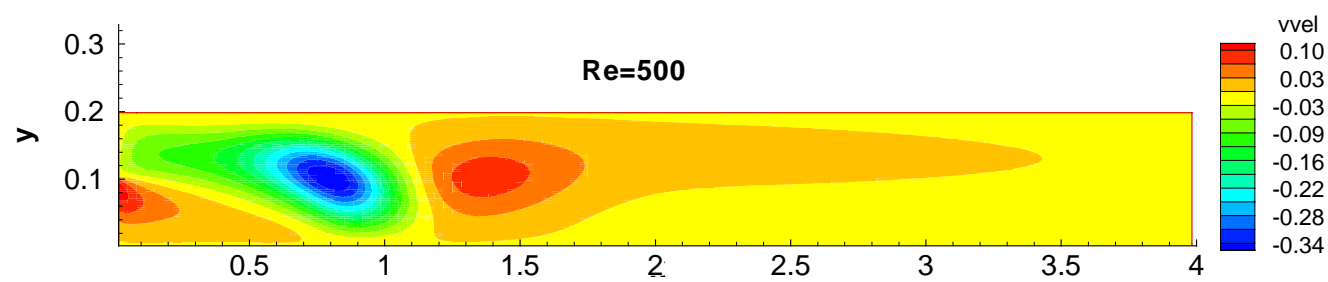

(a)

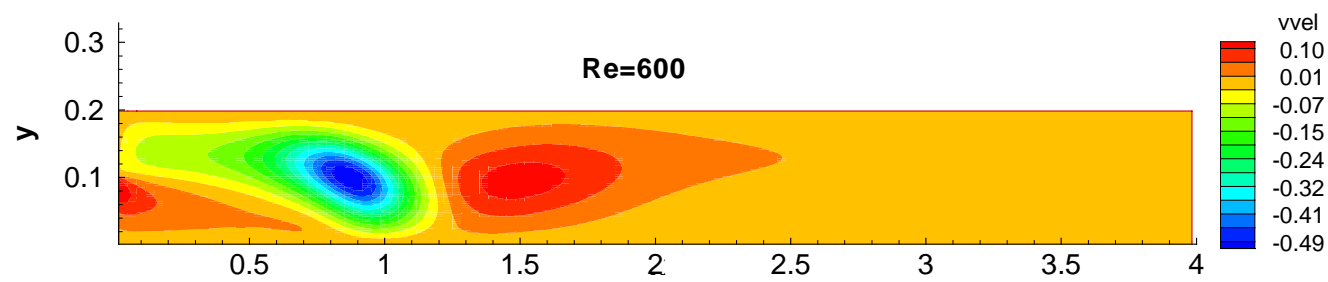

(b)

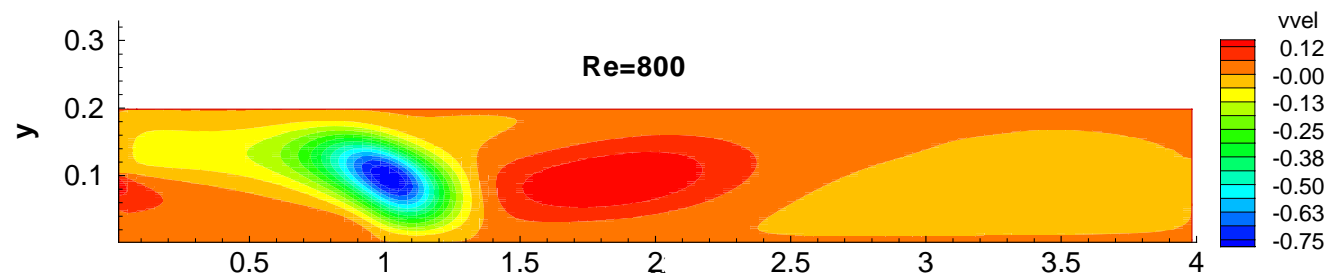

(c)

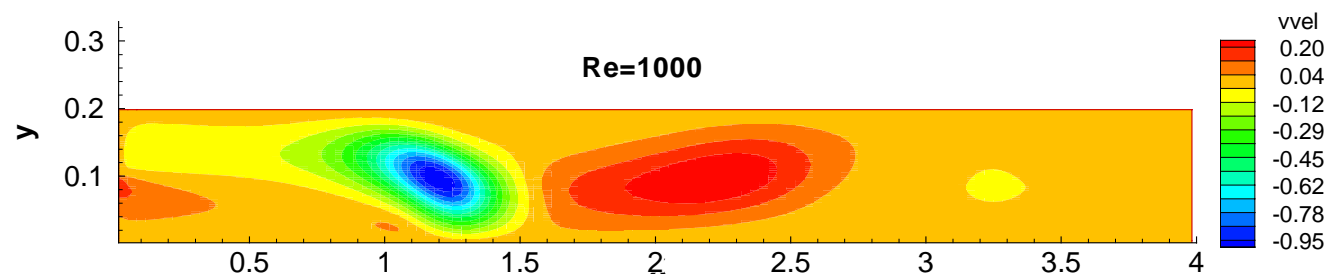

(d)

Figure 3-45 v-velocity contours of backward-facing step flow at various Reynolds numbers $(400<\mathrm{Re}$ $\leq$ 1000). 


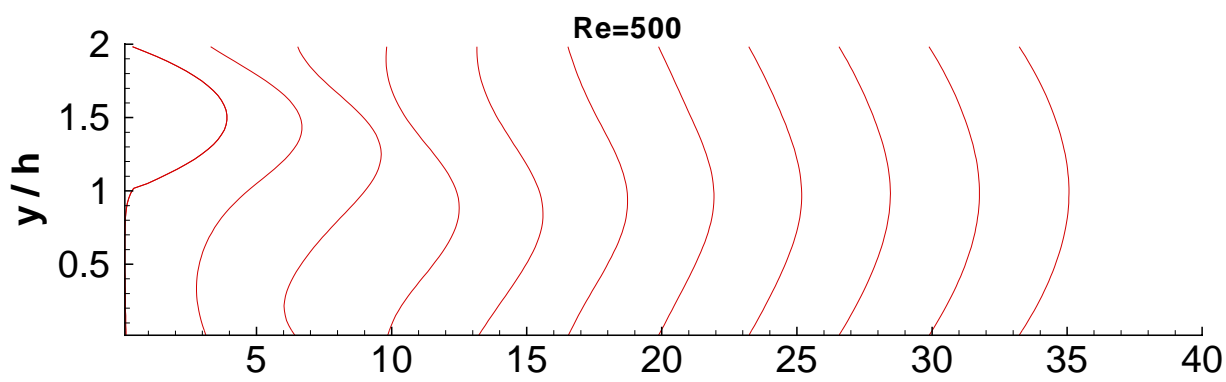

(a)

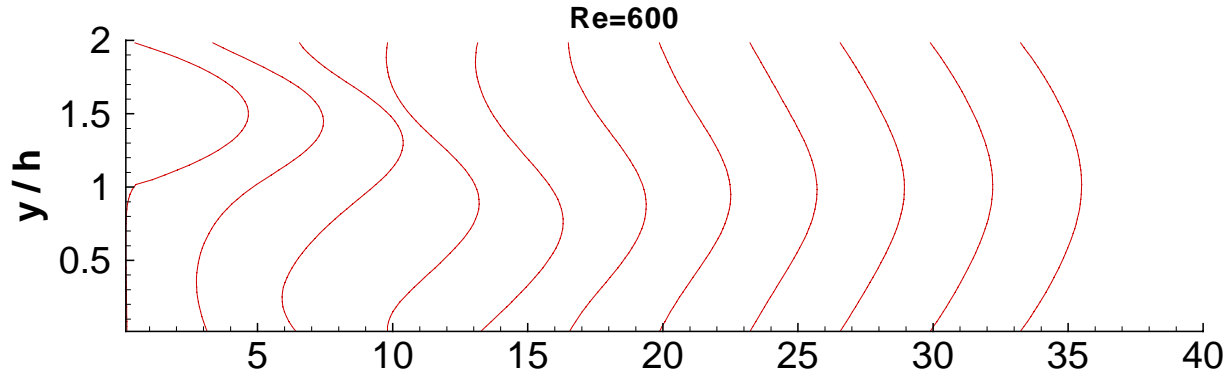

(b)

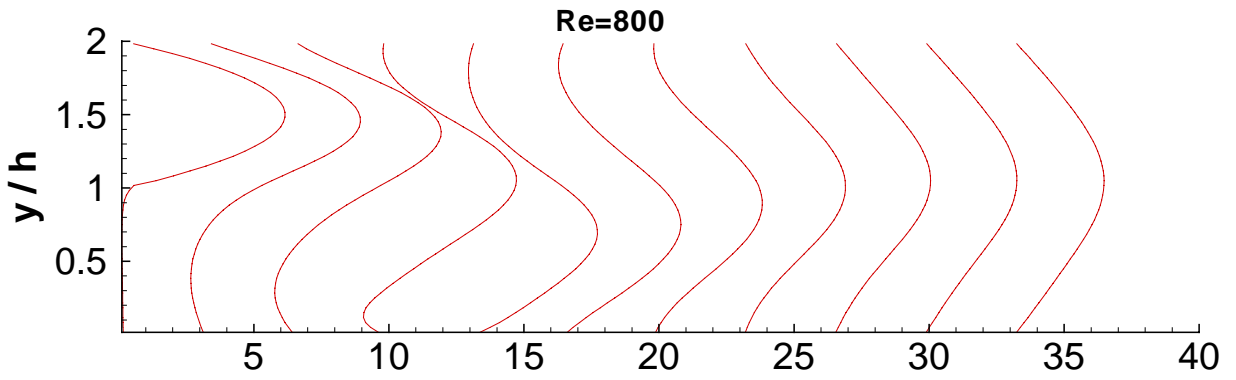

(c)

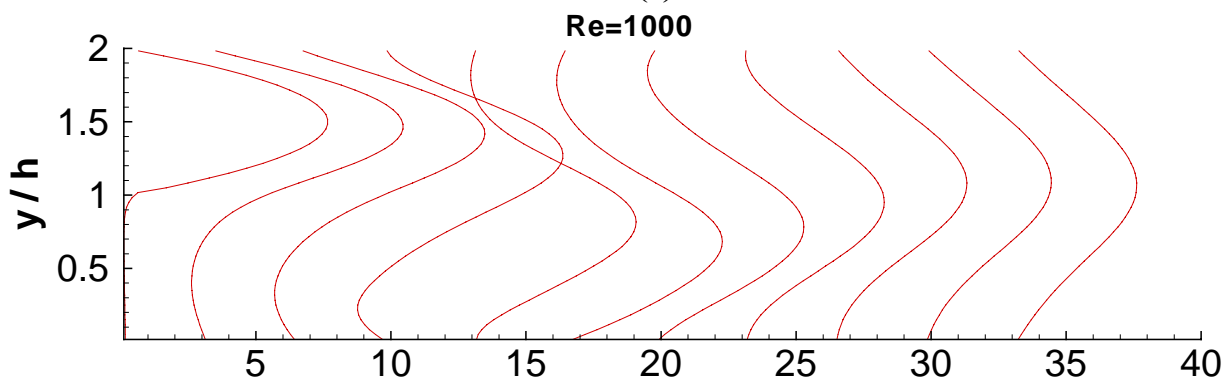

(d)

Figure 3-46 u-velocity profiles at various $x$-stations of a backward-facing step flow with different Reynolds number $(400<\operatorname{Re} \leq 1000)$. Both $x$ - and $y$-locations are in step-height unit. (a) $\operatorname{Re}=500$, (b) $\operatorname{Re}=600$, (c) $\operatorname{Re}=800$, (d) $\operatorname{Re}=1000$. 


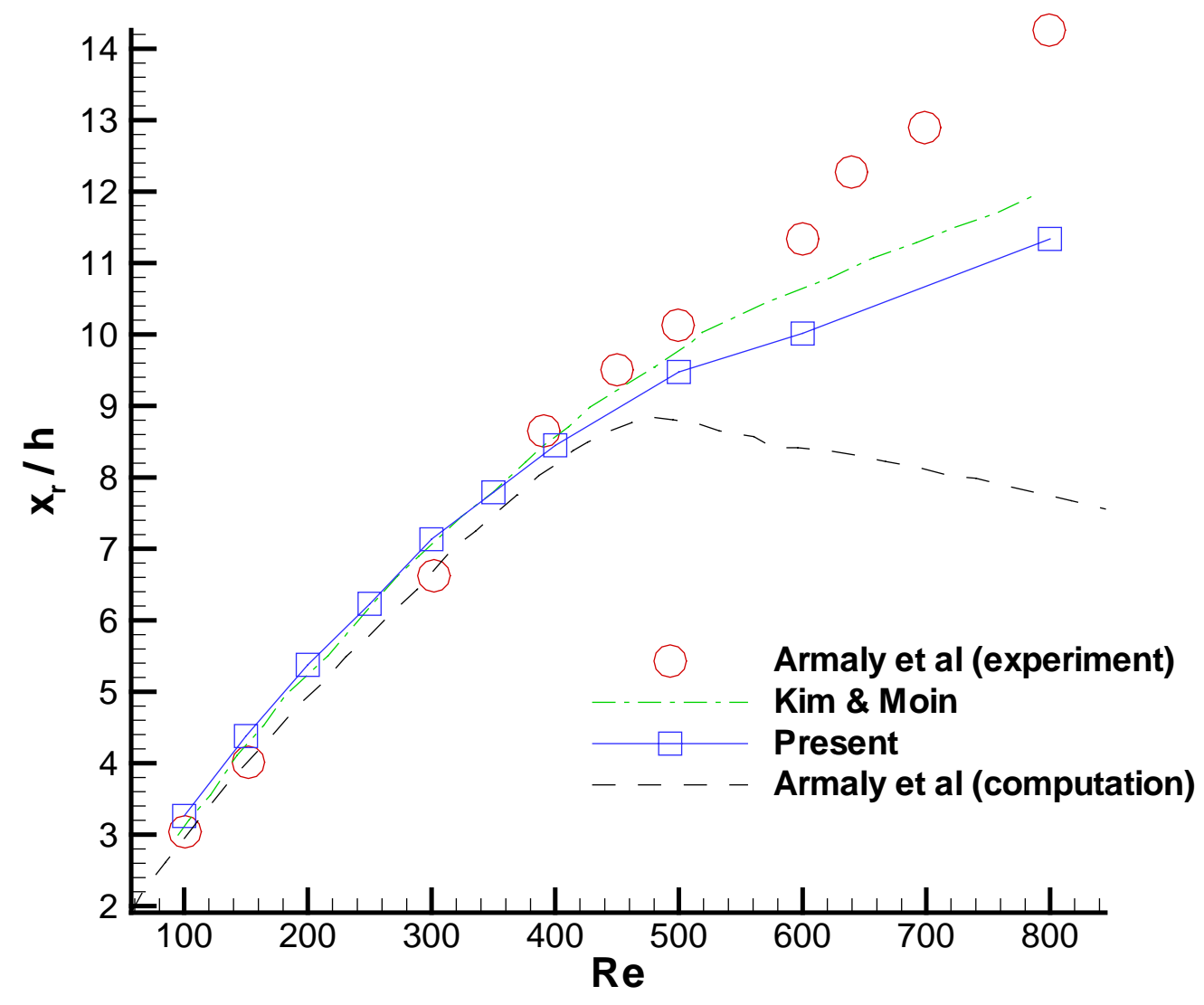

Figure 3-47 Comparison of reattachment length of a backward-facing step flow as a function of Reynolds number. Circle: experimental data from (Armaly et al. 1983); dash-dotted line: computation of (Kim and Moin 1985); square with solid line: present computation; dashed line: computation of (Armaly et al. 1983). 


\subsection{Square Cavity Flow}

In a lid-driven cavity flow the fluid contained inside a square cavity is set into motion by the upper wall (or plate) that is sliding at constant speed, owing to the action of shear stresses. Figure 3-48 shows a schematic of the flow. The Reynolds number defined for this flow is based on the plate velocity, $U$, and the side length of the cavity, $L$. Inside cavity several standing vortices may exist whose occurrence and characteristics are functions of Reynolds numbers.

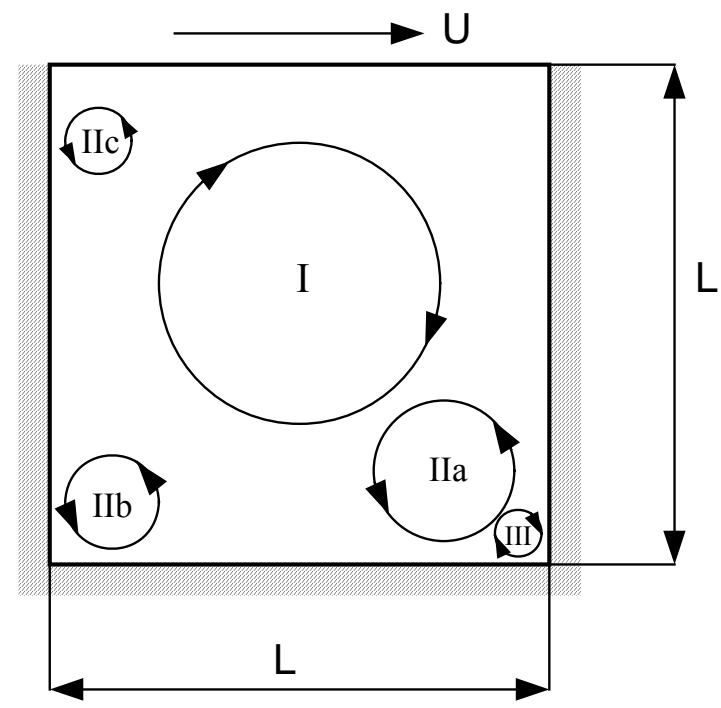

Figure 3-48 Schematic of a lid-driven cavity with possible vortices.

This phenomenon has been well explored through numerous investigations both experimentally and computationally (Nallasamy and Krishna Prasad 1977; Benjamin and Denny 1979; Goda 1979; Ghia et al. 1982; Schreiber and Keller 1983; Kim et al. 1987; Perng and Street 1989). For a two-dimensional problem, i.e., very thin in spanwise direction and the effect in that direction on the flow field is neglected, the moving lid creates a strong vortex, called the primary vortex (numbered I in Figure 3-48), and two secondary corner eddies (IIa and IIb). As the Reynolds number is raised to between 1000 and 2000, a third secondary eddy (IIc) is formed in the upper left corner, rotating in the counter direction of other secondary eddies. A further increase of Reynolds number (from about $\mathrm{Re}=5000$ ) may lead to the formation of a tertiary tiny eddy in the lower right corner (III). If the problem takes into account the effect from the third dimension, the transition to turbulence occurs in the range of $6000 \leq \mathrm{Re} \leq 8000$, and the flow becomes fully turbulent when Re $>10000$ (Zang et al. 1993; Jordan and Ragab 1994). Also, for a 3D problem but at moderate Reynolds numbers (before transition), Taylor-Goertler type vortices, which consist of counter-rotating vortices near the bottom wall, are observed in both experiment (Koseff et al. 1983) and numerical simulation (Kim et al. 1987).

This simple but excellent test case has served over and over again as a model problem to test and evaluate numerical techniques. An accurate scheme should be able to 
capture all these eddies described above. Herein quasi two-dimensional calculations with Reynolds numbers ranging between 100 and 5000 are considered. The widely referenced solution of (Ghia et al. 1982) will be used for comparison.

Consider a square computational domain with the side length $L=0.1 \mathrm{~m}$. The velocity of the sliding upper wall is kept at a constant value, $1 \mathrm{~m} / \mathrm{s}$. Computations are carried out at $\operatorname{Re}=1,100,400,1000,3200$ and 5000, where various Reynolds numbers are obtained by changing the kinematic viscosity. Non-uniform grid is used with an expansion ratio of 1.2 for $\mathrm{Re} \leq 1000$ and 1.1 for $\mathrm{Re}>1000$. The grid size will be reported when the results are addressed. Zero-slip wall boundary conditions are applied at the left, right and bottom wall. The upper boundary is set equal to the sliding wall velocity. The explicit QUICK is utilized for all simulations. One example, however, shall be shown that the semi-implicit power-law scheme, which produces diffusive results due to the absence of the $2^{\text {nd }}$ order CD part in the center region, is inefficient for the cavity calculation at moderate and higher Reynolds numbers $(\operatorname{Re} \geq 400)$. In all cases, simulation is started with a relatively large time step, typically $0.01 \mathrm{~s}$ or $0.001 \mathrm{~s}$, followed by an even smaller one. At steady state, the overall mass residuals are kept below $10^{-10}$.

From a numerical point of view, $\mathrm{Re}=400$ is a critical number above which a higher order scheme shows significant superiority over a lower-order schemes. This point is illustrated in Figure 3-49(a) where three u-velocity profiles from various grids and schemes are shown at the middle of the cavity. From the $21 \times 21$ 's and $31 \times 31$ 's curves calculated with the QUICK, a trend toward a grid independent solution is clearly seen, implying that the $31 \times 31$ 's results is closer to an extrapolated "exact" solution and thus more accurate. This point is verified by comparing the same $31 \times 31$ profile with other two independent calculations shown in Figure 3-49(b), in which excellent agreement is obtained. It should be stressed that the present results, as well as the one from (Kim and Moin 1985) are obtained on a $31 \times 31$ grid, and this is four times less than the one used by (Ghia et al. 1982). On the other hand, as already pointed out in the previous paragraph, the semi-implicit power-law produces rather diffusive solution, as depicted in Figure 3-49(a), and the results with this scheme will get even worse with higher Reynolds numbers. Therefore, in the following only the results calculated from the QUICK are presented.

First, two velocity profiles, namely, the u-velocity at $x=0.5 \mathrm{~L}$ and the v-velocity at $y=0.5 \mathrm{~L}$, are compared at several Reynolds numbers with (Ghia et al. 1982)'s $129 \times$ 129 fine grid solution. Figure 3-50(a) through (e) summarize u-velocity profiles at $\mathrm{Re}=$ 100, 400, 1000, 3200, and 5000, while Figure 3-51(a) through (e) summarize the vvelocity profiles. It can be seen from those figures that the present results calculated from a coarse, non-uniform grid generally agree well with Ghia's benchmark solution. Good agreement is still retained even at higher Reynolds numbers $(\operatorname{Re}=3200$ and 5000) with acceptable minor discrepancies occurring at some locations. In most cases, the peak values of both $\mathrm{u}$ - and v-profiles are calculated accurately. One sees, with increased Reynolds numbers, the magnitude of the maximum u-velocity increases only slightly, while its location gets much closer to the bottom wall, and the near-wall slope gets much sharpened. When the Reynolds number exceeds 3200, the u-velocity does not experience 
a further appreciable increase in its magnitude (see Figure 3-50(d) and (e)). Similar phenomena are observed with the v-velocity profile, as shown in Figure 3-51(d) and (e). The increase of the maximum v-velocity starts to slow down when Reynolds number reaches 3200 .

Next, the flow structures at representative Reynolds numbers, namely, at $\operatorname{Re}=1$, $100,400,1000,3200$, and 5000, are examined. For that the velocity vectors are plotted along with streamlines indicating flow direction and presence of eddies of various sizes. Those figures are grouped in Figure 3-52(a) through (f). In all figures the primary vortex can be clearly observed with its center located differently. At $\operatorname{Re}=1$, the flow is almost symmetric with respect to the center y-line, and two little secondary corner eddies are present even at this low Re (Figure 3-52(a)). As the Reynolds number increases, the center of the primary vortex first moves toward the upper downstream corner before it returns toward the center at higher Reynolds numbers. At $\mathrm{Re}=3200$, a third secondary eddy is formed (Figure 3-52(e)) at the upper left corner. (Kim and Moin 1985) obtained the third secondary eddy at $\mathrm{Re}=2000$, so it may be concluded that the formation of this upper left corner eddy occurs between $\operatorname{Re}=1000$ and 2000. At $\operatorname{Re}=5000$, besides the presence of the upper left corner eddy, a tertiary tiny corner eddy is also visible which rotates in a counter direction to its adjacent secondary lower right corner eddy (Figure 3-52(f)). All the observations obtained in the present study conform well with those reported in (Ghia et al. 1982) and (Kim and Moin 1985). As a last check, the extrapolated center location of the primary eddy is also listed in Table 3-9, in comparison with those given in (Ghia et al. 1982).

Table 3-9 Center locations of the primary vortex of cavity flow at various Reynolds number

\begin{tabular}{|l|c|c|c|c|c|}
\hline $\mathbf{R e}=$ & $\mathbf{1 0 0}$ & $\mathbf{4 0 0}$ & $\mathbf{1 0 0 0}$ & $\mathbf{3 2 0 0}$ & $\mathbf{5 0 0 0}$ \\
\hline \multirow{2}{*}{ Present } & $(0.619,0.742)$ & $(0.560,0.607)$ & $(0.532,0.564)$ & $(0.518,0.540)$ & $(0.502,0.514)$ \\
& $21 \times 21$ & $31 \times 31$ & $41 \times 41$ & $61 \times 61$ & $61 \times 61$ \\
\hline \multirow{2}{*}{ Ghia 82 } & $(0.617,0.734)$ & $(0.555,0.606)$ & $(0.531, x)$ & $(x, 0.547)$ & $(0.512,0.535)$ \\
& $129 \times 129$ & $257 \times 257$ & $257 \times 257$ & $257 \times 257$ & $257 \times 257$ \\
\hline
\end{tabular}




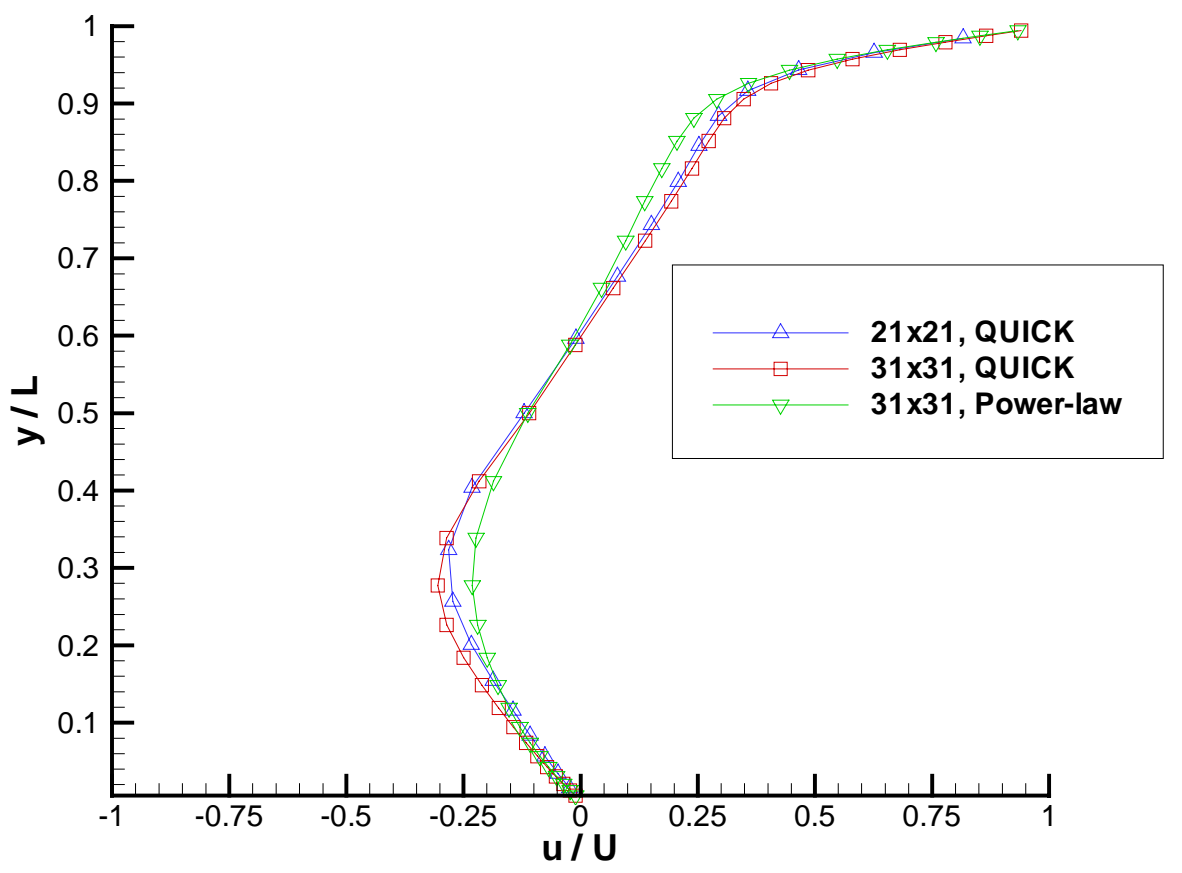

(a)

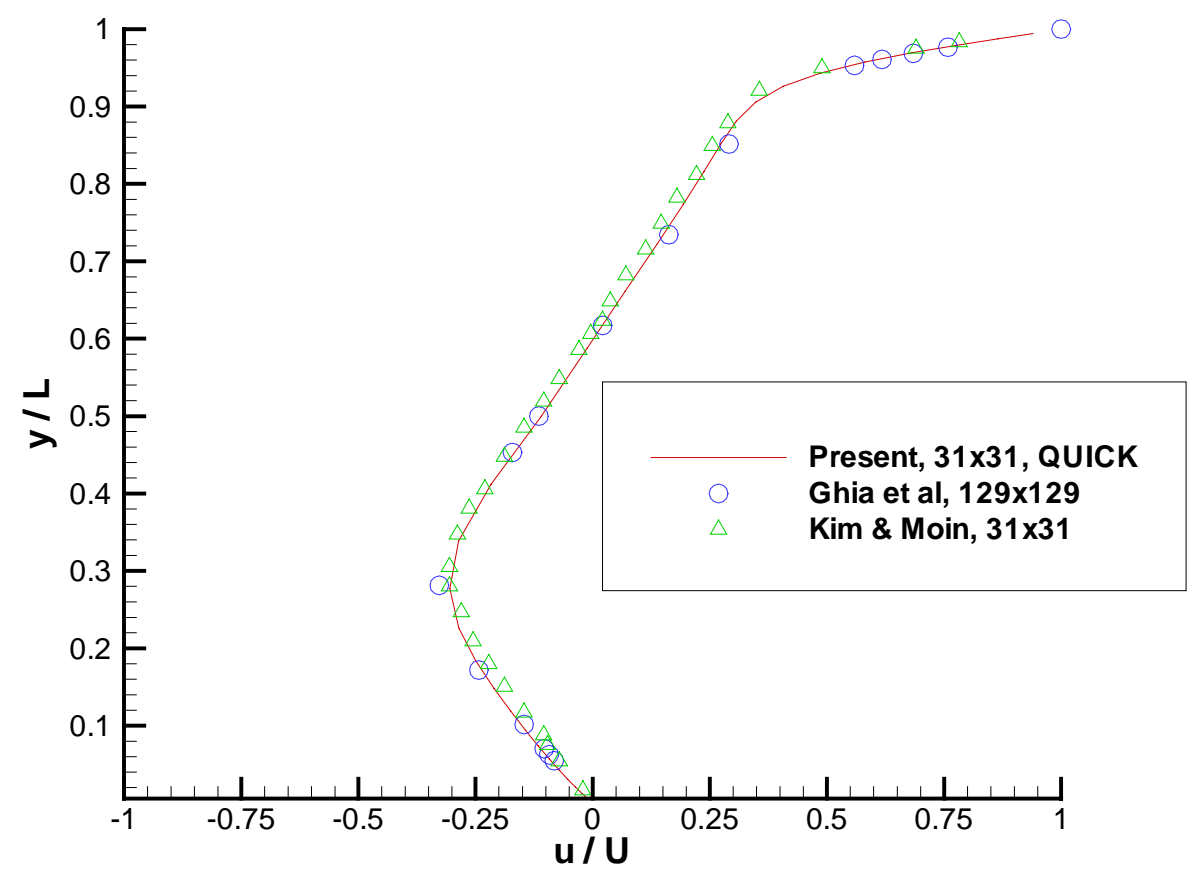

(b)

Figure 3-49 u-velocity profile of a cavity flow at $x=0.5 \mathrm{~L}$ for $\mathrm{Re}=400$. (a) Comparison of results using different scheme and grids, (b) comparison with the computation from (Ghia et al. 1982) and (Kim and Moin 1985). 


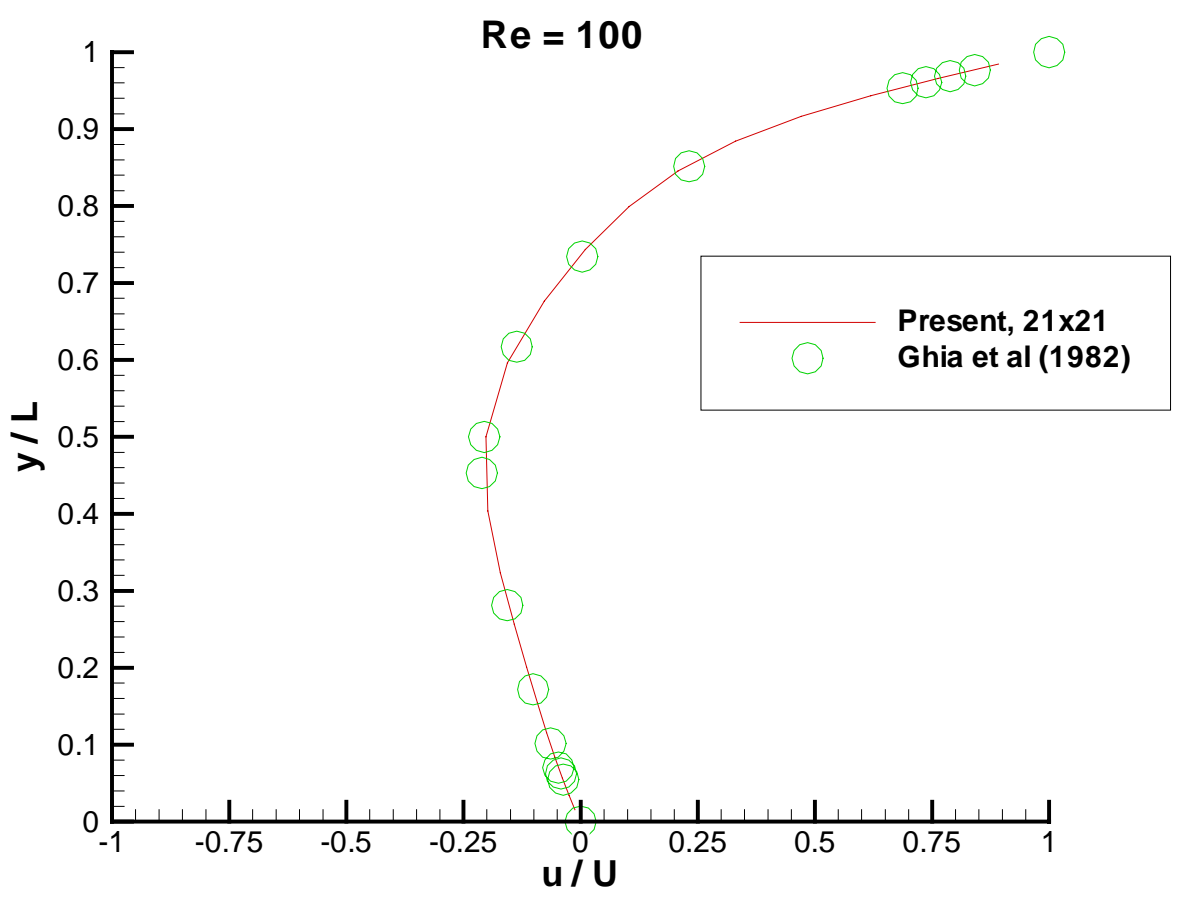

(a)

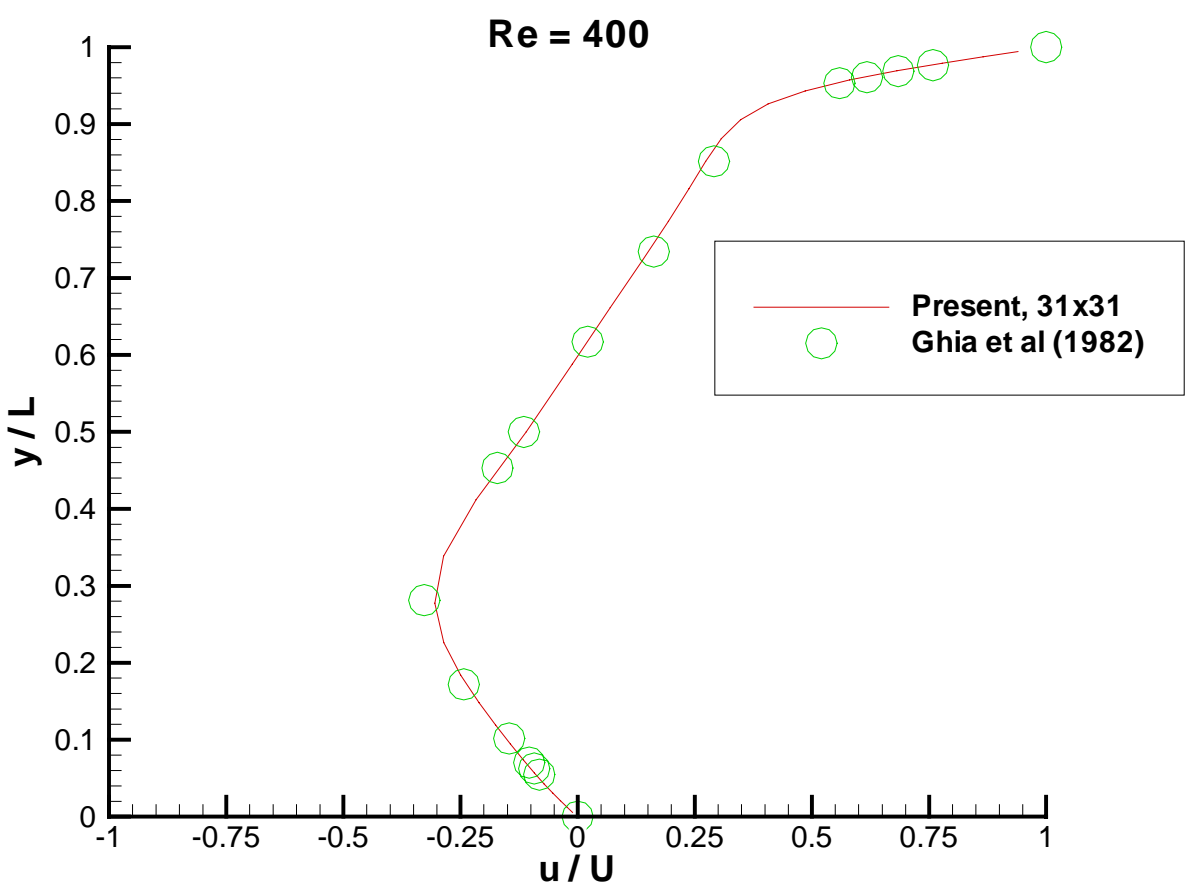

(b)

Figure 3-50 u-velocity profiles of a cavity flow at $x=0.5 \mathrm{~L}$ for various Reynolds numbers. $(\mathrm{a}) \mathrm{Re}=$ 100 , (b) $R e=400$. Solid line: present results; circles: results from (Ghia et al. 1982) with $129 \times 129$ grid nodes. 


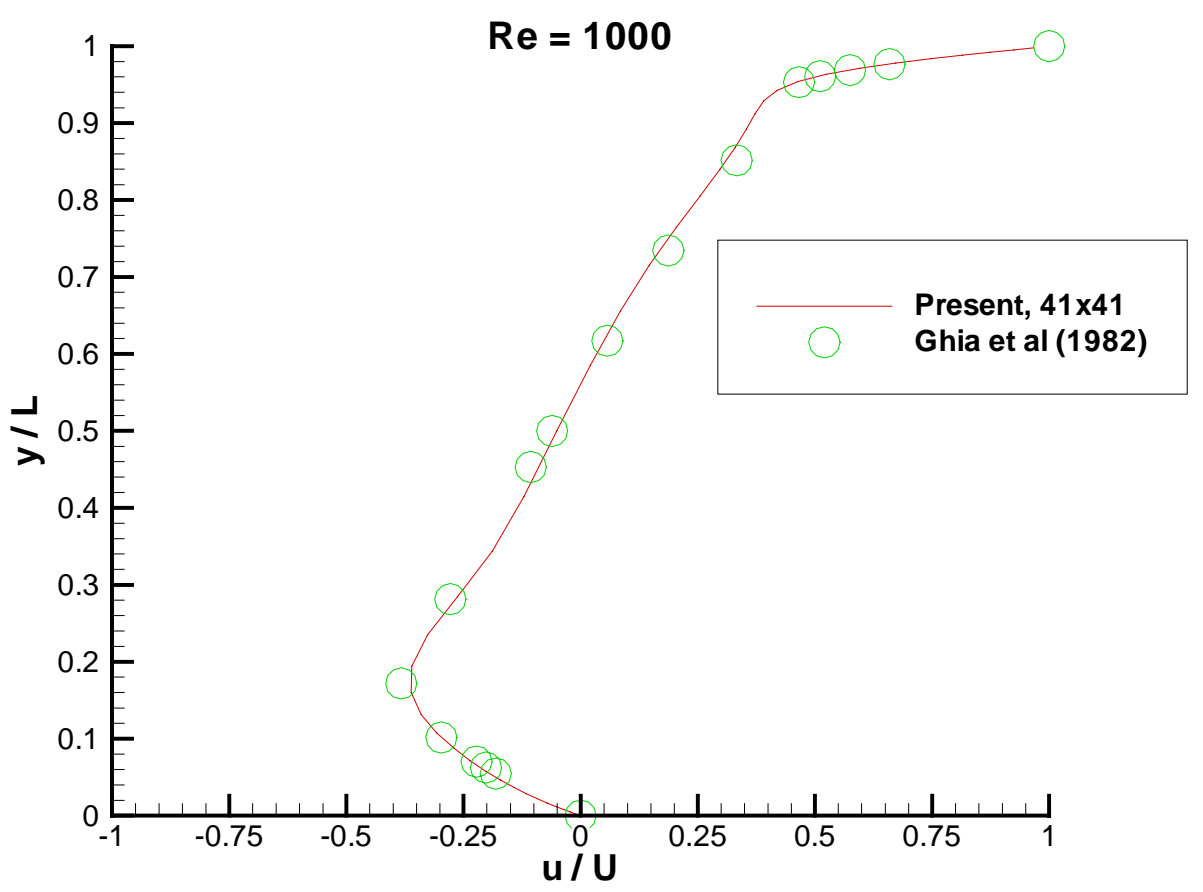

(c)

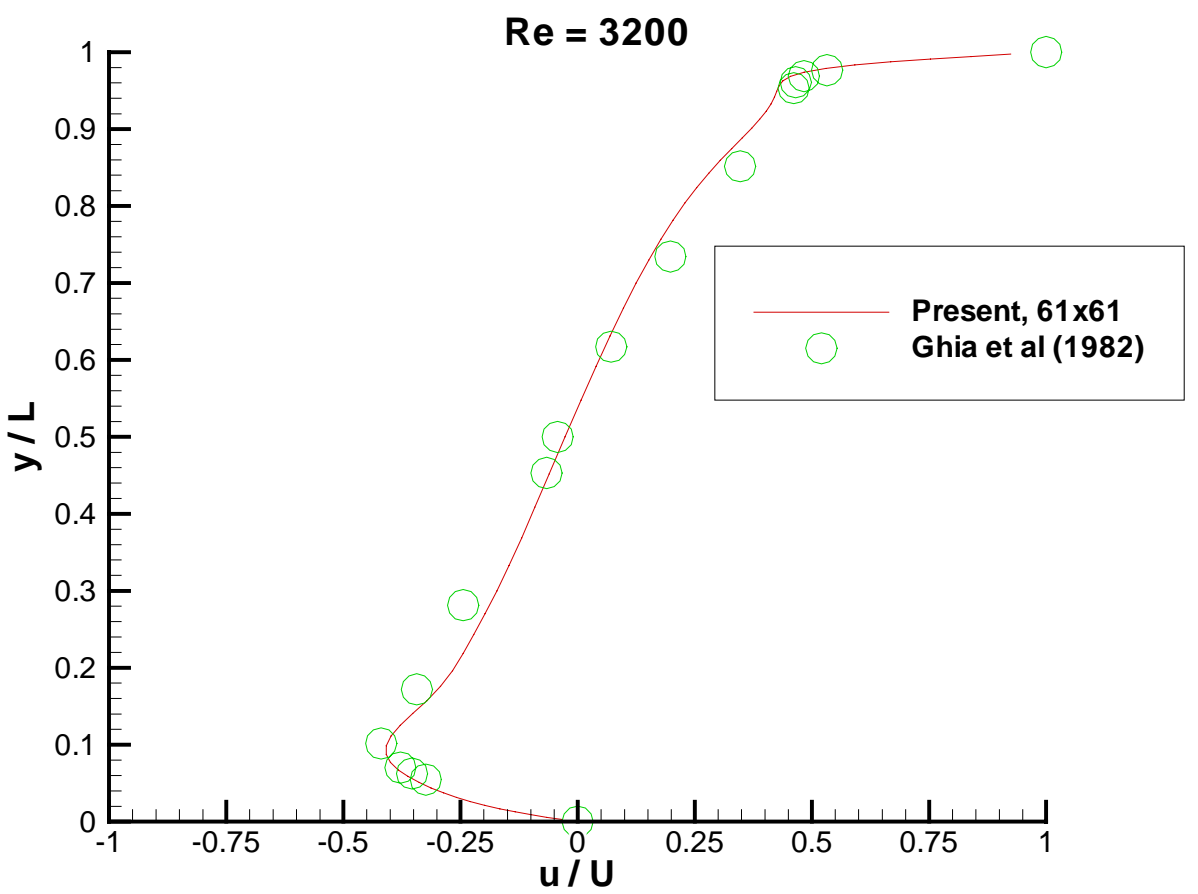

(d)

Figure 3-50 u-velocity profiles of a cavity flow at $x=0.5 \mathrm{~L}$ for various Reynolds numbers. (c) $\operatorname{Re}=$ 1000, (d) $\operatorname{Re}=3200$, (d) $\operatorname{Re}=5000$. Solid line: present results; circles: results from (Ghia et al. 1982) with $129 \times 129$ grid nodes. 


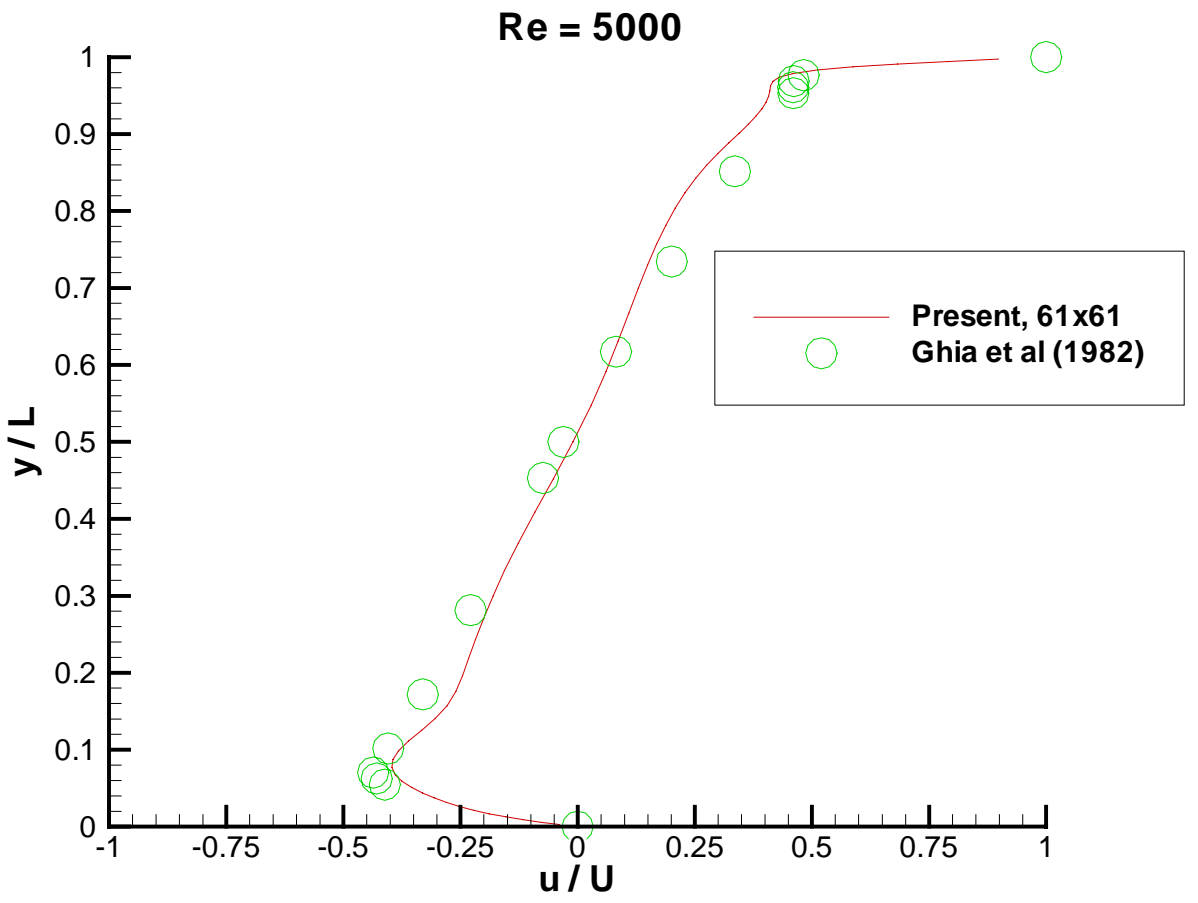

(e)

Figure 3-50 u-velocity profiles of a cavity flow at $x=0.5 \mathrm{~L}$ for various Reynolds numbers. (e) $\operatorname{Re}=$ 5000. Solid line: present results; circles: results from (Ghia et al. 1982) with $129 \times 129$ grid nodes. 


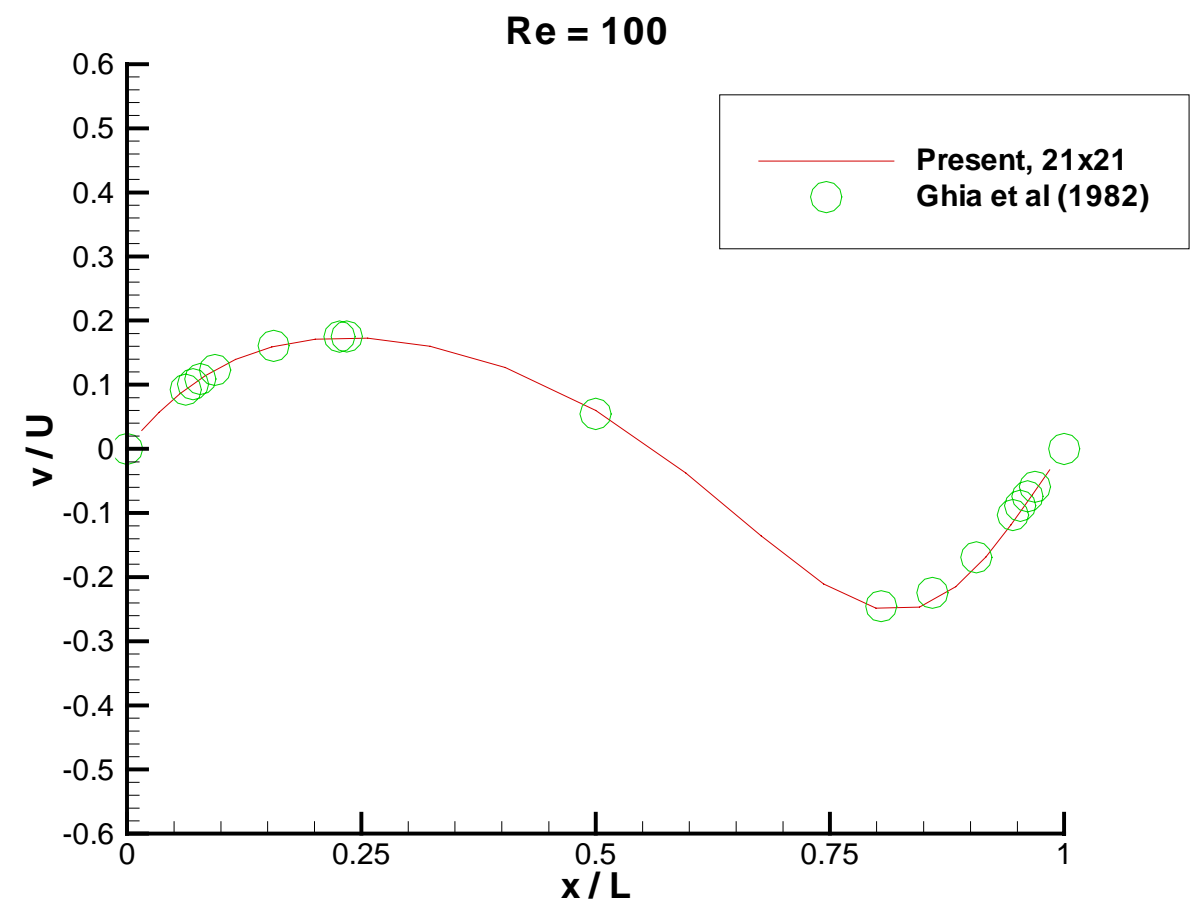

(a)

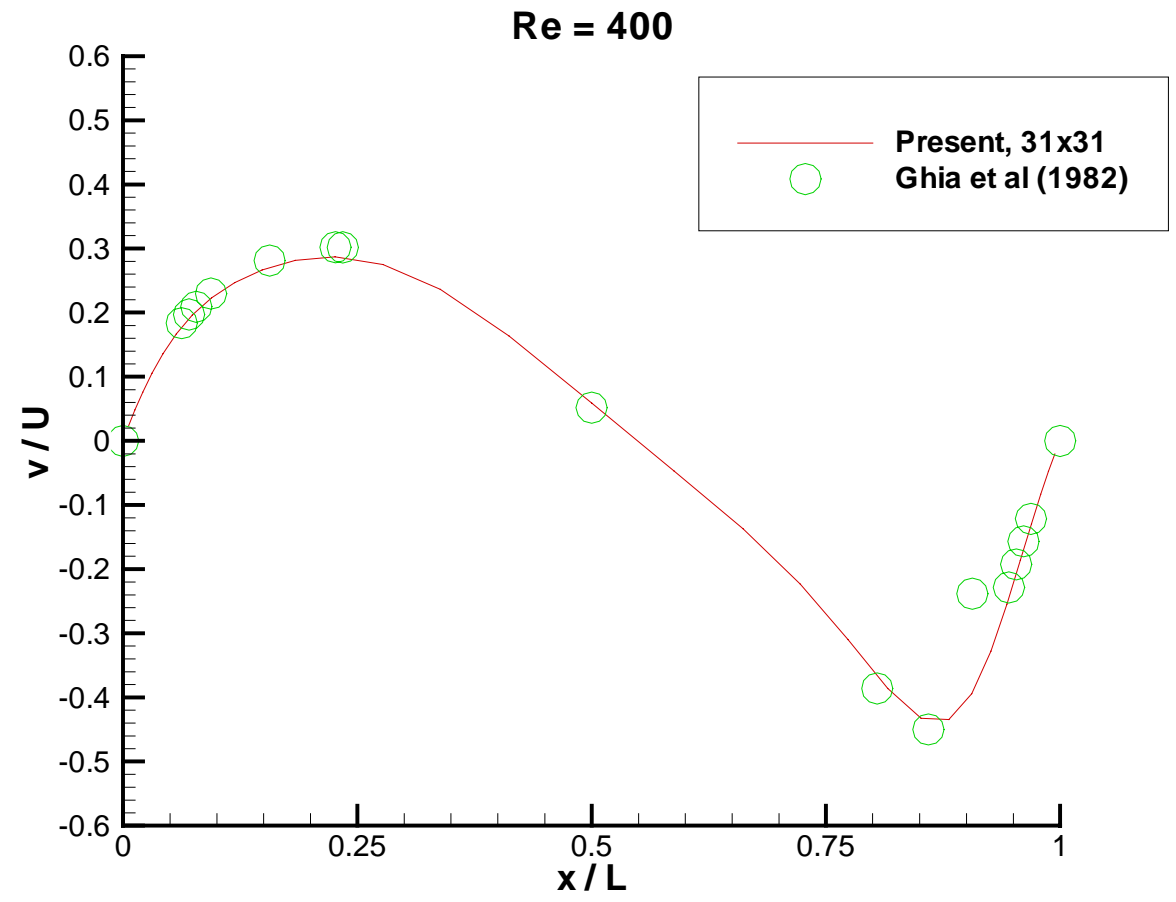

(b)

Figure 3-51 v-velocity profiles of a cavity flow at $y=0.5 \mathrm{~L}$ for various Reynolds numbers. $(\mathrm{a}) \mathrm{Re}=$ 100 , (b) $R e=400$. Solid line: present results; circles: results from (Ghia et al. 1982) with $129 \times 129$ grid nodes. 


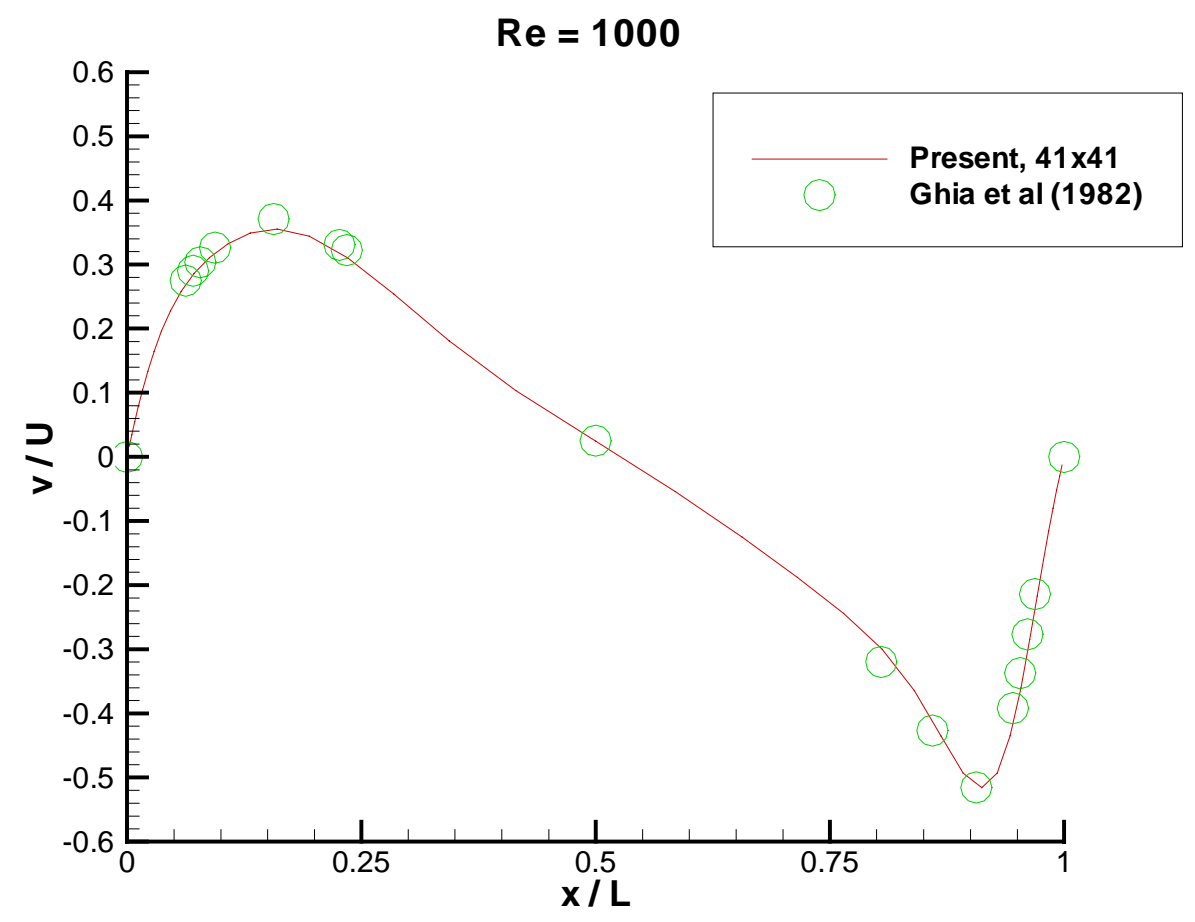

(c)

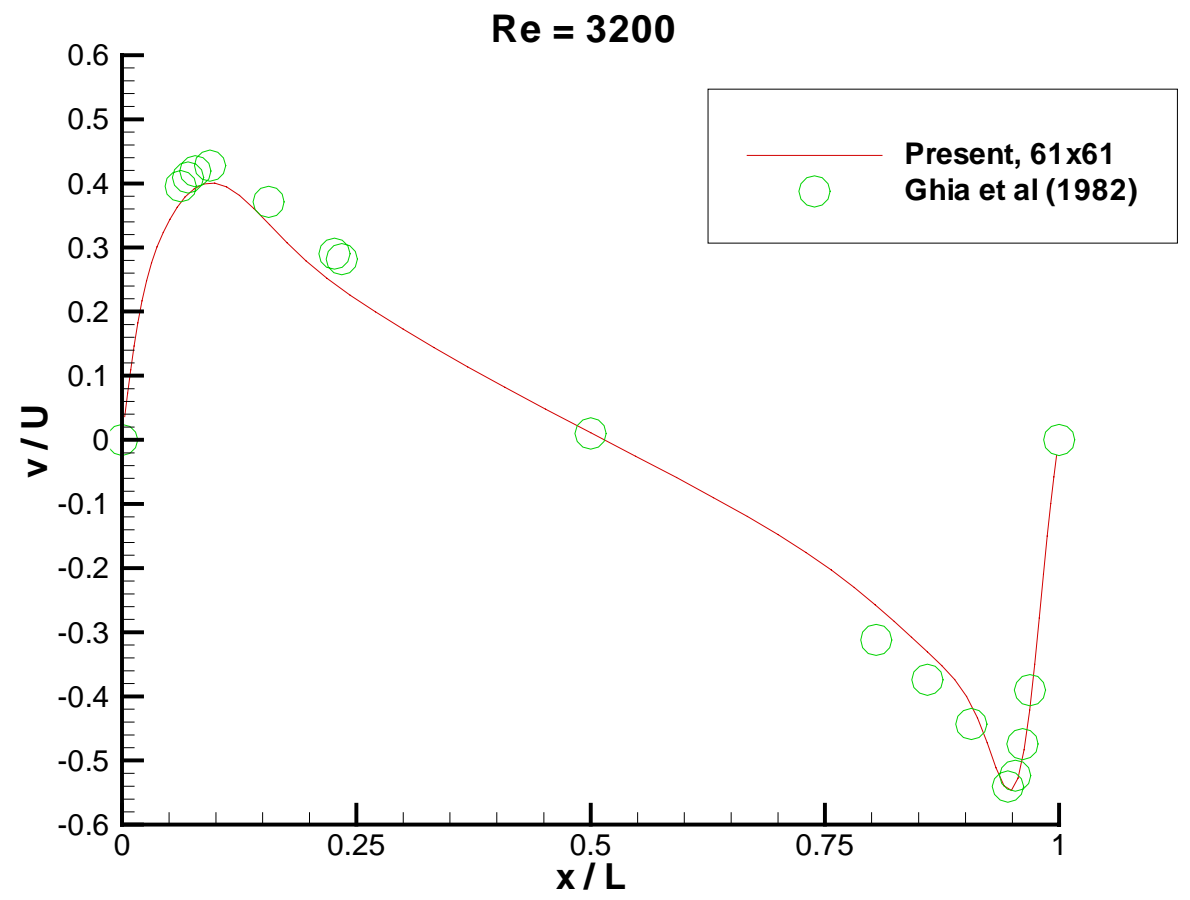

(d)

Figure 3-51 v-velocity profiles of a cavity flow at $y=0.5 \mathrm{~L}$ for various Reynolds numbers. (c) $\operatorname{Re}=$ 1000, $(d) \operatorname{Re}=3200$. Solid line: present results; circles: results from (Ghia et al. 1982) with $129 \times 129$ grid nodes. 


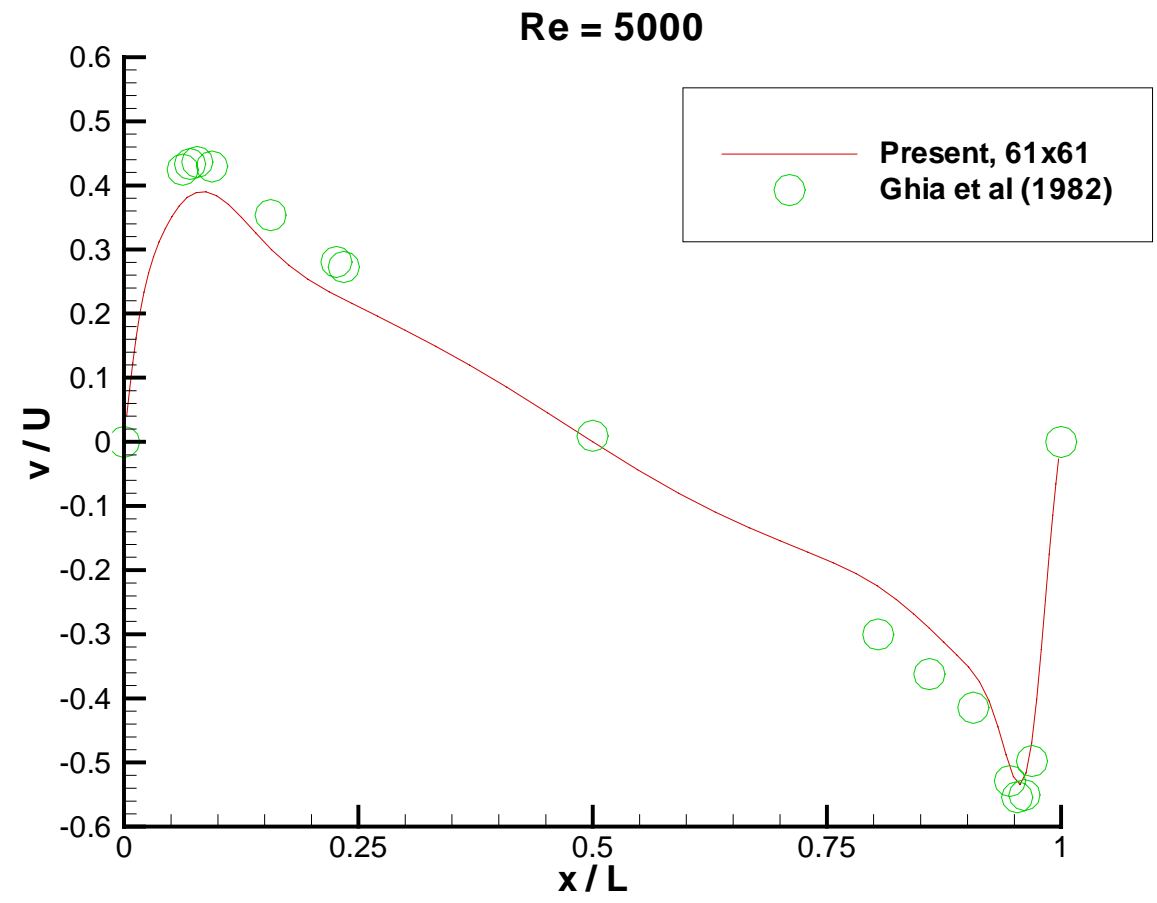

(e)

Figure 3-51 v-velocity profiles of a cavity flow at $y=0.5 \mathrm{~L}$ for various Reynolds numbers. (e) $R e=$ 5000. Solid line: present results; circles: results from (Ghia et al. 1982) with $129 \times 129$ grid nodes. 


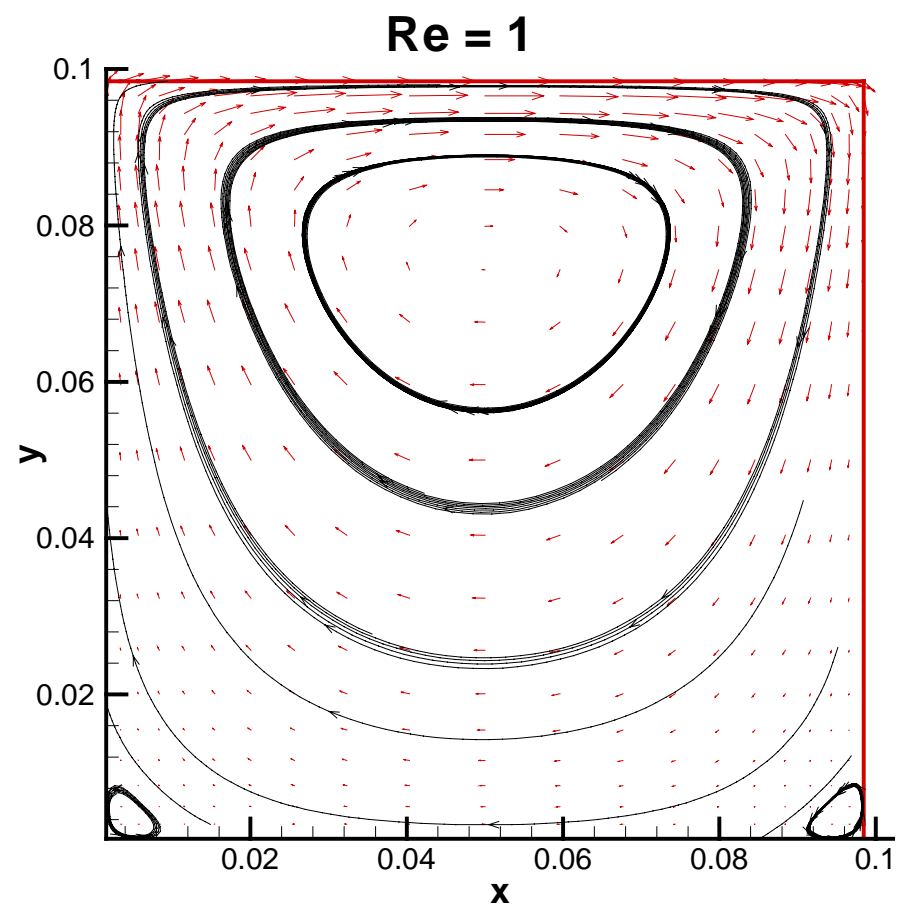

(a)

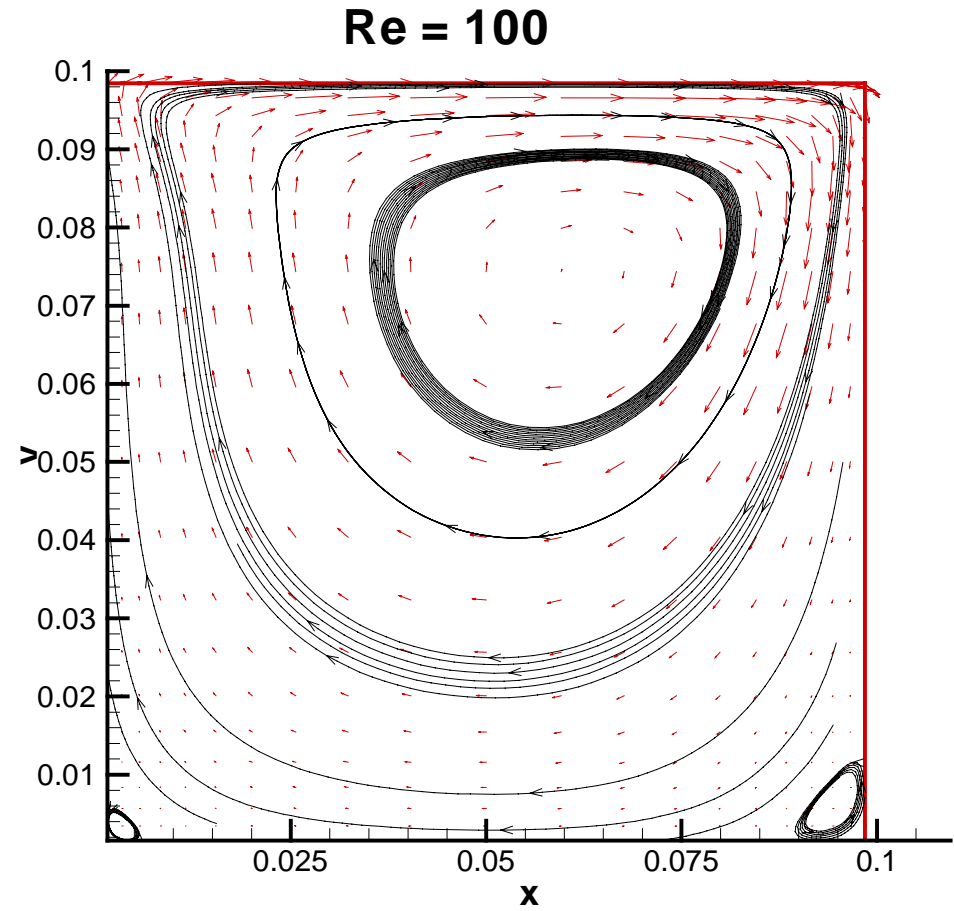

(b)

Figure 3-52 Velocity vectors and streamlines of cavity flow at various Reynolds number. (a) $R e=1$, $21 \times 21$, (b) $\operatorname{Re}=100,21 \times 21$. 


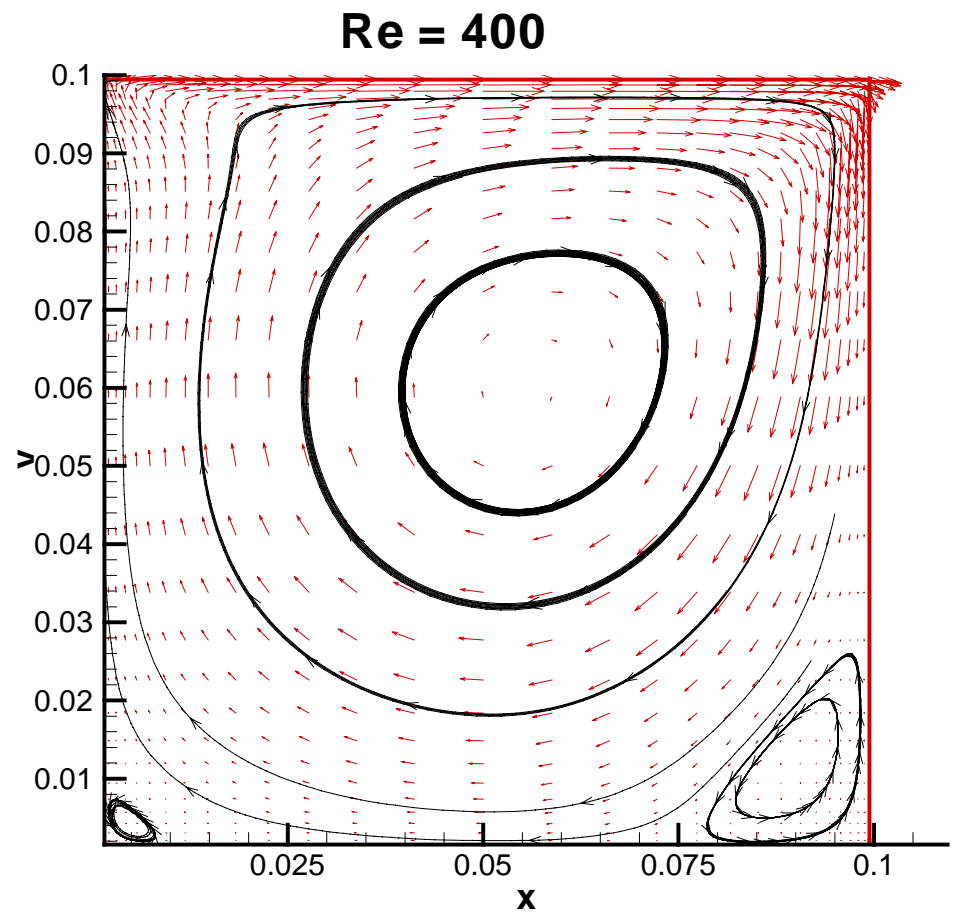

(c)

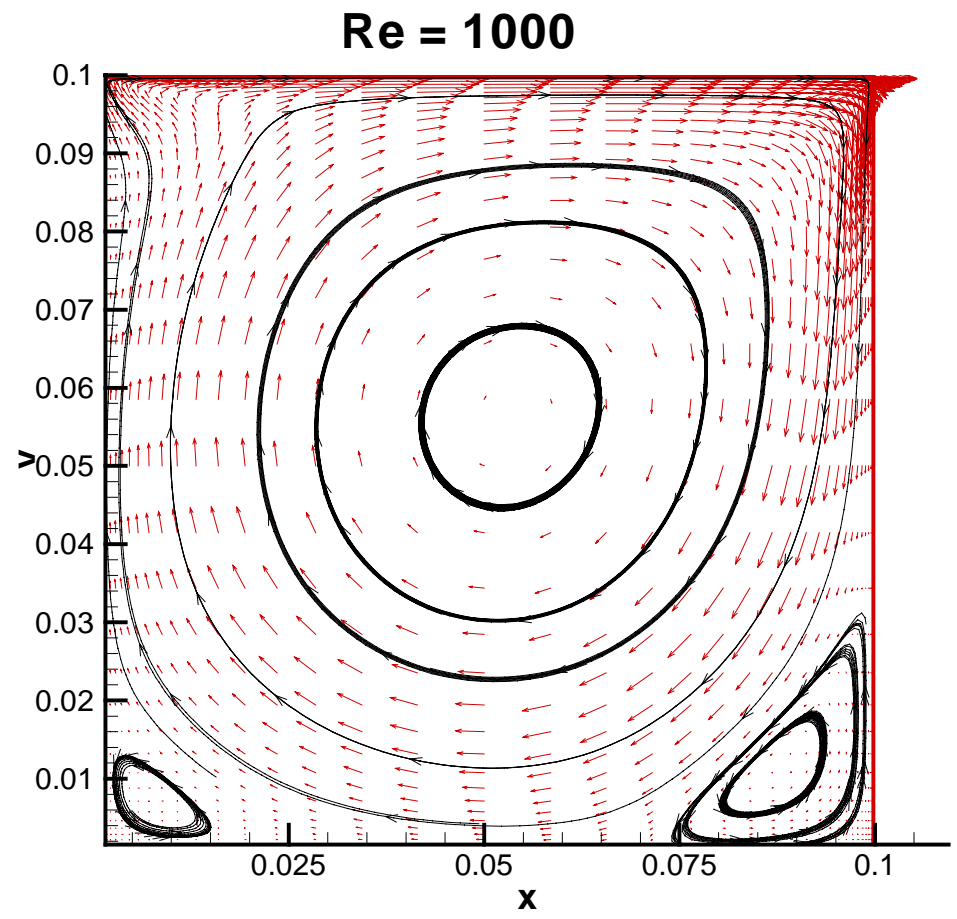

(d)

Figure 3-52 Velocity vectors and streamlines of cavity flow at various Reynolds number. (c) $\operatorname{Re}=400$, $31 \times 31,(d) \operatorname{Re}=1000,41 \times 41$. 


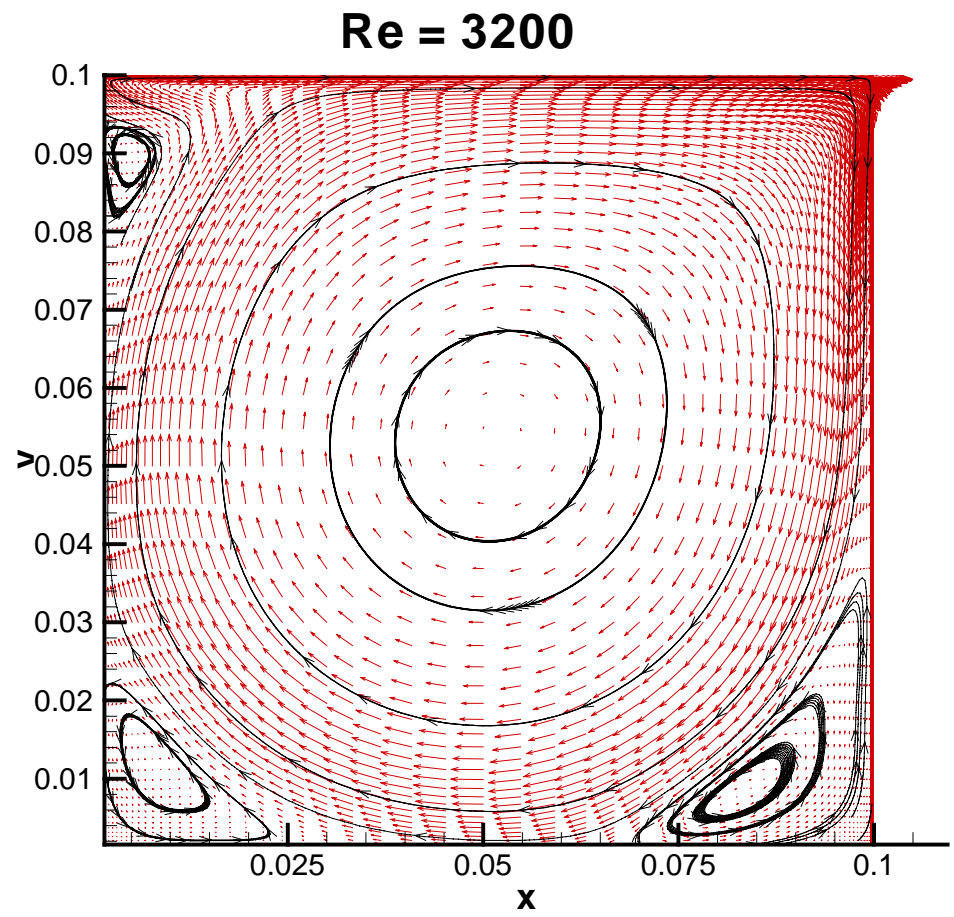

(e)

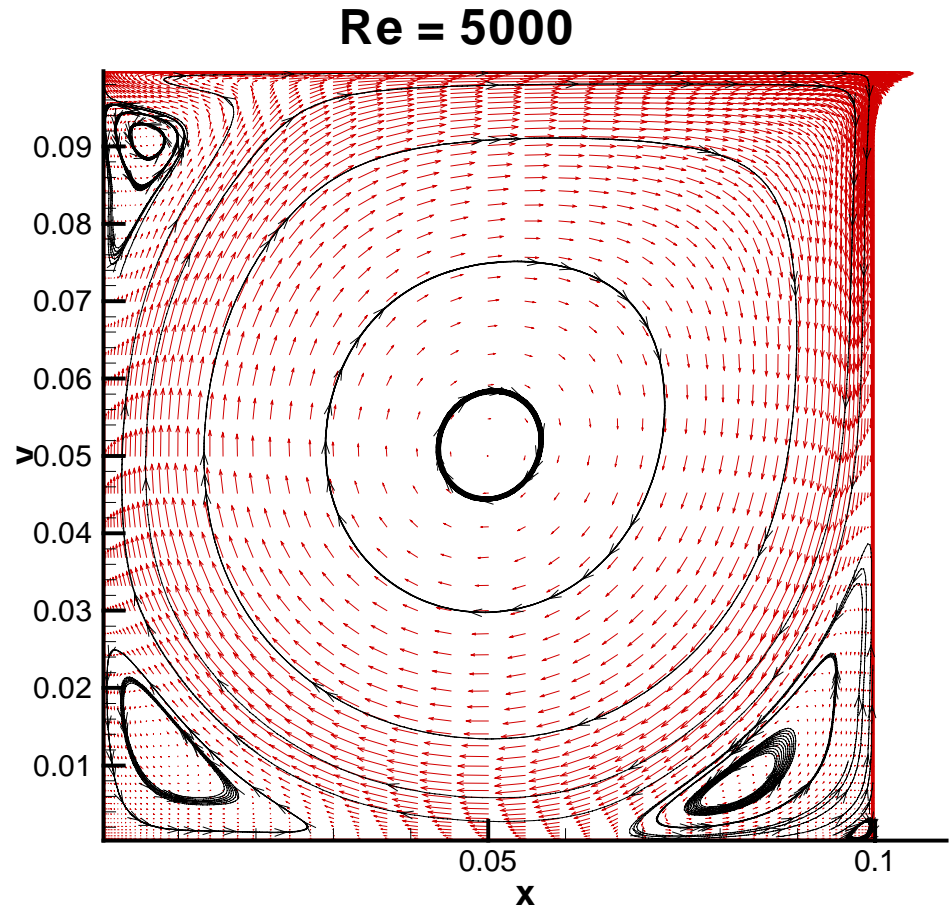

(f)

Figure 3-52 Velocity vectors and streamlines of cavity flow at various Reynolds number. (e) $R e=$ $3200,61 \times 61$, (f) $\operatorname{Re}=5000,61 \times 61$. 


\section{Part II Modeling and Simulation of Turbulent Flows}

\section{Chapter 4 Modeling Strategies for Turbulent Flows}

\subsection{Scales in Turbulent Flows and Direct Numerical Simulation}

In a turbulent flow, where the flow is highly unsteady, three-dimensional, contains a great deal of vorticity and coherent structures, exhibits enhanced mixing accompanied with increased dissipation of kinetic energy, there exists a wide range of length and time scales. As for example pointed out by (Tennekes and Lumley 1972), the smallest scales in a turbulent flow, called the Kolmogorov scales, are determined by the kinetic viscosity, $v$, and the dissipation rate, $\varepsilon$, i.e.,

$$
\begin{aligned}
& \eta \sim\left(v^{3} / \varepsilon\right)^{1 / 4}, \\
& u_{\eta} \sim(\varepsilon v)^{1 / 4} \\
& \tau_{\eta} \sim(v / \varepsilon)^{1 / 2}
\end{aligned}
$$

where $\eta, u_{\eta}, \tau_{\eta}$ denote the Kolmogorov length, velocity (fluctuation) and time scale, respectively. The inviscid estimate relates the dissipation rate $\varepsilon$ to the scales of largest eddies, $l_{0}$ and $u_{0}$ being their characteristic size (an integral length scale) and velocity fluctuations, by

$$
\varepsilon \sim u_{0}^{3} / l_{0} \text {. }
$$

Note that $l_{0}$ is comparable to the flow geometry, characterized by length scale $L$; and $u_{0}$ is of the order of the flow's RMS (root mean square) velocity fluctuation, $u^{\prime}=(2 k / 3)^{1 / 2}(k$ being the turbulent kinetic energy defined in Eq. (4.35)), and thus comparable to the mean flow velocity scale. With the help of Eq. (4.2) one deduces the ratios of the largest and smallest scales:

$$
\begin{aligned}
& \eta / l_{0} \sim \mathrm{Re}^{-3 / 4}, \\
& u_{\eta} / u_{0} \sim \mathrm{Re}^{-1 / 4}, \\
& \tau_{\eta} / \tau_{0} \sim \mathrm{Re}^{-1 / 2}
\end{aligned}
$$


where $\tau_{0}=l_{0} / u_{0}$, representing the lifetime (or turnover time) of large eddies, and $\mathrm{Re}=$ $u_{0} l_{0} / v$. One sees from Eq. (4.3) that relative to largest scales, the Kolmogorov length scale decreases as $\mathrm{Re}^{-3 / 4}$, and the Kolmogorov time scale as $\mathrm{Re}^{-1 / 2}$.

The direct numerical simulation (DNS) attempts to tackle the turbulent flow problem by solving the Navier-Stokes equations directly. Therefore, it must resolve all the turbulent scales down to the Kolmogorov scales. A direct implication of (4.3) is that the number of grid in each direction, $L / \eta$, or $l_{0} / \eta$, is proportional to $\mathrm{Re}^{3 / 4}$; consider the total number of grids in three directions, and also note that the time step is related to the grid size as well; the overall cost of simulation typically scales as $\mathrm{Re}^{3}$. Although the Reynolds number defined here is based on the fluctuation velocity of the largest eddies, there is proportionality between this Re and the macroscopic Reynolds number engineers usually use to describe a flow. Thus, the computational cost also scales with cube of a typical engineering Reynolds number.

A precise picture of the resolution requirement can be gained by examining the Fourier series representation of the turbulent velocity field $\mathbf{u}(\mathbf{x}, t)$. For this purpose consider the homogeneous isotropic turbulence in a cubic domain of side $L$, and suppose that the flow has a periodicity with period $L$ in all three dimensions. As such, the Fourier series expansion of $\mathbf{u}(\mathbf{x}, t)$ may be written out as:

$$
\mathbf{u}(\mathbf{x}, t)=\sum_{n_{3}=-\infty}^{\infty} \sum_{n_{2}=-\infty}^{\infty} \sum_{n_{1}=-\infty}^{\infty} \mathbf{c}_{n_{1}, n_{2}, n_{3}} e^{i \boldsymbol{\kappa}_{n_{1}, n_{2}, n_{3}} \cdot \mathbf{x}},
$$

where the wave number vector $\boldsymbol{\kappa}_{n 1, n 2, n 3}$ and the Fourier coefficient vector $\mathbf{c}_{n 1, n 2, n 3}$ are given by

$$
\begin{gathered}
\boldsymbol{\kappa}_{n_{1}, n_{2}, n_{3}}=\left(n_{1} \kappa_{0}, n_{2} \kappa_{0}, n_{3} \kappa_{0}\right), \kappa_{0}=\frac{2 \pi}{L}, \\
\mathbf{c}_{n_{1}, n_{2}, n_{3}}\left(\boldsymbol{\kappa}_{n_{1}, n_{2}, n_{3}}, t\right)=\frac{1}{L^{3}} \int_{0}^{L} \int_{0}^{L} \int_{0}^{L} \mathbf{u}(\mathbf{x}, t) e^{-i \boldsymbol{\kappa}_{1,1}, n_{2}, n_{3} \cdot \mathbf{x}} d \mathbf{x} .
\end{gathered}
$$

Notice that the formula for obtaining $\mathbf{c}_{n 1, n 2, n 3}$ (4.6) is essentially a "Fourier transform" operation (except for the multiplication factor and the integration limits, see, e.g., (Lighthill 1970; Bracewell 1978; Folland 1992) concerning Fourier transform) that transforms $\mathbf{u}(\mathbf{x}, t)$ from the physical space into a wave number space.

Now let $N$ be the number of grid nodes in each direction of the computational box, thus $N^{3}$ being the total number of nodes. For convenience, let $N$ be an even integer. The objective here is to determine a reasonable $N$ for a DNS. Of practical interest, $\mathbf{c}_{n 1, n 2, n 3}$ is expressed in the form of discrete Fourier transform (DFT, see, e.g., (Press et al. 1992)) based on the $N^{3}$ samples; as such, with $N^{3}$ numbers of input, no more than $N^{3}$ independent $\mathbf{c}_{n}$ 's will be produced in the wave number domain. Thus, Eq. (4.4) can be re-expressed in terms of the discrete inverse Fourier transform that consists of $N^{3}$ wave numbers (Fourier modes or Fourier coefficients):

$$
\mathbf{u}(\mathbf{x}, t)=\sum_{n_{3}=1-\frac{N}{2}}^{N / 2} \sum_{n_{2}=1-\frac{N}{2}}^{N / 2} \sum_{n_{1}=1-\frac{N}{2}}^{N / 2} \mathbf{c}_{n_{1}, n_{2}, n_{3}} e^{i \boldsymbol{k}_{\eta_{1}, n_{2}, n_{3}} \cdot \mathbf{x}} .
$$


It is seen that representing $\mathbf{u}(\mathbf{x}, t)$ in the physical space on an $N^{3}$ grid is equivalent to a spectral representation with $N^{3}$ wave numbers. Therefore, saying a higher resolution in the physical space is the same as saying more Fourier coefficients with higher wave numbers.

Similar to the understanding of Nyquist critical frequency $(=1 / 2 \Delta t, \Delta t$ being the sampling interval), a term used in the sampling theory (in the area of signal processing), the maximum wave number that can be represented, in each direction, is:

$$
\kappa_{\max }=\frac{1}{2} N \kappa_{0}=\frac{\pi N}{L}=\frac{\pi}{h},
$$

where $h$ is the uniform grid spacing. This important relation reveals that the cost of computation, characterized by either $N$ or $h$, is determined by the maximum resolved wave number, $\kappa_{\max }$. (Pope 2000) has demonstrated with model dissipation spectra that the dissipation effect becomes negligibly small beyond $\kappa \eta=1.5 \approx \pi / 2$. This sets up the following resolution criteria for a satisfactory DNS:

$$
k_{\max } \eta \geq \frac{\pi}{2}
$$

or equivalently,

$$
\frac{h}{\eta} \leq 2
$$

As a result, a DNS of, say, a channel flow at $\mathrm{Re}=10^{6}$ requires about $15 \mathrm{E} 12$ grid nodes, and would take around hundred years on a computer running at several gigaflops, flops being the number of floating-point operations within one second. For this reason the DNS is still restricted to low to moderate Reynolds numbers. The current highest Re of DNS channels being simulated is at around $\mathrm{Re}_{\tau}=600$ (Moser et al. 1999; Abe et al. 2001), based on the friction velocity, $u_{\tau}$, and the half channel height, $\delta$, or $\operatorname{Re}_{c}=13,000$ based on the center line velocity. To circumvent this great challenge, different approaches to the Navier-Stokes equations have been proposed, such as RANS (Reynolds-averaged Navier-Stokes) and LES (large-eddy simulation), but all at an additional expense of the so-called "turbulence modeling."

\subsection{Reynolds Averaged Navier-Stokes and Its Closure Models}

In the Reynolds-averaged Navier-Stokes (RANS) approach a time averaging, also called Reynolds averaging due to (Reynolds 1895), is performed to the Navier-Stokes (N-S) equations. A time-averaged quantity, indicated by an overbar, is defined as

$$
\bar{\phi}(\mathbf{x}, t)=\frac{1}{T} \int_{t}^{t+T} \phi(\mathbf{x}, t) d t
$$

where $T$ is a time interval much longer than all the time scales of the turbulent flow but much less than the time scale relative to the mean flow (e.g. period in an oscillating flow 
or wave). The averaging operation defined above permits one to decompose a timevarying quantity, say $\phi$, into its mean part and a fluctuation part, denoted by $\phi$, i.e.,

$$
\phi(\mathbf{x}, t)=\bar{\phi}(\mathbf{x}, t)+\phi^{\prime}(\mathbf{x}, t) .
$$

When the time (Reynolds) averaging is applied to the incompressible N-S equations, (Eq. (1.18) and (1.24)), one obtains the well-known RANS equations expressed on the Cartesian coordinates:

$$
\begin{gathered}
\frac{\partial \bar{u}_{i}}{\partial x_{i}}=0, \frac{\partial u_{i}^{\prime}}{\partial x_{i}}=0 \\
\frac{\partial}{\partial t}\left(\rho \bar{u}_{i}\right)+\frac{\partial}{\partial x_{j}}\left(\rho \bar{u}_{j} \bar{u}_{i}\right)=-\frac{\partial \bar{p}}{\partial x_{i}}+\frac{\partial}{\partial x_{j}}\left(\mu \frac{\partial \bar{u}_{i}}{\partial x_{j}}\right)+\frac{\partial}{\partial x_{j}}\left(-\rho \overline{u_{j}^{\prime} u_{i}^{\prime}}\right)+\rho \bar{b}_{i},
\end{gathered}
$$

or equivalently (by using Eq. (1.23))

$$
\frac{\partial}{\partial t}\left(\rho \bar{u}_{i}\right)+\frac{\partial}{\partial x_{j}}\left(\rho \bar{u}_{j} \bar{u}_{i}\right)=-\frac{\partial \bar{p}}{\partial x_{i}}+\frac{\partial}{\partial x_{j}}\left(2 \mu \bar{S}_{i j}\right)+\frac{\partial}{\partial x_{j}}\left(-\rho \overline{u_{j}^{\prime} u_{i}^{\prime}}\right)+\rho \bar{b}_{i},
$$

with $\bar{S}_{i j}$ being the mean strain rate tensor given by

$$
\bar{S}_{i j}=\frac{1}{2}\left(\frac{\partial \bar{u}_{i}}{\partial x_{j}}+\frac{\partial \bar{u}_{j}}{\partial x_{i}}\right)
$$

An important quantity that signifies the magnitude of $\bar{S}_{i j}$ is the characteristic mean strain rate, $\bar{S}$, which is defined as

$$
\bar{S}=\frac{1}{2}\left(\bar{S}_{i j} \bar{S}_{i j}\right)^{1 / 2}
$$

The extra term in Eq. (4.14) or (4.15), $-\rho \overline{u_{j}^{\prime} u_{i}^{\prime}}$, is known as the Reynolds stresses, which represents the crucial difference between the RANS and the Navier-Stokes equations, and needs to be modeled. In the derivation of Eq. (4.14) one has assumed negligible density fluctuations, i.e.,

$$
\rho=\bar{\rho}+\rho^{\prime} \cong \bar{\rho},
$$

and used the following facts (or rules) that are associated with the averaging operation:

$$
\begin{aligned}
& \overline{\bar{f}}=\bar{f}, \overline{f^{\prime}}=0, \frac{\overline{\partial f}}{\partial x_{i}}=\frac{\partial \bar{f}}{\partial x_{i}}, \overline{\frac{\partial f}{\partial t}}=\frac{\partial \bar{f}}{\partial t}, \\
& \overline{f+g}=\bar{f}+\bar{g}, \overline{\bar{f} g}=\bar{f} \bar{g}, \overline{\bar{f} g^{\prime}}=\bar{f} \overline{g^{\prime}}=0, \overline{f g}=\bar{f} \bar{g}+\overline{f^{\prime} g^{\prime}},
\end{aligned}
$$

where $f$ and $g$ are two arbitrary variables.

The kinetic energy equation of the mean flow and the equation of turbulent kinetic energy can be formed from the RANS equations (4.15) and the N-S equations (1.23). Albeit both energy equations are not relevant to the numerical procedure, they are of fundamental importance in understanding the mechanical energy transfer between the mean motion and the fluctuating part, thus providing critical insight into the modeling strategies. Let $K$ denote the kinetic energy of the mean flow, it is defined as

$$
K=\frac{1}{2} \overline{\mathbf{u}} \cdot \overline{\mathbf{u}}=\frac{1}{2} \bar{u}_{i} \bar{u}_{i} .
$$

The $K$-equation is obtained by multiplying Eq. (4.15) by $\bar{u}_{i}$. For brevity, the body force is neglected here. The resulting equation reads 


$$
\frac{\bar{D} K}{\bar{D} t} \equiv \frac{\partial K}{\partial t}+\bar{u}_{j} \frac{\partial K}{\partial x_{j}}=\frac{\partial}{\partial x_{j}}\left(\mathcal{T}_{i j} \bar{u}_{i}\right)-\mathcal{T}_{i j} \bar{S}_{i j}
$$

with the stress tensor

$$
\mathcal{T}_{i j}=-\frac{\bar{p}}{\rho} \delta_{i j}+2 v \bar{S}_{i j}-\overline{u_{i}^{\prime} u_{j}^{\prime}} .
$$

The first term on the RHS of Eq. (4.21) includes the diffusive transport (or molecular transport, see (Bird et al. 2002)) of the mean-flow kinetic energy, in contrast to the convective transport on the LHS. As is seen from Eq. (4.22) this transport mechanism is attributed to the normal stress, the viscous stress, and the Reynolds stress. The second term on the RHS of (4.21) is called deformation work, which can be expanded into:

$$
-\mathcal{T}_{i j} \bar{S}_{i j}=-\varepsilon_{m}-\mathcal{P}
$$

where

$$
\begin{gathered}
\varepsilon_{m}=2 v \bar{S}_{i j} \bar{S}_{i j}=v \bar{S}^{2}, \\
\mathcal{P}=-\overline{u_{i}^{\prime} u_{j}^{\prime}} \bar{S}_{i j} .
\end{gathered}
$$

Note that the deformation work performed by the normal stresses is zero for an incompressible flow, since

$$
\bar{p} \delta_{i j} \bar{S}_{i j}=\bar{p} \bar{S}_{i i}=\bar{p} \frac{\partial \bar{u}_{i}}{\partial x_{i}}=0 .
$$

The minus sign in Eq. (4.23) is to emphasize its dissipation nature, i.e., they usually act as sink. $\left(-\varepsilon_{m}\right)$ performs deformation work against the mean strain rate. Because it is always negative, this term is called viscous dissipation of the mean flow field. The deformation work done by the Reynolds stresses is represented by $(-\mathcal{P})$ term; and it is negative, i.e., dissipative in most flows (Tennekes and Lumley 1972), saying that $\overline{u_{i}^{\prime} u_{j}^{\prime}}$ tends to have the opposite sign of $\bar{S}_{i j}$. An important observation is that the $(-\mathcal{P})$ term serves as an interface for exchanging kinetic energy between the mean flow and turbulence. As a result, the turbulent kinetic energy (TKE) benefits from this work, since $\mathcal{P}$ becomes a source in the TKE transport equation. It is for this reason that this term is called turbulence energy production. It can be further shown from the scale analysis (Tennekes and Lumley 1972) that the viscous dissipation is negligible; this leaves $(-\mathcal{P})$ as the dominant sink.

The turbulent kinetic energy (TKE) is defined as

$$
k=\frac{1}{2} \mathbf{u}^{\prime} \cdot \mathbf{u}^{\prime}=\frac{1}{2} u_{i}^{\prime} u_{i}^{\prime},
$$

The $k$-equation can be obtained by multiplying the $\mathrm{N}-\mathrm{S}$ equations (1.23) by $u_{i}$, taking the Reynolds averaging and subtracting the $K$-equation (4.21). The result is

$$
\frac{\bar{D} k}{\bar{D} t} \equiv \frac{\partial k}{\partial t}+\bar{u}_{j} \frac{\partial k}{\partial x_{j}}=-\frac{\partial \mathcal{T}_{j}}{\partial x_{j}}+\mathcal{P}-\varepsilon
$$

where

$$
\mathcal{T}_{j}=\frac{1}{\rho} \overline{u_{j}^{\prime} p^{\prime}}+\frac{1}{2} \overline{u_{i}^{\prime} u_{i}^{\prime} u_{j}^{\prime}}-2 v \overline{u_{i}^{\prime} s_{i j}}
$$




$$
\begin{aligned}
\mathcal{P} & =-\overline{u_{i}^{\prime} u_{j}^{\prime}} \overline{S_{i j}}, \\
\varepsilon & =2 v \overline{s_{i j} s_{i j}} .
\end{aligned}
$$

The quantity $s_{i j}$ is the fluctuating rate of strain, defined by

$$
s_{i j}=\frac{1}{2}\left(\frac{\partial u_{i}^{\prime}}{\partial x_{j}}+\frac{\partial u_{j}^{\prime}}{\partial x_{i}}\right) .
$$

Thus, the three terms on the RHS of Eq. (4.28) represent the diffusive transport of TKE, the production of TKE and the viscous dissipation of TKE, respectively. The major task of the transport terms is to redistribute energy; they are not significant in creating and removing energy. Notice that the expression of $\mathcal{P}$, Eq. (4.30), is identical to (4.25); this confirms the assertion made in the proceeding paragraph that the major dissipation energy lost to the mean flow, $\mathcal{P}$, is transformed into a source in the $k$-equation. Also see that the dissipation term, $(-\varepsilon)$, is always negative, signifying a drain of energy. Unlike $\varepsilon_{\mathrm{m}}$ in the $K$-equation, $(-\varepsilon)$ is significant in its parent equation, since it is responsible for energy cascade (in which energy is transferred to smaller and smaller scales) and maintaining the dynamics of turbulence. A direct implication from Eq. (4.28) is that in a statistically steady, homogeneous, pure shear-driven turbulent flow,

$$
\mathcal{P}=\varepsilon,
$$

which (by scale analysis) leads to a further conclusion of local isotropy of small-scale structure (see (Tennekes and Lumley 1972)). This result is useful in building up certain turbulence models in the RANS approach as well as in the LES approach.

The main advantage in transforming N-S to RANS is that a detailed resolution for small turbulent scales is obviated; computationally it simply means a coarser grid and a larger time step can be used. It is the very term, $-\rho \overline{u_{j}^{\prime} u_{i}^{\prime}}$, that attributes to the very different behaviors of the mean flow field governed by Eq. (4.14) and the instantaneous field described by Eq. (1.24). Various models have been developed to close the RANS equations. They can be classified into four major categories: (i) algebraic models, (ii) one-equation models, (iii) two-equation models and (iv) second moment models. Because there is a very close connection between the statistical models in RANS and the subgrid scale models in LES (to be presented Section 4.8), and also to appreciate the difference between the two, in the following a concise summary is presented for the four typical classes of RANS models. The conceptual shortcomings of the RANS approach will be also commented in the next section, along with an introduction to LES.

\subsubsection{Boussinesq Hypothesis}

The first three classes of models (i, ii and iii) can be further grouped into the turbulent viscosity (or eddy viscosity) models, since they are all based on the turbulent viscosity hypothesis (Boussinesq 1877) which is formulated as

$$
-\overline{u_{i}^{\prime} u_{j}^{\prime}}=v_{t}\left(\frac{\partial \bar{u}_{i}}{\partial x_{j}}+\frac{\partial \bar{u}_{j}}{\partial x_{i}}\right)-\frac{2}{3} k \delta_{i j},
$$

where $k$ is the turbulent kinetic energy (TKE) defined as 


$$
k=\frac{1}{2}\left(\overline{u_{1}^{\prime 2}+u_{2}^{\prime 2}+u_{3}^{\prime 2}}\right)=\frac{1}{2}\left(\overline{u_{1}^{\prime 2}}+\overline{u_{2}^{\prime 2}}+\overline{u_{3}^{\prime 2}}\right)=\frac{1}{2}\left(\mathbf{u}^{\prime} \cdot \mathbf{u}^{\prime}\right),
$$

and $v_{t}$ is the so-called turbulent (or eddy) viscosity. Note that $v_{t}$ is not a physical property but rather a proportionality parameter that is dependent on the local turbulent characteristics and behaves quite differently from flow to flow. The inclusion of the second term in Eq. (4.34) assures that the sum of the normal Reynolds stresses matches the definition of $k$ given in (4.35). This $k$-term can be further absorbed into the pressure term in the RANS equations (4.14); as a result, a modified pressure will replace the static pressure in (4.14),

$$
p_{\text {new }}=p+\frac{2}{3} \rho k \text {. }
$$

In short, all the eddy viscosity models are aimed at a proper specification of the eddy viscosity, $v_{t}$, or equivalently, a turbulent velocity scale, $u^{*}$, and a turbulent length scale, $l^{*}$, satisfying

$$
v_{t}=u^{*} l^{*}
$$

\subsubsection{Mixing Length Models}

A representative algebraic model is the mixing length model, which originates from Prandtl's idea (Prandtl 1925) and later generalized by (Smagorinsky 1963) as

$$
v_{t}=l_{m}^{2} \bar{S}=l_{m}^{2}\left(2 \bar{S}_{i j} \bar{S}_{i j}\right)^{1 / 2},
$$

and (Baldwin and Lomax 1978) as

$$
v_{t}=l_{m}^{2} \bar{\Omega}=l_{m}^{2}\left(2 \bar{\Omega}_{i j} \bar{\Omega}_{i j}\right)^{1 / 2},
$$

where $\bar{S}$ and $\bar{S}_{i j}$ are the characteristic mean strain rate and mean strain rate tensor, respectively; $\bar{\Omega}$ and $\bar{\Omega}_{i j}$ are the characteristic mean rotation rate and mean rotation rate tensor, respectively; $l_{m}$ is the mixing length which is specified based on the flow type and local flow property (such as near-wall region). In this model $l^{*}=l_{\mathrm{m}}$.

\subsubsection{Turbulent Kinetic Energy (One-equation) Model}

The one-equation model (usually referred to as the turbulent kinetic energy model), originally proposed by (Kolmogorov 1942; Prandtl 1945), attempts to express the $u^{*}$ in terms of the turbulent kinetic energy, $k$, i.e.,

$$
u^{*}=C_{1} k^{1 / 2}
$$

hence,

$$
v_{t}=u^{*} l^{*}=C_{1} k^{1 / 2} l_{m}
$$

where $C_{1}$ is a constant, which value $(\cong 0.55)$ can be derived from the wall-region behavior (Pope 2000); and $k$ is obtained from the TKE equation (Eq. (4.28)), which is repeated in a short-hand form as follows:

$$
\frac{\bar{D} k}{\bar{D} t}+\nabla \cdot \mathcal{T}=\mathcal{P}-\varepsilon,
$$


Note that the $\bar{D} k / \bar{D} t$ and $\mathcal{P}$ are in closed form, i.e., they can be deduced from the "known" mean flow field, while the $\nabla \cdot \mathcal{T}$ and $\varepsilon$ are unknown, and need to be further modeled. Using Eq. (4.2) yields a modeled expression for $\varepsilon$ :

$$
\varepsilon=C_{2} k^{3 / 2} / l_{m}
$$

where $C_{2}$ is a model constant and $C_{2}=C_{1}{ }^{3}$ ( attained by setting $\mathcal{P}=\varepsilon$ and considering log-law). It is important to note that the model assumption (4.43) is based on the local equilibrium, i.e., the production and dissipation of TKE balance each other (Celik 2005). The quantity $\mathcal{T}$ is typically unburdened with a gradient-diffusion hypothesis, i.e.,

$$
\mathcal{T}=-\frac{v_{t}}{\sigma_{k}} \nabla k
$$

where $\sigma_{k}$ is known as the turbulent Prandtl-Schmidt number and generally taken to be one. Physically, Eq. (4.44) asserts that, due to velocity and pressure fluctuations, there is a flux formed from $k$ gradient that diffuses the $k$ field. Mathematically, the resulting equations ensure a smooth solution under the action of the Laplacian operator $\left(\nabla \cdot \nabla=\nabla^{2}=\Delta\right)$. So, the final modeled evolution equation for $k$ reads:

$$
\frac{\bar{D} k}{\bar{D} t}=\nabla \cdot\left(\frac{v_{t}}{\sigma_{k}} \nabla k\right)+\mathcal{P}-\varepsilon,
$$

with $v_{t}$ and $\varepsilon$ are provided by Eq. (4.41) and (4.43), respectively, and an empirical specification of $l_{m}$ (flow-dependent) is also needed.

\subsection{4. $\quad k-\varepsilon$ (Two-equation) Model}

To overcome the lack of a length scale in the one-equation models, the two-equation models were developed. This class of models are typified by the widely used $k$ - $\varepsilon$ model, which is formalized mainly by (Jones and Launder 1972; Launder and Spalding 1972). In this model two transport equations are solved for $k$ and $\varepsilon$. By dimensional analysis, the eddy viscosity can be accordingly formulated as

$$
v_{t}=C_{\mu} k^{2} / \varepsilon
$$

where $C_{\mu}$ is a model constant. Referring to the discussions in the one-equation model, one can show that $C_{\mu}=\mathrm{C}_{1}{ }^{4}$ and thus takes a standard value of 0.09 . Note that the relation (4.46) also implies that

$$
u^{*} \sim k^{1 / 2}, l^{*} \sim \frac{k^{3 / 2}}{\varepsilon} .
$$

The $k$-equation is same as the one used in the TKE equation model; although the $\varepsilon$ equation can also be rigorously derived from the RANS equations, similar to the $k$ equation, it is not a useful starting point due to its high complexity. Therefore, the $\varepsilon$ equation has been developed on a semi-empirical basis to mimic its energy-dissipating role; it is

$$
\frac{\bar{D} \varepsilon}{\bar{D} t}=\nabla \cdot\left(\frac{v_{t}}{\sigma_{\varepsilon}} \nabla \varepsilon\right)+C_{\varepsilon 1} \frac{\mathcal{P} \varepsilon}{k}-C_{\varepsilon 2} \frac{\varepsilon^{2}}{k}
$$


A well explained physical justification of the $\varepsilon$-equation could be found, for example, in (Pope 2000). It is important to stress that in the derivation of the $\varepsilon$-equation two major assumptions have been made: (i) local isotropy, i.e., $u$ ', $v^{\prime}$ and $w^{\prime}$ are locally equal, (ii) local equilibrium, i.e., the production $\mathcal{P}$ and dissipation $\varepsilon$ are approximately equal locally (see (Celik 2005) for more detail). Equations (4.46), (4.45) and (4.48) form a "standard" $k-\varepsilon$ model. The standard values of those model constants optimized by (Launder and Sharma 1974) are:

$$
C_{\mu}=0.09, \sigma_{k}=1.0, \sigma_{\varepsilon}=1.3, C_{\varepsilon 1}=1.44, C_{\varepsilon 2}=1.92 .
$$

Variants of the standard $k-\varepsilon$ model and other two-equation models similar to the form of $k-\varepsilon$ exist, such as the RNG (renormalization group) $k-\varepsilon$ model due to (Yakhot and Orszag 1986), the Realizable $k$ - $\varepsilon$ model due to (Shih et al. 1995) and the $k$ - $\omega$ model due to (Kolmogorov 1942; Saffman 1970).

\subsubsection{Second Moment Models}

Different from the above three classes of RANS models, the second moment models attacks the Reynolds stresses directly without relying on the eddy viscosity concept (Eq. (4.34)). Two related models in this class are the Reynolds stress model (RSM) developed by (Daly and Harlow 1970; Launder et al. 1975; Lumley 1978; Speziale 1987; Speziale et al. 1991), and the algebraic stress model (ASM). In the Reynolds stress model, the exact transport equations for the Reynolds stresses are derived, which amount to six equations for six unknown Reynolds stresses. They can be written in compact tensor notation as (Pope 2000):

$$
\frac{\bar{D}}{\bar{D} t}\left(\overline{u_{i}^{\prime} u_{j}^{\prime}}\right)+\frac{\partial}{\partial x_{k}} \mathcal{T}_{i j k}=\mathcal{P}_{i j}+\mathcal{R}_{i j}-\varepsilon_{i j}
$$

with

$$
\begin{aligned}
& \mathcal{P}_{i j}=-\overline{u_{i}^{\prime} u_{k}^{\prime}} \frac{\partial \bar{u}_{j}}{\partial x_{k}}-\overline{u_{j}^{\prime} u_{k}^{\prime}} \frac{\partial \bar{u}_{i}}{\partial x_{k}}, \\
& \varepsilon_{i j}=2 v \overline{\frac{\partial u_{i}^{\prime}}{\partial x_{k}} \frac{\partial u_{j}^{\prime}}{\partial x_{k}}}, \mathcal{R}_{i j}=\overline{\frac{p^{\prime}}{\rho}\left(\frac{\partial u_{i}^{\prime}}{\partial x_{j}}+\frac{\partial u_{j}^{\prime}}{\partial x_{i}}\right)},
\end{aligned}
$$

and

$$
\begin{gathered}
\mathcal{T}_{k i j}=\mathcal{T}_{k i j}^{(u)}+\mathcal{T}_{k i j}^{(p)}+\mathcal{T}_{k i j}^{(v)}, \\
\mathcal{T}_{k i j}^{(u)}=\overline{u_{i}^{\prime} u_{j}^{\prime} u_{k}^{\prime}}, \mathcal{T}_{k i j}^{(p)}=\frac{1}{\rho} \overline{u_{i}^{\prime} p^{\prime}} \delta_{j k}+\frac{1}{\rho} \overline{u_{j}^{\prime} p^{\prime}} \delta_{i k}, \mathcal{T}_{k i j}^{(v)}=-v \frac{\partial \overline{u_{i}^{\prime} u_{j}^{\prime}}}{\partial x_{k}} .
\end{gathered}
$$

In the above notations, $p^{\prime}$ is the fluctuating pressure or modified pressure, $\mathcal{P}_{i j}$ is the production tensor, $\varepsilon_{i j}$ is the dissipation tensor, $\mathcal{R}_{i j}$ is the pressure rate of strain tensor responsible for the pressure-strain redistribution, and $\mathcal{T}_{i j k}$ is the Reynolds stress flux representing the turbulent diffusion effect due to the velocity/pressure fluctuations. Further, a seventh transport equation for the dissipation $\varepsilon$, similar to Eq. (4.48), is usually added into the equation set to provide a length or time scale of turbulence. Therefore, 
there will be a total of eleven primary variables in this model, i.e., $\overline{\mathbf{u}}, \bar{p}, \overline{u_{i}^{\prime} u_{j}^{\prime}}$, and $\varepsilon$. Also notice that $\mathcal{P}_{i j}$ is in closed form, while $\varepsilon_{i j}$, $\mathcal{R}_{i j}$ and $\mathcal{T}_{k i j}$ are not expressed in terms of the primary variables, and thus must be modeled. An obvious consequence of this model is the increased complexity and computational cost. Based on the Reynolds stress model, the algebraic stress model is developed, in which the transport terms (LHS of Eq. (4.50)) as well as the derivatives of the Reynolds stresses on the RHS of (4.50) is approximated with algebraic expression, such that the six PDE's for the Reynolds stresses are turned into six coupled algebraic equations. Although the reduced set of equations is much easier to solve, due to the inherent approximation nature, it is less accurate and general than the RSM.

The readers are referred to some standard and recently published texts, e.g., (Launder and Spalding 1972; Rodi 1980; Wilcox 1993; Pope 2000; Durbin and Pettersson Reif 2001; Marden and Bakker 2002; Celik 2005), for a greater detail concerning the RANS turbulence modeling.

\subsection{Large Eddy Simulation}

Much of the pioneering work of large-eddy simulation (LES) is performed by (Smagorinsky 1963; Lilly 1967; Deardorff 1974; Schumann 1975; Moin and Kim 1982). Reviews at different stages of development of LES are provided by (Rogallo and Moin 1984; Galperin and Orszag 1993; Lesieur and Metais 1996; Piomelli 1999; Meneveau and Katz 2000). Detailed expositions on LES are presented in the texts written by (Pope 2000; Sagaut 2002; Celik 2005).

The idea of large-eddy simulation (LES) arises from the observation that in a turbulent flow the turbulent kinetic energy (TKE) and anisotropy are contained predominately in the larger scales of motion, while the smaller scales are only responsible for fine wiggles of velocity fluctuations. Thus, it is possible to characterize the flow mainly with larger scales, while the smaller scales motion is "anticipated" by some means. A loose phenomenological definition between small and large scales is provided by (Frisch 1995), in which eddies of scale larger than some critical length scale $l$ is said to be large, and those below that is said to be small. Henceforth, the two loose terms will be used without an explicit explaining.

The "segregation" of large scales from small scales can be achieved by way of space averaging, formally known as the filtering. The small scales that are removed after the filtering operation are called the subgrid scales, or SGS in short. In the LES approach a filtering operation is applied to the Navier-Stokes (N-S) equations to yield a set of filtered N-S equations with the primary variables being the filtered (smooth) quantities, representing the motion of large scales (or large eddies). Similar to the situation in RANS, where Reynolds stresses come out as extra burden after Reynolds averaging, filtered N-S equations also carry extra stress terms, called SGS stresses, which are related to small scales motion and must be modeled as well (SGS model). Different from RANS, 
LES has to be three-dimensional and transient (i.e., provide instantaneous information concerning turbulent flows) because the ultimate goal of LES is to predict turbulence itself rather than providing some statistical properties.

LES is a technique intermediate between DNS and RANS. In comparison with DNS, where a full resolution of scales is required and nearly all of the computational effort is expended on the smallest, dissipative motions (Pope 2000), LES resolves only large scales and take into account the influence of small scales via SGS models. The computational cost of LES is generally several orders of magnitude less than DNS, hence making it accessible for simulating high Reynolds number flows. Since LES also provides similar turbulence characteristics as DNS does, it is sometimes viewed as a relaxed version of DNS.

As for the RANS approach, although it is computationally cheaper than LES, RANS suffers from a principal shortcoming, the fact that a RANS model must represent a wide spectrum of scales, since a Reynolds-averaged quantity only tells a statistical mean. Further, due to the fact that small scales tend to depend only on viscosity and may be somewhat universal, whereas the large ones are very strongly affected by the boundary conditions, it does not seem possible to model the effect of large scales of turbulence in the same way in flows that are very different (Piomelli 1999). These are the primary reasons why all the RANS models, including the most widely used $k$ - $\varepsilon$ type models, cannot find its universal applicability. In practice, a careful selection of a particular model from a vast model collection in existence, and a further fine-tuning of model constants are usually necessary before performing a RANS simulation.

There is a large body of discussions in the literature which addresses the advantage of LES over RANS with respect to their performance in the turbulence prediction. For example, (Rodi 1997) pointed out using the example of flow past bluff bodies that statistical turbulence models have difficulties with the complex phenomena, such as separation and reattachment, unsteady vortex shedding, bimodal behavior, high turbulence, large-scale turbulent structures as well as curved shear layers. He further stated that the LES approach is conceptually more suitable for such flow situations. (Su et al. 2001) mentioned in their indoor airflow study that most of the developed RANS turbulence models, such as the mixing length theory, one-equation models, two-equation models, and second moment models may perform reasonably well in one case, but poorly in another.

Nevertheless, there are also situations where saying LES is the right approach is less convincing. One example given by (Pope 2004) is the turbulent combustion at high Reynolds number and Damkohler number, in which the essential rate-controlling processes of molecular mixing and chemical reaction occur at the smallest scales. But overall, the LES approach is receiving wide recognition and strong support; the use of LES in engineering applications will keep increasing, along with the exponential increase in computing power and advances in numerical algorithm. It is also anticipated that RANS will still survive LES for some time to come (Celik 2005), since by far the RANS is still the most economical way for industrial applications, and the statistical models are 
trying to catch up with new modeling concept, such as the non-linear eddy viscosity approach and multi-equation, multi-scale second moment closure.

From this section on and throughout the rest of this study, attention will be focused on the LES approach. LES will be the primary tool to simulate single-phase turbulent flows (Part II) as well as two-phase turbulent flows (Part III). In the following sections, the LES concept and its modeling methodology will be briefly presented, of course biased towards the author's own understanding.

\subsection{Filtering}

As mentioned above, in LES a scale is split into a resolved part, which is obtained via a spatial filtering operation, and an unresolved part, called the subgrid scale, i.e.,

$$
\phi(\mathbf{x}, t)=\bar{\phi}(\mathbf{x}, t)+\phi^{\prime}(\mathbf{x}, t),
$$

where the overbar indicates a filtered (resolved) quantity and prime denotes a subgrid (unresolved) scale. Note that the same notations have been used here as in RANS (cf. Eq. (4.12)) for the decomposed parts. But the meaning of decomposition in LES and RANS is conceptually different. It is agreed that in the LES context the overbar always denotes a filtered quantity. Under circumstances where a mean quantity needs to be distinguished from a filtered one, the angle bracket \langle\rangle will be reserved for the mean quantity with declaration (since symbol \langle\rangle will also be used to denote the volume averaging in Part III of this work).

A spatial filtering that operates on a space-time variable (or function) $\phi(\mathbf{x}, \mathrm{t})$ to yield a filtered quantity $\bar{\phi}(\mathbf{x}, t)$ (Leonard 1974) is defined by

$$
\bar{\phi}(\mathbf{x}, t)=\int_{\mathbf{R}^{3}} G(\xi) \phi(\mathbf{x}-\xi, t) d \xi,
$$

where $\mathbf{R}^{3}$ represents a three-dimensional space, $\mathrm{d} \xi$ is a shorthand for $d \xi_{1} d \xi_{2} d \xi_{3}$, and $G$ is called filter function, filter kernel or filter. Mathematically, Eq. (4.54) is known as the convolution integral (see, e.g., (Kreyszig 1993)); hence one may also write:

$$
\begin{aligned}
\bar{\phi}(\mathbf{x}, t) & =\left(G^{*} \phi\right)(\mathbf{x}, t)=\int_{\mathbf{R}^{3}} G(\mathbf{x}-\xi) \phi(\xi, t) d \xi \\
& =\left(\phi^{*} G\right)(\mathbf{x}, t)=\int_{\mathbf{R}^{3}} \phi(\mathbf{x}-\xi, t) G(\xi) d \xi,
\end{aligned}
$$

where $*$ is the standard notation for a convolution operation of two functions. The convolution given by (4.55) can be viewed twofold in terms of $\phi$ : the $G^{*} \phi$ implies moving weighted averages of $\phi$ with respect to the weight function $G(\mathbf{x}-\xi)$ that moves along $\mathbf{x} ; \phi^{*} \mathrm{G}$ can be interpreted as a continuous superposition of translates of $\phi$ by distances $\xi$ multiplied by a corresponding coefficient $G(\xi)$. The word "filtering" used in LES takes from the former understanding. By definition, linearity is automatically satisfied, i.e., for variable $\phi, \varphi$ and constant $c$, one has

$$
\overline{\phi+c \varphi}=\bar{\phi}+c \bar{\varphi} \text {. }
$$


Note that the filter function given in Eq. (4.54) and (4.55) is a function of $\xi$ only, and independent of $\mathbf{x}$. Such a filter is known as the homogeneous or uniform filter. The filtering operation that uses a homogeneous filter commutes with partial derivative with respect to both space and time, i.e.,

$$
\frac{\overline{\partial \phi}}{\partial x_{i}}=\frac{\partial \bar{\phi}}{\partial x_{i}}, \frac{\overline{\partial \phi}}{\partial t}=\frac{\partial \bar{\phi}}{\partial t}
$$

since

$$
\begin{aligned}
\frac{\partial \bar{\phi}}{\partial x_{i}}(\mathbf{x}, t) & =\frac{\partial}{\partial x_{i}} \int_{\mathbf{R}^{3}} G(\boldsymbol{\xi}) \phi(\mathbf{x}-\boldsymbol{\xi}, t) d \xi=\int_{\mathbf{R}^{3}} \frac{\partial}{\partial x_{i}} G(\boldsymbol{\xi}) \phi(\mathbf{x}-\xi, t) d \xi \\
& =\int_{\mathbf{R}^{3}} G(\boldsymbol{\xi}) \frac{\partial \phi}{\partial x_{i}}(\mathbf{x}-\boldsymbol{\xi}, t) d \xi=\frac{\overline{\partial \phi}}{\partial x_{i}}(\mathbf{x}, t),
\end{aligned}
$$

and similar to the time derivative, provided that $\partial(G \phi) / \partial x_{i}$ exists and is continuous (Leibniz's rule, see, e.g., (Parzynski and Zipse 1982)). In LES a filter function should be selected such that the normalization (or conservation) property is satisfied, i.e.,

$$
\int_{\mathbf{R}^{3}} G(\xi) d \xi=1 \text {. }
$$

The purpose of this constrain is to ensure a rapid decay of the filter function at infinity in the physical space. If a filter function is independent of the orientation of the reference frame in physical space, i.e., $G(\xi)$ depends only on $|\xi|$, it is called an isotropic filter.

Further insight can be gained by analyzing the filtering operation in the wave number space via Fourier transform. One of the Fourier transform pair (forward and inverse transform) in three-dimensional space is defined by

$$
\begin{gathered}
\hat{\phi}(\mathbf{\kappa}, t)=\mathcal{F}[\phi(\mathbf{x}, t)]=\int_{\mathbf{R}^{3}} \phi(\mathbf{x}, t) e^{-i \mathbf{\kappa} \cdot \mathbf{x}} d \mathbf{x}, \\
\phi(\mathbf{x}, t)=\mathcal{F}^{-1}[\phi(\mathbf{\kappa}, t)]=\frac{1}{(2 \pi)^{3}} \int_{-\infty}^{\infty} \hat{\phi}(\mathbf{\kappa}, t) e^{i \mathbf{\kappa} \cdot \mathbf{x}} d \mathbf{\kappa},
\end{gathered}
$$

where $^{\wedge}$ denotes the Fourier transfer function, $\boldsymbol{\kappa}$ denotes the wave number vector. Thus, The following holds for the Fourier transform of the convolution (see some standard texts, e.g., (Lighthill 1970; Bracewell 1978; Folland 1992):

$$
\hat{\bar{\phi}}=\mathcal{F}\left[G^{*} \phi\right]=\hat{G} \hat{\phi} \text {. }
$$

By Eq. (4.59)

$$
\hat{G}(\mathbf{0})=\int_{\mathbf{R}^{3}} G(\xi) e^{-i \mathbf{0} \cdot \xi} d \xi=\int_{\mathbf{R}^{3}} G(\xi) d \xi=1 .
$$

For illustration, three commonly used one-dimensional filter functions are listed in Table 4-1 along with their Fourier transfer functions. They are the box (top-hat) filter, the Gaussian filter and the sharp spectral (or Fourier cut-off) filter. Here $G_{1}$, in contrast to its three-dimensional counterpart, $G$, denotes a one-dimensional filter in the $x$-direction. The graphs of these filters in both physical and wave number space are depicted in Figure 4-1(a) and (b). Note that since $G_{1}$ considered here are real and even, so also are their transfer functions. See (Pope 2000) for other filters and their properties. 
The symbol, $\Delta$, which shows up in the definition of a filter function is called filter width. For a constant $\Delta$ all the filters are homogeneous and isotropic (equivalent to symmetric in one-dimension). By inspecting their transfer functions and their plots shown in Figure 4-1(b), one sees that, due to Eq. (4.62), the three filters will lend more weight to low frequency parts of a signal, while they annihilate or make less significant the high frequency components. Therefore, they are all low-pass (in frequency) filters. Interpreted in physical space it simply means that large length scales will be taken and small-scale fluctuations will be more or less ignored, thus high-pass in length. Here, the filter width $\Delta$ serves as a "controller" who distinguishes between large and small scales. Figure 4-2 shows an example in which a random signal is smoothed to a different degree as a result of the filtering operation by a same filter with different $\Delta$.

With the box filter, $\bar{\phi}(x)$ is simply the local average of $\phi(\xi)$ in the interval $\mathrm{x}$ $0.5 \Delta \leq \xi \leq x+0.5 \Delta$. The Gaussian filter is the Gaussian distribution with mean zero and variance equal to $\Delta^{2} / 12$, a value chosen by (Leonard 1974) to match the second moments $\left(\int_{-\infty}^{\infty} \xi^{2} G(\xi) d \xi\right)$ of the Gaussian and the box filter. The sharp spectral filter (or Fourier cut-off) has a cut-off wave number, $\kappa_{c}=\pi / \Delta$, above which all the Fourier modes will be removed, and below which no frequency information will be lost. The spectral filter is usually employed when the N-S equation is solved in the wave number space.

Table 4-1 Commonly used one-dimensional filter functions and their Fourier transfer functions

\begin{tabular}{|l|c|c|}
\hline \multicolumn{1}{|c|}{ Name } & Filter function & Fourier transfer function \\
\hline Box (top-hat) & $G_{1}(x-\xi)= \begin{cases}\frac{1}{\Delta} & x-\frac{1}{2} \Delta \leq \xi \leq x+\frac{1}{2} \Delta \\
0 & \text { elsewhere }\end{cases}$ & $\hat{G}_{1}(\kappa)=\frac{\sin \left(\frac{1}{2} \kappa \Delta\right)}{\frac{1}{2} \kappa \Delta}$ \\
\hline Gaussian & $G_{1}(x-\xi)=\left(\frac{6}{\pi \Delta^{2}}\right)^{1 / 2} \exp \left(-\frac{6(x-\xi)^{2}}{\Delta^{2}}\right)$ & $\hat{G}_{1}(\kappa)=\exp \left(-\frac{\kappa^{2} \Delta^{2}}{24}\right)$ \\
\hline $\begin{array}{l}\text { Sharp spectral } \\
\text { (Fourier cut-off) }\end{array}$ & $G_{1}(x-\xi)=\frac{\sin (\pi(x-\xi) / \Delta)}{\pi(x-\xi)}$ & $\hat{G}_{1}(\kappa)= \begin{cases}1 & |\kappa|<\kappa_{c} \\
0 & \text { elsewhere } \\
\kappa_{c}=\pi / \Delta\end{cases}$ \\
\hline
\end{tabular}




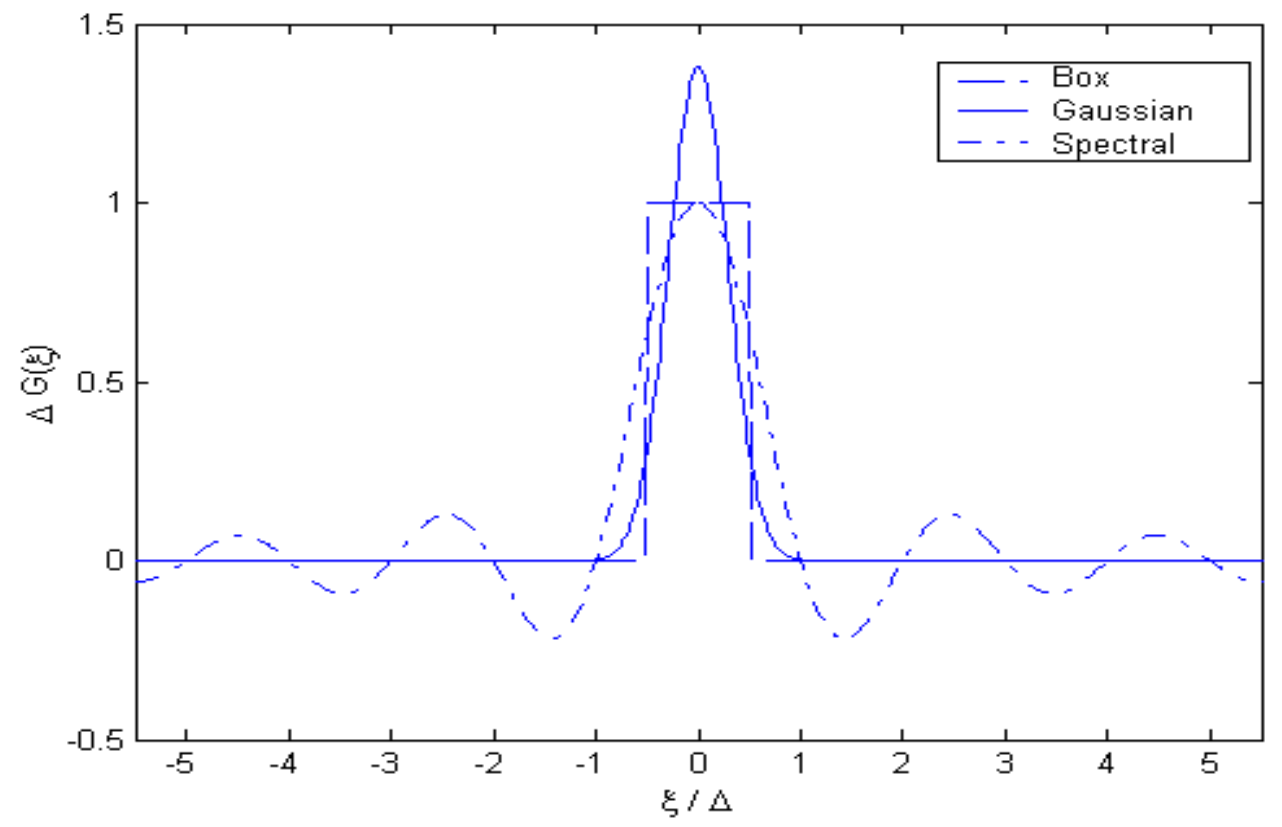

(a)

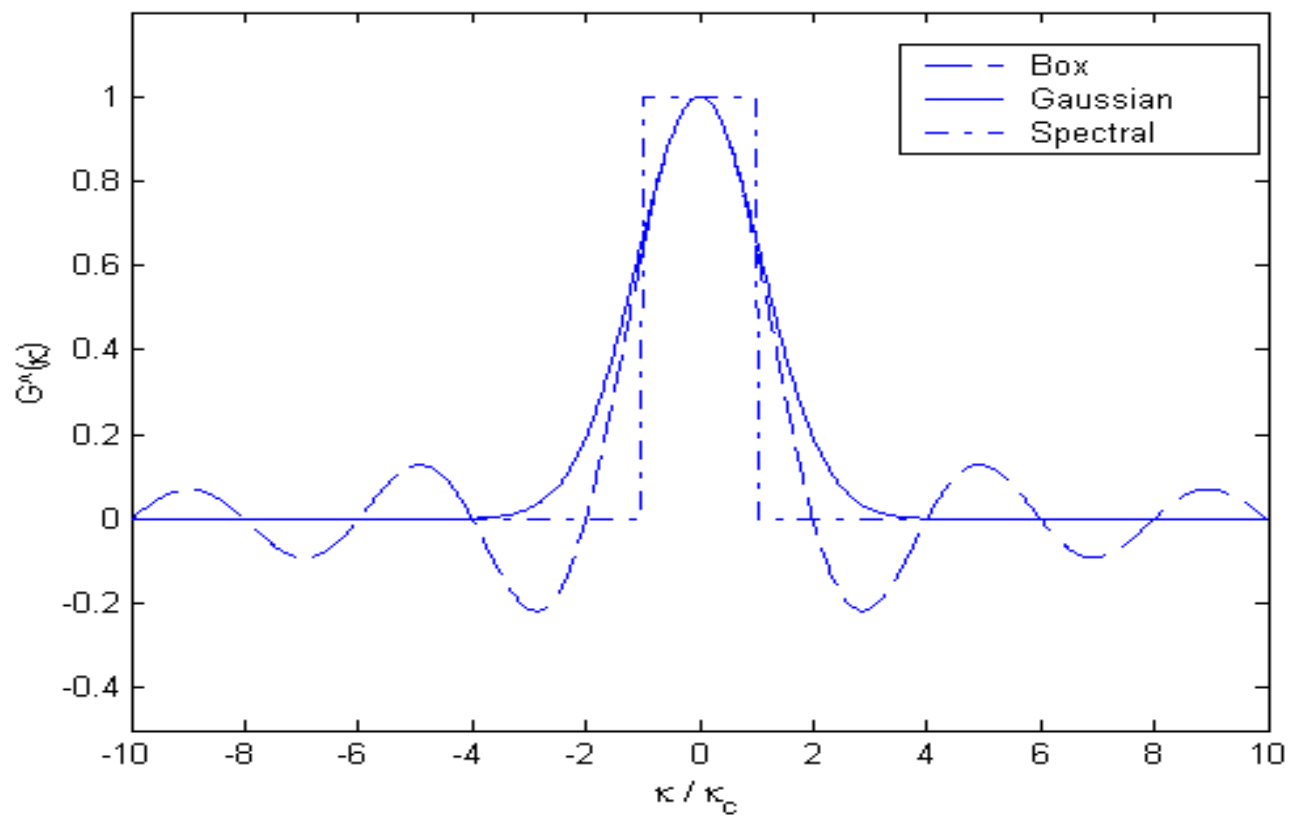

(b)

Figure 4-1 (a) One-dimensional filter functions, $G(\xi)$, (b) Filter transfer functions, $\hat{G}(\kappa)$ 


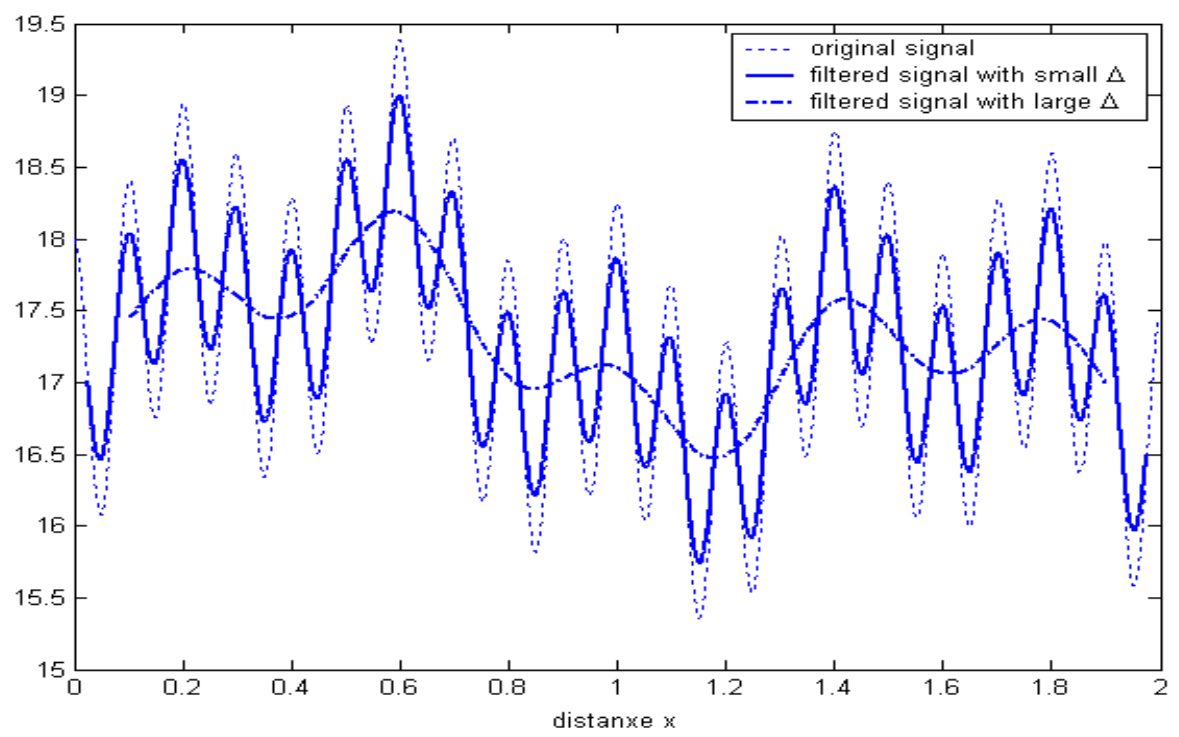

Figure 4-2 A random signal and its filtered signals

Generally, uniform and isotropic three-dimensional filters can be formulated based on their one-dimensional counterparts. For example, the box filter becomes a volume average over the spherical ball of radius $\Delta / 2$. On a rectangular grid, an easy way of constructing a high dimensional filter would be simply forming the product of onedimensional kernels (Pope 2000; Sagaut 2002), i.e.,

$$
G(\boldsymbol{\xi})=\prod_{i=1}^{3} G_{i}\left(\xi_{i}\right),
$$

where $G_{i}$ does not have to be of the same type, and can have different filter width $\Delta_{i}$ in each direction. Note that filters defined from Eq. (4.64) are generally anisotropic, even when $\Delta_{i}$ 's are the same, as $G(\xi)$ is dependent of the orientation of the reference frame. For such an anisotropic filter, a characteristic filter width $\Delta$ can be defined (Deardorff 1970; Scotti et al. 1997) as

$$
\Delta=\left(\Delta_{1} \Delta_{2} \Delta_{3}\right)^{1 / 3},
$$

which is useful in the LES modeling addressed later in this chapter.

There is an important distinction between the filtering in LES and the Reynolds averaging. Recall the properties associated with a Reynolds averaging (see Eq. (4.19)). In particular, the following is true in RANS:

$$
\overline{\bar{\phi}}=\bar{\phi}, \overline{\phi^{\prime}}=0, \overline{\bar{\phi} \varphi}=\bar{\phi} \bar{\varphi} .
$$

Yet, despite the same notation being used, the above relation does not hold for the case of filtering operation in general, i.e.,

$$
\begin{aligned}
& \overline{\bar{\phi}}=G^{*} G^{*} \phi \neq \bar{\phi}=G^{*} \phi, \\
& \overline{\phi^{\prime}}=G^{*}(1-G)^{*} \phi \neq 0, \overline{\bar{\phi} \varphi} \neq \bar{\phi} \bar{\varphi} .
\end{aligned}
$$

It is shown by (Sagaut 2002) that a filter which yields properties given by Eq. (4.66) is either trivial ( $G$ is identity function) or not possible. Moreover, only the spectral filter 
satisfies the first condition in Eq. (4.66), since $\hat{G}^{2}=\hat{G}$, and none of the filters will fulfill the second and third relation in Eq. (4.66) (Germano 1992; Froelich and Rodi 2002). However, it is still possible for some filters that satisfy the conditions (4.66) approximately. For example, the box filter satisfies Eq. (4.66) to the order of $O\left(\Delta^{2}\right)$ (Celik 2005).

So far, only the homogeneous filter $G(\xi)$ has been considered that is independent of $\mathbf{x}$. A filtering operation with an inhomogeneous filter, which takes both $\xi$ and $\mathbf{x}$ as its variable, can be written as

$$
\bar{\phi}(\mathbf{x}, t)=\int_{\mathbf{R}^{3}} G(\boldsymbol{\xi}, \mathbf{x}) \phi(\mathbf{x}-\boldsymbol{\xi}, t) d \xi .
$$

In this, the filter function $G$ will take different shape depending on the location $\mathbf{x}$. A sample application of an inhomogeneous filter is in the wall-bounded flows, where nonuniform grid is applied in the wall-normal direction and the filter width in that direction is taken to be proportional to the grid spacing. With an inhomogeneous filter the linearity (4.56) of the filtering and the normalization condition (4.59) are also satisfied. Unfortunately, the property of commutation with spatial differentiation gets lost (Ghosal and Moin 1995; Vasilyev et al. 1998; Pope 2000), since

$$
\begin{aligned}
\frac{\partial \bar{\phi}}{\partial x_{i}}(\mathbf{x}, t)= & \frac{\partial}{\partial x_{i}} \int_{\mathbf{R}^{3}} G(\xi, \mathbf{x}) \phi(\mathbf{x}-\xi, t) d \xi=\int_{\mathbf{R}^{3}} \frac{\partial}{\partial x_{i}} G(\xi, \mathbf{x}) \phi(\mathbf{x}-\xi, t) d \xi \\
= & \int_{\mathbf{R}^{3}} G(\xi, \mathbf{x}) \frac{\partial \phi}{\partial x_{i}}(\mathbf{x}-\xi, t) d \xi+\int_{\mathbf{R}^{3}} \phi(\mathbf{x}-\xi, t) \frac{\partial G}{\partial x_{i}}(\xi, \mathbf{x}) d \xi \\
& =\frac{\overline{\partial \phi}}{\partial x_{i}}(\mathbf{x}, t)+\int_{\mathbf{R}^{3}} \phi(\mathbf{x}-\xi, t) \frac{\partial G}{\partial x_{i}}(\xi, \mathbf{x}) d \xi \neq \frac{\overline{\partial \phi}}{\partial x_{i}}(\mathbf{x}, t) .
\end{aligned}
$$

A remedy is proposed by (Ghosal and Moin 1995) who introduced a class of filters which do not commute exactly, but commute at the second order with spatial differentiation. Such filters are given a name by (Sagaut 2002) as second order commuting filter. One example of such filter is the inhomogeneous box filter with variable filter width. With an anisotropy, inhomogeneous and three-dimensional filter, the characteristic filter width $\Delta$ can be similarly defined as Eq. (4.65), which varies with location.

Occasionally, e.g., in LES of channel flow with non-uniform grid in the wallnormal direction, this difficulty may be avoided by not filtering the inhomogeneous direction; the filtering is performed only in the plane parallel to the wall (Pope 2000). In fact, this practice is also supported by (Murray et al. 1996) who have shown that, for $y^{+}>$ 10 in a channel flow, filtering in the homogeneous plane is equivalent to threedimensional filtering.

It should also be mentioned that in the case of compressible flow, Favre-filtering is commonly used, which includes the density in the filtering operation. A Favre-filtered quantity can be written as

$$
\bar{\phi}(\mathbf{x}, t)=\int_{\mathbf{R}^{3}} G(\mathbf{x}-\xi) \rho(\mathbf{x}, t) \phi(\mathbf{x}, t) d \xi .
$$

Since in this study only incompressible flow is considered, no further details will be addressed for Favre-filtering. Interested reader is referred to (Favre 1983). 


\subsection{Filtered Navier-Stokes Equations}

When the filtering operation is applied to the N-S equations (1.18) and (1.24) (expressed on Cartesian coordinates) with a homogeneous filter, using the filter's commutation property, as addressed in the preceding section, one obtains the following filtered NavierStokes equations,

$$
\begin{gathered}
\frac{\partial \bar{u}_{i}}{\partial x_{i}}=0, \frac{\partial u_{i}^{\prime}}{\partial x_{i}}=0 \\
\frac{\partial}{\partial t}\left(\rho \bar{u}_{i}\right)+\frac{\partial}{\partial x_{j}}\left(\rho \overline{u_{j} u_{i}}\right)=-\frac{\partial \bar{p}}{\partial x_{i}}+\frac{\partial}{\partial x_{j}}\left(\mu \frac{\partial \bar{u}_{i}}{\partial x_{j}}\right)+\rho \bar{b}_{i}
\end{gathered}
$$

where $\bar{u}_{i}$ is the filtered velocity field, $u^{\prime}{ }_{i}$ is the subgrid scale (SGS) velocity. Similar to the argument used in RANS (see Eq. (4.18) and its assumptions), a negligible subgrid density fluctuation is also assumed here, i.e.,

$$
\rho=\bar{\rho}+\rho^{\prime} \cong \bar{\rho} .
$$

By defining the residual stress (also called subgrid scale stress) tensor

$$
\tau_{i j}^{R}=\rho\left(\overline{u_{i} u_{j}}-\bar{u}_{i} \bar{u}_{j}\right)
$$

one may rewrite Eq. (4.72) as

$$
\frac{\partial}{\partial t}\left(\rho \bar{u}_{i}\right)+\frac{\partial}{\partial x_{j}}\left(\rho \bar{u}_{j} \bar{u}_{i}\right)=-\frac{\partial \bar{p}}{\partial x_{i}}+\frac{\partial}{\partial x_{j}}\left(\mu \frac{\partial \bar{u}_{i}}{\partial x_{j}}\right)-\frac{\partial \tau_{i j}^{R}}{\partial x_{j}}+\rho \bar{b}_{i} .
$$

Thus, the effect of the small scales will appear through the residual stresses, $\tau_{i j}^{R}$, which must be modeled, and the model of which is called the $S G S$ model. A review of the SGS models will be given in Section 4.8. Introducing the residual kinetic energy

$$
k_{\Delta}=\frac{1}{2} \tau_{i i}^{R}
$$

allows for a decomposition of the residual stress into an isotropic part and a deviatoric (or anisotropic) part, i.e.,

$$
\tau_{i j}^{R}=\tau_{i j}^{r}+\frac{2}{3} k_{\Delta} \delta_{i j}
$$

The isotropic residual stress (second term in (4.77)) can be absorbed into the pressure term, so that Eq. (4.75) can be restated as

$$
\frac{\partial}{\partial t}\left(\rho \bar{u}_{i}\right)+\frac{\partial}{\partial x_{j}}\left(\rho \bar{u}_{j} \bar{u}_{i}\right)=-\frac{\partial \bar{p}}{\partial x_{i}}+\frac{\partial}{\partial x_{j}}\left(\mu \frac{\partial \bar{u}_{i}}{\partial x_{j}}\right)-\frac{\partial \tau_{i j}^{r}}{\partial x_{j}}+\rho \bar{b}_{i},
$$

with the understanding that the filtered pressure is a modified one, albeit the same symbol is used, and the deviatoric residual stress $\tau_{i j}^{r}$ is defined by Eq. (4.77). By considering the relation (1.16), Eq. (4.78) may also be written alternatively as

$$
\frac{\partial}{\partial t}\left(\rho \bar{u}_{i}\right)+\frac{\partial}{\partial x_{j}}\left(\rho \bar{u}_{j} \bar{u}_{i}\right)=-\frac{\partial \bar{p}}{\partial x_{i}}+\frac{\partial}{\partial x_{j}}\left(2 \mu \bar{S}_{i j}\right)-\frac{\partial \tau_{i j}^{r}}{\partial x_{j}}+\rho \bar{b}_{i},
$$

where $\bar{S}_{i j}$ is the filtered strain rate tensor given by 


$$
\bar{S}_{i j}=\frac{1}{2}\left(\frac{\partial \bar{u}_{i}}{\partial x_{j}}+\frac{\partial \bar{u}_{j}}{\partial x_{i}}\right),
$$

with its magnitude being quantified by the so-called characteristic filtered strain rate:

$$
\bar{S}=\left(2 \bar{S}_{i j} \bar{S}_{i j}\right)^{1 / 2} \text {. }
$$

Note that and both $\bar{S}_{i j}$ and $\bar{S}$ are two prominent quantities in the SGS modeling.

\section{Galilean invariance}

A quantity is said to be Galilean invariant if it does not change with respect to a frame moving at a constant velocity (see, e.g., (Pope 2000)). The moving inertial frame, denoted by $\mathbf{x}^{*}$, is related to the fixed inertial frame through

$$
\mathbf{x}^{*}=\mathbf{x}-\mathbf{V} t, t^{*}=t,
$$

where $\mathbf{V}$ is a constant velocity vector. By taking derivative of the first relation of (4.82) with respect to time, $t$, one obtains the relation of the two velocity fields:

$$
\mathbf{u}^{*}\left(\mathbf{x}^{*}, t^{*}\right)=\mathbf{u}(x, t)-\mathbf{V} \text {. }
$$

By recognizing the chain of dependences as

$$
\begin{array}{r}
\mathbf{u}^{*} \leftarrow \mathbf{u} \leftarrow \mathbf{x} \leftarrow \mathbf{x}^{*} \\
\nwarrow t \nwarrow t^{*}
\end{array}
$$

or

$$
\begin{array}{r}
\mathbf{u} \leftarrow \mathbf{u}^{*} \leftarrow \mathbf{x}^{*} \leftarrow \mathbf{x} \\
\nwarrow t^{*} \nwarrow t
\end{array}
$$

one arrives at the following partial derivative passages of the velocity field from one system to the other:

$$
\begin{gathered}
\frac{\partial u_{i}^{*}}{\partial x_{j}^{*}}=\frac{\partial u_{i}^{*}}{\partial u_{\lambda_{=1}}} \frac{\partial u_{i}}{\partial x_{j}} \frac{\partial x_{j}}{\partial x_{\lambda_{=1}^{*}}^{*}}=\frac{\partial u_{i}}{\partial x_{j}}, \\
\frac{\partial u_{i}^{*}}{\partial t^{*}}=\frac{\partial u_{i}^{*}}{\partial u_{i}} \frac{\partial u_{i}}{\partial t}+\sum_{j=1}^{3} \frac{\partial u_{i}^{*}}{\partial u_{\lambda_{=1}}} \frac{\partial u_{i}}{\partial x_{j}} \frac{\partial x_{j}}{\partial t\rangle_{=V_{j}}}=\frac{\partial u_{i}}{\partial t}+\sum_{j=1}^{3} V_{j} \frac{\partial u_{i}}{\partial x_{j}} .
\end{gathered}
$$

Note that in the above notation, dummy index summations do not apply. Using Eq. (4.86) , (4.87) and (4.83) the passage of the material derivative of a fluid can be deduced:

$$
\frac{D u_{i}^{*}}{D t^{*}}=\frac{\partial u_{i}^{*}}{\partial t^{*}}+u_{j}^{*} \frac{\partial u_{i}^{*}}{\partial x_{j}^{*}}=\frac{D u}{D t} \text {. }
$$

Therefore, it is seen that the velocity gradient and fluid acceleration are Galilean invariant, whereas the velocity and its partial time derivative are not. It can be further shown that the scalar $\phi(\mathbf{x}, t)$, pressure $p(\mathbf{x}, t)$, strain rate tensor $S_{i j}$, and vorticity $\omega$ are all Galilean invariant.

Based on the above discussion one can show that the Navier-Stokes equations are Galilean invariant; this tells that the behavior of fluid flows is the same in all inertial frames. This important physical property must be carried over if the filtered N-S equations could be used as the governing equations for the LES approach. This is shown, 
e.g., by (Sagaut 2002). Further observe that the residual stresses, i.e., Eq. (4.74), are Galilean invariant as well, since

$$
\begin{aligned}
\overline{u_{i} u_{j}}-\bar{u}_{i} \bar{u}_{j} & =\overline{\left(u_{i}^{*}+V_{i}\right)\left(u_{j}^{*}+V_{j}\right)}-\overline{\left(u_{i}^{*}+V_{i}\right)} \overline{\left(u_{j}^{*}+V_{j}\right)} \\
& =\overline{u_{i}^{*} u_{j}^{*}}-\overline{u_{i}^{*}} \overline{u_{j}^{*}} .
\end{aligned}
$$

Hence, a valid SGS model must be able to reflect this principle. Other invariance principles that a governing equation or turbulence model must follow include the time invariance (invariance under time reversal), rotation invariance and reflection invariance. See (Pope 2000) for a further description.

\section{Decomposition of residual stresses}

A better understanding of the residual stresses can be gained by looking into its decomposition. By noticing

$$
\overline{u_{i} u_{j}}=\overline{\left(\bar{u}_{i}+u_{i}^{\prime}\right)\left(\bar{u}_{j}+u_{j}^{\prime}\right)}=\overline{\bar{u}_{i} \bar{u}_{j}}+\overline{\bar{u}_{i} u_{j}^{\prime}}+\overline{\bar{u}_{j} u_{i}^{\prime}}+\overline{u_{i}^{\prime} u_{j}^{\prime}},
$$

one has

$$
\begin{aligned}
\frac{1}{\rho} \tau_{i j}^{R} & =\overline{u_{i} u_{j}}-\bar{u}_{i} \bar{u}_{j}=\left(\overline{\bar{u}_{i} \bar{u}_{j}}-\bar{u}_{i} \bar{u}_{j}\right)+\left(\overline{\bar{u}_{i} u_{j}^{\prime}}+\overline{\bar{u}_{j} u_{i}^{\prime}}\right)+\overline{u_{i}^{\prime} u_{j}^{\prime}} \\
& =L_{i j}+C_{i j}+R_{i j} .
\end{aligned}
$$

This is the Leonard decomposition, due to (Leonard 1974); the symbols $L_{i j}, \mathrm{C}_{i j}, R_{i j}$ denote the Leonard stresses, the cross stresses and the SGS Reynolds stresses, respectively. However, in this decomposition, $L_{i j}$ and $C_{i j}$ are not Galilean invariant (Speziale 1985). Therefore, (Germano 1986) proposed a Galilean-invariant version of the decomposition by a further operation on the $\bar{u}_{i} \bar{u}_{j}$ term, i.e.,

$$
\bar{u}_{i} \bar{u}_{j}=\overline{\left(\bar{u}_{i}+u_{i}^{\prime}\right)} \overline{\left(\bar{u}_{j}+u_{j}^{\prime}\right)}=\overline{\bar{u}}_{i} \overline{\bar{u}}_{j}+\overline{\bar{u}}_{i} \overline{u_{j}^{\prime}}+\overline{\bar{u}}_{j} \overline{u_{i}^{\prime}}+\overline{u_{i}^{\prime} \bar{u}_{j}^{\prime}} \text {. }
$$

Distributing terms in (4.92) into the Leonard's decomposition (4.91) yields a new set of definitions for $L_{i j}, \mathrm{C}_{i j}$, and $R_{i j}$ :

$$
\begin{aligned}
\frac{1}{\rho} \tau_{i j}^{R} & =\left(\overline{\bar{u}_{i} \bar{u}_{j}}-\overline{\bar{u}}_{i} \overline{\bar{u}}_{j}\right)+\left(\overline{\bar{u}_{i} u_{j}^{\prime}}+\overline{\bar{u}_{j} u_{i}^{\prime}}-\overline{\bar{u}}_{i} \overline{u_{j}^{\prime}}-\overline{\bar{u}}_{j} \overline{u_{i}^{\prime}}\right)+\left(\overline{u_{i}^{\prime} u_{j}^{\prime}}-\overline{u_{i}^{\prime} \bar{u}_{j}^{\prime}}\right) \\
& =L_{i j}+C_{i j}+R_{i j} .
\end{aligned}
$$

The physical interpretation of the three components in both versions is similar. They $\left(L_{i j}\right.$, $\left.C_{i j}, R_{i j}\right)$ represent, in order, the interactions between the large scales $\left(L_{i j}\right)$, large and small scales $\left(C_{i j}\right)$ and subgrid scales $\left(R_{i j}\right)$. Among the three stress components, only the Leonard stress is a resolved stress, i.e., it is known in terms of $\overline{\mathbf{u}}(\mathbf{x}, t)$. Note that if properties (4.66) are satisfied, $L_{i j}$ and $C_{i j}$ are identically zero; thus, both decompositions reduce to a single term of the same form of the Reynolds stresses in the RANS. More significance of the decomposed terms are discussed at length in (Pope 2000; Sagaut 2002).

As already mentioned in Section 4.4, the box filter approximately satisfies the property (4.66) to the order of $O\left(\Delta^{2}\right)$. This will simplifies the residual stress to:

$$
\tau_{i j}^{R} \cong \rho u_{i}^{\prime} u_{j}^{\prime},
$$

hence leading to a one-to-one correspondence of the filtered Navier-Stokes with the RANS equations. 
It should be stressed that the filtered equations (4.71) and (4.72) are derived based on a homogeneous filter. If an inhomogeneous filter is used, commutation error will occur; As a result, extra terms will be brought up in the filtered N-S equations. However, the commutation error can be reduced if a $2^{\text {nd }}$ order or a higher order commuting filters is employed. Unless a negligible commutation error is assumed, the closure of the filtered N-S will be more involved and more complicated. See (Sagaut 2002) for more details in this regard.

\section{6. $\quad$ Energy Spectra}

Investigation of energy spectra of a turbulent flow plays an important role in both DNS and LES. In this section a brief introduction to energy spectrum is presented. First recall the frequency spectrum, which refers to the Fourier transform of a time-dependent signal, say $u(t)$. A typical Fourier-transform pair (also see Eq. (4.60) and (4.61)) is defined as

$$
\begin{gathered}
\hat{u}(\omega)=\int_{-\infty}^{\infty} u(t) e^{-i \omega t} d t, \\
u(t)=\frac{1}{2 \pi} \int_{-\infty}^{\infty} \hat{u}(\omega) e^{i \omega t} d \omega .
\end{gathered}
$$

By plotting the modulus of $\hat{u}$ vs. $\omega$, one gets the picture in the frequency domain. The dominant frequencies of the sampled signal can then be identified by locating those frequencies at which $|\hat{u}|$ is relatively large. If $u(t)$ represents the flow velocity, then $\hat{u}-\omega$ plot may lend access to some inherit periodicity of the flow. From Parseval formula, it is also known that the energy conserves during the transform between the time domain and frequency domain:

$$
\int_{-\infty}^{\infty}|u(t)|^{2} d t=\frac{1}{2 \pi} \int_{-\infty}^{\infty}|\hat{u}(\omega)|^{2} d \omega
$$

In the context of analyzing a turbulent flow the energy spectrum is useful. Roughly speaking, an energy spectrum is created by replacing $u(t)$ in Eq. (4.95) with some correlation functions. This is described below.

Consider a stationary homogeneous fluctuating velocity field $u$ ' $(x, t)$. Note that in this section prime is used to denote turbulent fluctuation instead of a subgrid scale! A two-point spatial correlation function (also known as auto-covariance) of the fluctuation at a fixed time is defined as

$$
R(r)=\left\langle u^{\prime}(x, t) u^{\prime}(x+r, t)\right\rangle,
$$

where \langle\rangle denotes a mean quantity or expectation, e.g., the time mean. As usual, the Fourier transform pair of the correlation function can be written out:

$$
\begin{gathered}
\hat{R}(\kappa)=\int_{-\infty}^{\infty} R(r) e^{-i \kappa r} d r, \\
R(r)=\frac{1}{2 \pi} \int_{-\infty}^{\infty} \hat{R}(\kappa) e^{i \kappa r} d \kappa
\end{gathered}
$$


where $\kappa$ denotes the wave number. Because $R(r)$ is a real and even function, so is its Fourier transform. Since

$$
R(0)=\left\langle u^{\prime} u^{\prime}\right\rangle=\frac{1}{2 \pi} \int_{-\infty}^{\infty} \hat{R}(\kappa) d \kappa=\frac{1}{\pi} \int_{0}^{\infty} \hat{R}(\kappa) d \kappa,
$$

the one-dimensional energy (density) spectrum function can be defined as

$$
E(\kappa)=\frac{1}{\pi} \hat{R}(\kappa)=\frac{1}{\pi} \int_{-\infty}^{\infty} R(r) e^{-i \kappa r} d r
$$

so that the area enclosed by the $E(\kappa)$ curve and the positive $x$-axis gives the variance $\langle u$ ' $u$ '> (one half of the "1D" turbulent kinetic energy), i.e.,

$$
\left\langle u^{\prime} u^{\prime}\right\rangle=\int_{0}^{\infty} E(\kappa) d \kappa \text {. }
$$

Note that in the above the correlation function $R(r)$ is defined along one spatial dimension. If $R$ is calculated along the time line but at a fixed point, i.e.,

$$
R(s)=\left\langle u^{\prime}(x, t) u^{\prime}(x, t+s)\right\rangle ;
$$

it is named auto-correlation. In this situation Eq. (4.99) to (4.103) will still apply, and the $\kappa$-domain should be interpreted as the frequency domain. Also, $\kappa$ is usually replaced by the symbol $\omega$. If the fluctuating field $u$ ' $(x, t)$ is not homogeneous, the notation $R(r)$ should be changed to $R(r, x)$, indicating the correlation function is location dependent. Further, if $u^{\prime}(x, t)$ is not stationary, $R(r, x, t)$ should be used for it to be dependent on time as well.

If a filtered velocity field $\bar{u}(x, t)$ is considered, the auto-covariance of the filtered fluctuation can be defined as

$$
\bar{R}(r)=\left\langle\overline{u^{\prime}}(x, t) \overline{u^{\prime}}(x+r, t)\right\rangle .
$$

One can show (Pope 2000) that

$$
\bar{R}(r)=\int_{-\infty}^{\infty} \int_{-\infty}^{\infty} G(\xi) G(\zeta) R(r+\zeta-\xi) d \xi d \zeta,
$$

and further the energy spectrum of the filtered fluctuations,

$$
\begin{aligned}
\bar{E}(\kappa) & =\frac{1}{\pi} \int_{-\infty}^{\infty} \bar{R}(r) e^{-i \kappa r} d r \\
& =|\hat{G}(\kappa)|^{2} E(\kappa),
\end{aligned}
$$

where $|\hat{G}|^{2}$ serves as attenuation factor. Thus, while $E(\kappa)$ gives the characterization of the actual turbulence fluctuation, $\bar{E}(\kappa)$ offers smoothened turbulence fluctuations with respect to the filtered field $\bar{u}(x, t)$; the both are related by Eq. (4.107). Figure 4-3 shows a semi-log plot of the attenuation factor for the box filter, Gaussian filter and the sharp spectral filter. The fact that the box filter is very ineffective at annihilating high wave number modes implies that, in a filtered field with the box filter, a substantial amount of undesired turbulent kinetic energy may still persist.

When the derivative of the fluctuating field is of interest, the auto-covariance of the $n$th derivative of the fluctuating field can be defined as

$$
R^{(n)}(r)=\left\langle\frac{d^{n} u^{\prime}}{d x^{n}}(x, t) \frac{d^{n} u^{\prime}}{d x^{n}}(x+r, t)\right\rangle .
$$


The energy spectra of $R(r)$ and $R^{(n)}(r)$ are related by

$$
E^{(n)}(\kappa)=\kappa^{2 n} E(\kappa),
$$

the derivation of which can be found in (Pope 2000).

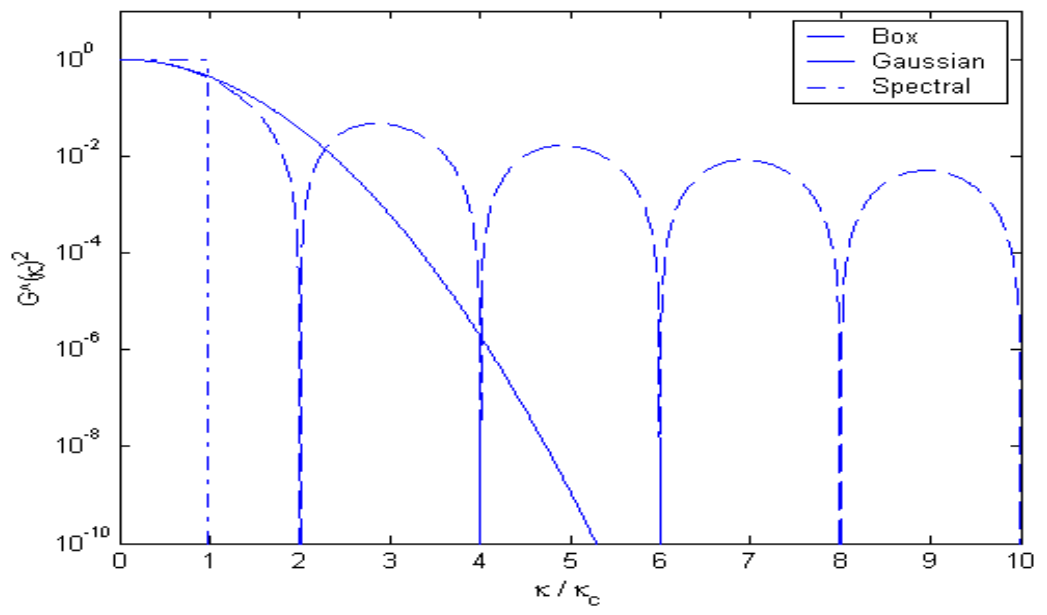

Figure 4-3 Attenuation factor of one-dimensional filters.

The one-dimensional energy spectrum can be further generalized to a threedimensional spectrum. For simplicity, consider a stationary, homogeneous and threedimensional fluctuating field, $\mathbf{u}^{\prime}(\mathbf{x}, t)$. The correlation tensor is defined as,

$$
R_{i j}(\mathbf{r})=\left\langle u_{i}^{\prime}(\mathbf{x}, t) u_{j}^{\prime}(\mathbf{x}+\mathbf{r}, t)\right\rangle .
$$

Fourier transform of $R_{i j}$ gives a (velocity) spectrum tensor $\hat{R}_{i j}$. The Fourier transform-pair formed by $R_{i j}$ and $\hat{R}_{i j}$ can be written:

$$
\begin{gathered}
\hat{R}_{i j}(\mathbf{\kappa})=\iint_{-\infty}^{\infty} \int R_{i j}(\mathbf{r}) e^{-i \mathbf{k} \cdot \mathbf{r}} d \mathbf{r}, \\
R_{i j}(\mathbf{r})=\frac{1}{(2 \pi)^{3}} \iint_{-\infty}^{\infty} \int \hat{R}_{i j}(\mathbf{\kappa}) e^{i \mathbf{k} \cdot \mathbf{r}} d \mathbf{\kappa} .
\end{gathered}
$$

Similar to (4.101),

$$
R_{i j}(0)=\left\langle u_{i}^{\prime} u_{j}^{\prime}\right\rangle=\iint_{-\infty}^{\infty} \int \hat{R}_{i j}(\mathbf{\kappa}) d \mathbf{\kappa}
$$

and in particular,

$$
\frac{1}{2} R_{i i}(0)=\frac{1}{2}\left\langle u_{i}^{\prime} u_{i}^{\prime}\right\rangle=k=\iint_{-\infty}^{\infty} \int_{-\infty}^{1} \frac{1}{2} \hat{R}_{i i}(\boldsymbol{\kappa}) d \mathbf{\kappa},
$$

where scalar $k$ is the turbulent kinetic energy. However, this fairly general spectral representation (Eq. (4.111)) with directional distinction (note the wave number $\kappa$ is a vector) gives too much information that is of practical interest. The directional information can be stripped off by integrating over spherical shells $\mathcal{S}(\kappa)$ in the wave number space, which is centered at the origin, and radius of which is $\kappa=|\boldsymbol{\kappa}|$, i.e., 


$$
E(\kappa)=\oiint \frac{1}{2} \hat{R}_{i i}(\kappa) d \mathcal{S}(\kappa)
$$

In this way, a energy spectrum $E(\kappa)$ is defined that is a function of the scalar wave number magnitude $\kappa$ and whose value represents the total energy at that wave number magnitude. Since this $E(\kappa)$ incorporates influences from all three dimensions, it is called a three-dimensional spectrum by (Tennekes and Lumley 1972). (Pope 2000) terms it as the energy spectrum function. The factor $1 / 2$ is there because

$$
\int_{0}^{\infty} E(\kappa) d \kappa=\int_{0}^{\infty}\left[\oiint \frac{1}{2} \hat{R}_{i i}(\boldsymbol{\kappa}) d \mathcal{S}(\kappa)\right] d \kappa=\iint_{-\infty}^{\infty} \int_{\frac{1}{2}} \hat{R}_{i i}(\mathbf{\kappa}) d \mathbf{\kappa}=\frac{1}{2}\left\langle u_{i}^{\prime} u_{j}^{\prime}\right\rangle=k
$$

Eq. (4.115) can also be alternatively expressed with the help of the sifting property of a Dirac delta function as:

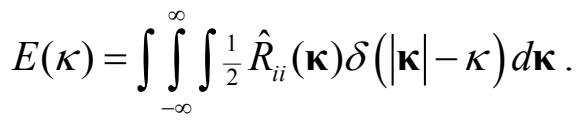

Figure 4-4 illustrates three representative sample energy spectra in a log-log plot, namely, the one-dimensional energy spectrum (Eq. (4.102)), the one-dimensional spectrum of filtered fluctuation (Eq. (4.107)) and the three-dimensional spectrum (Eq. (4.117)). Four qualitative observations can be made:

i. In the inertial sub-range, i.e., between the energy containing range (low wave number region) and the dissipation range (high wave number region), all three spectra exhibits power-law behavior with a power of $-5 / 3$, which conforms Kolmogorov's similarity hypothesis.

ii. In the dissipation (high wave number) range, the energy spectra decay more rapidly than a power of $\kappa$. This is consistent with the fact that a turbulent flow field is infinitely differentiable (Pope 2000).

iii. In the energy containing (low wave number) range, the three-dimensional spectrum tends to zero, while the one-dimensional spectrum attains maximum at origin. This inconsistency is due to aliasing, a problem that occurs when a 1D spectrum is obtained in a $3 \mathrm{D}$ turbulent field, since as such $E(\kappa)$ at wave number $\kappa$ contains contributions from wave numbers greater than $\kappa$.

iv. The spectrum of the filtered fluctuations demonstrates that the actual TKE gets lost in a filtered velocity field. By forming the ratio of the two areas beneath the 1D spectrum curve, and the 1D filtered spectrum curve respectively, one is able to tell the percentage of the total TKE being resolved by the filtered field. For example, from the figure, approximately $92 \%$ of actual TKE is resolved in 1D, or correspondingly $80 \%$ in $3 \mathrm{D}$.

The readers are referred to some turbulence texts (Tennekes and Lumley 1972; Hinze 1975; Frisch 1995; Mathieu and Scott 2000; Pope 2000) for further information concerning the spectrum. 


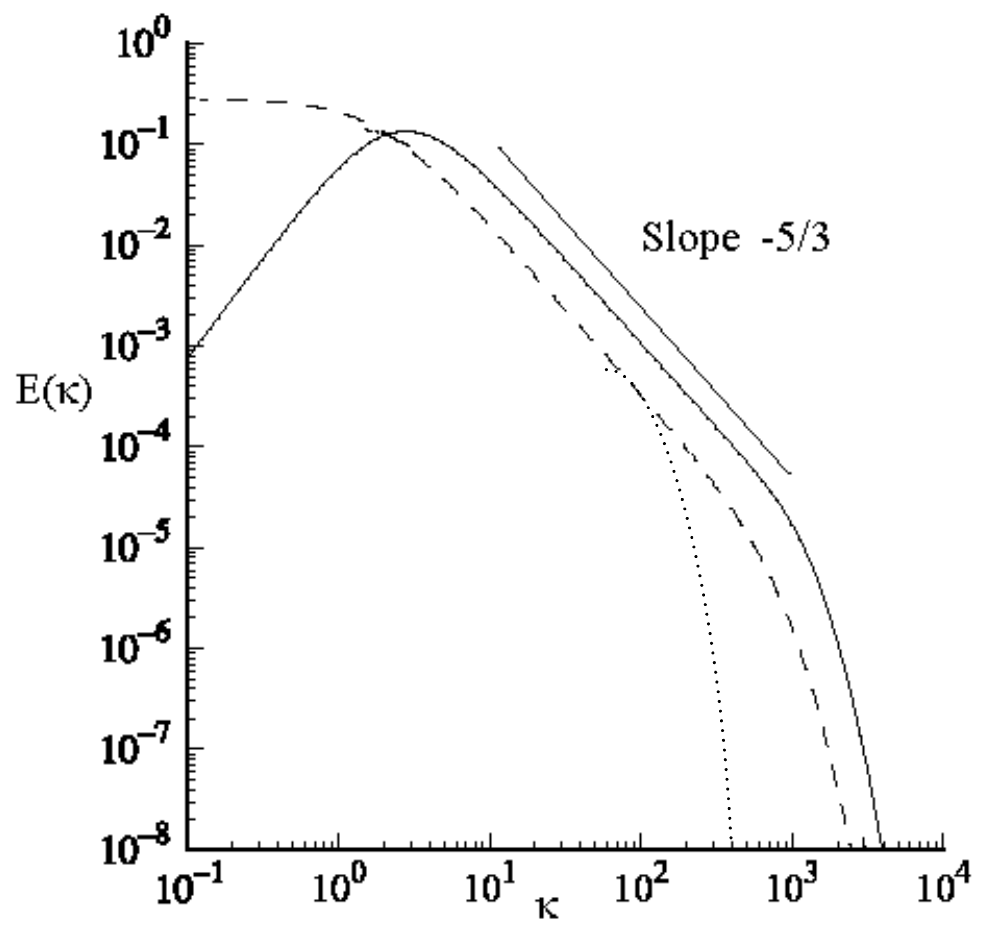

Figure 4-4 Sample energy spectra. Solid line: three-dimensional spectrum; dashed line: onedimensional spectrum; dotted line: filtered one-dimensional spectrum

\subsection{Grid Resolution Requirement in LES}

As aforementioned (Section 4.1), a DNS, aiming at resolving all turbulence scales, solves the N-S equations directly without Reynolds averaging or filtering; hence it demands a highly challenging resolution in both space and time. A natural question that follows up with LES will certainly be how many grid nodes, or put in other way, what grid spacing is needed, in order to resolve the filtered velocity field $\overline{\mathbf{u}}(\mathbf{x})$ governed by Eq. (4.71) and (4.75) adequately? The answer depends on (i) the choice of filter, (ii) the information to be extracted from $\overline{\mathbf{u}}(\mathbf{x})$, and (iii) the numerical method being used (Pope 2000). In what follows arguments provided by (Pope 2000) is addressed, blended with the author's own understanding.

Recall that the Fourier series representation of a turbulent velocity field has been used to deduce the resolution requirement in a DNS (see the end of Section 4.1). Again, it can be used for the same problem in LES. To facilitate understanding, consider a onedimensional statistically homogeneous and periodic velocity field $u(x)$ defined on the interval $0 \leq x<L$ with period of $L$. Without loss of generosity, further assume the mean velocity field is zero, so that $u(x)$ actually represents the velocity fluctuation field, i.e., $u(\mathrm{x})=u^{\prime}(\mathrm{x})$; if the mean field is not zero, one can always consider a new quantity by subtracting the mean field from the $u(x)$. 
Let $N_{\text {DNS }}$ and $N_{\text {LES }}$ denote the number of uniformly distributed grid nodes that should be used in a DNS and LES, respectively. The corresponding grid spacing is then $h_{\text {DNS }}$ and $h_{\text {LES }}$, respectively. Note that $N_{\text {DNS }}>N_{\text {LES }}$; and with $N_{\text {DNS }}$ the turbulence scales are sufficiently resolved, i.e., $h_{\mathrm{DNS}} / \eta \leq 2$ (see. Eq. (4.10)). The goal here is to find the $N_{\text {LES }}$ or $h_{\text {LES }}$ that can properly resolves $\bar{u}(x)$, which is obtained through a filtering process with a specified filter width $\Delta$. An obvious resolution constraint that can be drawn right away is

$$
h_{L E S} \leq \Delta
$$

As discussed in Section 4.1, the number of grid nodes corresponds exactly to the number of Fourier modes in a finite Fourier series representation. Similar to Eq. (4.7), the Fourier series for both $u(x)$ and $\bar{u}(x)$ can be written out in form of the discrete inverse Fourier transform:

$$
\begin{aligned}
& u(x)=\sum_{n=1-N_{D N S} / 2}^{N_{D N S} / 2} c_{n} e^{i \kappa_{n} x}, \kappa_{n}=n \kappa_{0}=n \frac{2 \pi}{L}, \\
& \bar{u}(x)=\sum_{n=1-N_{D N S} / 2}^{N_{D N S} / 2} \bar{c}_{n} e^{i \kappa_{n} x}, \kappa_{n}=n \kappa_{0}=n \frac{2 \pi}{L},
\end{aligned}
$$

with the understanding that $c_{n}$ and $\bar{c}_{n}$ are the $1 \mathrm{D}$ discrete Fourier transforms (DFT) of $u$ and $\bar{u}$, respectively, divided by period $L$, i.e.,

$$
\begin{aligned}
& \frac{1}{L} \int_{0}^{L} u(x) e^{-i \kappa_{n} x} d x \approx c_{n}\left(\kappa_{n}\right)=\frac{1}{L} \sum_{j=0}^{N_{D N S}-1} u\left(x_{j}\right) e^{-i \kappa_{n} x_{j}} h_{D N S}=\frac{1}{N_{D N S}} \sum_{j=0}^{N_{D N S}-1} u\left(x_{j}\right) e^{-i \kappa_{n} x_{j}}, \\
& \frac{1}{L} \int_{0}^{L} \bar{u}(x) e^{-i \kappa_{n} x} d x \approx \bar{c}_{n}\left(\kappa_{n}\right)=\frac{1}{L} \sum_{j=0}^{N_{D N S}-1} \bar{u}\left(x_{j}\right) e^{-i \kappa_{n} x_{j}} h_{D N S}=\frac{1}{N_{D N S}} \sum_{j=0}^{N_{D N S}-1} \bar{u}\left(x_{j}\right) e^{-i \kappa_{n} x_{j}} .
\end{aligned}
$$

Notice that the Fourier series for both $u$ and $\bar{u}$ involve $N_{\text {DNS }}$ modes, which sets an upper limit of the number of Fourier modes that can be reached. Since $N_{\text {DNS }}$ Fourier modes is sufficient to capture all the scales in the turbulent field $u(x)$, the same number of modes is certainly more than enough to represent the filtered field $u(x)$. By property (4.62) the following relation holds:

$$
\bar{c}_{n}=\hat{G}\left(\kappa_{n}\right) c_{n} .
$$

Eq. (4.123) becomes obvious if loosely think of $c_{n}$ and $\bar{c}_{n}$ as $\hat{u}$ and $\hat{\bar{u}}$, respectively. The relation (4.8) should also hold here, i.e., the maximum resolved wave number is determined by $N_{\mathrm{DNS}}$ or $h_{\mathrm{DNS}}$ :

$$
\kappa_{\max }=\frac{1}{2} N_{D N S} \kappa_{0}=\frac{\pi N_{D N S}}{L}=\frac{\pi}{h_{D N S}} .
$$

With the above preparation, it then comes to the question: how well the filtered field $\bar{u}(x)$ can be still represented by its Fourier series (Eq. (4.120)) if the number of Fourier modes is reduced from $N_{\text {DNS }}$ to some number $N_{\text {LES }}$ ? The answer is not unique.

\section{$\underline{\text { Resolution with sharp spectral filter }}$}

First consider the sharp spectral filter defined in Table 4-1. By Eq. (4.123), 


$$
\bar{c}_{n}=\left\{\begin{array}{ll}
c_{n} & \left|\kappa_{n}\right|<\kappa_{c} \\
0 & \left|\kappa_{n}\right| \geq \kappa_{c}
\end{array},\right.
$$

with the wave number $\kappa_{\mathrm{n}}$ given in Eq. (4.120) and the cut-off wave number $\kappa_{\mathrm{c}}$ being

$$
\kappa_{c}=\frac{\pi}{\Delta} \text {. }
$$

If one chooses the most economical resolution, i.e., $h=\Delta$ (cf. Eq. (4.118)), the inequality condition $\left|\kappa_{n}\right|<\kappa_{c}$ reads

$$
\begin{gathered}
\left|\kappa_{n}\right|=|n| \frac{2 \pi}{L}=|n| \frac{2 \pi}{N_{L E S} \Delta}<\kappa_{c}=\frac{\pi}{\Delta}, \text { or }, \\
|n|<\frac{1}{2} N_{L E S}
\end{gathered}
$$

(This also explains why one would like $\kappa_{c}$ to be defined as $\pi / \Delta$ ). Thus, Eq. (4.125) can be rewritten as

$$
\bar{c}_{n}=\left\{\begin{array}{ll}
c_{n} & |n|<N_{L E S} / 2 \\
0 & |n| \geq N_{L E S} / 2
\end{array},\right.
$$

and Eq. (4.120) becomes

$$
\bar{u}(x)=\sum_{n=1-N_{D N S} / 2}^{N_{D N S} / 2} \bar{c}_{n} e^{i \kappa_{n} x}=\sum_{n=1-N_{L E S} / 2}^{N_{L E S} / 2} c_{n} e^{i \kappa_{n} x} .
$$

Therefore, without loss of information, the sharp spectral filter allows $\bar{u}(x)$ to be exactly represented with only $N_{\text {LES }}$ Fourier modes, or equivalently, $N_{\text {LES }}$ grid nodes in the physical space. More grid points provide no further information. To summarize, with the grid resolution

$$
\begin{aligned}
& h_{L E S}=\Delta=\frac{\pi}{k_{c}}, \\
& N_{L E S}=L / h_{L E S},
\end{aligned}
$$

the filtered field using a sharp spectral filter is resolved exactly.

\section{$\underline{\text { Resolution with Gaussian filter }}$}

Next, consider the Gaussian filter (see Table 4-1 for its definition) with a specified filter width $\Delta$. The reference wave number $\kappa_{c}$ originally defined in the spectral filter (Eq. (4.126)) will be also useful here. Suppose for a large-eddy simulation $N_{\text {LES }}(<$ $\left.N_{\text {DNS }}\right)$ grid nodes are used, which corresponds to a grid spacing, $h_{\mathrm{LES}}=L / N_{\mathrm{LES}}$, then the highest wave number that can be resolved in a Fourier series representation (cf. Eq. $(4.124))$ is

$$
k_{r}=\frac{\pi}{h_{L E S}} .
$$

To measure the resolution, form the ratio of filter width $\Delta$ to $h_{\mathrm{LES}}$, in the physical space, or equivalently, the ratio of $\kappa_{r}$ to $\kappa_{c}$ in the wave number space, since

$$
\frac{\Delta}{h_{L E S}}=\frac{\kappa_{r}}{\kappa_{c}} \text {. }
$$


The higher this ratio, the higher (or equal) the resolution, and consequently the quality of a LES will be. For the case of sharp spectral filter, the lowest possible resolution, i.e., $\Delta$ / $h_{\mathrm{LES}}=1$, already yields exact representation of filtered field.

Look at Eq. (4.122) again. If the filtered field is obtained with a Gaussian filter, there exists no demarcating mode, after which the coefficients are identically zero. Thus, if $N_{\text {DNS }}$ modes are truncated to only $N_{\text {LES }}$ terms, loss of information cannot be avoided; the point is how much the information will be lost. This is usually measured with the help of an energy spectrum (see Section 4.6 for a brief summary of energy spectrum).

As an example, consider the filtered velocity derivative, $d \bar{u} / d x$, an important term in the filtered N-S equation. One would like to know the accuracy of the truncated Fourier series (up to $N_{\text {LES }}$ modes), as compared to the original one. A quantitative measure is to form a ratio of the integrals of the energy spectra (variance), which gives an idea of the amount of fluctuating "energy" being resolved, relative to the actual variance produced by a filtered field.

Suppose a turbulent (fluctuating) velocity field $u(x)$ possesses a one-dimensional Kolmogorov spectrum given by

$$
E(\kappa)=C_{1} \varepsilon^{2 / 3} \kappa^{-5 / 3}
$$

where $C_{1} \approx 0.49$. By Eq. (4.107) and (4.109) the spectrum of the filtered velocity derivative, $d \bar{u} / d x$, is:

$$
\bar{E}^{(1)}(\kappa)=\kappa^{2} \bar{E}(\kappa)=\kappa^{2}|\hat{G}|^{2} C_{1} \varepsilon^{2 / 3} \kappa^{-5 / 3}=C_{1} \varepsilon^{2 / 3} \kappa^{1 / 3} \exp \left(-\frac{\pi^{2} \kappa^{2}}{12 \kappa_{c}^{2}}\right) .
$$

This spectrum is plotted in Figure 4-5 with an assumed $\varepsilon$ and $\kappa_{c}$. If the Fourier series representation of $u(x)$ goes up to wave number $k_{r}$, the fraction of $\left\langle(d \bar{u} / d x)^{2}\right\rangle$ is

$$
\frac{\int_{0}^{\kappa_{r}} \kappa^{2} \bar{E}(\kappa) d \kappa}{\int_{0}^{\infty} \kappa^{2} \bar{E}(\kappa) d \kappa}=\Gamma\left(\frac{2}{3}, \frac{\pi^{2}}{12}\left(\frac{\kappa_{r}}{\kappa_{c}}\right)^{2}\right),
$$

where $\Gamma$ denotes the incomplete gamma function (the formula is provided in (Pope 2000)). Therefore, at the resolution of $\Delta / h_{\mathrm{LES}}=\kappa_{r} / \kappa_{c}=2$ and 1 , there is a neglect of $2 \%$ and $28 \%$ of the total variance, respectively (Figure $4-5$ ). Now, if the velocity is solved in the spectral space (as with the spectral method), i.e., the first $N_{\text {LES }}$ Fourier coefficients are known, the best one can get is that those known coefficients are exact; thus, with the resolution of 2 and 1 respectively, $98 \%$ and $72 \%$ of the total fluctuating "energy" of the filtered derivative field is resolved. If the velocity is known in the physical space, additional aliasing error will be introduced while obtaining coefficients through DFT. In fact, (Pope 2000) has demonstrated that with $\Delta / h_{\mathrm{LES}}=1$, the aliased spectrum is a poor approximation to (4.135). 


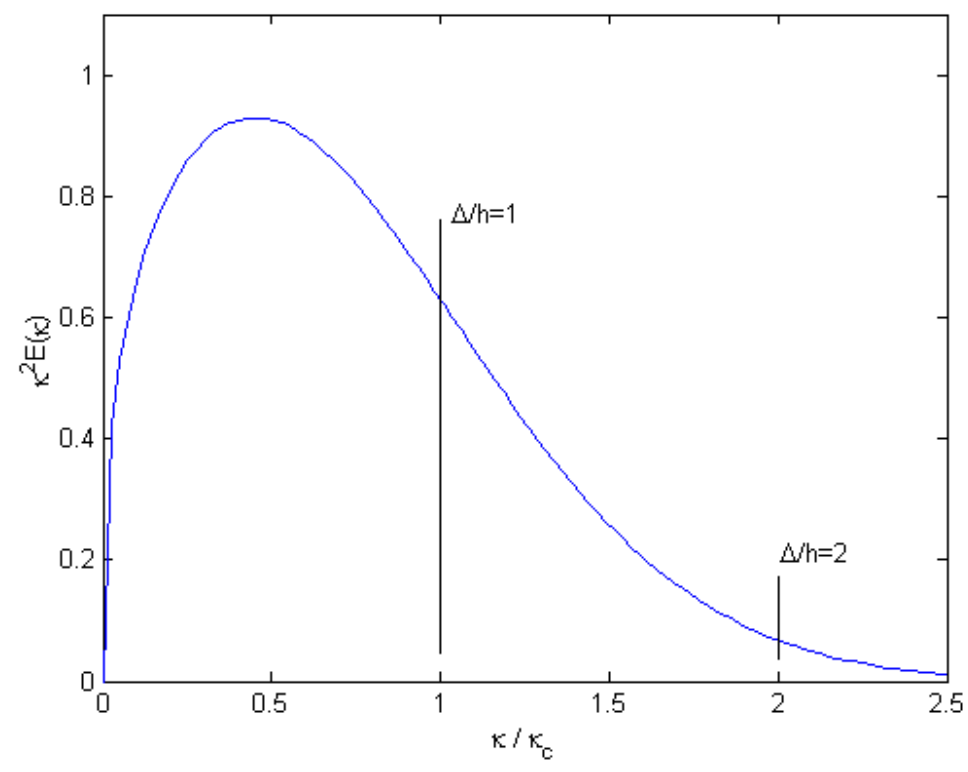

Figure 4-5 Energy spectrum of filtered velocity derivative

One may wish the grid resolution, $\Delta / h_{\mathrm{LES}}$, to be as high as possible to yield an accurate solution of the filtered field. The resulting computational cost increases roughly in a proportion of $\left(\Delta / h_{\mathrm{LES}}\right)^{4}$, considering three spatial dimension and one temporal dimension. But one should realize that a filtered field is mainly dependent on the filter width, not the grid itself (Pope 2004); As long as the grid resolution is sufficient (with respect to the resolved kinetic energy of the filtered fluctuations), say equal to 4, a further refinement of the grid does not necessarily improve the solution. Therefore, once $\Delta$ is fixed, one may say: "there is no such thing as a grid-independent LES." In fact, test calculations have been performed by (Vreman et al. 1997; Chow and Moin 2003) to study the effect of $\Delta \sim h_{\text {LES }}$ ratio. Their general conclusions are: for a scheme with $2^{\text {nd }}$ order spatial accuracy $\Delta / h_{\text {LES }} \geq 4$ is needed, and for a scheme with sixth order accuracy $\Delta / h_{\text {LES }} \geq 2$.

In this study, turbulent flow field is solved in the physical space. The numerical schemes to be employed are generally $2^{\text {nd }}$ order in both space and time. From the above example it is seen that if the Gaussian filter is used, $\Delta / h_{\text {LES }}=2$ seems to be an optimal grid resolution for a satisfactory and economical LES at some compromise of accuracy, while $\Delta / h_{\mathrm{LES}}=1$ is deemed to be a poor resolution. For economical reasons, $\Delta / h_{\mathrm{LES}}=1$ may sometimes also be adopted, but caution must be taken when interpreting the results. The same conclusions should also apply to the box filter, since it is less effective in attenuating energy (see Figure 4-3).

To emphasis, the above discussion is to address the proper grid resolution for the solution of a filtered field. It should not be confused with another technical term, the resolved kinetic energy (by a filtered field), which is defined as 


$$
k_{r}=\int_{0}^{\infty} \bar{E}(\kappa) d \kappa=\int_{0}^{\infty} \widehat{G}(\kappa)^{2} E(\kappa) d \kappa .
$$

The second equality is due to Eq. (4.107). Thus, the ratio

$$
\frac{k_{r}}{k}=\frac{\int_{0}^{\infty} \bar{E}(\kappa) d \kappa}{\int_{0}^{\infty} E(\kappa) d \kappa}
$$

gives the percentage of the resolved kinetic energy with respect to the actual TKE. With, e.g., the model spectrum (Kolmogorov spectrum),

$$
E(\kappa)=C \varepsilon^{2 / 3} \kappa^{-5 / 3}, C=1.5
$$

one can show that around $80 \%$ of the TKE is resolved (see (Pope 2000) for detail), or equivalently, around $20 \%$ TKE is carried by the residual motion.

\subsection{Subgrid Scale Modeling}

This section is not intended to give a comprehensive review of all the subgrid scale (SGS) models in existence. Such a work has already been done, e.g., by (Sagaut 2002). Rather, it picks several representative SGS models, and tries to provide a fundamental understanding of these models.

For clarity, the governing equations (Eq. (4.71) and (4.78) or (4.79)) used for large-eddy simulation (LES) is rewritten below:

$$
\begin{gathered}
\frac{\partial \bar{u}_{i}}{\partial x_{i}}=0, \frac{\partial u_{i}^{\prime}}{\partial x_{i}}=0 \\
\frac{\partial}{\partial t}\left(\rho \bar{u}_{i}\right)+\frac{\partial}{\partial x_{j}}\left(\rho \bar{u}_{j} \bar{u}_{i}\right)=-\frac{\partial \bar{p}}{\partial x_{i}}+\frac{\partial}{\partial x_{j}}\left(2 \mu \bar{S}_{i j}\right)-\frac{\partial \tau_{i j}^{r}}{\partial x_{j}}+\rho \bar{b}_{i} .
\end{gathered}
$$

where $\tau_{i j}^{r}$ is the deviatoric (or anisotropic) residual stress, a term resulting from the filtering operation and given by (cf. Eq. (4.77), (4.74) and (4.76))

$$
\begin{gathered}
\tau_{i j}^{r}=\tau_{i j}^{R}-\frac{2}{3} k_{\Delta} \delta_{i j}, \\
\tau_{i j}^{R}=\rho\left(\overline{u_{i} u_{j}}-\bar{u}_{i} \bar{u}_{j}\right),
\end{gathered}
$$

and the filtered strain rate tensor $\bar{S}_{i j}$ and its magnitude (characteristic filtered strain rate) $\bar{S}$ (see also Eq. (4.80), (4.81)) are given by

$$
\begin{gathered}
\bar{S}_{i j}=\frac{1}{2}\left(\frac{\partial \bar{u}_{i}}{\partial x_{j}}+\frac{\partial \bar{u}_{j}}{\partial x_{i}}\right), \\
\bar{S}=\left(2 \bar{S}_{i j} \bar{S}_{i j}\right)^{1 / 2} .
\end{gathered}
$$

A subgrid scale (SGS) model is aimed at providing a closure of the filtered N-S equations by constructing a modeled expression for $\tau_{i j}^{r}$. 


\subsubsection{Kinetic Energy Equations}

Similar to the study of kinetic energy transfer mechanism of TKE (see Section 4.2), fundamental insight can be gained into the mechanical energy transfer between the resolved (filtered) scales and the residual (or SGS) scales. Again, the phenomena can be best viewed by forming the kinetic energy equation of the filtered velocity field and the residual scales (or subgrid scales), similar to the kinetic energy equation of the mean flow and the TKE equation presented in Section 4.2.

The kinetic energy of the filtered velocity, $K_{f}$, is defined as

$$
K_{f}=\frac{1}{2} \overline{\mathbf{u}} \cdot \overline{\mathbf{u}}=\frac{1}{2} \bar{u}_{i} \bar{u}_{i} .
$$

Its transport equation is obtained by multiplying Eq. (4.141) by $\bar{u}_{i}$. The result (see, e.g., (Piomelli et al. 1991; Germano 1992; Pope 2000)) is

where

$$
\frac{\bar{D} K_{f}}{\bar{D} t} \equiv \frac{\partial K_{f}}{\partial t}+\bar{u}_{j} \frac{\partial K_{f}}{\partial x_{j}}=\frac{\partial \mathcal{T}_{j}}{\partial x_{j}}-\varepsilon_{f}-\mathcal{P}_{\Delta},
$$

$$
\begin{gathered}
\mathcal{T}_{j}=\bar{u}_{i} 2 v S_{i j}-\bar{u}_{i} \tau_{i j}^{r}-\bar{u}_{j} \frac{\bar{p}}{\rho}, \\
\varepsilon_{f}=2 v \bar{S}_{i j} \bar{S}_{i j}=v \bar{S}^{2}, \\
\mathcal{P}_{\Delta}=-\frac{1}{\rho} \tau_{i j}^{r} \bar{S}_{i j} .
\end{gathered}
$$

The three terms on the RHS of Eq. (4.147) represent, respectively, the diffusive transport, the viscous dissipation of the filtered velocity field, and the SGS dissipation or production. The energy transfer between the resolved and the subgrid scales occurs through the $\left(-\mathcal{P}_{\Delta}\right)$ term. If $\left(-\mathcal{P}_{\Delta}\right)$ is negative, the subgrid scales remove energy from the resolved ones (forward scatter); if it is positive, they release energy to the resolved scales (backscatter). However, in the mean, energy is transferred from large scales to subgrid scales (see discussion in Section 4.2), i.e., $\left\langle-\mathcal{P}_{\Delta}\right\rangle$ is negative, where \langle\rangle denotes a mean quantity in contrast to a filtered. It is for this reason that $\mathcal{P}_{\Delta}$ is called the (rate of) production of residual kinetic energy or SGS production. It can be shown (Pope 2000) that the $\varepsilon_{f}$ term is relatively small for a high Reynolds number flow with the filter width much larger than the Kolmogorov scale, so that $\left\langle-\mathcal{P}_{\Delta}\right\rangle$ becomes the dominant sink.

The residual kinetic energy, $k_{\Delta}$, is defined as

$$
k_{\Delta}=\frac{1}{2} \overline{\mathbf{u} \cdot \mathbf{u}}-\frac{1}{2} \overline{\mathbf{u}} \cdot \overline{\mathbf{u}}=\frac{1}{2}\left(\overline{u_{i} u_{i}}-\bar{u}_{i} \bar{u}_{i}\right) \text {. }
$$

The conservation equation for $k_{\Delta}$ is obtained by multiplying the N-S equation (1.24) by $u_{i}$, filtering, and subtracting the $e_{f}$ equation (4.147) (see, e.g., (Lilly 1967; Meneveau and O'Neil 1994)). It reads

where

$$
\frac{\bar{D} k_{\Delta}}{\bar{D} t} \equiv \frac{\partial k_{\Delta}}{\partial t}+\bar{u}_{j} \frac{\partial k_{\Delta}}{\partial x_{j}}=-\frac{\partial \mathcal{T}_{j}^{\Delta}}{\partial x_{j}}+\mathcal{P}_{\Delta}-\varepsilon_{\Delta}
$$




$$
\begin{gathered}
\mathcal{P}_{\Delta}=-\frac{1}{\rho} \tau_{i j}^{r} \bar{S}_{i j}, \\
\varepsilon_{\Delta}=2 v\left(\overline{S_{i j} S_{i j}}-\bar{S}_{i j} \bar{S}_{i j}\right),
\end{gathered}
$$

The expression for the transport term $T_{j}^{\Delta}$ is relatively complex and of less interest, thus it is abridged here. One sees that the SGS production, $\mathcal{P}_{\Delta}$, given by Eq. (4.153) has the same form as Eq. (4.150), meaning that the kinetic energy lost or added to the filtered field becomes the source or sink, respectively, in the residual kinetic energy equation. $\varepsilon_{\Delta}$ represents the dissipation of residual kinetic energy or SGS dissipation; as opposed to other terms in Eq. (4.152), $\varepsilon_{\Delta}$ is dominated by the unresolved small scales. If a filter satisfies condition (4.66) (for example, the box filter satisfies this condition to the order of $O\left(\Delta^{2}\right)$, see Section 4.4), there is one-to-one correspondence between the filtered N-S equation and the RANS; as such, $T_{j}^{\Delta}$ will be simplified to Eq. (4.29), and the SGS dissipation will have the same form as the TKE dissipation (Eq. (4.31)), i.e.,

$$
\varepsilon_{\Delta}=2 v \overline{s_{i j} s_{i j}}, s_{i j}=\frac{1}{2}\left(\frac{\partial u_{i}^{\prime}}{\partial x_{j}}+\frac{\partial u_{j}^{\prime}}{\partial x_{i}}\right),
$$

while only the interpretation of the overbar needs to be adjusted from a mean quantity to a filtered quantity, and similar for the quantities with prime. An important result from Eq. (4.152), due to (Lilly 1967), also pointed out by (Pope 2000), is that, in the mean, production and dissipation approximately balance each other if the Reynolds number is high and the filter width $\Delta$ is in the inertial subrange, i.e.,

$$
\left\langle\mathcal{P}_{\Delta}\right\rangle \approx\left\langle\varepsilon_{\Delta}\right\rangle \approx \varepsilon
$$

\subsubsection{Smagorinsky Model}

Recall the Boussinesq eddy viscosity concept (see Section 4.2 and Eq. (4.34). The standard Smagorinsky model due to (Smagorinsky 1963; Lilly 1967) is based on the conceptually same hypothesis, i.e., the deviatoric residual stress, $\tau_{i j}^{r}$, is expressed in terms of the filtered (or resolved) strain rate tensor $\bar{S}_{i j}$ through the relation

$$
\tau_{i j}^{r}=-2 \rho v_{t} \bar{S}_{i j}
$$

where $v_{t}$ is an artificial parameter, called the eddy (turbulent) viscosity. Analogous to the mixing length model (Eq. (4.38)), $v_{t}$ is constructed from

$$
v_{t}=l_{S}^{2} \bar{S}=\left(C_{S} \Delta\right)^{2} \bar{S},
$$

where $\bar{S}$ is defined in Eq. (4.145) and $l_{S}$ is the Smagorinsky length scale proportional to the characteristic filter width $\Delta$ (see Eq. (4.65)); $C_{S}$ is called Smagorinsky constant, whose value is usually between 0.05 and 0.25 , depending on the characteristics of the flow. Thus, the final model expression for $\tau_{i j}^{r}$ reads

$$
\tau_{i j}^{r}=-2 \rho\left(C_{S} \Delta\right)^{2} \bar{S} \bar{S}_{i j} \text {. }
$$


The expression for the eddy viscosity $v_{t}$, given by Eq. (4.158), can be formally derived from the production equals dissipation (in the mean) relation (4.156). Writing the production $\mathcal{P}_{\Delta}$ (Eq. (4.153)) with the hypothesis (4.157) gives

$$
\mathcal{P}_{\Delta}=-\frac{1}{\rho} \tau_{i j}^{r} \bar{S}_{i j}=2 v_{t} \bar{S}_{i j} \bar{S}_{i j}=v_{t} \bar{S}^{2}
$$

Estimating the dissipation $\varepsilon$ with Eq. (4.2) and further using the characteristic filtered strain rate $\bar{S}$ in place of the velocity scale yields:

$$
\varepsilon \sim \frac{u_{0}{ }^{3}}{l_{0}} \sim \frac{u_{0}{ }^{3}}{l_{S}} \sim \bar{S}^{3} l_{S}{ }^{2},
$$

where $l_{S}$ is the Smagorinsky length scale. By equating Eq. (4.160) and (4.161) the desired relation (4.158) is obtained. The assumption that $l_{S}$ scales linearly with the filter width can also be loosely confirmed by considering the mean balance equation (4.156) and the Kolmogorov spectrum good in the inertial subrange; this yields a proportionality constant $C s \approx 0.17$ with a sharp spectrum filter (for details see analysis provided by (Lilly 1967; Pope 2000)).

Notably, even in the isotropic turbulence, analysis provided by (Voke 1996; Meneveau and Lund 1997; Pope 2000) show that, as $\Delta \rightarrow \eta, C_{S}$ must vary with $\Delta$ in order to reproduce the correct SGS dissipation rate in the viscous (dissipation) range. From Eq. (4.160) one also sees that the SGS production offered by Smagorinsky model is always positive, thus there is no backscatter in this model and the kinetic energy is transferred everywhere from the filtered motion to the residual motion. The optimal value of $C_{S}$ will be dependent on the regime and type of flow, Reynolds number, and discretization scheme; therefore in practical use a calibration is usually needed. In the near-wall region, the specification of $l_{S}=C_{S} \Delta$ with constant $C_{S}$ is not justifiable, since it incorrectly leads to a non-zero residual viscosity and shear stress at the wall. A common remedy is use of a damping function which ensures the value of $C_{S}$ vanishes at the wall. The van Driest damping, for example, is defined by

$$
l_{S}=C_{S} \Delta\left(1-e^{y^{+} / A^{+}}\right),
$$

with $A^{+}=25$ or 26 .

\subsubsection{Dynamic Smagorinsky Model}

Motivated by alleviating the drawbacks of the standard Smagorinsky model, the dynamic Smagorinsky model or the dynamic eddy-viscosity model or simply the dynamic model, was proposed by (Germano et al. 1991), followed by an important modification made by (Lilly 1992) and valuable extension provided by (Ghosal et al. 1995) and (Meneveau et al. 1996). The dynamic model uses the Smagorinsky model as a basis model; with a proposed procedure the Smagorinsky constant $C_{S}$ is determined locally in a dynamic fashion. Note that, strictly speaking, a dynamic model (or procedure) does not have to use Smagorinsky as its basis model, but in practice one usually treats it as a default. 
The basic idea here is to make use of the "known" field $\overline{\mathbf{u}}(\mathbf{x}, t)$; by performing a second filtering operation on this "known" field one may extract information useful for determining $C_{S}$. For this purpose two filters of the same type with different filter widths are introduced, namely, a grid filter and a test filter. The grid filter has a filter width $\bar{\Delta}$, which is proportional to the grid spacing, e.g., $\bar{\Delta}=h$ or $\bar{\Delta}=2 h$; the test filter has a filter width $\tilde{\Delta}$, which is larger than $\bar{\Delta}$ and typically taken to be twice of $\bar{\Delta}$. Let $\bar{\phi}$ and $\tilde{\phi}$ denote a filtered quantity obtained using a grid filter and a test filter, respectively. Also, let $\tilde{\bar{\phi}}$ denote a double-filtered quantity obtained using first the grid filter and then the test filter. If $\tilde{\bar{\phi}}$ is equivalently obtained through a single filtering operation with a filter of the same type, the associated filter width is called effective double-filter width, denoted by $\tilde{\bar{\Delta}}$. It can be shown (e.g., (Germano et al. 1991; Germano 1992; Pope 2000)) that, for the sharp spectral filters,

and for the Gaussian filters,

$$
\tilde{\bar{\Delta}}=\tilde{\Delta},
$$

$$
\tilde{\bar{\Delta}}=\left(\tilde{\Delta}^{2}+\bar{\Delta}^{2}\right)^{1 / 2}=\sqrt{5} \bar{\Delta} .
$$

Note that in Eq. (4.164) $\tilde{\Delta}=2 \bar{\Delta}$ has been assumed. In what follows a brief derivation is presented for the dynamically determined model coefficient, $C_{S}$.

A single-filtering operation (with a grid filter) on the N-S equations produces a residual stress given by Eq. (4.143) or (4.74). Similarly, when performing a double filtering (with a grid filter and a test filter) on the N-S equations, one obtains a new residual stress given by

$$
T_{i j}^{R}=\rho\left(\widetilde{\bar{u}_{i} u_{j}}-\tilde{\bar{u}} \tilde{\bar{u}}_{j}\right) .
$$

Filtering Eq. (4.143) with the test filter and taking difference between the resulting equation and Eq. (4.165) yield so-called Germano identity (Germano et al. 1991):

$$
\mathcal{L}_{i j}=T_{i j}^{R}-\widetilde{\tau_{i j}^{R}}=\rho\left(\widetilde{\overline{\bar{u}}_{i} \bar{u}_{j}}-\tilde{\bar{u}}_{i} \tilde{\bar{u}}_{j}\right) .
$$

Its deviatoric part is formed correspondingly as

$$
\mathcal{L}_{i j}^{\mathrm{dev}} \equiv \mathcal{L}_{i j}-\frac{1}{3} \mathcal{L}_{k k} \delta_{i j}=T_{i j}^{r}-\widetilde{\tau_{i j}^{r}},
$$

where $\tau_{i j}^{r}$ is defined in Eq. (4.142) and $T_{i j}^{r}$ defined in a similar manner. The symbol $\mathcal{L}$ is used for this identity since when $\bar{\Delta}=\tilde{\Delta}$ it is identical to the Leonard stress defined in Eq. (4.93). The significance of the Germano identity is that it can be explicitly computed using the known resolved field $\overline{\mathbf{u}}(\mathbf{x}, t)$. Physically, it can be loosely interpreted as a resolved turbulent stress (Germano et al. 1991) contributed by scales intermediate between the grid filter width and the test filter width.

Next, write down the Smagorinsky model (Eq. (4.159)) for the residual stresses at both the grid level and test level:

$$
\begin{aligned}
& \tau_{i j}^{r}=\tau_{i j}^{R}-\frac{1}{3} \tau_{k k}^{R} \delta_{i j}=-2 \rho C_{S} \bar{\Delta}^{2} \bar{S} \bar{S}_{i j}, \\
& T_{i j}^{r}=T_{i j}^{R}-\frac{1}{3} T_{k k} \delta_{i j}=-2 \rho C_{S} \tilde{\bar{\Delta}}^{2} \tilde{\bar{S}} \tilde{\bar{S}}_{i j} .
\end{aligned}
$$


Notice that the Smagorinsky model formulated above is slightly different from the one given in Eq. (4.159). Here instead of $C_{S}^{2}, C_{S}$ is used, so that local backscatter can be realized via negative $C_{S} . \tilde{\bar{S}}_{i j}$ and $\tilde{\bar{S}}$ in the above equations are the double-filtered strain rate tensor and its magnitude, respectively; they are defined similarly to the definition of $\bar{S}_{i j}$ and $\bar{S}$ (Eq. (4.144) and (4.145)). Further assume $C_{S}$ to be a local constant at both filter levels. Filtering Eq. (4.168) with the test filter, subtracting the resulting equation from Eq. (4.169) and using the Germano identity (4.167) gives

$$
\mathcal{L}_{i j}^{\mathrm{dev}}=T_{i j}^{r}-\widetilde{\tau_{i j}^{r}}=C_{S} M_{i j}
$$

with the tensor $M_{i j}$ defined by

$$
M_{i j}=2 \rho\left(\bar{\Delta}^{2} \overline{\bar{S}} \overline{\bar{S}}_{i j}-\tilde{\bar{\Delta}}^{2} \tilde{\bar{S}} \tilde{\bar{S}}_{i j}\right) .
$$

Mathematically viewed, Eq. (4.170) is ill-posed since a single constant $C_{S}$ can not be determined from five independent stress relations. Thus, a way is proposed by (Lilly 1992), who adopts the least square approach to minimize the error. This yields:

$$
C_{S}=\frac{\mathcal{L}_{i j} M_{i j}}{M_{k l} M_{k l}} .
$$

Therefore, Eq. (4.168), (4.172), (4.166) and (4.171) provide a complete description of this model, in which the model constant $C_{S}$ is obtained in a self-contained manner with no empirical specification.

Through the dynamic specification of $C_{S}$ value (Eq. (4.172)), the dynamic model makes a backscattering process possible. However, as pointed out by many researchers, the resulting $C_{S}$ field is found to be highly variable and may contain a significant portion of negative values, which makes a LES unstable. To circumvent this difficulty, the usual practice is to perform an additional averaging for both the numerator and the denominator of Eq. (4.172) over directions of statistically homogeneity (see, e.g., (Germano et al. 1991; Piomelli 1993)). This is further given formal ground by (Ghosal et al. 1995), who showed that this averaging procedure minimizes the total error in the homogeneous plane.

A problem still exists in the inhomogeneous flows, such as flows with complex geometries, since no plane can be used for the averaging procedure. However, the inhomogeneous flows are of high interest for practical applications. To this end, two approaches are prominent. (Ghosal et al. 1995)'s localized dynamic model transforms determining a proper local average value of $C_{S}$ into a variational problem, which can be solved to determine a non-negative $C_{S}$ field (thus no backscatter). (Meneveau et al. 1996) proposed a Lagrangian dynamic model, in which a weighted average is accumulated over flow pathlines rather than over homogeneous directions. Superior results were obtained in their sample LES calculations.

A further development of the dynamic model is the so-called mixed models. A representative model in this category is the one proposed by (Zang et al. 1993), which is partially based on an early work, known as the scale similarity model (Bardina et al. 1980). Recall the Germano decomposition presented in Section 4.5 (Eq. (4.93)): 


$$
\frac{1}{\rho} \tau_{i j}^{R}=L_{i j}+C_{i j}+R_{i j},
$$

where

$$
L_{i j}=\overline{\bar{u}_{i} \bar{u}_{j}}-\overline{\bar{u}}_{i} \overline{\bar{u}}_{j} .
$$

In the scale similarity model $\tau_{i j}^{R}$ is modeled directly with the resolved Leonard stress:

$$
\tau_{i j}^{R}=-\rho L_{i j}=-\rho\left(\overline{\bar{u}}_{i} \overline{\bar{u}}_{j}-\overline{\bar{u}_{i} \bar{u}_{j}}\right) .
$$

The supporting argument for the model expression (4.175) is that the smallest resolved scale motions and the largest subgrid scale motions are similar in structure. Yet, this model hardly dissipates any energy and cannot serve as a "stand alone" SGS model (Ferziger and Peric 1996). As a logical improvement, Zang's mixed model combines the scale similarity and the dynamic model, i.e., it uses the dynamic model to express the unresolved stress components $\left(C_{i j}\right.$ and $\left.R_{i j}\right)$ in Germano's decomposition of residual stress, and computes the resolved stress $L_{i j}$ explicitly using Eq. (4.174). The combined model reads

$$
\tau_{i j}^{r}=\rho\left(L_{i j}-\frac{1}{3} L_{k k} \delta_{i j}\right)-2 \rho C_{S} \bar{\Delta}^{2} \bar{S} \bar{S}_{i j} .
$$

In a turbulent mixing layer simulation (Vreman et al. 1997), this model was found to be the most successful among six models being evaluated.

\subsubsection{One-equation SGS Model}

SGS models are very much related to the RANS modeling, as many SGS models can find their counterpart in the RANS models. For example, the Smagorinsky model is an analog to the mixing length model, and out of the one-equation RANS model the one-equation SGS model is created. The development and application of the one-equation SGS model is pioneered by the meteorological community, with the representative work performed by (Deardorff 1974; Deardorff 1980).

Recall that the one-equation RANS model lacks a turbulence length scale and thus involves a flow-dependent specification of $l_{m}$ (see Section 4.2). For this reason the twoequation RANS model, e.g., the $k-\varepsilon$ model, was developed, to make itself complete. However, in the SGS modeling a similar two-equation model usually is not necessary, since the turbulence length scale is already available in terms of the characteristic filter width $\Delta$.

A typical one-equation SGS model is created from the residual (or SGS) kinetic energy equation (4.152). Modeling of unclosed terms proceeds in a similar fashion as the one-equation RANS model. The production term $\mathcal{P}_{\Delta}$ is closed through the eddy-viscosity assumption (Eq. (4.157)). The SGS dissipation term is taken to be isotropic and is modeled according to Eq. (4.2) as

$$
\varepsilon_{\Delta}=C_{\varepsilon} \frac{k_{\Delta}^{3 / 2}}{\Delta} .
$$


The transport term (first term on the RHS of (4.152)) is typically assumed to be comparable with a diffusion process with its diffusivity dependent on the residual kinetic energy, i.e.,

$$
\begin{gathered}
\mathcal{T}^{\Delta}=-\frac{v_{t}}{\sigma_{k}} \nabla k_{\Delta}, \\
v_{t}=C_{v} k_{\Delta}^{1 / 2} \Delta,
\end{gathered}
$$

where $\sigma_{k}$ is the turbulent Prandtl-Schmidt number whose value is commonly taken to be one. Thus, the complete one-equation model reads:

$$
\frac{\partial k_{\Delta}}{\partial t}+\bar{u}_{j} \frac{\partial k_{\Delta}}{\partial x_{j}}=-\frac{\partial}{\partial x_{j}}\left(\frac{v_{t}}{\sigma_{k}} \frac{\partial k_{\Delta}}{\partial x_{j}}\right)+v_{t} \bar{S}-C_{\varepsilon} \frac{k_{\Delta}^{3 / 2}}{\Delta},
$$

with $v_{t}$ provided by Eq. (4.179). The constants associated with $v_{t}$ and the $\varepsilon_{\Delta}$ term are chosen to be

$$
C_{v} \approx 0.1, C_{\varepsilon} \approx 0.7
$$

based on an analysis for high-Reynolds-number homogeneous turbulence with the sharp spectral filter (Pope 2000). The model constants provided by (Yoshizawa and Horiuti 1985) and cross-references by (Menon et al. 1996) are

$$
C_{v} \approx 0.05, C_{\varepsilon} \approx 1.0 \text {. }
$$

The above one-equation model may also be combined with the dynamic procedure presented in the previous subsection (dynamic Smagorinsky model) to allow a variable model constant, $C_{v}$ and $C_{\varepsilon}$. This is called dynamic one-equation model. Variants of dynamic one-equation model exist, depending on a specific dynamic procedure. See (Ghosal et al. 1995; Menon et al. 1996; Sohankar et al. 1999).

Unlike the algebraic eddy viscosity models (e.g. the Smagorinsky model and the dynamic model), which only utilize the local flow information to close the residual stress, the one-equation model takes into account the time history and non-local effects through a transport equation. Also, it assumes no local balance between the SGS production and dissipation. It could be expected that this model performs better than the algebraic eddy viscosity model, especially in regions where local balance is violated (Menon et al. 1996). In spite of this, general experience shows that, in LES, with two exceptions, the additional computational cost is not justified by an assured increase in accuracy (Pope 2000). The two exceptions mentioned by (Pope 2000) are (i) the application to meteorological flow, where one-equation models have proved to be advantageous, and (ii) the application to the reacting flow, where the important processes of mixing and reaction occur at the subgrid scale level.

In the literature extensive comparison study of various SGS models can be found. To enumerate some, they are (Fureby et al. 1997; Vreman et al. 1997; Sohankar and Davidson 2000; Su et al. 2001). However, as commented by (Pope 2000), no general conclusion can be drawn, since a model performance is in general a function of many known and unknown factors, such as the Reynolds number, local flow characteristics, filter type and width, and influence of numerical methods. 


\subsubsection{Implicit Turbulence Modeling}

In the implicit turbulence modeling (ITM), also referred to as the implicit SGS modeling, monotone integrated LES (MILES), or "no model," no SGS models are explicitly defined. A big assumption with the ITM is that the residual stresses are represented by the spatial truncation errors of the numerical scheme being used; as a consequence, the "SGS model" is intrinsically embedded in the numerical solution. To see this, one may write the modified equation corresponding to the LES momentum equations (4.141):

$$
\frac{\partial}{\partial t}\left(\rho \bar{u}_{i}\right)+\frac{\partial}{\partial x_{j}}\left(\rho \bar{u}_{j} \bar{u}_{i}\right)=-\frac{\partial \bar{p}}{\partial x_{i}}+\frac{\partial}{\partial x_{j}}\left(2 \mu \bar{S}_{i j}\right)-\frac{\partial}{\partial x_{j}}\left(\tau_{i j}^{r}+\tau_{i j}^{h}\right)+\rho \bar{b}_{i},
$$

where $\tau_{i j}^{h}$ appears as an additional numerical stress, and it includes spatial truncation errors with the leading error term giving the order of the method, i.e.,

$$
\tau_{i j}^{h} \sim h^{p},
$$

where $h$ is the representative grid size. Note that, the modified equation is typically derived from the Taylor series expansion of the discretized equation, and hence it can be viewed as a partial differential equation satisfied by the numerical solution. Now ITM says: let us ignore the residual stress $\tau_{i j}^{r}$ completely, a term difficult to model in general, and let $\tau_{i j}^{h}$ play the role in place of $\tau_{i j}^{r}$. The implication of this statement is twofold: firstly,

$$
\tau_{i j}^{r}=0 \text {; }
$$

and secondly, the numerical scheme used should be designed in such a way that its corresponding truncation error is capable of mimicking the function played by the residual stress, mainly being the kinetic energy withdrawal from the resolved motion. As such, the energy removal mechanism can be expresses as

$$
\mathcal{P}_{\Delta}=-\frac{1}{\rho} \tau_{i j}^{h} \bar{S}_{i j}=\varepsilon_{\text {num }}
$$

(cf. Eq. (4.150) and (4.153)), where $\varepsilon_{\text {num }}$ represents the numerical dissipation rate. Thus, effectively, the ITM solves the N-S equation directly, without pre-filtering and explicit modeling of the residual stresses; yet, the solved velocity is still interpreted as a filtered field, due to the action of numerical dissipation.

A key distinction should be made between the SGS models presented in the previous sections and the ITM approach. With the formers, where a SGS model is explicitly built on a physical basis, the filtered N-S equation is solved accurately, so that the numerical error is negligibly small $\left(\tau_{i j}^{h} \ll \tau_{i j}^{r}\right)$. This requires (i) a scheme typically of at least second order in both space and time, and (ii) a sufficiently small grid size, $h$, for a specified filter width $\Delta$ (see discussions in Section 4.7). On the other hand, the ITM, while completely avoiding an explicit SGS modeling, produces a numerical solution on a relative coarse grid, and with the numerical dissipation being an essential part of the LES. Thus, the resolved field is fundamentally linked to the grid size $h$ and the numerical method being employed. Due to the conceptually very different properties (Pope 2004) called one "physical LES," and the other "numerical LES." 
With that being said, one realizes that the central task in the ITM is not the modeling of SGS stresses itself, but rather, the design of a suitable numerical scheme. Looking into the literature, work in this regard is predominantly based on so-called nonoscillatory numerical schemes. An oscillatory scheme is a one that produces spurious (or unphysical) oscillations when applied across a discontinuity (e.g., shockwave); a wellknown example is the central differencing. On the other hand, a non-oscillatory scheme is designed to prevent or abate unphysical oscillations. Most of these non-oscillatory schemes used in ITM are in essence based on flux limiting technique and/or higher-order upwind differencing, because these schemes usually carry a leading higher-order diffusion error term, which works for the removal of kinetic energy, while still retaining a higher order of accuracy.

(Boris et al. 1992; Oran and Boris 1993) were the first who proposed so-called monotone-integrated LES (MILES). The concept is further developed and formalized by (Fureby and Grinstein 1999, 2002; Grinstein and Fureby 2002). In MILES the discretization is carried out using flux-limiting method (see e.g. (Hirsch 1988) for greater details regarding this class of method), for which the numerical flux is decomposed as the weighted sum of a higher-order flux $F_{f}^{H}$ that works well in smooth regions, and a lowerorder flux $F_{f}^{L}$ that effectively damps the spurious oscillations in sharp-gradient regions:

$$
F_{f}=F_{f}^{H}-(1-\Gamma)\left(F_{f}^{H}-F_{f}^{L}\right),
$$

where $f$ denotes the cell face and $\Gamma$ is the flux limiter. There are generally two types of limiters, namely slope limiters, represented by (van Leer 1974) and flux limiters, represented by (Boris and Book 1973). A nice summary of explicit as well as implicit limiters is provided in (Yee 1987; Chung 2002). To achieve desirable physical properties in the associated implicit SGS model, such as frame-invariance, symmetry, non-negative dissipation of SGS kinetic energy, some guidelines (or constraints) were suggested in choosing flux limiters. They can be mainly summarized as monotonicity, contraction, positivity, total variation diminishing (TVD), monotonicity preservation, and local monotonicity preservation. In fact, these properties are all related to the nonlinear stability analysis (for nonlinear conservation law). To be self-contained, a brief review is presented for the definitions of these technical terms.

For simplicity consider the one-dimensional situation. Let $\phi\left(x_{i}, t_{n}\right)$ and $\varphi\left(x_{i}, t_{n}\right)$ be numerical solutions to the same scalar conservation equation with two different initial conditions. The monotonicity, first proposed by (Harten et al. 1976), is defined as follows: if $\phi\left(x_{i}, 0\right) \leq \varphi\left(x_{i}, 0\right)$ for all $x_{i}$, then $\phi\left(x_{i}, t_{n}\right) \leq \varphi\left(x_{i}, t_{n}\right)$ for all $x_{i}$ and $t_{n}$; similar arguments also holds for $\phi\left(x_{i}, 0\right) \geq \varphi\left(x_{i}, 0\right)$. If the scheme is an explicit forward-time method, i.e.,

$$
\phi_{i}^{n+1}=H\left(\phi_{i-k}^{n}, \phi_{i-k+1}^{n}, \ldots, \phi_{i+k}^{n}\right)=\sum_{j=i-k}^{i+k} a_{j} \phi_{j}^{n},
$$

where $H$ represents a discretization with a $(2 k+1)$ elements stencil. It can be shown that the monotonicity condition in this case is simply: 


$$
\frac{\partial H}{\partial \phi_{j}^{n}} \geq 0 \text { for all } i-k \leq j \leq i+k ;
$$

this is equivalently to saying that the coefficients $a_{j}$ 's of the linear combination of $\phi_{j}^{n}$ must be all positive. A scheme that satisfies the monotonicity condition is called monotone scheme or monotone method. It has been proved that a monotone scheme in conservation form is at most first order (see e.g., (Sod 1985)). The contraction property requires

$$
\left\|\phi^{n+1}-\varphi^{n+1}\right\|_{1} \leq\left\|\phi^{n}-\varphi^{n}\right\|_{1},
$$

where $\|\cdot\|$ denotes the $l_{1}$ norm defined by

$$
\left\|\phi^{n}\right\|_{1}=\sum_{j}\left|\phi_{j}^{n}\right| .
$$

It tells that any two solutions with different initial conditions always go closer and closer. Next, consider a scheme that is split in the form of

$$
\phi_{i}^{n+1}=\phi_{i}^{n}+C\left(\phi_{i+1}^{n}-\phi_{i}^{n}\right)-D\left(\phi_{i}^{n}-\phi_{i-1}^{n}\right) .
$$

The positivity condition, first suggested by (Harten 1983), is satisfied if

$$
C \geq 0, D \geq 0, C+D \leq 1 \text {. }
$$

The total variation (TV) is defined as

$$
\operatorname{TV}\left(\phi^{n}\right)=\sum_{j}\left|\phi_{j+1}^{n}-\phi_{j}^{n}\right| .
$$

A numerical scheme is said to be $T V$-stable if its TV is bounded. It can be shown (see e.g. (LeVeque 1992)) that for numerical schemes in conservation form with consistent numerical flux TV-stability is a sufficient condition for convergence (convergence means the numerical solution tends to exact solution as grid size approaches zero). The total variation diminishing (TVD) is then defined as

$$
\operatorname{TV}\left(\phi^{n+1}\right) \leq \operatorname{TV}\left(\phi^{n}\right),
$$

due to (Harten 1983, 1984). TVD is a special instance of TV-stability, and it is a useful condition, since schemes satisfying TVD can effectively damp spurious oscillations. However, it should be stressed that in theory TVD may still allow large spurious oscillations, although it is rarely the case in practice. Also, as pointed out by (Laney 1998), few outside of the mathematics community recognize that TVD refers to stability condition, although it is widely used. Under circumstances the TVD condition and positivity condition have "if and only if" relation. The condition of Monotonicity preservation, first suggested by (Godunov 1959), says if $\phi\left(x_{i}, 0\right)$ is a monotone increasing (or decreasing) function, then $\phi\left(x_{i}, t_{n}\right)$ is also monotone increasing (or decreasing) for all $t$, i.e.,

$$
\begin{aligned}
& \text { if } \phi_{i}^{0} \leq \phi_{i}^{0} \text { for all } i \text {, then } \phi_{i}^{n} \leq \phi_{i}^{n} \text { for all } n \text { and } i \text {; } \\
& \text { if } \phi_{i}^{0} \geq \phi_{i}^{0} \text { for all } i \text {, then } \phi_{i}^{n} \geq \phi_{i}^{n} \text { for all } n \text { and } i \text {. }
\end{aligned}
$$

However, this property does not address a non-monotone solution. A little relaxed version of Eq. (4.196) is the local monotonicity preservation. One common definition given in is:

$$
\begin{aligned}
& \text { if } \phi_{i-1}^{n} \leq \phi_{i}^{n} \leq \phi_{i+1}^{n} \text { then } \phi_{i-1}^{n} \leq \phi_{i}^{n+1} \leq \phi_{i+1}^{n}, \\
& \text { if } \phi_{i-1}^{n} \geq \phi_{i}^{n} \geq \phi_{i+1}^{n} \text { then } \phi_{i-1}^{n} \geq \phi_{i}^{n+1} \geq \phi_{i+1}^{n} .
\end{aligned}
$$


A stronger local constraint is

$$
\begin{array}{ll}
\text { if } \quad \phi_{i-1}^{n} \leq \phi_{i}^{n} \leq \phi_{i+1}^{n} \leq \phi_{i+2}^{n} \quad \text { then } \quad \phi_{i-1}^{n} \leq \phi_{i}^{n+1} \leq \phi_{i+1}^{n+1} \leq \phi_{i+2}^{n} ; \\
\text { if } \phi_{i-1}^{n} \geq \phi_{i}^{n} \geq \phi_{i+1}^{n} \geq \phi_{i+2}^{n} \text { then } \phi_{i-1}^{n} \geq \phi_{i}^{n+1} \geq \phi_{i+1}^{n+1} \geq \phi_{i+2}^{n} .
\end{array}
$$

For example, the quasi-second-order-upwind (QSOU) scheme (Amsden et al. 1989) is strong locally monotonicity preserving.

It has been known that the following relations hold between the above conditions: monotonicity implies contraction; the contraction condition implies TVD; the positivity also implies TVD; TVD implies monotonicity preservation. Although local monotonicity preservation is related to monotonicity preservation condition, as well as to the TVD condition, no implication can be drawn from one to the other. This hierarchy is shown in Figure 4-6. From bottom to top the conditions become stronger, an upper condition implies a lower condition, directed by an arrow. Some reference texts (Sod 1985; Laney 1998; Chung 2002) are suggested for an in-depth understanding of those definitions and their related properties.

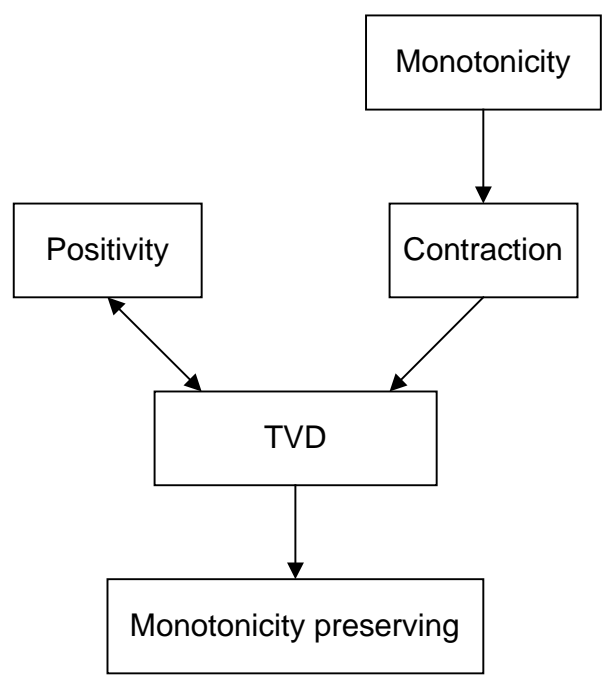

Figure 4-6 A summary of nonlinear stability conditions for scalar conservation laws

As already mentioned in the previous discussion, a monotone method, whose accuracy is only limited to first order, will generally produce too diffusive results and hence rarely used in practice; the same for a scheme that satisfies the contraction condition. Since schemes with locally monotonicity preserving constraint has inherently less-diffusive nature. Experience from (Grinstein and Fureby 2002) shows that a TVD methods, together with locally monotonicity preserving schemes, such as FCT (fluxcorrected transport, (Boris and Book 1973)) and PPM (piecewise parabolic method (Colella and Woodward 1984)), works well for MILES.

(Fureby and Grinstein 1999; Grinstein and Fureby 2002) also analyzed the leading error term with their proposed flux-limiter based scheme. They showed that one of the leading error terms appears as a general subgrid-viscosity model with a tensorial 
diffusivity, while the other leading term mimics the Leonard tensor. Effectively, this becomes an implicit mixed model!

Recently, an even more courageous move is made by (Margolin and Rider 2002; Margolin et al. 2002). In contrast to the flux-limiter based discretization schemes, as those with the MILES, the scheme they were using is more directly based on upwinding. It is for this reason that they introduced a new name, "implicit turbulence modeling" (ITM), to make this implicit SGS model category more general, and to allow more diverse discretization method to be used in this approach. Also, the leading truncation error in their scheme is analyzed for an example of the one-dimensional Burger's equation, and these error terms are further justified with physical rationales. In fact, as pointed out by (Grinstein and Guirguis 1992) the numerical dissipations produced by those upwind-based schemes, such as QUICK, PPM, TVD and FCT, can all in certain cases be very close to that introduced by a physical model.

In the current study the third order upwind-based QUICK scheme will be used without any explicit models as part of the toolkit to simulate turbulent flows. It is important to note that in the framework of finite volume method, discretization (cf. Section 2.2) is essentially focused on approximating cell face fluxes. While doing so, the conservation property at each computational cell must be always followed. This very fundamental principle also serves in the flux-limiting based schemes. 


\section{Chapter 5 Large Eddy Simulation of Building-Block Turbulent Flows}

In this chapter focus is placed on the large eddy simulation (LES) of turbulent flows. The subgrid scale (SGS) models implemented in this study are the Smagorinsky model, dynamic Smagorinsky model and "no model" (implicit turbulence modeling, ITM). Three classical building-block flows, namely, the turbulent channel flow, flow past a square cylinder and plane mixing layer, are simulated and validated. It is important to note that making the turbulent flow right is a vital step towards the two-phase flow simulation (Part III). The obtained results establish confidence in the fidelity of the developed simulation code and the SGS model.

\subsection{Turbulent Channel Flow}

\subsubsection{Introduction}

Investigation of turbulent channel flow has been carried out in great extent and depth in the literature, using both experimental and numerical techniques. Standard results, such as the mean velocity profiles and Reynolds shear stresses in the near wall region, have been well established. Earlier experimental contribution are mainly made by (Clark 1968; Hussain and Reynolds 1975; Kreplin and Eckelmann 1979). Numerical approaches include RANS (Wilcox 1993), LES (Deardorff 1970; Schumann 1975; Moin and Kim 1982) and DNS (Kim et al. 1987; Mansour et al. 1988; Moser et al. 1999; Abe et al. 2001). Experimental and DNS databases exist in abundance; they are commonly used for the verification of a numerical simulation that involves turbulence modeling, such as the RANS and LES.

Three Reynolds numbers, based on different velocity and length scales, are often used to characterize the turbulent channel flow. They can be written down as:

$$
\operatorname{Re}=\frac{U_{a v e}(2 \delta)}{v}, \operatorname{Re}_{c}=\frac{U_{c} \delta}{v}, \operatorname{Re}_{\tau}=\frac{u_{\tau} \delta}{v},
$$

where $\delta$ is the channel half-width, $U_{\text {ave }}$ is the average streamwise velocity (or bulk velocity), $U_{c}$ is the streamwise center-line velocity and $u_{\tau}$ is the friction velocity. As a reminder, $u_{\tau}$ is defined as

$$
u_{\tau}=\sqrt{\frac{\tau_{w}}{\rho}},
$$

where $\tau_{w}$ is the wall shear stress given by

$$
\tau_{w}=\left.\mu \frac{d\langle u\rangle}{d y}\right|_{y=0} .
$$


In Eq. (5.3) as well as in the following appearance it is agreed that $y$ represents the wallnormal direction. Further, a length scale in the a turbulent channel is commonly normalized by either the half channel width ( $\delta$-units), or the wall units (denoted by a plus sign), which is defined, e.g. for the y-position, as

$$
y^{+}=\frac{y u_{\tau}}{v} .
$$

Limited by the computational capacity, the current highest $\mathrm{Re}_{\tau}$ of a DNS, is 590 (Moser et al. 1999) and 640 (Abe et al. 2001). Their corresponding $\mathrm{Re}_{r}$ and $\mathrm{Re}_{c}$ are summarized in Table 5-1. The present channel simulation will be validated with (Abe et al. 2001)'s (referred to as AKM henceforth) DNS data.

Table 5-1 Reynolds numbers of available DNS channel calculations

\begin{tabular}{|c|c|c|c|}
\hline & $\operatorname{Re}_{\tau}$ & $\mathrm{Re}$ & $\operatorname{Re}_{c}$ \\
\hline (Moser et al. 1999) & 590 & 21870 & 12485 \\
\hline (Abe et al. 2001) & 640 & 24326 & 13984 \\
\hline
\end{tabular}

\subsubsection{Computational Details}

Consider a computational domain of $2 \pi \times 2 \times \pi$, in $\delta$ units, in streamwise, vertical and spanwise directions, respectively (Figure 5-1). This selection of domain lengths is guided by the comments made by (Moin and Kim 1982). In that, it is pointed out that the twopoint correlation of velocity fluctuation in the streamwise direction and away from the wall becomes negligibly small after $3.2 \delta$, according to a previous measurement. Similarly, the two-point correlation in the spanwise direction becomes weak after $1.6 \delta$. Thus, if a periodic boundary condition is applied in these two directions, the computational domain should be at least twice as large as these dimensions.

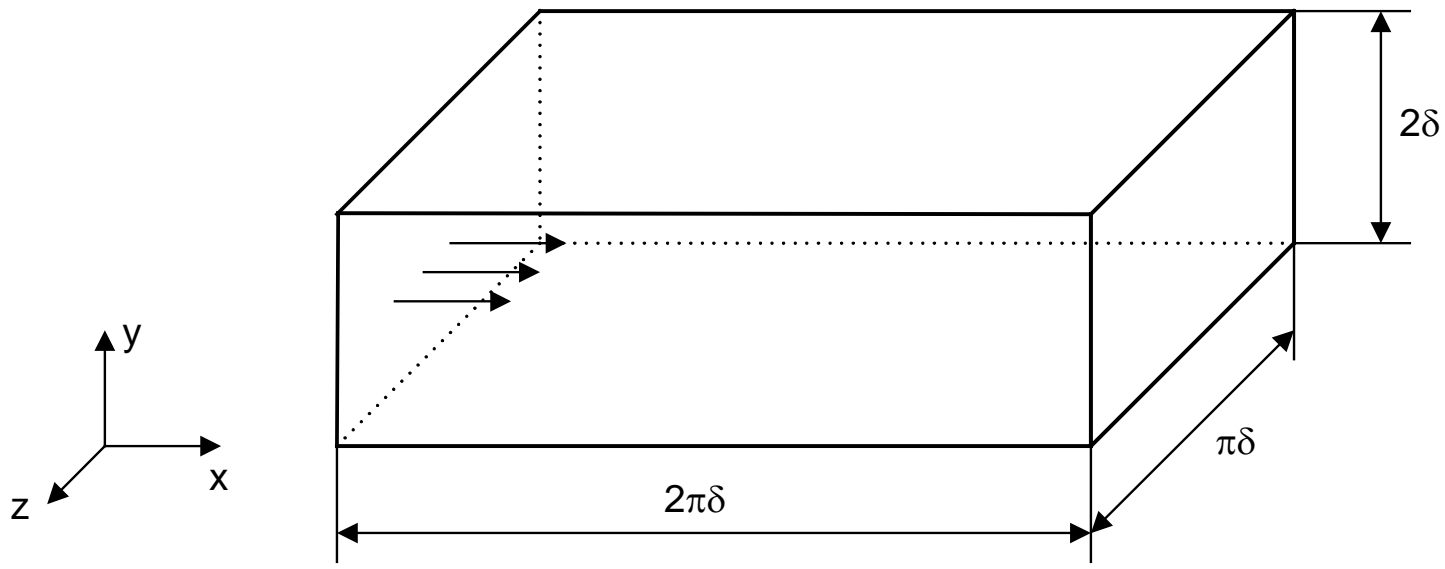

Figure 5-1 Geometry of the channel flow

The factors in the selection of grid resolution are twofold. First, the grid size should be small enough to accommodate important scales of motion in the flow. Second, 
the total number of computational nodes should be within the doable range in accordance with the available computational resource. In the wall-normal direction sufficient grid nodes should be placed within $\mathrm{y}^{+}<5$ in order to properly resolve the viscous sublayer. From previous experiences, accommodating two computational cells in this layer can already yield satisfactory results. Due to the very fine resolution used in the near wall region, a non-uniform grid distribution in this direction is inevitable. Near the wall the important large-scale structures are the "streaks" (Kline et al. 1967), these structures are relatively finely spaced in the spanwise direction, with a mean spanwise distance of 100 and the most probable spanwise spacing of about 80, in wall units. Their mean spacing characterizes the length scale of eddies in the viscous sublayer; thus, a calculation with inadequate resolution in the spanwise direction may result in an overprediction of the viscous sublayer thickness. Moreover, in the experimental studies of (Kline et al. 1967; Clark and Markland 1970), they occasionally observed U-shaped vortices in the inner region $(\mathrm{y} / \delta<0.1)$; (Clark and Markland 1970) reported that the average streamwise spacing of these structures is 440 wall units. Based on the above guidelines, a $64 \times 64 \times$ 64 grid system is adopted in this study. The grid nodes are uniformly distributed in the $x-$ and z-directions, while in the y-direction a non-uniform grid with a constant expansion ratio equal to 1.12 is employed. The expansion ratio is selected in such a way that two computational cells are located within $\mathrm{y}^{+}<5$. A finer resolution calculation with $64 \times 96$ $\times 112$ computational cells and a smaller expansion ratio of 1.08 is also carried out in this study to examine the mesh size influence. Table 5-2 summarizes the domain length (denoted by $L$, in $\delta$-units), number of grid nodes (denoted by $N$ ), and the grid spacing (denoted by $h$, in wall units) used in the present simulations.

Table 5-2 Spatial resolution of the channel flow simulation, $\operatorname{Re}_{\tau}=640$

\begin{tabular}{|c|c|c|c|c|c|c|c|c|c|}
\hline Case & $L_{\mathrm{x}}(\delta)$ & $L_{\mathrm{y}}(\delta)$ & $L_{\mathrm{z}}(\delta)$ & $N_{\mathrm{x}}$ & $N_{\mathrm{y}}$ & $N_{\mathrm{z}}$ & $h_{\mathrm{x}}^{+}$ & $h_{\mathrm{y} 1}{ }^{+}$ & $h_{\mathrm{z}}{ }^{+}$ \\
\hline 1 & 6.4 & 2 & 3.2 & 64 & 64 & 64 & 64 & 2.18 & 32 \\
\hline 2 & 6.4 & 2 & 3.2 & 64 & 92 & 112 & 64 & 1.01 & 18.3 \\
\hline
\end{tabular}

In the present simulation, periodic boundary conditions are applied in the streamwise and spanwise directions. At two channel walls no-slip conditions are specified to ensure a wall-resolving solution. Note that other boundary conditions in the spanwise direction are possible, such as the slip-wall condition that mimics a laboratory setting.

Three modeling approaches are considered herein: the standard Smagorinsky model, dynamic eddy-viscosity model and implicit turbulence modeling (ITM). In the ITM approach the third order QUICK scheme is employed without any explicit SGS formulation; it is assumed that the dissipation of SGS kinetic energy is implicitly performed by the third-order upwind scheme. For the Smagorinsky model, Gaussian filter (see Table 4-1) is used in planes parallel to the walls in which the flow is statistically homogeneous. For the reasons addressed in Section 4.7, the filter width of the Gaussian filter is taken to be twice as the grid spacing, i.e.,

$$
\Delta_{x}=2 h_{x}, \Delta_{z}=2 h_{z} \text {. }
$$


Owing to the variation of turbulence length scale in the wall-normal direction, a piecewise continuous "top-hat" filter with a variable filter width is used. The filter width can be expressed as

$$
\Delta_{y}(y)=y_{j+1}-y_{j-1} \quad \text { for } \frac{1}{2}\left(y_{j+1}-y_{j}\right)<y<\frac{1}{2}\left(y_{j}-y_{j-1}\right) .
$$

An important consequence of this definition is that the filtering operation and the partial differentiation commutes in open interval defined in Eq. (5.6), and in particular at the computational grid points $y_{j}$ (Moin and Kim 1982). The characteristic filter width $\Delta$ is then defined as

$$
\Delta=\left(\Delta_{x} \Delta_{y} \Delta_{z}\right)^{1 / 3},
$$

which is used as a local variable in the Smagorinsky model expression (Eq. (4.158)). The Smagorinsky constant used by (Deardorff 1970) is 0.1 with $\Delta_{i}=h_{i}, h_{i}$ being the grid size. (Moin and Kim 1982) used 0.065 for this constant since their $\Delta$ is $4^{1 / 3}$ larger than Deardorff's one. In the current study, the defined $\Delta$ is approximately twice as large as the Deardorff's one, therefore, $C_{s}$ is taken to be 0.05 . It should be noted that in the Smagorinsky model no explicit filtering operation is performed; the filtering effect is implicitly reflected through the model expression (4.158), which is linked to the grid size through the characteristic filter width, $\Delta$. As usual, a damping function (Eq. (4.162)) is applied to ensure a proper behavior of residual stresses at the wall region.

The dynamic model is employed in conjunction with a box filter that applies filtering operation in all three directions at both the grid level and the test level. There are three reasons for the use of a box filter instead of a Gaussian filter. First, it is easy to implement in the computational code. Second, a non-uniform Gaussian filter will have difficulty in commuting with differentiation in the wall-normal direction along which the grid distribution is non-uniform. Third, it has been shown that in the physical space the Gaussian and top-hat filter are indeed very similar (Vreman et al. 1994; Vreman et al. 1997); their sample computation further verifies that the results obtained from a dynamic model do not change much if a Gaussian filter is used in place of the box filter. Given a dynamic model that uses box filters, the definition of their filter widths at the two levels can be given as follows: at the grid filter level, one shall still use Eq. (5.5), (5.6) and (5.7) ; at the test filter level, the filter width is then defined as twice (in the $\mathrm{x}$ - and z-direction) or approximately twice (in the y-direction) as the grid filter width, i.e.,

$$
\begin{gathered}
\tilde{\Delta}_{x}=2 \bar{\Delta}_{x}=4 h_{x}, \tilde{\Delta}_{z}=2 \bar{\Delta}_{z}=4 h_{z}, \\
\tilde{\Delta}_{y}(y) \cong 2 \bar{\Delta}_{y}=y_{j+2}-y_{j-2} \text { for } \frac{1}{2}\left(y_{j+1}-y_{j}\right)<y<\frac{1}{2}\left(y_{j}-y_{j-1}\right) .
\end{gathered}
$$

Recall that in the dynamic model the effective double-filter width $\tilde{\bar{\Delta}}$ is needed, in order to evaluate $M_{i j}$ (see Eq. (4.171)). As already mentioned in Section 4.8.3, the consecutive application of two spectral or Gaussian filters can be replaced by an effective filter of the same type, whose filter width can be determined exactly (see Eq. (4.163), (4.164)). Unfortunately, two consecutive filtering using the box filters does not yield an effective top-hat filter, and the resulting filter width is difficult to be expressed analytically. However, estimation can be made which minimizes the error. In (Vreman et al. 1997) it is shown that if

$$
\tilde{\bar{\Delta}}=\sqrt{5} \bar{\Delta}
$$


the error norm becomes minimal. Eq. (5.10) is used here for the dynamic procedure.

In LES where an explicit model is employed the filtered N-S equations should be solved as accurately as possible. This requires (i) a sufficiently large $\Delta / h$ ratio, and (ii) the dissipation caused by the numerical scheme is small. Condition (i) can be met by proper definition of the filter width. In regard with condition (ii) a high-order accurate scheme shall be used for the convective terms, typically the $2^{\text {nd }}$ or $4^{\text {th }}$ order CD (central differencing), or QUICK. In the present study, the baseline discretization methods are summarized as follows: the diffusion and convection terms are discretized using the $2^{\text {nd }}$ order CD; time integration is advanced using the semi-implicit (Crank-Nicolson) scheme for the diffusion terms, and the $2^{\text {nd }}$ order explicit Adams-Bashforth for the convective terms, respectively; and the pressure Poisson equation is solved using the $4^{\text {th }}$ order deferred correction (see Section 2.8). Also, other spatial discretization for the convective terms will also be considered. It is henceforth agreed that, terms such as " 2 nd order CD," " 4 th order CD" and "QUICK" always refer to a discretization method applied to the convective terms, while other discretization details follows the baseline setup.

At this point it is worthwhile to mention that, to prevent numerical diffusion, the nonlinear convection terms in the momentum equation is sometimes recast into the skewsymmetric form (Gresho 1991), which is a linear combination of half of the standard divergence form, $\nabla \cdot(\mathbf{u u})$, and half of the advective form, $\mathbf{u} \nabla \cdot \mathbf{u}$. When the skewsymmetric form is discretized it is relatively easier than the other forms to conserve kinetic energy. When a central scheme is applied to the skew-symmetric form, it is called a kinetic-energy-conserving scheme, meaning that the total kinetic energy is conserved apart from the viscous and compressibility effects. Although this class of scheme prevents "blow up" of kinetic energy, it does loose momentum conservation property and exhibits possible instabilities, such as spurious wiggles (Vreman et al. 1997). Hence, the divergence form of the momentum equations (see Section 1.1) is used here along with the central differencing to achieve certain energy-conserving property.

A constant time step of 6.4E-4, non-dimensionalized by $\delta / u_{\tau}$, is used in the simulation. The CFL (Courant-Friedrichs-Lewy) number, defined by

$$
\mathrm{CFL}=\max \left(\Delta t\left|\frac{\bar{u}}{h_{x}}\right|+\Delta t\left|\frac{\bar{v}}{h_{y}}\right|+\Delta t\left|\frac{\bar{w}}{h_{z}}\right|\right),
$$

is monitored throughout the computations, and it never exceeds 0.1 . The simulation is considered complete when the numerical solution reaches statistically steady state. The equilibrium state can be identified by total mean shear stress and turbulent kinetic energy of the velocity field.

\subsubsection{Results}

The results of the Smagorinsky model and dynamic model are very similar. The ITM with QUICK yields fairly diffusive turbulence quantities. Therefore, in what follows, only the results obtained from the standard Smagorinsky model will be presented. For 
simplicity, in this section, let $\phi$ denote a generic filtered quantity (without the overbar), $\langle\phi\rangle$ the corresponding mean (long-time averaged) quantity, and $\phi$ ' the resolved total fluctuation defined by $\phi^{\prime}=\phi-\langle\phi\rangle$. Therefore, $\langle u\rangle$, for example, denotes the mean streamwise velocity, and $u^{\prime}$ the instantaneous fluctuation about $\langle u\rangle$.

Figure 5-2 shows the mean streamwise velocity profile over an entire channel cross-section. The presented mean quantity is normalized by the centerline velocity $U_{c}$, and averaged over the spanwise direction to yield a smooth curve. The profile shown in Figure 5-2 is typical in a turbulent channel flow, as it has sharp gradient near the wall while it is relative flat in the center region. When the mean velocity profile is expressed in the wall units, i.e., in terms of $y^{+}$(Eq. (5.4)) and $u^{+}\left(=u / u_{\tau}\right)$, and the $y^{+}$is plotted in the logarithmic scale (Figure 5-3), one obtains a close-up view of the near-wall region. It is seen that the linear variation in the viscous sublayer $\left(y^{+}<5\right)$ as well as the classical log-law profile in the outer layer $\left(y^{+}>30\right)$ is accurately recovered. As a reminder, the log-law, due to(von Karman 1930), can be expressed as

$$
u^{+}=\frac{1}{\kappa} \ln y^{+}+B,
$$

with a typical value of $\kappa=0.41$ (von Karman constant) and $B=5.2$. For comparison, also included in Figure 5-3 is the data from a DNS (Abe et al. 2001), which employed $512 \times$ $256 \times 256$ grid nodes, 128 times as many as that in the LES computation. The resolved turbulence intensity, represented by the root mean square (RMS) of the velocity fluctuations, is depicted in Figure 5-4. The RMS values are normalized by the wall friction velocity $u_{\tau}$. The overall agreement of results from the LES and DNS is good. It is common that in LES of a turbulent channel flow the $u_{\text {rms }}$ is overpredicted while the $v_{\text {rms }}$ and $w_{\text {rms }}$ are underpredicted, the reason being the insufficient grid resolution in all three directions. In fact, the uncertainty in the predicted turbulence levels is the tradeoff a LES usually has to take at the convenience of largely reduced computational grid nodes.

The detailed flow structures can be examined by looking at contour plots of instantaneous velocity, pressure and vorticity field. In the following presentation attempt is made to recover some important flow features discussed in (Moin and Kim 1982). Note that the velocity components used herein are normalized by $U_{c}$, and pressure by $\rho U_{c}$. It is well recognized that a distinct feature of the flow pattern is the existence of highly elongated regions of high-speed fluid ("streaks"), in the vicinity of the wall. This is evident in the $u$ ' contour plot in the (x, z)-plane cut through a viscous sublayer $y^{+}=5.77$ (Figure 5-5). Consistent with the findings of (Kline et al. 1967) and (Moin and Kim 1982), these high- and low-speed structures alternate in the spanwise direction, and there are several localized regions ("pockets") of very high-speed fluid inside the pockets, identifiable by the large concentrations. When the (x, z)-plane moves away from the wall (Figure 5-6, at $y=0.59 \delta$ ), the streaky pattern disappears, confirming that it is a unique characteristic of the wall-layer turbulence. In all the contour plots shown here, positive values are marked by solid lines and negative values by dashed lines. Figure 5-7 shows the pressure contours, again at $y^{+}=5.77$. In contrast to $u^{\prime}$, the pressure patterns are not stretched in the streamwise direction. It is also seen that the regions of high-pressure 
fluctuations (with large gradient) are generally located in the vicinity of the pockets of the streaks, an important observation reported in (Falco 1978) and (Moin and Kim 1982).

Next, locate a high-speed streak at about $\mathrm{z}=0.83 \delta$ in the lower left corner of Figure 5-5. Then make a vertical (x,y)-plane cut through the center of this streak. The resultant contour plot of $u$ ' and vertical velocity is shown in Figure 5-8 and Figure 5-9, respectively. It is seen that, in near the lower wall, the high-speed streak $(u>0)$ between $\mathrm{x}=0$ and $2.5 \delta$ has a negative vertical velocity $\left(v^{\prime}<0\right)$, implying a sweep event. On the other hand, an ejection ( $\mathrm{v}^{\prime}>0$ ) event can be observed between $\mathrm{x}=2.5 \delta$ and $3.2 \delta$ for a low-speed region $\left(u^{\prime}<0\right)$. A further distinct feature captured in Figure 5-8 is that the above-identified high-speed structure is inclined at an oblique angle with respect to the wall. As pointed out by (Moin and Kim 1982), this is an evidence of the action of mean shear on the fluid elements that moves from the outer layer towards the wall. The mean angle of inclination of these structures reported by (Rajagopalan and Antonia 1979) is $13^{\circ}$.

A close look should also be taken at the (y, z)-plane. Figure 5-10 and Figure 5-11 show the contour plots of $u$ ' and $v$, respectively, in the lower half (y, z)-plane sliced at $\mathrm{x}$ $=3 \delta$. The flow patterns are then magnified in Figure 5-12 through Figure 5-14 for the near-wall region with the vertical extent up to $y^{+}=50$. Note that the contour lines in these enlarged views are highly stretched in the vertical direction. From Figure 5-10 and Figure 5-11 one sees, throughout a large portion of half- channel displayed, there is a negative correlation between $u$ ' and $v$, including both in the wall layer and in the channel center region. In Figure 5-12 the alternating array of the high- and low-speed structure along the spanwise direction is clearly discernible. The mean spacing between two adjacent highspeed streaks is about $0.24 \delta$, or 150 in wall units. Compared to the experimental value of 100 wall units, this distance is still a little large. Similar overprediction is also reported by (Moin and Kim 1982), whose simulation yields about 250 wall units. Figure 5-13 displays the alternating feature of positive and negative regions of the vertical velocity, identifying the fluid portion moving away or towards the wall. An important message it conveys is that, due to the strong slip, shear layers will be formed in the respective region interfaces, and these shear layers may further undergo Helmholtz-type instabilities in the $(\mathrm{y}, \mathrm{z})$-plane. As a consequence, the streamwise vortices can be formed. This is evident through the contour plot of the streamwise vorticity and the streamlines plot on the same $(\mathrm{y}, \mathrm{z})$-plane (Figure 5-15). Conforming to Figure 5-13 and Figure 5-12, the most intense streamwise vortices, $\omega_{x}$, are concentrated near the wall, and they are separated by a distance on the order of the mean streak-spacing. From Figure 5-15 it is further seen that the eddy size in the wall region is significantly smaller than that in the region away from the wall. Figure 5-13 and Figure 5-14 together demonstrate that, the high-speed streams moving towards the wall produce a splatting effect, forcing the fluid elements at the impinging location to leave with opposite velocities in the spanwise direction. Such flow pattern is very similar to that of a jet impingement on a plate. 


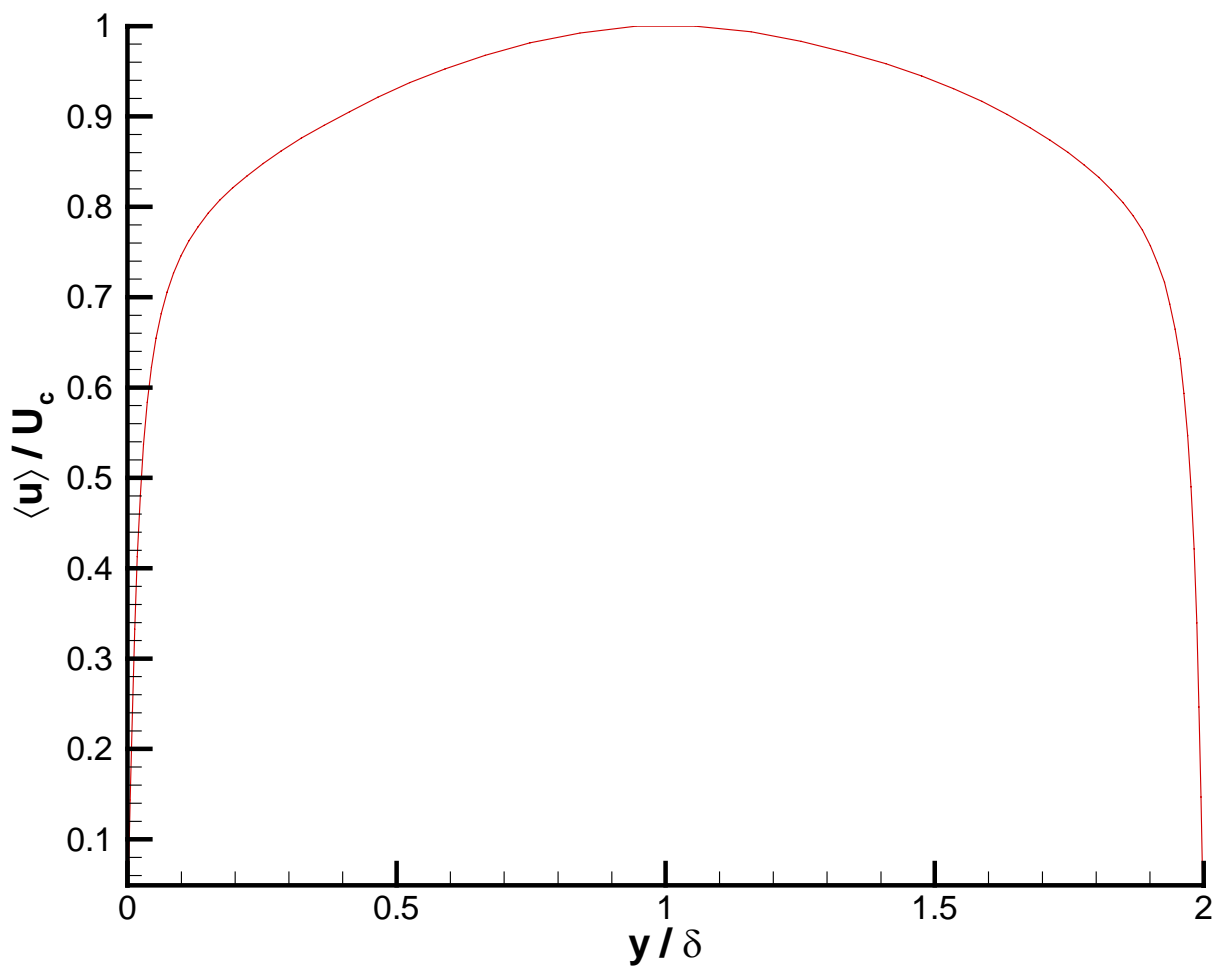

Figure 5-2 Mean streamwise velocity profile in a turbulent channel flow. 


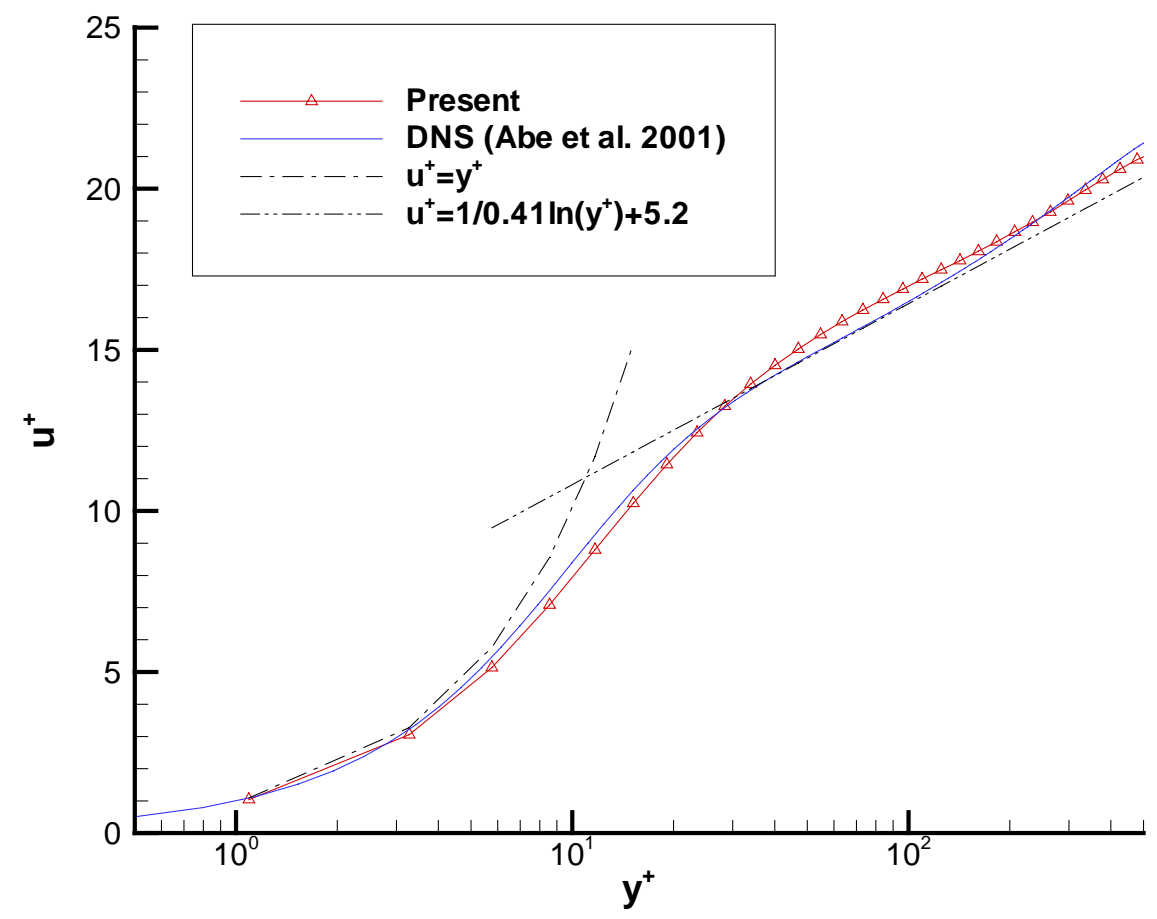

Figure 5-3 Mean-velocity logarithmic profile in a turbulent channel flow.

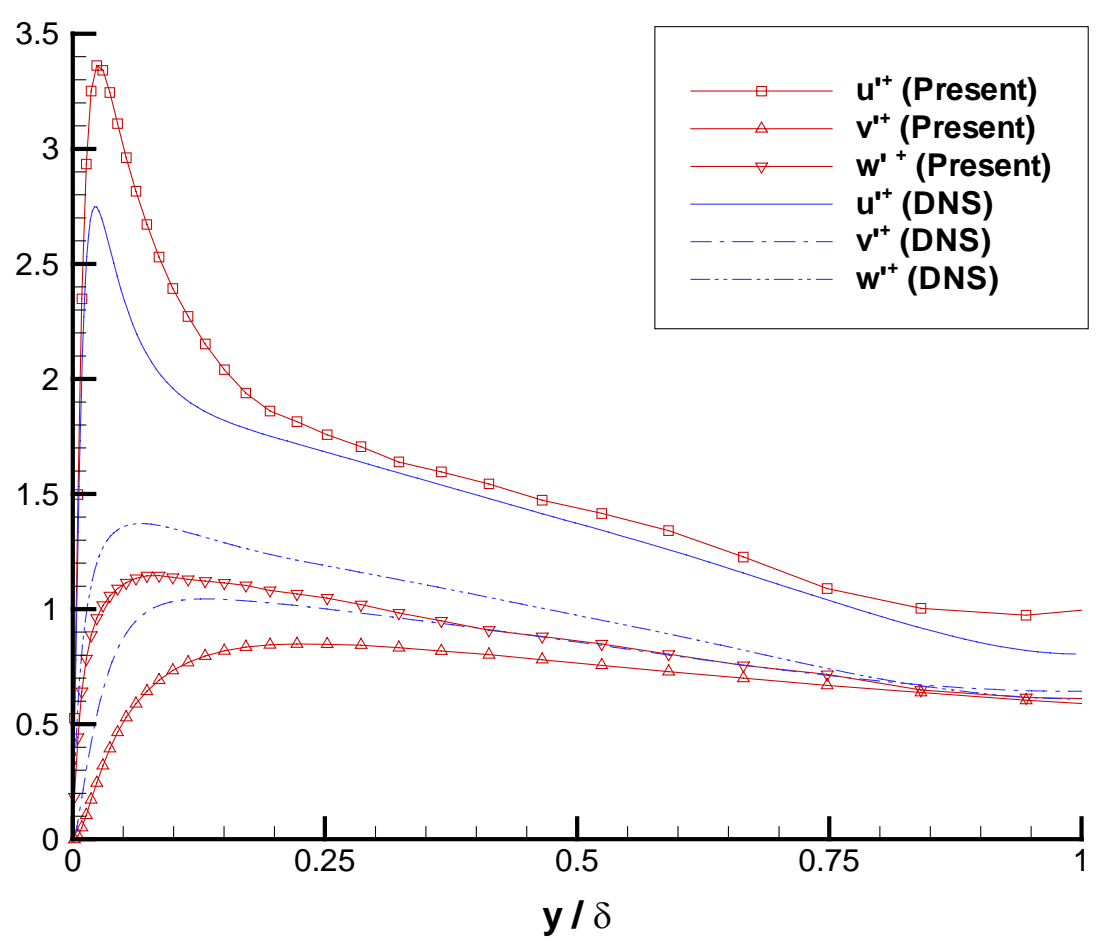

Figure 5-4 Resolved turbulence intensity in a turbulent channel flow. 


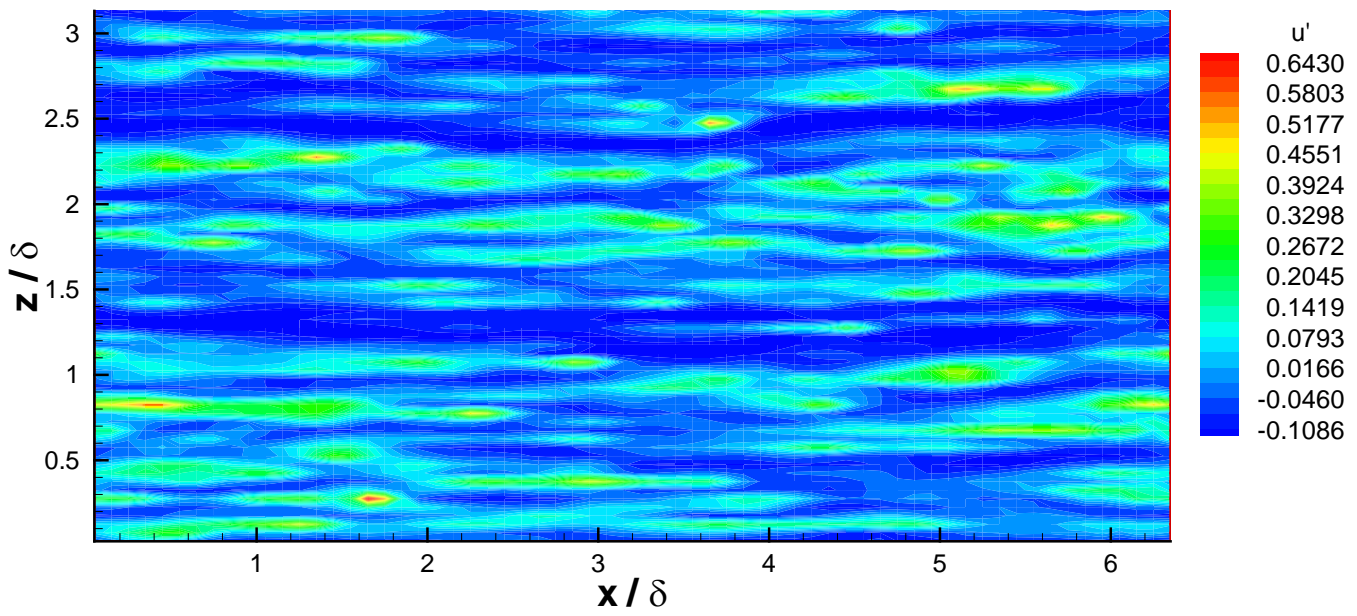

Figure 5-5 Contours of $u^{\prime}$ in the $(x, z)-p l a n e$ at $y^{+}=5.77$.

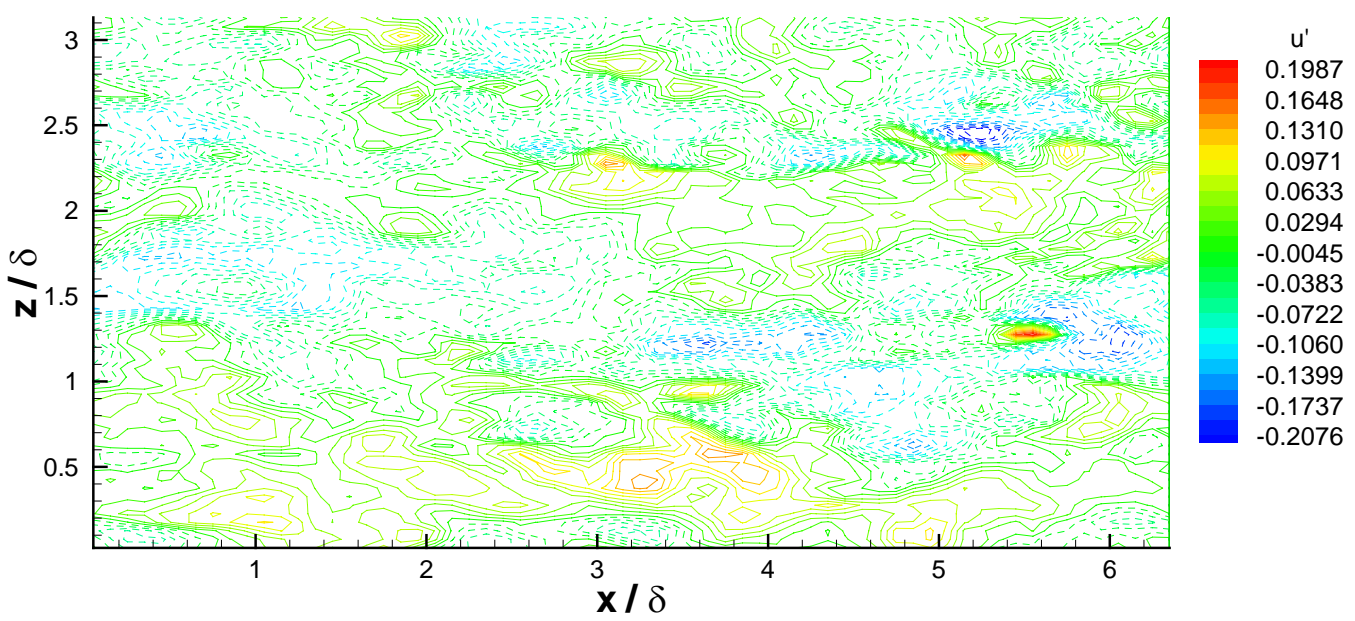

Figure 5-6 Contours of $u^{\prime}$ in the $(x, z)-p l a n e$ at $y=0.59 \delta$.

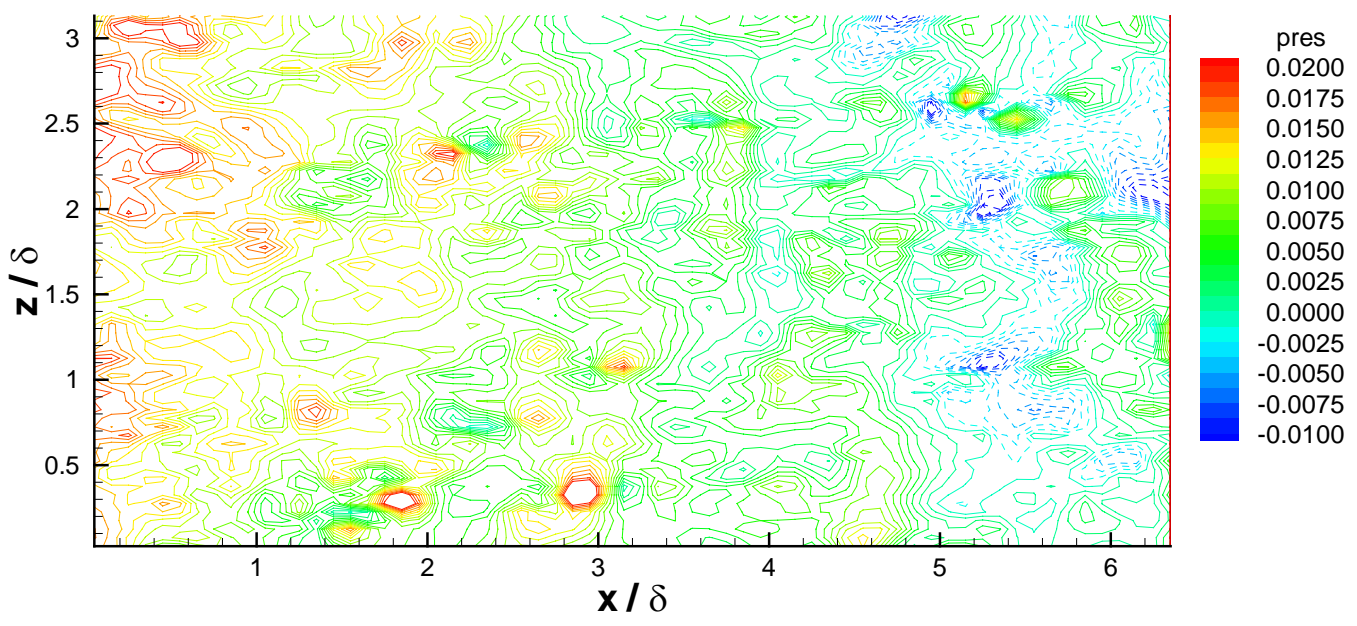

Figure 5-7 Contours of pressure in the $(x, z)$-plane at $y^{+}=5.77$. 


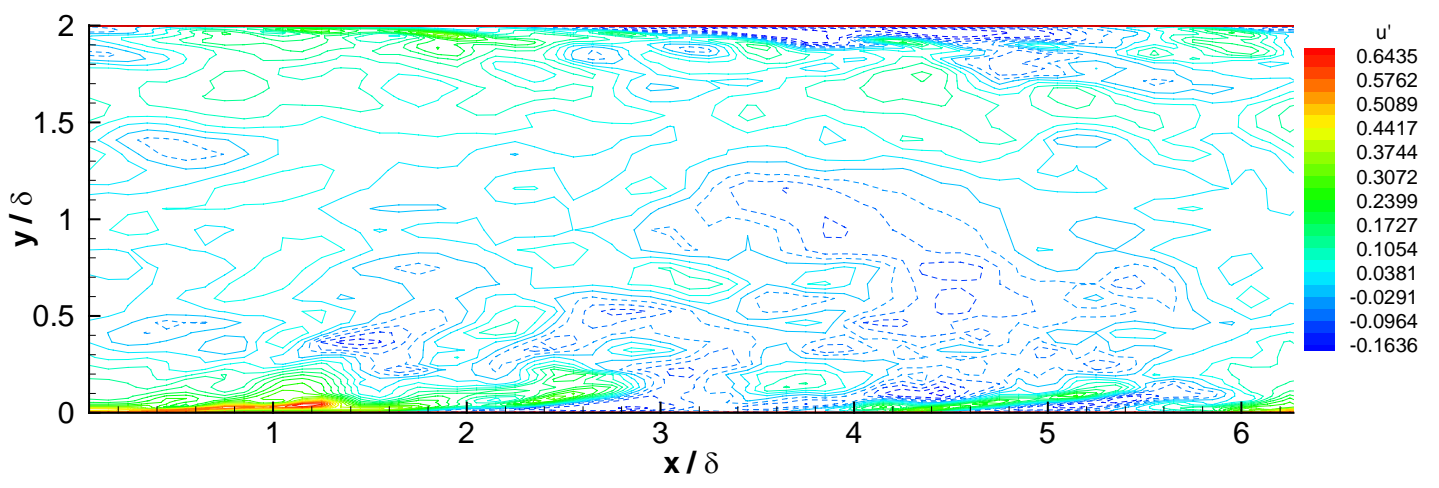

Figure 5-8 Contours of $u^{\prime}$ in the $(x, y)$-plane at $z=0.83 \delta$.

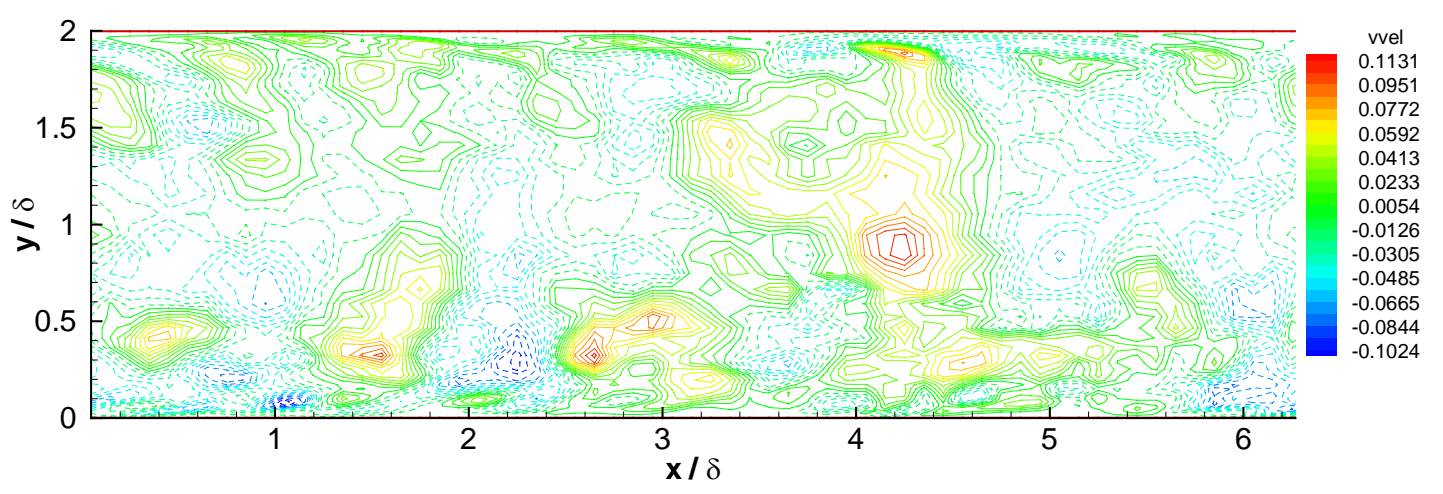

Figure 5-9 Contours of $v$ in the $(x, y)$-plane at $z=0.83 \delta$. 


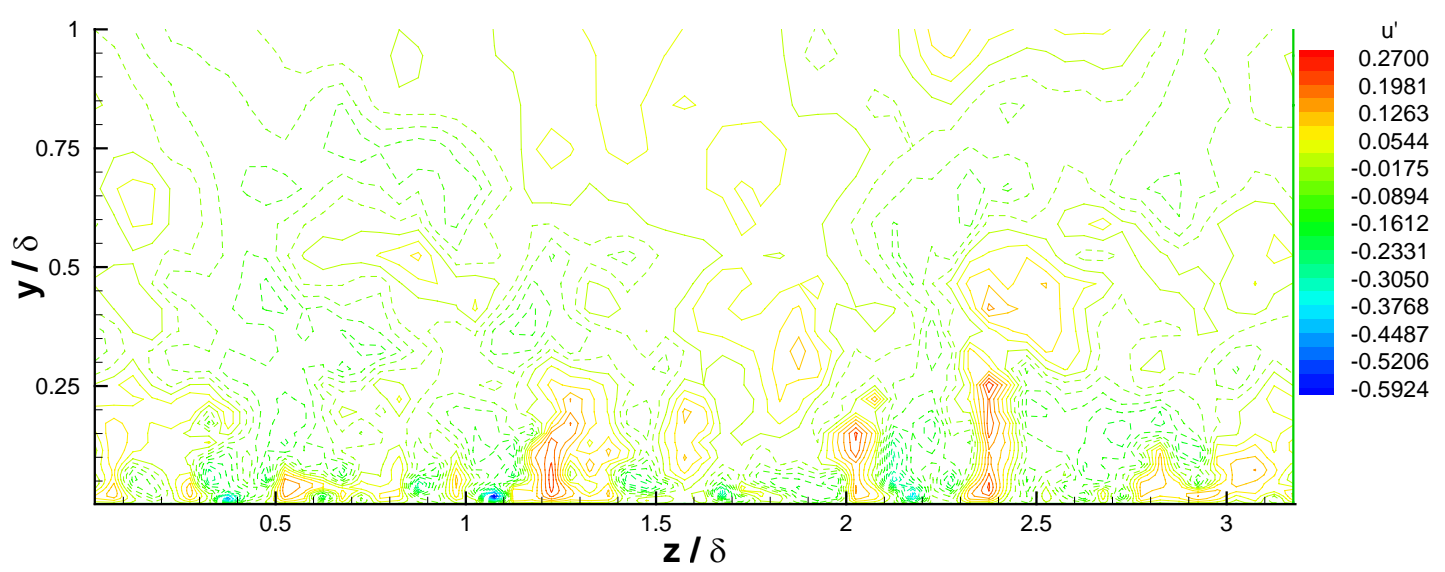

Figure 5-10 Contours of $u^{\prime}$ in the lower half $(y, z)$-plane at $x=3 \delta$.

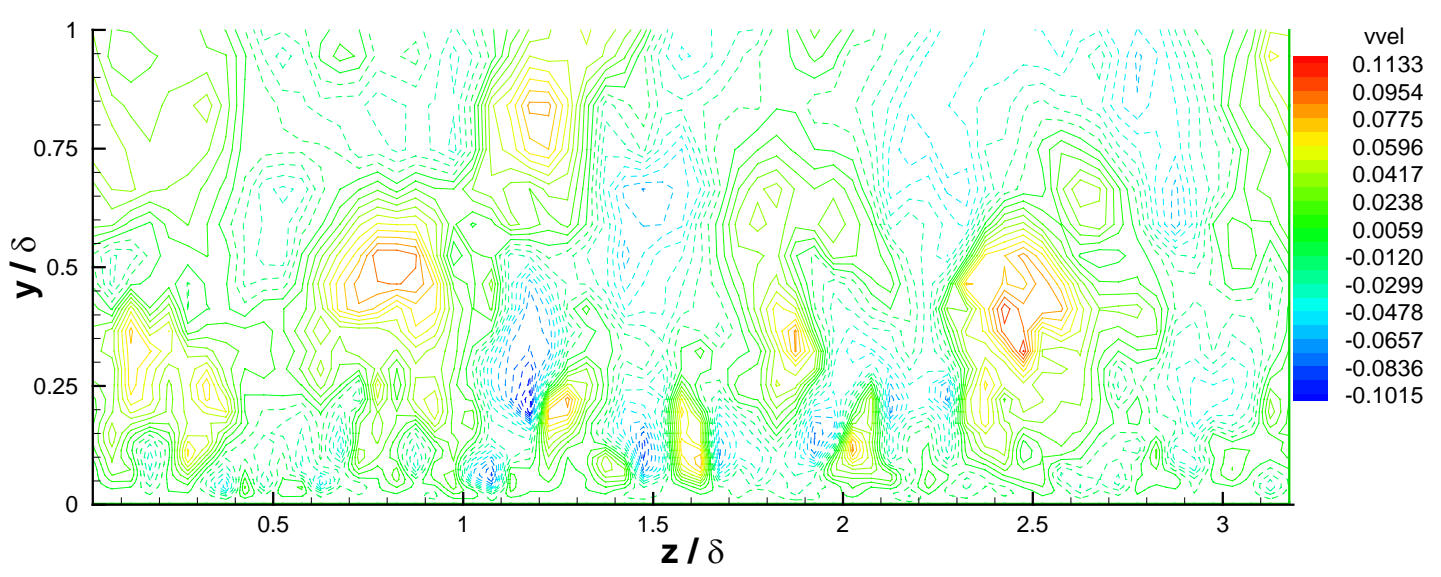

Figure 5-11 Contours of $v$ in the lower half $(y, z)$-plane at $x=3 \delta$.

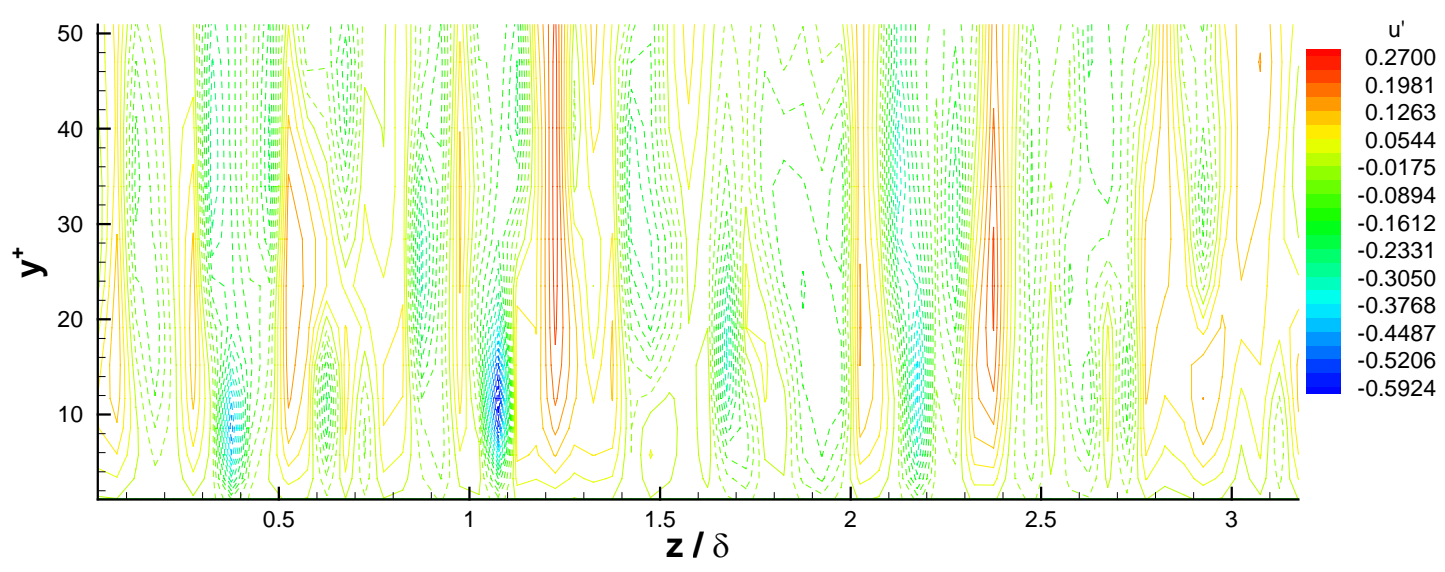

Figure 5-12 Contours of $u^{\prime}$ in the $(y, z)-p l a n e$ at $x=3 \delta, y^{+}<50$. 


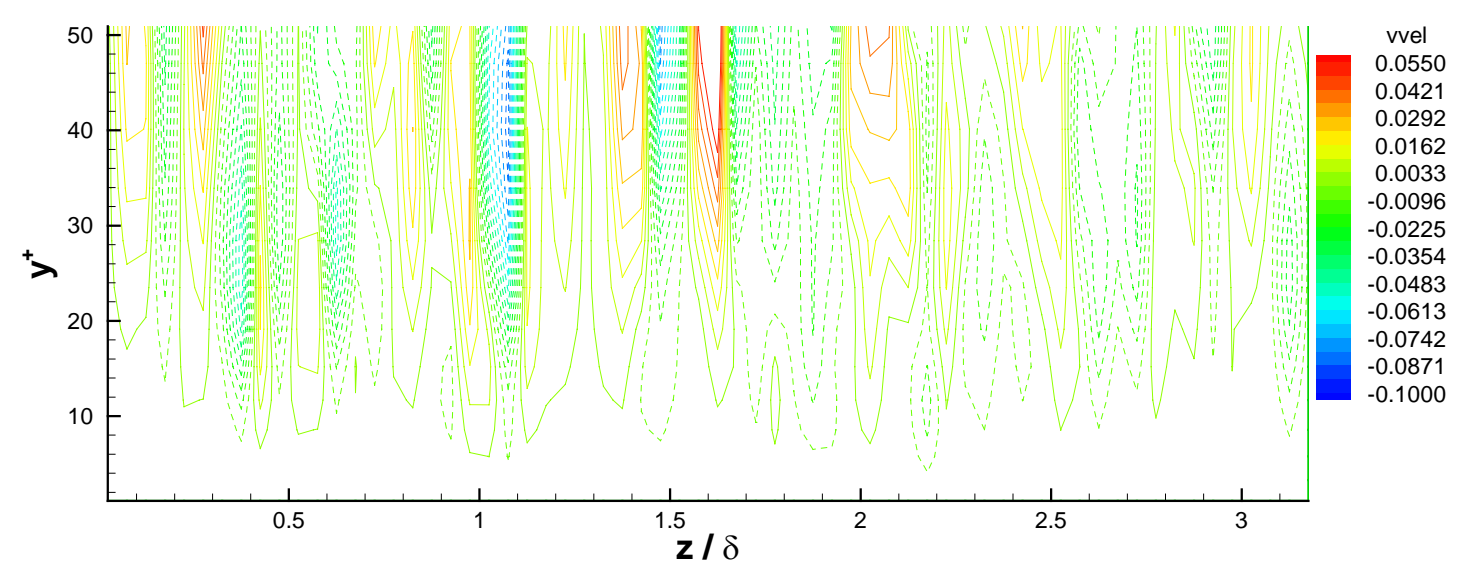

Figure 5-13 Contours of $v$ in the $(y, z)-p l a n e$ at $x=3 \delta, y^{+}<50$.

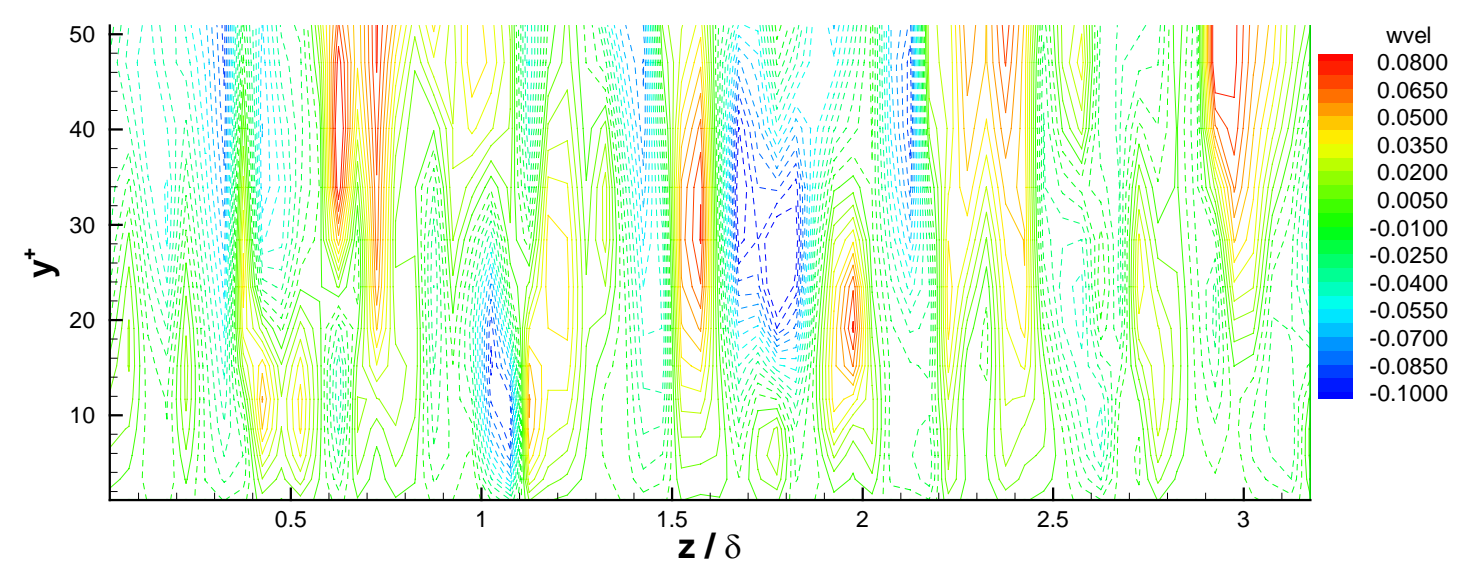

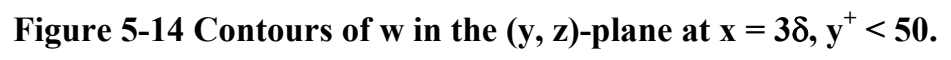

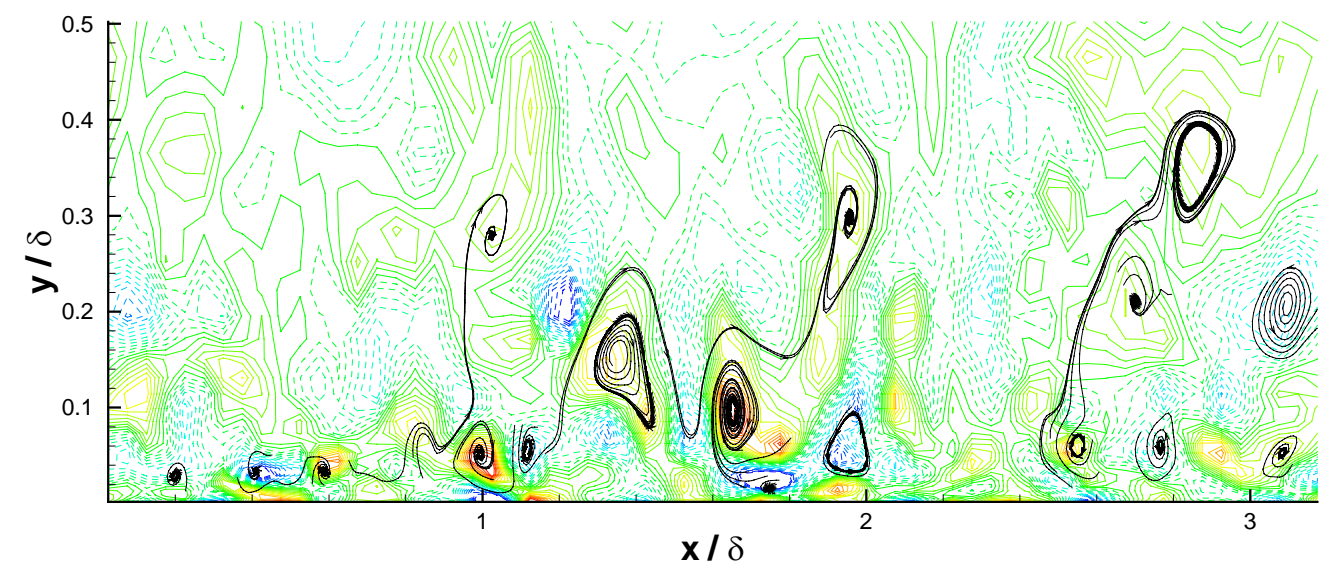

Figure 5-15 Contours of streamwise vorticity and streamlines in the $(y, z)-p l a n e$ at $x=3 \delta$. 


\subsection{Plane Mixing Layer}

\subsubsection{Introduction}

A plane mixing layer is a turbulent flow that forms between two parallel fluid streams of different velocities. It is a particular (but canonical) case of a broader flow class named free shear flows. As the name "free" implies, this type of flows are remote from walls. Other examples of free shear flows are jets and wakes. In this section sought is an understanding some fundamental features and LES of the plane mixing layer.

Since (Brown and Roshko 1974)'s landmark discovery of large, coherent and apparently two-dimensional structures in turbulent mixing layers, there has been considerable research aimed at exploring the mechanism for their formation, the role of the coherent vortical structures, and their eventually breakdown into random turbulent motion (see a review provided by (Ho and Huerre 1984)). These coherent structures have been said to engulf fluid into the mixing layer, and they are related to the spanwise vortices, called "rollers," which form as a result of Kelvin-Helmholtz instability in a laminar shear layer.

Before and in the early stage of the transition to turbulence, the rollers may undergo the "pairing" process (Winant and Browand 1974), whereby neighboring rollers rotate about each other and amalgamate (see Figure 5-16). Pairings generally lead to an increase in vortex size and a decrease in number of vortices, and they are responsible for the shear layer growth. Also possible is the so-called "tearing" (Moore and Saffman 1975), in which one small vortex located between two larger ones is torn apart and its vorticity is redistributed to its larger neighbors (Figure 5-17). It should be noted that the two-dimensional array of rollers, along with the possible pairing and tearing process, is commonly viewed as a kind of two-dimensional turbulence, a stage before the fully turbulent mixing layer. In fact, it is probably known by far as the only exception of the more universally defined three-dimensional turbulence.

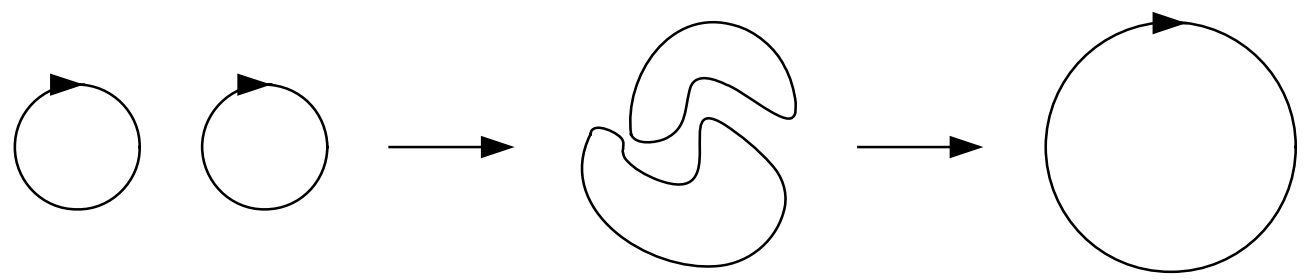

Figure 5-16 Illustration of vortex "pairing" in a plane mixing layer.

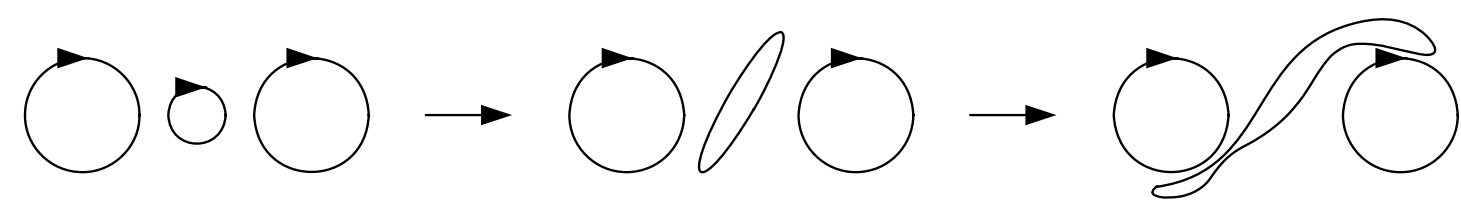

Figure 5-17 Illustration of vortex "tearing" in a plane mixing layer. 
During the late stage of the transition to a fully turbulent mixing layer, several secondary instability mechanisms can operate, giving rise to the streamwise vortices, called "rib-vortices" (Figure 5-18). These rib-vortices are mainly located in the "braid" region (the region between rollers), counter-rotating, and extend from the bottom of one roller to the top of the next. One possible cause is described by (Corcos and Lin 1984; Lin and Corcos 1984): the vorticity in the braid region is strained by the neighboring vortices and undergoes an instability that stretches it into longitudinal vortices. Also, a single two-dimensional vortex is itself unstable to three-dimensional disturbances. Suppose the vortex develops a slight kink. The self-induced velocity created by the kink can result in a stretching of the vortex in the streamwise direction, forming a so-called hairpin vortex or vortex tube. The lengthening of a single kink can further induce kinking of the neighboring vortices, producing a kind of chain reaction. The existence of the streamwise vortical structures have been evident from a number of experiments (Pierrehumbert and Widnall 1982; Jimenez 1983; Bernal and Roshko 1986; Lasheras et al. 1986; Lasheras and Choi 1988), as well as from DNS calculations (Rogers and Moser 1992, 1994).

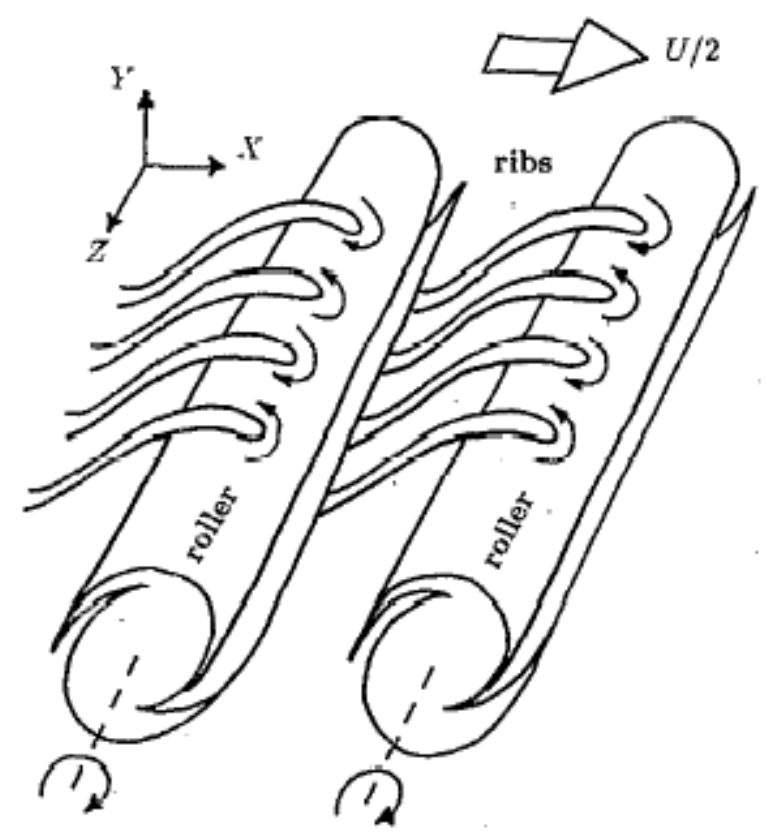

Figure 5-18 Illustration of "rib-vortices" in a plane mixing layer (provided by (Lopez and Bulbeck 1993)).

Besides the occurrence of the rib-vortices, the three-dimensional instability may also lead to a different type of pairing, known as the local pairing or helical pairing. The concept of the local pairing is primarily based on the experiments of (Chandrsuda et al. 1978) and the analytical work of (Pierrehumbert and Widnall 1982). In contrast to (Winant and Browand 1974)'s classical pairing, local pairings are fairly localized in space, usually occur in the spanwise direction, and they do not necessarily evolve into a single merged vortex. In addition to the above cited references, the local pairing has also been observed and reported in other transitional mixing layers, for example in the 
experiments of (Nygaard and Glezer 1991; Nygaard and Glezer 1994) and the DNS calculations of (Comte et al. 1992; Moser and Rogers 1993; Collis et al. 1994; Rogers and Moser 1994). On the other hand, it is fully possible that the local pairing does not exist, such as in (Brown and Roshko 1974) and (Hussain and Zaman 1985)'s experiments, as well as in one of (Rogers and Moser 1994)‘s simulations.

Quite often, an ordinary mixing layer is perturbed in form of forcing functions either initially, for a temporal evolving mixing layer, or continuously at the inlet boundary for a spatially developing one. A forced mixing layer could exhibit very different behavior as compared to an unforced one. Studying the forced mixing layer is itself an extensive topic because the forcing can be implemented in a variety of ways, according to its dimensionality, direction, frequency, strength, phase variation, uniformity, and randomness. The primary purpose of adding disturbance is to excite instabilities that occur naturally in the mixing layer, so that the processes, such as the roller formation and pairing, will occur in a controlled manner. A prominent result in this regard is that the subharmonic disturbances, i.e., the subharmonic modes of the fundamental frequency of instabilities in the mixing layer, can help trigger and sustain the classical pairing process, and subsequently expedite the transition to turbulence (Kelly 1967; Ho and Huang 1982; Pierrehumbert and Widnall 1982). In a further experimental study of (Ho et al. 1991), it is found that the subharmonic modes can also cause phase decorrelation (or phase jitter) which leads to a more random merging location of the a vortex pair. In the numerical simulation of (Sandham and Reynolds 1989), they noted that by "jittering" the inlet forcing, i.e., randomly varying the phases of the exciting modes, very realistic results were obtained. (Wilson and Demuren 1996) performed a simulation excited with a broad spectrum of modes, aiming at emulating a realistic experimental condition; they observed that the vortex pairing in this kind of forcing occurs over a region but not at a fixed location, as is the case with the subharmonic forcing. Using the three-dimensional direct numerical simulation, (Collis et al. 1994) examined the influence of a spanwise nonuniform forcing on the vortical structure. In (Lazaro and Lasheras 1992b; Rightley and Lasheras 2000)'s experiments they studied developing mixing layers and pointed out that a single-wave periodic perturbation operating at the fundamental frequency of instability with very small amplitudes lead to a rapid growth of the Kelvin-Helmholtz billows, and greatly enhance the coherence of the large-scale structures. Given above reviews it is seen that the seemingly simple plane mixing-layer indeed contains a wealth of complex physical information.

For clarity, some basic quantities often used to characterize a mixing layer should be summarized here. Let $U_{h}$ denote the fast-speed stream, and $U_{l}$ the low-speed stream. Their difference is denoted by $\Delta U$, i.e.,

$$
\Delta U=U_{h}-U_{l} .
$$

The average convective velocity $U_{c}$ is then

$$
U_{c}=\frac{1}{2}\left(U_{h}+U_{l}\right) .
$$

The normalized velocity difference, $R$, can be defined as (Ho and Huerre 1984) 


$$
R=\frac{\Delta U}{2 U_{c}} .
$$

It measures the relative magnitude of the total shear, $\Delta U$. When $R=0$, it reduces to a wake; when $R=1$, only one stream is present.

From the linear stability analysis, it is known that the mixing layer has three inherent instability frequencies. The most unstable one is known as the fundamental frequency, denoted by $f_{0}$; Its corresponding wavelength is

$$
\lambda_{0}=\frac{U_{c}}{f_{0}} .
$$

Thus, the streamwise station (x-direction) can be normalized as

$$
x^{*}=\frac{x}{\lambda_{0}}, \text { or } x^{*}=R \frac{x}{\lambda_{0}},
$$

which gives the approximate location of the $x^{*}$ th Kelvin-Helmholtz billow. The $y-$ coordinate can be non-dimensionalized by the mixing a length thickness, for example, $\delta_{\omega}$.

The mixing layer width, $\delta$, can be defined in many ways. The frequently used ones are the level thickness, momentum thickness, and vorticity thickness. At each xstation, one can define a non-dimensional longitudinal velocity profile normalized with the free stream velocities, i.e.,

$$
\beta(y)=\frac{u(y)-U_{l}}{U_{h}-U_{l}} .
$$

Clearly, $0<\beta<1$, and a large and small $\beta$ indicates a cross-stream location close to the high-speed stream edge and the low-speed edge, respectively. The level thickness is then defined as

$$
\delta_{L}=y(\beta=0.9)-y(\beta=0.1) ;
$$

The momentum thickness is defined as

$$
\delta_{\theta}=\int_{-\infty}^{\infty} \beta(y)[1-\beta(y)] d y
$$

The definition for the vorticity thickness (or shear thickness) is given by

$$
\delta_{\omega}=\left.\frac{\Delta U}{\partial U / \partial y}\right|_{y=0},
$$

where $U$ is the mean velocity profile.

The Reynolds numbers used in a mixing layer can be defined accordingly based on the initial or inlet mixing layer thickness $\left(\delta_{L 0}, \delta_{\theta 0}, \delta_{\omega 0}\right.$ etc.), i.e.,

$$
\operatorname{Re}_{\delta}=\frac{U_{c} \delta_{l 0}}{v}, \operatorname{Re}_{\theta}=\frac{U_{c} \delta_{\theta 0}}{v}, \operatorname{Re}_{\omega}=\frac{U_{c} \delta_{\omega 0}}{v} .
$$

In general, the two parameters, $R$ and the Reynolds number, provide an overall characterization of a mixing layer.

The rate of change of the mixing layer thickness can be quantified through the spreading rate (or growth rate) defined by 


$$
r=\frac{d \delta}{d x} \quad \text { or } \quad r=\frac{U_{c}}{\Delta U} \frac{d \delta}{d x} .
$$

Note that the second definition uses $R / 2$ as its normalization factor, and it is more general since it eliminates the effect of different velocity ratios (Abramovich 1963; Sabin 1965; Brown and Roshko 1974). The spreading rate tells how fast a mixing layer grows.

In certain development stage of a mixing layer, the flow, albeit turbulent, may undergo self-similarity, i.e., the mean velocity profiles taken at different streamwise locations collapse into an identical one if they are properly scaled. The scaled crossstream coordinate $\xi$ can be defined as

$$
\xi=\frac{y-y_{\text {ref }}}{\delta},
$$

with $y_{\text {ref }}=\frac{1}{2}[y(f=0.9)+y(f=0.1)]$; and the scaled mean u-velocity is given by

$$
f(\xi)=\frac{\langle u\rangle-U_{c}}{\Delta U}
$$

The similarity region is typically seen in the far field, followed by an exponential growth of the layer thickness. It can be shown (e.g., see (Pope 2000)) that a direct implication of the self-similarity is the presence of the linear spreading rate. In the near field (developing region) of a forced mixing layer, the evolution of the layer thickness can undergo two stages as well: an initial non-linear growth and a linear spreading. However, such a two-region growth is distinct from the one in an unforced mixing layer, and, the linear spreading region in a forced mixing layer usually does not imply a self-similar flow (Lazaro 1989; Lazaro and Lasheras 1992b; Rightley and Lasheras 2000).

In the present study LES of a spatially developing mixing layer will be performed, and the obtained flow solution will be compared with the experimental measurements made by (Rightley 1995). Note that the Rightley's original thesis was later published in (Rightley and Lasheras 2000). For brevity this experiment will occasionally be referred to as R\&L. The primary reason of choosing this benchmark is that the original work not only details the flow field measurements, but also provides a reliable study of a bubbly two-phase flow. Thus, once the carrier-phase flow field is verified, it may be utilized for the two-phase flow simulation in Part III. Other related mixing-layer studies published by Professor Lasheras's group include (Lasheras et al. 1986; Lasheras and Choi 1988; Lazaro and Lasheras 1992a, b). The evolution of the forced mixing layer towards an asymptotic, self-similar state is often significantly delayed (Ho and Huang 1982).

\subsubsection{Computational Details}

In the original experiment (Rightley 1995; Rightley and Lasheras 2000), the study was focused on the developing region of a forced, turbulent mixing layer. The low-speed stream, placed in the upper layer, is separated with the high-speed stream in the lower layer with a splitter plate (Figure 5-19). This arrangement (fast stream below slow stream) is due to practical considerations in the experiment. The flow is forced in the inlet region of the lower layer using a sinusoidal function operating at a frequency $(2.1 \mathrm{~Hz})$ 
near the fundamental frequency and with very small amplitude. With the help of forcing, the Kelvin-Helmholtz instability is triggered at a location very close to the plate tip, and the flow evolves rapidly into a pattern composed of an array of large, coherent spanwise vortices. The mixing layer thickness undergoes an initial non-linear development, followed by a linear spreading. As aforementioned, the linear spreading region seen in the near field of a forced mixing layer should not be taken as an indication of the selfsimilar state. Since the study is confined in the developing region, processes associated with the secondary instability, such as the rib-vortices and local pairing, are not to be expected, and the flow is essentially two-dimensional in the mean.

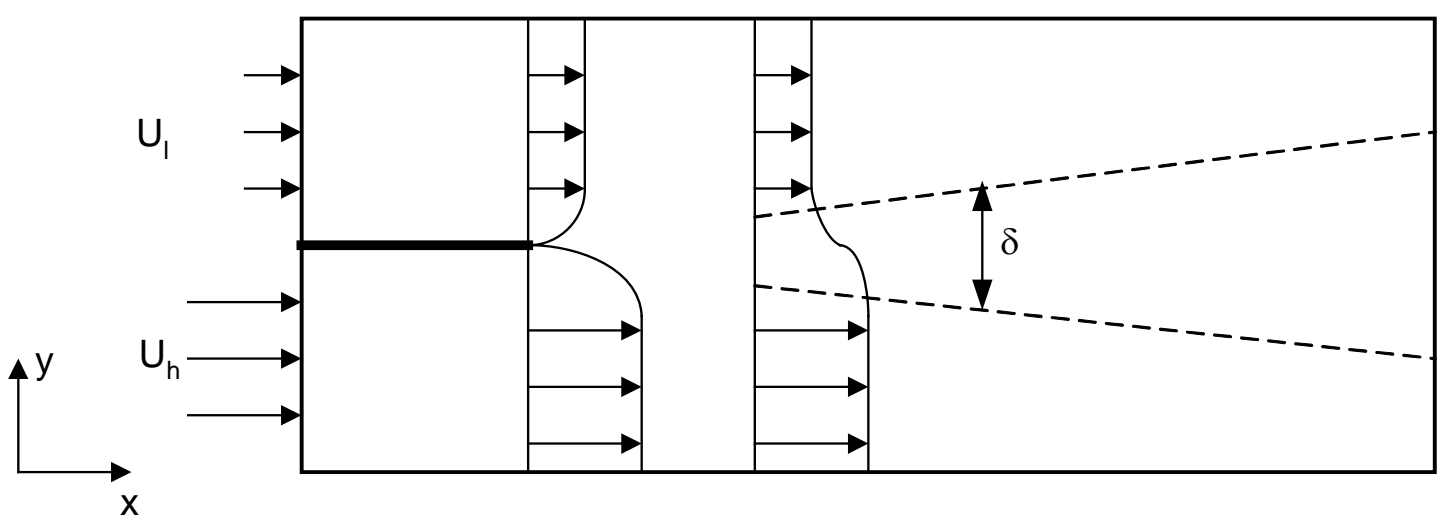

Figure 5-19 Schematic of a plane mixing layer

The computational domain starts from the trailing edge of the splitter plate, and it is sized as $0.4 \mathrm{~m} \times 0.2 \mathrm{~m} \times 0.04 \mathrm{~m}$ in the $\mathrm{x}-, \mathrm{y}$ - and $\mathrm{z}$-directions, respectively. Since the flow is statistically two-dimensional, a relatively small dimension is used in the spanwise direction. The grid contains $N_{x}=128, N_{y}=64$ and $N_{z}=10$ nodes. The grid points are distributed evenly in the $\mathrm{x}$ - and z-directions, yielding a uniform cell size of $\Delta \mathrm{x}=3.125$ $\mathrm{mm}$ and $\Delta \mathrm{z}=4.0 \mathrm{~mm}$, respectively. In the vertical direction, to resolve the mixing region with sufficient accuracy, the cells are concentrated around the center and expanded with a constant ratio of 1.08 towards the lower and upper bound. This yields a smallest cell size of about $0.37 \mathrm{~mm}$.

Two uniform streams with $\mathrm{U}_{l}=0.28 \mathrm{~m} / \mathrm{s}$ and $\mathrm{U}_{h}=0.06 \mathrm{~m} / \mathrm{s}$ are imposed at the inlet of the lower and upper layers, respectively. Other inlet profiles, such as the one displayed at the plate tip in Figure 5-19, is also possible. Numerical experimentations show that two uniform streams help trigger an early instability, as compared to other inlet profiles, and the Kelvin-Helmholtz vortical structures as well as the turbulence statistics in a forced mixing layer is relatively insensitive to the inlet condition. As a result, the choice of two constant inlet velocities reduces the streamwise extent of the computational domain and saves unnecessary computational cells that would have been used to accompany the generation of instability. At the east outlet the standard outlet boundary condition is used. In the vertical direction two slip-walls are imposed, which moves at $\mathrm{U}_{l}$ in the lower layer and $\mathrm{U}_{h}$ in the upper layer. As usual, the periodic boundary condition is applied in the spanwise direction. 
To emulate the forcing effect in the experiment, a two-dimensional sinusoid perturbation is superimposed to the high-speed stream at the lower layer's inlet. The perturbation function can be expressed as

$$
\begin{aligned}
& u_{\text {perturb }}=A \sin (2 \pi f t+\varphi), \\
& v_{\text {perturb }}=B \sin (2 \pi f t+\varphi) .
\end{aligned}
$$

where the frequency $f=2.1 \mathrm{~Hz}$, and $\varphi$ is a random phase that mimics noises existing under the experimental condition. Following (Sandham and Reynolds 1989; Druzhinin and Elghobashi 2001), $\varphi$ is obtained at each time step as

$$
\varphi(t+\Delta t)=\varphi(t)+q \frac{\pi}{12}
$$

$q$ being a random number between -1 and 1 with uniform distribution. The artificial phase jittering (Eq. (5.27)) plays an important role in reproducing a realistic mixing layer. On the other hand, a strictly periodic forcing gave erratic, anomalous results (Sandham and Reynolds 1989; White 1991). In the experimental study of Rightley and Lasheras, it is reported that the small perturbation amounts to about $0.5 \%$ of the lower layer's volume flux. Therefore, in the present simulation, set the forcing amplitude to be $\mathrm{A}=\mathrm{B}=0.0015$ $\mathrm{m} / \mathrm{s}$.

Fluid properties are taken from those of water: $1.0 \mathrm{E} 3 \mathrm{~kg} / \mathrm{m}^{3}$ for the density and $1.0 \mathrm{E}-3 \mathrm{~N} \cdot \mathrm{s} / \mathrm{m}^{2}$ for the dynamic viscosity. The Reynolds number, defined with the longitudinal coordinate $\mathrm{x}$ as $\mathrm{Re}=\Delta \mathrm{Ux} / v$, varies from $2.2 \mathrm{E} 4$ at $\mathrm{x}=0.1 \mathrm{~m}$ upwards. According to the experiment, the Reynolds number at the first measuring station is about 2100 based on the level thickness, and is 430 based on the momentum thickness.

Large-eddy simulation is carried out using the ITM approach and the dynamic SGS model. In both cases, QUICK scheme is used for discretization of the convective terms. For the case with the dynamic model, the box filter is applied for both the grid filtering and test filtering. The relevant definition of the filter width follows that discussed in Section 5.1. Note that the Smagorinsky model is found to (Vreman et al. 1997) be too dissipative during transition, thus is avoided here.

The flow is initialized with the inlet velocity profile in the absence of forcing. The integration is performed with time step $\Delta \mathrm{t}=0.001 \mathrm{~s}$. After the initial flow field is "washed" out of the computational domain sufficient number of times by the mean advection, it can be regarded as nearly stationary. The mean and RMS quantities are then calculated from the stationary flow field.

\subsubsection{Results}

Figure 5-20 (a) shows contours of the mean streamwise velocity, $\langle u\rangle$. In that, the growth of the mixing layer, as well as the tendency of growing into the low-speed stream is clearly seen. This familiar structure also reminds us of a laminar shear layer (cf. Figure $3-36(a)$ ). In the outlet region from $x=0.36 \mathrm{~m}$ the growth rate of the shear thickness is 
hindered, owing to the influence of numerical implementation of the outlet condition. Thus, analysis including this region may give some error. Figure 5-20(b) provides a zoomed view of the first half domain. In that three regions can be identified: an initial induction region (marked by I) where the Kelvin-Helmholtz instability occurs, and a high and a low growth region (II and III, respectively). A slowed-down growth rate is signified by an inflection point discernible between region II and III. Similar process is also observed in the R\&L's experiment shown in Figure 5-21 with about the same extent. It should be noted that a comparison between Figure 5-20(b) and Figure 5-21 can be only qualitative, since the latter picture is obtained in the presence of a dispersed phase (bubbles), and, the experiment has a different inlet condition (with splitter plate), which may let the layer undergo a different distance for respective regions.

In the R\&L's experiment, measurements are taken at five stations, i.e., $x / \lambda=0.31$, $0.63,1.25,1.88$ and 2.50 , where $\lambda=0.08 \mathrm{~m}$ is the most unstable wavelength, or billow-tobillow distance. In the present simulation, due to the not completely identical inlet and boundary conditions, instability and initial development may occur at a different location. Therefore, a shift of $\mathrm{x}$-coordinate is necessary to allow for a direct comparison with the profiles taken from the measurements. Here, the $\mathrm{x}$-axis is displaced such a distance that the level thickness at the first measuring station $(\mathrm{x} / \lambda=0.31)$ matches the computed value. This yields a shift of $\mathrm{x}$-coordinate for about $0.1 \mathrm{~m}$ in the present data set.

Based on the shifted coordinates, the mean and RMS profiles in the vertical direction are generated at those five x-stations (Figure 5-22 through Figure 5-27), where results in Figure 5-22 through Figure 5-24 are obtained from the dynamic model, and those in Figure 5-25 through Figure 5-27 are from ITM (LES without a SGS model). The measured profiles for the streamwise component are displayed in Figure 5-28(a) and (b). The following can be seen from the comparison: (i) the results with the dynamic model and ITM are similar in terms of mean and RMS quantities. (ii) The overall trend of the shear thickness development presented in the $\langle u\rangle$-profiles is well captured. (iii) The overall increasing tendency of the RMS values from the $\mathrm{x} / \lambda=1.25$ station is well predicted. (iv) There is underprediction of RMS values at the first two stations, and overprediction of RMS far downstream. The underprediction can be due to high turbulence level present in the inlet region (close to the tip of the splitter plate) in the actual experiment. The possible causes of the overprediction will be addressed in the next paragraph. (v) A shift of the peak RMS values towards the low-speed stream is well predicted.

For a better comparison, the results with the dynamic model, "no model" and experiment are put together for the stations $x / \lambda=0.63$ (Figure 5-29 through Figure 5-31) and $x / \lambda=2.50$ (Figure 5-32 through Figure 5-34). From these plots, it is seen that the overall agreement with the experiment is satisfactory. The RMS quantities from the dynamic model yield closer agreement with the measurements. Notably, the numerical solutions generally overpredict the RMS values in the mixing core region with respect to the experiments, while give underestimation in the regions away from the center (see Figure 5-31, Figure 5-33 and Figure 5-34). The differences could be attributed to several factors. First, the mixing layer experiment was conducted in an open channel; the 
computational domain to the interest of the simulation is away from the walls and free surface. This suggests that the boundary conditions needed by a numerical simulation, especially in the vertical direction, could be difficult to specify, since flow variables at those boundaries are in general time- and location-dependent. An accurate solution will thus demand a full knowledge of temporal-spatial evolution of flow variables at those boundaries. In practice, one commonly uses either a slip-wall (used in the present simulation) or a stress-free condition $\left(\partial u_{\mathrm{i}} / \partial y=0\right)$ to serve as an approximate vertical boundary condition. Due to the fact that possible influence of the wall turbulence and turbulence at free surface on the fluctuation intensity in the mixing core region is not well known, it is speculated that the channel wall and free surface may have to be resolved to yield a better emulation of the actual experiment. A second cause could be related to the forcing. In the experiment, the forcing effect is realized via a loudspeaker that is placed far upstream of the inlet and drives a small cylindrical plug of water into and out of the lower stream. A macroscopic quantity, the volume flux in the lower stream, is reported to oscillate according to a sinusoid function. Nevertheless, how the forcing actually propagates through the long entrance region, and what form the perturbation actually takes at the inlet of the computational domain, remain open questions. From the numerical point of view, the overestimation may also point to two plausible causes: the turbulent energy dissipation supplied by the SGS model or the numerical scheme could be insufficient; the resolution in the third dimension (only ten computational cells) could be underweighted, since the effect of the third dimension on the fluctuating field could be significant. In summary, the deviations of the RMS values between the simulation and experiment are possibly due to insufficient characterization of the boundary conditions, and aspects of in the numerical methods.

Figure 5-35 gives a comparison of the predicted $\mathrm{u}_{\mathrm{rms}}$ along the center $\mathrm{x}$-line obtained from the dynamic model and the ITM, respectively. The x-axis has been shifted following the aforementioned principle. Overall, the two curves are in good accord with each other. However, in the far field, the fluctuation level with the ITM is higher than that of the dynamic model. Considering the generally overpredicted $u_{\mathrm{rms}}$ with reference to the experiment, this picture implies that, for this particular flow case, the energy dissipation mechanism operated by the dynamic model is superior over ITM. This conclusion also conforms to (Vreman et al. 1997)'s finding.

An integral quantity, namely, the level thickness (cf. Eq. (5.19)) is calculated at selected locations. Its variation along the streamwise direction is depicted in Figure 5-36. $\mathrm{R} \& \mathrm{~L}$ fitted a straight line based on the measured thicknesses in the linear spreading region; and it is given by

$$
\delta_{L}=0.134 x+9.1 \text {, }
$$

where $x$ and $\delta_{L}$ are in mm. For a comparison, this line is also shown on the same figure. It is seen that the two curves are in excellent agreement in terms of both the magnitude and slope. The close agreement of the slope simply tells the growth rate of the mixing layer is correctly predicted.

Next, the instantaneous flow structure is examined. Figure 5-37(a) depicts instantaneous contours of the spanwise vortices. The vector field and streamlines at the 
same instant are shown in Figure 5-37(b). The large, coherent vortical structures are clearly seen in these pictures. In Figure 5-37(b) the mean convective velocity $(0.17 \mathrm{~m} / \mathrm{s})$ has been subtracted from the streamwise velocity component. In doing so, the recirculating eddy structures become visible to an observer who travels along with the flow at a speed equal the mean streamwise velocity. In the region of interest, i.e., between $0.1 \mathrm{~m}$ and $0.35 \mathrm{~m}$, the average billow-to-billow distance is around $0.08 \mathrm{~m}$.

The R\&L's experimental study also provided phase-averaged velocity vector fields (Figure 5-38 (a) and (b)). These fields record the time evolution of velocity vectors along a vertical line at a designated $\mathrm{x}$-station. The recording is started when the phase angle of the forcing function is zero, and lasts for two full periods. After recording, a phase averaging is performed over the instantaneous data, with one period broken evenly into ten phase bins. Finally, the mean velocity is subtracted from the streamwise velocity component to yield the two temporal velocity fields. The phase angle on the abscissa is related to elapsed time according to

$$
\theta=2 \pi f t,
$$

where $f=2.1 \mathrm{~Hz}$. In essence, the vector history generated in this way may also be viewed as a prediction of possible spatial structure extending from the recording $x$-location up to a distance advected by the mean velocity for two periods, i.e., two wavelengths. Therefore, in the present simulation, two instantaneous vector fields are also created conditioned on the zero phase angle of the forcing function. The domain of Figure 5-39(a) extends from $x / \lambda=1.25$ to 3.25 , and that of Figure $5-39$ (b) from $x / \lambda=1.88$ to 3.88. Again, the constant mean streamwise velocity has been subtracted. By comparing the experiments and simulation, very similar topological flow structure can be found. At the $\mathrm{x} / \lambda=1.88$ station, for example, it is seen (i) two Kelvin-Helmholtz billows with their cores located at about $\theta=120^{\circ}$ and $480^{\circ}$, (ii) the free stagnation point at about $350^{\circ}$, and (iii) perturbations in the vertical velocity that extend into the lower free stream. Note that this spatial-temporal link can be justified by Taylor's hypothesis that essentially approximates spatial correlations by temporal ones. Yet, the accuracy of the hypothesis will depend both upon the properties of the flow and the statistics being measured. In free shear flows, many experiments have shown that Taylor's hypothesis fails (Pope 2000). Therefore, caution must be taken when interpreting this surprisingly good agreement. 


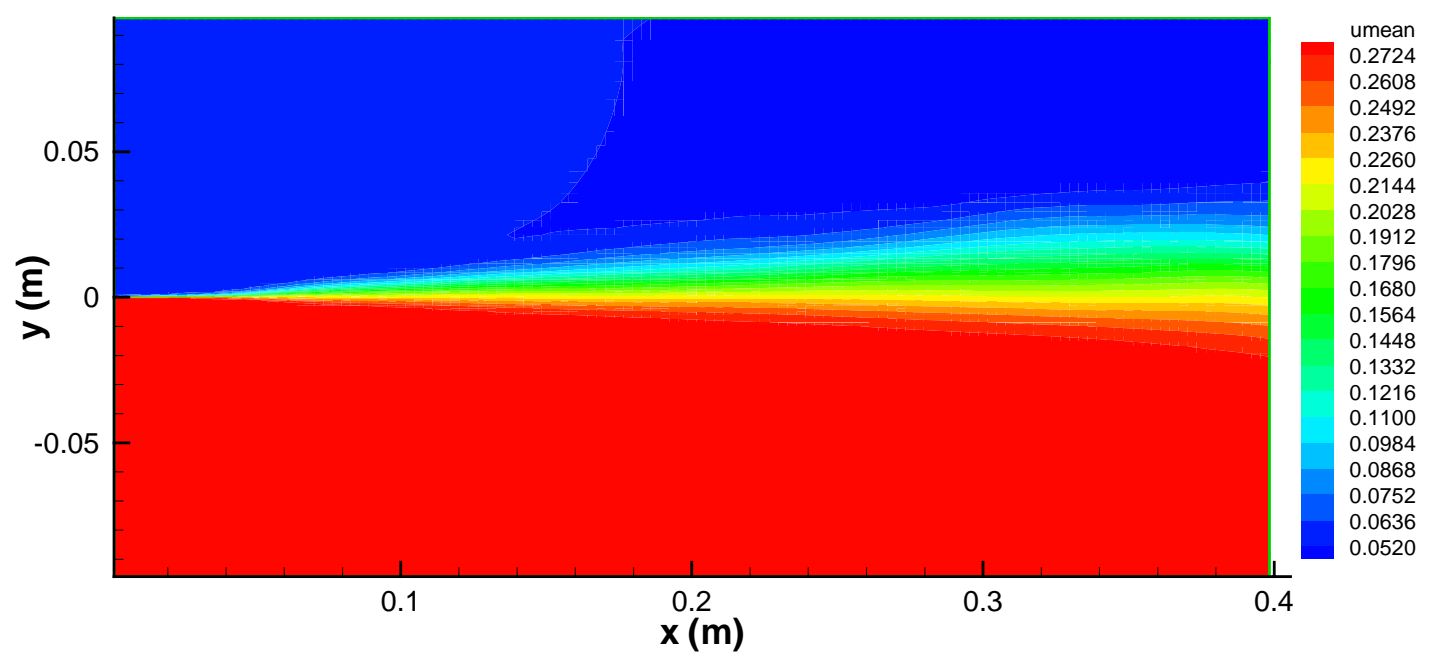

(a)

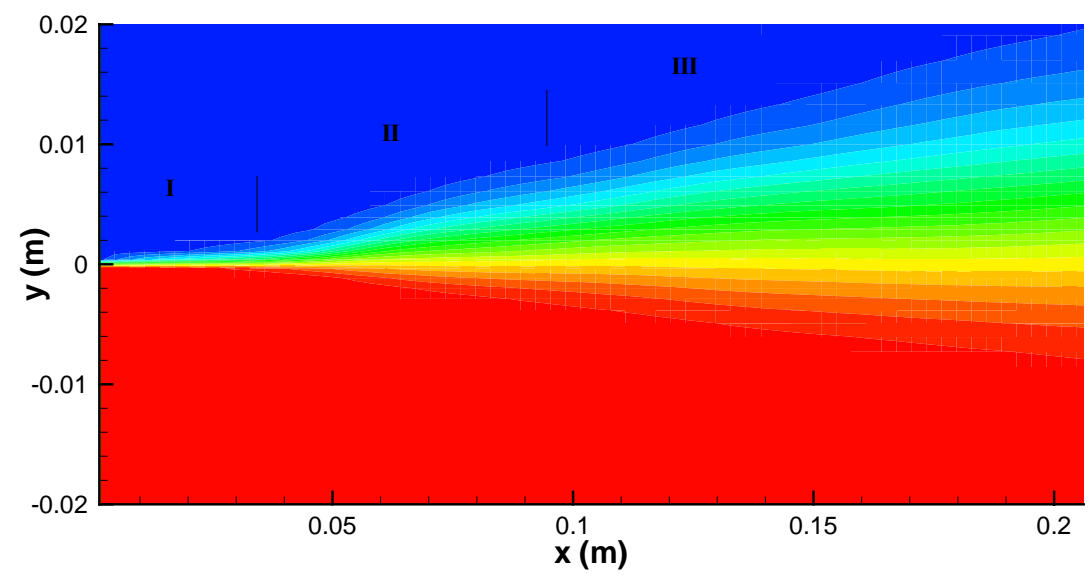

(b)

Figure 5-20 Contours of mean streamwise velocity of a mixing layer: (a) entire domain, (b) zoomed view of near field.

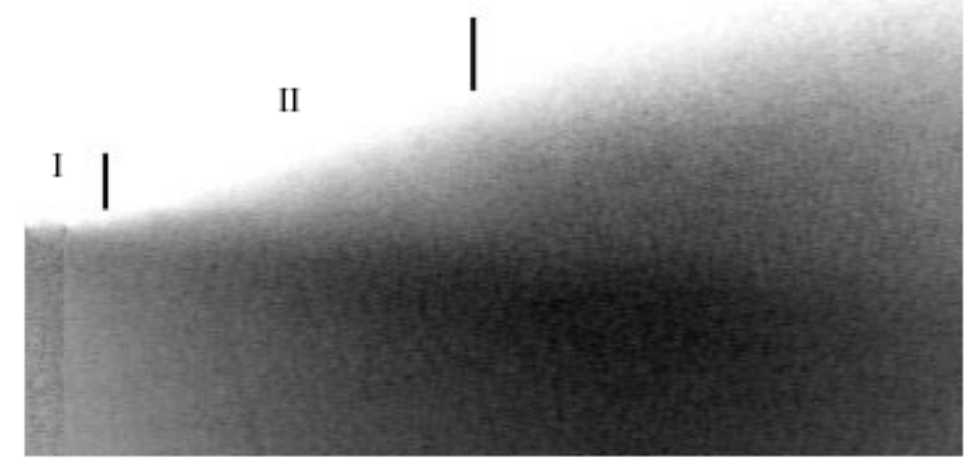

Figure 5-21 Mean flow visualization from Rightley \& Lasheras (2000). 


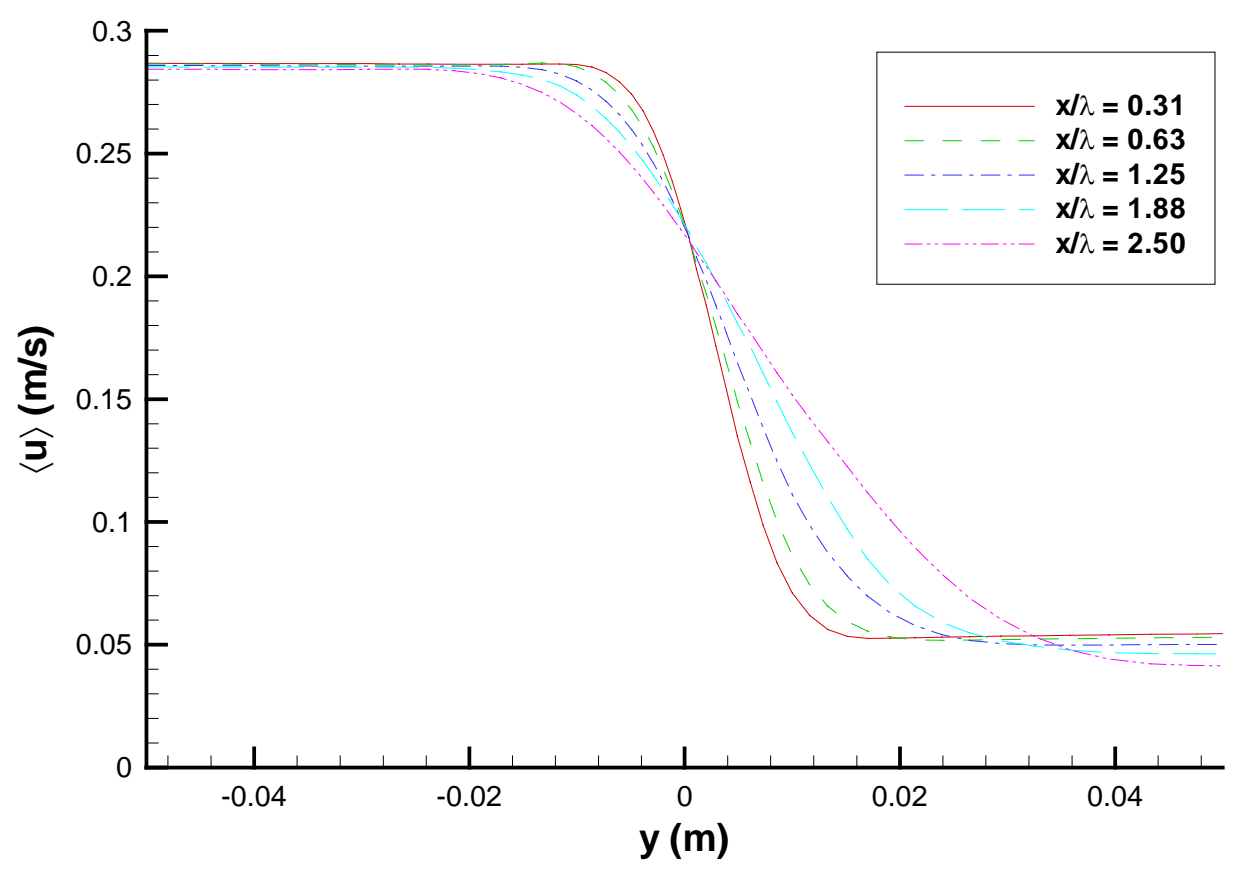

Figure 5-22 Mean streamwise velocity profiles in a mixing layer. Dynamic model is used.

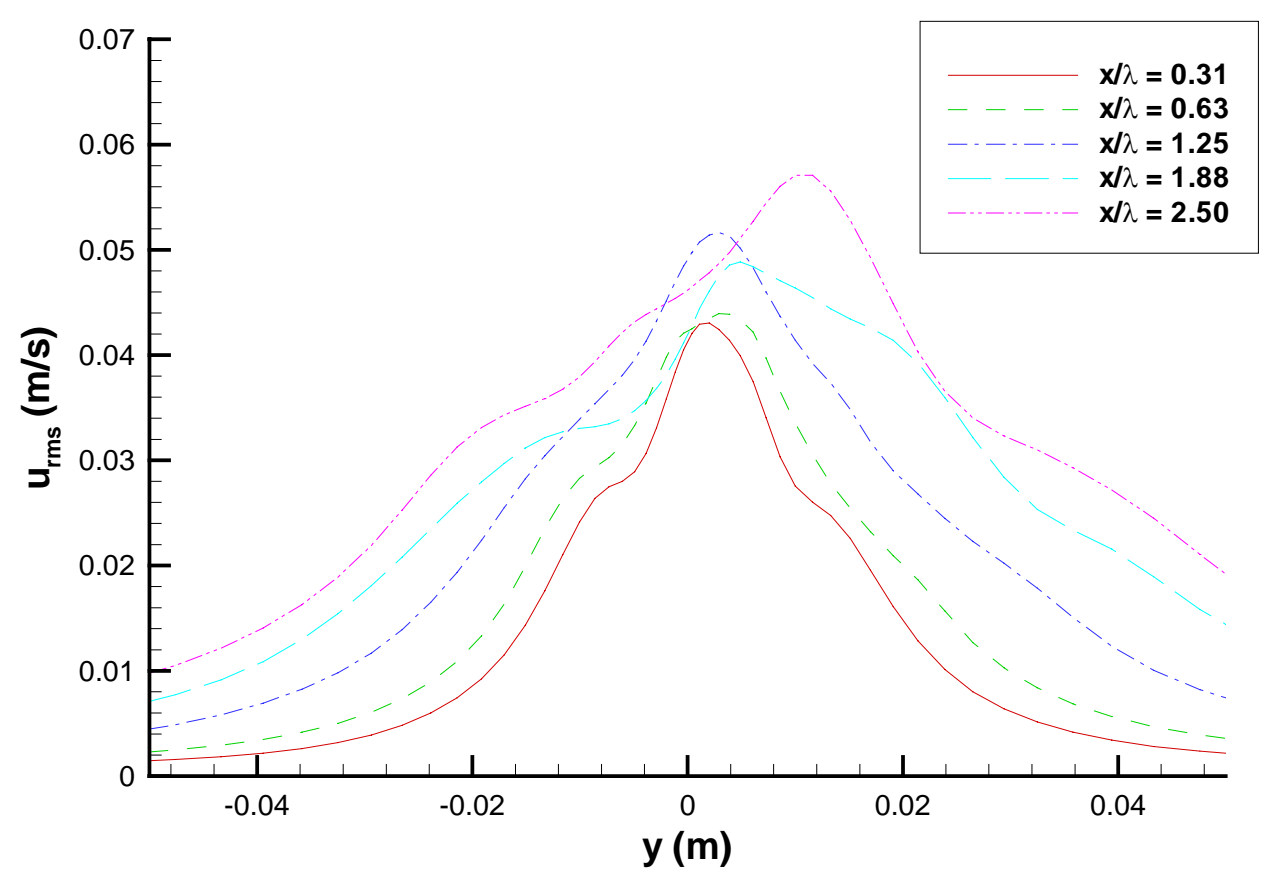

Figure 5-23 RMS streamwise velocity profiles in a mixing layer. Dynamic model is used. 


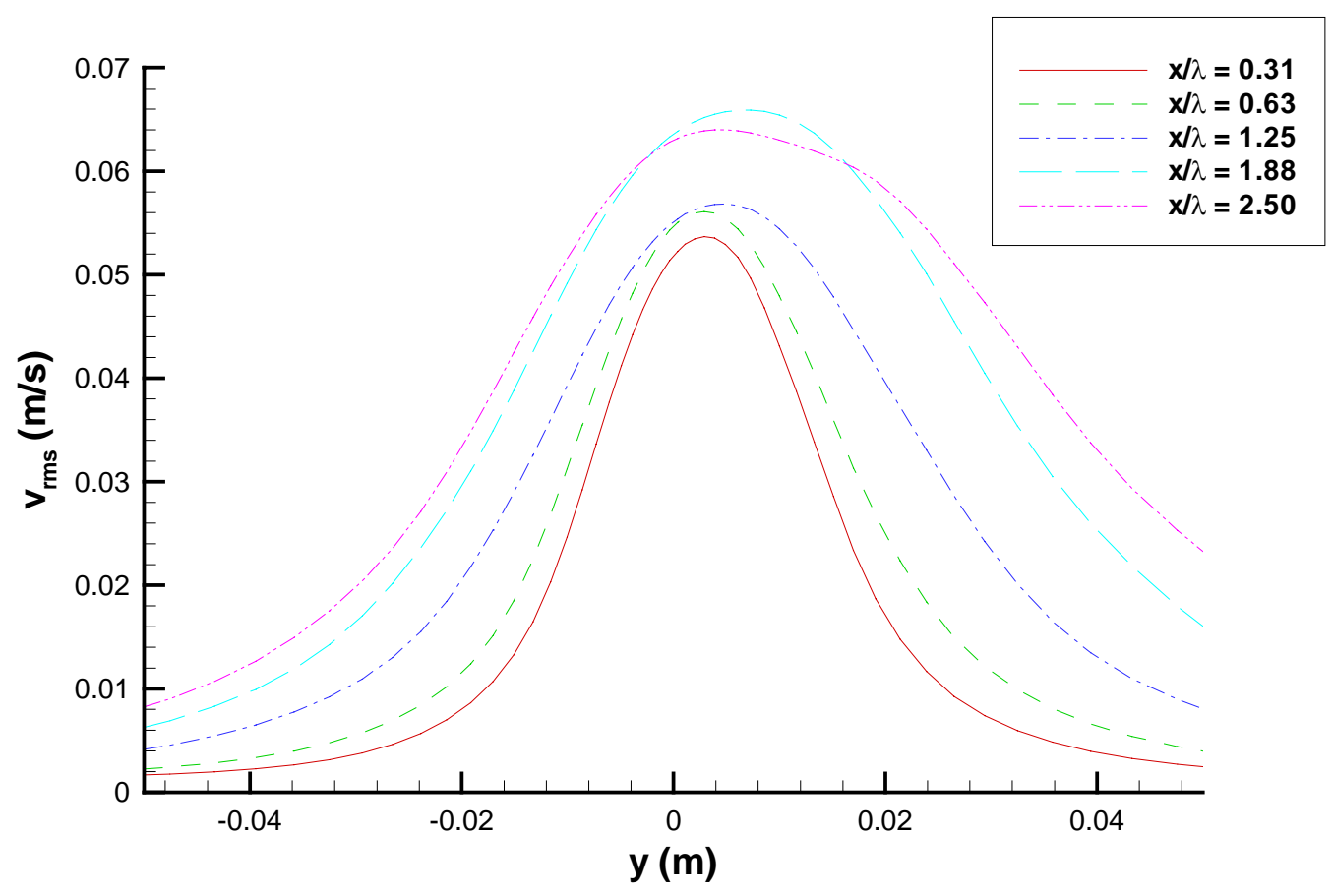

Figure 5-24 RMS vertical velocity profiles in a mixing layer. Dynamic model is used.

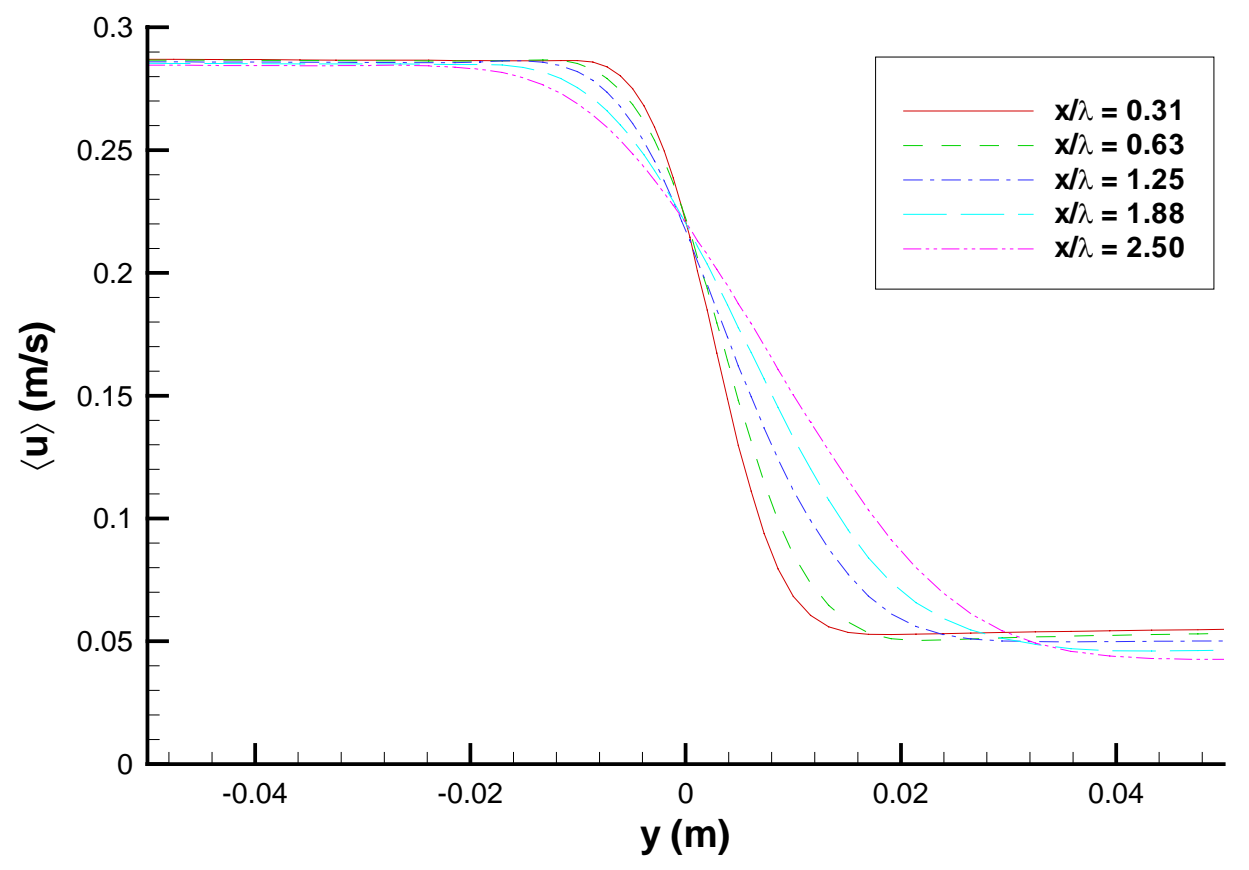

Figure 5-25 Mean streamwise velocity profiles in a mixing layer. No SGS model is used. 


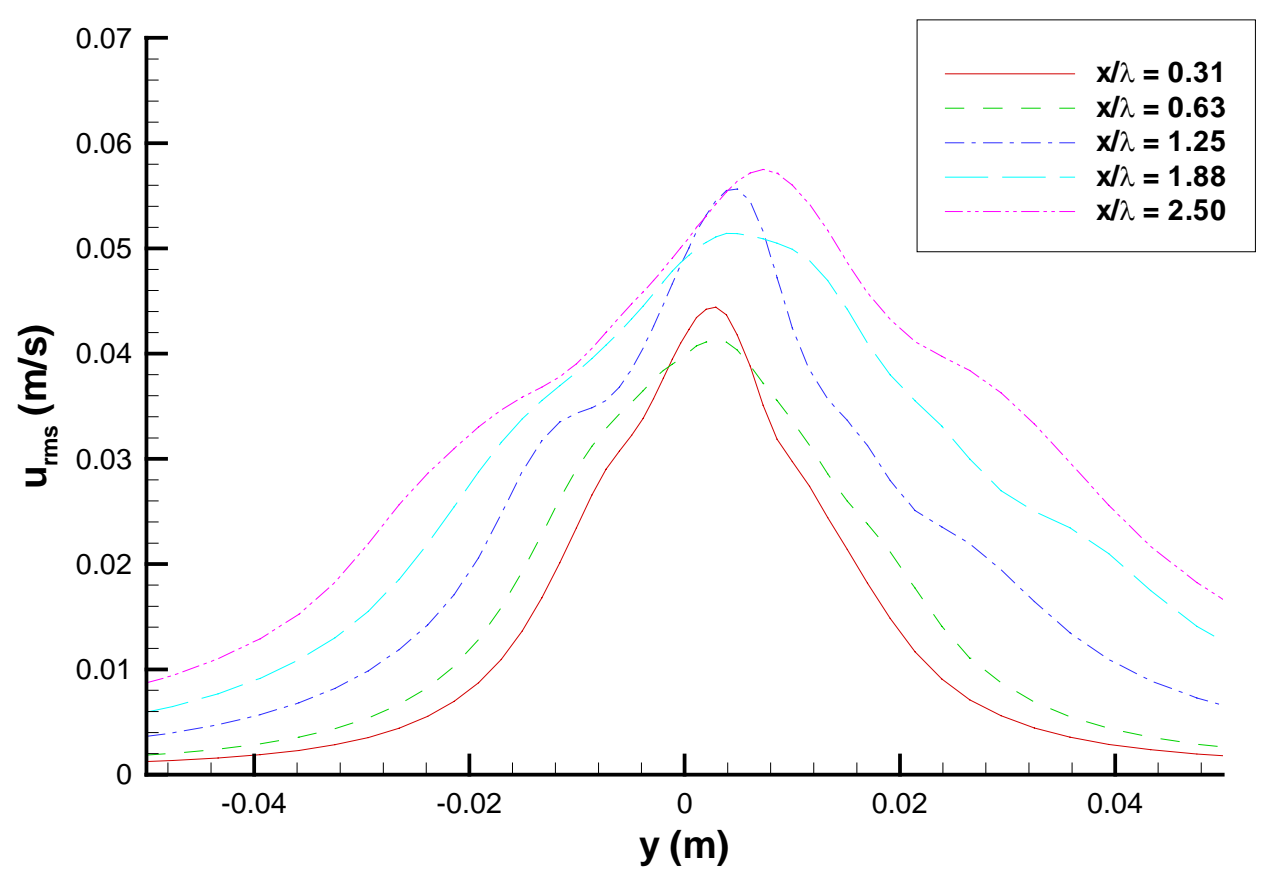

Figure 5-26 RMS streamwise velocity profiles in a mixing layer. No SGS model is used.

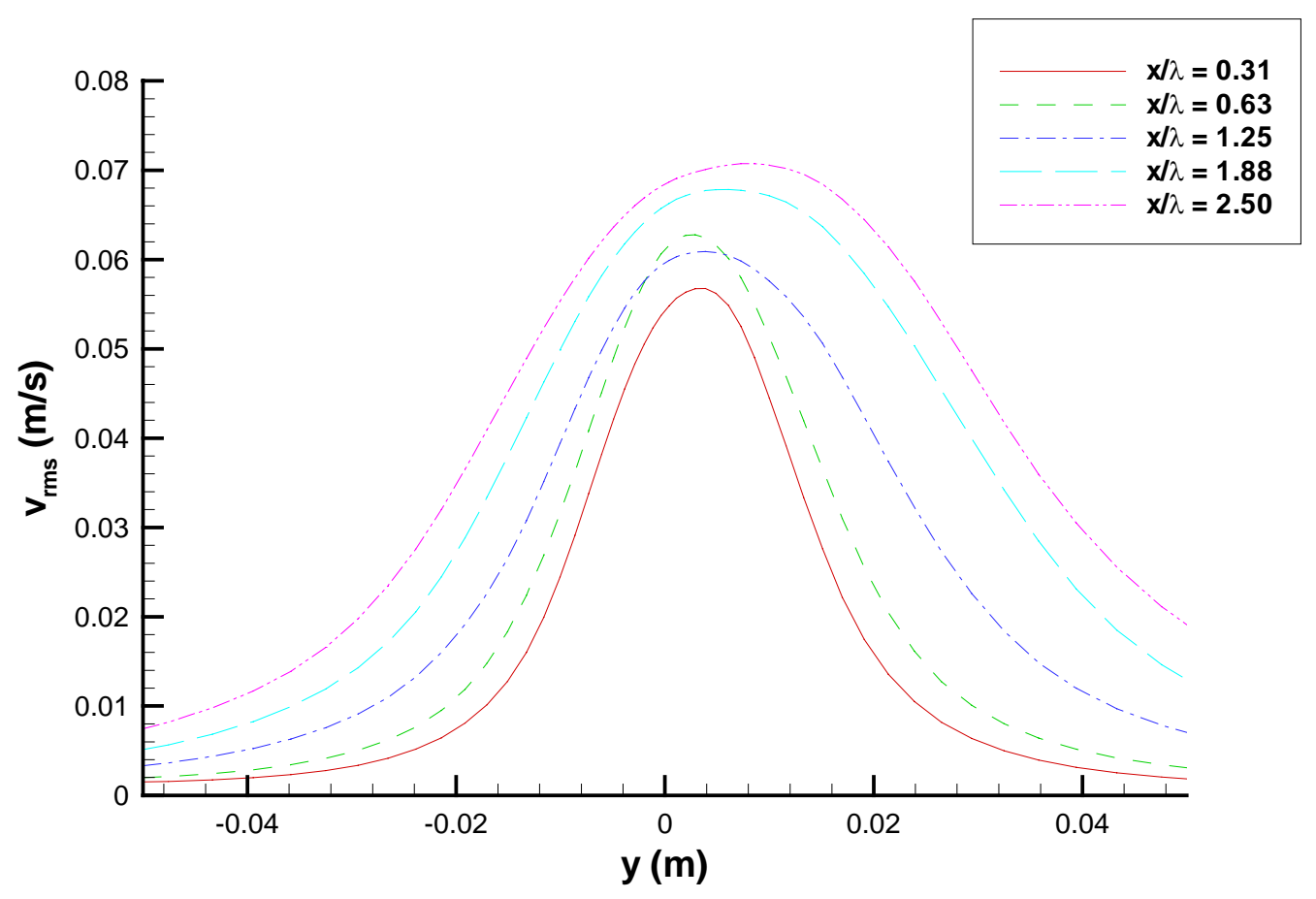

Figure 5-27 RMS vertical velocity profiles in a mixing layer. No SGS model is used. 

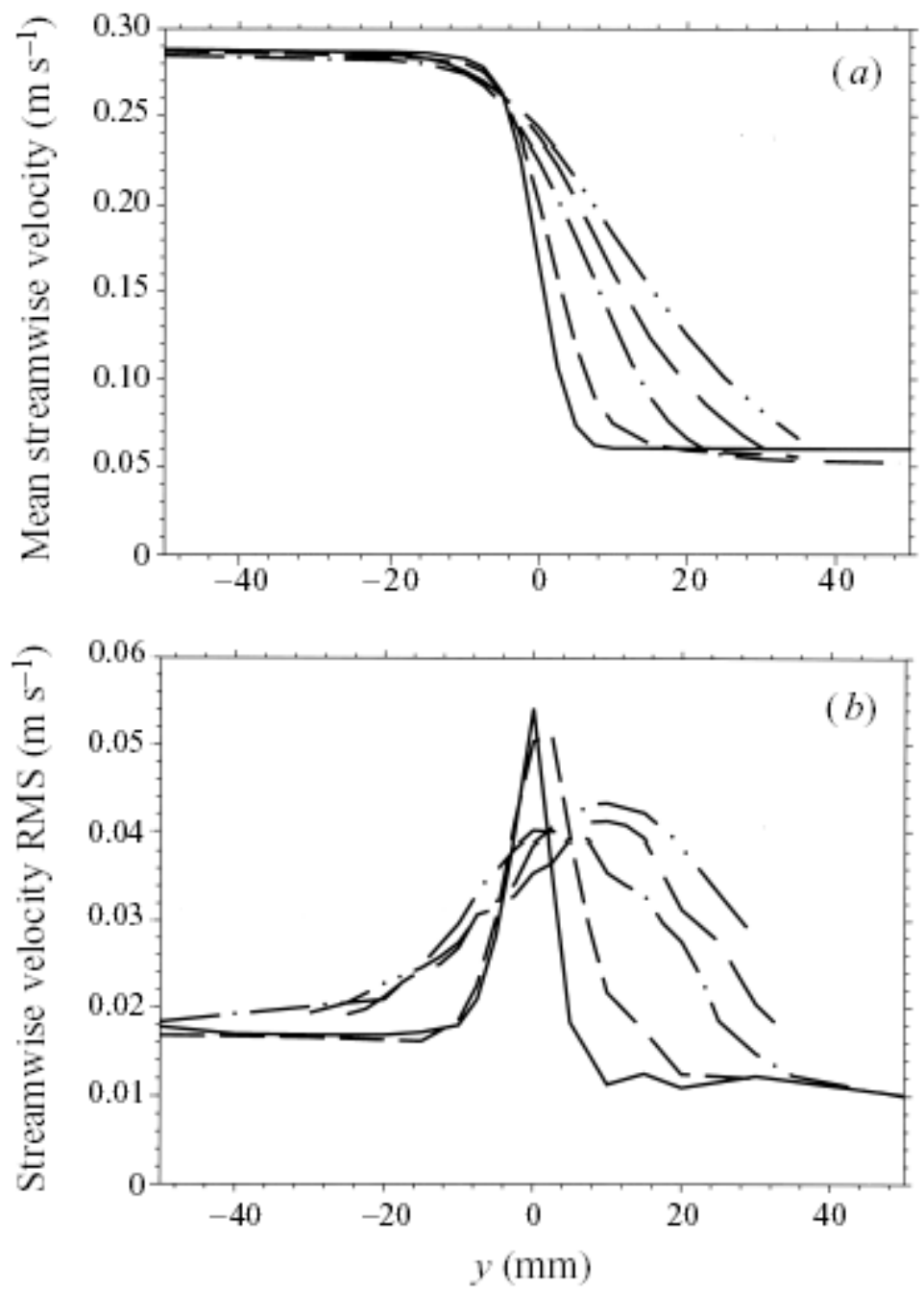

Figure 5-28 Streamwise (a) mean and (b) RMS velocity profiles from Rightley \& Lasheras (2000). Solid line, $x / \lambda=0.31 ;$ dash, $x / \lambda=0.63 ;$ dash-dot, $x / \lambda=1.25 ;$ long-dash, $x / \lambda=1.88 ;$ dash-dot-dot, $x / \lambda=$ 2.50. 


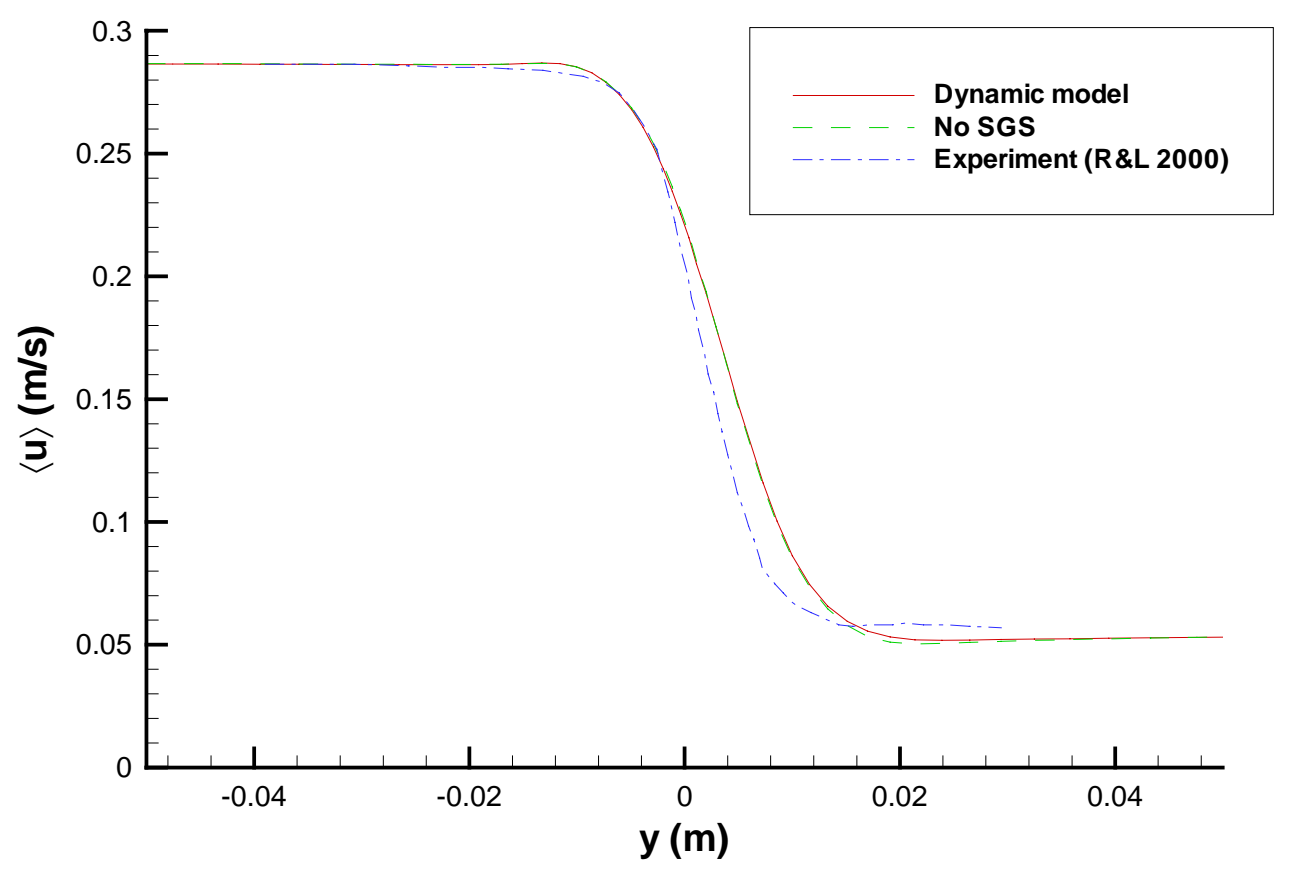

Figure 5-29 Comparison of mean streamwise velocity profiles at $x / \lambda=0.63$.

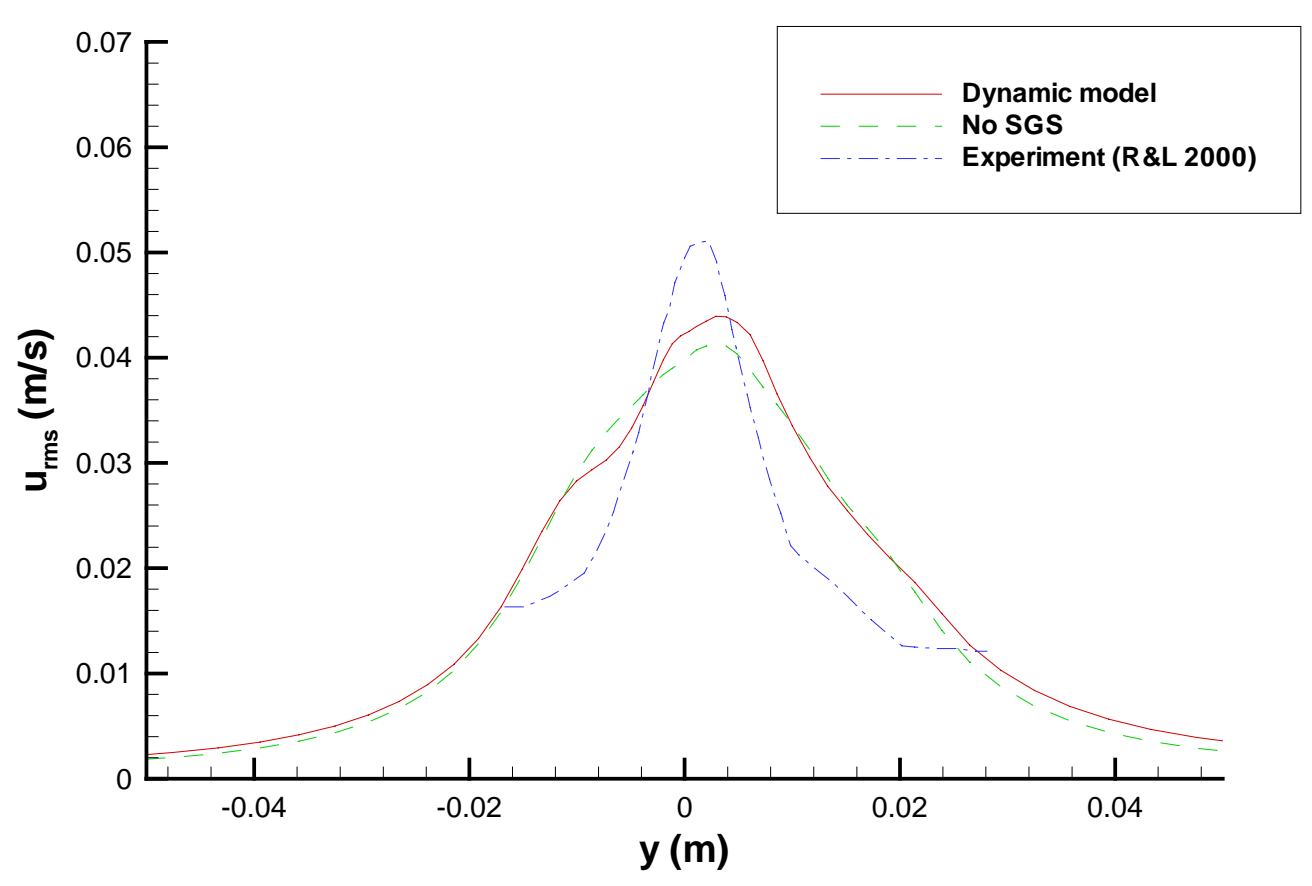

Figure 5-30 Comparison of $u_{\text {rms }}$ profiles at $x / \lambda=0.63$. 


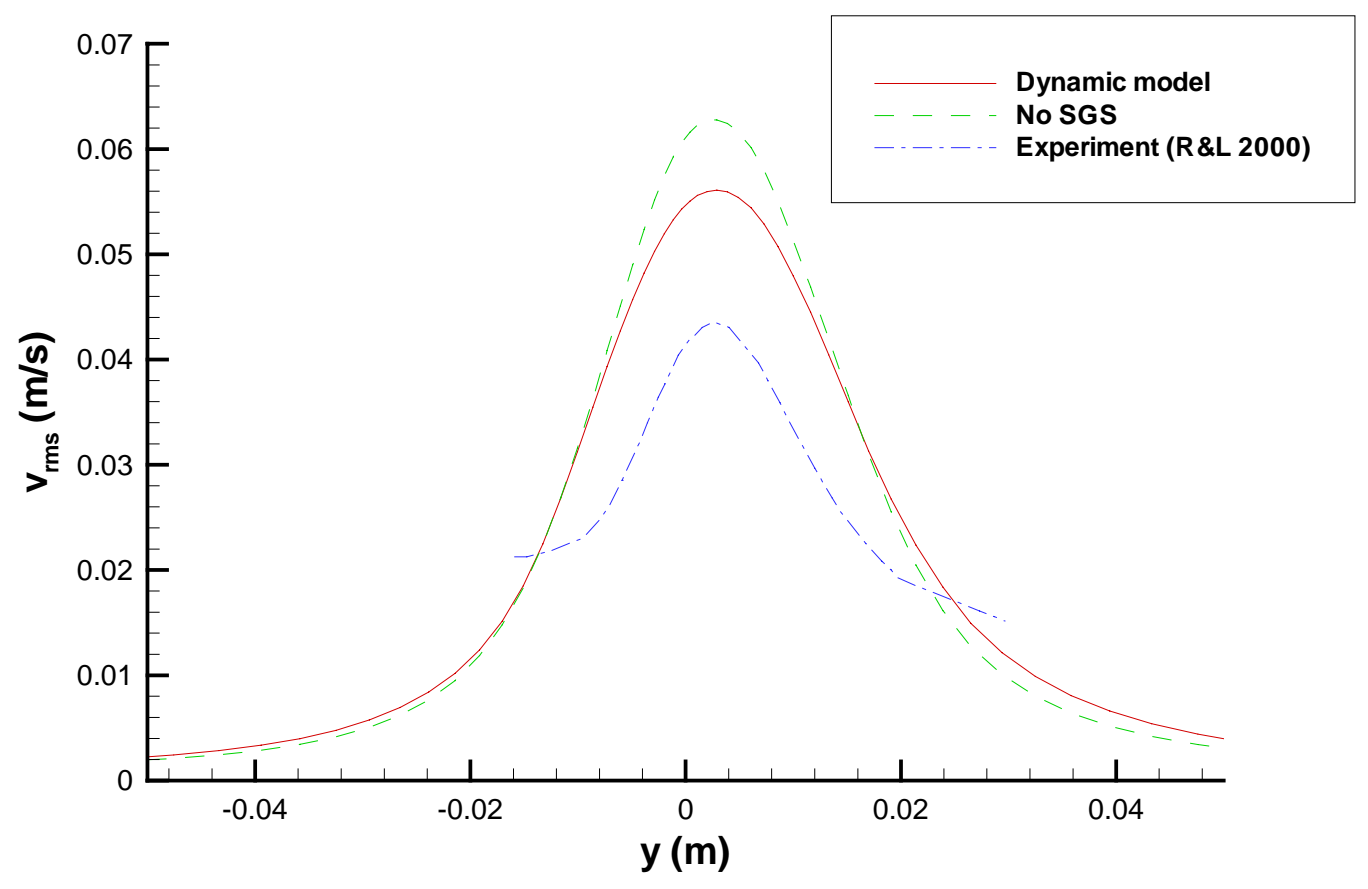

Figure 5-31 Comparison of $v_{\text {rms }}$ profiles at $x / \lambda=0.63$.

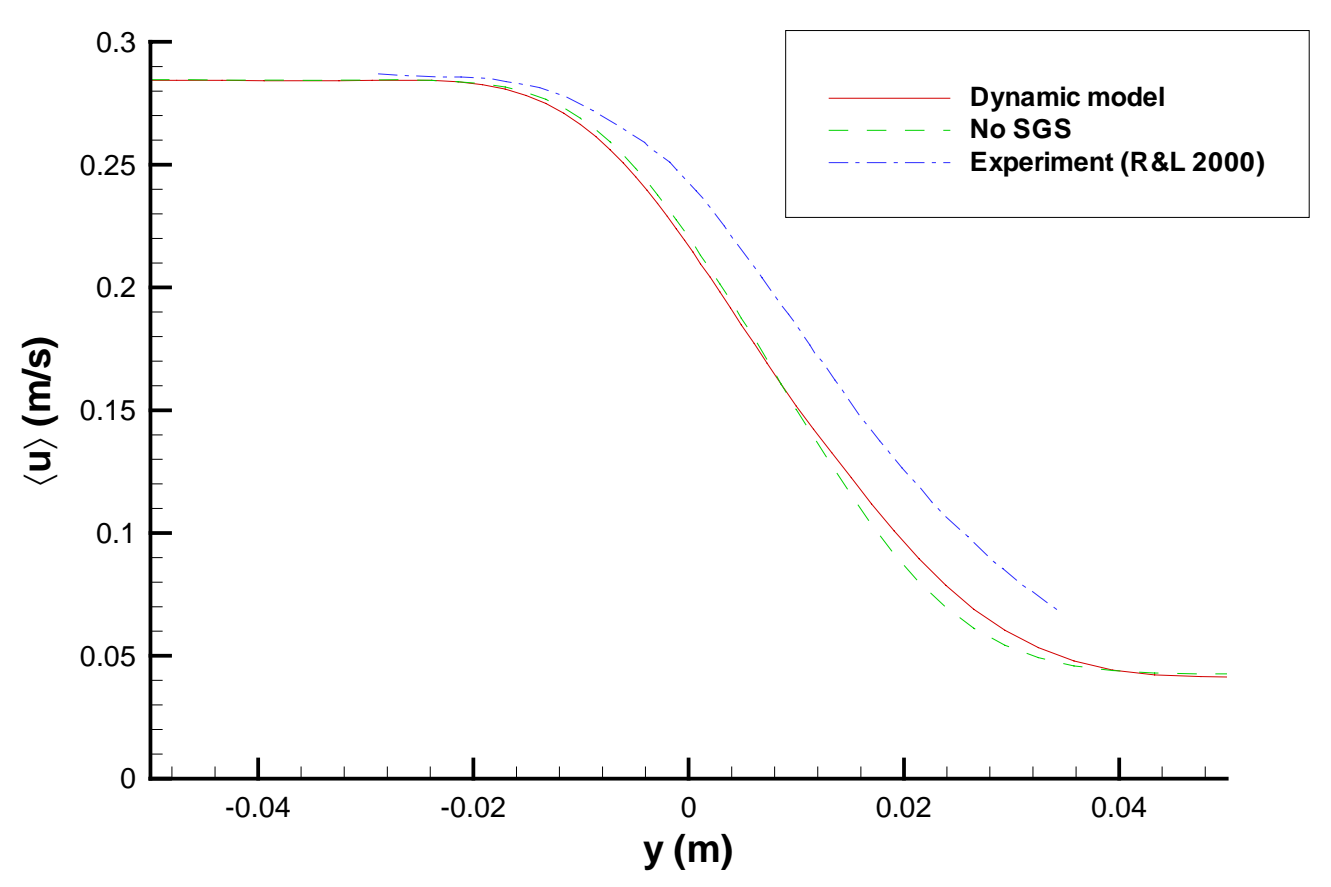

Figure 5-32 Comparison of mean streamwise velocity profiles at $x / \lambda=2.5$. 


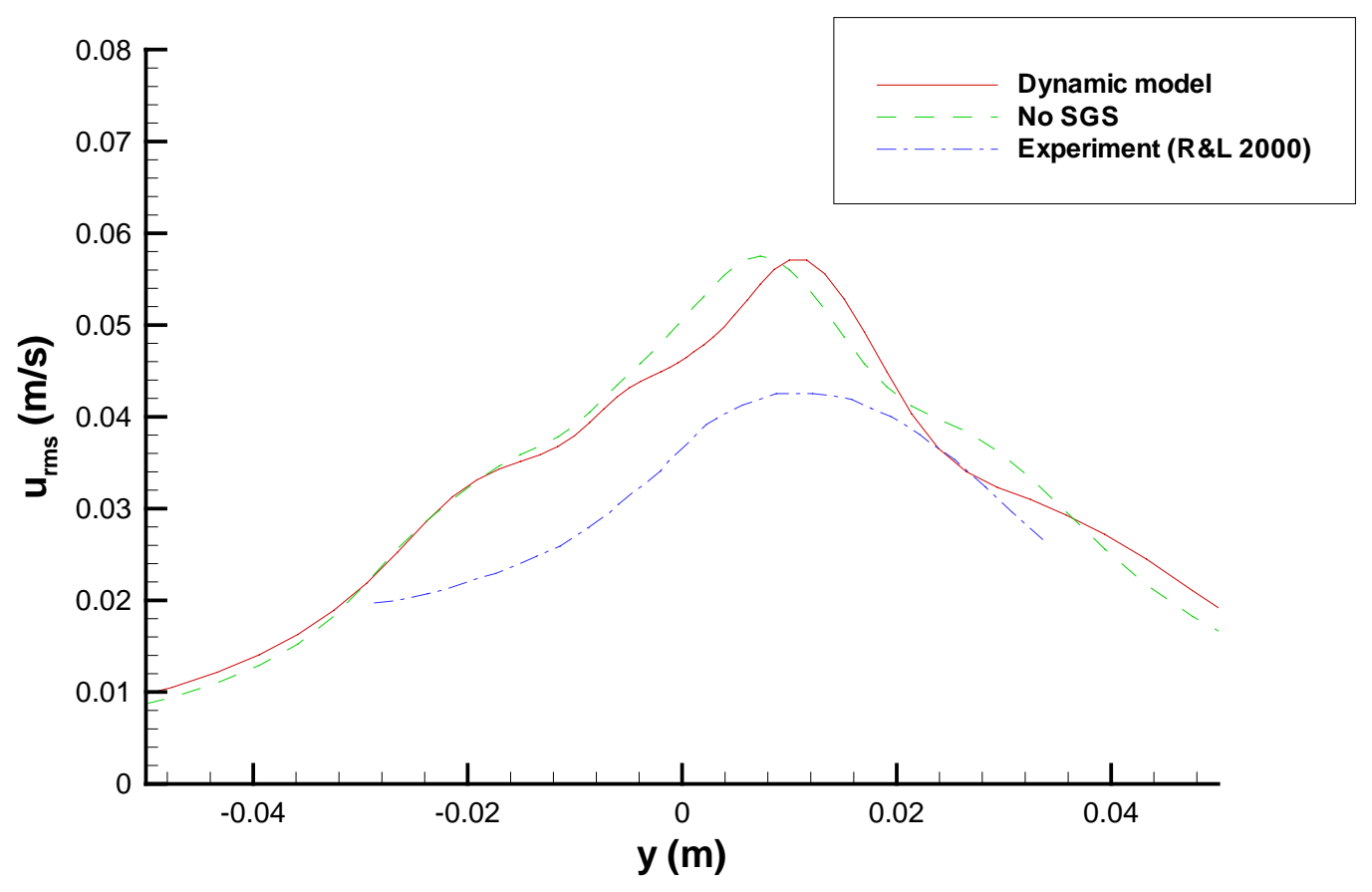

Figure 5-33 Comparison of $u_{r m s}$ profiles at $x / \lambda=2.5$.

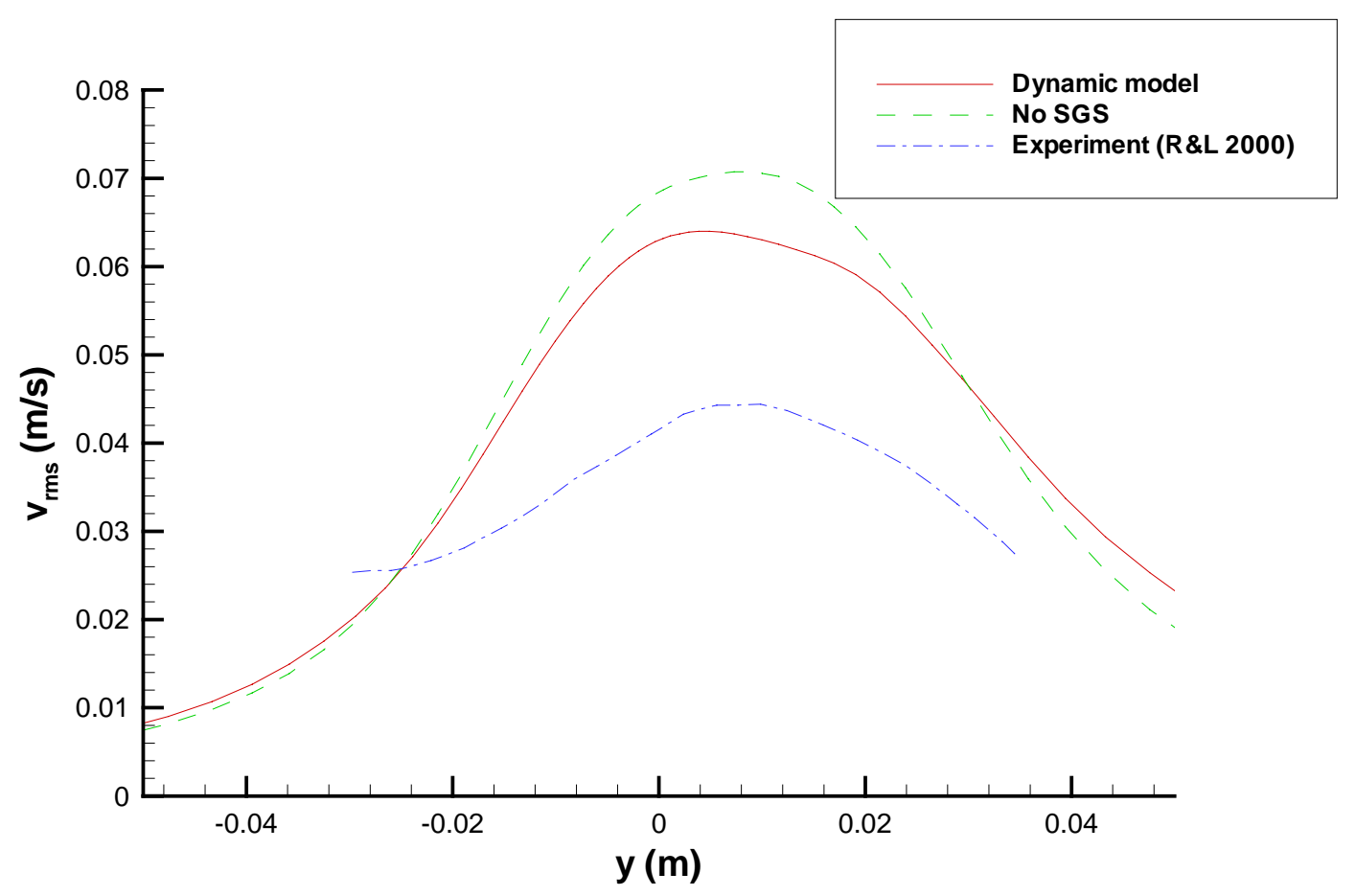

Figure 5-34 Comparison of $v_{\text {rms }}$ profiles at $x / \lambda=2.5$. 


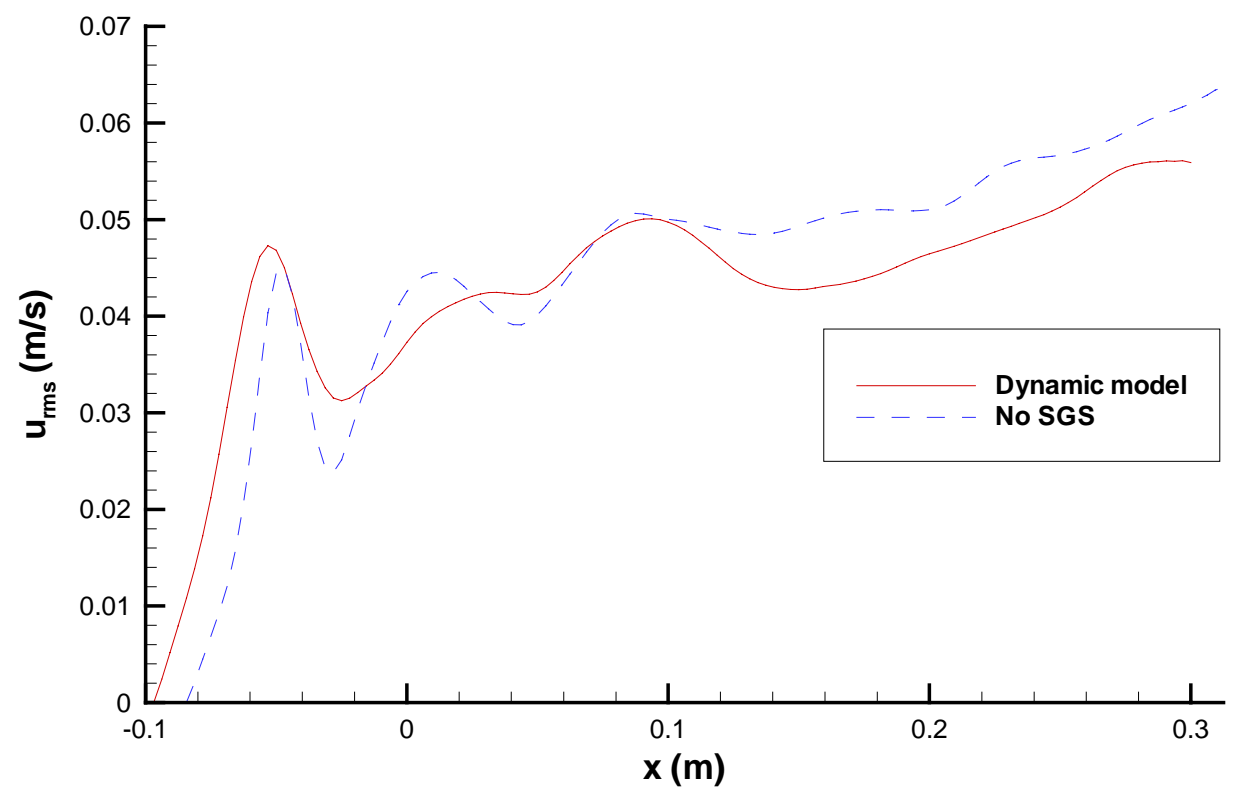

Figure 5-35 Comparison of $u_{r m s}$ along centerline computed with dynamic model and without SGS model.

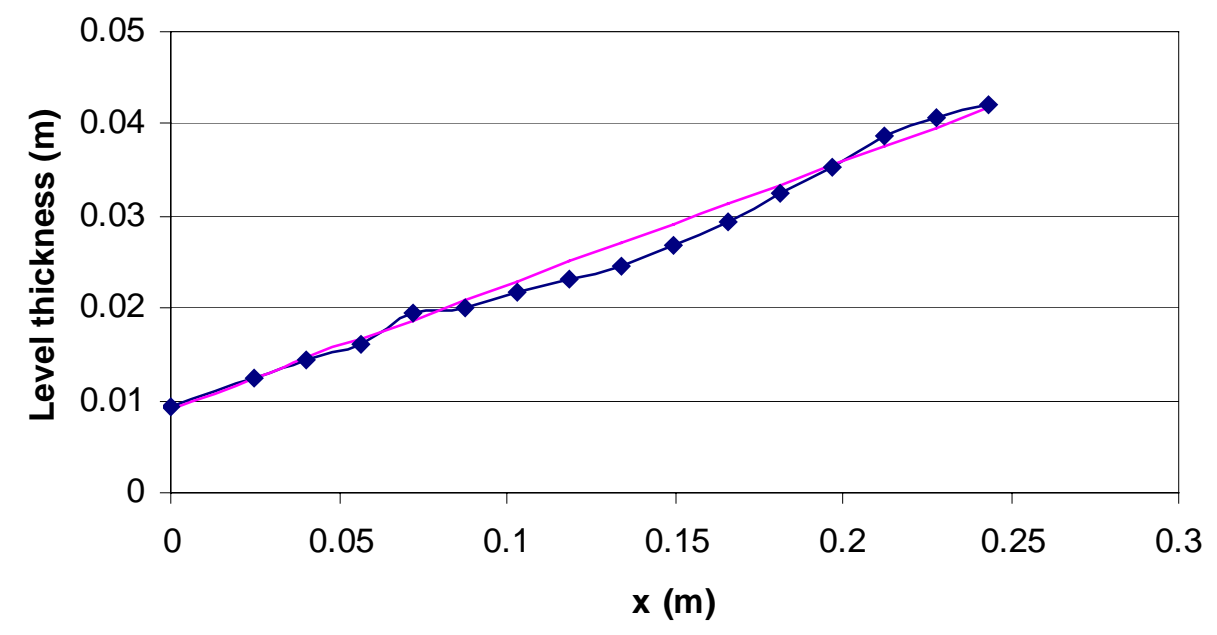

$$
\multimap \multimap \text { Present __ Experiment (R\&L 2000) }
$$

Figure 5-36 Comparison of level thickness between prediction and measurements. 


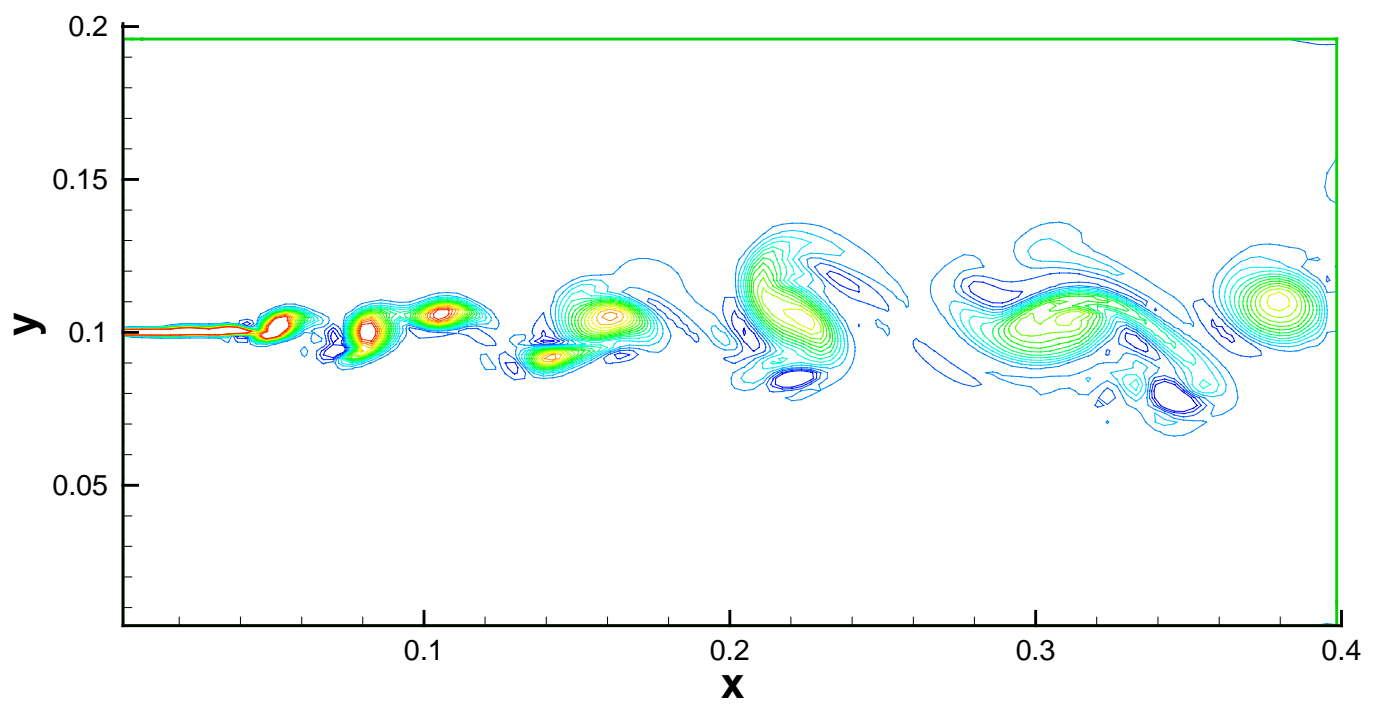

(a)

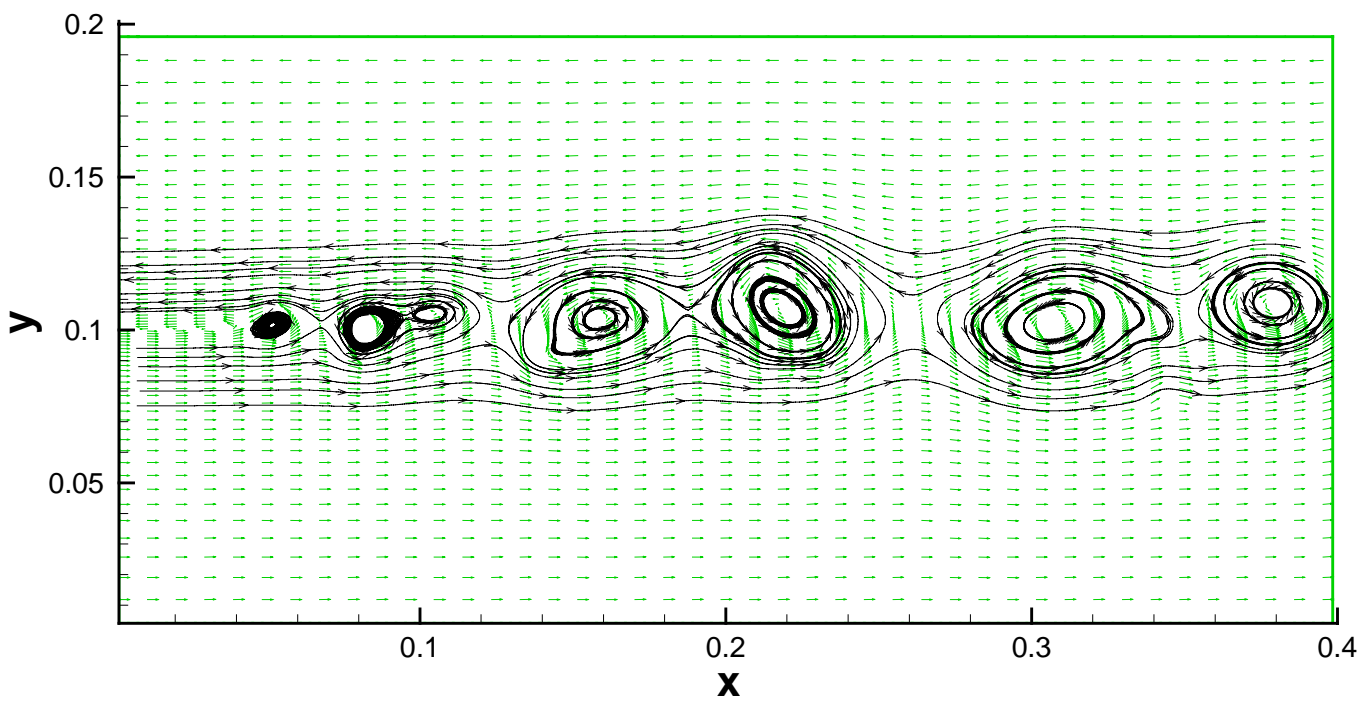

(b)

Figure 5-37 A snapshot of (a) spanwise vorticity contours, and (b) streamlines and velocity vectors, mean convective velocity is subtracted from the streamwise velocity component. 


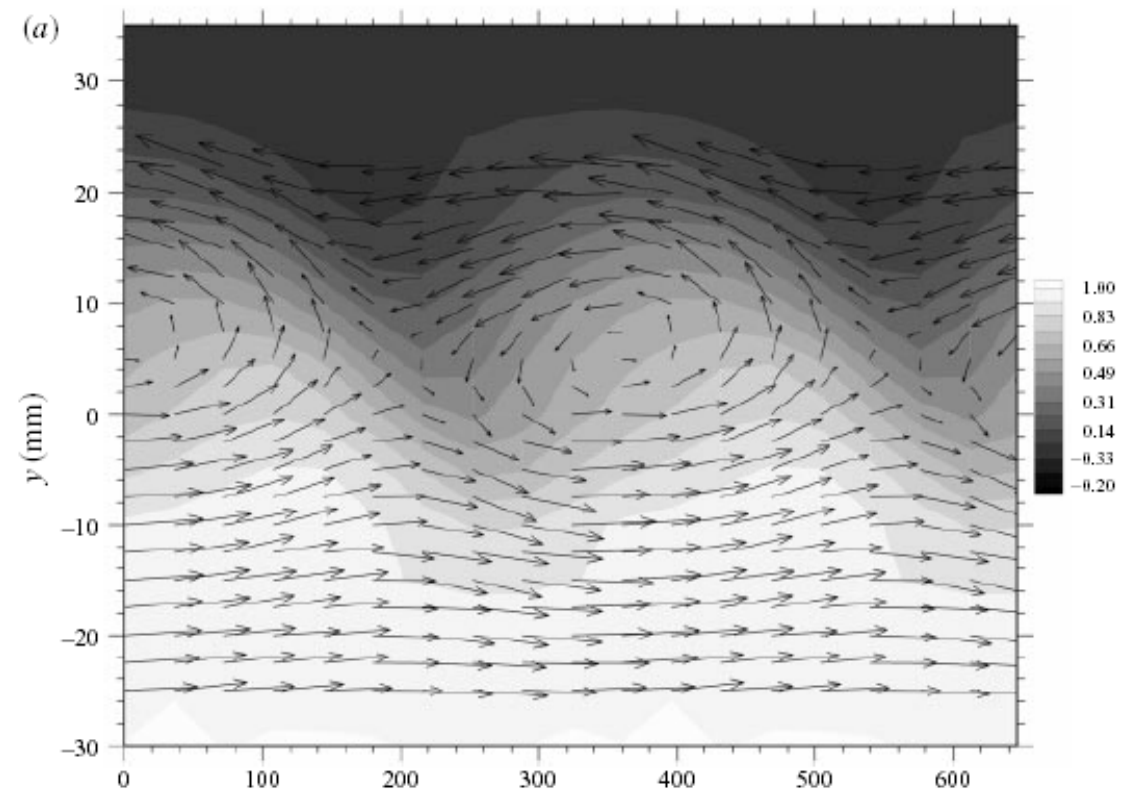

(a)

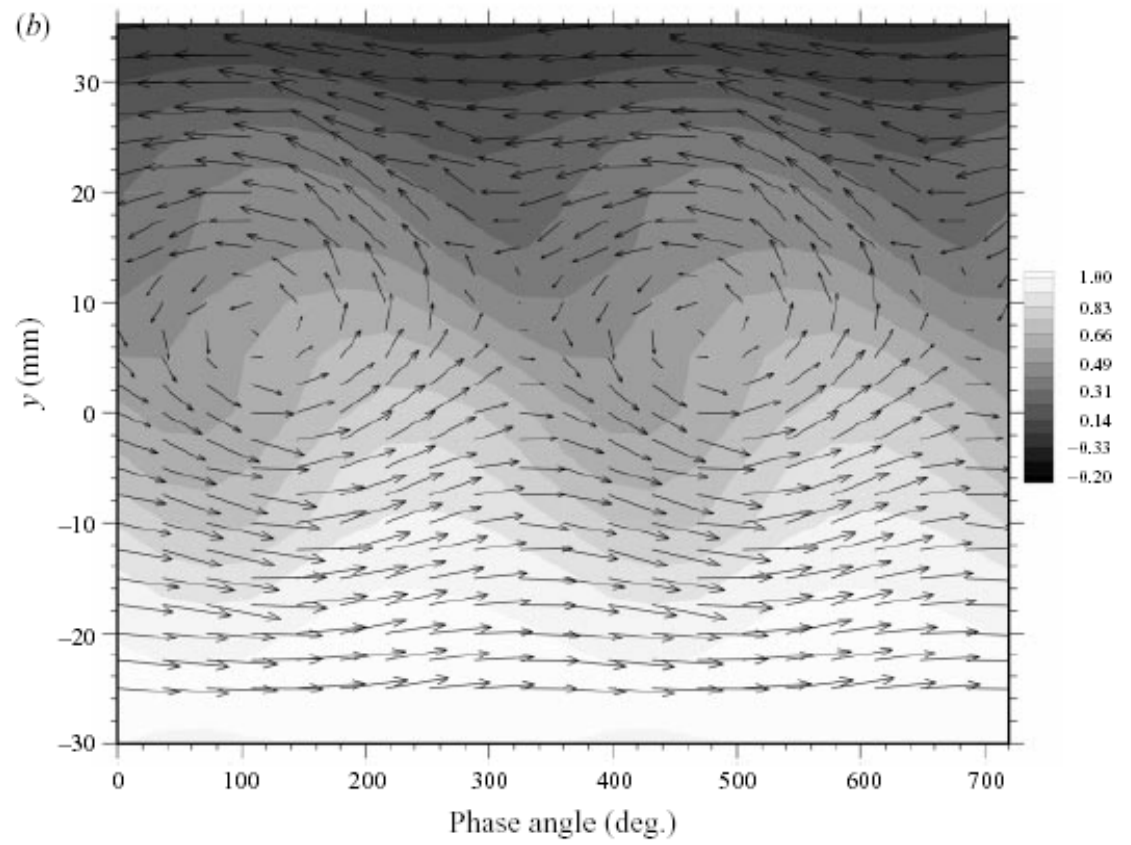

(b)

Figure 5-38 Phase-averaged velocity field at (a) $x / \lambda=1.25$ and (b) $x / \lambda=1.88$ from Rightley $\&$ Lasheras (2000). Mean velocity is subtracted from the streamwise velocity component. 


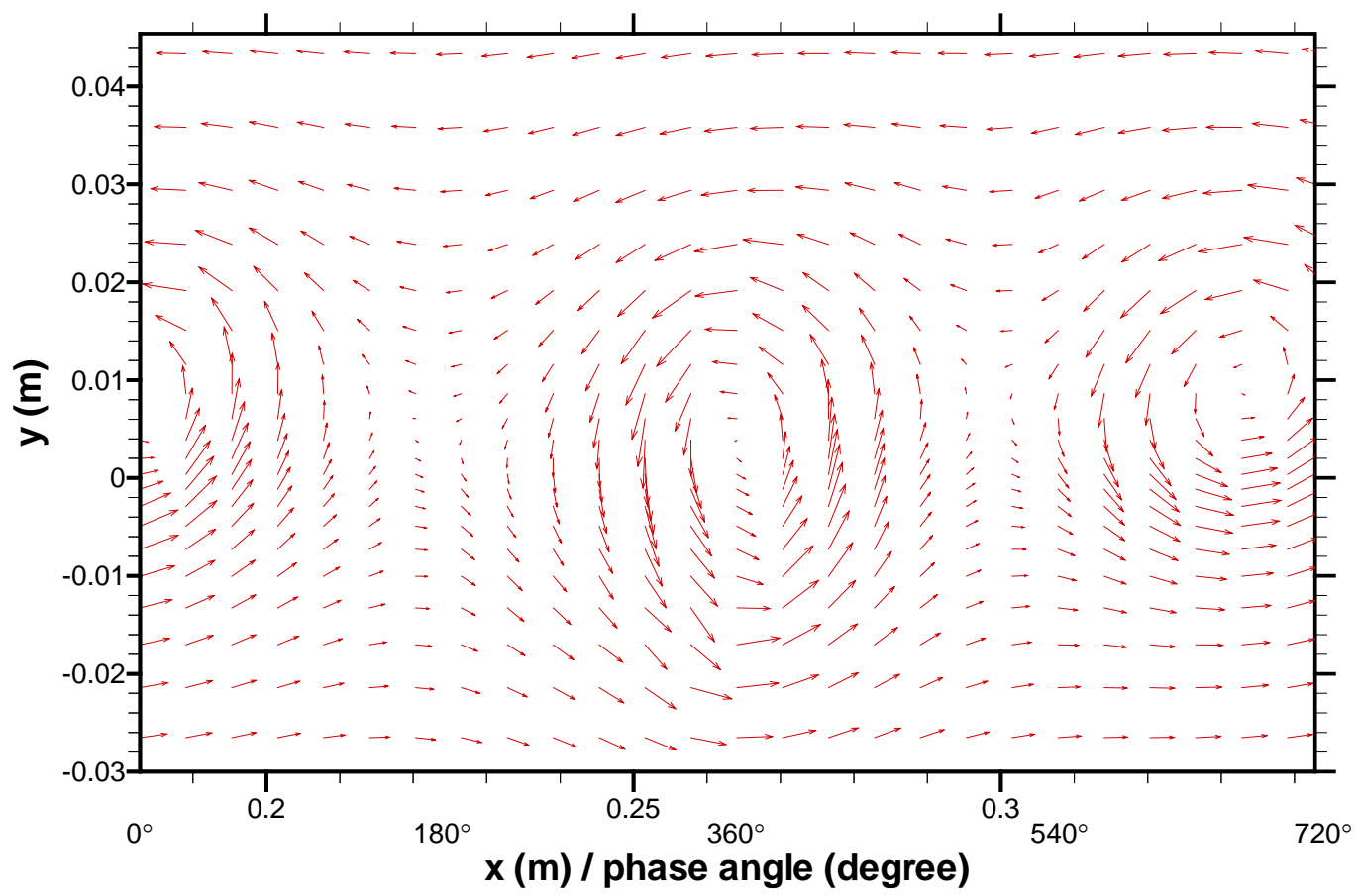

(a)

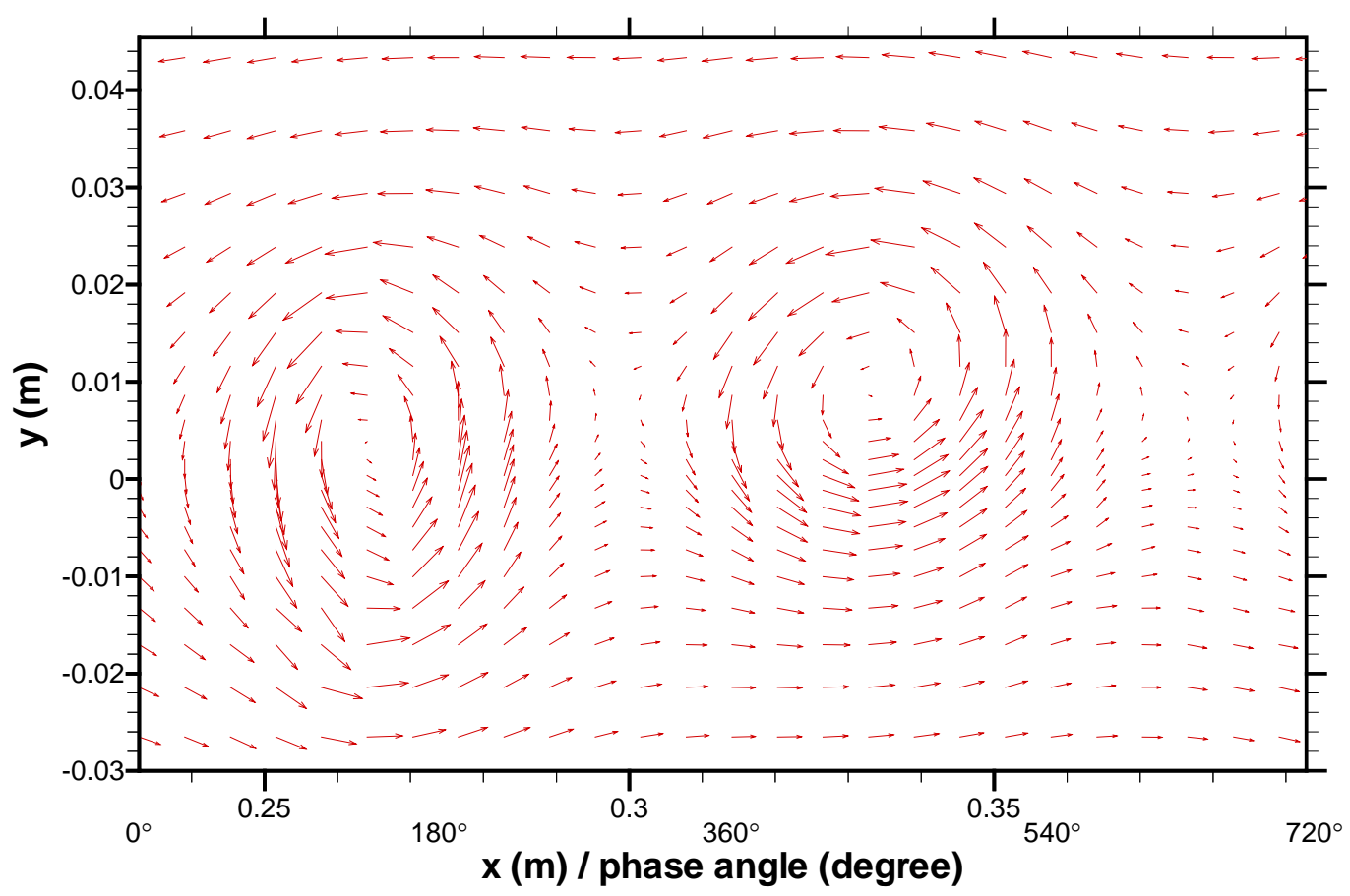

(b)

Figure 5-39 Instantaneous velocity vector field (a) between $x / \lambda=1.25$ and $x / \lambda=3.88$, (b) between $x / \lambda$ $=1.88$ and $x / \lambda=3.88$. Instant is taken at zero phase angle of the forcing function. Mean convective velocity is subtracted from the streamwise velocity component. 


\subsection{Flow Past a Square Cylinder}

\subsubsection{Introduction}

Studying flow past bluff bodies, such as circular or square cylinders, is of important technical relevance to many industrial applications, e.g., the vehicle aerodynamics. At high Reynolds number the flow phenomena, even with very simple body geometry like a square cylinder, is rather complex in general. It often involves separation and reattachment, multiple separations with partial reattachment, recirculation, unsteady vortex shedding, curved shear layers, bimodal flow behavior, transition from laminar to turbulent flow, high turbulence level and three-dimensional large-scale structures (Rodi 1997). In this section LES is to be conducted and verified for the case of flow past a square cylinder. Note that many complicated flow situations can be essentially regarded as a synthesis of wall boundary layers and shear layers, among others. Therefore, the three selected turbulent flow cases, i.e., the turbulent channel flow, the plane mixing layer and the flow past a square cylinder are representative, and well suited for benchmarking purpose.

A classical LDV (Laser-Doppler velocimetry) measurement for the flow past a square cylinder was performed by (Lyn et al. 1995). A LES workshop, aimed at exploring the LES capability in reproducing this experiment's results, was held in 1995 in Germany. Results from the workshop are published in (Rodi et al. 1995; Rodi et al. 1997; Rodi 1998). Using the same flow configuration a second workshop was further organized, and the results are available at (Voke 1997). A comparison between RANS and LES calculation has also been made in (Rodi 1997); and a comparison among different SGS models in the framework of LES has been done by (Sohankar et al. 2000). Regarding RANS, the general conclusions are that the RANS calculations severely underpredict turbulence fluctuations. This is easily understood since in RANS the entire length-scale spectrum of the turbulence is modeled, while in this complex flow largescale eddy structures dominate the turbulent transport and unsteady processes like vortex shedding and bistable behavior prevail. As pointed out by (Rodi 1998), the LES approach for simulating complex flows is conceptually more suitable.

\subsubsection{Computational Details}

The geometry used for the simulation is shown in Figure 5-40. To avoid ambiguity, it has been assumed that the origin of the coordinates is at the center of the cylinder. All the lengths are scaled with the side length of the square cylinder, D. The calculation domain extends 4.5D upstream, 14.5D downstream of the cylinder, 6.5D on either side of the cylinder, and 4D in the spanwise direction. The Reynolds number, based on the uniform inlet flow velocity and the side length of the square cylinder, is 22000. This flow configuration, both the geometry and the Reynolds number, is identical to the one used in the 1995's workshop. 


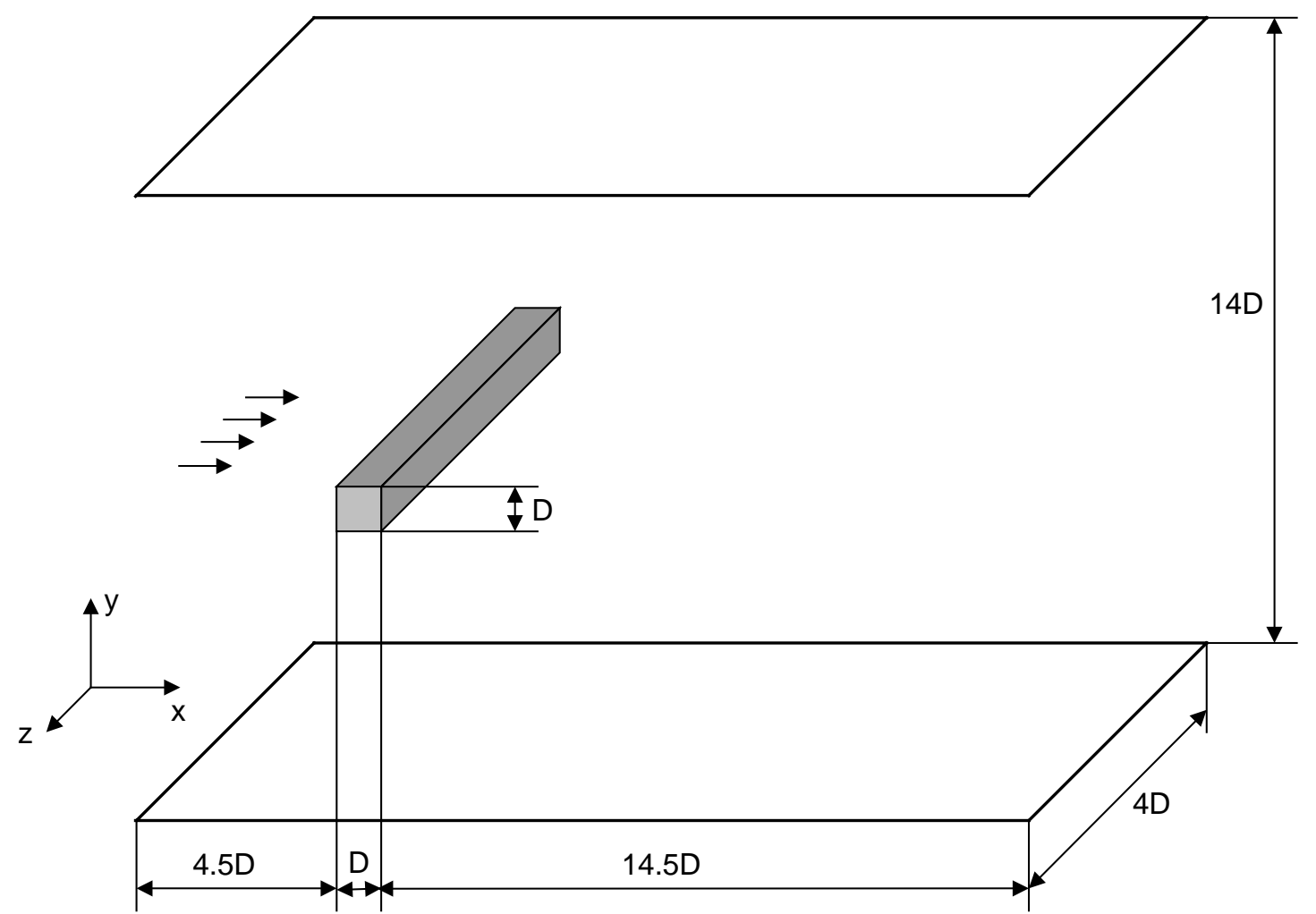

Figure 5-40 Schematic of flow past a square cylinder

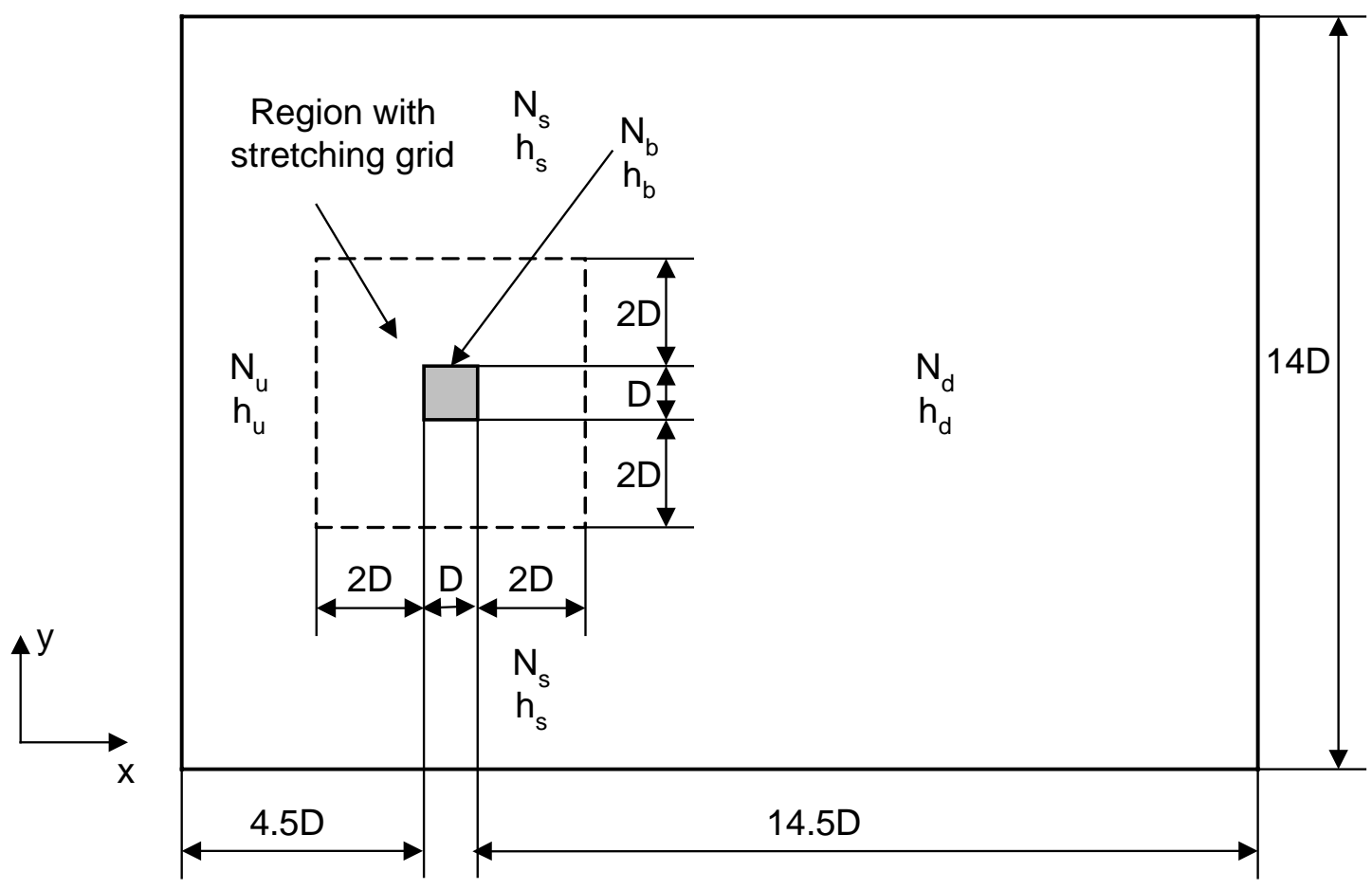

Figure 5-41 Grid distribution on the x-y plane for flow past a square cylinder 
A grid system with $160 \times 112 \times 16$ nodes in the streamwise, vertical and spanwise direction is used. In the spanwise direction, the grid distribution is uniform, with a grid spacing of $h_{z}=0.25 \mathrm{D}$. On the $\mathrm{x}-\mathrm{y}$ plane, the grid distribution (Figure 5-41) is made as follows: in the regions near the cylinder surface, i.e., from the surface to $2 \mathrm{D}$ away from the surface, the computational cells are stretched with a constant expansion ratio of 1.1. The purpose of doing so is to better resolve the wall boundary layer and to properly capture the boundary layer separation phenomena. In this study, the nearest grid point away from the cylinder surface is located at about $0.0113 \mathrm{D}$. Outside of the stretching region, a uniform distribution is applied. The symbolic notations for the number of nodes (denoted by $N$ with a subscript) and the grid spacing (denoted by $h$ with a subscript) in their corresponding uniform regions are shown in (Figure 5-41). In the x-direction, the cell size at upstream of the left stretching region is $h_{u}=0.25 \mathrm{D}$, and the cell size at downstream of the right stretching region is $h_{d}=0.123 \mathrm{D}$. In the y-direction the uniform cell size at sideways of the lower and upper stretching region is $h_{s}=0.225 \mathrm{D}$. Also, the number of cells distributed over one side of the cylinder surface is 24 , corresponding to $h_{b}=0.0417 \mathrm{D}$. These details of the grid information are summarized in Table 5-3.

Table 5-3 Summary of grid information for flow past a square cylinder

\begin{tabular}{|c|c|c|c|c|c|}
\hline \multicolumn{3}{|c|}{ X-direction $\left(N_{x}=160\right)$} & \multicolumn{2}{c|}{ y-direction $\left(N_{y}=112\right)$} & z-direction \\
\hline$N_{u}$ & $N_{d}$ & $N_{b}$ & $N_{s}$ & $N_{b}$ & $N_{z}$ \\
\hline 10 & 102 & 24 & 20 & 24 & 16 \\
\hline$h_{u}$ & $h_{d}$ & $h_{b}$ & $h_{s}$ & $h_{b}$ & $h_{z}$ \\
\hline 0.25 & 0.123 & 0.0417 & 0.225 & 0.0417 & 0.25 \\
\hline
\end{tabular}

It should be noted that although the non-uniform grid is used in the vicinity of cylinder surface, the nearest node away from the surface is still not close enough in order to deliver a full wall-resolving solution. This can be seen by making an estimate of the required distance at which $y^{+}=5$. Note that the drag coefficient $C_{D}$ measured by (Lyn et al. 1995)'s is around 2.1. As a first approximation let the pressure distribution around the cylinder be uniform, so that only the friction drag contributes to the total drag force. Hence, by definition of $C_{D}$ and further noticing that the frontal area and the side-surface area are equal for the case of a square cylinder, one has

$$
C_{D}=\frac{F_{D}}{\frac{1}{2} \rho U_{0}^{2} A} \approx \frac{2 \tau_{w} A}{\frac{1}{2} \rho U_{0}^{2} A}=\frac{\tau_{w}}{\frac{1}{4} \rho U_{0}^{2}},
$$

where $U_{0}$ is the uniform inlet velocity, $A$ is the front area, $F_{D}$ is the total drag force, and $\tau_{w}$ is the averaged shear stress at the upper and lower cylinder surface. With Eq. (5.30) $y^{+}$ can be expressed as

$$
y^{+}=\frac{u_{\tau} \delta}{v}=\frac{\sqrt{\frac{\tau_{w}}{\rho}} \delta}{v}=\frac{1}{2} \sqrt{C_{D}} \frac{U_{0} D}{v} \frac{\delta}{D}=\frac{1}{2} \sqrt{C_{D}} \operatorname{Re} \frac{\delta}{D},
$$

where $\delta$ is the distance from the cylinder surface to the nearest grid point. Thus, given $\mathrm{Re}$ $=22000$ and $C_{D}=2.1$, at $y^{+}=5$ one obtains $\delta=3.1 \mathrm{E}-4 \mathrm{D}$. This implies that for a wallresolving solution at high $\mathrm{Re}$ a rather demanding resolution near the wall is required, which, from computational point of view, is prohibitive. Even in a relatively recent calculation performed by (Sohankar et al. 2000) the $\delta$ only reaches $0.008 \mathrm{D}$. Nevertheless, 
the no-slip condition has been used in most of the previous LES's. It may appear to be appropriate to use a suitable near-wall model similar to the well-known wall functions employed in RANS calculations. However, almost all the wall models proposed in the literature assume a phase coincidence between the instantaneous tangential velocity inside the first cell and the instantaneous wall shear stress; and they were all basically developed for attached flows, the validity of their application to a separated flow, e.g., the flow under consideration here, remains to be an open question (Rodi et al. 1997; Rodi 1998). Developing a well-suited wall model for separated flow is beyond the scope of this study and will be left as a future work.

As to the boundary conditions, a uniform flow velocity is prescribed at the inlet. At the outlet an outlet boundary condition is used which ensures global mass conservation. The symmetry boundary conditions are applied in the normal direction at the upper and lower surfaces of the domain. In the spanwise direction the periodic boundary condition is applied. Also, the no-slip conditions are employed on the four cylinder surfaces. It is kept aware that the wall layer on the cylinder surfaces is not adequately resolved with the current grid resolution, albeit a stretched mesh is used. However, as a commonly accepted practice, an enforcement of the wall-condition is always better than doing nothing!

Two type SGS models, namely the ITM (implicit turbulence model) and the dynamic eddy-viscosity model, are used. The standard Smagorinsky model is avoided here because it requires the specification of a wall-damping function, which is not known a priori. For the ITM approach, the $3^{\text {rd }}$ order QUICK scheme is employed, which supposedly provides the methods-embedded damping effect of the TKE. In the calculation with the dynamic SGS model, the box filter is used for both grid filtering and the test filtering. The definition of the filter widths, as well as their justification, has already been given in the proceeding section. A fourth order central differencing is applied to the convection terms, combined with a switching to QUICK (due to numerical stability) once the grid Peclet number exceeds 2.

The time marching calculation is started with the fluid at rest. A constant time step of $2.2 \mathrm{E}-3$, non-dimensionalized by $D / U_{0}$, is used. The calculation is considered complete when a fully developed state is reached, in which the turbulence quantities do not change in the mean. Results are then obtained by analyzing the flow data in the fully developed state.

To be consistent, let $\langle\phi\rangle$ denote a long-time averaged quantity, and $\phi$ ' the resolved total fluctuation, i.e.,

$$
\phi=\langle\phi\rangle+\phi^{\prime} .
$$

When a flow involves periodic vortex shedding, such as the case under consideration, it is appropriate (Hussain and Reynolds 1970; Rodi 1997; Bosch and Rodi 1998; Liou et al. 2002) to further decompose the total fluctuation, $\phi$, into a periodic component, $\phi^{\sim}$ and a component of stochastic turbulent fluctuation, $\phi$ '. Thus,

$$
\phi=\langle\phi\rangle+\phi^{\sim}+\phi^{\prime \prime} \text {. }
$$


Combining the first two quantities on the RHS of Eq. (5.33) yields the ensemble- (or phase-) averaged quantity, $\langle\phi\rangle$, i.e.,

$$
\phi=\langle\phi\rangle^{e}+\phi^{\prime \prime} .
$$

The phase averaging is often employed in an unsteady RANS calculation as well as in experimental measurements. In summary, Eq. (5.32), (5.33) and (5.34) represent three ways of decomposing an instantaneous signal.

\subsubsection{Results}

Figure 5-42 shows the variation of the mean (long-time averaged) streamwise velocity along the centerline of the square cylinder. Presented are the current results obtained using the dynamic model and ITM, in a comparison with the experimental data of (Lyn et al. 1995) and the data from two representative LES performed in the Rottach-Egern workshop (Rodi et al. 1995). The results reported from the Rottach-Egern workshop (Figure 5-43) generally exhibit a great variance in both near-wall and downstream regions. In contrast to Lyn's data, most of the LES results from that workshop, as well as the present result using ITM produce a fast recovery of the upstream velocity in the downstream region. In this regard, the present simulation with the dynamic model appears to give a closer agreement with the measurements. An inspection of the nearcylinder region tells that the dynamic model and ITM yield similar predictions, and, with respect to the experimental data, they both underpredict the mean recirculation length to some extent, a commonly observed result in a non-wall-resolving LES.

Figure 5-44, Figure 5-45 and Figure 5-46 show the RMS values of total fluctuations (periodic plus turbulent) of the streamwise and vertical velocity components, and the kinetic energy, respectively, along the centerline of the square cylinder. The overall agreement between the simulation and the experiment is good. Both the peak values and their locations are correctly predicted. In Figure 5-44 it is seen that there is an overprediction of $u_{\mathrm{rms}}$ with the ITM approach around the peak location as well as further downstream. This may reveal a possible problem associated with the ITM that the energy dissipation supplied by the numerical methods only can be not sufficient. Between about 4D and 10D from the sidewall, the dynamic model also overpredicts the measured $u_{\text {rms }}$ and $v_{\text {rms. }}$. The results could be improved by limiting the backscatter mechanism in the dynamic model. For a comparison with other LES, also included are the results from the Rottach-Egern workshop (Figure 5-47). Except TAMU2, which yields excessive fluctuations, all other LES results underpredict the kinetic energy to a different level. It is important to note that there is a connection between the fluctuation level and the separation length (Rodi 1998): excessive fluctuations generally cause an underprediction of the separation length, and vice versa.

Figure 5-48 and Figure 5-49 show the distribution of the mean $u$-velocity and its RMS fluctuations along the $y$-direction at the cylinder center $(x=0)$. The vertical extent displayed starts from a near wall location at $y=0.5 \mathrm{D}$ up to $y=2.5 \mathrm{D}$. It is seen, both dynamic model and ITM yield a profile that is in fairly good accord with the measured data. In particular, the magnitude and location $(y \approx 0.75 \mathrm{D})$ of the near-wall peak are 
accurately captured by both curves. Very close to the wall $(y<0.6 \mathrm{D})$ both calculations indicate that there might exist a secondary peak which is much less in magnitude than the primary one, and possibly related to a profile commonly seen in a turbulent channel flow. Since the wall-layer is not sufficiently resolved in the present calculation, it is difficult to judge which trend is more plausible. In this problem, the near-wall resolution can be of critical significance, because the characteristics of the boundary layer separation and its evolution on the sides of the cylinder is responsible for the overall behavior of this vortex-shedding flow.

The global pattern of the $\langle u\rangle, u_{\mathrm{rms}}, v_{\mathrm{rms}}$ and total fluctuating kinetic energy are depicted in the contour plots (Figure 5-50 through Figure 5-53) in the center (x, y)-plane. It is seen that the flow pattern is symmetric in the mean with respect to the cylinder centerline. In Figure 5-50 a large mean recirculation zone (contoured by dashed lines) right behind the cylinder, and two small boundary layer separation zones on both sides of the cylinder are clearly visible. Figure 5-51 indicates that the highest $u$-fluctuations occur near the sidewalls, due to the boundary layer separation. However, the largest $k$-region is found in the near wake of the square cylinder, owing to the evolution of the vertical fluctuating component.

Figure 5-54 presents streamlines of phase-averaged flow field at three phases (phase 1,9 and 17). The left column is from (Lyn et al. 1995)'s experiment, and the right column is the current LES simulation. In Lyn's experiment a total of 20 phases are defined within a vortex-shedding period. The starting phase is determined from a pressure signal measured by a pressure sensor placed at the midpoint of a sidewall. Ensemble (or phase) averaging is undertaken in the measurements, so that the streamlines are created from the phase-averaged field, i.e., $\langle\mathbf{u}\rangle+\mathbf{u}^{\sim}$. In the simulation, the phase averaged plots are obtained by post-processing the instantaneous flow field solved by LES. Notice that, the shedding motion is qualitatively well reproduced by the present simulation.

Figure 5-55 through Figure 5-58 show a typical snapshot of the instantaneous velocity components and the spanwise vorticity. The illustrated flow pattern clearly shows the existence of large coherent vortical structures, as well as fine turbulence in the near wake of the cylinder.

Figure 5-59, Figure 5-60 and Figure 5-61 show the time history of the velocity components and pressure sampled at three selected locations along a vertical line in the near wake of the cylinder. The three points are at (1D, 0D), (1D, 0.5D) and (1D, 3D). The sampled signals are subtracted by their corresponding mean values to yield the total fluctuation. From these plots it is evident that the signals contain a periodic component superimposed by stochastic turbulent fluctuations.

The sampled signals for $u$ ' and $v^{\prime}$ at the three locations are then transformed into the frequency domain to yield the corresponding power spectra (Figure 5-62 and Figure 5-63). The power spectra offer a view of the frequency content of a signal. The Strouhal number, defined by 


$$
\mathrm{St}=\frac{f D}{U_{0}}
$$

is used in place of frequency in the spectra plots. The three $v$ '-spectra in Figure 5-63 give unanimously a dominant Strouhal number of 0.1387. In the $u$-spectra (Figure 5-62) the dominant St at the first two locations is 0.1248 , and again equal to 0.1387 at the (1D, 3D) station. Overall, these predicted St's are close to the experimental value of 0.132 reported by (Lyn et al. 1995). If the average is calculated from the predicted St's, it then yields 0.132 , thus recovering the experimental one. As mentioned by (Rodi 1998), the Strouhal number appears to be not very sensitive to the parameters as well as SGS models of the simulation. Also notable in Figure 5-62 is a secondary frequency peak that exists at (1D, $0 \mathrm{D})$ and $(1 \mathrm{D}, 0.5 \mathrm{D})$ stations (in the recirculation zone). To the author's knowledge, this secondary frequency is generally not reported in the literature, and it could be an important indication of a secondary vortex shedding occurring near the rear edge of the cylinder. The secondary Strouhal number found in the current study is 0.0832 . 


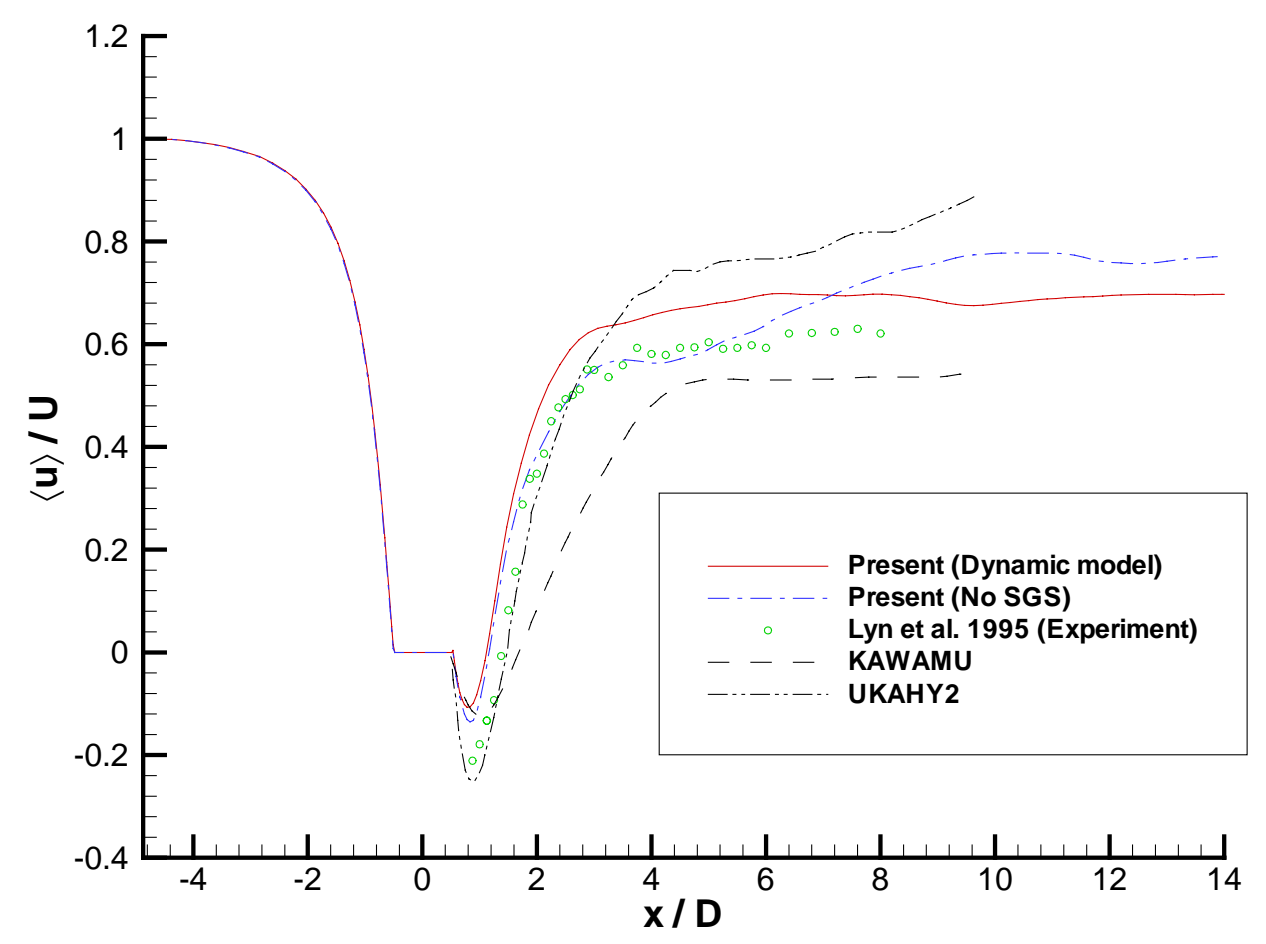

Figure 5-42 Mean streamwise velocity along centerline of square cylinder.

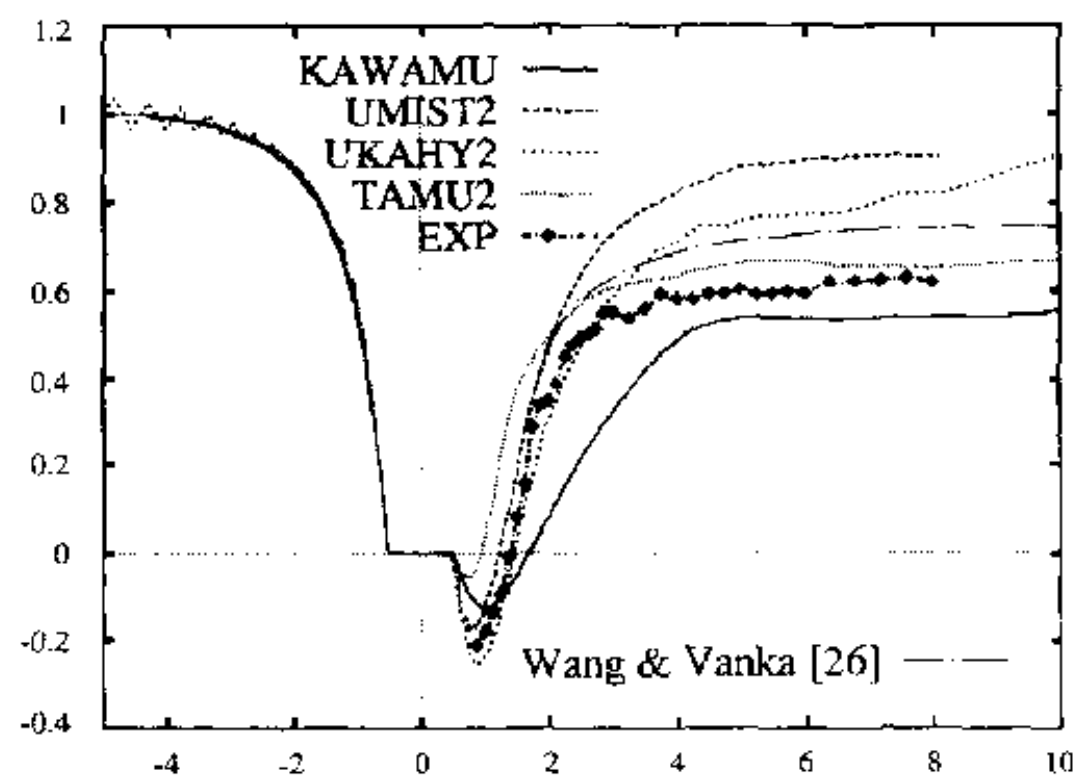

Figure 5-43 Results from Rottach-Egern workshop: mean streamwise velocity along centerline of square cylinder. (Reprinted from (Rodi 1997) with permission) 


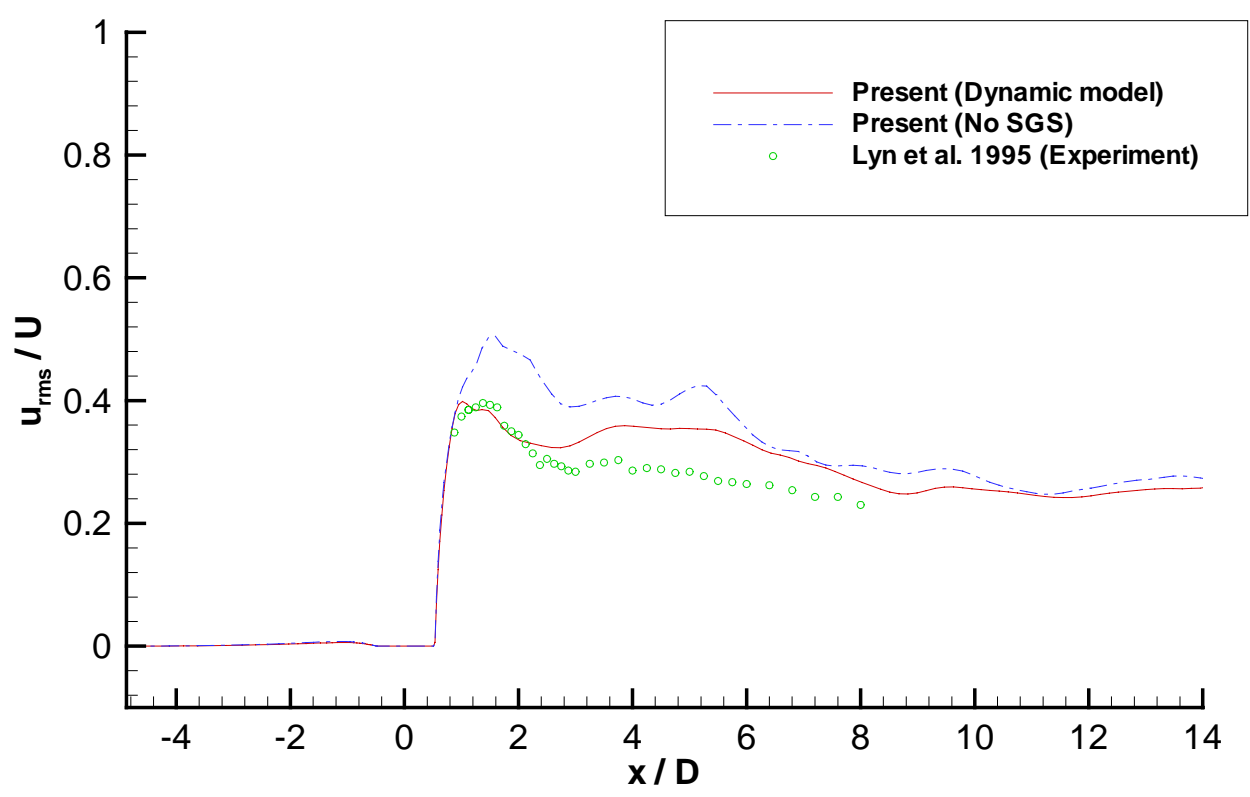

Figure 5-44 Total $u_{r m s}$ fluctuations (periodic + turbulent) along centerline of square cylinder.

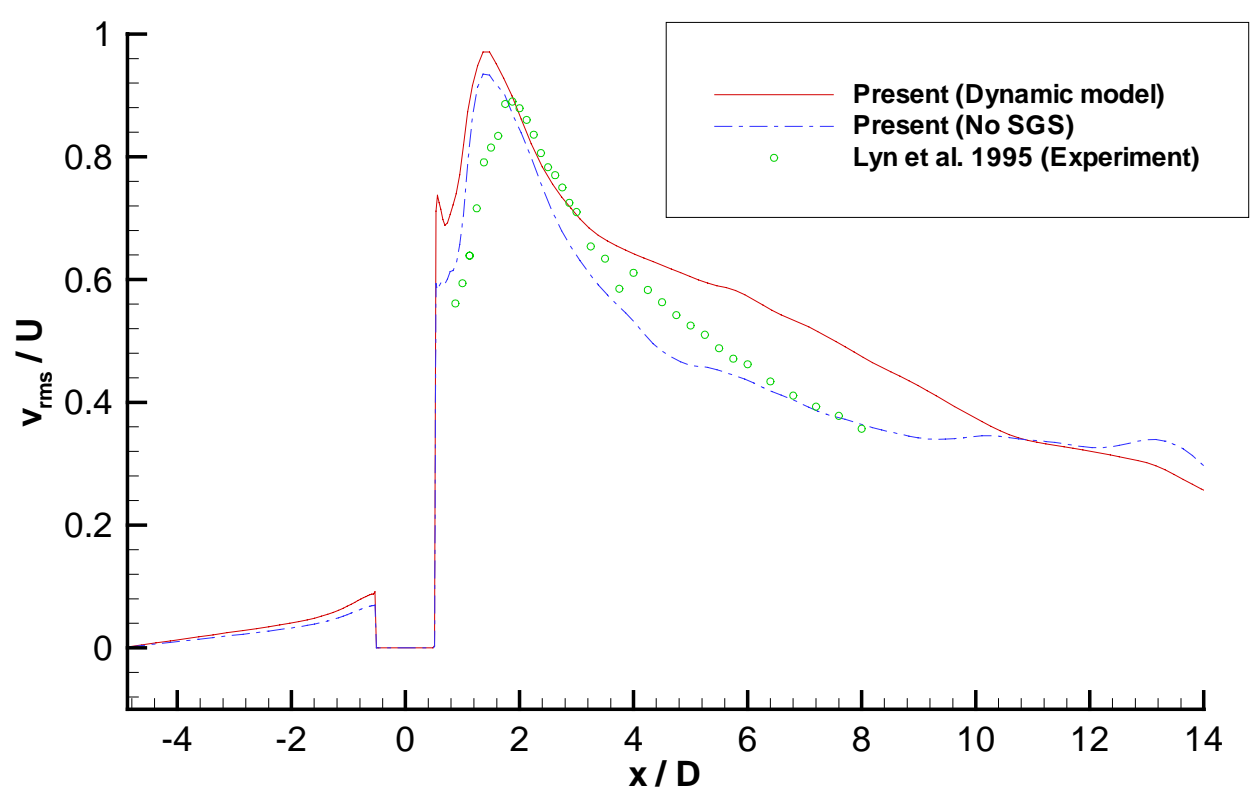

Figure 5-45 Total $v_{\text {rms }}$ fluctuations (periodic + turbulent) along centerline of square cylinder. 


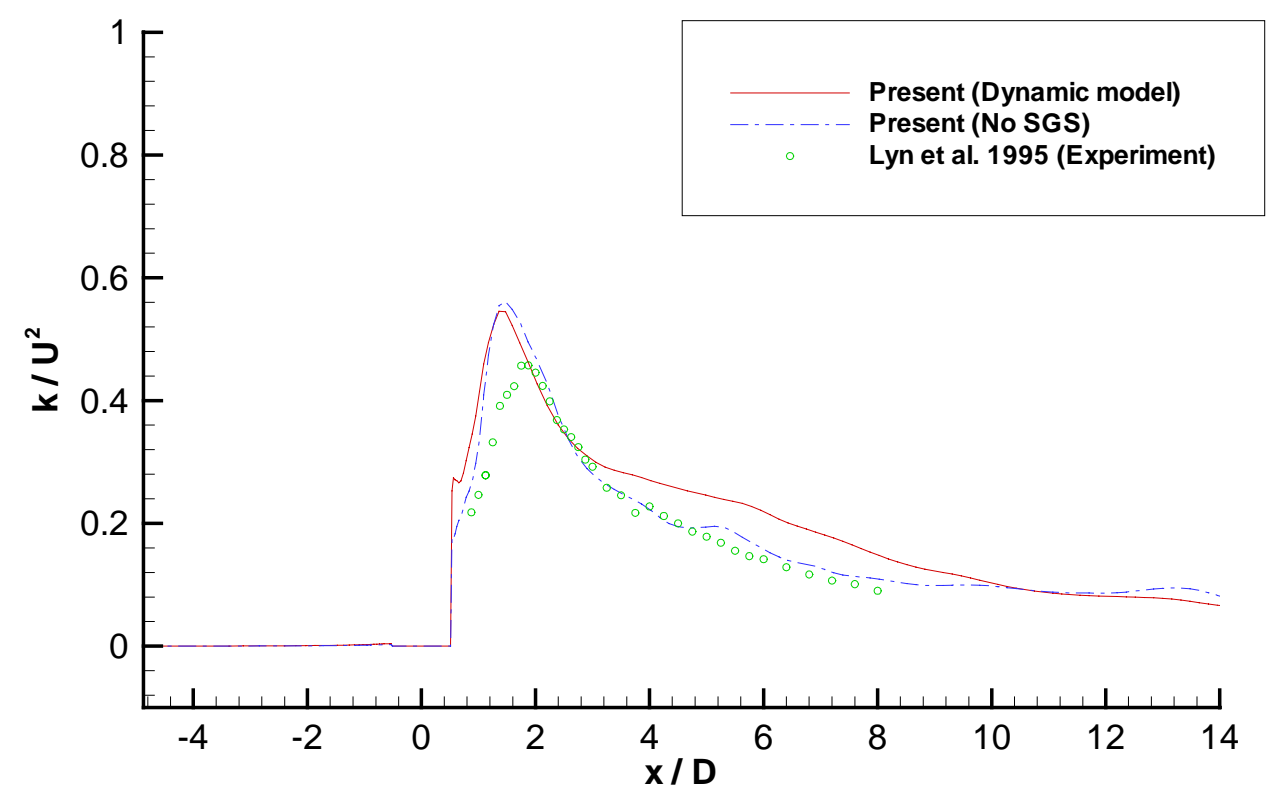

Figure 5-46 Total kinetic energy of fluctuations (turbulent + periodic) along centerline of square cylinder.

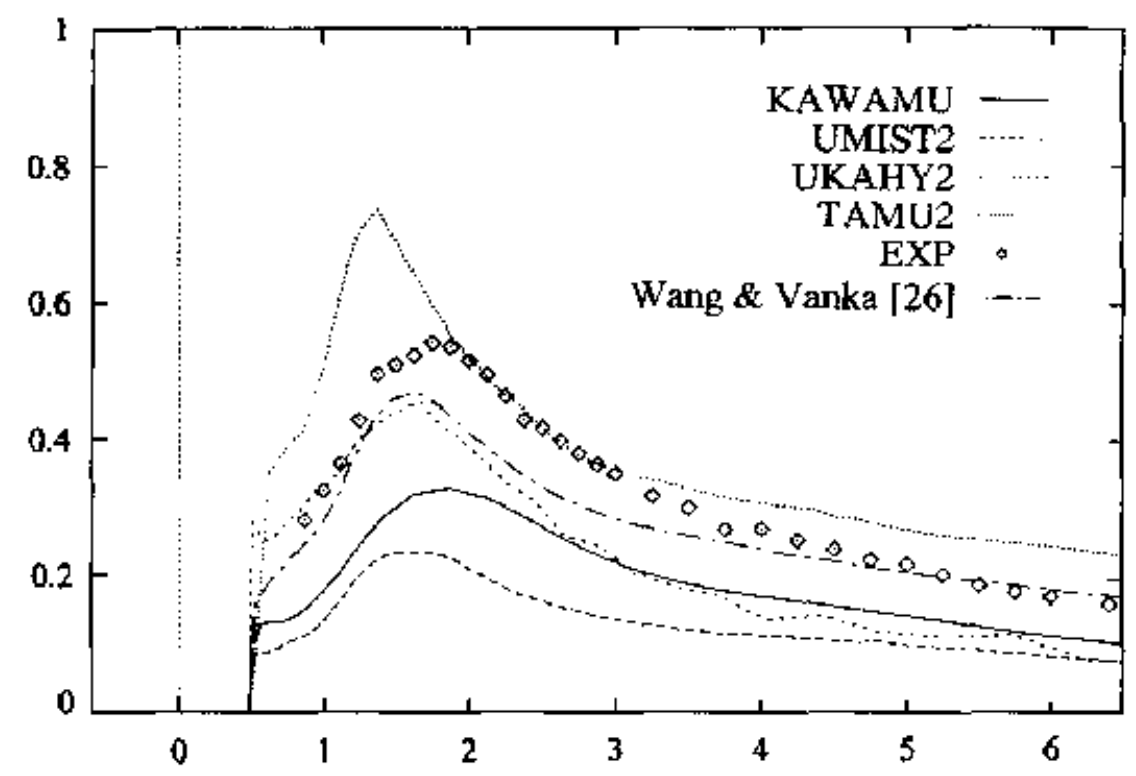

Figure 5-47 Results from Rottach-Egern workshop: total kinetic energy of fluctuations along centerline of square cylinder. (Reprinted from (Rodi 1997) with permission) 


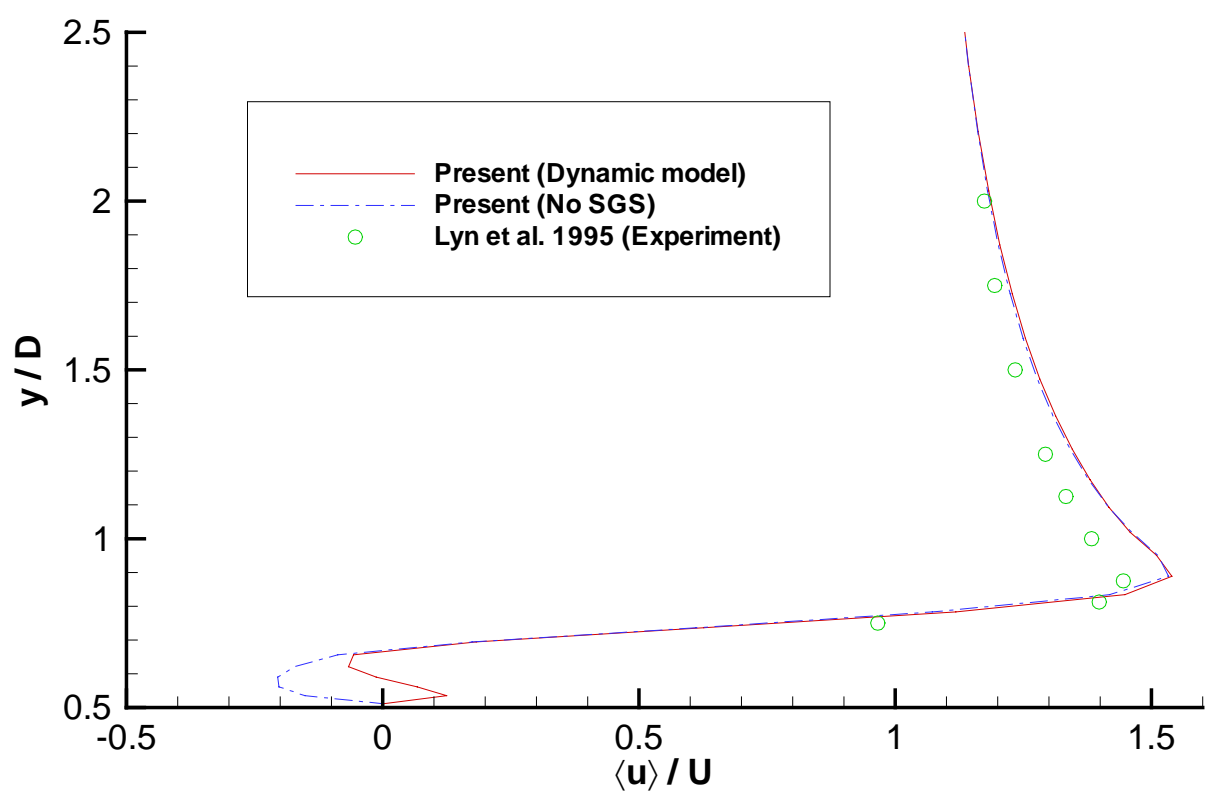

Figure 5-48 Mean streamwise velocity profile along $y$-direction at cylinder center $(x=0)$.

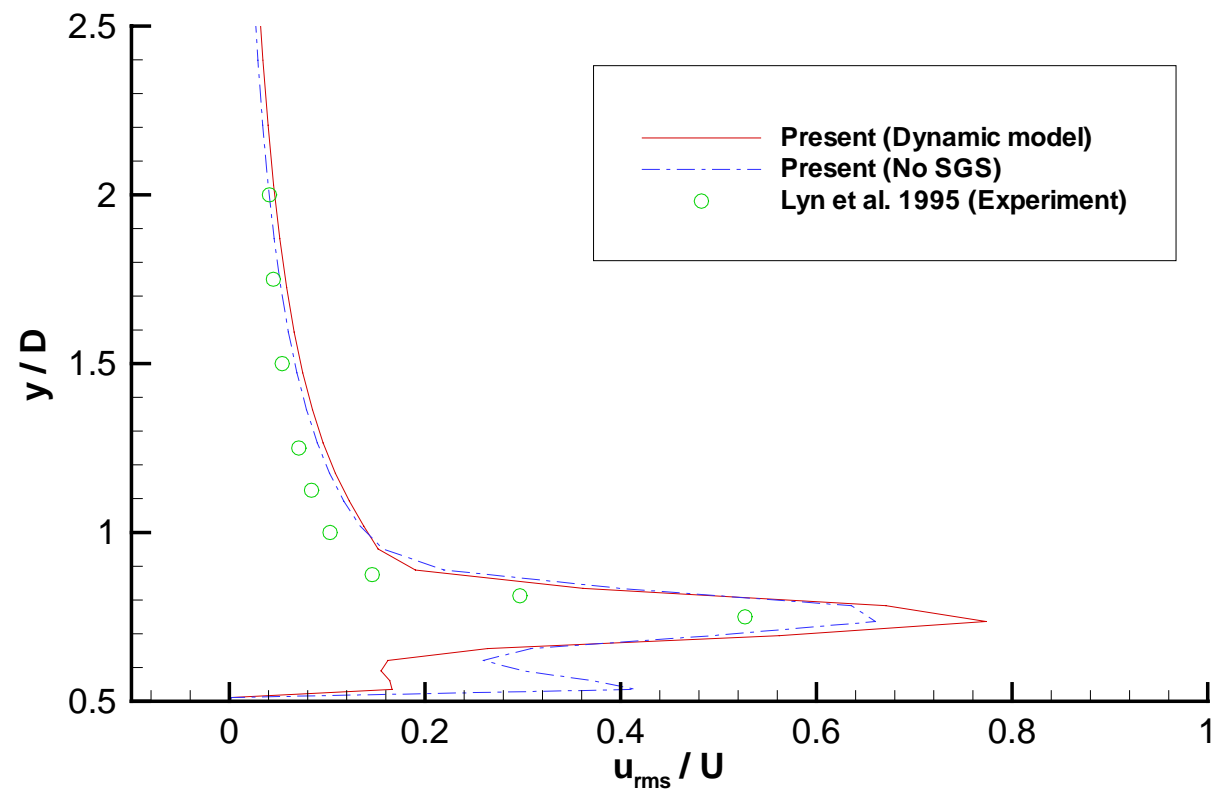

Figure 5-49 Total $u_{r m s}$ fluctuations (periodic + turbulent) along $y$-direction at cylinder center $(x=0)$. 


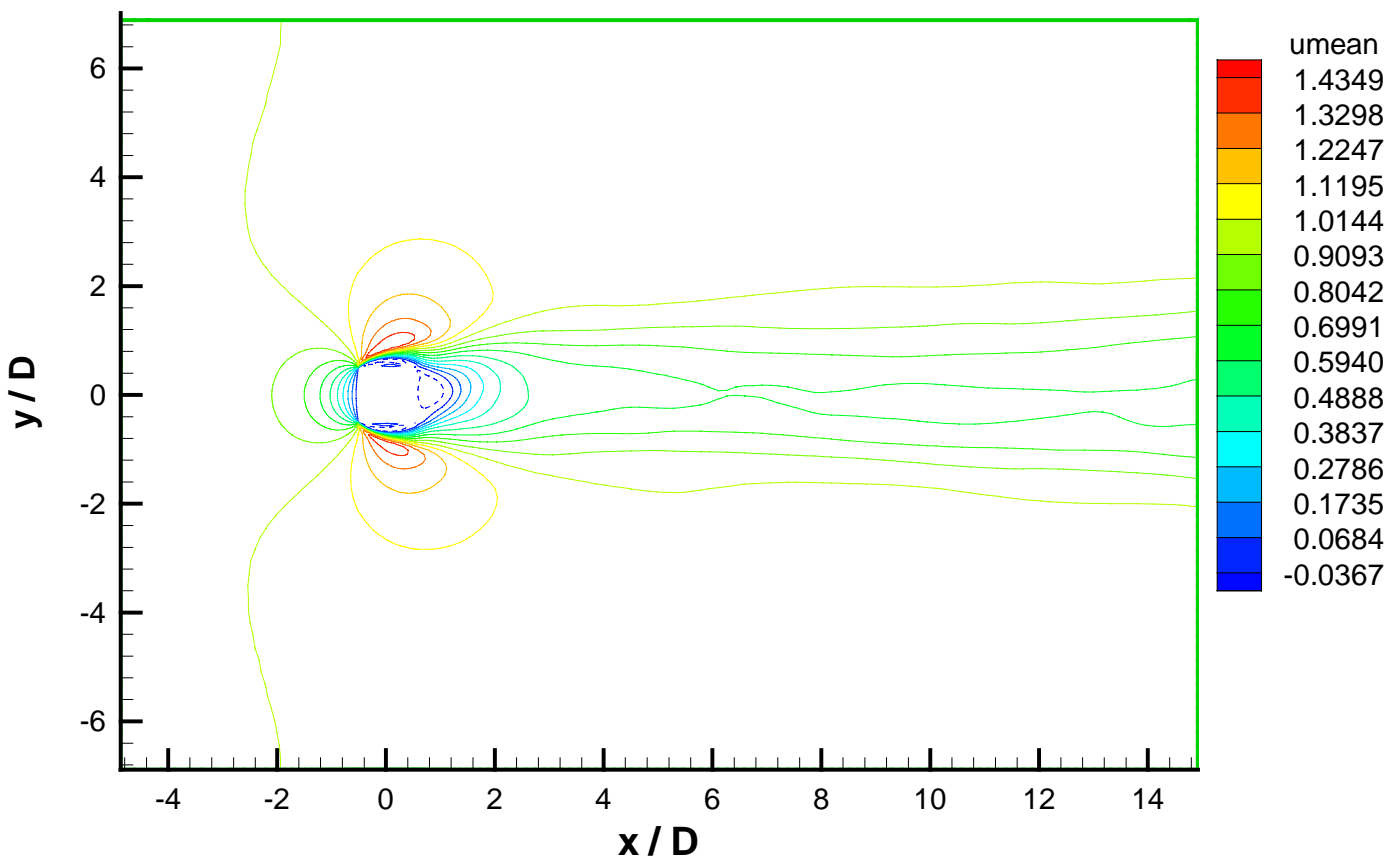

Figure 5-50 Contours of mean streamwise velocity in the center $(x, y)$-plane

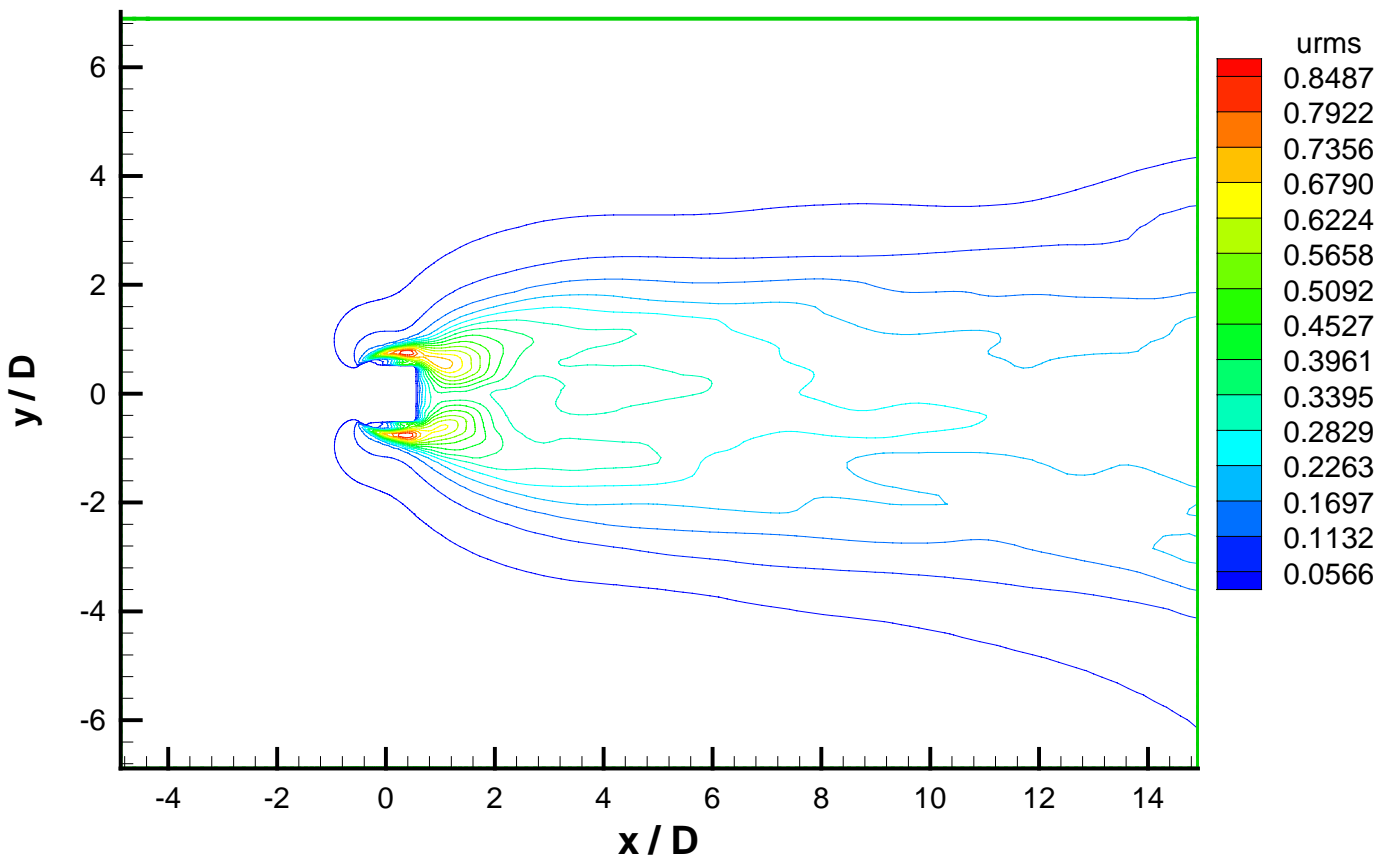

Figure 5-51 $u_{r m s}$ contours in the center $(x, y)$-plane. 


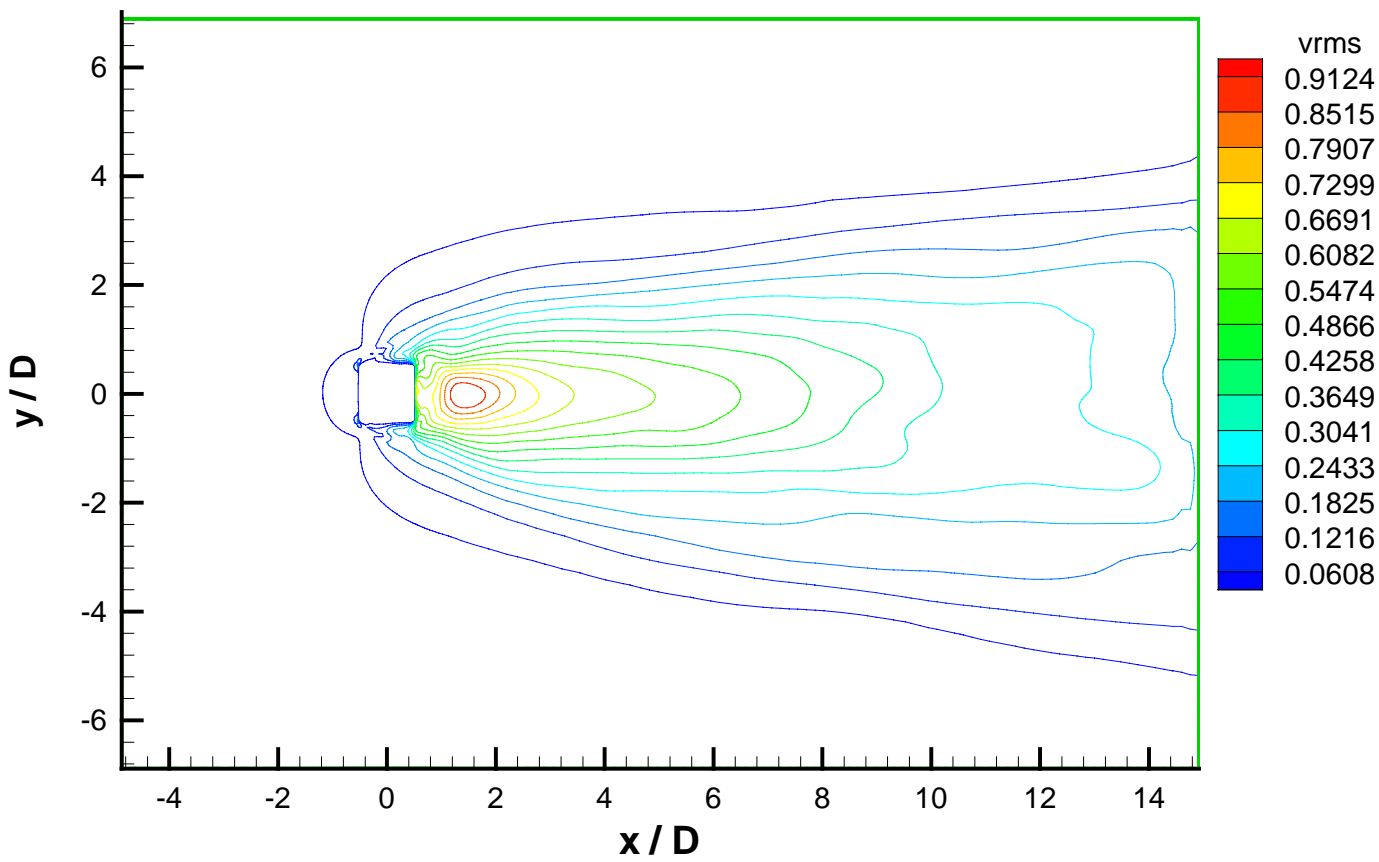

Figure 5-52 $v_{r m s}$ contours in the center $(x, y)$-plane.

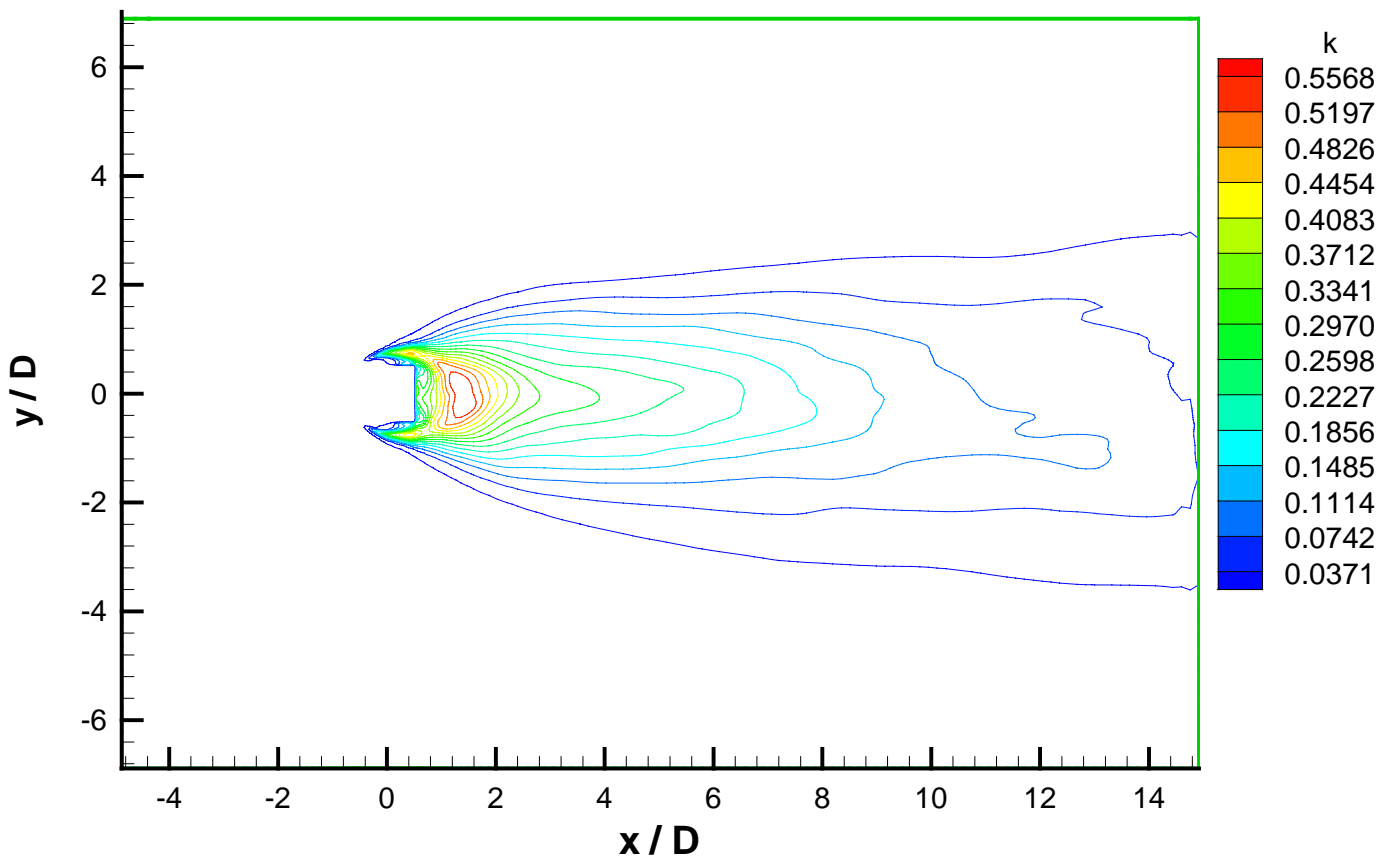

Figure 5-53 Contours of total kinetic energy of fluctuations in the center $(x, y)$ plane. 

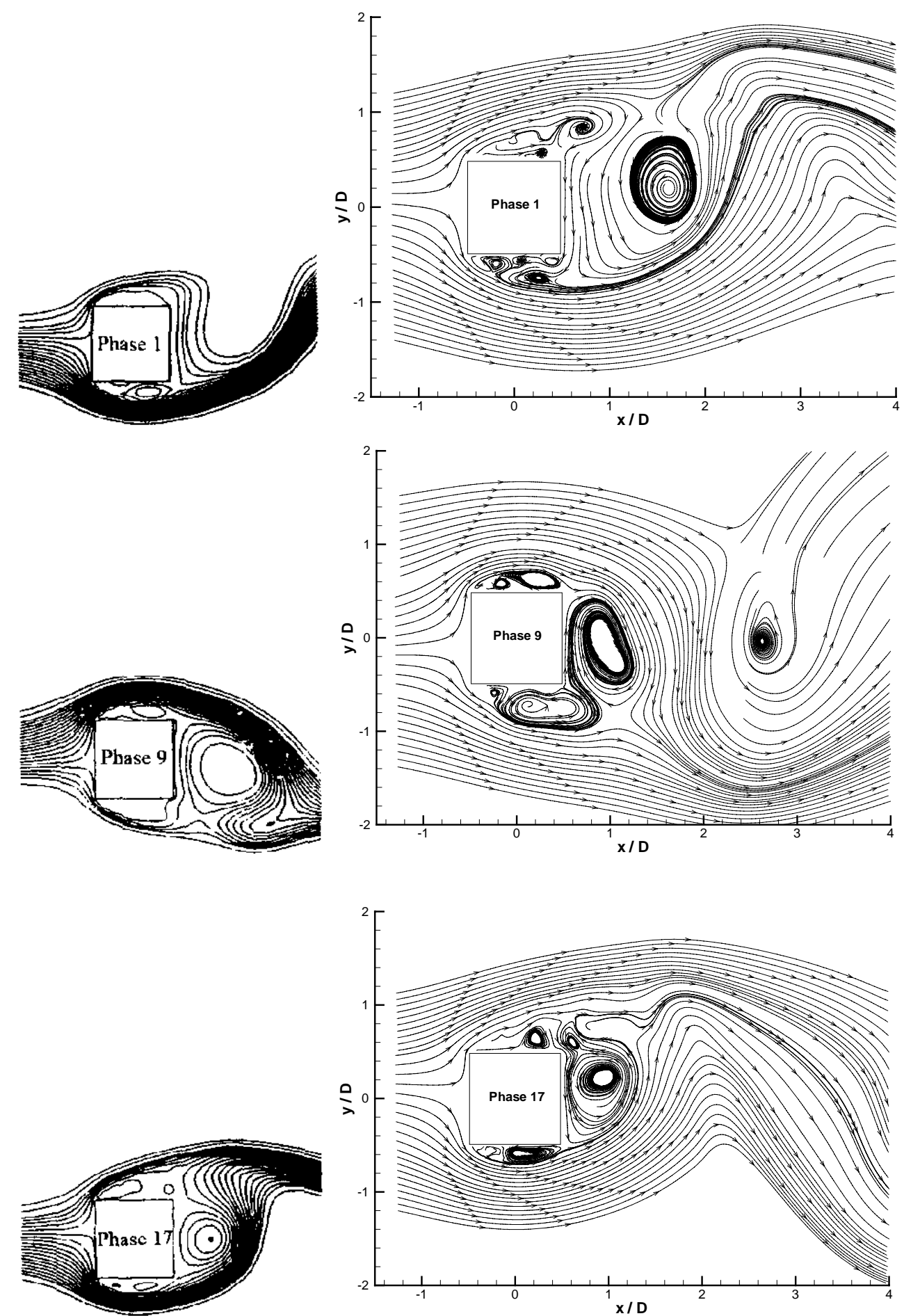

Figure 5-54 Phase-averaged streamlines of flow past a square cylinder at three phases (phase 1, 9 and 17). Left column is from experiment of Lyn et al. (1995), right column is present simulation. 


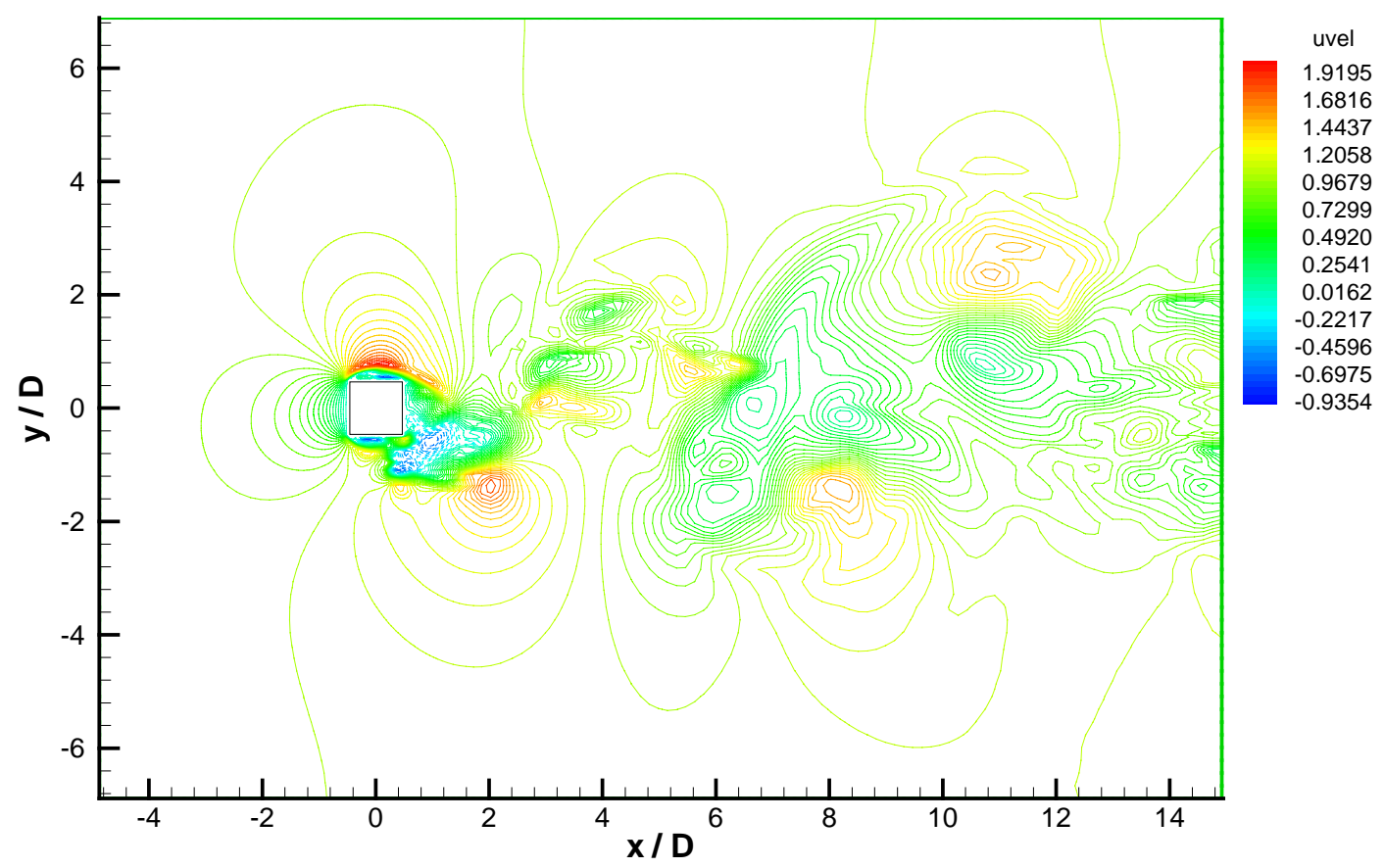

Figure 5-55 Instantaneous streamwise velocity in the center $(x, y)$ plane.

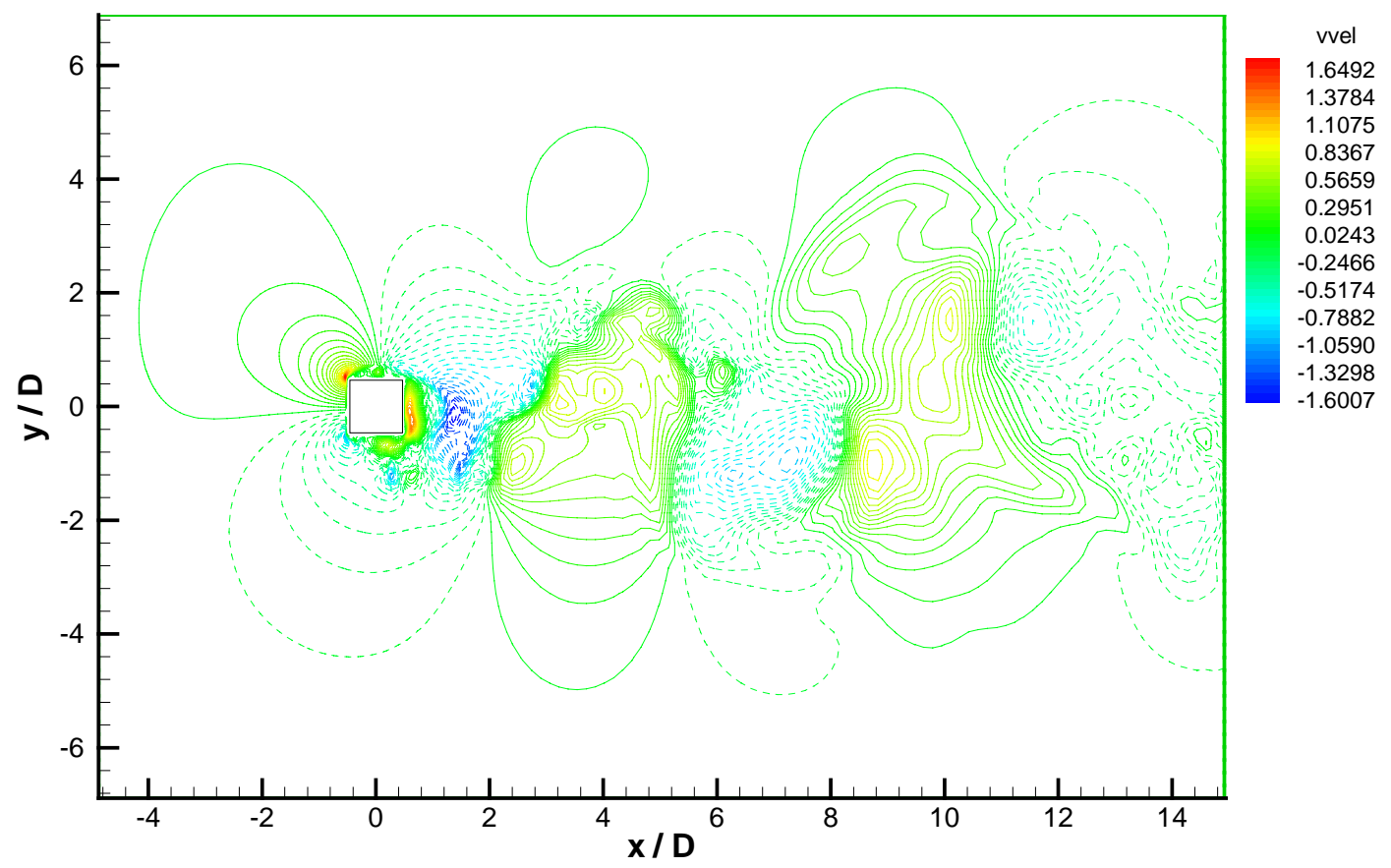

Figure 5-56 Instantaneous vertical velocity in the center $(x, y)$ plane. 


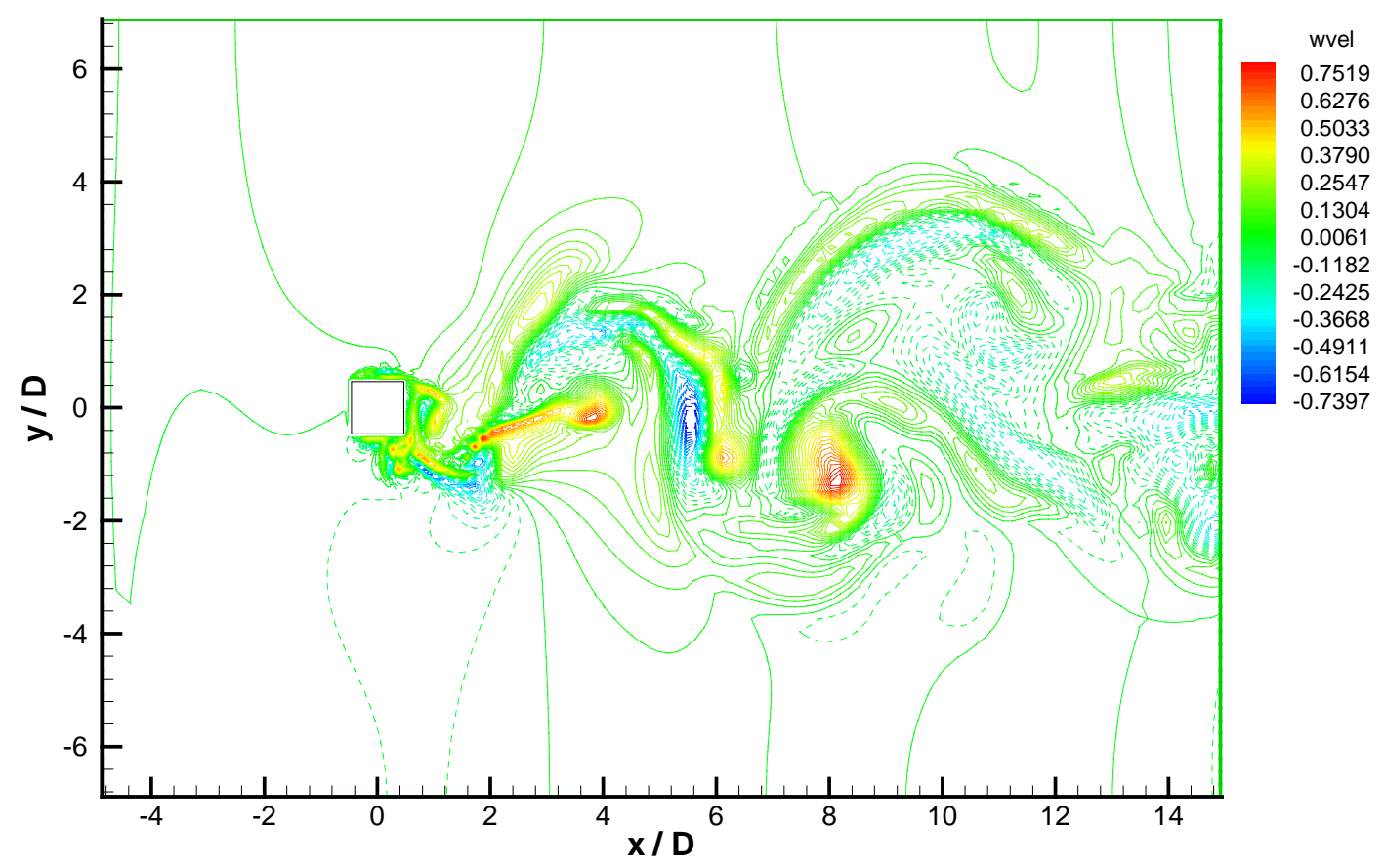

Figure 5-57 Instantaneous spanwise velocity in the center $(x, y)$ plane.

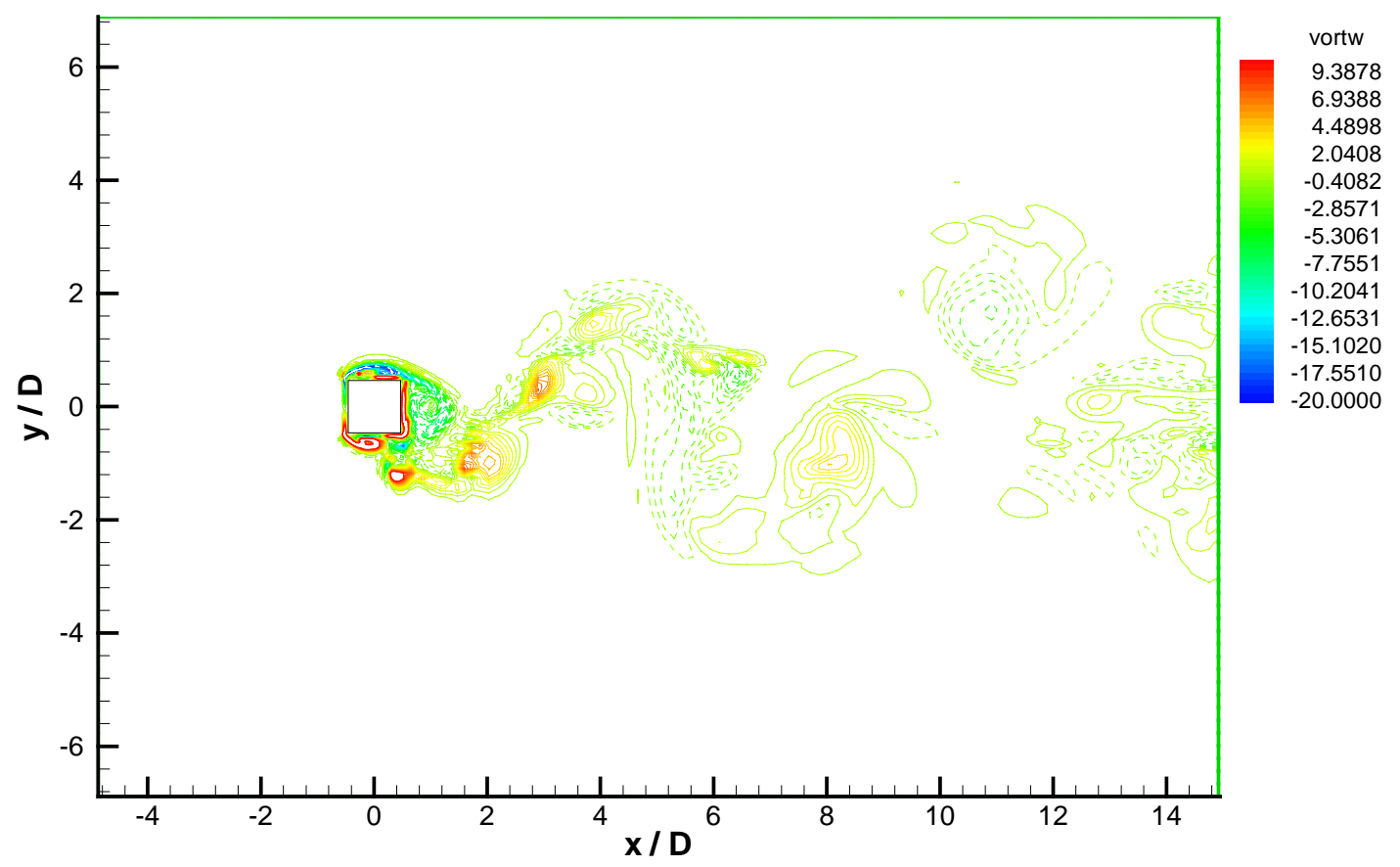

Figure 5-58 Instantaneous spanwise vorticity in the center $(x, y)$ plane. 

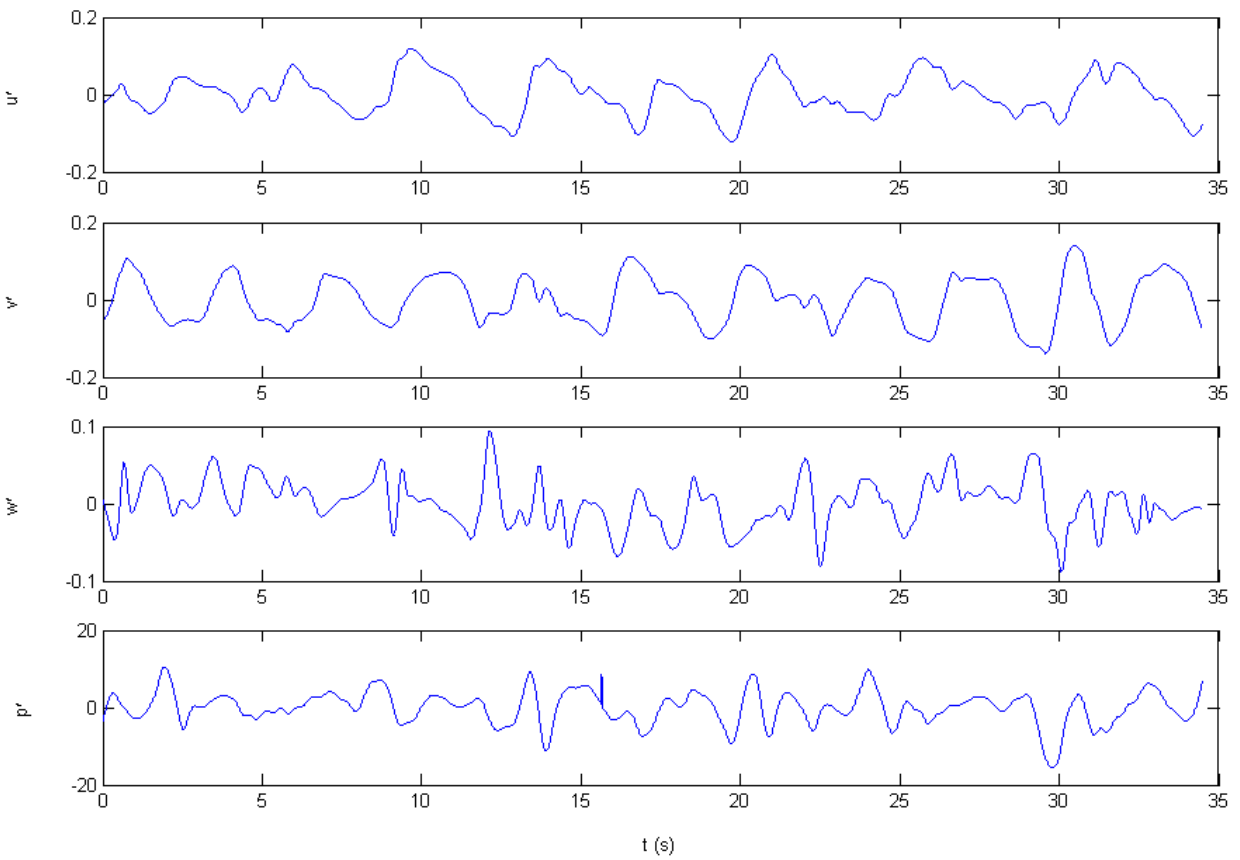

Figure 5-59 Time history of fluctuating signals sampled at (1D, 0D).
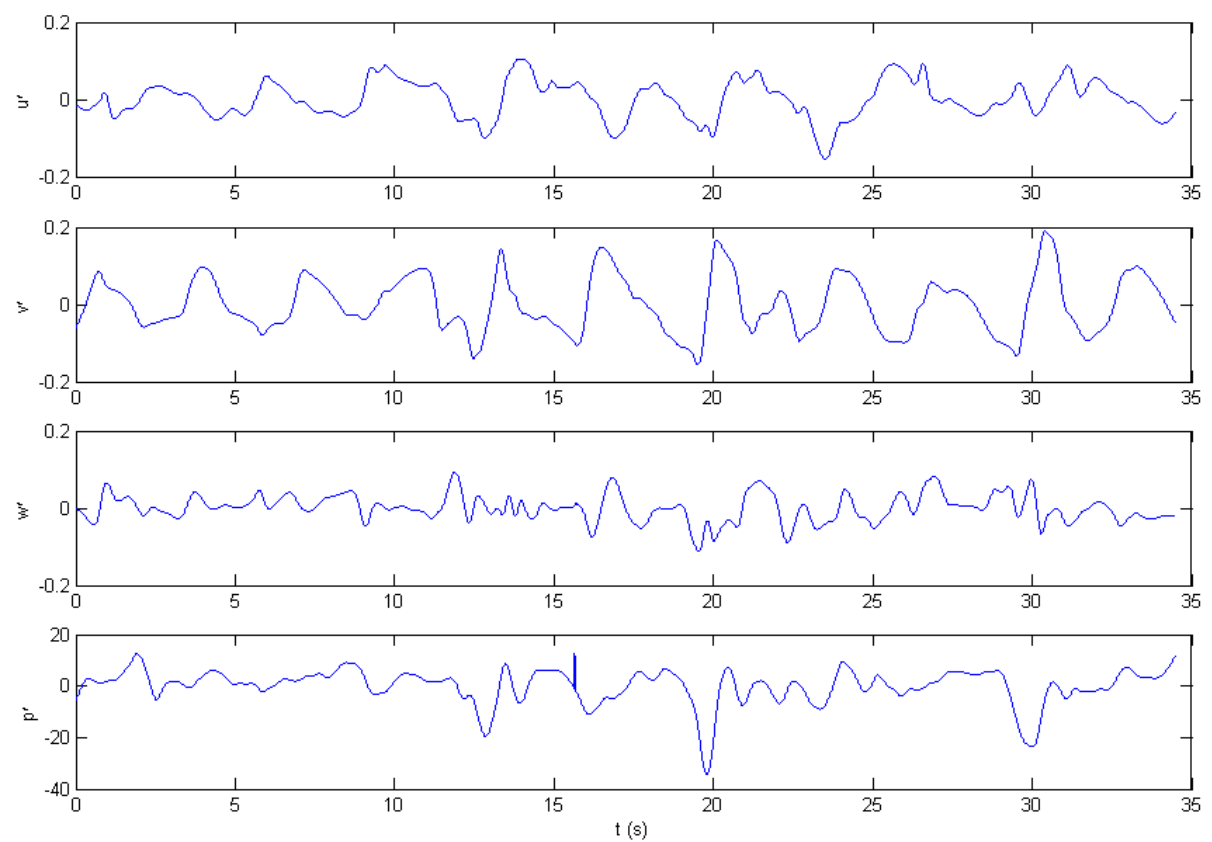

Figure 5-60 Time history of fluctuating signals sampled at (1D, 0.5D). 

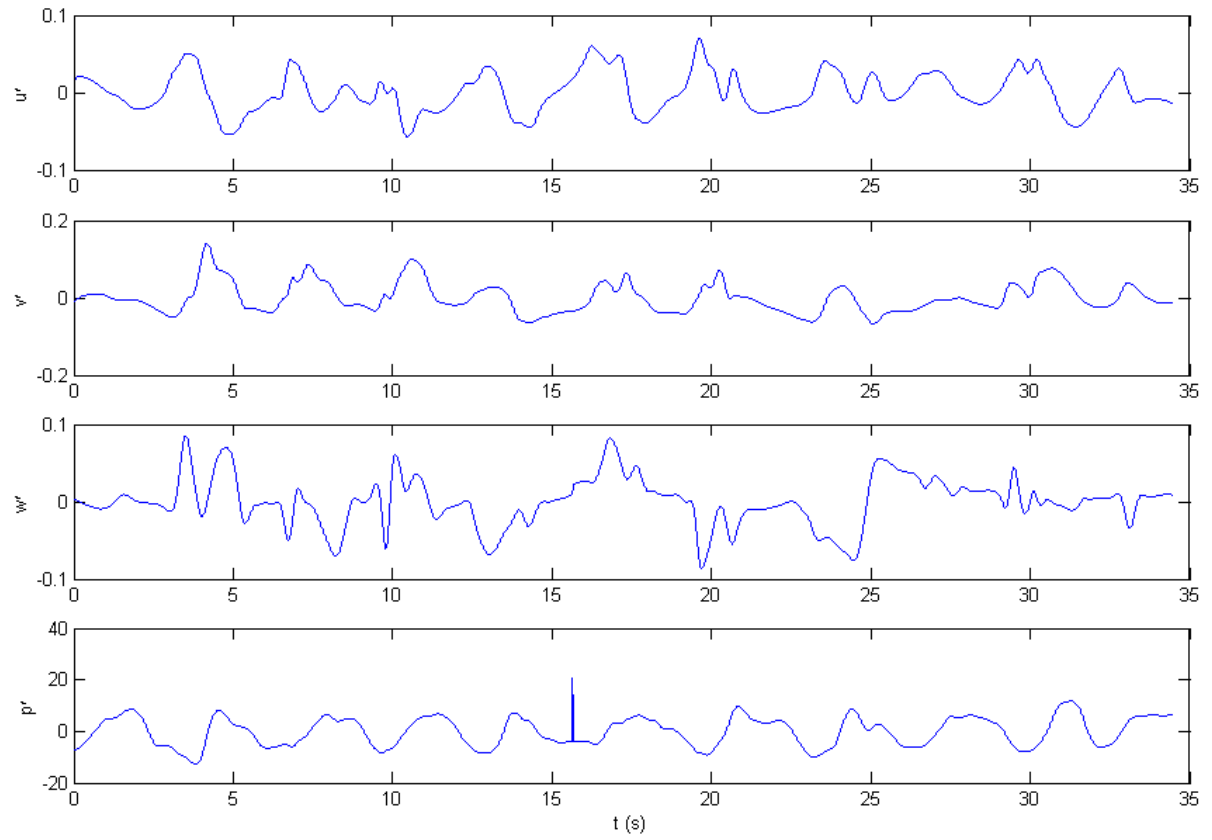

Figure 5-61 Time history of fluctuating signals sampled at (1D, 3D). 

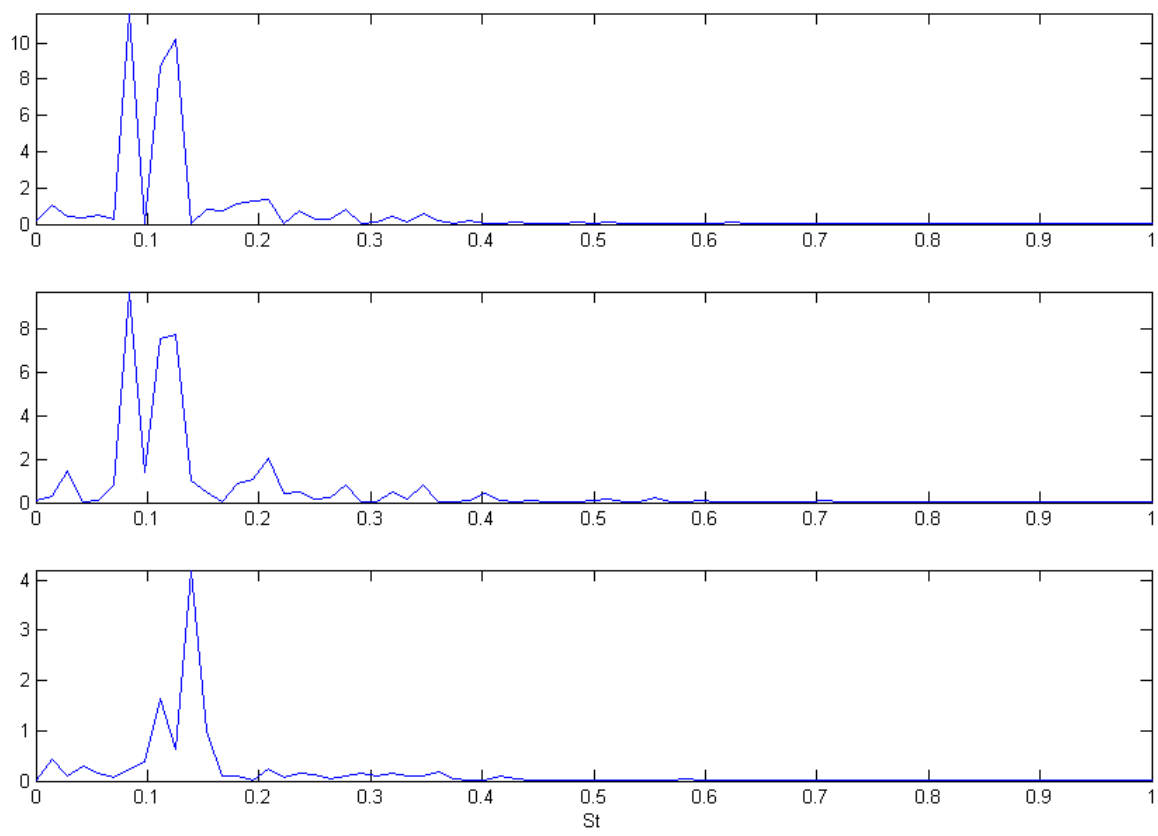

Figure 5-62 Power spectra of u'-signals sampled at, in order, (1D, 0D), (1D, 0.5D) and (1D, 3D).
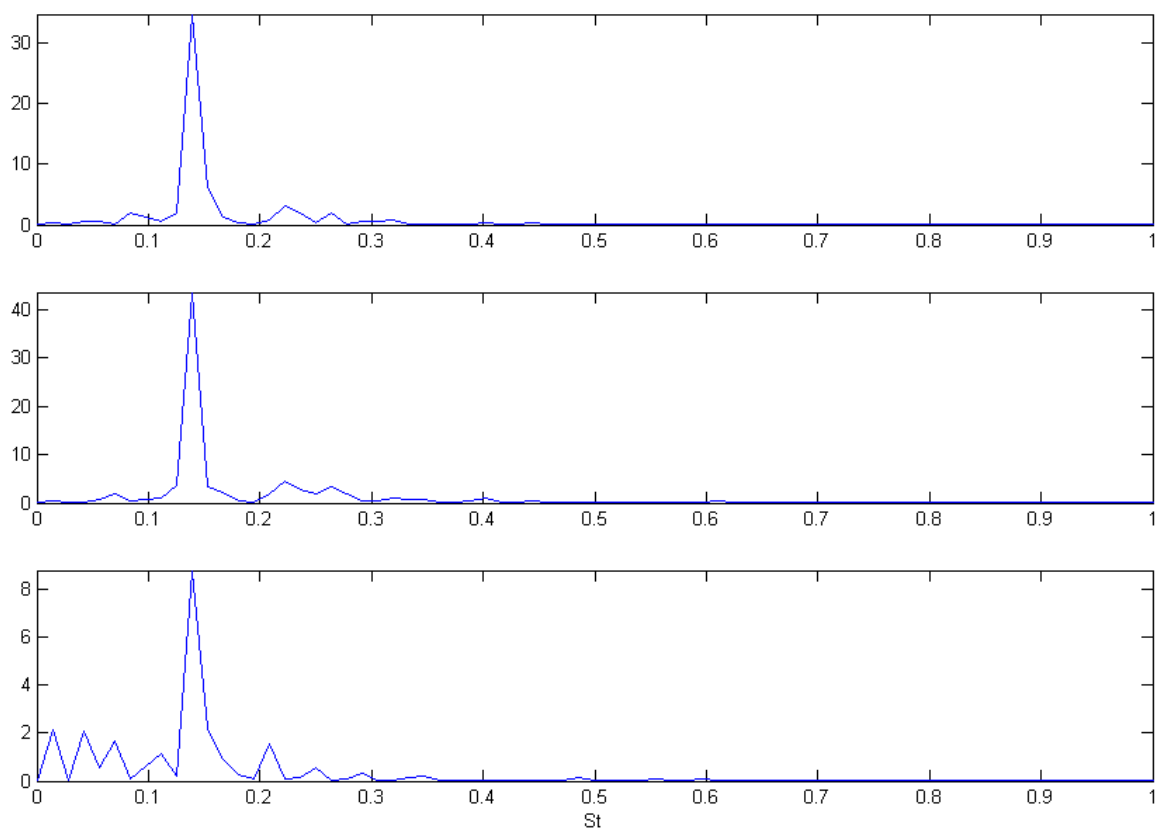

Figure 5-63 Power spectra of v'-signals sampled at, in order, (1D, 0D), (1D, 0.5D) and (1D, 3D). 


\section{Part III Modeling and Simulation of Gas-Liquid Dispersed Two-Phase Flows}

\section{Chapter 6 Verification of Lagrangian Tracking with a Single Rigid Particle in Imposed Flow Fields}

In this chapter the dynamics of a rigid spherical particle is studied. A particle is said to be rigid when its shape is not subject to deformation. The equation of motion of such a particle can usually be described by a linear ordinary differential equation (ODE), which is analytically solvable. This unique opportunity shall be used here to (i) gain some fundamental insight into the dynamics of a single spherical particle, and (ii) to verify the current Lagrangian particle tracking (LPT) implementation crucial for the two-phase flow simulation. Some general references used for the presentation in this chapter are (Clift et al. 1978; Rudinger 1980; Munson et al. 1994; Crowe et al. 1998; Fan and Zhu 1998).

\subsection{Steady-state Drag and Momentum Relaxation Time}

Let vector $\mathbf{u}$ denote flow velocity and $\mathbf{v}$ denote particle velocity. Recall in fluid mechanics, if the particle velocity is different from the conveying fluid velocity, their velocity difference (also called slip-velocity) defined by

$$
\mathbf{v}_{r e l}=\mathbf{v}-\mathbf{u}
$$

leads to viscous stress and unbalanced pressure distribution on the particle surface, which yield a force known as the drag force acting in the direction of upstream velocity. The part of drag due to viscous stresses is called friction drag, and that due to uneven pressure distribution around the body is called pressure drag, or form drag. Since obtaining detailed information of the stresses and pressure distribution around the body is difficult in general, the drag force, $\mathbf{F}_{D}$, is conventionally quantified as a whole by an empirical drag coefficient, $C_{D}$, through the equation 


$$
\mathbf{F}_{D}=-\frac{1}{2} C_{D} \rho A_{f r}\left|\mathbf{v}_{r e l}\right| \mathbf{v}_{r e l},
$$

where $\rho$ is the fluid density, $A_{f r}$ is the frontal area of the particle exposed to the direction of the incoming flow. For a spherical particle

$$
A_{f r}=\frac{1}{4} \pi d_{p}^{2}
$$

The drag force is measured under two general flow conditions: (i) there is no acceleration of the relative velocity between the particle and conveying fluid, i.e., the particle reaches a steady state. And (ii) the pressure field is uniformly distributed in the absence of particle; this excludes the influence of an existing pressure gradient on the pressure drag. The drag force measured in this setting is often referred to as the steadystate drag or quasi-steady drag in the case of turbulent flow.

In general, the steady state drag force is a function of the particle shape, the orientation with respect to the flow as well as of the particle Reynolds number. In the current study only spherical particle is considered. The particle Reynolds number for a spherical particle is defined as

$$
\operatorname{Re}_{p}=\frac{\rho d_{p}\left|\mathbf{v}_{r e l}\right|}{\mu},
$$

where $d_{p}$ is the particle diameter, $\rho$ and $\mu$ are properties of the continuous phase. The frontal area and the volume of a spherical particle are

$$
\begin{aligned}
& A_{f r}=\frac{1}{4} \pi d_{p}{ }^{2}, \\
& V_{p}=\frac{1}{6} \pi d_{p}{ }^{3} .
\end{aligned}
$$

At very low particle Reynolds numbers, i.e., Re $<1$, the flow is called creeping flow or Stokes flow, in which the inertial terms in the N-S equations can be neglected. In this flow regime, the reduced N-S equations were solved analytically by Stokes (Stokes 1851) who transformed the equation into spherical coordinates and further introduced a stream function for axisymmetric flow to turn the PDE into a $4^{\text {th }}$ order ODE. The solved velocities can be written as

$$
\begin{aligned}
& v_{\theta}=-U \sin \theta\left[1-\frac{3}{4} \frac{a}{r}-\frac{1}{4}\left(\frac{a}{r}\right)^{3}\right], \\
& v_{r}=-U \cos \theta\left[1-\frac{3}{2} \frac{a}{r}+\frac{1}{2}\left(\frac{a}{r}\right)^{3}\right],
\end{aligned}
$$

where $a$ is the particle radius, $U$ is the free stream velocity. The pressure $\operatorname{drag}, F_{D, p}$, and friction drag, $F_{D, \tau}$, can be readily deduced from the obtained velocity field:

$$
\begin{aligned}
& F_{D, p}=\pi d_{p} \mu U, \\
& F_{D, \tau}=2 \pi d_{p} \mu U,
\end{aligned}
$$

with the total drag force being the summation of the both: 


$$
F_{D}=F_{D, \tau}+F_{D, p}=3 \pi d_{p} \mu U .
$$

Eq. (6.8) may be rewritten in an equivalent vector form:

$$
\mathbf{F}_{D}=3 \pi \mu d_{p}(\mathbf{u}-\mathbf{v}),
$$

which can be used to obtain the expression for drag coefficient, $C_{D}$, defined in Eq. (6.2):

$$
C_{D}=\frac{24 \mu}{\rho d_{p} \mathbf{v}_{r e l}}=\frac{24}{\operatorname{Re}_{p}} .
$$

This $C_{D}$ expression is often referred to as the Stokes drag coefficient as it is derived under the Stokes flow condition. It is seen that the Stokes drag varies linearly with the slip velocity (therefore it is also called linear drag), and the Stokes drag coefficient is proportional to the reciprocal of the particle Reynolds number. For small particles, say less than 100 micron, the particle Reynolds number usually is quite small, and the Stokes linear drag can be assumed to apply.

When the flow is beyond the Stokes regime, correlations based on experimental data are usually employed to determine $C_{D}$. It is a common practice to express $C_{D}$ as the product of the Stokes drag coefficient (Eq. (6.10)) and a correction factor, $f$, known as the drag factor or the Stokes correction:

$$
C_{D}=\frac{24}{\operatorname{Re}_{p}} f\left(\operatorname{Re}_{p}\right),
$$

where $f$ is a function of $\operatorname{Re}_{p}$. Expressions for $f$ can be found in most texts on fluid mechanics or particle dynamics (for instance, (Clift et al. 1978; Schlichting 1979)). Some

\begin{tabular}{|c|c|c|c|}
\hline $\begin{array}{l}\text { (Shiller and } \\
\text { Naumann 1933) }\end{array}$ & $f=\left\{\begin{array}{cc}1+0.15 \mathrm{Re}_{p}^{0.687} & \operatorname{Re}_{p} \leq 800 \\
0.44\left(\operatorname{Re}_{p} / 24\right) & \operatorname{Re}_{p}>800\end{array}\right.$ & & $(6.12)$ \\
\hline $\begin{array}{l}\text { (Clift et al. 1978; } \\
\text { Loth 2000) }\end{array}$ & $f=\left\{\begin{array}{l}1+0.1875 \operatorname{Re}_{p} \\
1+0.1935 \operatorname{Re}_{p}^{0.6305} \\
1+0.015 \operatorname{Re}_{p}+0.2283 \operatorname{Re}_{p}^{0.427} \\
0.44\left(\operatorname{Re}_{p} / 24\right)\end{array}\right.$ & $\begin{array}{l}\operatorname{Re}_{p} \leq 1 \\
1<\operatorname{Re}_{p} \leq 285 \\
285<\operatorname{Re}_{p} \leq 2000 \\
\operatorname{Re}>2000\end{array}$ & (6.13) \\
\hline (Schuh et al. 1989) & $f=\left\{\begin{array}{l}1+0.15 \operatorname{Re}_{p}^{0.687} \\
0.914 \operatorname{Re}_{p}^{0.282}+0.0135 \operatorname{Re}_{p} \\
0.4008\left(\operatorname{Re}_{p} / 24\right)\end{array}\right.$ & $\begin{array}{l}0<\operatorname{Re}_{p} \leq 200 \\
200<\operatorname{Re}_{p} \leq 2500 \\
\operatorname{Re}_{p}>2500\end{array}$ & $(6.14)$ \\
\hline $\begin{array}{l}\text { (Morsi and } \\
\text { Alexander 1972) }\end{array}$ & $\begin{array}{l}f=C_{1}+\frac{C_{2}}{\operatorname{Re}_{p}}+\frac{C_{3}}{\operatorname{Re}_{p}^{2}} \\
\text { where } C_{1}, C_{2}, C_{3} \text { are functions of } \mathrm{I}\end{array}$ & & $(6.15)$ \\
\hline
\end{tabular}
popular correlations are listed in Table 6-1. If $f=1$, the Stokes drag coefficient is retained.

Table 6-1 Commonly used drag correction factor $f$. 
If the drag force is the only force that applies on a particle, the equation of motion for the particle can be written as

$$
\rho_{p} V_{p} \frac{d \mathbf{v}}{d t}=-\frac{1}{2} C_{D} \rho A_{f r}|\mathbf{v}-\mathbf{u}|(\mathbf{v}-\mathbf{u}),
$$

where $\rho_{\mathrm{p}}, V_{p}$ stand for particle density and volume respectively. Using the general expression of $C_{D}$ (Eq. (6.11)) and the geometric properties of a sphere (Eq. (6.5)), the above equation can be rewritten as

$$
\frac{d \mathbf{v}}{d t}=-\frac{f\left(\mathrm{Re}_{p}\right)}{\tau_{p}}(\mathbf{v}-\mathbf{u}),
$$

where

$$
\tau_{p}=\frac{\rho_{p} d_{p}^{2}}{18 \mu} .
$$

is called the momentum (velocity) relaxation time, or response time of the particle. It is related to the time required for a particle to respond to a change in velocity. For Stokes flow where $f=1$, Eq. (6.17) is simplified to

$$
\frac{d \mathbf{v}}{d t}=-\frac{(\mathbf{v}-\mathbf{u})}{\tau_{p}} .
$$

For a constant $\mathbf{u}$, Eq. (6.19) is readily integrated with the result

$$
\mathbf{v}-\mathbf{u}=\left(\mathbf{v}_{0}-\mathbf{u}\right) \exp \left(-t / \tau_{p}\right),
$$

where $\mathbf{v}_{0}$ is the particle velocity at $t=0$. From the above relation it is seen that if $\mathbf{v}_{0}=\mathbf{0}$, $\tau_{v}$ reflects the time required for a particle released from its initial condition to achieve $63 \%$, i.e., $(e-1) / e$, of the initial velocity difference (slip velocity).

In the following sections studied are some classical examples characterizing dynamics of a single spherical particle in an imposed flow field. In most of the cases a small particle with small $\operatorname{Re}_{p}$ is assumed so that the Stokes drag can apply. Symbols $u_{p}, v_{p}$ and $w_{p}$ shall be used to denote the three components of the particle velocity, whereas $u, v$, $w$ are still reserved for the fluid velocities. Similarly, $x_{p}, y_{p}$ and $z_{p}$ represents the particle position in Cartesian coordinates.

\subsection{Gravitational Settling}

Consider a particle falling into a quiescent fluid (Figure 6-1(a)). At sufficiently large time gravity is balanced by drag force. The particle motion is described by

$$
\rho_{p} V_{p} \frac{d u_{p}}{d t}=\rho_{p} g V_{p}-\frac{1}{2} C_{d} \rho A_{f r}\left|u_{p}\right| u_{p},
$$

and

$$
\frac{d x_{p}}{d t}=u_{p}
$$

where $g=-9.81 \mathrm{~m} / \mathrm{s}^{2}$. Note that the drag force always acts in the opposite direction of the particle velocity, thus the minus sign in front of it. Applying Stokes drag coefficient yields 


$$
\frac{d u_{p}}{d t}=-\frac{u_{p}}{\tau_{p}}+g
$$

where $\tau_{p}$ is defined in Eq. (6.18). One may wish to recast Eq. (6.23) into a mathematically more legible form,

$$
\frac{d u_{p}}{d t}+P u_{p}=Q
$$

with

$$
P=\frac{1}{\tau_{p}} \text { and } Q=g
$$

The general solution of this first order nonhomogeneous ODE is a combination of the general solution of its corresponding complementary equation (the ODE without the gravity term) and a particular solution, i.e.,

$$
u_{p}(t)=C \exp (-P t)+\frac{Q}{P}
$$

where the integration constant $C$ is determined from the initial particle velocity $u_{p}=u_{p, 0}$. The final solution is

$$
\begin{aligned}
u_{p}(t) & =\left(u_{p, 0}-\frac{G}{P}\right) \exp \left(-t / \tau_{p}\right)+\frac{Q}{P} \\
& =\left(u_{p, 0}-g \tau_{p}\right) \exp \left(-t / \tau_{v}\right)+g \tau_{p} .
\end{aligned}
$$

The terminal velocity of the particle, which is defined as $t$ goes to infinity, is thus

$$
u_{p, \infty}=g \tau_{p} \text {. }
$$

Given the initial particle position, $x_{p}=x_{p, 0}$, the exact trajectory can be readily obtained by integrating Eq. (6.27) from zero to time $t$ :

$$
x_{p}(t)=\left(u_{p, 0}-g \tau_{p}\right) \tau_{p}\left[1-\exp \left(-t / \tau_{p}\right)\right]+g \tau_{p} t+x_{p, 0} .
$$

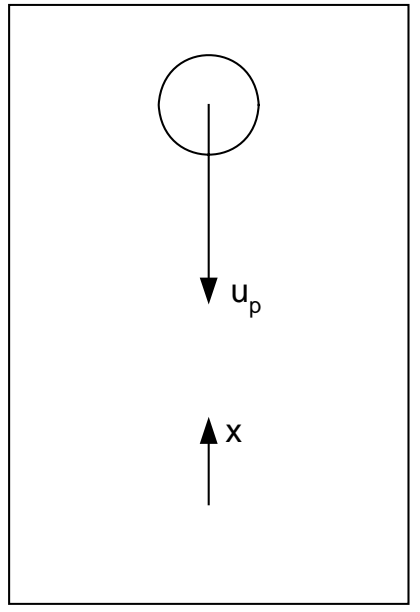

(a)

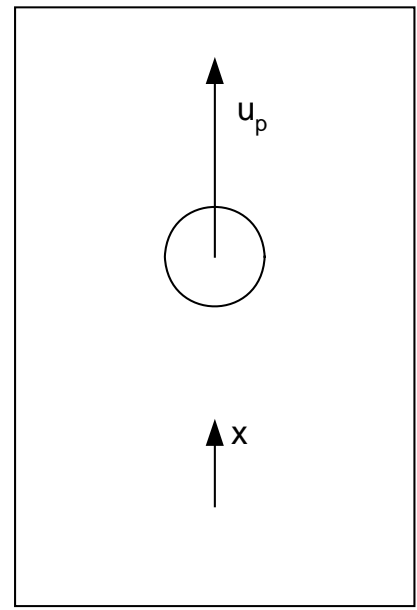

(b)

Figure 6-1 (a) Gravitational settling, (b) buoyant settling. 
To compute the trajectory numerically, the classical Runge-Kutta (RK) methods can be used. A detailed discussion and related formulae on the RK methods have been presented in Section 2.5. Here the $2^{\text {nd }}$ order (Eq. (2.65)) and $4^{\text {th }}$ order RK (Eq. (2.67)) formulae will be applied.

It should be noted that the program for particle tracking is not specifically written for this simple problem formulated in 1D space. In fact, it is capable of tracking the transient motion of particle(s) in a truly 3D space, and can be used in conjunction with the main N-S solver. Therefore, the numerical computations presented throughout this chapter for a single particle can be regarded as a rigorous verification procedure for this general Lagrangian tracking program, rather than testing the capability of the RK methods.

As a sample computation consider a $50 \mu \mathrm{m}$ water drop (density $=1 \mathrm{E} 3 \mathrm{~kg} / \mathrm{m}^{3}$ ) falling in quiescent air. The dynamic viscosity of the air is set to $1.0 \mathrm{E}-5 \mathrm{~N} \cdot \mathrm{s} / \mathrm{m}^{2}$. The momentum relaxation time of the drop is thus, by Eq. (6.18), $0.0139 \mathrm{~s}$, and its theoretical terminal velocity is $-0.136 \mathrm{~m} / \mathrm{s}$, according to Eq. (6.28). The particle Reynolds number based on the theoretical terminal velocity is then 0.681 , which verifies the validity of the Stokes regime assumption $\left(\operatorname{Re}_{p}<1\right)$. Figure 6-2(a) and (b) show the velocity and position history in the first 60 seconds for a drop starting from an initial velocity of $0 \mathrm{~m} / \mathrm{s}$. The scheme used is the $2^{\text {nd }}$ order Runge-Kutta (RK2) with a time step of $1 \mathrm{E}-3 \mathrm{~s}$. At the end of 60 s the computed drop velocity reaches $98.7 \%$ of the terminal velocity.

More accurate results can be achieved by employing the $4^{\text {th }}$ order RK method (RK4). This is shown through a comparison of the exact error of the drop velocity computed using the RK2 and RK4 schemes with the same time step (1E-3s). As it is seen in Figure 6-3(a) and (b), the $4^{\text {th }}$ order RK reduces the maximum absolute error by at least two orders of magnitude.

An error analysis to verify the order of the method is also performed. The exact absolute error at $t=60 \mathrm{~s}$ with three different time steps, i.e., $0.0005 \mathrm{~s}, 0.001 \mathrm{~s}$ and $0.002 \mathrm{~s}$, are collected for the RK2 scheme, and the post-processed data for the order of the method is listed in Table 6-2, as well as in Figure 6-4. It is clearly seen that the implemented RK2 is truly a $2^{\text {nd }}$ order scheme.

Table 6-2 Order of method analysis of RK2 for the calculation of gravitational settling.

$\begin{array}{cccccc}\text { Step size } & \mathrm{h} / \mathrm{h}_{\text {ref }} & \varepsilon(\mathrm{t}=50 \mathrm{~s}) & \ln \left(\mathrm{h} / \mathrm{h}_{\text {ref }}\right) & \ln (\varepsilon) & \text { slope } \\ 0.0005 & 1 & 1.7732 \mathrm{E}-06 & 0.0000 & -13.2427 & \\ 0.001 & 2 & 7.1526 \mathrm{E}-06 & 0.6931 & -11.8480 & \\ 0.002 & 4 & 3.0383 \mathrm{E}-05 & 1.3863 & -10.4016 & \mathbf{2 . 0 4 9 4}\end{array}$

It should be noted that although the RK4 scheme provides more accurate results than RK2, its accuracy is limited by the order of interpolation method with respect to the continuous flow velocity field, and thus, it does not necessarily yield a desired $4^{\text {th }}$ order if a similar order of method analysis is performed. On the other hand, as long as the time 
step is kept relatively small, the results given by RK2 are satisfactory. And, the number of evaluations at each time step required by RK4 is twice as much as by RK2. For a single particle one hardly "feels" the difference, but when thousands of particles are to be tracked for a long time, the difference in simulation time between the two is significant. Given above, RK2 is chosen to be the default method for the particle tracking.

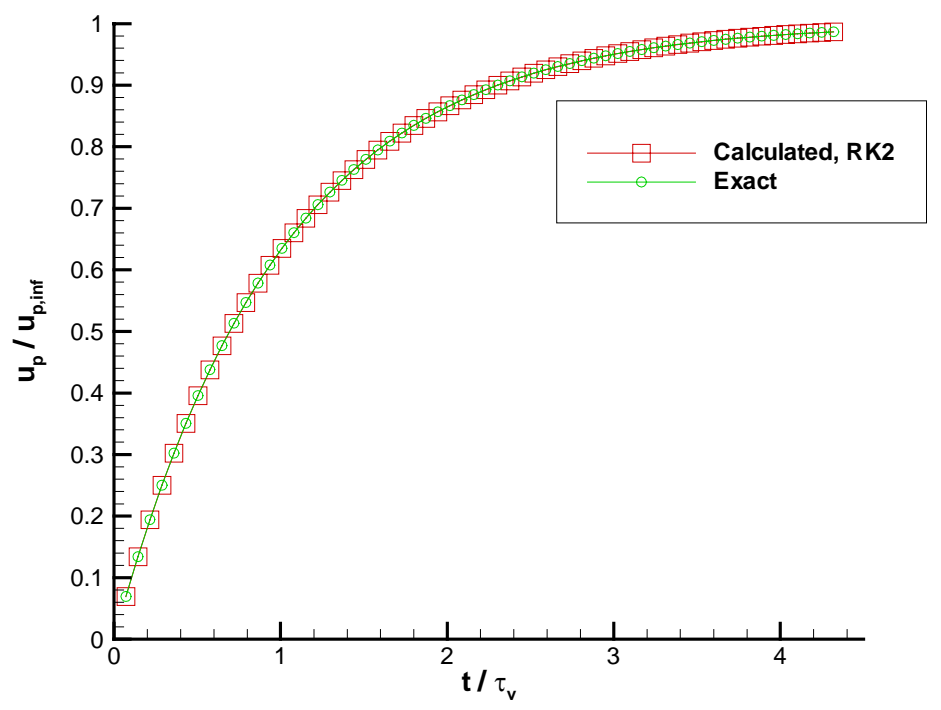

(a)

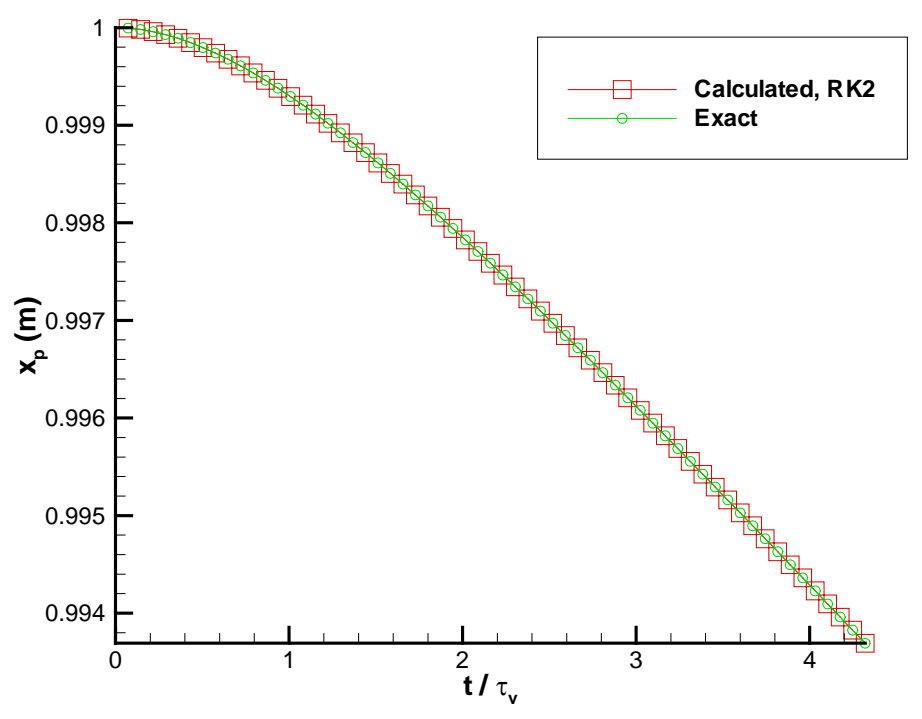

(b)

Figure 6-2 Gravitational settling of water drop in quiescent air: (a) velocity evolution, (b) position evolution. 


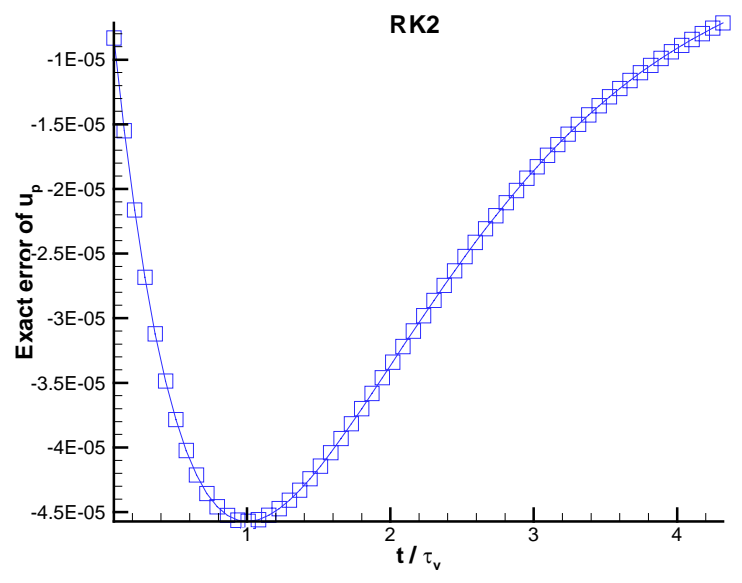

(a)

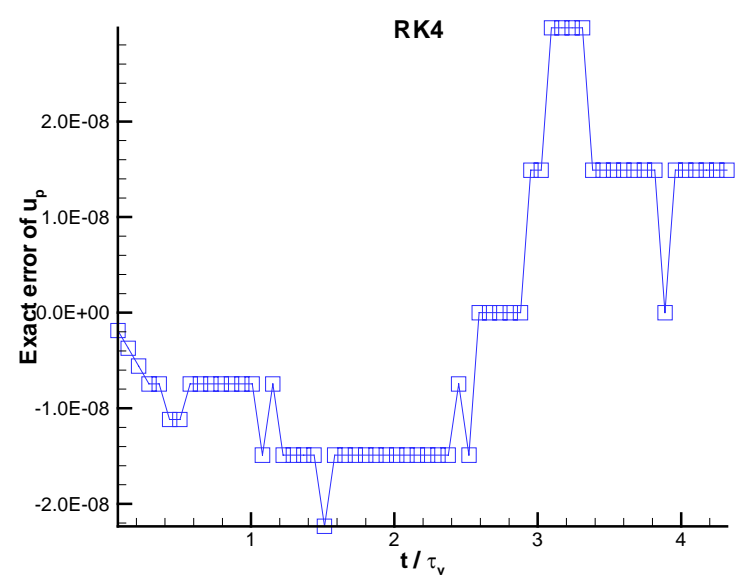

(b)

Figure 6-3 Comparison of exact error of the calculated water drop velocity with two different RungeKutta schemes; (a) $2^{\text {nd }}$ order RK, (b) $4^{\text {th }}$ order RK.

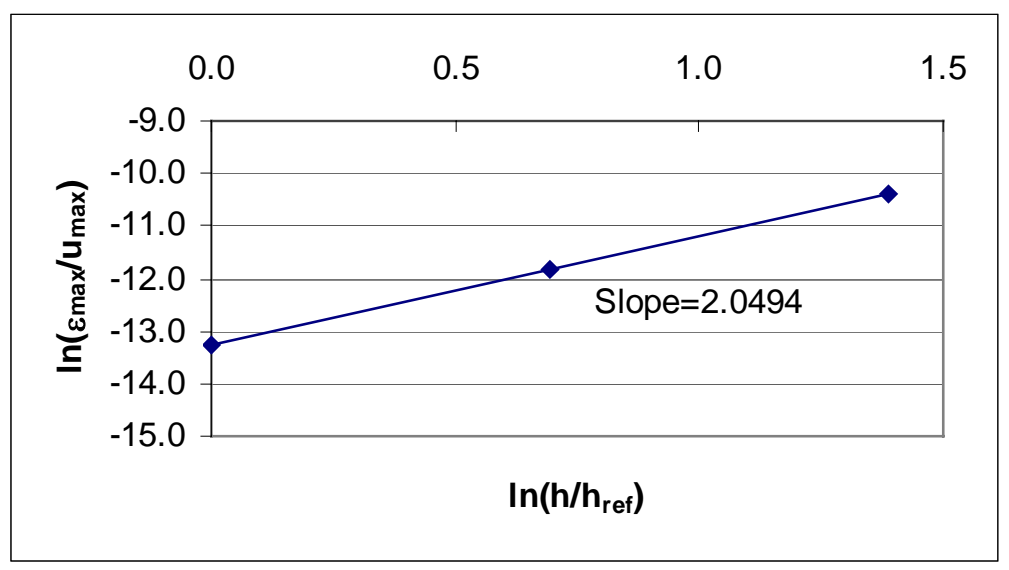

Figure 6-4 Order of method of RK2 for the calculation of gravitational settling. 


\subsection{Buoyant Settling}

Consider a single rising bubble in quiescent fluid where the driving force, buoyancy, is balanced by the drag force (Figure 6-1(b)). The equation of motion by including buoyant and drag force is

$$
\rho_{p} V_{p} \frac{d u_{p}}{d t}=-\rho g V_{p}-\frac{1}{2} C_{D} \rho A_{f r}\left|u_{p}\right| u_{p} .
$$

With Stokes drag Eq. (6.30) becomes

or equivalently,

$$
\frac{d u_{p}}{d t}=-\frac{u_{p}}{\tau_{p}}-\frac{\rho}{\rho_{p}} g
$$

$$
\frac{d u_{p}}{d t}+P u_{p}=Q
$$

with

$$
P=\frac{1}{\tau_{p}} \text { and } Q=-\frac{\rho}{\rho_{p}} g
$$

The analytical solution for $u_{p}$ is obtained as

$$
u_{p}(t)=\left(u_{p, 0}+\frac{\rho}{\rho_{p}} g \tau_{p}\right) \exp \left(-t / \tau_{v}\right)-\frac{\rho}{\rho_{p}} g \tau_{p} .
$$

The particle terminal velocity and the trajectory are then

$$
u_{p, \infty}=-\frac{\rho}{\rho_{p}} g \tau_{p}
$$

and

$$
x_{p}(t)=\left(u_{p, 0}+\frac{\rho}{\rho_{p}} g \tau_{p}\right) \tau_{p}\left[1-\exp \left(-t / \tau_{p}\right)\right]-\frac{\rho}{\rho_{p}} g \tau_{p} t+x_{p, 0}
$$

To verify numerical solution consider a $1 \mathrm{~mm}$ air bubble with density equal to $1.0 \mathrm{E}-3$. The dynamic viscosity of the primary phase, water, is $1.0 \mathrm{E}-3 \mathrm{~N} \cdot \mathrm{s} / \mathrm{m}^{2}$. This yields a bubble relaxation time of $5.556 \mathrm{E}-5 \mathrm{~s}$. The particle Reynolds number at the terminal velocity is 0.545 , which is well within the Stokes regime. Simulation is carried out with RK2 and a time step of 1.E-5s for 50 time steps. The results for the velocity and position variation is shown in Figure 6-5(a) and (b). The agreement with the exact solution is again very good. The calculated velocity at $t=5.0 \mathrm{E}-4 \mathrm{~s}$ is $0.54493 \mathrm{~m} / \mathrm{s}$, which matches the theoretical terminal velocity of $0.545 \mathrm{~m} / \mathrm{s}$, or the terminal particle Reynolds number of 0.545 . 


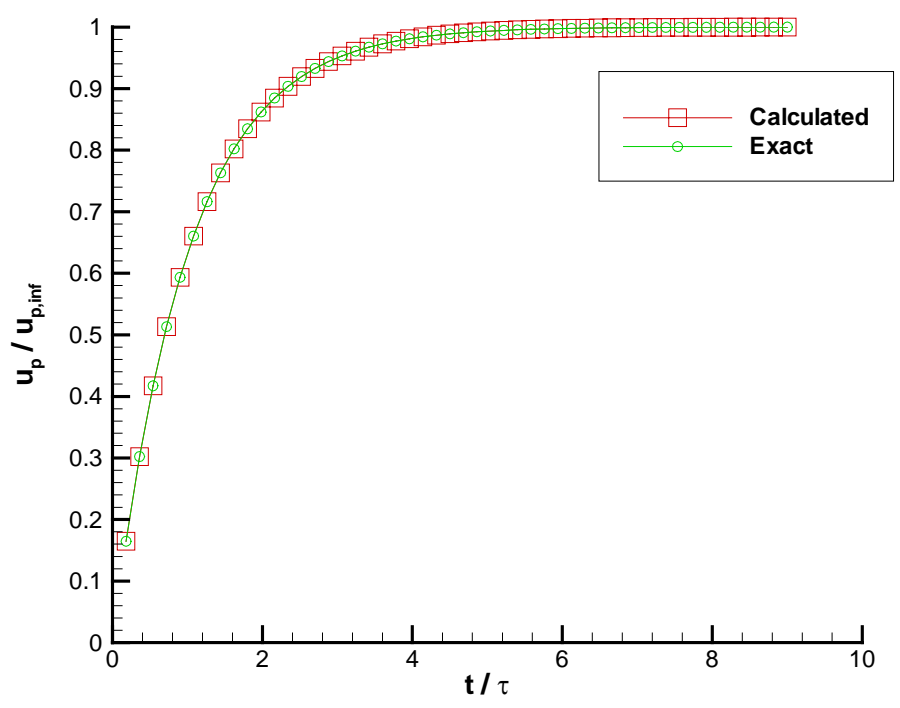

(a)

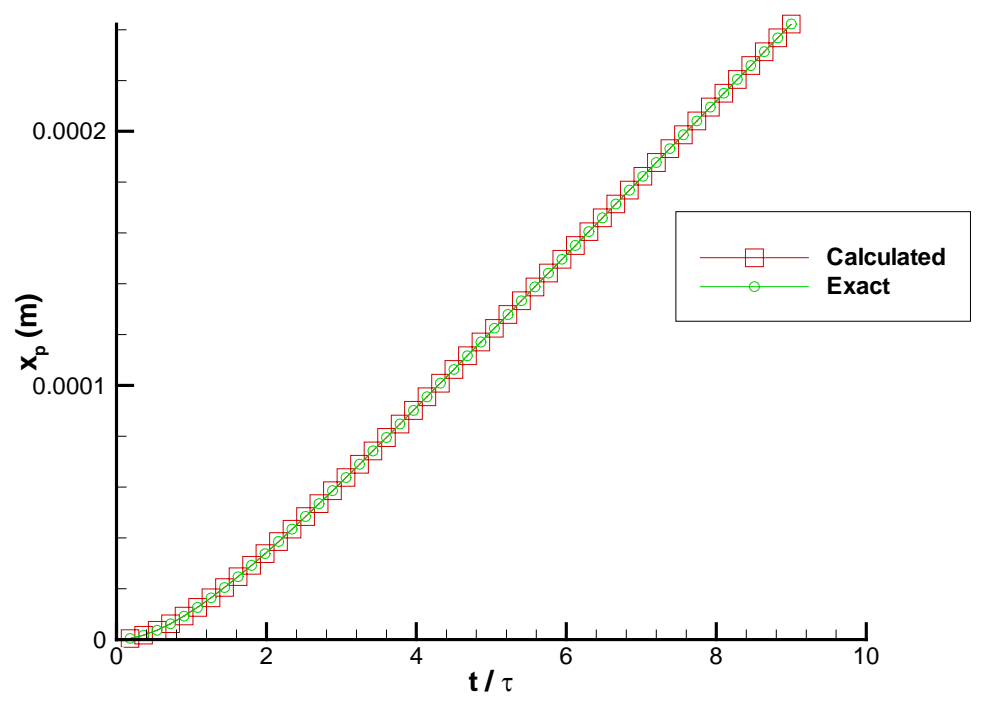

(b)

Figure 6-5 Buoyant settling of air bubble rising in quiescent water: (a) velocity evolution, (b) position evolution.

\subsection{Particle Injected across a Uniform Flow}

Consider a particle that is injected at some angle into a uniform flow $U$ which is orientated in the x-direction (Figure 6-6). The resulting motion then is no longer onedimensional as in the previous cases. 


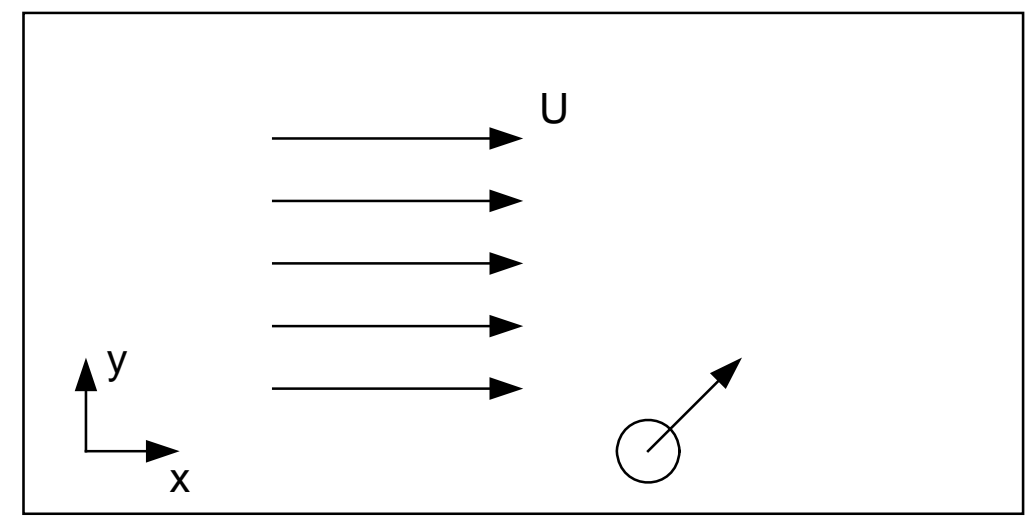

Figure 6-6 Particle injected across a uniform flow.

The system of equations describing the two-dimensional motion of the particle in the absence of gravity can be formulated as

$$
\begin{aligned}
& \frac{d u_{p}}{d t}=\frac{U-u_{p}}{\tau_{p}}, \\
& \frac{d v_{p}}{d t}=-\frac{v_{p}}{\tau_{p}},
\end{aligned}
$$

where only the Stokes drag is assumed. The two uncoupled ODEs can be readily solved with the following results

$$
\begin{aligned}
& u_{p}(t)-U=\left(u_{p, 0}-U\right) \exp \left(-t / \tau_{p}\right), \\
& v_{p}(t)=v_{p, 0} \exp \left(-t / \tau_{p}\right) .
\end{aligned}
$$

It is easily seen the uniform flow velocity $U$ also becomes the terminal velocity of the particle. The corresponding trajectory in parametric form is then

$$
\begin{aligned}
& x_{p}(t)=\tau_{p}\left(u_{p, 0}-U\right)\left[1-\exp \left(-t / \tau_{p}\right)\right]+U t+x_{p, 0}, \\
& y_{p}(t)=\tau_{p} v_{p, 0}\left[1-\exp \left(-t / \tau_{p}\right)\right]+y_{p, 0} .
\end{aligned}
$$

Different from the two examples in the previous sections, this problem allows for an evaluation of the tracking technique for a particle moving in a two-dimensional space. For test, consider a $1 \mathrm{~mm}$ water drop that is injected across a water cross flow which operates at a constant speed of $1 \mathrm{~m} / \mathrm{s}$. The density and dynamic viscosity used here for water are $1.0 \mathrm{E} 3 \mathrm{~kg} / \mathrm{m}^{3}$ and $1.0 \mathrm{E}-3 \mathrm{~N} \cdot \mathrm{s} / \mathrm{m}^{2}$. The momentum relaxation time of this water drop is $5.556 \mathrm{E}-2 \mathrm{~s}$. Initially, the water drop is injected with a velocity magnitude of $5 \mathrm{~m} / \mathrm{s}$ perpendicular to the cross flow direction which is aligned in x-direction. Simulation is performed using the RK2 for a period of 0.5 seconds.

Figure 6-7(a) and (b) show the calculated u- and v- velocity history of the water drop along with the exact solution given by Eq. (6.38). Figure 6-8(a) and (b) show the calculated $\mathrm{x}$ - and $\mathrm{y}$-location of the water drop along with the analytical locations given by (6.39). Figure 6-9 gives a two-dimensional view on the evolution of the particle position. 
It is seen that at $t=0.5 \mathrm{~s}$ the water drop almost reaches its terminal velocity equal to the constant cross flow velocity $1 \mathrm{~m} / \mathrm{s}$. Afterwards, the water drop will simply follow the main stream, acting like a fluid particle.

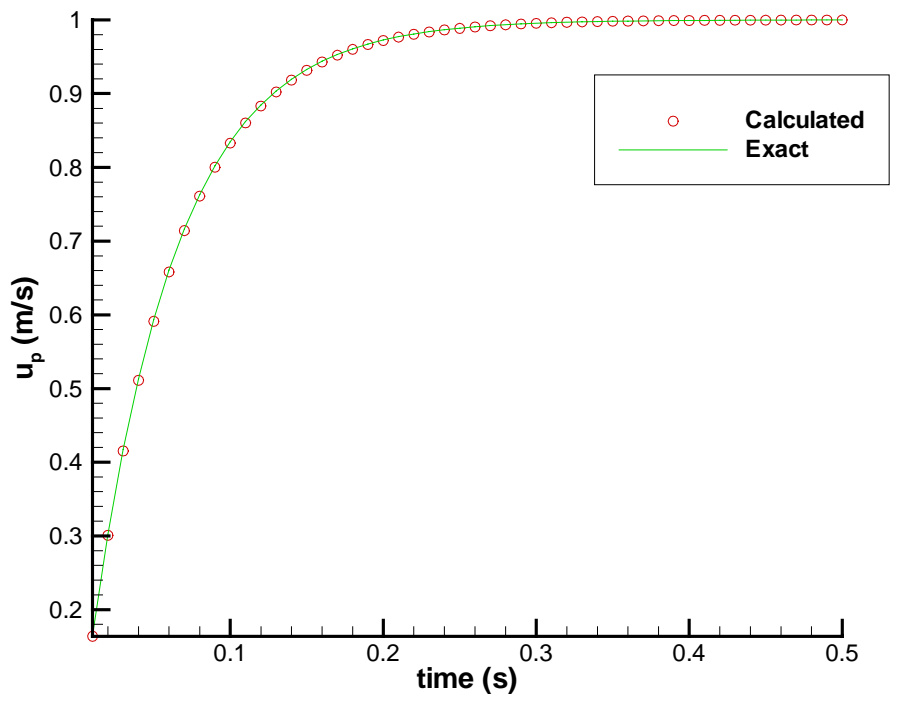

(a)

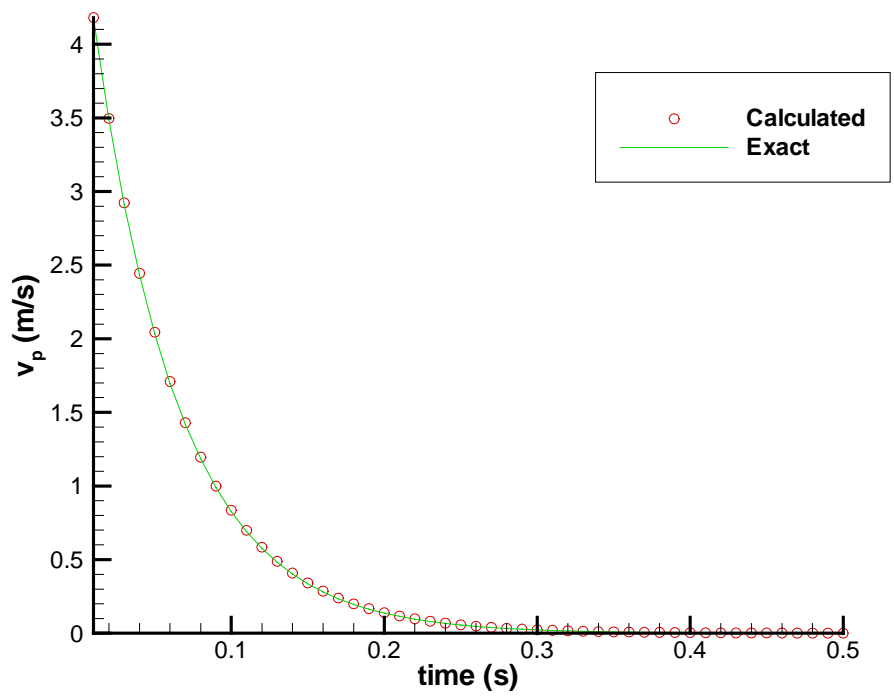

(b)

Figure 6-7 Water drop injected across a uniform flow. (a) u-velocity, and (b) v-velocity evolution of the water drop. 


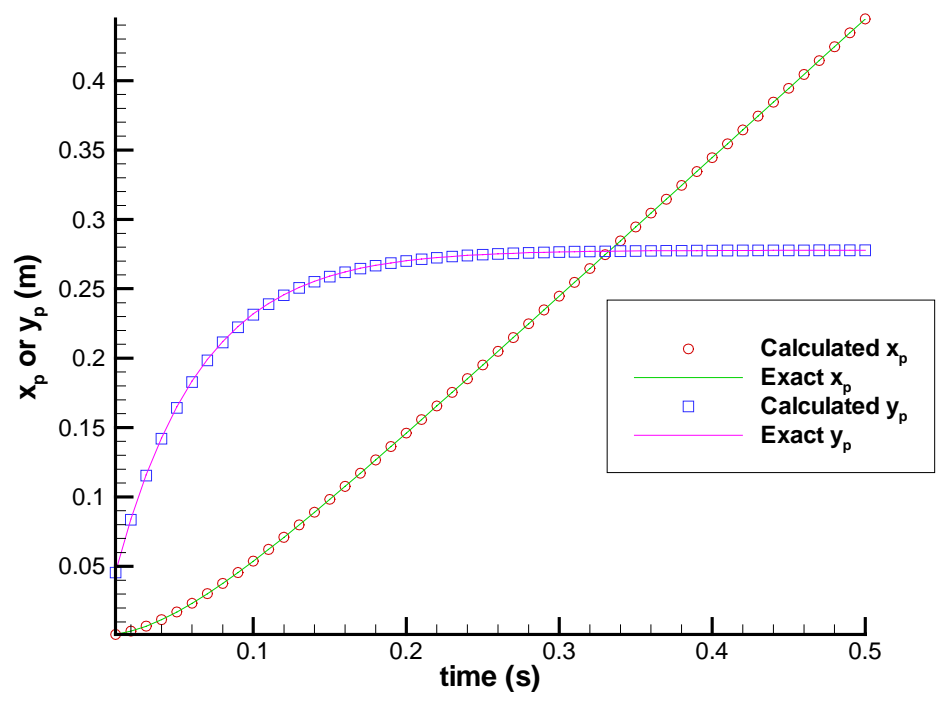

Figure 6-8 Parametric trajectory of a water drop injected across a uniform flow.

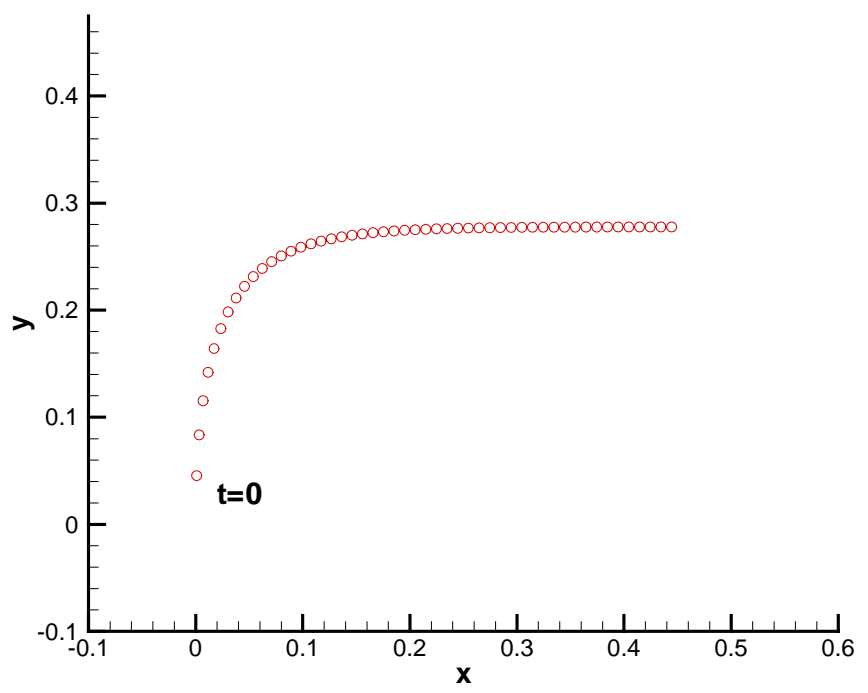

Figure 6-9 Two-dimensional view of the trajectory of a water drop injected across a uniform flow. Circles represent locations passed by the drop.

\subsection{Particle in an Oscillating Flow}

Consider a particle released in a flow that oscillates according to

$$
u=U \sin \left(\omega t+\varphi_{0}\right) \text {. }
$$

Such problems arise in a study of particle behavior in an acoustic field. The analysis outlined here essentially follows the treatment of (Rudinger 1980). 
The equation of motion is written as

$$
\frac{d u_{p}}{d t}=\frac{U \sin \left(\omega t+\varphi_{0}\right)-u_{p}}{\tau_{p}}
$$

where again Stokes drag is applied and $\tau_{v}$ is defined in Eq. (6.18). Finding a particular solution directly is not an easy task in this case; instead, one assumes a possible solution form as

$$
u_{p}(t)=C(t) \exp \left(-t / \tau_{p}\right)
$$

Substituting Eq. (6.42) into (6.41), after some algebraic manipulation, the expression for $C(t)$ can be obtained:

$$
C(t)=\frac{U \exp \left(t / \tau_{p}\right)}{1+\left(\omega \tau_{p}\right)^{2}}\left[\begin{array}{c}
\sin \omega t\left(\cos \varphi_{0}+\omega \tau_{p} \sin \varphi_{0}\right)+ \\
\cos \omega t\left(\sin \varphi_{0}-\omega \tau_{p} \cos \varphi_{0}\right)
\end{array}\right]+\text { const } .
$$

With prescribed initial conditions the integration constant can be determined, and the final solution for $u_{p}$ is thus found as

$$
\begin{aligned}
u_{p}(t) & =\frac{U}{\left[1+\left(\omega \tau_{p}\right)^{2}\right]^{1 / 2}} \sin (\omega t+\varphi) \\
& +\left[u_{p, 0}+\frac{U}{1+\left(\omega \tau_{p}\right)^{2}}\left(\omega \tau_{p} \cos \varphi_{0}-\sin \varphi_{0}\right)\right] \exp \left(-t / \tau_{p}\right),
\end{aligned}
$$

where

$$
\tan \varphi=\frac{\sin \varphi_{0}-\omega \tau_{p} \cos \varphi_{0}}{\cos \varphi_{0}+\omega \tau_{p} \sin \varphi_{0}}
$$

Comparing Eq. (6.44) with (6.40) it is seen the particle oscillates at the same frequency as the conveying fluid, but with reduced amplitude and different phase angle. Superimposed on this oscillating part of the particle velocity there is also a drift velocity that decays with the relaxation time $\tau_{p}$.

Integrating Eq. (6.44) yields a particle trajectory that looks more complicated. However, an asymptotic if only the particle motion at sufficiently large $t$ is interested:

$$
x_{p}(t)=\bar{x}-\frac{U}{\omega\left[1+\left(\omega \tau_{p}\right)^{2}\right]^{1 / 2}} \cos (\omega t+\varphi),
$$

where $\bar{x}$ is the mean position, given by

$$
\bar{x}=\tau_{p}\left[u_{p, 0}+\frac{U}{1+\left(\omega \tau_{p}\right)^{2}}\left(\omega \tau_{p} \cos \varphi_{0}-\sin \varphi_{0}\right)\right] .
$$

The first term in Eq. (6.47) represents a drift due to the initial injection velocity of the particle; the second term is an additional displacement caused by the oscillations with both positive and negative sign possible. For the case $\tan \left(\varphi_{0}\right)=\omega \tau_{p}$, the second part of 
the mean drift disappears. It follows from calculus that the maximum mean displacement is obtained when

$$
\tan \varphi_{0}=\tan \varphi_{m}=-\frac{1}{\omega \tau_{p}}
$$

which corresponds to

$$
\bar{x}_{\max }=\tau_{p}\left(u_{p, 0}+\frac{U}{\omega \tau_{p}} \cos \varphi_{m}\right)=\tau_{p}\left(u_{p, 0} \pm \frac{U}{\left[1+\left(\omega \tau_{p}\right)^{2}\right]^{1 / 2}}\right) .
$$

Therefore, particles which are released at the same injection point but at random instants will spread over a band of width $2 U /\left[1+\left(\omega \tau_{p}\right)^{2}\right]^{1 / 2}$ with its center located at the distance $u_{p, 0} \tau_{p}$ from the injection point. Rudinger (1980) pointed out that this behavior exhibits exemplary features of particle dispersion in a turbulent flow, but the assumption that the turbulent fluctuations can be represented by a simple sinusoidal oscillation is oversimplified. For a more detailed discussion for this example the reader is referred to (Rudinger 1980).

With this problem it is possible to verify the numerical accuracy of the tracking routines for a single particle in an unsteady flow environment. Again, considerer here is a $1 \mathrm{~mm}$ water drop with its density equal to $1.0 \mathrm{E} 3 \mathrm{~kg} / \mathrm{m}^{3}$. Further, let the carrier phase be air (dynamic viscosity equal $1.0 \mathrm{E}-3 \mathrm{~N} \cdot \mathrm{s} / \mathrm{m}^{2}$ ) which oscillates according to Eq. (6.40) with $U$ $=1 \mathrm{~m} / \mathrm{s}, \omega=\pi / 2, \varphi_{0}=0$. The relaxation time for the water drop is 5.556 seconds. The time step size used for the simulation is $0.1 \mathrm{~s}$. The trajectory of the water drop is calculated with an initial injection velocity of $1 \mathrm{~m} / \mathrm{s}$ for a total of 250 time steps, corresponding an evolution time of 25 seconds.

The velocity evolution of the water drop is shown in Figure 6-10 in comparison with the exact solution (Eq. (6.44)). The position evolution is plotted in Figure 6-11 along with the analytical solution (Eq. (6.46)) which is valid at sufficiently large time. It is seen that the particle velocity variation in an unsteady flow is accurately captured. As time goes along, the spatial oscillation of the water drop starts to engage in the exact oscillatory trajectory for large $t$. This observation is further evidenced in Figure 6-12 where the calculation is performed for a longer time (50 seconds). 


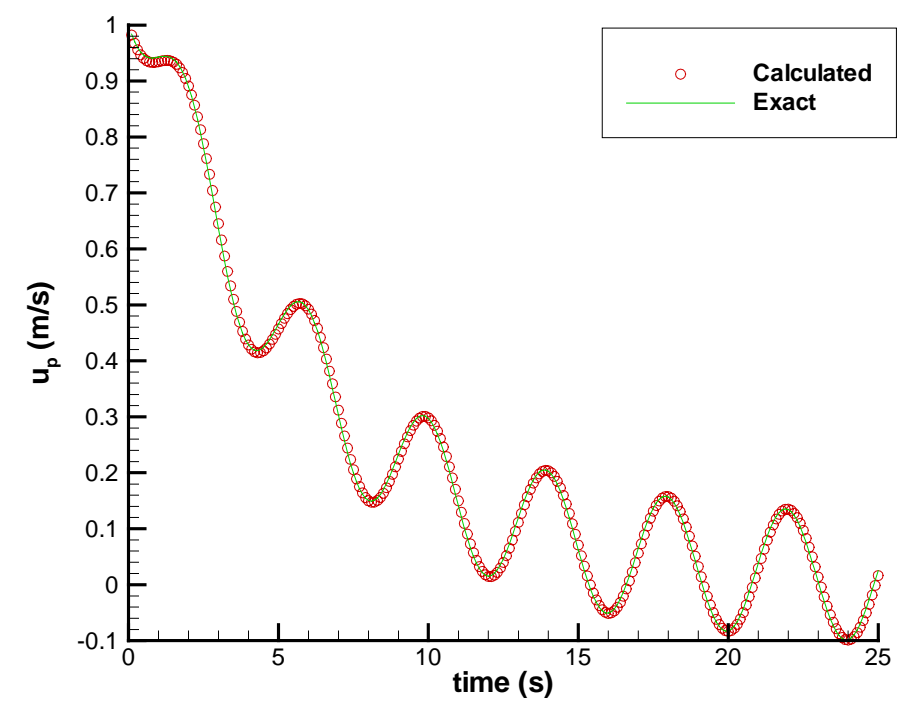

Figure 6-10 Velocity evolution of a water-drop in an oscillating air flow.

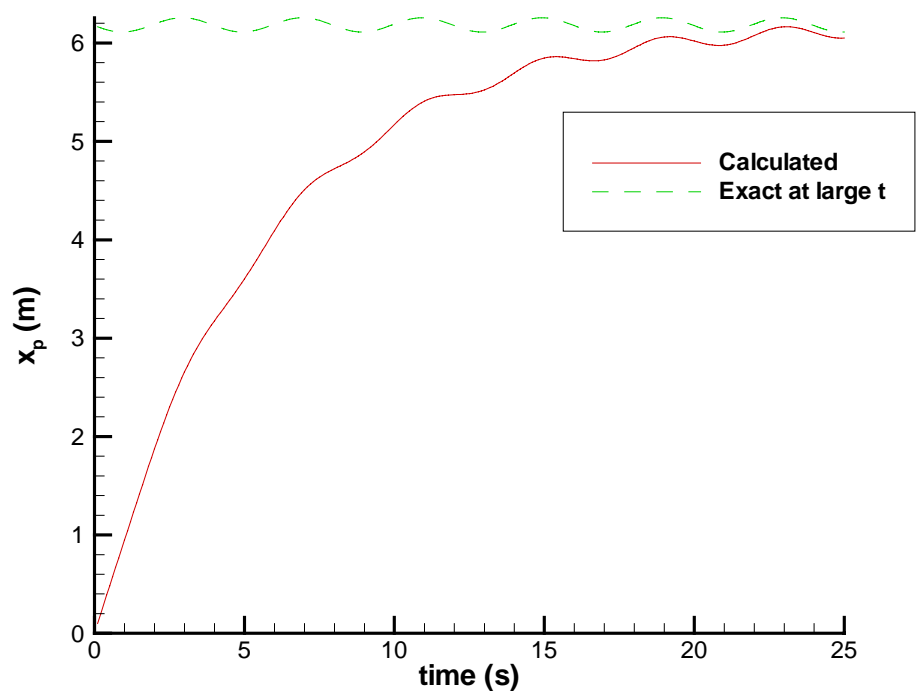

Figure 6-11 Position evolution of a water-drop in an oscillating air flow. Dashed line is the analytical location at sufficiently large time. 


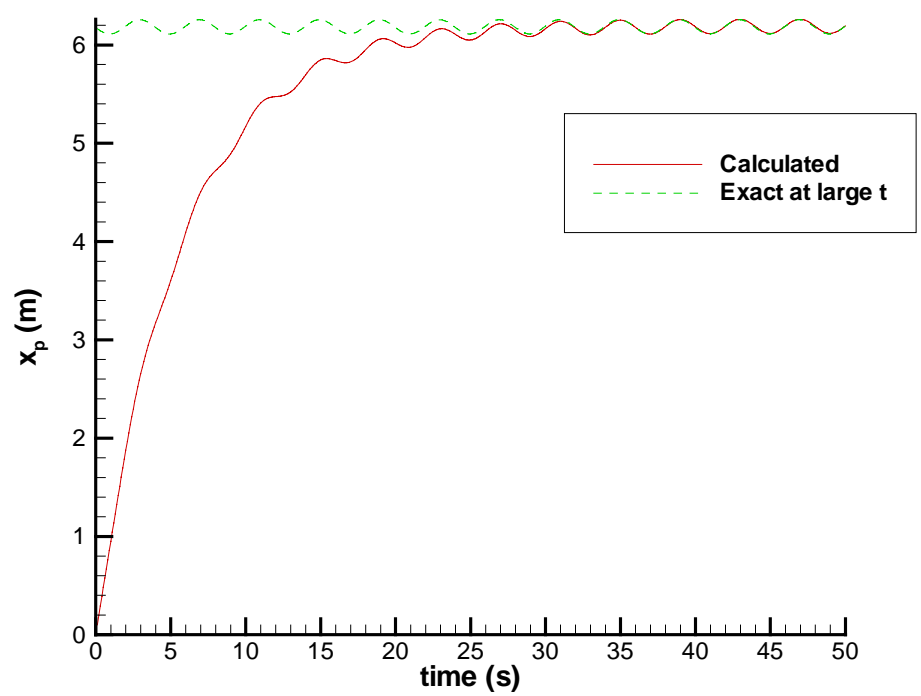

Figure 6-12 Position evolution of a water-drop in an oscillating air flow. Dashed line is the analytical location at sufficiently large time. Total time of evolution is $50 \mathrm{~s}$.

\subsection{Particle in a Rotating Flow}

This section considers the particle motion in a flow field which rotates at constant angular velocity, $\omega$, in counter-clockwise direction. Figure 6-13 shows the schematic for this problem with the corresponding notations. The angle of rotation here is $\theta=\omega t$. The velocity components of the flow can be expressed as

$$
u=-(\omega R) \frac{y}{R}=-\omega y, v=(\omega R) \frac{x}{R}=\omega x .
$$

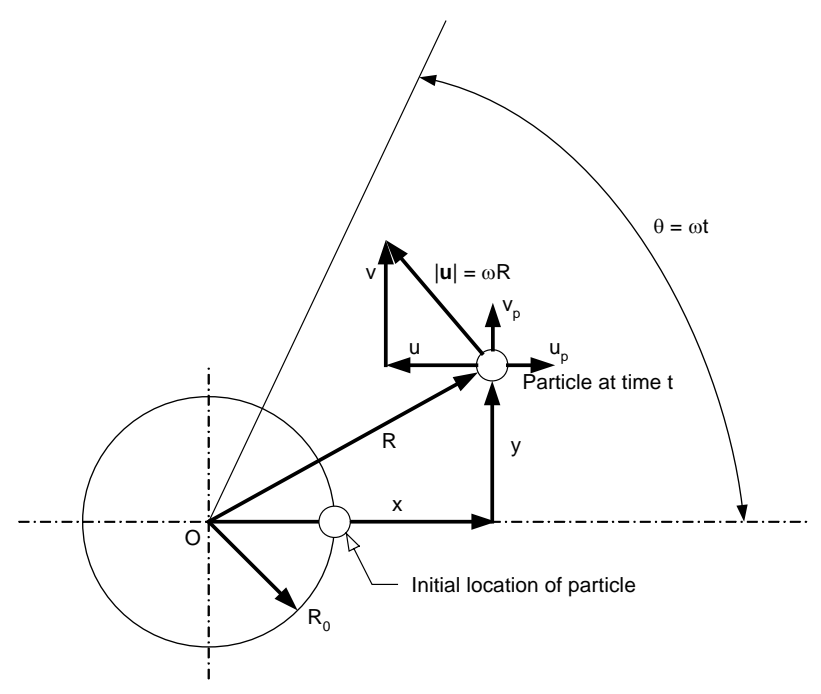

Figure 6-13 Particle in a rotating flow. 
The particle equation of motion with Stokes drag is then given by

$$
\begin{aligned}
& \frac{d u_{p}}{d t}=\frac{-\omega y-u_{p}}{\tau_{p}}, \\
& \frac{d v_{p}}{d t}=\frac{\omega x-v_{p}}{\tau_{p}} .
\end{aligned}
$$

Using the fact that

$$
\begin{array}{ll}
\dot{x}_{p}=u_{p}, & \dot{y}_{p}=v_{p}, \\
\ddot{x}_{p}=\frac{d u_{p}}{d t}, & \ddot{y}_{p}=\frac{d v_{p}}{d t},
\end{array}
$$

where $\operatorname{dot}(\mathrm{s})$ represents the derivative(s) with respect to time, and

$$
\frac{d}{d t}=\omega \frac{d}{d \theta}
$$

two coupled second order linear ODEs can be obtained (Lapple and Shepherd 1940):

$$
\begin{aligned}
& A^{2} \frac{d^{2} x_{p}}{d \theta^{2}}+A \frac{d x_{p}}{d \theta}+A y_{p}=0, \\
& A^{2} \frac{d^{2} y_{p}}{d \theta^{2}}+A \frac{d y_{p}}{d \theta}-A y_{p}=0 .
\end{aligned}
$$

where the symbol $A$ is a shorthand of

$$
A=\omega \tau_{p}=\frac{(1 / 6) \pi d_{p}^{3} \rho_{p} \omega^{2} R}{3 \pi d_{p} \mu \omega R},
$$

representing the ratio of the centrifugal force to Stokes drag. Analytical solution of Eq. (6.54) was originally provided by (Kriebel 1961), and re-addressed in (Rudinger 1980). In that, a Laplace transformation was first employed to formulate and solve the two unknowns in a transformed space; the desired solution is then obtained by the inverse transformation. The final solution reads

$$
\begin{aligned}
x_{p}(\theta)= & R_{0} e^{-\frac{\theta}{2 P}}\left\{\cos \frac{C_{2} \theta}{2 A}\left[\cosh \frac{C_{1} \theta}{2 A}+\frac{C_{1}(1+2 A B)+2 A C_{2}}{C_{3}^{2}} \sinh \frac{C_{1} \theta}{2 A}\right]\right. \\
& \left.+\sin \frac{C_{2} \theta}{2 A}\left[\frac{C_{2}(1+2 A B)-2 A C_{1}}{C_{3}^{2}} \cosh \frac{C_{1} \theta}{2 A}\right]\right\}, \\
y_{p}(\theta)= & R_{0} e^{-\frac{\theta}{2 P}}\left\{\begin{array}{l}
{\left[\frac{C_{1}}{2}+\frac{C_{1}}{2 C_{3}^{2}}(1+4 A B)\right] \sin \frac{C_{2} \theta}{2 A} \cosh \frac{C_{1} \theta}{2 A}+\sin \frac{C_{2} \theta}{2 A} \sinh \frac{C_{1} \theta}{2 A}} \\
+\left[\frac{C_{2}}{2}-\frac{C_{2}}{C_{3}{ }^{2}}(1+4 A B)\right] \cos \frac{C_{2} \theta}{2 A} \sinh \frac{C_{1} \theta}{2 A}
\end{array}\right\},
\end{aligned}
$$

where 


$$
\begin{aligned}
& C_{1}=\left[\left(\frac{1}{4}+4 A^{2}\right)^{1 / 2}+\frac{1}{2}\right]^{1 / 2}, \\
& C_{2}=\left[\left(\frac{1}{4}+4 A^{2}\right)^{1 / 2}-\frac{1}{2}\right]^{1 / 2}, \\
& C_{3}^{2}=C_{1}^{2}+C_{2}^{2}=\left(1+16 A^{2}\right)^{1 / 2}, \\
& B=\frac{u_{p, 0}}{\omega R_{0}}
\end{aligned}
$$

The initial conditions at $t=0$, or $\theta=0$, are given as

$$
\begin{aligned}
& x_{p, 0}=R_{0}, y_{p, 0}=0, \\
& \dot{x}_{p, 0}=u_{p, 0}, \dot{y}_{p, 0}=v_{p, 0}=\omega R_{0}, \\
& \left(\frac{d x_{p}}{d \theta}\right)_{0}=u_{p, 0} / \omega,\left(\frac{d y_{p}}{d \theta}\right)_{0}=R_{0} .
\end{aligned}
$$

Similar to the example in Section 6.4, this problem also requires a tracking of particle in a two-dimensional space. However, the flow field considered here is no longer constant, and this gives rise to the need of accurate interpolation of the flow field onto where the particle is located at every calculation instant. Any inaccurate interpolation practice may lead to inaccurately evaluated drag forces, and consequently, pollutes the results.

For verification, consider a particle with a diameter of 10 micron and a density of $3.0 \mathrm{E}-3 \mathrm{~kg} / \mathrm{m}^{3}$. The rotating fluid is air with a dynamic viscosity of $1.81 \mathrm{E}-5 \mathrm{~N} \cdot \mathrm{s} / \mathrm{m}^{2}$. Thus, the particle relaxation time is $9.208 \mathrm{E}-4$ seconds. This configuration for the material property of the two phases is the same as the one used by (Kriebel 1961). Further assume the air rotate at a speed of $7200 \mathrm{rpm}$ (rotations per minute), or equivalently, $753.98 \mathrm{rad} / \mathrm{s}$. At $t=0$, a single particle is released at $\left(\mathrm{R}_{0}, 0\right)$ with an initial velocity equal to the local flow velocity. The particle is then followed for 150 time steps with a step size of $1.0 \mathrm{E}-4 \mathrm{~s}$, which corresponds to 1.8 rotations of the air- flow. The calculated and exact $\mathrm{x}-$ and $\mathrm{y}-$ position are plotted in Figure 6-14, showing a good agreement between the computation and theory. A two-dimensional view of the particle trajectory is also shown in Figure 6-15, where one sees the particle is driven outward due to the centrifugal field, and its trajectory forms a nice outward directed spiral. 


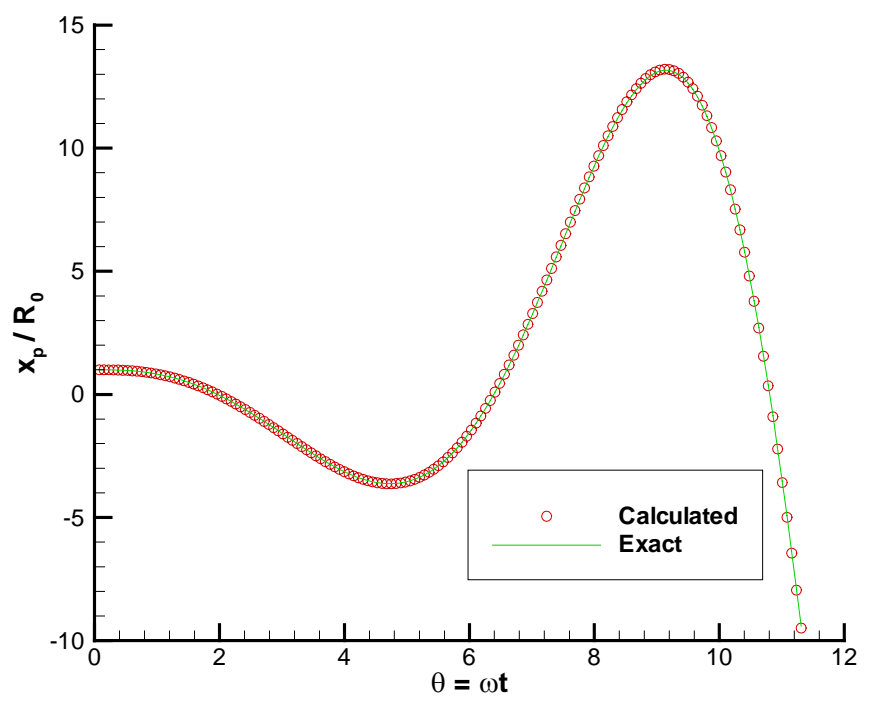

(a)

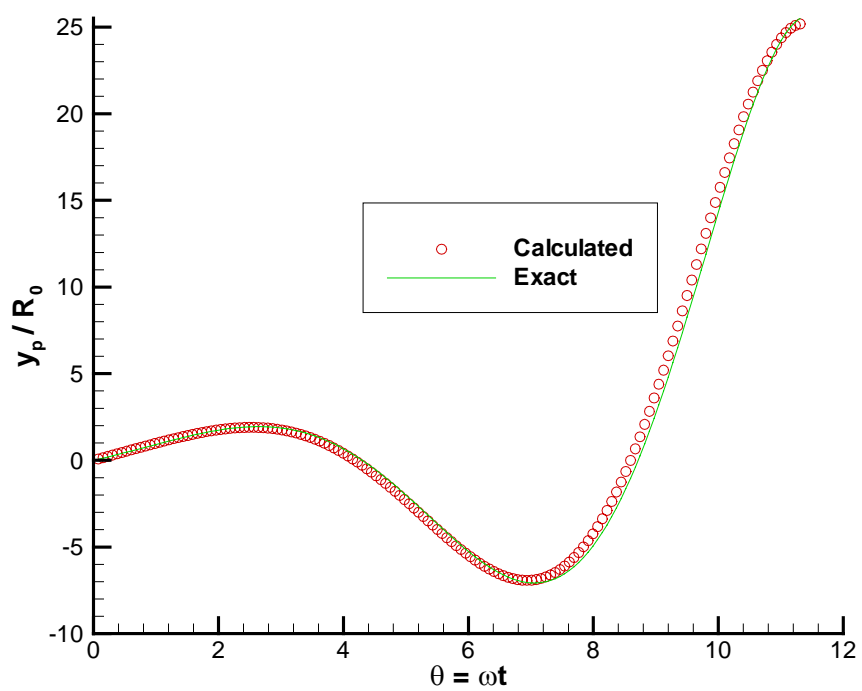

(b)

Figure 6-14 $x$ - and y-position evolution of a particle in a rotating air. (a) $x$-location, (b) $y$-location 


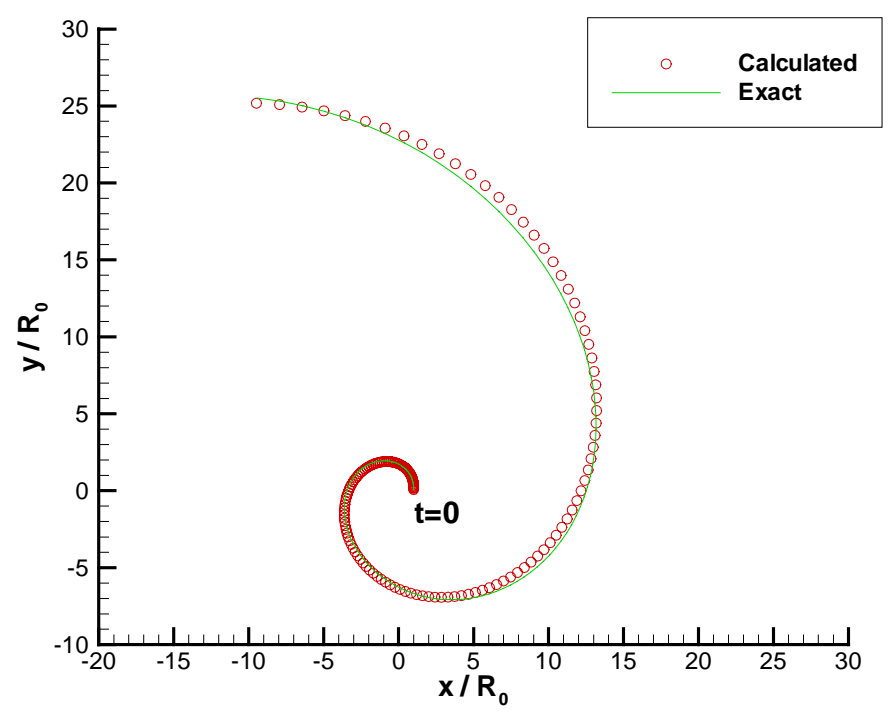

Figure 6-15 Two-dimensional view of the trajectory of a particle in a rotating air. Circles represent locations passed by the particle.

\subsection{Bubble in a Swirling Flow}

In this section an isolated bubble in a swirling flow (Figure 6-16) is considered. Singlephase swirling flows are widely used in industrial devices such as cyclonic separators, combustion chambers or Francis turbines. In a swirling flow system, the flow field can be thought of as solid-body rotation with a constant angular velocity $\omega$ superposed to a uniform axial velocity. The velocity components can be written in Cartesian coordinate system as

$$
u=-\omega y, v=\omega x, w=w_{0},
$$

where $w_{0}$ denotes a constant axial (or bulk) velocity. Notice that the first two components in Eq. (6.59) are the same as in the example of rotating flow field (6.50). In cylindrical coordinates Eq. (6.59) becomes

$$
u=0, v=\omega r, w=w_{0},
$$

with $u, v$ and $w$ denoting radial, azimuthal, and axial velocity, respectively. The existence of such a flow field characterized by the solid body rotation and constant axial velocity can be justified for up to a height $\mathrm{z} / 2 \mathrm{R} \cong 10$ when the Rossby number

$$
\text { Ro }=\frac{w_{0}}{R \omega}
$$

is larger than 0.6 and the Reynolds number is sufficiently large (Weske and Sturov 1974). This assertion is also confirmed by the experiment of (Magaud et al. 2003), who generated nearly uniform axial velocity profile in the vicinity of the pipe axis. 


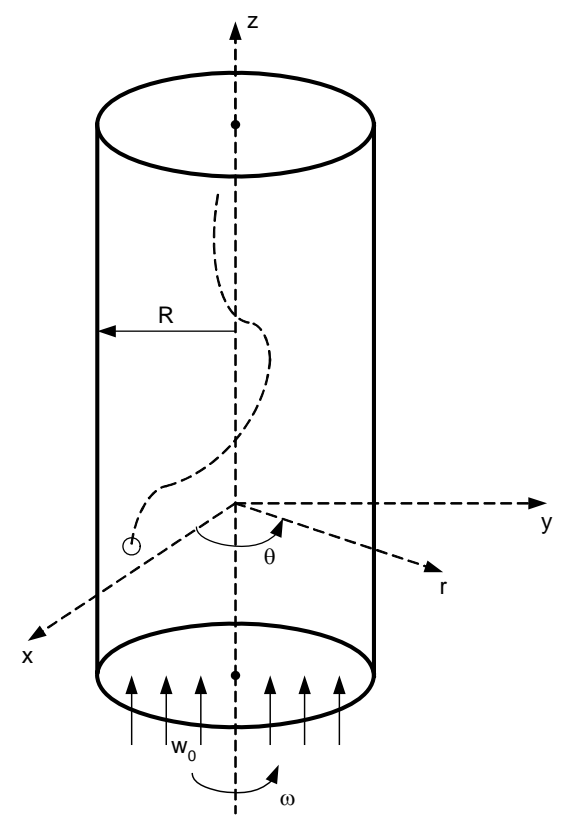

Figure 6-16 Bubble in a swirling flow.

The dynamics of a single bubble in a fluid flow is complex in general. Even for an isolated bubble, i.e., without a reverse effect on the flow field, the forces that the conveying fluid act on the bubble still remains a big subject to be studied (Michaelides 1997, 2003). Through extensive investigation in the past, both experimentally and numerically, some empirical and semi-empirical forces were made known to the researchers. Some of these forces that are significant to the bubble are the buoyancy force $\left(\mathbf{F}_{G}\right)$, drag force $\left(\mathbf{F}_{D}\right)$, lift force $\left(\mathbf{F}_{L}\right)$, added-mass force $\left(\mathbf{F}_{A}\right)$, and the force due to fluid stresses present in the flow field $\left(\mathbf{F}_{S}\right)$. The reader is referred to the next chapter (Section 7.5) for a detailed discussion regarding these forces. For the problem of this section the bubble equation formulated in Cartesian coordinates simply follows the one used in (Magaud et al. 2003):

$$
\begin{aligned}
\frac{d \mathbf{v}}{d t}= & -\frac{\left(1-\gamma_{\rho}\right)}{\left(\gamma+C_{A}\right)} \mathbf{g}+\frac{3}{8 a} \frac{C_{D}}{\left(\gamma_{\rho}+C_{A}\right)}|\mathbf{u}-\mathbf{v}|(\mathbf{u}-\mathbf{v}) \\
& +\frac{\left(1+C_{A}\right)}{\left(\gamma_{\rho}+C_{A}\right)} \frac{D \mathbf{u}}{D t}+\frac{C_{L}}{\left(\gamma_{\rho}+C_{A}\right)}(\mathbf{u}-\mathbf{v}) \times \mathbf{\omega}
\end{aligned}
$$

where $a$ is the bubble diameter, $C_{A}$ is the added mass coefficient equal to $1 / 2, C_{L}$ is the lift coefficient and its value is approximately $1 / 2$ (Auton 1981), $\gamma_{\rho}=\rho_{p} / \rho$ is the density ratio between two phases, $\omega$ is the curl of the flow vector field (vorticity), i.e., $\omega=\nabla \times \mathbf{u}$. Note that the bold written vorticity vector field $\omega$ should not be confused with the angular velocity $\omega$, which is a scalar.

In a swirling flow, the last two terms of the RHS of Eq. (6.62) can be explicitly expressed if they are formulated in cylindrical coordinates, since by Eq. (6.59), 


$$
\frac{D \mathbf{u}}{D t}=\left(-\omega^{2} r, 0,0\right)
$$

and

$$
\boldsymbol{\omega}=(0,0,2 \omega) .
$$

Also, if the position of the bubble at time $t$ is $(r(t), \theta(t), z(t))$, then in polar coordinates,

$$
\begin{aligned}
& \mathbf{v}=(\dot{r}, r \dot{\theta}, \dot{z}), \\
& \frac{d \mathbf{v}}{d t}=\left(\ddot{r}-r \dot{\theta}^{2}, 2 \dot{r} \dot{\theta}+r \ddot{\theta}, \ddot{z}\right) .
\end{aligned}
$$

With the above preparation Eq. (6.62) is ready for a coordinates transformation. Using the following non-dimensional variables,

$$
r^{*}=\frac{r}{R}, z^{*}=\frac{z}{R}, t^{*}=\omega t, a^{*}=\frac{a}{R}
$$

and

$$
\begin{aligned}
& u^{*}=\frac{u}{R \omega}, v^{*}=v, w^{*}=\frac{w}{R \omega}, \\
& u_{p}{ }^{*}=\frac{u_{p}}{R \omega}, v_{p}{ }^{*}=v_{p}, w_{p}{ }^{*}=\frac{w_{p}}{R \omega},
\end{aligned}
$$

Eq. (6.62) in cylindrical coordinates reads (Magaud et al. 2003):

$$
\begin{aligned}
& \frac{d^{2} r^{*}}{d t^{* 2}}-r^{*} \frac{d^{2} \theta}{d t^{* 2}}=-C_{1} r^{*}-\frac{3}{8} C_{3}\left|\mathbf{v}_{s}\right| \frac{d r^{*}}{d t^{*}}-2 C_{4} r^{*}\left(\frac{d \theta}{d t^{*}}-1\right), \\
& 2 \frac{d r^{*}}{d t^{*}} \frac{d \theta}{d t^{*}}+r^{*} \frac{d^{2} \theta}{d t^{* 2}}=-\frac{3}{8} C_{3}\left|\mathbf{v}_{s}\right| r^{*}\left(\frac{d \theta}{d t^{*}}-1\right)+2 C_{4} \frac{d r^{*}}{d t^{*}}, \\
& \frac{d^{2} z^{*}}{d t^{* 2}}=\frac{C_{2}}{\mathrm{Fr}}-\frac{3}{8} C_{3}\left|\mathbf{v}_{s}\right|\left(\frac{d z^{*}}{d t^{*}}-\mathrm{Ro}\right),
\end{aligned}
$$

where

$$
\begin{aligned}
& C_{1}=\frac{1+C_{A}}{\gamma_{\rho}+C_{A}} \cong 3, C_{2}=\frac{1-\gamma_{\rho}}{\gamma_{\rho}+C_{A}} \cong 2, \\
& C_{3}=\frac{C_{D}}{\left(\gamma_{\rho}+C_{A}\right) a^{*}} \cong \frac{2 C_{D}}{a^{*}}, C_{4}=\frac{C_{L}}{\gamma_{\rho}+C_{A}} \cong 1,
\end{aligned}
$$

since for bubble and water, $\gamma=\mathrm{O}\left(10^{-3}\right)$; also

$$
\mathbf{v}_{s}=\left(\frac{d r^{*}}{d t^{*}}, r^{*}\left(\frac{d \theta}{d t^{*}}-1\right), \frac{d z^{*}}{d t^{*}}-\mathrm{Ro}\right)
$$

is the non-dimensional slip velocity, and

$$
\mathrm{Fr}=\frac{R \omega^{2}}{g}
$$

is the pseudo-Froude number representing the ratio of inertia to gravity.

If the drag force is linear, i.e., $C_{D}$ can be expressed as 


$$
C_{D}=\frac{k}{\operatorname{Re}_{p}}
$$

for some proportionality constant $k$ ( $k=24$ yields Stokes drag coefficient), Eq. (6.68) can be reduced to

$$
\begin{aligned}
& \ddot{r}+f \dot{r}+2 r=0, \\
& 2 \dot{r}(\dot{\theta}-1)+r \ddot{\theta}=-f r(\dot{\theta}-1), \\
& \ddot{z}+f \dot{z}-f \operatorname{Ro}=\frac{2}{\mathrm{Fr}},
\end{aligned}
$$

where

$$
f=\frac{3 k}{8} \frac{v}{\omega a^{2}}
$$

is related to some rotation frequency (Magaud et al. 2003). The set of the three simplified equations (6.73) are then solved by (Magaud et al. 2003), who first tackled the second ODE. For the initial conditions of

$$
\begin{aligned}
& r=r_{0}, \theta=\theta_{0}, \quad z=z_{0}, \\
& u_{p}=0, v_{p}=\omega r_{0}, w_{p}=w_{0},
\end{aligned}
$$

the results are

$$
\begin{gathered}
\theta=t^{*}+\theta_{0}, \\
z^{*}\left(t^{*}\right)=z_{0}-\frac{2}{f^{2} \mathrm{Fr}}\left(1-\exp \left(-f t^{*}\right)\right)+\left(\frac{2}{f \mathrm{Fr}}+\mathrm{Ro}\right) t^{*},
\end{gathered}
$$

and

$$
r^{*}\left(t^{*}\right)= \begin{cases}r_{0} \exp \left(-f t^{*} / 2\right)\left(\cosh \frac{\sqrt{\Delta}}{2} t^{*}+\frac{f}{\sqrt{\Delta}} \sinh \frac{\sqrt{\Delta}}{2} t^{*}\right) & \text { if } \omega<\omega_{c}, \\ r^{*}\left(t^{*}\right)=r_{0} \exp \left(-f t^{*} / 2\right)\left(\cos \frac{\sqrt{\Delta}}{2} t^{*}+\frac{f}{\sqrt{\Delta}} \sin \frac{\sqrt{\Delta}}{2} t^{*}\right) & \text { if } \omega>\omega_{c} .\end{cases}
$$

where

$$
\Delta=\left|f^{2}-8\right|
$$

and

$$
\omega_{c}=\frac{3 k}{16 \sqrt{2}} \frac{v}{a^{2}}
$$

is the critical angular velocity (at which $f=2 \sqrt{2}$, or $169.7 \mathrm{rpm}$ ) that characterize two solution regimes, a non-oscillating regime and an oscillating regime. In particular, the non-dimensional terminal vertical velocity of the bubble is

$$
w_{p, \infty}^{*}=\mathrm{Ro}+\frac{2}{f \mathrm{Fr}},
$$

which corresponds the dimensional terminal velocity of

$$
w_{p, \infty}=w_{0}+\frac{16 g a^{2}}{3 k v_{c}} .
$$


The above analysis mainly summarized from (Magaud et al. 2003) presents an excellent test case for the verification of a Lagrangian particle tracking (LPT) program, as (i) the particle motion is three-dimensional, and (ii) not only the drag and buoyancy force, but also other type of hydrodynamic forces are included in the particle equation of motion. Forces, such as the fluid stress and lift forces, are generally difficult to evaluate due to heavily involved interpolation practice from the Eulerian fluid grid to the discrete particle location. Overall, verifying the LPT routine with this benchmark is challenging.

The air bubble being considered for the verification has a diameter of $1 \mathrm{~mm}$ and density of $1.0 \mathrm{~kg} / \mathrm{m}^{3}$. The continuous phase is water whose dynamic viscosity is $1 \mathrm{E}-3$ $\mathrm{N} \cdot \mathrm{s} / \mathrm{m}^{2}$. The momentum relaxation time for this air bubble based on Stokes drag is 5.556E-5 seconds. However, the actual relaxation time of the bubble differs from the Stokes relaxation time. An estimate for the actual response time can be deduced from the drag term in the governing equation (6.62), which yields approximately $2.778 \mathrm{E}-2$ seconds.

Consider a cylindrical domain with base radius $R=0.03 \mathrm{~m}$ and height of $1.2 \mathrm{~m}$. The reader is referred to the original paper (Magaud et al. 2003) for a justification of this choice of radius. Since the simulation is carried out on Cartesian coordinates, for simplicity, the cylindrical domain is replaced with a $0.06 \mathrm{~m} \times 0.06 \mathrm{~m} \times 1.2 \mathrm{~m}$ cuboid. A 10 $\times 10 \times 10$ non-staggered grid is used to store the imposed exact flow velocities. Thus, the interpolation of flow variables, such as derivatives and vorticity, will be merely based on the known nodal values. No exact flow properties are imposed at where the bubble is located. The air bubble is initially located at $(0.8 \mathrm{R}, 0)$ and injected into the flow field at $t$ $=0$ with its velocity equal to the local flow velocity. The $2^{\text {nd }}$ order Runge-Kutta is used for all simulations. Also, the bulk velocity $w_{0}$ is assumed to be $1 \mathrm{~m} / \mathrm{s}$. Simulation is carried out for four various angular velocities, i.e., 104, 209, 313 and $522 \mathrm{rpm}$. The time steps used for each rpm are $0.01 \mathrm{~s}, 0.005 \mathrm{~s}, 0.0033 \mathrm{~s}$ and $0.002 \mathrm{~s}$, respectively; and the corresponding consumed time iterations are 100, 200, 300 and 500.

Figure 6-17 (a) through (d) show the decay of the absolute r-coordinate for the aforementioned four different runs. The calculated bubble positions agree well with the exact solution at both low and high rpm's. As predicted by the theory, the critical angular velocity is at around $170 \mathrm{rpm}$, below which a monotonic convergence of the bubble location toward center is expected, and above which the bubble approaches the center in an oscillatory manner. This is confirmed in these plots as well. It is also seen that the higher the rpm is, the more numbers of circulation the bubble undergoes, and the slower it converges oscillatory to the center. A top view of the bubble trajectory is shown in Figure 6-18(a) through (d), and a 3D view is provided in Figure 6-19(a) through (d), for the four runs respectively. 


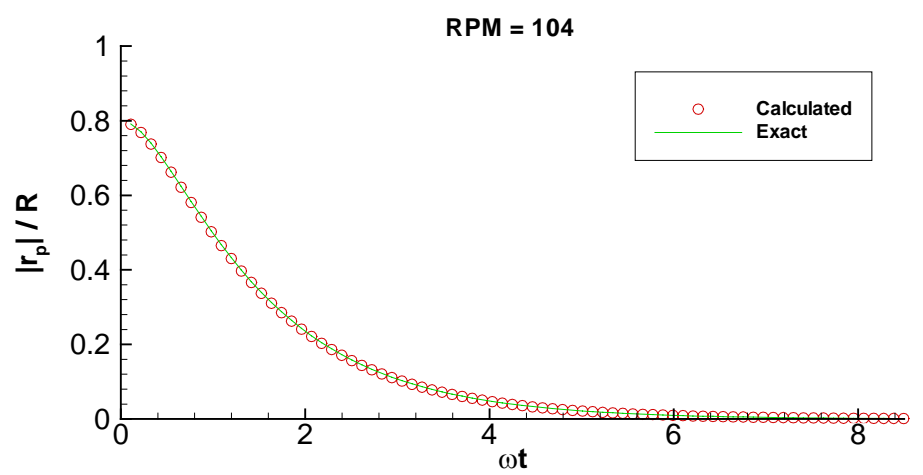

(a)

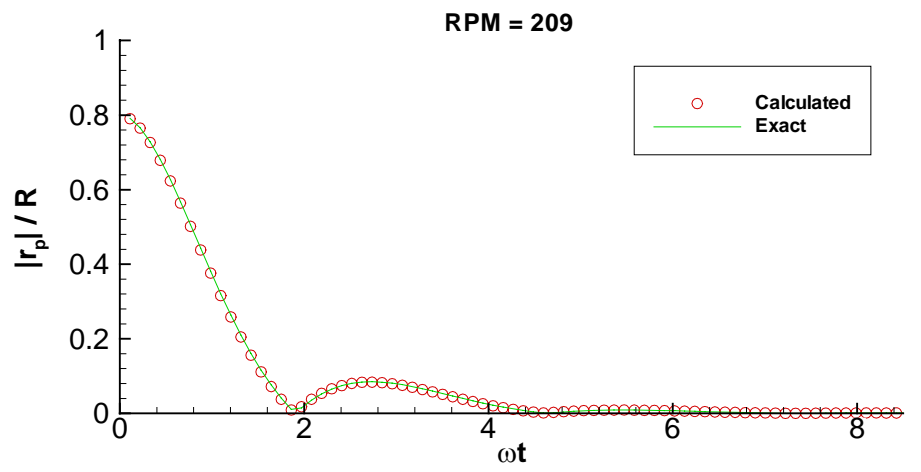

(b)

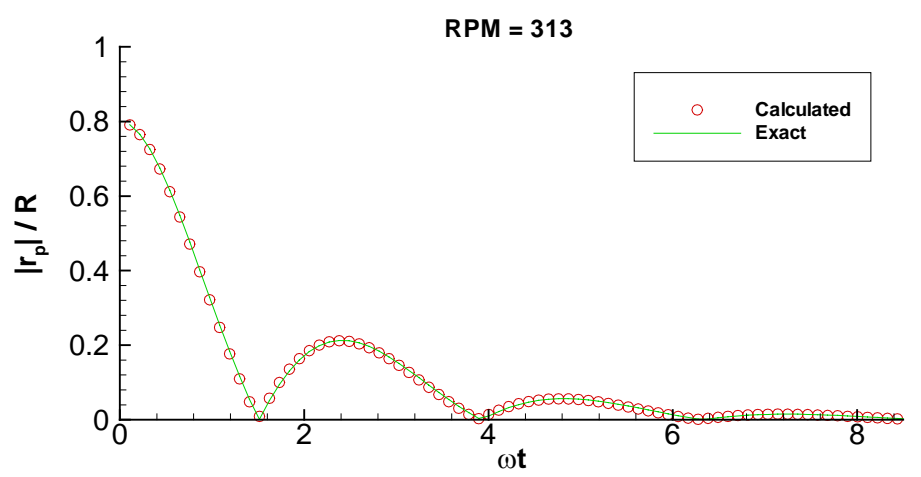

(c)

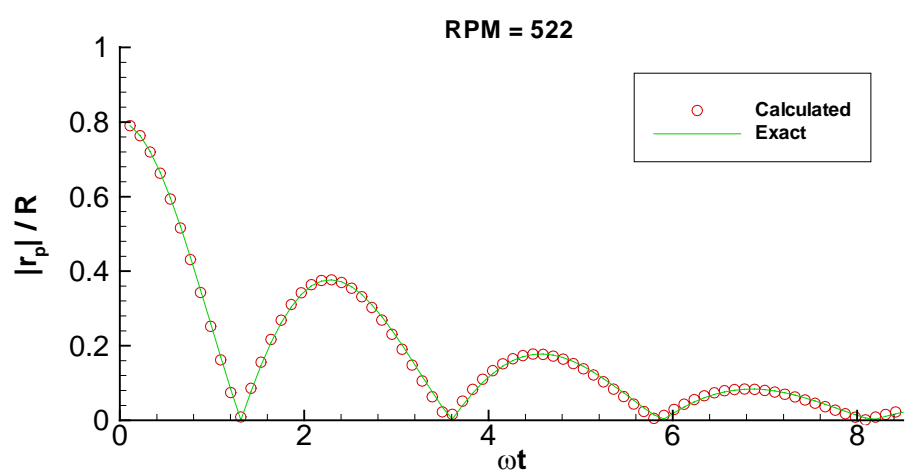

(d)

Figure 6-17 Decay of absolute r-coordinate of a bubble in a swirling flow. The angular velocity of the swirling flow is (a) $104 \mathrm{rpm}$, (b) $209 \mathrm{rpm}$, (c) $313 \mathrm{rpm}$, (d) $522 \mathrm{rpm}$. 


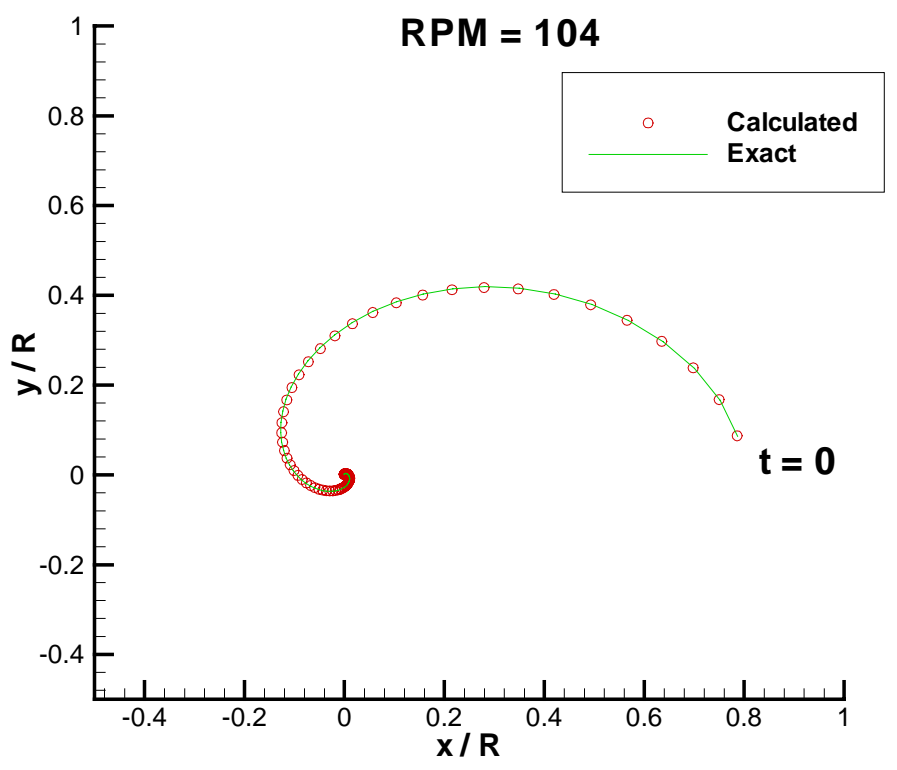

(a)

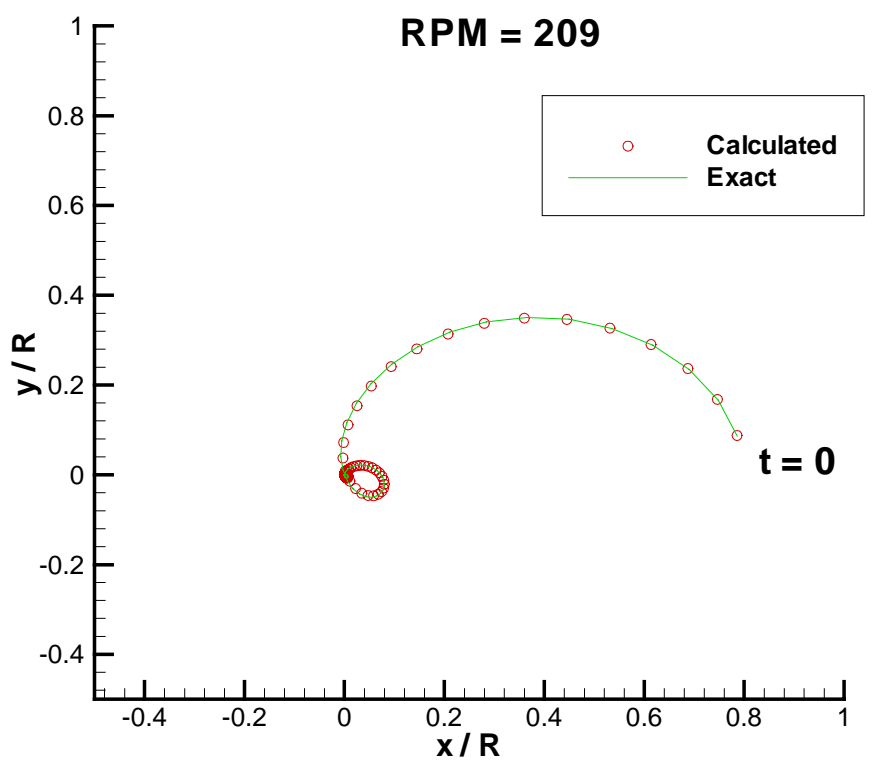

(b)

Figure 6-18 Top view of bubble trajectory in a swirling flow. The angular velocity of the swirling flow is (a) $104 \mathrm{rpm}$, (b) $209 \mathrm{rpm}$. Circle: calculated, line: exact. 


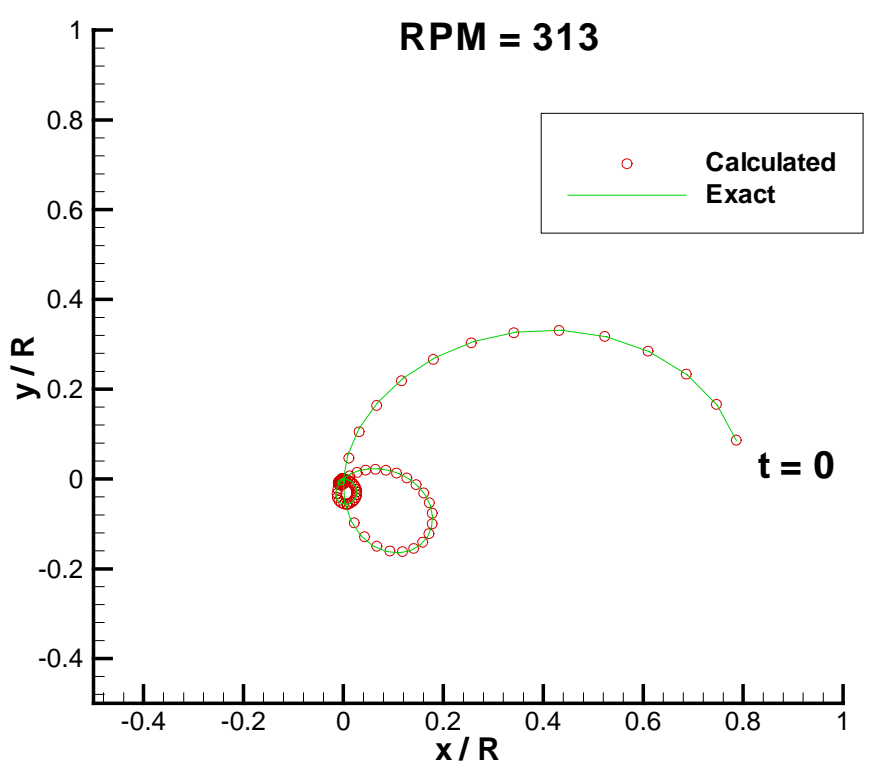

(c)

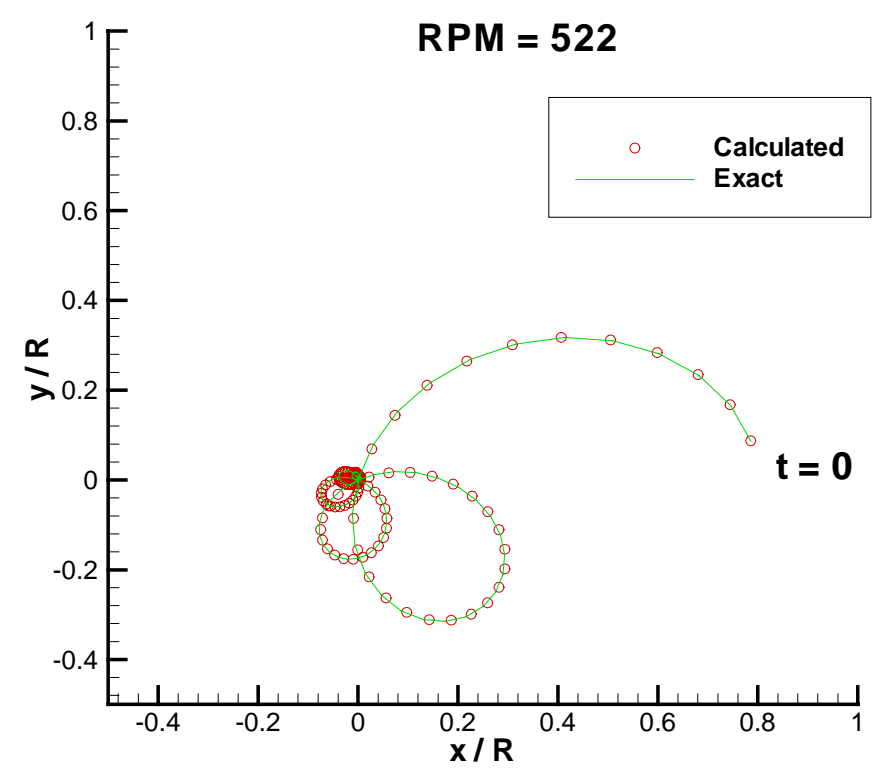

(d)

Figure 6-18 Top view of bubble trajectory in a swirling flow. The angular velocity of the swirling flow is (c) $313 \mathrm{rpm}$ (d) $522 \mathrm{rpm}$. Circle: calculated, line: exact. 


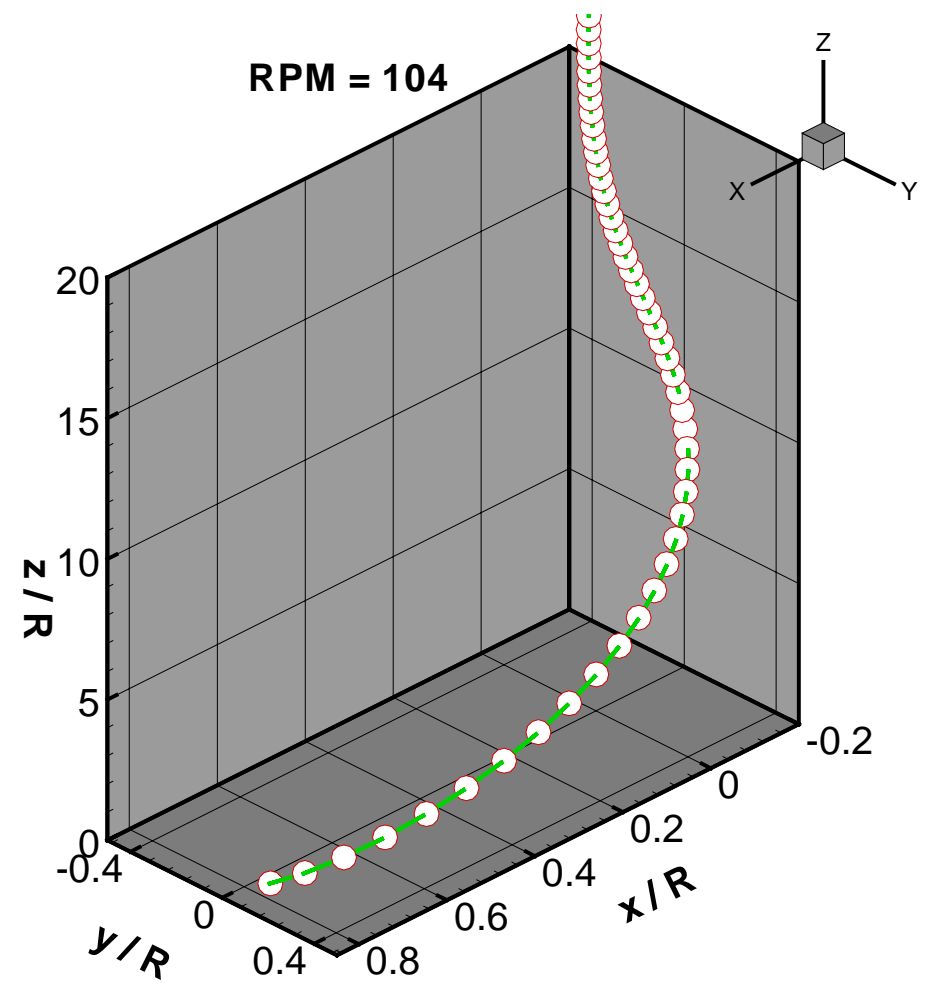

(a)

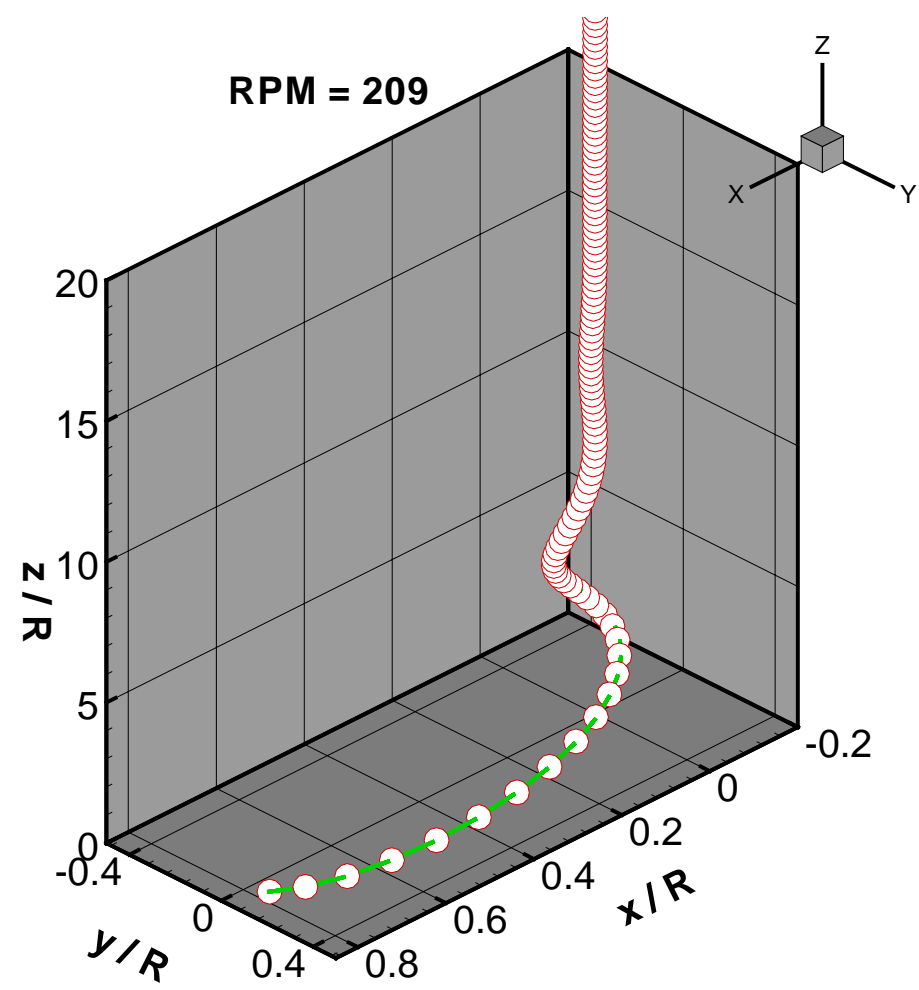

(b)

Figure 6-19 3D view of bubble trajectory in a swirling flow. The angular velocity of the swirling flow is (a) $104 \mathrm{rpm}$, (b) $209 \mathrm{rpm}$. Circle: calculated, line: exact. 


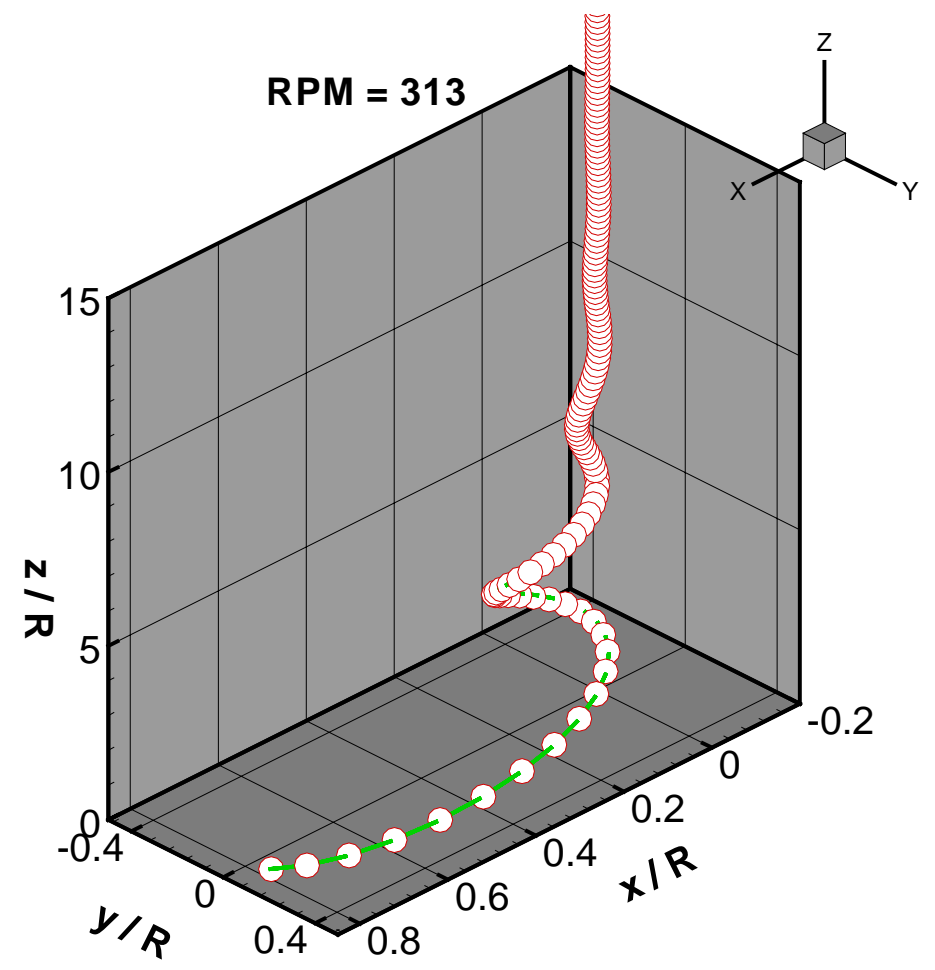

(c)

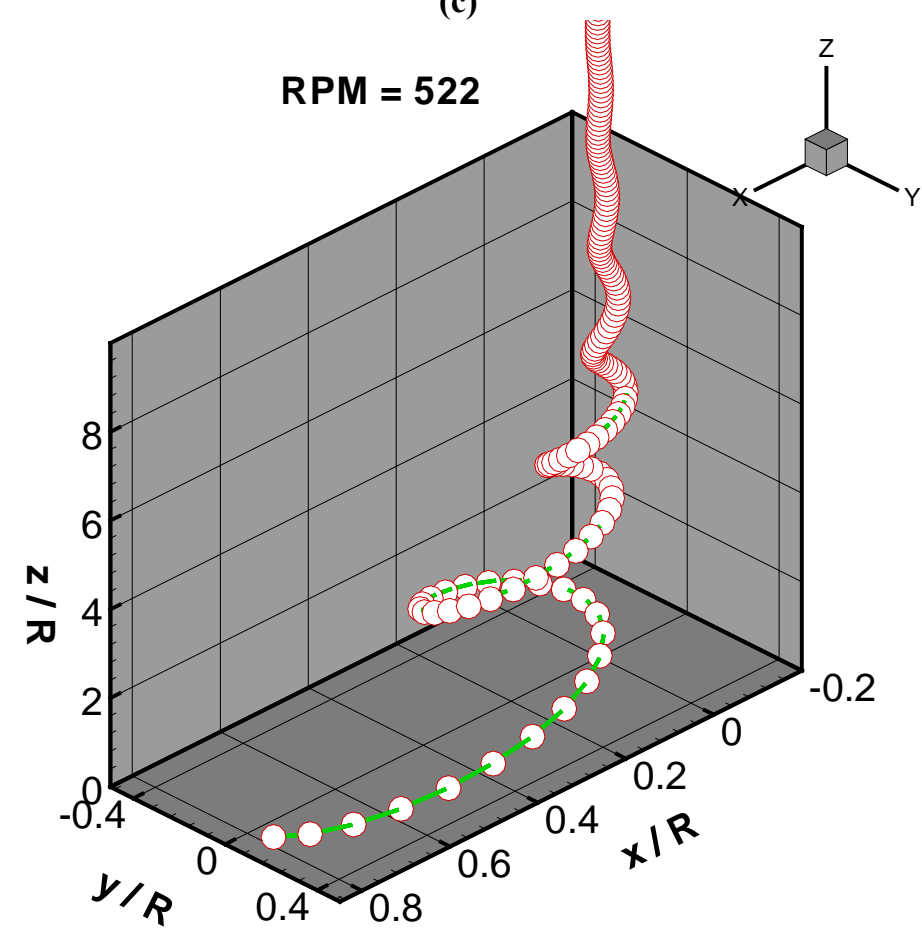

(d)

Figure 6-19 3D view of bubble trajectory in a swirling flow. The angular velocity of the swirling flow is (c) $313 \mathrm{rpm}$, (d) $522 \mathrm{rpm}$. Circle: calculated, line: exact. 


\section{Chapter 7 Formulations of Turbulent Gas-Liquid Dispersed Two-Phase Flows and Modeling Strategies}

In general, a two-phase flow system can be classified according to the configurations of the interfaces of phases into the dispersed system, stratified system and the system that lies between the two. It is well known that in the different regimes of two-phase systems, the flow exhibits very different physical behaviors (Soo 1989). For instance, the gradient of concentration of smoke will lead to diffusion of smoke particles; yet in a stratified airwater system at room condition, the diffusion effect of air through the interface will not occur. Also, in a dispersed system a common speed of sound exists, while in a pure stratified system, sound waves propagate through each phase at their own characteristic speeds. The scope of the current study is restricted to the dispersed two-phase flow systems.

The definition of turbulence in a multiphase flow is more complicated than in a single-phase flow. For example, a cloud of particles can set a fluid into a laminar-like motion; yet, the momentum flux associated with the displaced fluid may act like a Reynolds stress in the carrier phase, due to the fluctuating velocity of the particles. The present study adopts the convention stated in (Crowe et al. 1996), by which a two-phase flow is regarded as turbulent if the carrier phase exhibits random velocity fluctuations.

\subsection{Parameters and Criteria for Dispersed Two- phase Flow}

In this section a brief review is presented of the preliminaries and some characterizing parameters of a dispersed two-phase flow system. A phase is defined as a state of the matter, i.e., solid, liquid, gas or vapor. A dispersed two-phase flow consists of a primary phase, or carrier phase, which is always continuous, and a secondary phase, or dispersed phase, which is dispersed in the primary phase. The dispersed phase is usually materially disconnected, and can be of the form of bubbles, drops, droplets (small drops) and solid particles. In this text, for the sake of simplicity, these different forms of corpuscles shall be referred to as a single word, "particle," and this convention shall be always adopted unless otherwise mentioned. To distinguish the meaning of the "particle" in a multiphase flow study from the connotation used in other scientific disciplines, such as quantum and nuclear physics, a definition provided by (Clift et al. 1978) is cited herein: "a particle is a self-contained body with maximum dimension between $0.5 \mu \mathrm{m}$ and $10 \mathrm{~cm}$, separated from the surrounding medium by a recognizable interface." A particle is said to be heavy if the density ratio $\gamma_{\rho}$ defined by 


$$
\gamma_{\rho}=\frac{\rho_{p}}{\rho}
$$

is greater than 1, and said to be light or buoyant if $\gamma_{\rho}$ is less than 1. Here, $\rho_{p}$ and $\rho$ are the density of the dispersed phase and the carrier phase, respectively.

The continuous carrier phase is generally treated as a viscous Newtonian fluid governed by the Navier-Stokes equations. Although the dispersed phase (particles, drops, bubbles) are separated by the surrounding fluid, the flow field inside each individual particle, if exists, is still governed by the N-S equations, whose boundary conditions are defined at the interface between the two phases. Thus, it is possible to treat both phases as a whole with an additional interphase transferring term added to the N-S equations (see Section 7.2). However, this demands a detailed description at the phase interfaces. Of practical interest, simplifications are pursued mainly in two directions: one is to perform a volume averaging on the entire flow field to yield a set of equations expressed in terms of the so-called mesoscale quantities (see Section 7.3); and the other is to treat individual particle as a point source (see Section 7.4.4).

\subsubsection{Five Parameters}

For a characterization of dispersed two-phase flow systems, five non-dimensional parameters are of primary importance. They are the particle Reynolds number, the Stokes number, the Froude number, the length scale ratio and the particle drift parameter (velocity ratio). The definitions of these parameters are given by

$$
\operatorname{Re}_{p}=\frac{\left|\mathbf{v}_{r e l}\right| d_{p}}{v}, \mathrm{St}=\frac{\tau_{p}}{\tau}, \mathrm{Fr}=\frac{\left|\mathbf{v}_{r e l}\right|^{2}}{g l}, \gamma_{l}=\frac{d_{p}}{l}, \gamma_{v}=\frac{v_{\infty}}{u^{\prime}} .
$$

where $\mathbf{v}_{\text {rel }}$ is the relative velocity between the continuous phase and the dispersed phase, i.e., $\mathbf{v}_{\text {rel }}=\mathbf{v}-\mathbf{u} ; d_{p}$ is the characteristic dimension of the dispersed phase, such as the particle diameter; $\tau_{p}$ is the momentum response (or relaxation) time of the particle. For a spherical solid particle or droplet, the drag-induced response time is (see Eq. (6.18))

$$
\tau_{p}=\frac{\rho_{p} d_{p}^{2}}{18 \mu}
$$

A more general $\tau_{p}$-expression which also takes into account buoyant particles can be written as (Hinze 1972, 1975; Loth 2000)

$$
\tau_{p}=\frac{\rho_{p} d_{p}^{2}\left(1+C_{A} / \gamma_{\rho}\right)}{18 \mu f}
$$

where $C_{A}$ is the added-mass coefficient, and $f$ is the drag factor (or Stokes correction) defined in Eq. (6.11); Further, $\tau$ and $l$ is a characteristic time scale and length scale of the continuous phase, respectively; $v_{\infty}$ is the particle terminal velocity, and $u$ ' represents the RMS velocity scale of the carrier phase.

Next, the physical significance is addressed for the five parameters. The particle Reynolds number, $\mathrm{Re}_{p}$, is based on the magnitude of the velocity difference between the 
two phases, and it determines whether the flow around the particle can be assumed to be in the Stokes regime.

The Stokes number, St, gives the ratio of the particle response time to some time scale of the continuous phase, typically the eddy turnover time. It measures the kinetic influence of the carrier phase on the dispersed phase. If $\mathrm{St}<<1$, i.e., $\tau_{p}<<\tau$, the particle will have ample time to reach its terminal velocity before the ambient continuous flow field is subject to an appreciable change; in other words, it responds quickly to the surrounding flow field, and thus the local velocity of the two phases will be nearly equal or at a kinetic equilibrium most of the time. In this capacity, the particle will act nearly as a passive tracer of the primary fluid. In contrast, if $\mathrm{St}>>1$, the particle then will appear to be little affected by the velocity changes of the carrier phase, and its trajectory in a turbulent flow will be primarily controlled by the mean convection and gravity, not the fluctuations of an eddy. Example for the former is air bubbles in water, and for the latter is relatively large solid particles in a gas. When the Stokes number is intermediate, a heavy particle $\left(\gamma_{\rho}>1\right)$ tends to centrifuge out of eddy cores, while a buoyant particle $\left(\gamma_{\rho}\right.$ $<1$ ) is apt to be attracted by the low-pressure eddy cores (Crowe et al. 1988; Tang et al. 1992; Crowe et al. 1998; Loth 2000).

The Froude number, Fr, is based on the relative velocity as well, and it relates the inertia (or convection) to gravitation. Other velocity and length scales can be chosen for the definition of the Froude number. In a bubbly flow, this form of Froude number is also found to be the ratio of the vortical entrapment velocity to the bubble terminal rise velocity, thus providing the likelihood of bubble entrapment by the vortex (Rightley 1995).

The length scale ratio, $\gamma_{l}$, provides a particle size relative to the surrounding flow scales. If $l$ is taken to be the smallest turbulence scale, i.e., the Kolmogorov length scale, and if $\gamma_{l}<1$, the system is said to be highly dispersed (Soo 1989), and the particle may be regarded as a point source whose motion is controlled by the carrier phase (Elghobashi 1994). If $l$ is the mean free path of the molecules of the carrier phase, a criterion can then be established of whether continuum assumption of the primary phase around the particle is justified. This gives (Loth 2000)

$$
\frac{d_{p} a \rho}{\mu} \gg 1,
$$

where $a$ is the speed of sound of the carrier phase, and the following approximation has been used for expressing the mean free path (White 1991):

$$
v \approx 0.67 \mathrm{al} \text {. }
$$

If $l$ represents the integral length scale of turbulence, then $\gamma_{l}$ determines whether the turbulence will be enhanced or suppressed through the action of particles. (Gore and Crowe 1989) found that for gas-solid flows the critical value is $\gamma_{l} \approx 0.1$, above which turbulence intensity is increased and below which it is suppressed.

The particle drift parameter, $\gamma_{v}$, due to (Stock 1996), gives the ratio of particle terminal velocity to the turbulent fluctuation. It reflects a particle's capability of drifting 
away from a characteristic turbulent eddy. When $\gamma_{v}<<1$, the particle will behave as a passive tracer and tend to be trapped within the eddy; and a large value of $\gamma$ will provide the particle with more drifting capability. Previous studies (Stock 1996; Loth 2000) have shown that $\gamma_{v}$, via the so-called crossing trajectory effect, is the main controlling parameter for the mean particle diffusion. This point will become clearer as the particle diffusion and dispersion are addressed later in this section. It will be further mentioned in Section 7.4 that this parameter can also help justify the assumption of an approximate point-mass particle with respect to its carrying flow.

For the definition of St, Fr and $\gamma_{l}$, it is also possible to use different time and length scales of the carrier fluid. Let $\eta, \Lambda$ and $L$ denote the Kolmogorov length scale, turbulent integral length scale and the macroscopic length scale, respectively. When a parameter comes with a subscript $\eta, \Lambda$ or $L$, it simply means the corresponding definition is based on the Kolmogorov scales, turbulent integral scales and the macroscopic scales, respectively. For example, $\mathrm{St}_{\eta}$ refers to the Stokes number based on the Kolmogorov time scale, while $\mathrm{St}_{\Lambda}$, based on the eddy turnover time, is the one discussed in the above paragraph.

Further notice that the three parameters St, Fr and $\gamma_{\mathrm{v}}$ are interrelated. Consider a heavy particle, whose response time is given as $\tau_{p}=v_{\infty} / g$ (see Eq. (6.28)). When written down based on the $\Lambda$-scale, the three definitions read:

$$
\mathrm{St}_{\Lambda}=\frac{\tau_{p}}{\tau_{\Lambda}}, \mathrm{Fr}_{\Lambda}=\frac{u^{\prime 2}}{g \Lambda}, \gamma_{v}=\frac{v_{\infty}}{u^{\prime}} .
$$

it is seen

$$
\mathrm{St}_{\Lambda}=\gamma_{v} \mathrm{Fr}_{\Lambda}
$$

\subsubsection{Number Density and Volume Fraction}

Another two parameters important to the definition of dispersed phase flows are the number density and the volume fraction. The number density of the dispersed phase at a local point is defined as

$$
n_{p}=\lim _{\delta V \rightarrow \delta V^{0}} \frac{\delta N}{\delta V},
$$

where $\delta V$ is a local control volume that contains the mixture of both phases, $\delta N$ is the number of particles included inside $\delta V, \delta V^{0}$ is a limiting volume that is small enough but still contains enough particles to yield a stationary average. Similarly, the volume fraction of the dispersed phase is defined by

$$
\alpha_{p}=\lim _{\delta V \rightarrow \delta V^{0}} \frac{\delta V_{d}}{\delta V}
$$

where $\delta V_{d}$ is the volume of the dispersed phase in $\delta V$. An alternative but equivalent definition for the volume fraction is also given in Section 7.3. Note that the number density and the volume fraction defined above are a local quantity in general, and they 
may vary considerably throughout the flow. If let $\delta V^{0}$ be a macroscopic volume, such as the flow domain under consideration, the two quantities then become the global number density and the global volume fraction, respectively.

\subsubsection{Dilute System}

A dispersed two-phase flow system can be dilute or dense. A system is said to be dilute if the particle-particle interactions are not significant; as such, the particle motion is controlled predominantly by the forces of the carrier phase. The negligible particleparticle interactions refer to two separate mechanisms (Loth 2000): the particle-particle collision is not significant, and the particle-particle fluid dynamic interaction, which occurs indirectly through the surrounding carrier phase, is not significant.

The first criterion can be met if the average particle-particle collision time, $\tau_{p p}$, is much longer than the particle response time $\left(\tau_{p}\right)$ or the particle-eddy interaction time $\left(\tau_{p e}\right)$, i.e. (Loth 2000$)$,

$$
\frac{\tau_{p}}{\tau_{p p}} \ll 1, \frac{\tau_{p e}}{\tau_{p p}} \ll 1 .
$$

The collision frequency (number of collisions per unit time per particle), $f_{p p}$, can be approximated by the product of the number density, the average inter-particle relative velocity $\left(v_{p p}\right)$, and the area swept out by a single particle (Crowe et al. 1998):

$$
f_{p p}=n_{p} \pi d_{p}^{2} v_{p p} \text {. }
$$

For a preliminary estimation, $n_{p}$ can be replaced by the global number density and $v_{p p}$ replaced by an upper bound velocity, the quiescent terminal velocity $v_{\infty}$. Thus, the inverse of the collision frequency gives the collision time scale $\left(\tau_{p p}=1 / f_{p p}\right)$, and Eq. (7.11) can be equivalently re-expressed as:

$$
n_{p} \pi d_{p}^{2} v_{\infty}<<\frac{1}{\tau_{p}}, n_{p} \pi d_{p}^{2} v_{\infty}<<\frac{1}{\tau_{p e}} .
$$

With the substitution of the relaxation time for a spherical particle (Eq. (7.3)), the first inequality in Eq. (7.13) becomes

$$
\frac{\tau_{p}}{\tau_{p p}}=\frac{n_{p} \pi \rho_{p} d_{p}^{4} v_{\infty}}{18 \mu} \ll 1 .
$$

Further, the time scale of the particle-eddy interaction can be approximated as (Csanady 1963):

$$
\frac{1}{\tau_{p e}^{2}}=\frac{1}{\tau_{\Lambda}^{2}}+\frac{1}{\tau_{\text {trav }}^{2}},
$$

where

$$
\tau_{\Lambda}=\frac{\Lambda}{u_{\Lambda}}, \tau_{\text {trav }} \sim \frac{\Lambda}{v_{\infty}}
$$

are the turbulent integral time scale, i.e., the eddy turnover time or eddy lifetime, and the particle traversal time scale (time for a particle to traverse an eddy), respectively. By 
substituting Eq. (7.15) and (7.16) into Eq. (7.13) and applying the definition of the drift parameter, one obtains a second working inequality out of Eq. (7.13):

$$
\frac{n_{p} \pi d_{p}^{2} \Lambda \gamma_{\rho}}{\left(1+\gamma_{\rho}^{2}\right)^{1 / 2}}<<1 .
$$

In short, Eq. (7.14) and (7.17) can be used together for an estimation of the first dilute condition.

The second criterion is related to the mean inter-particle spacing, $L_{p p}$, and further the particle volume fraction, $\alpha_{p}$, since

$$
\alpha_{p}=\frac{\pi d_{p}^{3}}{6 L_{p p}^{3}} .
$$

Studies (Sirignano 1993; Yuan and Prosperetti 1994; Chen and Wu 1999) have shown that if particle spacing exceeds five diameters they have only secondary effects with respect to drag unless they are moving directly in-line with each other. This yields the second criterion for a dilute system:

$$
\alpha_{p} \ll 1
$$

or more specifically

$$
\alpha_{p}<0.42 \% \text {. }
$$

In summary, with the conditions of (7.14) and (7.20) being satisfied, the effect of particle-particle collision, as well as the indirect particle-particle interaction through the carrier phase can be neglected; as such, the dispersed two-phase flow system can be considered as a dilute system. The second criterion is typically more physically restrictive than the first one; sometimes, the small volume fraction $0.42 \%$ is relaxed with a slightly larger value, say $1 \%$ or even $2 \%$. On the other hand, if the particle-particle interactions are significant, the system is then said to be dense. In this study, when a system is said to be dilute, it always refers to the "globally dilute", which does not rule out the possibility of a high local volume fraction of the dispersed phase.

\subsubsection{Phase Coupling}

Phase coupling is an important concept in the analysis of multiphase flows. One-way coupling refers to the situation when the flow of one phase affects the other but not vice versa. When the interphase transfers of mass, momentum and energy occur between the two phases, the flow system has a two-way coupling. Further, if the particle-particle interaction in the dispersed phase has to be taken into account, it is then a four-way coupling problem. The present study is primarily interested in a dilute isothermal system, therefore, the particle-particle interaction is neglected, and the mass and energy transfer between the two phases are neglected.

It is known that the continuous phase's (momentum) influence on the dispersed phase can be characterized with the Stokes number. The reverse effect of the two-way coupling, i.e., the influence of the dispersed phase on the carrier phase, can be estimated via a momentum-coupling parameter $\Pi$ (Loth 2000). Depending on the dominant force 
that drives the particle relative velocity, $\Pi$ can be defined correspondingly. If gravity is the primary force balancing the velocity non-equilibrium $\left(\mathbf{v}_{r e l}\right)$, $\Pi$ then can be formulated as

$$
\Pi=\frac{\alpha_{p}|\Delta \rho| g l^{3}}{\rho\left|\mathbf{v}_{r e l}\right|^{2} l^{2}}=\frac{\alpha_{p}|\gamma-1|}{\mathrm{Fr}},
$$

where $\Delta \rho$ is the density difference between the two phases; the numerator of the second term represents the particle's net restitution force on the fluid, due to gravity, and it is normalized by the momentum of the carrier phase. If $l$ here represents a macroscopic length scale, then when $\Pi<<1$, the particle coupling on the mean flow can be neglected. One further sees, given a fixed $\gamma$ and Fr, when the system is dilute $\left(\alpha_{p}<<1\right)$, the two-way effect can also be not significant. Again, different length scales can be chosen to allow for $\Pi$ assessing the two-way coupling significance at different levels in a turbulent flow, e.g., the mean flow level, integral scale level and Kolmogorov scale level. Nevertheless, one should not completely rule out the possibility of significant reverse coupling even when $\Pi<<1$. An example is to introduce discrete bubbles located far apart into a still water (i.e., bubble columns), and the resulting motion of the liquid is due to the presence of bubbles. Thus, in this case, there is always two-way coupling present and it cannot be neglected.

\subsubsection{Turbulent Dispersion and Diffusion}

Particles in a turbulent flow are subject to dispersion due to the underlying turbulence. The dispersion phenomena can be observed, for example, by dropping a spoon of coffee powder into a cup of stirred water. In general, turbulent dispersion for two-phase flows can be separated into two different aspects, i.e., structure dispersion and mean diffusion (Loth 2000). The structure dispersion refers to evolution structure of instantaneous particle concentration generated by local instantaneous features of the flow. The mean diffusion characterizes the time-averaged particle concentration, which may appear to spread uniformly with respect to time in a turbulent flow. Note that the instantaneous particle concentration can differ substantially from the mean particle concentration profile, but both are also closely related since the particle dispersion structure can consequently influence the mean diffusion profile. Figure 7-1, reprinted from the original paper (Loth 2000) with permission, provides a graphical illustration of the difference between the two phenomena using the example of particles injected into a turbulent boundary layer.

The dispersion of particles is controlled by the local velocity fluctuations due to turbulence and by the ordered motion of large-scale turbulent structures. In order to describe the structure dispersion, detailed knowledge of the local flow field, primarily the spatio-temporal turbulent eddy features, needs to be known. In addition, particle-particle interaction may also change the local particle distribution where local particle concentration is high.

For the mean diffusion, whose concentration profiles can be assumed to vary smoothly over space on macroscopic length scales (and not the turbulent eddies), the 
turbulence particle diffusion coefficient, $D_{p t}$, can be introduced. Its non-dimensional version is the particle diffusion ratio (Loth 2000), $\gamma_{D}$, defined by:

$$
\gamma_{D}=\frac{D_{p t}}{D_{t}},
$$

where $D_{t}$ is the scalar (mass) turbulent diffusion coefficient. In the example of mixing layer, $\gamma_{D}$ can be taken as the ratio of the particle spreading rate and the spreading rate of the layer. Analogous to the Prandtl-Schmidt number for the momentum diffusion, the particle Prandtl-Schmidt number, $\sigma_{p}$, can be introduced as well:

$$
\sigma_{p}=\frac{v_{t}}{D_{p t}}
$$
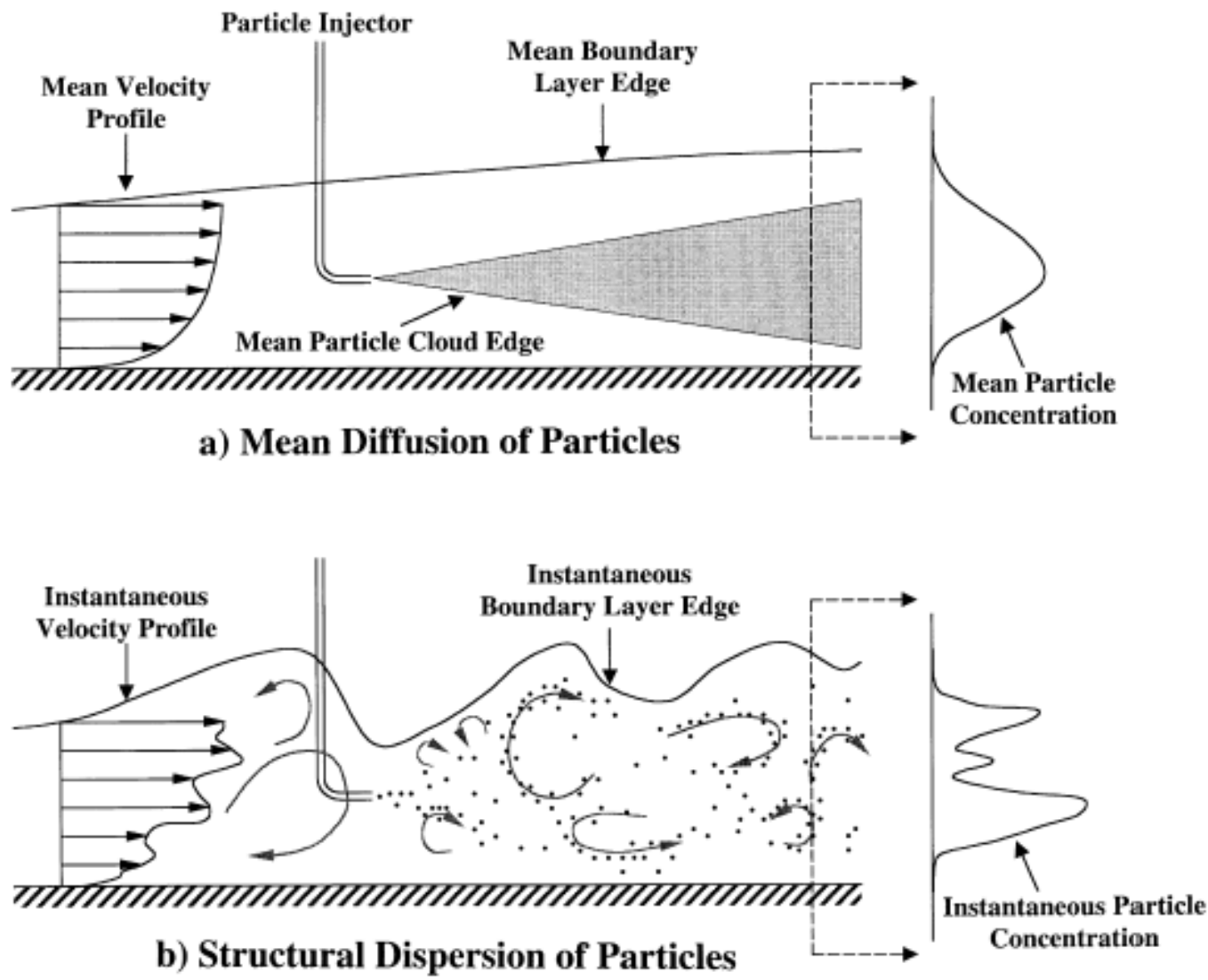

Figure 7-1 Difference between particle diffusion and dispersion in a turbulent boundary layer. (Reprinted from (Loth 2000) with permission)

In general, the significance of turbulent dispersion and diffusion of particles is a function of several non-dimensional parameters, such as the St, $\operatorname{Re}_{p}, \gamma_{\mathrm{v}}$ and $\gamma_{\rho}$. The Stokes number, however, is commonly used alone to give a rule-of-thumb estimate. If $\mathrm{St}_{\eta}<<1$, then the turbulent dispersion and diffusion is similar to that of a scalar; if $\mathrm{St}_{\Lambda}>>1$, then the turbulent dispersion is negligible. 
For heavy particles (Loth 2000) summarizes four features associated with the mean diffusion. The first feature is the Stokes number dependency. It has been found that particles with small St values will yield a particle diffusion ratio of order one $\left(\gamma_{D} \approx 1\right)$, however, very larger particles diffuse at much slower rate than that of a scalar field $\left(\gamma_{D}\right.$ $<<1$ ), owing to the slow response to the turbulent dispersion. The second feature is the so-called crossing-trajectory effect (Csanady 1963) which is caused by the local velocity non-equilibrium (due to particle gravity) between the heavy particle and its surrounding fluid. Under this effect a particle tends not to participate in the higher frequency oscillations in a turbulent eddy, and continuously changes its eddy-particle neighborhood, leading to a reduced eddy residence time. Later studies (Reeks 1977; Wells and Stock 1983; Stock 1996) have shown that given value of $\gamma$ and $C_{A}$, the crossing-trajectory effect is controlled by any two of the three parameters (only two are independent): the eddy Stokes number, the eddy Froude number and the drift parameter, since

$$
\mathrm{St}_{\Lambda}=\frac{\operatorname{Fr}_{\Lambda} \gamma_{v}\left(\gamma_{\rho}+C_{A}\right)}{\left|\gamma_{\rho}-1\right|}
$$

where $\gamma_{v}$ is given in Eq. (7.2), and

$$
\operatorname{Fr}_{\Lambda}=\frac{u^{\prime 2}}{g \Lambda}
$$

An important finding is that as $\gamma_{v}$ increases under constant Stokes number, the mean diffusion will be reduced by the traversal motion of the particles. The third and fourth features are the inertial-limit behavior and the continuity effect. See (Loth 2000) for further details.

\subsubsection{Turbulence Modulation}

In a particle-laden turbulent flow field, the level of turbulence affects the effective viscosity of the fluid, the particle dispersion, and the particle-fluid transfer coefficients, e.g., the drag coefficient. Particles, in turn, can modify the turbulence level of the conveying fluid through its own fluctuating motion and the fluid-dynamic interaction with the surrounding. The result is either an increased or attenuated turbulence level, depending on whether the particles have participated in enhancing the turbulent fluctuations or enhancing the dissipation rate. This phenomenon is commonly called turbulence modulation, an important subcategory of the two-way coupling.

Although a thorough understanding of this subject is still an ongoing effort, some preliminary results with respect to solid particles have been made available through experimental and numerical investigations (Gore and Crowe 1989; Hetsroni 1989; Squires and Eaton 1990; Gore and Crowe 1991; Tsuji 1991; Elghobashi and Truesdell 1993; Elghobashi 1994; Sundaram and Collins 1999; Crowe 2000), and they are summarized here. (i) Particle size effect. "Small" particles will attenuate turbulence and "large" ones will generate turbulence. The reason for the generation may be well attributed to the wake formation and the streamline distortion by large particles. The demarcation between small and large particles is suggested at a size of about 1/10 of the integral length scale of turbulence. (ii) Volume fraction $\left(\alpha_{p}\right)$ or mass loading effect. 
When $\alpha_{p}<10^{-6}$, the presence of particles would have no effect on turbulence. When $10^{-6}$ $<\alpha_{p}<10^{-3}$, the particles can augment the turbulence if $\mathrm{St}>1$, or attenuate turbulence if St $<1$. When $\alpha_{p}>10^{-3}$, the turbulence can be modulated through particle-particle collisions. Fundamental information regarding bubbles in homogeneous turbulence or in free shear layer comes at present from experiments such as (Lance and Bataille 1991; Roig et al. 1998; Rightley and Lasheras 2000), and direct numerical simulations such as (Maxey et al. 1994; Reutsch and Meiburg 1994).

At this point, it is necessary to define a scope of the current study. Considered are dispersed two-phase flow systems with an emphasis placed on the gas-liquid flows. In particular, bubbly flows are of the interest where interactions between gas bubble and its carrying liquid are of crucial importance. The study is further confined to dilute systems, where particle-particle interactions can be neglected (so, no four-way coupling). A major aim is to capture the particle dispersion structure using the large-eddy simulation (LES) approach. The two-way coupling is achieved through an interphase transfer term in the momentum equation, with the possible turbulence modulation indirectly generated by this term. Nevertheless, an explicit modeling for the turbulence modulation effect will not be considered in this study. Also note that formulations presented in Section 7.2 and 7.3 are fairly general, and they should be useful for a general dispersed system.

\subsection{General Formulations of Two-phase Flow System}

Consider a flow system that contains two distinct phases (materials). By making the same assumptions used in deriving the Navier-Stokes equations for a Newtonian fluid, each phase can be considered as a continuum and governed by the N-S equations of the same form. Again, incompressibility and isothermal system are assumed for the system under consideration. Thus, the conservation of mass and momentum equation can be written in conservative form for the primary continuous phase as

$$
\begin{gathered}
\nabla \cdot \mathbf{u}=0 \\
\frac{\partial \rho_{c} \mathbf{u}}{\partial t}+\nabla \cdot\left(\rho_{c} \mathbf{u} \mathbf{u}\right)=-\nabla p_{c}+\nabla \cdot \mu_{c}\left(\nabla \mathbf{u}+\nabla \mathbf{u}^{T}\right)+\rho_{c} \mathbf{g}
\end{gathered}
$$

and for the secondary dispersed phase as

$$
\begin{gathered}
\nabla \cdot \mathbf{v}=0 \\
\frac{\partial \rho_{d} \mathbf{v}}{\partial t}+\nabla \cdot\left(\rho_{d} \mathbf{v v}\right)=-\nabla p_{d}+\nabla \cdot \mu_{d}\left(\nabla \mathbf{v}+\nabla \mathbf{v}^{T}\right)+\rho_{d} \mathbf{g} .
\end{gathered}
$$

Here the velocity vector $\mathbf{u}$ and the subscript $c$ are reserved for the primary phase, while $\mathbf{v}$ and subscript $d$ for the secondary one. Although a single phase and a two-phase flow system possess the same governing equations, the analysis of a two-phase flow problem is highly complicated by the complex boundary geometries defined by the interface between the two phases. As such, the boundary conditions consist of the flow boundaries and the jump conditions for mass and momentum transfer across the interface. The jump conditions at the interface of zero thickness can be expressed as 


$$
\begin{gathered}
{\left[\rho_{c}(\mathbf{u}-\mathbf{w})\right] \cdot \mathbf{n}-\left[\rho_{d}(\mathbf{v}-\mathbf{w})\right] \cdot \mathbf{n}=0,} \\
{\left[\rho_{c} \mathbf{u}(\mathbf{u}-\mathbf{w})\right] \cdot \mathbf{n}-\left[\rho_{d} \mathbf{v}(\mathbf{v}-\mathbf{w})\right] \cdot \mathbf{n}=\left(\mathbf{T}_{c}-\mathbf{T}_{d}\right) \cdot \mathbf{n}+\sigma \kappa \mathbf{n},}
\end{gathered}
$$

where $\mathbf{w}$ denotes interface velocity, $\mathbf{n}$ is the unit vector normal to the interface surface pointing from the primary phase to the secondary phase, $\sigma$ is the surface tension coefficient whose unit is $\mathrm{N} \cdot \mathrm{m}^{-1}, \kappa$ is the local curvature with the unit of $\mathrm{m}^{-1}$ (strictly speaking, $\kappa$ is the curvature in two-dimensional case, and twice the mean curvature in three dimension), and $\mathbf{T}$ is the total stress tensor given by

$$
\begin{gathered}
\mathbf{T}_{c}=-p_{c} \mathbf{I}+\mu_{c}\left(\nabla \mathbf{u}+\nabla \mathbf{u}^{T}\right), \\
\mathbf{T}_{d}=-p_{d} \mathbf{I}+\mu_{d}\left(\nabla \mathbf{u}+\nabla \mathbf{u}^{T}\right),
\end{gathered}
$$

where I is the unitary tensor. Note that in the above interface expressions (Eq. (7.30) and (7.31)) the two phases have been treated as miscible fluids, i.e., one fluid is allowed to penetrate into the other through the interface. In order for the formulation of the flow field of two miscible fluids to be complete, an additional mass diffusion equation and eventually the energy equation need to be added. If the two fluids are immiscible, i.e., the flow moves along (not through) the interface, at the interface,

and the jump conditions simplifies to

$$
\mathbf{u}=\mathbf{w}, \mathbf{v}=\mathbf{w},
$$

$$
\left(\mathbf{T}_{c}-\mathbf{T}_{d}\right) \cdot \mathbf{n}+\sigma \kappa \mathbf{n}=0 .
$$

This suggests that the total stress difference across the interface is balanced by the surface tension. For further details of conservation equations see (Aris 1962; Soo 1989).

In this formulation, the flow field of either phase must be solved on a highly complex domain defined by the phase boundaries. If the domain of the dispersed phase is disjoint, their solutions are then coupled through the interfaces, meaning a highly coupled equations system. Also, a direct solution to the set of equations asks for a full knowledge of the flow field of both phases at all scales as well as the specific geometry of the interface. This is equivalent to saying (i) the smallest turbulence scales in both phases need to be resolved, and (ii) the interface geometry needs to be tracked. Therefore, a full numerical solution is not feasible in the practice.

In fact, when the two phases are immiscible, by recognizing that the N-S equations govern the fluid motion in the domain of either phase, one can combine Eq. (7.26) through (7.29) into a single set of equations for the entire flow domain. In this way one must (i) allow for discontinuous material properties in the formulation, and (ii) add a singular term, the surface tension term, into the momentum equations, so that the correct phase boundaries are implicitly ensured. The equations read

$$
\begin{gathered}
\nabla \cdot \mathbf{u}=0 \\
\frac{\partial \rho \mathbf{u}}{\partial t}+\nabla \cdot(\rho \mathbf{u} \mathbf{u})=-\nabla p+\nabla \cdot \mu\left(\nabla \mathbf{u}+\nabla \mathbf{u}^{T}\right)+\rho \mathbf{g}+\sigma \int_{F} \kappa \mathbf{n} \delta(\mathbf{x}-\mathbf{x}) d \mathcal{S} .
\end{gathered}
$$

Here, the velocity vector $\mathbf{u}$ and the pressure $p$ are understood as field variable for the entire flow domain, the density $\rho$ and the viscosity $\mu$ are allowed being discontinuous. One also sees the presence of the surface tension at the interface $F$ is achieved with the help of the sifting property of the three-dimensional Dirac delta function, $\delta$. Further, the 
following equations are needed to ensure a constant density and viscosity within each phase:

$$
\frac{D \rho}{D t}=0, \frac{D \mu}{D t}=0 .
$$

An essential task in this formulation is that the interface between the two phases must be tracked in order to obtain proper local values of $\rho, \mu$, and surface tension. Note that this formation is not limited to a two-phase system; it is indeed good for any number of noninterpenetrating phases (Tryggvason et al. 1997).

Theoretically, the two formulations with immiscible fluids, Eq. (7.36) through (7.38) plus Eq. (7.34), (7.35) being the one, and Eq. (7.36) through (7.38) being the other, are equivalent, since they maintain the identical details at the phase boundaries. From computational point of view, however, they can be very much different. In the first formulation the flow field must be solved with complex interface boundaries, while in the second approach the flow field can be solved on the entire domain, which can be a regular one, and the interface can be tracked in an explicit fashion. Thus, the latter formalism grants researchers an efficient way to perform a direct numerical simulation of a two-phase flow system. Work in this relevance has been pioneered by the research group led by Tryggvason (Bunner and Tryggvason 1997; Tryggvason et al. 1997; Esmaeeli and Tryggvason 1998, 1999). In their direct numerical simulations, they devised and successfully employed a so-called front-tracking method which tracks the interface explicitly by using a second-layer moving grid. See (Unverdi and Tryggvason 1992; Tryggvason et al. 1998a; Tryggvason et al. 1998b; Tryggvason et al. 1998c) for details of this method.

\subsection{Volume-averaged Conservation Equations}

For a general multiphase system consisting of interacting phases dispersed randomly in space and time, detailed solutions, which maintain full knowledge of the interface between the phases, are neither feasible nor needed in many applications. A realistic approach is to express the essential physical and dynamic quantities of such a system in terms of averages. A variety of phasic averaging methods for a multiphase system have been proposed including time averaging (Ishii 1975), ensemble averaging (Drew 1983b, a), and volume averaging (Soo 1967; Whitaker 1969; Slattery 1972; Delhaye et al. 1981; Soo 1989; Whitaker 1999). In some sense, the space average and the time average are two special cases of the ensemble average (Drew 1983a). For a dispersed multiphase system, the volume averaging seems to be most appropriate, because the dynamic and thermodynamic properties of a mixture are cumulative with volume fractions, but they are not with the fraction residence time arising from the time averaging (Soo 1989). Further, if high-frequency components in a multiphase system are significant but only the time-mean quantities are of interest, the time averaging can be carried out on top of the volume averaging. In this section a brief account is provided of the volume-averaged governing equations for a dispersed system. 
The length scale over which the volume averaging is performed is given a name mesoscale and denoted by $L_{\text {meso }}$ (see, e.g., (Rightley 1995)). The mesoscale is intermediate between the microscopic continuum scale and the macroscopic flow scale, and it should meet certain restrictions. First, the mesoscale volume should be large enough to contain sufficient number of dispersed phase elements to yield a locally stationary average; thus, the mesoscale must be larger than the characteristic length of the dispersed phase, e.g., the particle diameter, and the mean interparticle distance. Second, the mesoscale must be sufficiently small compared to the typical flow length scales, $L$, such as the eddy sizes, so that important flow structure can be retained. Third, in the interest of the current study, in which primarily dilute dispersed systems are considered, the particle size should be much smaller than the mean interparticle spacing, $d_{p} / a^{1 / 3}$ (see Eq. (7.18)). Summarized from the above discussion, one obtains the following relation:

$$
d_{p} \ll \frac{d_{p}}{\alpha^{1 / 3}} \ll L_{\text {meso }} \ll L,
$$

where $d_{p}$ represents the length scale of the dispersed phase, $\alpha$ is the global volume fraction of the dispersed phase, and $d_{p} / \alpha^{1 / 3}$ gives the order of the mean interparticle spacing. This scale relation is illustrated in Figure 7-2.

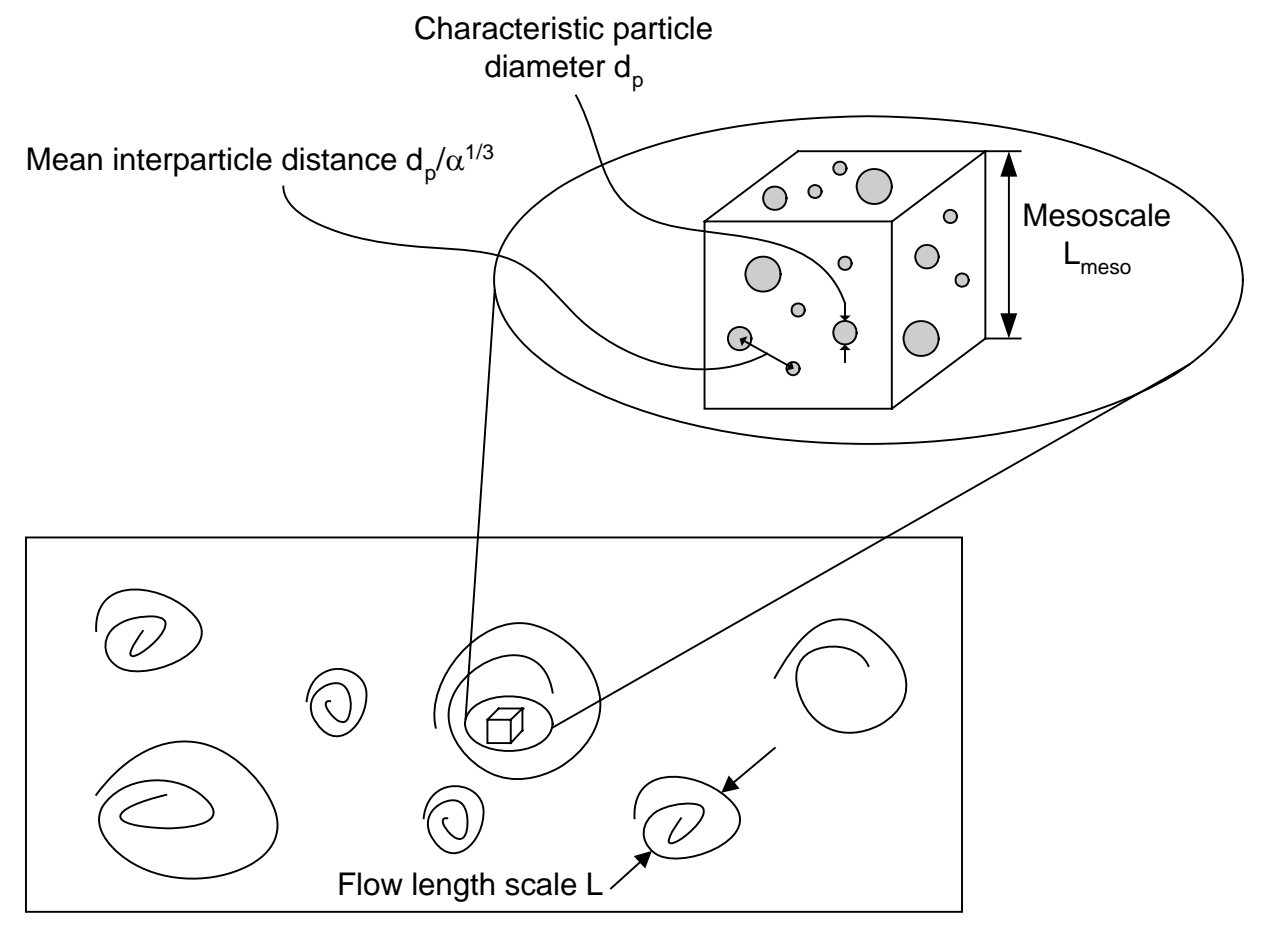

Figure 7-2 Illustration of mesoscale

Now, consider a mesoscale averaging volume, $V$. Let $\phi_{k}$ denote a generic field variable of phase $k$ per unit volume. The extensive volume average, or simply volume average, of $\phi_{k}$ is defined as

$$
\left\langle\phi_{k}\right\rangle=\frac{1}{V} \int_{V} \phi_{k} d V,
$$


where \langle\rangle denotes the volume averaging operator, and this notation shall be adopted throughout Part III of this study. Further let $V_{k}$ denote the volume of phase $k$ inside $V$, and define the intrinsic phasic average of $\phi_{k}$ as

$$
\left\langle\phi_{k}\right\rangle^{i}=\frac{1}{V_{k}} \int_{V} \phi_{k} d V
$$

where the superscript $i$ indicates an intrinsic averaging. The local volume fraction of phase $k, \alpha_{k}$, whose original definition is given in Eq. (7.10), can also be alternatively expressed through the phase indication function $\chi_{k}$ :

$$
\alpha_{k}(\mathbf{x}, t)=\left\langle\chi_{k}\right\rangle=\frac{1}{V} \int_{V} \chi_{k} d V
$$

where

$$
\chi_{k}(\mathbf{x}, t)= \begin{cases}1 & \mathbf{x} \in \text { phase } k \\ 0 & \text { otherwise }\end{cases}
$$

It is not difficult to see that

$$
\left\langle\phi_{k}\right\rangle=\alpha_{k}\left\langle\phi_{k}\right\rangle^{i}
$$

Note that the volume averaging can only be applied to quantities per unit volume or area, which include density, momentum per unit volume and gradients of stresses and fluxes. Thus, using Eq. (7.41) for the definition of, say, an intrinsic volume-averaged velocity of phase $k$, is not appropriate. Instead, define $\left\langle\mathbf{u}_{k}\right\rangle^{i}$ as

$$
\left\langle\mathbf{u}_{k}\right\rangle^{i} \equiv \frac{\left\langle\rho_{k} \mathbf{u}_{k}\right\rangle^{i}}{\left\langle\rho_{k}\right\rangle^{i}}=\frac{\left\langle\rho_{k} \mathbf{u}_{k}\right\rangle}{\left\langle\rho_{k}\right\rangle},
$$

and similarly for $\left\langle\mathbf{u}_{k} \mathbf{u}_{k}\right\rangle^{i}$,

$$
\left\langle\mathbf{u}_{k} \mathbf{u}_{k}\right\rangle^{i} \equiv \frac{\left\langle\rho_{k} \mathbf{u}_{k} \mathbf{u}_{k}\right\rangle^{i}}{\left\langle\rho_{k}\right\rangle^{i}}=\frac{\left\langle\rho_{k} \mathbf{u}_{k} \mathbf{u}_{k}\right\rangle}{\left\langle\rho_{k}\right\rangle} .
$$

Note that the volume averaging generally does not commute with spatial and time derivatives. The standard theorems for the volume averages with respect to time and spatial derivatives are summarized for example in (Gray and Lee 1977; Howes and Whitaker 1985; Soo 1989) as:

$$
\begin{gathered}
\left\langle\frac{\partial \phi}{\partial t}\right\rangle=\frac{\partial\langle\phi\rangle}{\partial t}-\frac{1}{V} \int_{S_{k}} \phi_{k} \mathbf{w} \cdot \mathbf{n}_{k} d S, \\
\langle\nabla \phi\rangle=\nabla\langle\phi\rangle+\frac{1}{V} \int_{S_{k}} \phi_{k} \mathbf{n}_{k} d S, \\
\langle\nabla \cdot \phi\rangle=\nabla \cdot\langle\phi\rangle+\frac{1}{V} \int_{S_{k}} \phi_{k} \cdot \mathbf{n}_{k} d S,
\end{gathered}
$$

where $\mathbf{w}$ is the interface velocity, $\mathbf{n}_{k}$ is the unit normal vector pointing outwards from phase $k, \mathbf{w} \cdot \mathbf{n}_{k}$ is the speed of displacement of interface, $S_{k}$ is the total interface area of phase $k$ within the volume $V$. Performing the volume averaging on the conservation equations of phase $k$, and also applying the rules of (7.47) through (7.49) yields the volume-averaged equations for phase $k$ : 


$$
\begin{gathered}
\frac{\partial\left\langle\rho_{k}\right\rangle}{\partial t}+\nabla \cdot\left\langle\rho_{k} \mathbf{u}_{k}\right\rangle=\Gamma_{k}, \\
\frac{\partial}{\partial t}\left\langle\rho_{k} \mathbf{u}_{k}\right\rangle+\nabla \cdot\left\langle\rho_{k} \mathbf{u}_{k} \mathbf{u}_{k}\right\rangle=\nabla \cdot\left\langle\mathbf{T}_{k}\right\rangle+\left\langle\rho_{k}\right\rangle \mathbf{g}+\mathcal{I}_{k}+\mathcal{J}_{k},
\end{gathered}
$$

where the total stress tensor $\mathbf{T}_{k}$ is a shorthand of

$$
\mathbf{T}_{k}=-p_{k} \mathbf{I}+\mu_{k}\left(\nabla \mathbf{u}+\nabla \mathbf{u}^{T}\right)
$$

and $\Gamma_{k}, \mathcal{I}_{k}$ and $\mathcal{J}_{k}$ are three integral terms arising from the volume averaging which are responsible for the interphase mass and momentum transfer:

$$
\begin{gathered}
\Gamma_{k}=-\frac{1}{V} \int_{S_{k}} \rho_{k}\left(\mathbf{u}_{k}-\mathbf{w}\right) \cdot \mathbf{n}_{k} d S, \\
\mathcal{I}_{k}=\frac{1}{V} \int_{S_{k}} \mathbf{T}_{k} \cdot \mathbf{n}_{k} d S, \\
\mathcal{J}_{k}=-\frac{1}{V} \int_{S_{k}} \rho_{k} \mathbf{u}_{k}\left(\mathbf{u}_{k}-\mathbf{w}\right) \cdot \mathbf{n}_{k} d S .
\end{gathered}
$$

To be specific, $\Gamma_{k}$ gives the rate of total mass generation of phase $k$ per unit volume, $\mathcal{I}_{k}$ and $\mathcal{J}_{k}$ together account for the transfer of pressure, viscous stresses, and inertial forces across the interface. By Eq. (7.44), Eqs (7.50) and (7.51) can be equivalently expressed with the intrinsic phasic quantities as

$$
\begin{gathered}
\frac{\partial \alpha_{k}\left\langle\rho_{k}\right\rangle^{i}}{\partial t}+\nabla \cdot \alpha_{k}\left\langle\rho_{k} \mathbf{u}_{k}\right\rangle^{i}=\Gamma_{k}, \\
\frac{\partial}{\partial t} \alpha_{k}\left\langle\rho_{k} \mathbf{u}_{k}\right\rangle^{i}+\nabla \cdot \alpha_{k}\left\langle\rho_{k} \mathbf{u}_{k} \mathbf{u}_{k}\right\rangle^{i}=\nabla \cdot \alpha_{k}\left\langle\mathbf{T}_{k}\right\rangle^{i}+\alpha_{k}\left\langle\rho_{k}\right\rangle^{i} \mathbf{g}+\mathcal{I}_{k}+\mathcal{J}_{k} .
\end{gathered}
$$

By definitions (7.45) and (7.46), the following volume- averaged conservation equation for a dispersed multiphase system is finally reached:

$$
\begin{gathered}
\frac{\partial \alpha_{k}\left\langle\rho_{k}\right\rangle^{i}}{\partial t}+\nabla \cdot \alpha_{k}\left\langle\rho_{k}\right\rangle^{i}\left\langle\mathbf{u}_{k}\right\rangle^{i}=\Gamma_{k} \\
\frac{\partial}{\partial t} \alpha_{k}\left\langle\rho_{k}\right\rangle^{i}\left\langle\mathbf{u}_{k}\right\rangle^{i}+\nabla \cdot \alpha_{k}\left\langle\rho_{k}\right\rangle^{i}\left\langle\mathbf{u}_{k} \mathbf{u}_{k}\right\rangle^{i}=\nabla \cdot \alpha_{k}\left\langle\mathbf{T}_{k}\right\rangle^{i}+\alpha_{k}\left\langle\rho_{k}\right\rangle^{i} \mathbf{g}+\mathcal{I}_{k}+\mathcal{J}_{k} .
\end{gathered}
$$

In this formulation one notes that the primary variables are expressed on some enlarged scales (mesoscales), and the phases appear as two interpenetrating continua with interactions represented via the volume fraction, $\alpha_{k}$, and the interphase transfer integrals. The major trade-off from the volume averaging is twofold: first, the averaged equations contain averages of products, which are additional unknowns. Second, complex interphase coupling integrals are created; these integral terms are expressed with nonaveraged local quantities, and take over almost the entire burden of explaining the interphase "activities" within the mesoscale volume. Therefore, solution of these equations calls for a closure model which can express additional unknowns in terms of the primary intrinsic averaged quantities, i.e., $\langle\mathbf{u}\rangle^{I},\langle p\rangle^{i}$ and $\alpha_{k}$. 
In turbulent flow problems, in order to eliminate high frequency fluctuations, a further averaging, namely the time averaging can be performed on top of the volume averaging (Lahey and Drew 1988; Soo 1989). With the time averaging one is able to express averages of products in terms of products of averages, and to define quantities for each phase analogous to Reynolds stresses, so that a RANS type closure model can be utilized. A conceptual difference between the two is that the volume averaging is a phasic averaging while the time averaging is a turbulence averaging, a tool used to facilitate turbulence analysis.

Further simplification of Eqs (7.58) and (7.59) can be made for the case of a highly dispersed system with the dispersed phase being spheres. In particular, if the system is composed of two phases, the simplified time-volume averaged equations for the carrier phase, denoted by $c$, can be written down as (Soo 1989).

$$
\begin{gathered}
\frac{\partial}{\partial t}\left(\alpha_{c} \rho_{c}\right)+\nabla \cdot\left(\alpha_{c} \rho_{c} \mathbf{u}_{c}\right)=\Gamma_{c}, \\
\frac{\partial}{\partial t}\left(\alpha_{c} \rho_{c} \mathbf{u}_{c}\right)+\nabla \cdot\left(\alpha_{c} \rho_{c} \mathbf{u}_{c} \mathbf{u}_{c}\right)=\nabla \cdot\left(\alpha_{c} \mathbf{T}_{c}\right)+\alpha_{c} \rho_{c} \mathbf{g}+\mathcal{I}_{c}+\mathbf{u}_{c} \Gamma_{c} .
\end{gathered}
$$

Here, for simplicity, the averaging operator has been dropped with the understanding that the quantities are expressed on mesoscales. If the two phases are immiscible and incompressible, then the mass transfer $\Gamma_{c}$ vanishes and the density can be treated as a constant. This gives

$$
\begin{gathered}
\frac{\partial \alpha_{c}}{\partial t}+\nabla \cdot\left(\alpha_{c} \mathbf{u}_{c}\right)=0, \\
\rho_{c} \frac{D}{D t}\left(\alpha_{c} \mathbf{u}_{c}\right)=\nabla \cdot\left(\alpha_{c} \mathbf{T}_{c}\right)+\alpha_{c} \rho_{c} \mathbf{g}+\mathcal{I}_{c},
\end{gathered}
$$

with

$$
\mathcal{I}_{c}=\frac{1}{V} \int_{S_{c}} \mathbf{T}_{c} \cdot \mathbf{n}_{c} d S,
$$

where $\mathrm{V}$ is the mesoscale volume.

\subsection{Eulerian and Lagrangian Description of Dispersed Phase}

In the previous two sections two descriptions have been presented for a general dispersed two-phase flow system. One is fine (Section 7.2), aimed at capturing every detail in a mixed system, and the other is coarse (Section 7.3), in which quantities are expressed on mesoscales. To emphasize, a true direct numerical simulation (DNS) of two-phase flow system solves the Navier-Stokes equations for either phase directly without resorting to any empirical closure assumptions; and it resolves not only the smallest turbulence scales in either phase, but also tracks the interface between the two. Technically, it needs to solve the standard "single-phase" N-S equations on a domain occupied by the fluid, with the effect of the secondary phase, e.g., particles, being formally taken into account through the fluid boundary conditions on the surface of particles. According to the 
current computer resources, the true DNS is still not feasible, or at most limited to a small number of particles.

A variety of other approaches have been proposed for the simulation of multiphase flows. Some good reviews can be found in (Faeth 1987; Elghobashi 1994; Crowe et al. 1996; Shirolkar et al. 1996; Loth 2000; Lakehal 2002). These simulation approaches, including the DNS, fall mainly into two classes: the Eulerian-Eulerian (E-E) approach and the Eulerian-Lagrangian (E-L) approach. The E-E approach considers both phases as a continuum and solves them on a fixed Eulerian reference frame; the E-L approach solves the continuous fluid on the Eulerian frame, but treats the dispersed phase as discrete particles or particle clouds, whose position and possibly shape are tracked in the Lagrangian reference frame. Note that all the conservation equations for a field quantity seen in this study are expressed on the Eulerian frame, such as Eqs (7.36) to (7.37) and Eqs (7.58) to (7.59). The equations of motion for a single particle used in Chapter 6 are set up on the Lagrangian frame.

The E-E and E-L approaches have in common in that the continuous phase is always described on the Eulerian frame. However, the level of the description for the continuous phase can vary, depending on the simulation techniques being used. DNS captures the smallest turbulence scales in the continuous fluid. LES (large-eddy simulation) ignores those smallest Kolmogorov scales, but retains relatively larger eddies important to the transport mechanism of turbulent energy. RANS (Reynolds-averaged Navier-Stokes) only concerns the time-averaged quantities, and most of the turbulent eddy structures get lost. Other simulation methods also exist. (Loth 2000) grouped them into two categories: the resolved-eddy description and the unresolved-eddy description. Thus, The DNS and LES belong to the former while the RANS falls in the latter class.

The Eulerian treatment for the dispersed phase(s) can be further subdivided into the mixed-fluid (or one-fluid) approach and the two-fluid approach. They will be addressed in the following two subsections.

\subsubsection{Mixed-fluid Eulerian Approach}

As pointed out by (Landau and Lifschitz 1971), the Navier-Stokes equation system can be applied to a two-phase flow if (i) the dispersed phase is small in size (compared to the geometry of the primary flow field), and (ii) it does not significantly change the overall fluid density. By doing so, one has implicitly agreed that the two phases forms a mixedfluid, which contains interpenetrating continuum components (phases). Different from the single-phase N-S equation, the density here becomes the mixture density (or effective density), $\rho_{m}$, and similarly, the fluid viscosity must be replaced by some effective viscosity, $\mu_{m}$, where subscript $m$ denotes the mixture. When the momentum of the dispersed phases cannot be neglected, such as the case of heavy particles settling in a gas flow, an inertia slip term must be added to the equation set. In general, one may write a single set of conservation equations for the mixture (not necessarily limited to only two phases) as: 


$$
\begin{gathered}
\frac{\partial \rho_{m}}{\partial t}+\nabla \cdot\left(\rho_{m} \mathbf{u}_{m}\right)=0 \\
\frac{\partial}{\partial t}\left(\rho_{m} \mathbf{u}_{m}\right)+\nabla \cdot\left(\rho_{m} \mathbf{u}_{m} \mathbf{u}_{m}\right)=\nabla p+\nabla \cdot\left[\mu_{m}\left(\nabla \mathbf{u}_{m}+\nabla \mathbf{u}_{m}^{T}\right)\right]+\rho_{m} \mathbf{g}+\mathcal{J}_{s l i p}
\end{gathered}
$$

where

$$
\begin{gathered}
\rho_{m}=\sum_{k} \alpha_{k} \rho_{k}, \mu_{m}=\sum_{k} \alpha_{k} \mu_{k}, \\
\mathcal{J}_{\text {slip }}=\nabla \cdot\left(\sum_{k} \alpha_{k} \rho_{k} \mathbf{v}_{\text {slip }, k} \mathbf{v}_{\text {slip }, k}\right), \mathbf{v}_{\text {slip }, k}=\mathbf{u}_{k}-\mathbf{u}_{m},
\end{gathered}
$$

and the mixture velocity is given by

$$
\mathbf{u}_{m}=\frac{\sum_{k} \alpha_{k} \rho_{k} \mathbf{u}_{k}}{\rho_{m}} .
$$

Note that Eq. (7.69) only presents a formal definition of the mixture velocity and it does not participate in the actual solution procedure. In particular, if the primary and the dispersed phases are in local kinetic equilibrium, that is, the relative velocities between the phases are small in comparison to variations of the overall flow field, the slip term in Eq. (7.66) drops and the mixture becomes the locally homogeneous flow (Faeth 1987), which distinguishes only the local volume fractions of each phase in a mixed volume. It is seen, in this mixed-fluid formulation the two-way coupling effects are accounted for through the void fraction and possibly the interphase slip term. The system of equations contains five primary unknowns $\mathbf{u}_{m}, p$, and $\rho_{m}$, and it can be closed with additional transport equations for respective $\alpha_{k}$, i.e.,

$$
\frac{\partial}{\partial t}\left(\rho_{m} \alpha_{k}\right)+\nabla \cdot\left(\rho_{m} \mathbf{u}_{m} \alpha_{k}\right)=\nabla \cdot\left(\frac{\mu_{m}}{\sigma_{\alpha}} \nabla \alpha_{k}\right),
$$

where $\sigma_{\alpha}$ is the Prandtl-Schmidt number for the $\alpha_{k}$ transport. The RHS term in Eq. (7.70) represents dispersion effects in the phase $k$ due to random fluctuations; if the carrier phase can be characterized as laminar, this term then equals zero.

\subsubsection{Two-fluid Eulerian Approach}

The often referred two-fluid approach is based on the volume-averaged equations, or the time and volume-averaged equations in the presence of turbulence (see Section 7.3). In this approach the two phases are assumed to be interpenetrating continua as well, but each has its own kinetic attributes. It follows that the volume-averaged conservation equations are solved for each phase, with the coupling between the phases to be achieved through the interphase exchange terms (see Eq. (7.58) and (7.59)). The turbulence closure models are usually derived from those used in single-phase RANS, typically the $k-\varepsilon$ model and its variants. In addition, approximation to the interphase integral will also be needed to close the equation set. See (Drew 1983b; Ishii 1987; Drew and Lahey 1993; Zhang and Prosperetti 1994) for more details of this method. An extension of the twofluid formalism to a more general multiphase system with more than two phases is straightforward, in which $n$ (instead of two) sets of equations need to be solved for $n$ (instead of two) phases simultaneously. In such a case, it is given a more general name, 
the separated-fluid approach (Loth 2000). Similar to the mixed-fluid approach, this separated-fluid approach is inherently two-way coupled. Also note that since this approach solves the time and volume-averaged equations, the description for the continuous phase is essentially a RANS type.

It is important to note that the smallest resolvable length scale in the volumeaveraged two-fluid approach is the size of the averaging volume, which is generally much larger than the smallest length scale of turbulence. Hence, one cannot expect that volumeaveraged equations will lead to detailed description of turbulence of the carrier phase. Now one might wonder if there is a two-fluid DNS or a two-fluid LES. As a true DNS not only resolves all the flow scales, but also keeps track of the phase interface. It is obvious to see that the latter must be a Lagrangian practice and cannot be achieved in a Eulerian frame. Therefore, an E-E two-fluid DNS does not appear to be the right terminology.

Before answering if a two-fluid LES is possible, some conceptual differences between the filtering and volume averaging must be clarified. For LES of single-phase flow a predefined filter is usually used to filter the N-S equations. If the filter used is a box filter, the filtered equations are then equivalent to the single-phase volume-averaged $\mathrm{N}-\mathrm{S}$. However, in the context of multiphase flows, filtering and volume averaging is conceptually different. The filtering is aimed at filtering out the high frequency portion of spectrum associated with small turbulence length scales, and it does not necessarily meet the criteria set by Eq. (7.39). On the other hand, the mesoscale used in the volume averaging must be large enough to yield an asymptotic statistical average, but also not too large to violate the condition (7.39). Further, the filter width in LES can be designed as a variable, for example, proportional to non-uniform grid spacing according to the local turbulence feature, while the mesoscale is typically understood as a "constant." Unlike the filter, whose size and shape is clearly defined in the physical or spectral spaces, the literature on the development of the averaged equations for two-phase flows (Soo 1967; Marble 1970; Bear 1972; Drew 1983b; Sirignano 1999) has not been specific about the volume size or shape, over which the volume averaging occurs. But it is possible that an overlap portion exist between the two length scales, i.e., filter width and the mesoscale.

Now, if a two-fluid LES is possible, one will gain the advantage of capturing detailed turbulence, while still avoiding a resolution of the phase interfaces. Notably, (Alajbegovic 2001) proposed a LES formalism applied to multiphase flows, which essentially treats the filtering as a generalization of the volume averaging. However, the author did not address the fundamental difference of the scales involved in the respective filtering and volume-averaging operation, which may eventually lead to a failure of the theory. Another useful attempt is made by (Pandya and Mashayek 2002) which deserves further attention. In view of the present author, the two-fluid LES in the framework of EE approach is theoretically feasible; however, its formalism must be subject to further elaboration and a rigorous development. Such work (Lakehal et al. 2002; Sirignano 2005) has started emerging in the literature, with the aim of unifying the volume averaging and filtering. 


\subsubsection{Resolved-volume Lagrangian Approach}

The Lagrangian reference frame is the natural frame for treating particles. The basic idea of this approach is to consider the dispersed phase as discrete objects and keep track of their motion as they move through the flow field. The discrete particles can have their own shape, whose boundary geometry forms the interface with the carrying fluid, or they can be approximated with a point-volume under certain assumptions. Note that the pointvolume is also referred to as the point-mass when dealing with heavy particles. However, the point-volume is a more general term since an air bubble, for example, is usually "massless" with respect to the liquid, but not "volume-less." Based on the two types of representation of the discrete particles, the Lagrangian formulation for the dispersed phase can be subdivided into the resolved-volume approach and the point-volume approach (Loth 2000).

The resolved-volume approach tracks information of the particle's geometric shape and its pertinent motion. The shape of the particle can be rigid or deformable. The flow field inside a particle, e.g., airflow in a bubble, can be taken into account or neglected. Thus, there will be four possible configurations with different level of physical complexity. If the particle is non-deformable and without internal flow field, e.g., a solid particle, only the standard N-S equations on the domain occupied by the carrier fluid need to be solved, and the rigid body will be advanced according to the calculated fluid stress around the particle surface. If the non-deformable particle has an internal flow field, then the N-S equations will also be solved inside each individual particle, but with the interphase treatment conforming to the rigid shape condition (i.e., the carrier fluid velocity at the particle surface is zero, and for the flow field inside the particle, the normal component is zero at the interface but the tangential component is shear-free). The motion of the rigid particle can be tracked in a similar manner by considering the integrated shear forces applied on the particle surface. When the particle is deformable, the formulations including the standard jump conditions presented in Section 7.2 apply, and the particle surface will be deformed and advected according to the resultant shear stress difference across the interface. The front-tracking method mentioned there belongs to this category.

It is clear that herein the two-way coupling has been considered in the greatest detail, since describing the phase interaction at the interface is an integral part in the entire formulation. One of the primary goals of using this approach is to sufficiently resolve the spatial and temporal scales associated with the particles' local external and internal flow field, so that the dynamics of the particle as well as their interaction with the continuous flow field can be accurately captured. Therefore, the resolved-volume Lagrangian simulation is usually accompanied with the DNS level of description for the Eulerian carrier flow field.

Despite the fact that this method can offer a full and detailed description of the multiphase system, it is challenged by two major technical difficulties: first, the continuous flow field will have to be solved on a highly complex domain due to the presence of the particle geometry; this will require a more sophisticated gridding 
technique with moving-grid capability. Second, the resolution requirement, especially for resolving the details in the interfaces region, is overly demanding. Given such, the resolved-volume approach is currently only limited to simple flow configuration with one or a few particles, and generally not applicable yet to engineering two-phase problems. However, limited knowledge gained from the direct volume-resolving simulations, similar to the relation between the single-phase DNS and LES, can be very useful in developing and validating the point-volume formulations, which are addressed next.

\subsubsection{Point-volume Lagrangian Approach}

The point-volume approach neglects the particle's geometric information and assumes the particle's entire volume is represented by a single point. As such, the details of the hydrodynamic stresses around, as well as the mass transfer across the particle surface get lost. Instead, they are "distilled" into particle-averaged point quantities. By this simplification, a detailed resolution of the flow around the particle surface is no longer necessary. The dynamics of the particle is then controlled by point forces, which include body forces and those resulting from the particle surface stresses, among others. The equation of motion of a single point-volume particle, following Newton's second law, is then,

$$
\begin{gathered}
\frac{d \mathbf{y}}{d t}=\mathbf{v}, \\
\frac{d\left(m_{p} \mathbf{v}\right)}{d t}=\mathbf{F}_{p} .
\end{gathered}
$$

where $\mathbf{y}$ and $\mathbf{v}$ denote the position and velocity, respectively, of a Lagrangian particle (note that they are not Eulerian fields here), $m_{p}$ is the particle mass, $\mathbf{F}_{p}$ represents the total point forces acting on the particle, which is generally a function of the particle's position, properties and the local feature of the carrier fluid. Detailed formulations of $\mathbf{F}_{p}$ will be addressed in Section 7.5. If the particle's mass is constant, i.e., no mass transfer takes place across the interface, Eq. (7.72) becomes

$$
m_{p} \frac{d \mathbf{v}}{d t}=\mathbf{F}_{p} .
$$

It should be stressed that this equation set is not an exact representation of the motion of an actual particle, but rather, they are approximate equations with the involved quantities being understood as either surface-averaged (e.g., forces resulting from particle surface stresses), or volume-averaged (e.g., particle mass).

With the point-volume treatment, the task of simulating the dispersed phase simplifies to tracking the temporal evolution of each particle's position (trajectory) and velocity by solving two coupled ordinary differential equations (ODE), i.e., (7.71), and (7.72) or (7.73). This can be easily done using some explicit numerical integration, such

as the Runge-Kutta class of methods. The computational expense mainly depends on the number of particles to be tracked. For $n$ particles, which correspond to $n$ independent trajectories, $2 n$ ODEs will need to be solved; but keep in mind that these $2 n$ ODEs are only pairwise coupled. 
To be computationally more economical, one may let a single particle represent, instead of itself, a group (cluster) of particles that share a same or prescribed set of physical attributes, such as location, velocity, diameter, density etc. Such a representation is known as parcel of particles, discrete element, or simply trajectory (Crowe et al. 1998; Loth 2000). The representing particle is also called computational particle. Suppose there are $n$ computational particles (or parcels) in the simulation, each representing $m$ particles possessing the same properties. Thus, the statistical sample size is $n \times m$ physical particles; however, only $n$ trajectories need to be computed since all the members in a parcel move in the same manner. If the members of a parcel have varying attributes, one may employ the so-called random sampled approach, which randomly selects a representative member in the parcel during each time-integration. Refer to (Loth 2000) for more details of the parcel method. Also, the parcel size is not arbitrary. It should be large enough to yield a statistically sufficient sample size, but also not too large to impair the statistical reliability. The total number of computational particles is limited by the computer resources. A point-volume E-L simulation typical involves 10,000 - 100,000 computational particles. The discussion presented by (Graham and Moyeed 2002) can be useful in determining the parcel size.

Unlike the resolved-volume approach and those E-E approaches, where the twoway coupling is a built-in feature, in the point-volume E-L approach one needs to distinguish if the formulation is one-way or two-way coupled. Equations (7.71) through (7.73) reflect the influence of the carrier fluid on the particle kinetics. The reverse effects from particle to the continuous phase, if any, can be taken into account in the carrier phase's formulation.

When using the point-volume approach to simulate the dispersed phase, the choice of the simulation method for the continuous phase is flexible: RANS, LES and DNS are all possible candidate. In the case of a one-way problem, simulation of the continuous phase will use the standard single-phase governing equations associated with each method, e.g., time-averaged N-S for RANS and filtered N-S for LES; the turbulence models for RANS and LES addressed in Chapter 4 can fully apply. Simply put, the continuous field will do its normal job as if it doesn't "see" the presence of particles. If two-way coupling is of significance, a reverse-coupling (from particle to continuous fluid) model must be introduced. Section 7.6 will provide a detailed discussion on the two-way coupling issue in the framework of the point-volume E-L LES simulation.

From the above discussions it is seen that the point-volume approach features simplicity in its formalism (two ODEs), ability of accommodating a sufficiently large body of particles (with the parcel concept), and flexibility in the choice of its Eulerian partner. Thus, provided that the particle equation of motion with the modeled point forces is a good approximation in describing the particle dynamics, this Lagrangian approach has opened its unique access to various real-world engineering problems. But, before one starts using this method to simulate complex two-phase flows and relying on the results it provides, it is extremely important to know under what assumptions and restrictions this very point-volume approach can lead to a successful application. This is explained next. 
A rigorous condition for the validity of the point-volume assumption is provided by (Loth 2000). It consists of two parts. First, the particle diameter is sufficiently small that the fully resolved continuous fluid characteristics (e.g., velocity and pressure) around the particle can be considered to vary linearly. In the one-dimensional situation, this is equivalent to saying that the change of a continuous function at a distance of particle size, $d_{p}$, is a linear function of $d_{p}$; clearly, this is true only when $d_{p}$ is sufficiently small. A direct implication of this assumption is that the length scales with which the smallest flow structure of the continuous phase is associated must be larger than the particle diameter, since otherwise a function can vary linearly across the flow structure, which is not possible in general. In a turbulent flow, it requires the particle diameter is small compared to the Kolmogorov length scale, $\eta$. This gives

$$
\frac{d_{p}}{\eta}<1 \text {. }
$$

A similar temporal criterion can also be formed where the particle time scale, $d_{p} / v_{\infty}$ or $d_{p}^{2} / v$, is smaller than the Kolmogorov time scale, $\tau_{\eta}$, i.e.,

$$
\frac{d_{p} / v_{\infty}}{\tau_{\eta}}<1 \text { or } \frac{d_{p}^{2} / v}{\tau_{\eta}}<1,
$$

where $v_{\infty}$ is the particle terminal velocity and $v$ is the fluid viscosity. Second, the instantaneous turbulence velocity field of the continuous phase is known in the immediate vicinity of the particle. This simply sets a resolution requirement for the continuous flow, i.e., the turbulent eddies must be sufficiently resolved down to the Kolmogorov scales. It is necessary because the point force (RHS of Eq. (7.72)) will need as an input the local instantaneous characteristics of the continuous field, upon which the accuracy of the evaluated $\mathbf{F}_{p}$ depends. In summary, in order for the point-volume treatment to be applicable in a turbulent flow, the requirements set by Eq. (7.74) and (7.75) for the dispersed phase must be met, and the turbulent fluctuations of the continuous phase must be captured down to the Kolmogorov scales. Under these conditions, the point-volume approach will be theoretically exact.

Physically, these restrictions ensures that a particle can be "immersed" into the smallest turbulent eddies, so that the particle motion is primarily controlled by its surrounding continuous fluid, while the continuous flow is not disturbed by the presence of the particles. From this point of view, it leads to a one-way problem, which precludes the approach's applicability to a two-way coupled flow. Further, Eq. (7.74) and (7.75) set extremely restrictive physical conditions on the particle size, especially for bubbles in turbulent flow where diameters tend to be larger. Moreover, the continuous flow's resolution requirement essentially calls for a DNS approach; problems may arise when tracking particles using a field supplied by LES or RANS. Therefore, as pointed out by (Loth 2000), these ideal criteria are too restrictive and generally will not be met by most engineering two-phase flow problems.

To relax the original restrictions, (Loth 2000) further proposed an alternative set of assumptions, which provides looser restrictions on the particle size and the flow field resolution. In that, one assumes that the point-volume treatment is appropriate in an "approximate" sense if the instantaneous variations of the continuous-fluid velocity, $u$, 
in the immediate vicinity of the particle, if not fully, are resolved to within some small fraction of the particle relative velocity quantified by $v_{\infty}$. Thus, the criterion becomes

$$
\gamma_{v}=\frac{v_{\infty}}{u^{\prime}} \gg 1 .
$$

In addition, (Loth 2000) relates the particle size restriction to the spatial and temporal grid resolution from the numerical computation, i.e.,

$$
\frac{d_{p}}{h}<1, \frac{d_{p} / v_{\infty}}{\Delta t}<1,
$$

where $h$ is the characteristic grid size and $\Delta \mathrm{t}$ is the time step. This argument is based on the assumption that the resolution of the particle wake should be smaller than that of the continuous-fluid, so that the resolved fluid properties may be interpolated to the particle location. Given particle type and size (i.e., $v_{\infty}$ and $d_{p}$ is fixed), the criteria of Eq. (7.76) and (7.77) ask for a resolution of the continuous-fluid field down to the scales where the turbulent fluctuations are much less than $v_{\infty}$; but at the same time the mesh size can not be arbitrarily fine, since it is bounded below by the particle diameter. If LES is used for the continuous phase simulation, it simply tells that the filter width and its related mesh size should be controlled in such a way that the smallest resolved eddies fluctuate at an order much less than $v_{\infty}$ besides ensuring $h>d_{p}$. On the other hand, if the fluctuation level of the continuous-fluid field is already known, the maximum grid size $h$ can be determined in an eddy-resolving simulation; thus, it sets constraints on the particle, whose size must be smaller than $h$, and whose terminal velocity must be sufficiently large. In this situation, heavy and small particles can easily pass the test while the buoyant particles, such as bubbles, will have difficulties to survive, since large rising velocity is always accompanied with large bubble size. Given above discussion, it seems that LES, as a resolved-eddy approach, may qualify for representing the continuous-fluid field; However, this LES must be carefully designed, in terms of its resolution and its dispersed "partner," in order to truly satisfy the a little relaxed criteria (7.76) and (7.77). It should be noted that further elaborations on these criteria will be given in Section 7.6, which finally leads to the two-layer concept proposed by the present author. For the time being, they are good enough.

One may further notice that Eq. (7.76) and (7.77) still cannot be met if the continuous-phase field is represented by RANS quantities, or quantities from a lowresolution LES. In such cases, missing is the detailed information of instantaneous velocity field of the continuous phase, which is crucial to the particle dispersion. Therefore, an empirical model must be employed to account for the spatiotemporal variations of the velocity field. Typically, these models are based on stochastic, probabilistic or deterministic diffusion methods. See (Crowe et al. 1996) for a detailed review on the diffusion models under the RANS approach. The turbulence dispersion associated with LES will be addressed in Section 7.6.

In general, to answer when the resolved-volume approach is appropriate is a subtle issue. Nevertheless, to judge when this approach is not appropriate is relatively easier. It is known that at large $\mathrm{Re}_{p}$, when $\mathrm{St}_{\Lambda}$ or $\gamma_{l}$ approach unity, particle can have strong interaction with the carrier turbulence by shedding wake (Hetsroni 1989). In such 
case, wake turbulence may dominate the dynamics of the particles. Additional complication arises when the particle is deformable. For example, large deformable bubbles subjected to eddy fluctuations develop fundamentally different wakes as opposed to non-deformable ones (Kojima et al. 1975; Chahine et al. 1993; Loth et al. 1997). To fully describe such conditions, one may have to resort to the resolved-volume approach.

\subsection{Hydrodynamic Forces for a Single Particle}

The problem considered here is that of a rigid spherical particle. A fluid particle, such as bubbles and droplets, may be subjected to deformation. To judge if a fluid particle can be assumed to be deformable, three additional non-dimensional groups can be introduced. They are the Weber number (We), the Eötvös number (Eo, also called Bond number), and the Morton number (Mo):

$$
\mathrm{We}=\frac{\rho U^{2} d_{p}}{\sigma}, \mathrm{Eo}=\frac{\Delta \rho g d_{p}^{2}}{\sigma}, \mathrm{Mo}=\frac{g \Delta \rho \mu^{4}}{\rho^{2} \sigma^{3}},
$$

where $\Delta \rho=\left|\rho-\rho_{p}\right|$. The Weber number represents the ratio of inertia forces to surface tension; the Eötvös number gives the ratio between the buoyancy forces and surface tension forces; the Morton number contains only fluid properties, and it ranges between $10^{-4}$ for gas bubbles in liquid metals and $10^{8}$ for gas bubbles in viscous oils. For air bubbles in pure water $(\sigma=0.0728 \mathrm{~N} / \mathrm{m})$ the value of Mo is $2.48 \mathrm{E}-11 \mathrm{~N} / \mathrm{m}$. Also, the Morton number can be written in terms of We and Eo as

$$
\mathrm{Mo}=\frac{\mathrm{EoWe}^{2}}{\mathrm{Re}^{4}} .
$$

For a fluid particle to remain essentially spherical, one needs

$$
\text { We } \ll 1,
$$

which indicates the surface tension forces outweigh those tending to deform them. A more robust characterization of deforming fluid particles is to employ the $\mathrm{Re}_{p}$-Eo-Mo chart (Clift et al. 1978; Fan and Tsuchiya 1990), which is included in this manuscript (Figure 7-3) merely for convenience in late uses. From that, (Loth 2000) developed an approximate criteria for significant deformation of low-density fluid particles (i.e., droplets / bubbles) under terminal velocity conditions:

$$
\begin{array}{ll}
\mathrm{Mo}<1.2 \times 10^{-7} \mathrm{Eo}^{8.15} & \text { for } \mathrm{Eo}<5 \\
\mathrm{Mo}<0.21 \mathrm{Eo}^{2.83} & \text { for } \mathrm{Eo} \geq 5 .
\end{array}
$$

In a gas-liquid dispersed system, for example, it is necessary to check the above criteria before the sphericity is assumed for the gas bubbles. In an air-water system bubbles smaller than $1 \mathrm{~mm}$ in diameter typically can be assumed to be spherical and behave like a rigid particle. 


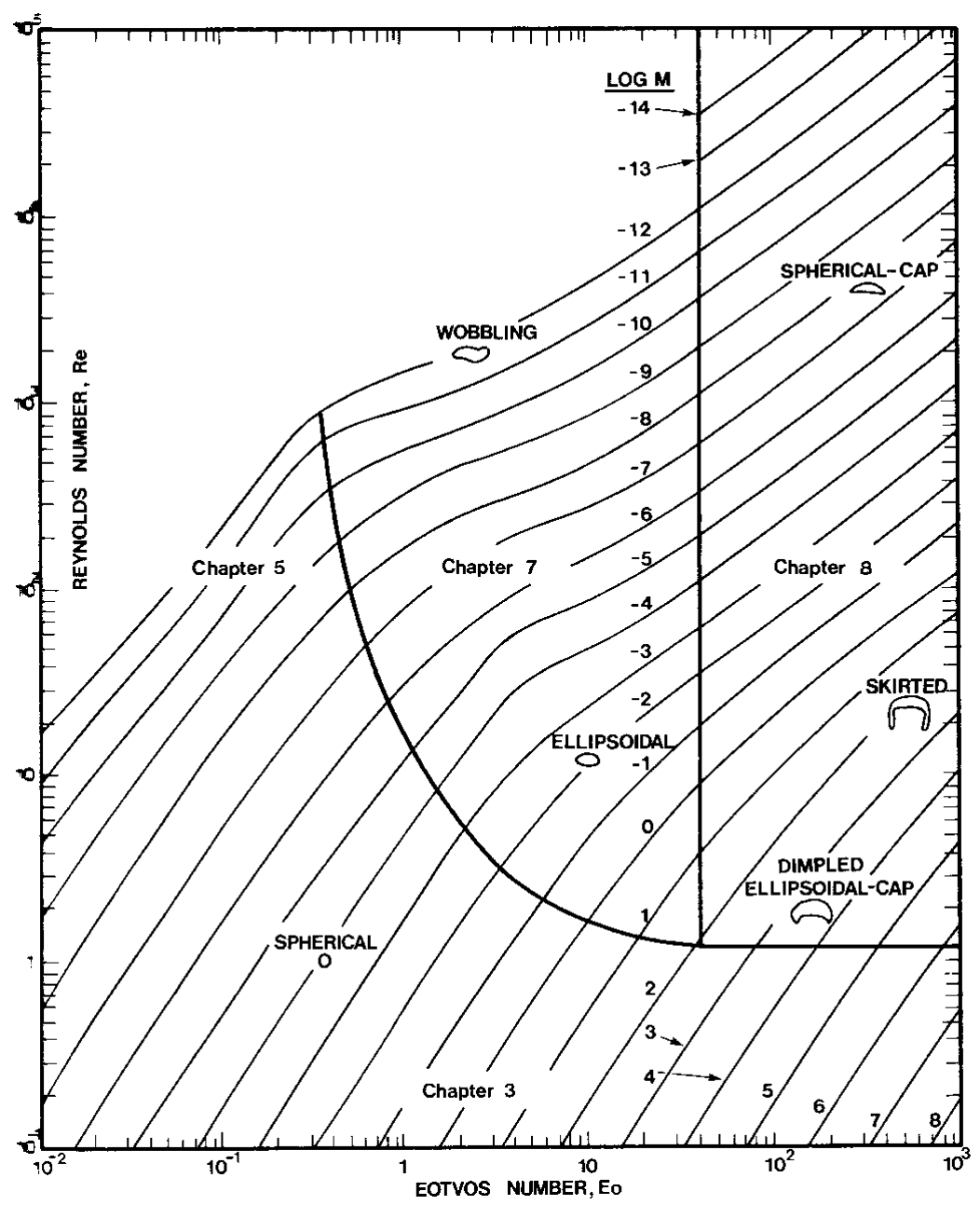

Figure 7-3 Shape regimes for bubbles and drops rising or falling freely in quiesent liquids. (From (Clift et al. 1978) with permission, chapter numbers annotated on the figure refer to those of the original textbook, not of the present thesis)

In the point-volume approach, the motion of a single particle is governed by (also see Eq. (7.72)):

$$
m_{p} \frac{d \mathbf{v}}{d t}=\mathbf{F}_{p} .
$$

The expression for $\mathbf{F}_{p}$, which represents all significant forces imposed on the particle, must be determined. It is to reiterate that $\mathbf{F}_{p}$ includes surface-averaged representations resulting from fluid stresses on the particle surface, as well as volume-averaged body forces. The goal is to find simple analytical expressions that are suitable for repetitive calculation in a Lagrangian simulation, and to reproduce particle behaviors that can match laboratory measurements or volume-resolved DNS with reasonable fidelity. It is certainly not a good idea to start deriving the expressions from the scratch; but rather, a thorough review is made based upon a great deal of past studies. This is presented below as well as in the following sections. 
In the effort of deriving such hydrodynamic forces, focus has been placed on a single small, rigid sphere immersed in an unsteady, non-uniform flow field under the Stokesian flow condition, i.e., particle Reynolds number much less than unity. The sphere can be a solid particle, a bubble or a drop. A single article of (Maxey and Riley 1983), which have assembled many of the previous research findings (Poisson 1831; Boussinesq 1885; Basset 1888b, a; Oseen 1910, 1913; Faxen 1922; Corrsin and Lumley 1956; Auton 1981), seems to be the dominant source for most of the recent publications in regard with the expression of $\mathbf{F}_{p}$. (e.g., (Auton 1987; Auton et al. 1988; Elghobashi and Truesdell 1992; Katz and Meneveau 1996; Michaelides 1997; Loth 2000; Armenio and Fiorotto 2001; Michaelides 2003)). In general, $\mathbf{F}_{p}$ can be considered a synthesis of several forces due to gravity $\left(\mathbf{F}_{G}\right)$, fluid stresses $\left(\mathbf{F}_{S}\right)$, steady-state $\operatorname{drag}\left(\mathbf{F}_{D}\right)$, lift $\left(\mathbf{F}_{L}\right.$, including Saffman lift and Magnus lift), added mass $\left(\mathbf{F}_{A}\right)$, history effect $\left(\mathbf{F}_{H}\right)$, and wall interaction $\left(\mathbf{F}_{W}\right)$, i.e.,

$$
\mathbf{F}_{p}=\sum_{l} \mathbf{F}_{l}=\mathbf{F}_{G}+\mathbf{F}_{S}+\mathbf{F}_{D}+\mathbf{F}_{L}+\mathbf{F}_{A}+\mathbf{F}_{H}+\mathbf{F}_{W} .
$$

Note that the linear addition of different forces given in Eq. (7.83) is not always valid, since different forces can act in a coupled manner. Yet, the non-linear interaction among these forces, albeit less understood, are typically small enough to be neglected in most situations (Loth 2000).

In the view of (Maxey and Riley 1983), these hydrodynamic forces acting on a particle can also be grouped into three contributions, i.e.,

$$
\mathbf{F}_{p}=\mathbf{F}_{p}^{(0)}+\mathbf{F}_{p}^{(1)}+m_{p} \mathbf{g},
$$

arising from an undisturbed flow, a disturbed flow and gravity, respectively. Note that an undisturbed flow is the original background flow field in the absence of particles, and a disturbed flow is the flow field perturbed by the presence of particles. The first contribution, $\mathbf{F}_{p}{ }^{(0)}$, arising from the undisturbed flow, would apply on a fluid element that coincides with the particle position. The pressure force and the viscous stresses existing in a undisturbed fluid belong to $\mathbf{F}_{p}{ }^{(0)}$. The second contribution, $\mathbf{F}_{p}{ }^{(1)}$, arises from the disturbed flow field. For a rigid particle moving in the fluid, the perturbation of the surrounding unsteady, non-uniform flow results in the drag, lift, added mass and history forces. The third contribution is made by the particle gravity.

In what follows, these forces are addressed in a greater detail. Note that $d_{p}, V_{p}$, and $\rho_{p}$ are used denote particle diameter, volume and density, respectively.

\subsubsection{Forces due to Gravity}

The particle gravity force and hydrostatic pressure, i.e., the buoyancy, are chosen to be included in the $\mathbf{F}_{G}$ term:

$$
\mathbf{F}_{G}=\left(\rho_{p}-\rho\right) \mathbf{g} V_{p},
$$

where $\mathbf{g}$ is the gravitational acceleration. Note that the buoyancy is part of $\mathbf{F}_{p}{ }^{(0)}$, the other part of $\mathbf{F}_{p}^{(0)}$ is known as fluid stress force which will be addressed in a later section. 


\subsubsection{Steady-state Drag Force}

The basics of steady-state or quasi-steady (in the case of turbulent flow) drag force $\mathbf{F}_{D}$ has been reviewed in Section 6.1. It is defined as

$$
\mathbf{F}_{D}=-\frac{1}{2} C_{D} \rho A_{f r}\left|\mathbf{v}_{r e l}\right| \mathbf{v}_{r e l},
$$

where

$$
\mathbf{v}_{r e l}=\mathbf{v}-\mathbf{u}
$$

is the relative velocity between the two phases. Note that the drag expression given by Eq. (7.86) is not limited to the Stokes flow or low- $\operatorname{Re}_{p}$ flow, because the drag coefficient $C_{D}$ here represents an empirical fit that can reproduce the particle drag behavior from a laboratory measurement. Commonly, $C_{D}$ is expressed as the product of the Stokes drag coefficient and the drag factor, $f$, i.e.,

$$
C_{D}=\frac{24}{\operatorname{Re}_{p}} f\left(\operatorname{Re}_{p}\right) .
$$

The expression for the drag factor is provided in Table 6-1. But some further aspects are addressed here.

First, the drag force given by Eq. (7.86) is derived in a uniform flow. A so-called Faxen term ( $2^{\text {nd }}$ term in parenthesis of Eq. (7.89)), due to (Faxen 1922), can be introduced to account for the non-uniformity of flow around the sphere. This yields (Maxey and Riley 1983)

$$
\mathbf{F}_{D}=-\frac{1}{2} C_{D} \rho A_{f r}\left|\mathbf{v}_{r e l}\right|\left(\mathbf{v}_{r e l}-\frac{1}{24} d_{p}^{2} \nabla^{2} \mathbf{u}\right) .
$$

Second, the expression for $f$ can be extended to account for other factors, such as particle non-sphericity and compressibility of the flow. A crude approximation is to write $f$ as the linear product of various correction ratios (Loth 2000):

$$
f=\prod_{l} f_{l}=f_{\text {Re }} f_{\text {Sphere }} f_{\text {Mach }} \cdots,
$$

where $f_{\mathrm{Re}}$ is the standard drag factor, same as the one in Eq. (7.88). To this end, see (Clift et al. 1978; Loth 2000) for more details. The present study will only consider the case where $f=f_{\mathrm{Re}}$.

Third, basically, the drag factor $f$ given in Table 6-1 is measured for a solid particle. For a fluid particle, e.g., droplets or air bubble, complications may arise due to internal recirculation and deformability; thus proper modifications for $f$ values are necessary. Table 7-1 summarizes five sets of drag factor for the case of spherical bubble in clean (lightly contaminated) water, contaminated water (tap water), and grossly contaminated water, as well as ellipsoidal bubble and spherical-cap bubble in contaminated water. All these correlations are expressed in dependency of the particle Reynolds number. The bubble diameter used in Eq. (7.94) represents a nominal (or effective) diameter. Due to surface-active impurities (surfactants) in the tap water, the bubble surface is immobilized; as a result small bubbles (whose diameter less than $1 \mathrm{~mm}$ ) in tap water behave very much like a solid sphere, and their drag factor is thus comparable to that of a rigid sphere (cf. Eq. (6.12)). For bubbles with diameter above 3 
$\mathrm{mm}$ freely rising in tap water, experimental evidence (Fan and Tsuchiya 1990) shows that use of the drag factor given by Eq. (7.92) yields greatly overpredicted bubble terminal velocity. To remedy this, additional correlations have been proposed and used in the literature. Two examples are provided in Table 7-2. Further correlations can be found in (Sokolichin et al. 2004).

Table 7-1 Drag correction factor f for air bubble in clean or contaminated water.

\begin{tabular}{|c|c|c|}
\hline $\begin{array}{l}\text { Spherical bubble in clean water } \\
\text { (Loth et al. 1997; Loth 2000) }\end{array}$ & $f= \begin{cases}1+0.1875 \mathrm{Re}_{p} & \mathrm{Re}_{p} \leq 0.1 \\
1+0.0565 \mathrm{Re}_{p}^{0.525} & 0.1<\mathrm{Re}_{p} \leq 500\end{cases}$ & $(7.91)$ \\
\hline $\begin{array}{l}\text { Spherical bubble in tap water } \\
\text { (Shiller and Naumann 1933) }\end{array}$ & $f= \begin{cases}1+0.15 \mathrm{Re}_{p}^{0.687} & \mathrm{Re}_{p} \leq 800 \\
0.44\left(\mathrm{Re}_{p} / 24\right) & \mathrm{Re}_{p}>800\end{cases}$ & $(7.92)$ \\
\hline $\begin{array}{l}\text { Spherical bubble in grossly } \\
\text { contaminated water } \\
\text { (Clift et al. 1978; Loth 2000) }\end{array}$ & $f= \begin{cases}1+0.1875 \mathrm{Re}_{p} & \operatorname{Re}_{p} \leq 1 \\
1+0.1935 \mathrm{Re}_{p}^{0.6305} & 1<\operatorname{Re}_{p} \leq 78 \\
1+0.03875 \mathrm{Re}_{p} & 78<\mathrm{Re}_{p} \leq 300\end{cases}$ & (7.93) \\
\hline $\begin{array}{l}\text { Ellipsoidal bubble in tap water } \\
\text { (Loth et al. 1997) }\end{array}$ & $\begin{array}{l}f=\frac{\operatorname{Re}_{p}^{1 / 2}}{24}\left[8.85+13.24\left(\frac{d_{p}}{1.1}-1\right)-0.203 \operatorname{Re}_{p}\right. \\
\text { for } 1.1 \mathrm{~mm} \leq d_{p}<4.5 \mathrm{~mm} \text { and } \operatorname{Re}_{p} \leq 200\end{array}$ & (7.94) \\
\hline $\begin{array}{l}\text { Bubble with spherical-cap shape } \\
\text { in tap water (Clift et al. 1978) }\end{array}$ & $f=\frac{\operatorname{Re}_{p}}{9} \quad \operatorname{Re}_{p}>150$, Eo $>40$ & $(7.95)$ \\
\hline
\end{tabular}

Table 7-2 Drag coefficient for air bubble in tap water

\begin{tabular}{|c|c|c|c|c|}
\hline $\begin{array}{l}\text { (Tomiyama et al. } \\
1995)\end{array}$ & $C_{D}=\max$ & $\frac{24}{\operatorname{Re}}(1+0$ & $\left.5 \mathrm{Re}^{0.687}\right), \frac{8}{3} \frac{\mathrm{Eo}}{\mathrm{Eo}+4}$ & $(7.96)$ \\
\hline $\begin{array}{l}\text { (Boisson and Malin } \\
\text { 1996) }\end{array}$ & $C_{D}=\left\{\begin{array}{l}24 \\
20 . \\
6.3 \\
\mathrm{We} \\
8 / 3\end{array}\right.$ & $\begin{array}{l}\mathrm{Re} \\
68 / \mathrm{Re}^{0.643} \\
/ \mathrm{Re}^{0.385} \\
/ 3\end{array}$ & $\begin{array}{l}\operatorname{Re}<0.49 \\
0.49<\operatorname{Re}<100 \\
\operatorname{Re}>100, \mathrm{We} \leq 8, \mathrm{R} \\
\operatorname{Re}>100, \mathrm{We} \leq 8, \mathrm{R} \\
\operatorname{Re}>100, \mathrm{We}>8 \\
(7.97)\end{array}$ & \\
\hline
\end{tabular}

\subsubsection{Lift Force}

The quasi-steady lift force can be subdivided into Saffman lift force and Magnus lift force. The Saffman lift force is caused by velocity gradient (shear-induced), and the Magnus lift force is due to the solid body rotation (spin-induced). Both the local velocity gradient of the continuous fluid and the rotation of particle can result in an uneven pressure distribution around the particle in the disturbed flow field, thus generating a lift 
in the direction perpendicular to the relative velocity between the fluid and the particle, and to rotation of fluid or particle.

The Magnus force derived by (Rubinow and Keller 1961) under the condition of $\operatorname{Re}_{p} \leq 1$ is

$$
\mathbf{L}_{M a g}=\frac{\pi}{8} \rho d_{p}^{3}(\mathbf{u}-\mathbf{v}) \times\left(\boldsymbol{\omega}_{p}-\frac{1}{2} \boldsymbol{\omega}\right)
$$

where $\omega_{p}$ is the particle angular velocity, $\omega$ is the vorticity of the flow, i.e.,

$$
\boldsymbol{\omega}=\nabla \times \mathbf{u}
$$

thus $0.5 \omega$ gives the rate of rotation of the fluid, and $\left(\omega_{p}-0.5 \omega\right)$ is the relative rotation of the particle with respect to the flow rotation. Similar to the general definition of the drag force, the Magnus force at finite particle Reynolds number can also be cast into the following form with an empirical lift coefficient, $\mathrm{C}_{L, M a g}$, due to rotation (Crowe et al. 1998):

$$
\mathbf{F}_{M a g}=-\frac{1}{2} C_{L, M a g} \rho A_{f r}\left|\mathbf{v}_{r e l}\right| \mathbf{v}_{r e l} \times \frac{\boldsymbol{\omega}_{r e l}}{\left|\boldsymbol{\omega}_{r e l}\right|},
$$

where $\mathbf{v}_{r e l}$ is defined in Eq. (7.87), and $\omega_{r e l}=\omega_{p}-0.5 \omega$. Equation (7.100) and (7.98) are linked through the following relation

$$
\mathbf{F}_{\text {Mag }}=\frac{C_{L, M a g}}{\text { Ro }} \mathbf{L}_{M a g}
$$

where Ro is the rotation parameter or Rossby number given by

$$
\mathrm{Ro}=\frac{d_{p}\left|\boldsymbol{\omega}_{r e l}\right|}{\left|\mathbf{v}_{r e l}\right|} .
$$

Thus, if $\mathrm{C}_{L, M a g}=$ Ro, Eq. (7.98) is recovered. For particles rotating in a uniform flow at higher $\operatorname{Re}_{p}$ and higher Ro, the Magnus lift coefficient has been measured, for example, by (Maccoll 1928; Davies 1949; Barkla and Auchterlonie 1971; Tsuji et al. 1985; Tanaka et al. 1990; Tri et al. 1990; Sridhar and Katz 1995), and summarized in (Loth 2000). According to the measurements a simple approximate expression for $\mathrm{C}_{L, \text { Mag }}$ is suggested by (Tanaka et al. 1990) and cross-referenced by (Crowe et al. 1998; Loth 2000) as

$$
C_{L, \text { Mag }}=\min (\mathrm{Ro} / 4,0.5) \text {, }
$$

with the applicable range of $500<\operatorname{Re}_{p}<100,000$ and $0.1<\mathrm{Ro}<20$. In a torque-free environment, the Magnus lift force can be generally neglected, since the particle spin tends to approach the fluid rotation after some time exceeding $\tau_{p}$ (Loth 2000).

The original Saffman force, due to (Saffman 1965, 1968), can be generally expressed as

$$
\mathbf{L}_{\text {Saff }}=-1.61 \rho d_{p}^{2} \sqrt{\nu|\boldsymbol{\omega}|} \mathbf{v}_{r e l} \frac{\mathbf{v}_{r e l} \times \boldsymbol{\omega}}{\left|\mathbf{v}_{r e l} \times \boldsymbol{\omega}\right|} .
$$

It was derived under a condition more restrictive than that of a creeping flow, i.e.,

$$
\operatorname{Re}_{p} \ll \sqrt{\operatorname{Re}_{\omega}} \ll 1,
$$

where $\operatorname{Re}_{\omega}$ is the shear Reynolds number defined as 


$$
\operatorname{Re}_{\omega}=\frac{|\boldsymbol{\omega}| d^{2}}{v}
$$

Under the condition of

$$
\operatorname{Re}_{\omega}<\operatorname{Re}_{p}<1,
$$

the Saffman force given by Eq. (7.104) can be corrected according to McLaughlin's theory (McLaughlin 1991; Cherukat et al. 1994):

$$
\mathbf{L}_{M c L}=\mathbf{L}_{\text {saff }} \frac{\left(\gamma_{\mathrm{Re}}-0.28\right)^{1.7}}{\left(\gamma_{\mathrm{Re}}-0.28\right)^{1.7}+0.16},
$$

where

$$
\gamma_{\mathrm{Re}}=\frac{\sqrt{\operatorname{Re}_{\omega}}}{\operatorname{Re}_{p}}, \gamma_{\mathrm{Re}}>0.3 .
$$

At high particle Reynolds numbers, (Auton 1981; Auton et al. 1988) derived an expression for the shear-induced lift force in an inviscid flow:

$$
\mathbf{L}_{\text {Auton }}=-C_{L, \text { Auton }} \rho V_{p} \mathbf{v}_{\text {rel }} \times \boldsymbol{\omega},
$$

where $C_{L, A u t o n}=1 / 2$. A similar derivation for the inviscid condition is performed by (Drew and Lahey 1987; Drew and Lahey 1990). For different type of flows the measured lift coefficients are found to deviate from the inviscid one (Wang et al. 1987; Moraga 1998; Tomiyama 1998; Tomiyama et al. 2002). For example, in a fully developed upward bubbly flow in a pipe, $C_{L, A u t o n}$ is found to be around 0.1 (Wang 1986; Wang et al. 1987). The correlation developed in (Tomiyama et al. 2002) provides a useful resource for numerical simulation.

Similar to Eq. (7.100), the general Saffman lift force can also be written based on the hydrodynamic pressure and projected area as

$$
\mathbf{F}_{\text {Saff }}=-\frac{1}{2} C_{L, \text { Saff }} \rho A_{f r}\left|\mathbf{v}_{r e l}\right|^{2} \frac{\mathbf{v}_{r e l} \times \boldsymbol{\omega}}{\left|\mathbf{v}_{r e l} \times \boldsymbol{\omega}\right|},
$$

(Sridhar and Katz 1995) produced from their experimental investigation a well-known fit for the lift coefficient:

$$
C_{L, S a f f}=0.5 \mathrm{Ro}^{0.25},
$$

where the fluid rotation number is defined as

$$
\mathrm{Ro}=\frac{d_{p}|\boldsymbol{\omega}|}{\left|\mathbf{v}_{\text {rel }}\right|} .
$$

This approximation is consistent with (Naciri 1992)'s results, and also later confirmed by (Felton and Loth 2001)'s study. Notably, (Sridhar and Katz 1995) finds that, if $20<\operatorname{Re}_{p}<80$ and $0.02<\operatorname{Ro}<0.2, C_{L, \text { Saff }}$ only depends on $\mathrm{Ro}$, and it is independent of $\operatorname{Re}_{p}$. This conclusion is consistent with Auton's inviscid lift expression, as well as with (Barkla and Auchterlonie 1971) and (Tsuji et al. 1985)'s data. On the other hand, studies of (Naciri 1992) and (Felton and Loth 2001) indicate a decreasing lift coefficient with increasing $\operatorname{Re}_{p}$. This discrepancy may stem from different flow regimes (laminar or turbulent), and will remain an open question (Felton and Loth 2001). Also note that in most experimental studies, the measured shear-induced lift force tends to be larger than that predicted by the original Saffman lift as well as Auton's inviscid lift for values of Ro 
below 0.2. Often times, Eq. (7.104) and (7.110) can serve as a conservative lower bound of an actual lift force.

\subsubsection{Fluid-stress Force}

The fluid stress force, $\mathbf{F}_{S}$, is a force arising from the undisturbed flow. In particular, it refers to the pressure and the viscous stresses that originally act in the undisturbed flow, and would apply on the particle as if on a fluid element in place of the particle. Since the buoyancy force has already been included in the $\mathbf{F}_{G}$ term (see Section 7.5.1), $\mathbf{F}_{S}$ should exclude the contribution from the hydrostatic pressure. That is, only the dynamic pressure would make part of $\mathbf{F}_{S}$.

The expression of $\mathbf{F}_{S}$ can be derived as follows. Let $\sigma^{(0)}$ denote the surface stresses comprising the dynamic pressure, $p_{d y n}$, and viscous stresses in the undisturbed flow, i.e.,

$$
\boldsymbol{\sigma}^{(0)}=-p_{d y n} \mathbf{I}+\mu\left(\nabla \mathbf{u}+\nabla^{T} \mathbf{u}\right) .
$$

The net fluid force acting on a rigid sphere can be determined from the integral of $\sigma^{(0)}$ over the particle surface $S$ :

$$
\mathbf{F}_{S}=\int_{S} \boldsymbol{\sigma}^{(0)} \cdot \mathbf{n} d \mathcal{S},
$$

where $\mathbf{n}$ is unit outward normal. Eq. (7.115) can be converted to the volume integral using Gauss's divergence theorem:

$$
\mathbf{F}_{S}=\int_{V_{p}} \nabla \cdot \boldsymbol{\sigma}^{(0)} d V,
$$

where $V_{p}$ is the volume occupied by the particle. If the particle dimension is less than all the characteristic length scales of the underlying flow, the integrand in Eq. (7.116) can be considered constant over the particle volume, i.e.,

$$
\mathbf{F}_{S}=V_{p} \nabla \cdot \boldsymbol{\sigma}^{(0)} .
$$

By noticing

$$
\rho \frac{D \mathbf{u}}{D t}=-\nabla p+\nabla \cdot \mu\left(\nabla \mathbf{u}+\nabla^{T} \mathbf{u}\right)+\mathbf{g}=\nabla \cdot \boldsymbol{\sigma}^{(0)},
$$

one obtains the working expression of the fluid-stress force as (Maxey and Riley 1983)

$$
\mathbf{F}_{S}=m_{f} \frac{D \mathbf{u}}{D t} .
$$

Thus, the fluid-stress force is in fact related to the fluid acceleration. The seemingly simple form of this force, Eq. (7.119), implicitly represents the action of the pressure gradient and the viscous stresses inside the flow.

Here, it is necessary to make a distinction. Notice that some other forces, such as the drag and lift, are in principle also generated by the viscous stresses. However, the surface stresses associated with the fluid-stress force are fundamentally different from those related to the drag and lift forces: the former is present in an undisturbed flow while the latter is created in a disturbed flow setting. 
Further recall that the drag force is usually measured in a uniform pressure field in the absence of the particles. Therefore, the significance of the fluid-stress force can be appreciated when there exists in the flow large local pressure gradient, which causes additional acceleration or deceleration of the particle. In fact, the role of the pressure gradient in contributing to the net fluid force acting on the particle was originally emphasized by (Corrsin and Lumley 1956), who, however, missed the contribution of the viscous stresses. (Maxey and Riley 1983) made the addition with the argument that, for a particle that is small compared to the flow scales, the effects of the undisturbed fluid stresses both from pressure and viscosity are well comparable.

It should be stressed that, in deriving equation (7.119), a critical restriction has been imposed: the particle dimension must be smaller than any of the characteristic flow length scales. In a turbulent flow, it simply means

$$
d_{p} \ll \eta \text {. }
$$

Therefore, caution must be taken when applying Eq. (7.119) to a Eulerian-Lagrangian calculation where condition (7.120) is violated.

\subsubsection{Added-mass Force}

Note that the drag and lift force are usually measured at steady state where there is no relative acceleration between the fluid and the immersed object. Thus, they can be grouped into steady-state forces. When a particle accelerates through a uniform fluid, there is a corresponding acceleration of the surrounding fluid at the expense of work done by the particle. By Newton's third law, the acceleration of the fluid will in turn generate a transient force that acts on the particle in the opposite direction of the particle acceleration. This force is a type of unsteady forces and known as the added-mass force, or virtual mass force, it is given by

$$
\mathbf{F}_{A}=-C_{A} m_{f} \frac{d}{d t}(\mathbf{v}-\mathbf{u}),
$$

where $m_{f}$ is the mass of the fluid which has the same volume as the sphere, i.e., $m_{f}=\rho V_{p}$; $C_{A}$ is the added-mass coefficient and equals $1 / 2$ for a rigid sphere in inviscid Stokes flow. The derivative $d / d t$ used here denotes the time derivative following the moving sphere. When $d / d t$ is applied to the continuous flow field, at low Reynolds number,

$$
\frac{d \mathbf{u}}{d t} \approx \frac{D \mathbf{u}}{D t}
$$

where $\mathrm{D} / \mathrm{D} t$ denotes the material derivative following a fluid element (Auton et al. 1988; Maxey et al. 1996). Thus Eq. (7.121) can also be written as

$$
\mathbf{F}_{A}=-C_{A} m_{f}\left(\frac{d \mathbf{v}}{d t}-\frac{D \mathbf{u}}{D t}\right) .
$$

An equivalent understanding of the origin of this force can be gained by looking at a uniform inviscid flow past a sphere fixed in space. An acceleration of the fluid will result in a change of the kinetic energy in the flow field, which creates a transient force (Eq. (7.121)) exerting on the sphere. Similar to the Faxen correction in the drag expression 
(see Eq. (7.89)), a similar Faxen term can also be added to the added-mass force to take account of the spatial variation in the flow. This gives (Maxey and Riley 1983)

$$
\mathbf{F}_{A}=-C_{A} m_{f} \frac{d}{d t}\left(\mathbf{v}-\mathbf{u}-\frac{1}{40} d_{p}^{2} \nabla^{2} \mathbf{u}\right) .
$$

The reason of calling it added-mass force is that the effective mass subjected to acceleration by the particle momentum equation is $\left(m_{p}+C_{A} m_{f}\right)$, instead of $m_{p}$. This is readily seen by moving the particle acceleration part of Eq. (7.121) to the LHS of Eq. (7.82). This yields

$$
\left(m_{p}+C_{A} m_{f}\right) \frac{d \mathbf{v}}{d t}=\mathbf{F}_{p}^{\prime},
$$

where $\mathbf{F}_{p}{ }_{p}$ is modified from the original $\mathbf{F}_{p}$ accordingly. It should be noted that, for heavy particles where $m_{p}>>m_{f}$, the added mass effect can be neglected, while for light particles $\left(m_{p}<<m_{f}\right)$, such as air bubbles, the influence of added mass is significant and must be included.

The added-mass force can be derived from the inviscid flow theory; In fact, it was first deduced by (Poisson 1831), who solved the potential flow equation around a sphere and determined the correct added-mass coefficient (equal to 1/2) about 20 years before the publication of what we now call "the Navier-Stokes equations." A detailed derivation can also be found in a recent book by (Crowe et al. 1998). (Green 1833) extended the results to flow around an ellipsoid, and obtained the same value for the added-mass coefficient. Even for moderately ellipsoidal air bubbles in water with diameters up to 3.5 $\mathrm{mm}$, this coefficient is still approximately valid based on (Bataille et al. 1991). Strictly speaking, $C_{A}=1 / 2$ is derived under the creeping flow condition. At finite particle Reynolds numbers, an empirical relation was suggested by (Odar and Hamilton 1964; Odar 1966)

$$
C_{A}=1.05-\frac{0.066}{0.12+A c^{2}}
$$

where $A c$ is the proposed acceleration number defined by

$$
A c=\frac{\left|\mathbf{v}_{r e l}\right|^{2}}{d_{p}\left|\frac{d \mathbf{v}}{d t}\right|} .
$$

But, there is no unified agreement on this correction, as discussed in (Sridhar and Katz 1995; Michaelides 1997). For example, (Tsuji et al. 1991) confirmed the accuracy of Eq. (7.126) with an extensive experimental study. On the other hand, the results of (Chang 1992) indicates that $C_{A}$ is independent of both Reynolds number and acceleration number, provided the particle is nearly spherical.

\subsubsection{History Force}

Another unsteady force due to the acceleration of the relative velocity is the history force or sometimes the Basset force. While the added-mass force relates to the force required to accelerate the surrounding fluid, the history force describes the force due to the temporal 
delay in boundary layer development as the relative velocity changes with time. The analytical expression of the history force was first seen in (Boussinesq 1885), and also independently derived by (Basset 1888b, a). The name "Basset force" is sort of ironic, as pointed out by (Michaelides 2003), since Boussinesq's work has precedence.

To understand the origin of the history force one may consider an infinite flat plate below a viscous fluid subject to a step velocity change from zero to $U_{0}$ (Stokes' first problem, see, e.g., (White 1991)). The one-dimensional velocity field is

$$
u=U_{0} \operatorname{erfc}(\eta)
$$

where erfc is the complementary error function, $\eta$ is the similarity variable defined by

$$
\eta=\frac{y}{2 \sqrt{v t}}
$$

$y$ is the direction perpendicular to the plate, and $t$ is the time. The shear stress at the plate $(y=0)$ given by this velocity profile is then

$$
\tau=U_{0} \sqrt{\frac{\rho \mu}{\pi t}} .
$$

Thus, a viscous shear force is generated by the sudden acceleration of the plate. Note that this force does not arise from a steady-state boundary layer, such as the laminar flow past a flat plate. Also, it reaches its maximum right after the step change, and as time goes to infinity, it approaches zero. Now, a general temporal variation in plate velocity can be assumed to consist of a series of such step changes. The shear stress at the plate is then an accumulative effect of Eq. (7.130), which leads to the following expression (Crowe et al. 1998)

$$
\tau=\sqrt{\frac{\rho \mu}{\pi}} \int_{0}^{t} \frac{\frac{d u}{d t^{\prime}}}{\sqrt{t-t^{\prime}}} d t^{\prime} .
$$

If the same principle is applied to the unsteady Stokes flow over a sphere, one obtains the expression for the history force as

$$
\mathbf{F}_{H}=-\frac{3}{2} d_{p}^{2} \sqrt{\pi \rho \mu} \int_{0}^{t} \frac{d}{\frac{d}{d t^{\prime}}(\mathbf{v}-\mathbf{u})} \sqrt{t-t^{\prime}} d t^{\prime} .
$$

In Maxey's formulation (Maxey and Riley 1983), a Faxen-type term is also added:

$$
\mathbf{F}_{H}=-\frac{3}{2} d_{p}^{2} \sqrt{\pi \rho \mu} \int_{0}^{t} \frac{\frac{d}{d t^{\prime}}\left(\mathbf{v}-\mathbf{u}-\frac{1}{24} d_{p}^{2} \nabla^{2} \mathbf{u}\right)}{\sqrt{t-t^{\prime}}} d t^{\prime} .
$$

By Eq. (7.132) or (7.133) the "historical" nature of this term is evident; the value of $\mathbf{F}_{H}$ is a function of the acceleration history up to the present time, and it decays as $t^{-1 / 2}$. Note that the history force, in contrast to the steady-state drag mentioned in Section 7.5.2, is essentially an unsteady drag force associated with the unsteady motion of the particle in a viscous medium. Also, a different but equivalent notion of this history integral suggests that it arises due to the temporal diffusion of vorticity around the particle surface with the decay rate proportional to $t^{-1 / 2}$ (Clift et al. 1978).

To make it clear, it is to reiterate that the unsteadiness of the relative velocity produces two main effects: a pure inertial (added-mass) effect and a viscous (history) 
effect. In the Stokes regime, the linearity of the Stokes equation makes it possible to identify unambiguously the added-mass and history force as two separate forces. At finite particle Reynolds numbers, however, the distinction between the two is unclear, because various mechanisms can act in a coupled manner (Magnaudet and Eames 2000); as such, this decomposition of the unsteady forces can only be viewed in an empirical fashion.

Examples showing significance of the history force are particle falling under gravity through still fluid from an initial state of rest, and particle suspended in a fluid oscillating uniformly at high frequency (Maxey and Riley 1983). In both cases, the history force is important owing to the strong unsteadiness of the relative velocities. For the latter example, (Maxey and Riley 1983) pointed out that the fluid oscillation limit the diffusion of vorticity around the sphere and confine it to a thin Stokes layer. Thus, effectively, the history force gives an augmented (total) drag force. Additional studies (Rizk and Elghobashi 1985; Thomas 1992; Armenio and Fiorotto 2001) also show that the unsteady history force can be many times larger than the quasi-steady drag.

One must realize that although the history term can be important in some situations, the evaluation of this term is often difficult. A novel way of computing this force has been proposed by (Michaelides 1992; Vojir and Michaelides 1994), which utilizes the Laplace transformation. On the other hand, the neglect of this term brings significant computational conveniences in the point-volume Lagrangian approach, as long as a sound justification exists.

In general, the history force becomes important when there are strong fluid accelerations at particle convective time scales on the order of $d_{p} / v_{r e l}$ (Loth 2000). In a turbulent flow, the acceleration can be characterized by the turbulent energy fluctuations $u$ ' at the $d_{p}$-wavelength. If

$$
u^{\prime} \ll v_{r e l},
$$

the unsteadiness is comparably small with respect to the particle relative velocity, and the history force can therefore be assumed to be negligible. In the case of $d_{p} / \Lambda<<1$, the criterion given in (7.134) typically holds. In the laminar flow, the history effects is likewise insignificant if $d_{p} / L<<1$, where $L$ is the characteristic length scale related to the flow geometry, e.g., the pipe diameter. It is important to note that, theoretically, the history force in the form of Eq. (7.132) or (7.133) is only valid at the creeping flow regime, i.e., $\operatorname{Re}_{p}<<1$. At Reynolds number greater than one, the actual history force is expected to be smaller than the result from Eq. (7.132) or (7.133). This point is discussed by (Mei et al. 1991), who used a resolved-volume approach and showed that the integration kernel behaves as $t^{-1 / 2}$ only for short times and decays at a much faster rate (as $t^{-2}$ ) at larger times and Reynolds numbers. For these reasons, the history force of a microscopic air bubbles is typically neglected. This is also experimentally confirmed by the study of (Sridhar and Katz 1995), in which Eq. (7.132) was used as an upper limit of the actual history force for a $707 \mu \mathrm{m}$ microbubble, and it was found that this force is $6 \%$ less than the buoyancy force.

Other criteria have also been proposed for different flow regimes. According to (Hjelmfelt and Mockros 1966, 1967), the history term is insignificant if $v / \omega d_{p}{ }^{2}>36$ for 
solid particles with $\gamma_{\rho} \sim 10^{-3}$ suspended in an oscillating flow. This is further approximately confirmed by (Vojir and Michaelides 1994), who showed negligible history effect if $\gamma_{\rho}>0.002$ and $\omega \tau_{p}<0.5$. For very buoyant particles of Stokes number near unity and high Froude numbers (e.g., relatively large bubbles in high convective flows), this term can be important (Mei and Klausner 1992; Loth 2000). But as long as time-averaged or integral quantities are of interest, it is again possible to neglect this term (Vojir and Michaelides 1994; Loth 1997; Armenio and Fiorotto 2001).

\subsubsection{Wall Effect}

Particle-wall interaction can be important for boundary layer flows and wall-bounded flows. It is well observed that in such flow configurations high concentrations of solid particles (Basset 1888b; Young and Hanratty 1991; Kaftori et al. 1995; Young and Leeming 1997) as well as gas bubbles (Zun et al. 1992; Liu and Bankoff 1993; Nakoryakov et al. 1996; Marie et al. 1997; Riviere et al. 1999; Felton and Loth 2001) can be produced very near the wall, i.e., on the order of particle diameter. The wall-peaking phenomenon is generally due to the modified continuous-fluid resistance in the proximity of the wall. However, its exact cause is not quite clear yet.

The effect of wall is commonly taken into account by either modifying the drag and lift coefficient in their respective force expressions, or introducing an additional wall force. For Stokesian flow (Clift et al. 1978) obtained an analytical correction for the drag factor under the assumption that the particle diameter is much smaller than the distance from the wall. As such, the drag factor should be considered a vector, which can be decomposed into components tangential and normal to the wall, respectively:

$$
\frac{1}{f_{\text {tang }}}=1-\frac{9}{32} f\left(\frac{d_{p}}{y}\right), \frac{1}{f_{\text {norm }}}=1-\frac{9}{16} f\left(\frac{d_{p}}{y}\right),
$$

where $f$ is the standard drag factor defined in Eq. (7.88), $f_{\text {tang }}$ and $f_{\text {norm }}$ are drag factor corrections in wall-parallel and wall-normal directions, respectively, $y$ represents the normal distance away from the wall. Thus, the drag coefficient, $C_{D}$, can be modified as

$$
C_{D, \text { tang }}=f_{\text {tang }} C_{D}, C_{D, \text { norm }}=f_{\text {norm }} C_{D} \text {. }
$$

While Clift's approximation assumes $d_{p}<<\mathrm{y}$, (Young and Hanratty 1991) proposed another drag factor correction for the situation when the particle position from the wall becomes on the order of the particle diameter. Their expression is based on a resolved-volume simulation under the Stokesian flow condition, and it reads

$$
f_{\text {tang }}=1+0.35\left(\frac{d_{p}}{y}\right), f_{\text {norm }}=1+1.1\left(\frac{2 y}{d_{p}}-1\right)^{-1} \text {. }
$$

Similarly, $C_{D}$ should be changed according to Eq. (7.136).

Instead of modifying the drag force, (Soo 1989) formulated a hydrodynamic wall interaction model in terms of a separate wall force, $\mathbf{F}_{W}$. The derivation is for solid 
spherical particles and it is based on the potential flow theory. Again, the wall force is decomposed into a tangential component and a normal component:

$$
\begin{aligned}
& F_{W, \text { tang }}=\left(\frac{1}{8}\right)^{3} \pi \rho \frac{d_{p}^{6}}{y^{4}}\left(y \frac{d v_{\text {tang }}}{d t}-3 v_{\text {tang }} v_{\text {norm }}\right), \\
& F_{W, \text { norm }}=\left(\frac{1}{8}\right)^{3} \pi \rho \frac{d_{p}^{6}}{y^{4}}\left(2 y \frac{d v_{\text {norm }}}{d t}-3 v_{\text {norm }}^{2}-1.5 v_{\text {tang }}^{2}\right) .
\end{aligned}
$$

For bubbles, (Tsao and Koch 1997) conducted an experiment on relatively large bubbles (with radii of $0.5-0.7 \mathrm{~mm}$ ) rising along an oblique wall at high $\operatorname{Re}_{p}$ (in the range of 45 - 200). Their results indicate that the drag coefficient in the bubble sliding direction is consistent with that of the contaminated formula given in Eq. (7.93) or (7.92), suggesting $f_{\text {tang }} \approx 1$. This is attributed to the thin lubrication film formed between the bubble and the wall, which appears to counterbalance the enhanced drag force due to the wall proximity (Loth 2000).

With regard to the wall correction for the shear lift force, limited information is available in the open literature. For small solid particles (Wang et al. 1997) formulated an "optimum" lift force, which compiles many previous results (mainly of (Saffman 1965, 1968; Cox and Hsu 1977; Vasseur and Cox 1977; McLaughlin 1991, 1993)). The application of the resulting formula (or formula collection) appears not to be very straightforward. Although no direct measurements of the wall influence on the lift force can be found, one do notice that there exist the lift reversal effect (where lift decrease and change its sign as the particle moves towards the wall), and the wall-peaking phenomena, which may be attributed to the modified lift force.

\subsubsection{Assemblies of Forces}

The forces described in the previous sections have different level of significance when applied to different particle types. Even for the same type of particle, different flavors exist in selecting significant forces and their respective expressions. In this subsection the classical assemblies of those forces are summarized. It is to stress that the superposition of different forces should be understood as a modeling approach with the assumption that the nonlinear interaction between different types of forces is insignificant.

In the very early stage of the work, (Boussinesq 1885) and (Basset 1888a) independently derived the equation of motion for a sphere moving in a stagnant fluid or a fluid of uniform velocity at the creeping flow condition. Their equation includes the steady-state drag, the added-mass force and the history integral:

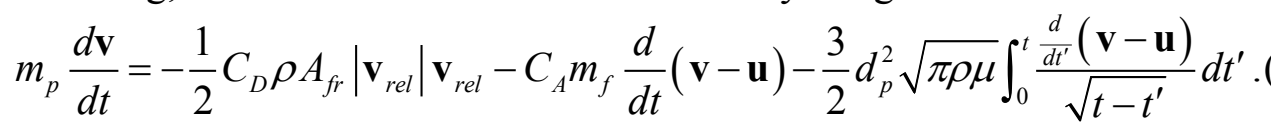

Substituting the Stokesian $C_{D}$ expression (Eq. (7.88)) and geometric information of a sphere, Eq. (7.139) becomes 


$$
m_{p} \frac{d \mathbf{v}}{d t}=-3 \pi d_{p} \mu(\mathbf{v}-\mathbf{u})-\frac{1}{2} m_{f} \frac{d}{d t}(\mathbf{v}-\mathbf{u})-\frac{3}{2} d_{p}^{2} \sqrt{\pi \rho \mu} \int_{0}^{t} \frac{d}{\frac{d t^{\prime}}{}(\mathbf{v}-\mathbf{u})} \sqrt{t-t^{\prime}} d t^{\prime}
$$

The well-known and widely used Basset-Boussinesq-Oseen (BBO) equation for solid particles or droplets is an extension of Eq. (7.140), in which the gravity and fluidstress force are added:

$$
\begin{aligned}
m_{p} \frac{d \mathbf{v}}{d t} & =m_{p} \mathbf{g}+m_{f}(-\nabla p+\nabla \cdot \boldsymbol{\tau}) \\
& -3 \pi d_{p} \mu(\mathbf{v}-\mathbf{u})-\frac{1}{2} m_{f} \frac{d}{d t}(\mathbf{v}-\mathbf{u})-\frac{3}{2} d_{p}^{2} \sqrt{\pi \rho \mu} \int_{0}^{t} \frac{\frac{d}{d t^{\prime}}(\mathbf{v}-\mathbf{u})}{\sqrt{t-t^{\prime}}} d t^{\prime}
\end{aligned}
$$

where $\tau$ is the viscous stress tensor of undisturbed flow. Note that here the buoyancy force is implicitly represented in the $-\nabla p$ term, as $p$ includes both dynamic and static pressure.

It is beneficial to know that the equation proposed by (Corrsin and Lumley 1956) is very similar to the $\mathrm{BBO}$ equation. However, in their formulation they only considered the pressure stress, and did not include the viscous fluid-stress effect. Their equation reads:

$$
\begin{aligned}
m_{p} \frac{d \mathbf{v}}{d t}= & \left(m_{p}-m_{f}\right) \mathbf{g}+m_{f}\left(\frac{D \mathbf{u}}{D t}-\nabla \cdot \boldsymbol{\tau}\right) \\
& -3 \pi d_{p} \mu(\mathbf{v}-\mathbf{u})-\frac{1}{2} m_{f} \frac{d}{d t}(\mathbf{v}-\mathbf{u})-\frac{3}{2} d_{p}^{2} \sqrt{\pi \rho \mu} \int_{0}^{t} \frac{\frac{d}{d t^{\prime}}(\mathbf{v}-\mathbf{u})}{\sqrt{t-t^{\prime}}} d t^{\prime}
\end{aligned}
$$

where

$$
\rho \frac{D \mathbf{u}}{D t}=-\nabla p+\nabla \cdot \boldsymbol{\tau}+\rho \mathbf{g}=-\nabla p_{d y n}+\nabla \cdot \boldsymbol{\tau} .
$$

It is noticed that in the above formulation the static pressure (buoyancy) is excluded from the pressure stress term, and combined with the particle gravity.

The BBO equation (7.141) and Eq. (7.142) underwent a landmark revision made by (Maxey and Riley 1983). They rigorously derived the equation of motion for small particles valid in the limits of $d_{p}<<\eta, d_{p}{ }^{2} / v<<\tau_{\eta}, \operatorname{Re}_{p}<<1$. The viscous fluid-stress force missing in Eq. (7.142) is corrected, and the steady-state drag, added-mass and history term in Eq. (7.141) are complemented with additional Faxen corrections:

$$
\begin{aligned}
m_{p} \frac{d \mathbf{v}}{d t}= & \left(m_{p}-m_{f}\right) \mathbf{g}+m_{f} \frac{D \mathbf{u}}{D t} \\
& -3 \pi d_{p} \mu\left(\mathbf{v}-\mathbf{u}-\frac{1}{24} d_{p}^{2} \nabla^{2} \mathbf{u}\right)-\frac{1}{2} m_{f} \frac{d}{d t}\left(\mathbf{v}-\mathbf{u}-\frac{1}{40} d_{p}^{2} \nabla^{2} \mathbf{u}\right) \\
& -\frac{3}{2} d_{p}^{2} \sqrt{\pi \rho \mu} \int_{0}^{t} \frac{\frac{d}{d t^{\prime}}\left(\mathbf{v}-\mathbf{u}-\frac{1}{36} d_{p}^{2} \nabla^{2} \mathbf{u}\right)}{\sqrt{t-t^{\prime}}} d t^{\prime} .
\end{aligned}
$$

Again, in (Maxey and Riley 1983)'s expression, the buoyancy force is represented in the gravity term. Notice that the Faxen corrections associated with the added-mass, the history and drag force are on the order of $d_{p}^{5}, d_{p}{ }^{4}$ and $d_{p}^{3}$, respectively. Although the 
correction to the drag term is $O\left(d_{p}{ }^{3}\right)$, it is $O\left(d_{p}^{2} / 8\right)$ compared to the rest of the drag term. Therefore, in practical use, these corrections are often neglected. Thus one writes

$$
\begin{aligned}
m_{p} \frac{d \mathbf{v}}{d t}= & \left(m_{p}-m_{f}\right) \mathbf{g}+m_{f} \frac{D \mathbf{u}}{D t} \\
& -3 \pi d_{p} \mu(\mathbf{v}-\mathbf{u})-\frac{1}{2} m_{f} \frac{d}{d t}(\mathbf{v}-\mathbf{u})-\frac{3}{2} d_{p}^{2} \sqrt{\pi \rho \mu} \int_{0}^{t} \frac{\frac{d}{d t^{\prime}}(\mathbf{v}-\mathbf{u})}{\sqrt{t-t^{\prime}}} d t^{\prime} .
\end{aligned}
$$

It is not difficult to see that Eq. (7.145) and the BBO equation are identical.

For light particles, such as air bubbles, the most significant forces are buoyancy, drag, lift, and added-mass forces. It is always a good idea to also include the fluid-stress force and history force. Although gravity force is negligibly small in this case, it will not hurt to put it together with the buoyancy. Note that the added-mass must be included for light particles because otherwise the drag is balanced only by buoyancy, and as a result, the calculated particle relaxation time will not be accurate. Given these, the equation of motion for light particles can be expressed as follows, where the lift force is adopted from the Auton's shear-induced analytical lift, i.e., Eq. (7.110):

$$
\begin{aligned}
& m_{p} \frac{d \mathbf{v}}{d t}=\left(m_{p}-m_{f}\right) \mathbf{g}+m_{f} \frac{D \mathbf{u}}{D t} \\
& -\frac{1}{2} C_{D} \rho A_{f r}|\mathbf{v}-\mathbf{u}|(\mathbf{v}-\mathbf{u})-C_{L} m_{p}(\mathbf{v}-\mathbf{u}) \times \boldsymbol{\omega} \\
& -C_{A} m_{f} \frac{d}{d t}(\mathbf{v}-\mathbf{u})-\frac{3}{2} d_{p}^{2} \sqrt{\pi \rho \mu} \int_{0}^{t} \frac{\frac{d}{d t^{\prime}}(\mathbf{v}-\mathbf{u})}{\sqrt{t-t^{\prime}}} d t^{\prime} .
\end{aligned}
$$

One may further split the added-mass term and combine the resulting two terms into the particle acceleration term on the LHS and the fluid stress term on the RHS, respectively. If the history force is neglected, the following equation is obtained

$$
\begin{aligned}
\left(m_{p}+C_{A} m_{f}\right) \frac{d \mathbf{v}}{d t} & =\left(m_{p}-m_{f}\right) \mathbf{g}+\left(m_{f}+C_{A} m_{f}\right) \frac{D \mathbf{u}}{D t} \\
& -\frac{1}{2} C_{D} \rho A_{f r}\left|\mathbf{v}_{r e l}\right| \mathbf{v}_{r e l}-C_{L} m_{p} \mathbf{v}_{r e l} \times \boldsymbol{\omega} .
\end{aligned}
$$

Dividing both sides by $\left(m_{p}+C_{A} m_{f}\right)$ and assuming a spherical bubble yields the reduced form of the bubble equation of motion:

$$
\frac{d \mathbf{v}}{d t}=-\frac{1-\gamma_{\rho}}{\gamma_{\rho}+C_{A}} \mathbf{g}+\frac{1+C_{A}}{\gamma_{\rho}+C_{A}} \frac{D \mathbf{u}}{D t}-\frac{3}{4} \frac{C_{D}}{d_{p}\left(\gamma_{\rho}+C_{A}\right)}\left|\mathbf{v}_{r e l}\right| \mathbf{v}_{r e l}-\frac{C_{L}}{\gamma_{\rho}+C_{A}} \mathbf{v}_{r e l} \times \boldsymbol{\omega},(7
$$

where $\gamma_{\rho}=\rho_{p} / \rho$ is the density ratio. For an air bubble in liquid $\gamma_{\rho}$ is negligibly small. Further taking $C_{A}$ to be 0.5 for a sphere one arrives at a simpler expression:

$$
\frac{d \mathbf{v}}{d t}=-2 \mathbf{g}+3 \frac{D \mathbf{u}}{D t}-\frac{3}{2 d_{p}} C_{D}\left|\mathbf{v}_{r e l}\right| \mathbf{v}_{r e l}-2 C_{L} \mathbf{v}_{r e l} \times \boldsymbol{\omega} .
$$

Eq. (7.149) is used, for example, in the study of (Magaud et al. 2003).

(Sridhar and Katz 1995) also summarized the forces for microscopic bubbles but with the lift force taking the form of Eq. (7.111): 


$$
\begin{aligned}
m_{p} \frac{d \mathbf{v}}{d t}= & \left(m_{p}-m_{f}\right) \mathbf{g}+m_{f} \frac{D \mathbf{u}}{D t} \\
& -\frac{1}{2} C_{D} \rho A_{f r}\left|\mathbf{v}_{r e l}\right| \mathbf{v}_{r e l}-\frac{1}{2} C_{L} \rho A_{f r}\left|\mathbf{v}_{r e l}\right| \mathbf{v}_{r e l} \times \frac{\boldsymbol{\omega}}{|\boldsymbol{\omega}|}-C_{A} m_{f} \frac{d}{d t}(\mathbf{v}-\mathbf{u}) .
\end{aligned}
$$

In this situation the reduced form of bubble equation gives

$$
\begin{aligned}
\frac{d \mathbf{v}}{d t} & =\frac{\gamma_{\rho}-1}{\gamma_{\rho}+C_{A}} \mathbf{g}+\frac{1+C_{A}}{\gamma_{\rho}+C_{A}} \frac{D \mathbf{u}}{D t} \\
& -\frac{3}{4} \frac{C_{D}}{d_{p}\left(\gamma_{\rho}+C_{A}\right)}\left|\mathbf{v}_{r e l}\right| \mathbf{v}_{r e l}-\frac{3}{4} \frac{C_{L}}{d_{p}\left(\gamma_{\rho}+C_{A}\right)}\left|\mathbf{v}_{r e l}\right| \mathbf{v}_{r e l} \times \frac{\mathbf{\omega}}{|\boldsymbol{\omega}|},
\end{aligned}
$$

or equivalently,

$$
\frac{d \mathbf{v}}{d t}=-2 \mathbf{g}+3 \frac{D \mathbf{u}}{D t}-\frac{3}{2 d_{p}} C_{D}\left|\mathbf{v}_{r e l}\right| \mathbf{v}_{r e l}-\frac{3}{2 d_{p}} C_{L}\left|\mathbf{v}_{r e l}\right| \mathbf{v}_{r e l} \times \frac{\boldsymbol{\omega}}{|\boldsymbol{\omega}|} .
$$

Note that Eq. (7.152) is the same as (7.149) except for the lift force expression. Also, $C_{L}$ $=0.5$ if the fluid is inviscid.

In the framework of the point-volume approach, the particle equations of motion presented above can serve as reasonably accurate model. Their respective applicability mainly depends on the type of particle and flow configurations. It should be emphasized that most of the force terms in those equations are derived at low $\mathrm{Re}_{p}$ limit, and the particle length and time scales must be smaller than the characteristic scales of the flow. In a turbulent flow, the latter condition asks $d_{p}<\eta$ and $d_{p}{ }^{2} / v<<\tau_{\eta}$. When this restriction is not satisfied, these equations can however still serve as an approximate model, but caution must be taken when interpreting the simulation results.

\subsubsection{Bubble Terminal Rise Velocity and Relaxation Time}

A straightforward application of the bubble equation is the computation of the terminal rise velocity of the bubble, $v_{\infty}$. For steady motion of a bubble in a quiescent fluid, all the unsteady forces as well as the lift force will be canceled out, only the buoyancy and steady-state drag force remains, which must be in balance, i.e.,

$$
0=2 g-\frac{3}{2 d_{p}} C_{D} v_{\infty}^{2}
$$

where $g$ is the gravitational constant. By assuming Stokes drag coefficient the following estimate for the terminal rise velocity is obtained:

$$
v_{\infty}=\frac{g d_{p}^{2}}{18 v} .
$$

Notice that this result is identical to Eq. (6.35) derived in the buoyant settling section (Section 6.3). For a handy reference, which can be useful in this study, the terminal velocity and the terminal Reynolds number of an air bubble in water are plotted with respect to bubble diameter in Figure 7-4 (a) and (b). For representative bubble diameters, the corresponding terminal values are also listed in Table 7-3. Note that the calculated values are based on $v=1 . \mathrm{E}-6 \mathrm{~m}^{2} / \mathrm{s}$ for water and $\rho_{p}=1.2 \mathrm{~kg} / \mathrm{m}^{3}$ for air bubble. 


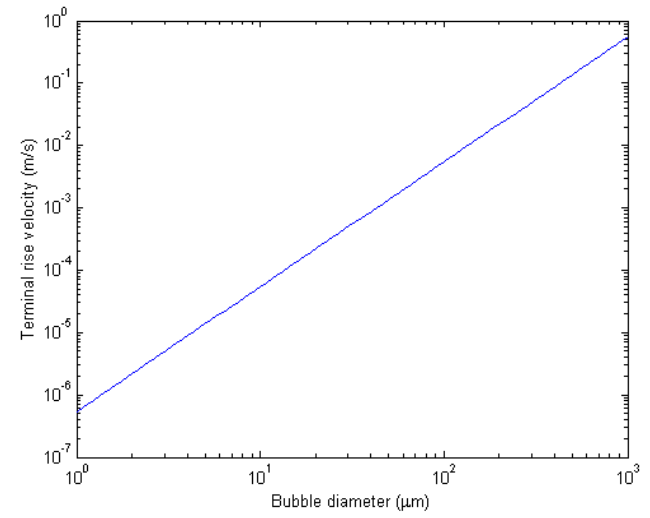

(a)

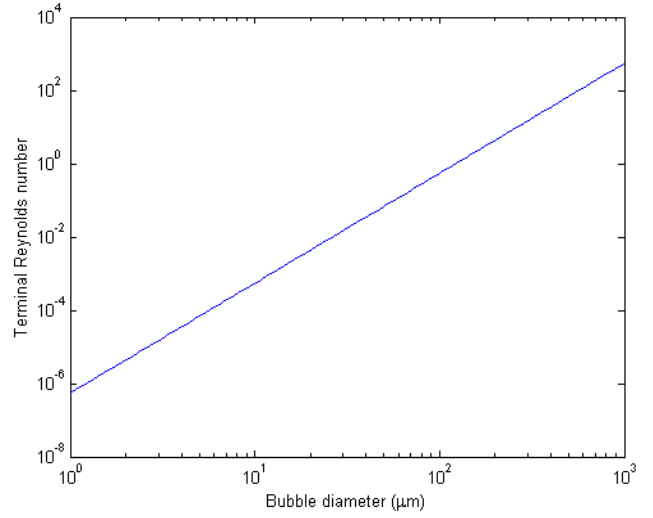

(b)

Figure 7-4 Bubble terminal rise velocities and terminal Reynolds numbers

Table 7-3 Bubble terminal rise velocities, terminal Reynolds numbers and relaxation time

\begin{tabular}{|c|c|c|c|}
\hline $\begin{array}{c}\text { Particle } \\
\text { diameter }\end{array}$ & $\begin{array}{c}\text { Terminal rise } \\
\text { velocity }(\mathrm{m} / \mathrm{s})\end{array}$ & $\begin{array}{c}\text { Terminal Reynolds } \\
\text { number }\end{array}$ & Relaxation time (s) \\
\hline $1 \mu \mathrm{m}$ & $5.450 \mathrm{E}-7$ & $5.450 \mathrm{E}-7$ & $2.784 \mathrm{E}-8$ \\
\hline $10 \mu \mathrm{m}$ & $5.450 \mathrm{E}-5$ & $5.450 \mathrm{E}-4$ & $2.784 \mathrm{E}-6$ \\
\hline $100 \mu \mathrm{m}$ & $5.450 \mathrm{E}-3$ & $5.450 \mathrm{E}-1$ & $2.784 \mathrm{E}-4$ \\
\hline $500 \mu \mathrm{m}$ & $1.363 \mathrm{E}-1$ & $6.813 \mathrm{E}-1$ & $6.961 \mathrm{E}-3$ \\
\hline $1 \mathrm{~mm}$ & $5.450 \mathrm{E}-1$ & $5.450 \mathrm{E} 2$ & $2.784 \mathrm{E}-2$ \\
\hline $2 \mathrm{~mm}$ & 2.180 & $4.360 \mathrm{E} 3$ & $1.114 \mathrm{E}-1$ \\
\hline
\end{tabular}

Another application is the calculation of effective bubble relaxation time. The relaxation time defined by

$$
\tau_{p}=\frac{\rho_{p} d_{p}^{2}}{18 \mu}
$$

(also see Eq. (6.18) or (7.3)) is derived only based on the particle response to the drag force. Therefore, this definition is usually used for solid particles or droplets in a $\gamma_{\rho}>>1$ flow, where the drag is the dominant force. For bubbles in liquid, the buoyancy and added-mass force are important. As such, an estimate of the effective bubble relaxation time can be obtained from the following simplified bubble equation:

$$
\left(m_{p}+C_{A} m_{f}\right) \frac{d v_{p}}{d t}=-\left(m_{p}-m_{f}\right) g-3 \pi d_{p} \mu v_{p}
$$

where the Stokes drag is again assumed. Thus, it is easily seen

$$
\tau_{p}=\frac{m_{p}+C_{A} m_{f}}{3 \pi d_{p} \mu}=\frac{d_{p}^{2}\left(\rho_{p}+C_{A} \rho\right)}{18 \mu}=\frac{\rho_{p} d_{p}^{2}\left(1+C_{A} / \gamma_{\rho}\right)}{18 \mu} .
$$


In fact, Eq. (7.157) is a generalization of Eq. (7.155), and this point has already been mentioned in Eq. (7.4). For convenience, the bubble relaxation times calculated from Eq. (7.3) are also included Table 7-3.

\subsection{Two-way Coupling}

This section concerns two-way coupling formulations for a two-phase flow system. The two-way coupling involves the forward coupling from fluid to particles, and the backward coupling from particles to fluid. It may take different modeling formalism, depending on the simulation approach, i.e., Eulerian-Eulerian (E-E) or EulerianLagrangian (E-L), and the level of description of the continuous phase, i.e., DNS, LES or RANS.

For a "true" direct numerical simulation (DNS) of two-phase flow, where the phase interfaces are resolved and tracked, the two-way interaction is automatically taken care of in its solution; no two-way model needs to be introduced. Often times, DNS is used for the solution of the continuous carrier phase only, while the discrete phase is computed relying on some simplified approach, typically the point-volume approach. In order not to cause confusion, such a single-phase DNS can be referred to as quasi DNS (a term invented by the present author) in the context of multiphase simulation. In a quasi DNS the two-way coupling effect needs to be addressed.

\subsubsection{A Simplified Two-way Model from Two-fluid Approach}

Under the class of Eulerian-Eulerian (E-E) method the mixed fluid approach and the twofluid approach have been discussed in previous sections (cf. Section 7.4.1 and 7.4.2). Two-way coupling in the mixed-fluid approach is achieved via void fraction and possibly a slip term in the mixture momentum equation (see Eq. (7.66)). In the two-fluid approach volume-averaged mass and momentum equations are obtained for each phase. Phases are coupled not only through the void fraction, but also the interaction integrals as a result of the volume averaging operation in the respective mass and momentum equations (see Eq. (7.60) and (7.61)).

In this subsection the aim is to understand the analysis of a simplified two-way coupling model discussed in (Rightley 1995; Rightley and Lasheras 2000). Though the model is derived from a two-fluid formulation for buoyant particles (air bubbles) in a dilute system, it does provide useful insight that would benefit later discussions on the two-way model in the E-L approach (to be presented in Section 7.6.3). In what follows, effort is made to recap their analysis, but with some proper rearrangements, modifications and supplements.

Here, one starts with the volume-averaged equations of two immiscible fluids given by Eq. (7.62) through (7.64). In the dilute limit, the void fraction of the continuous phase approach unity, the equation set for the continuous phase thus can be simplified as 


$$
\begin{gathered}
\nabla \cdot\langle\mathbf{u}\rangle=0, \\
\rho \frac{D\langle\mathbf{u}\rangle}{D t}=\nabla \cdot\langle\mathbf{T}\rangle+\rho \mathbf{g}+\mathcal{I},
\end{gathered}
$$

where

$$
\begin{gathered}
\mathbf{T}=-p \mathbf{I}+\mu\left(\nabla \mathbf{u}+\nabla \mathbf{u}^{T}\right), \\
\mathcal{I}=\frac{1}{V} \int_{S} \mathbf{T} \cdot \mathbf{n} d S .
\end{gathered}
$$

Note that the operator \langle\rangle denotes a volume-averaged quantity, and $\mathcal{I}$ is the interphase exchange term. For a dilute system, the influence of the dispersed phase on the continuous phase can be assumed relatively weak. Thus, the ambient carrier phase flow field in the region near an individual particle is governed by

$$
\rho \frac{D \mathbf{u}}{D t}=\nabla \cdot \mathbf{T}+\rho \mathbf{g},
$$

or expressed on the mesoscale as

$$
\rho \frac{D\langle\mathbf{u}\rangle}{D t}=\nabla \cdot\langle\mathbf{T}\rangle+\rho \mathbf{g} .
$$

This is known as the weak two-way coupling, meaning the coupling is important only at those locations where the particles reside.

Recall that (Section 7.5) the forces acting on an individual mircoparticle $\left(d_{p}<<\eta\right)$ can be decomposed into forces due to the undisturbed flow $\mathbf{F}_{p}{ }^{(0)}$, forces due to the disturbed flow $\mathbf{F}_{p}{ }^{(1)}$, and the particle gravity:

$$
m_{p} \frac{d \mathbf{v}}{d t}=\mathbf{F}_{p}=\mathbf{F}_{p}^{(0)}+\mathbf{F}_{p}^{(1)}+m_{p} \mathbf{g} .
$$

Further recall that the buoyancy force and the fluid stress force are in $\mathbf{F}_{p}{ }^{(0)}$, while the other forces except the gravity belong to $\mathbf{F}_{p}^{(1)}$. Taking the bubble equation (7.146) as an example, its RHS forces may be decomposed as follows:

$$
\begin{gathered}
\mathbf{F}_{p}^{(0)}=-m_{f} \mathbf{g}+m_{f} \frac{D \mathbf{u}}{D t}, \\
\mathbf{F}_{p}^{(1)}=-3 \pi d_{p} \mu(\mathbf{v}-\mathbf{u})-\frac{1}{2} m_{p}(\mathbf{v}-\mathbf{u}) \times \mathbf{\omega} \\
\left.-\frac{1}{2} m_{f} \frac{d}{d t}(\mathbf{v}-\mathbf{u})-\frac{3}{2} d_{p}^{2} \sqrt{\pi \rho \mu} \int_{0}^{t} \frac{d t^{\prime}}{\sqrt{t-t^{\prime}}} d t^{\prime} . \mathbf{u}\right)
\end{gathered}
$$

Some general remarks should be made at this point: (i) Since the derivation of these forces is based on the microscale, so the decomposed ones; (ii) The forces resulting from the disturbed flow are due to the relative motion between the particle and the carrier fluid. (iii) As explained in Section 7.5, $\mathbf{F}_{p}^{(0)}$ is in fact the fluid stresses that would act upon the spherical inclusion of a fluid element in place of the particle. Therefore, $\mathbf{F}_{p}{ }^{(0)}$ simplify refers to the $\mathbf{T}$ term in Eq. (7.162), or $\langle\mathbf{T}\rangle$ in Eq. (7.163) on the mesoscale, suggesting that this force has already been included in the standard momentum equation of the carrier phase. A direction implication is then that, in the weak two-way coupling limit, the balance equation of the carrier phase at the location of a particle must include an 
additional term which counterbalances the forces arising from the disturbed flow, i.e., the $\mathbf{F}_{p}{ }^{(1)}$. As a result, the momentum equation for the carrier phase under the influence of an individual particle can be written on the microscale as

$$
\rho \frac{D \mathbf{u}}{D t}=\nabla \cdot \mathbf{T}+\rho \mathbf{g}-\mathbf{F}_{p}^{(1)}\left(\mathbf{y}^{n}\right) \delta\left(\mathbf{x}-\mathbf{y}^{n}\right),
$$

where $\delta$ is the three-dimensional Dirac delta function that isolates $-\mathbf{F}_{p}{ }^{(1)}$ at the particle location $\mathbf{y}^{n}$. Note that the delta function has the unit of reciprocal volume (reciprocal length cubed), so that the resultant unit of the coupling term is $\mathrm{N} / \mathrm{m}^{3}$. The consistent mesoscale-averaged form of the momentum transfer integral comes from averaging over all the particles in the mesoscale volume $V$,

$$
\mathcal{I}=\frac{1}{V} \int_{V} \sum_{n=1}^{N} \mathbf{F}\left(\mathbf{y}^{n}\right) \delta\left(\mathbf{x}-\mathbf{y}^{n}\right) d \mathbf{x},
$$

where $\mathbf{y}^{n}$ is the location of the $n$th particle, $\mathbf{y}^{n} \in V ; N$ is the number of particles within $V$, and $\mathbf{F}\left(\mathbf{y}^{n}\right)$ is the reacting force exerted by the $n$th particle on the fluid, i.e.,

$$
\mathbf{F}\left(\mathbf{y}^{n}\right)=-\mathbf{F}_{p}^{(1)}\left(\mathbf{y}^{n}\right) .
$$

Eq. (7.168) can be further reduced using the properties of the delta function:

$$
\begin{aligned}
\mathcal{I} & =\frac{1}{V} \sum_{n=1}^{N} \int_{V} \mathbf{F}\left(\mathbf{y}^{n}\right) \delta\left(\mathbf{x}-\mathbf{y}^{n}\right) d \mathbf{x} \\
& =\frac{1}{V} \sum_{n=1}^{N} \int_{V} \mathbf{F}(\mathbf{x}) \delta\left(\mathbf{x}-\mathbf{y}^{n}\right) d \mathbf{x} \\
& =\frac{1}{V} \sum_{n=1}^{N} \mathbf{F}\left(\mathbf{y}^{n}\right) .
\end{aligned}
$$

This yields the following instantaneous volume-averaged momentum equation for the carrier phase valid in the entire domain and including the influence of all particles in the domain:

$$
\rho \frac{D\langle\mathbf{u}\rangle}{D t}=\nabla \cdot\langle\mathbf{T}\rangle+\rho \mathbf{g}+\mathcal{I}(\mathbf{x}, t)
$$

with

$$
\mathcal{I}(\mathbf{x}, t)=\frac{1}{V} \sum_{n=1}^{N(\mathbf{x}, t)} \mathbf{F}\left(\mathbf{y}^{n}, t\right)=-\frac{1}{V} \sum_{n=1}^{N(\mathbf{x}, t)} \mathbf{F}_{p}^{(1)}\left(\mathbf{y}^{n}, t\right) .
$$

It is important to note that arriving at Eq. (7.172) is based on a series of assumptions by taking advantage of the unique configuration of a dilute, dispersed system of microparticles.

For the case of massless particles, such as air bubbles, the particle inertial is negligible and Eq. (7.164) becomes

$$
0=\mathbf{F}_{p}^{(0)}+\mathbf{F}_{p}^{(1)} \text { or } \mathbf{F}_{p}^{(1)}=-\mathbf{F}_{p}^{(0)} .
$$

By substituting Eq. (7.173) into (7.172) and noticing that 


$$
\begin{aligned}
\frac{1}{V} \sum_{i=1}^{N} \mathbf{F}_{p}^{(0)} & =\frac{1}{V} \sum_{i=1}^{N}\left(m_{f} \frac{D \mathbf{u}}{D t}-m_{f} \mathbf{g}\right) \cong \frac{1}{V} \sum_{i=1}^{N} m_{f}\left(\frac{D\langle\mathbf{u}\rangle}{D t}-\mathbf{g}\right) \\
& =\alpha_{d} \rho\left(\frac{D\langle\mathbf{u}\rangle}{D t}-\mathbf{g}\right),
\end{aligned}
$$

one arrives at a simplified two-way coupling model for a dilute bubbly flow:

$$
\rho \frac{D\langle\mathbf{u}\rangle}{D t}=\nabla \cdot\langle\mathbf{T}\rangle+\rho \mathbf{g}+\alpha_{d} \rho\left(\frac{D \mathbf{u}}{D t}-\mathbf{g}\right),
$$

where $\alpha_{d}$ is the void fraction of the dispersed phase, and $\rho$ is the density of the continuous phase ( $\rho$ and $\rho_{c}$ are used interchangeably in this text).

As noted by (Rightley 1995; Rightley and Lasheras 2000), this result (Eq (7.175)) for a dilute bubbly flow is similar to that given by (Maxey et al. 1994), and it also resembles, with the exception of the fluid stress term $\mathrm{Du} / \mathrm{D} t$, that derived by (Reutsch and Meiburg 1994), in which only the buoyancy coupling is considered. Notably, by omitting the fluid stress term in the coupling expression, it reminds us of a buoyancy-driven fluid of variable density under the Boussinesq approximation. The Boussinesq approximation (Turner 1973) assumes that density variations are small enough so that the density appears as a constant in all terms except the buoyancy term. However, the density (or temperature) field in a buoyancy-driven flow is determined by a scalar transport equation, whereas for the two-phase bubbly flow the mixture density $\rho_{m}$ is a function of the void fraction, as

$$
\rho_{m}=\alpha_{c} \rho_{c}+\alpha_{d} \rho_{d} \cong \alpha_{c} \rho_{c}=\left(1-\alpha_{d}\right) \rho_{c} .
$$

\subsubsection{Forward Coupling in E-L Approach}

Next, consider the two-way coupling in the framework of Eulerian-Lagrangian (E-L) approach where the discrete phase is calculated using the point-volume assumption. In that, the forward effect of fluid on particle and the effect in the reverse direction must be addressed separately. The forward coupling is considered in this present section, while the backward coupling will be addressed in the next subsection. Again, the dispersed system considered here (and in this study) is dilute, meaning that the particle concentrations are dilute enough for the particle-particle interaction to be neglected, but, if the turbulence modulation of the carrier phase is of interest, also large enough for cumulative effects of particles to influence the carrier flow. Also note that, if only the forward coupling is considered without the account of the backward influence, the problem then reduces to a one-way coupling problem.

With respect to the forward coupling from the carrier phase on the dispersed phase, it is simply realized through the equation of motion of the point mass (Eq. (7.71) and (7.72)). The models for the forces, $\mathbf{F}_{p}$, have been reviewed in Section 7.5. In general, the RHS of Eq. (7.72) is a function of particle position, velocity and local properties of the continuous phase, such as fluid velocity, stress and vorticity. If the continuous phase is computed using single-phase DNS (or quasi DNS), the fluid properties made available 
by the DNS solution are instantaneous and can be directly used in the evaluation of RHS of Eq. (7.72). This has been the standard approach in the study of many one-way coupled problems. Some representative one-way coupled DNS have been performed for isotropic, homogeneous turbulence (Squires and Eaton 1991b, a; Elghobashi and Truesdell 1992; Wang and Maxey 1993), turbulent channel flows (McLaughlin 1989; Pdeinotti et al. 1993), and plane mixing layer (Crowe et al. 1977; Crowe et al. 1985).

However, if LES or RANS is used for the primary phase calculation, the solved continuous field, available to the particle calculation, is either filtered or time-averaged. As such, in order to accurately describe the particle dispersion or diffusion induced by the background turbulent flow, a model may have to be introduced, which accounts for the subgrid scale fluctuations (in LES), or the instantaneous fluctuations (in RANS). Three common approaches are: (i) adding a modeled fluctuation, u', to the continuous velocity field, $\mathbf{u}$, before using it in Eq. (7.72), i.e.,

$$
\mathbf{u}^{*}=\mathbf{u}+\mathbf{u}^{\prime}
$$

where $\mathbf{u}^{*}$ is to be used for particle integration; (ii) adding a modeled fluctuation directly to the computed particle velocity or particle trajectory, i.e.,

$$
\mathbf{v}=\mathbf{v}+\mathbf{v}^{\prime} \text { or } \mathbf{y}=\mathbf{y}+\mathbf{y}^{\prime}
$$

(iii) adding a modeled force, $\mathbf{F}_{p, f l u c}$, to the RHS of Eq. (7.72), representing particle's random motion due to unresolved flow fluctuations:

$$
m_{p} \frac{d \mathbf{v}}{d t}=\mathbf{F}_{p}+\mathbf{F}_{p, f l u c} .
$$

Note that Eq. (7.179) is similar in form to the Langevin equation.

Obtaining those unknown fluctuations usually falls into the general category of stochastic modeling. See (Crowe et al. 1996; Crowe et al. 1998) for a general review. Three models in category (i) are worth mentioning. First, the eddy lifetime model selects u' from a Gaussian distribution with a variance proportional to the turbulence energy, and assumes the fluid velocity $\mathbf{u}^{*}$ encountered by a particle is constant during the eddy lift time. The model is originally proposed by (Yuu et al. 1978) and later improved by (Gosman and Ioannides 1981) and many others working along this line. Second, a random flow generation (RFG) technique developed by (Smirnov et al. 2002) based on the idea of (Kraichnan 1970) may be used to produce a random velocity fluctuation field which satisfies continuity. A principle drawback in RFG is that the generated random field cannot reflect local turbulence features, such as TKE, which is crucial in properly driving the particle's local randomness. Third, (Wang and Squires 1996a) modeled their SGS fluctuations by solving a transport equation for the SGS kinetic energy. Examples in category (ii) are (Dukowicz 1980; Smith et al. 1981), and an example in category (iii) is (Fukagata et al. 1997).

Any additional modeling will introduce extra inaccuracy and uncertainty. It is the author's belief that an empirical model should be employed when it has to be and should be avoided whenever the situation allows. In a RANS, modeling the particle fluctuating velocity is a must, because the mean flow field provided by the RANS is generally too "numb" to reproduce the physical behavior of particle's random motion. However, when LES is used for the continuous phase calculation, the effect of SGS fluctuations on the 
particle random motion could be insignificant (Armenio et al. 1999). They found from a systematic investigation for particles with and without inertia that, in a statistical sense, the particle dynamics is mainly governed by large-scale, energy-carrying fluid motion, and is not very sensitive to small-scale velocity field. Further, when the filter width is small, the particle dispersion statistics obtained with the filtered field from DNS data and with the DNS field are very close, with the maximum difference being less than $8 \%$. In a wall-resolving LES, using the filtered velocity field to advance the particles in time yields satisfactory results. In particular, when the dynamic model is employed, the modeling errors do not affect the particle statistics as mush as the filtering itself. Nevertheless, inaccuracy can occur when a significant percentage of energy is removed from the velocity field, for example, due to insufficient resolution of the LES in the wall layer. (Armenio et al. 1999)'s general conclusion is that a "careful" (in terms of grid resolution and SGS model) LES can provide fairly accurate particle statistics. The findings of (Armenio et al. 1999) are also partially supported by some earlier studies of (Yeh and Lei 1991; Elghobashi and Truesdell 1992; Wang and Squires 1996b; Yang and Lei 1998) with a focus on finite-inertia particles. For example, (Elghobashi and Truesdell 1992)'s DNS results suggest that the inertia makes the particle less sensitive to the small-scale fluctuating velocity field. (Wang and Squires 1996b) shows negligible effect of SGS fluctuations on the dispersion and deposition in a particle-laden turbulent channel. (Yang and Lei 1998) investigated particle's settling velocity in homogeneous isotropic turbulence, they conclude that the large, energy-carrying scales are the controlling parameter of the settling velocity. Based on the above considerations, the present study chooses not to introduce a SGS fluctuation model for the particle trajectory calculation. However, when the particle dynamics in the wall region is of importance, special treatment would be necessary; this can be achieved, for example, through the modeled wall force detailed in Section 7.5.7.

\subsubsection{Backward Coupling in E-L Approach: Force Coupling}

In general, the influence of the presence of the particles on the fluid motion has not yet been fully understood. For example, in a DNS study of bubble columns (Esmaeeli and Tryggvason 1998, 1999) it is found that the velocity fluctuation of the carrier phase may increase with the number of bubbles, and eventually produce a wavelength smaller than the bubble diameter. There also exist extensive discussions on the role of the solid particles in modulating the carrier phase turbulence. It appears that the addition of particles may either increase or decrease the turbulent kinetic energy of the continuous phase. Although some observations suggest "large" particles enhance turbulence, whereas "small" particles suppress turbulence (Gore and Crowe 1989; Hetsroni 1989), the particle size may not be the only cause (Pan and Banerjee 1996).

As mentioned earlier, the ideal approach to account for the reverse coupling would be the volume-resolving DNS, which resolves fully the disturbance flow generated by each particle, such as the wall boundary layer and the wake around the particle surface. However, such an approach is rarely feasible in most practical applications where the particle dimensions are below the resolution scale of the unladen turbulent flow calculation, or, the number of particles to be tracked is large. The approximate 
point-volume Lagrangian approach has found its wide acceptance in calculating the particulate phase. Based on the information gained from the point-volume Lagrangian tracking, simplified representations must also be introduced to approximate the reverse influences. This becomes the central task in this subsection. In particular, the investigation of the reverse coupling in a large-eddy simulation for the continuous phase is one of the core parts of the entire study.

An important feature to be reproduced by a reverse coupling model is the cumulative effects of particles on the carrier phase flow, and further on the motion of individual particles. When the volumetric fraction of the particle phase is negligible, and the length $\left(d_{p}\right)$ and time $\left(d_{p}{ }^{2} / v\right)$ scales associated with the particle are smaller than or comparable to the Kolmogorov scales, one can represent the feedback mechanism of small particles on the flow by a set of point forces acting on the flow, each being the reaction force against the force of fluid on the particle. These point forces together form an interphase momentum exchange term in the Navier-Stokes equations of the carrier phase,

$$
\begin{gathered}
\nabla \cdot \mathbf{u}=0, \\
\rho \frac{D \mathbf{u}}{D t}=-\nabla p+\nabla \cdot(\mu \nabla \mathbf{u})+\rho \mathbf{g}+\mathcal{I},
\end{gathered}
$$

where the force coupling term $\mathcal{I}$ can be expressed as

$$
\mathcal{I}=\sum_{n=1}^{N} \mathbf{F}^{n} \delta\left(\mathbf{x}-\mathbf{y}^{n}\right),
$$

$\mathbf{F}^{n}$ being the resultant point force exerted by the $n$th particle on the fluid at the particle location $\mathbf{y}^{n}$, i.e., $\mathbf{F}^{n} \equiv \mathbf{F}\left(\mathbf{y}^{n}\right), N$ the total number of particles in the domain, and $\delta$ the threedimensional Dirac delta function, satisfying

$$
\int_{R^{3}} \delta(\mathbf{x}) d \mathbf{x}=1 .
$$

Notice that the continuity equation is same as that of a single phase, as the direct effect of the particles presence on the continuity can be neglected in a dilute system. This form of coupling has already been seen in Eq. (7.167) in Section 7.6.1, and it has a sound theoretical support, so long as the required assumptions are met. In fact, this formalism has served as a fundamental assumption and starting point in deriving most coupling models in the two-fluid E-E approach, as well as in the E-L category. Work in the latter regard includes the classical non-volume-resolving DNS (Squires and Eaton 1990; Elghobashi and Truesdell 1993; Reutsch and Meiburg 1994; Maxey et al. 1997; Boivin et al. 1998; Sundaram and Collins 1999), some explorative LES (Wang and Squires 1996b; Boivin et al. 2000; Fukagata 2000; Yamamoto et al. 2001; Milelli 2002; Apte et al. 2003b), and RANS or "laminar" two-phase flow simulations (Becker et al. 1994; Celik and Wang 1994; Hoomans et al. 1995; Delnoij et al. 1997a; Delnoij et al. 1997b; Kuo et al. 1997; Sokolichin et al. 1997). This present section primarily concerns the point-force based coupling used in (quasi) DNS, i.e., the N-S equations for the carrier phase is solved in its original form. The backward coupling is LES will be addressed in Section 7.8 and 7.9 . 
In the interest of computation, the exact point-force coupling represented by Eq. (7.182) is neither possible, nor desirable. Variants based on Eq. (7.182) exist. An obvious choice is to integrate the coupling term over a computational cell of volume $\Delta V$. This yields the following integral form of the coupling force:

$$
\mathbf{F}^{v} \equiv \int_{\Delta V} \mathcal{I} d V=\sum_{n \in \Delta V} \mathbf{F}^{n}
$$

where $\mathbf{F}^{v}$ is used to denote the resultant force acting on a Eulerian grid node, or vertex $v$. Thus, it suggests that all the discrete forces enclosed by a computational cell will produce a cumulative forcing effect on the hosting cell (Figure 7-5). This is the so-called ParticleSource-In Cell (PSI-Cell), or simply Particle-In-Cell (PIC) method, due to (Migdal and Agosta 1967), (Crowe et al. 1977), and (Crowe 1982). Notably, the original PSI-Cell implementation of (Crowe et al. 1977) is a little different from the current one in that the coupling source is calculated by tracking the momentum budge of particles into and out of the cell.

Instead of letting a point force be associated with just one cell, one may also assume that the influence of a point force is distributed over its surrounding Eulerian grid nodes according to a properly defined weighting (Figure 7-6). The weights used in the distribution operation can be based on cell volumes (or areas in 2D case) as in (Squires and Eaton 1990; Boivin et al. 1998), or on the distances between the particle and the surrounding nodes as employed by (Elghobashi and Truesdell 1993). For illustration, consider a two-dimensional grid. With reference to Figure 7-7, $\phi$ is a quantity given at the particle location, the four volume- (or area-) weighted Eulerian quantities, $\phi_{1}$ to $\phi_{4}$, can be calculated from

$$
\phi_{1}=\frac{A_{1}}{A} \phi, \phi_{2}=\frac{A_{2}}{A} \phi, \phi_{3}=\frac{A_{3}}{A} \phi, \phi_{4}=\frac{A_{4}}{A} \phi
$$

where

$$
\begin{aligned}
& A_{1}=(\Delta x-\delta x)(\Delta y-\delta y), \\
& A_{2}=\delta x(\Delta y-\delta y), \\
& A_{3}=(\Delta x-\delta x) \delta y, \\
& A_{4}=\delta x \delta y, A=\Delta x \Delta y .
\end{aligned}
$$

The schematic of the distance-based weighting is depicted in Figure 7-8. The formula for the inverse-distance-weighted distribution (Shepard 1968) is given by

$$
\begin{gathered}
\phi_{i}=w_{i} \phi, i=1,4 \\
w_{i}=\frac{d_{i}^{-p}}{\sum_{j=1}^{4} d_{j}^{-p}},
\end{gathered}
$$

where $d_{1}$ to $d_{4}$ are distances between the particle and the corresponding Eulerian grid nodes, $p$ is an positive real number called the power parameter, and typically $p=2$. Note that the distribution process can be interpreted more generally as a reverse interpolation. It is recommended (Sundaram and Collins 1996) that the reverse interpolation should use the same scheme as the forward one. This methodology was then adopted, for example, in the turbulence modulation study of (Sundaram and Collins 1999), where a third-order Lagrangian polynomial was used for both forward and backward interpolations. 
Nevertheless, a problem it faces is that using a higher order interpolation in the reverse direction causes unphysical "spreading" of the assumed point force. These points will be discussed in more detail in Section 7.7.

Now, look at a Eulerian grid node $v$. The resultant coupling force acting on the node, denoted by $\mathbf{F}^{v}$, is an accumulation of a portion of each point force, whose location is near $v$. Mathematically, this can be written as

$$
\mathbf{F}^{v}=\sum_{n \in \Delta V^{\prime}} \operatorname{proj}\left(\mathbf{F}^{n}\right) .
$$

where proj denotes a projection operation that takes part of $\mathbf{F}^{n}$ and gives it to $\mathbf{F}^{v}, V^{\prime}$ is the region confined by the dotted lines in Figure 7-6. Thus, $\mathbf{F}^{v}$ can be contributed by a particle not only from inside the computational cell $\Delta V$, but also from a nearby one.

It is seen, the first method (7.184) requires a summation operation within each cell, while the second method (7.189) involves force distribution and then summation. (Boivin et al. 1998) evaluated the two approaches with a statistically stationary flow, and found that the second method is much superior over the first one, in terms of recovered initial kinetic energy and accuracy in the high-wave number spectrum. Also, with regard to the second method, (Boivin 1996) compared the two distribution scheme explained above (i.e., the volume-weighted and the distance-weighted), and found that both yield similar results.

Another method to deal with the numerical representation of the point force is proposed by (Maxey et al. 1997), in which the $\delta$-distribution of the point force (cf. Eq. (7.182)) is modified into a numerically resolvable envelope function, $g$, centered at the particle location, $\mathbf{y}^{n}$. With that,

$$
\mathcal{I}=\sum_{n=1}^{N} \mathbf{F}^{n} g\left(\mathbf{x}-\mathbf{y}^{n}\right) .
$$

A suggested envelope function is the three-dimensional normal distribution

$$
g(|\mathbf{x}|)=\left(2 \pi \sigma^{2}\right)^{-3 / 2} \exp \left(|\mathbf{x}|^{2} / 2 \sigma^{2}\right),
$$

satisfying

$$
\int_{R^{3}} g(\mathbf{x}) d \mathbf{x}=1 .
$$

thus, the dominant influence region of the point force is controlled by adjusting the dispersion parameter, $\sigma$, relative to the grid spacing. The formulation given by Eq. (7.190) can be thought of as a generalization of (7.182), as the $g$-function can be the delta function, Eq. (7.191) or some other well defined envelops.

Recall that in Section 7.4.4 the parcel concept has been reviewed, in which a computational particle is allowed to represent a group of particles, usually of the same type and properties. With this method a large statistical sample size can be achieved on limited computational resources. For simplicity, assume a constant parcel size, and each parcel contains same number of particles. As such, the backward coupling incorporating the parcel representation can be simply extended from Eq. (7.182) as 


$$
\mathcal{I}=n_{p} \sum_{n=1}^{N_{p}} \mathbf{F}^{n} \delta\left(\mathbf{x}-\mathbf{y}^{n}\right),
$$

where $N_{p}$ is the total number of computational particles, $n_{p}$ the constant parcel size. Thus, the total number of actual particles considered is $N_{p} \times n_{p}$. Often, it is convenient to express $n_{p}$ in terms of volume fraction, $\alpha$, for bubbly flows, or mass loading, $\phi\left(=\alpha \rho_{d}\right.$ / $\rho_{c}$ ), for flows involving heavy particles. These yield, respectively,

$$
\begin{aligned}
& \mathcal{I}=\frac{\alpha V}{N_{p} V_{p}} \sum_{n=1}^{N_{p}} \mathbf{F}^{n} \delta\left(\mathbf{x}-\mathbf{y}^{n}\right), \\
& \mathcal{I}=\frac{\phi \rho_{c} V}{N_{p} m_{p}} \sum_{n=1}^{N_{p}} \mathbf{F}^{n} \delta\left(\mathbf{x}-\mathbf{y}^{n}\right) .
\end{aligned}
$$

Next, we ask the question: what point forces should be accounted for in the $\mathbf{F}^{n}$ term. As mentioned earlier, $\mathbf{F}^{n}$ is a resultant force in reaction to the hydrodynamic forces that the $n$th particle takes from the fluid. This suggests that $\mathbf{F}^{n}$ should stem from some of those forces addressed in Section 7.5. To this end, different blending and flavors can be found in the literature, depending on the particle type and the flow problems being studied. For solid particles with density large compared to the fluid density, typically included in the $\mathbf{F}^{n}$ is the drag force, as in (Squires and Eaton 1990; Boivin et al. 1998; Sundaram and Collins 1999). For example, (Sundaram and Collins 1999) used the following expression for $\mathbf{F}^{n}$ :

$$
\mathbf{F}^{n} \equiv-\mathbf{F}_{D}^{n}=m_{p} \frac{\mathbf{v}^{n}-\mathbf{u}\left(\mathbf{y}^{n}\right)}{\tau_{p}},
$$

where $\mathbf{v}^{n}$ is the instantaneous velocity of the $n$th particle, $\mathbf{u}\left(\mathbf{y}^{n}\right)$ is the fluid velocity evaluated at the particle location $\mathbf{y}^{n}, \tau_{p}$ the particle response time defined in Eq. (7.3). Note that $\mathbf{F}^{n}$ is always the opposite of that applied to particle by the fluid, therefore, $\mathbf{F}^{n}=$ - $\mathbf{F}_{D}^{n}$. In the study of (Elghobashi and Truesdell 1993), more interaction forces are included:

$$
\mathbf{F}^{n} \equiv-\left(\mathbf{F}_{D}^{n}+\mathbf{F}_{S}^{n}+\mathbf{F}_{A}^{n}+\mathbf{F}_{H}^{n}\right) .
$$

(Reutsch and Meiburg 1994) investigated the buoyancy coupling effect of bubbles on a free shear layer, and his buoyancy-coupling term is expressed as

$$
\sum_{n=1}^{N} \mathbf{F}^{n} \delta\left(\mathbf{x}-\mathbf{y}^{n}\right)=-\alpha_{d} \rho \mathbf{g},
$$

where $\alpha_{d}$ is the local void fraction of the dispersed phase.

At this point, one may wish to recall the derivation presented in Section 7.6.1, which utilized (Maxey and Riley 1983)'s decomposition of forces into $\mathbf{F}_{p}{ }^{(0)}$ (forces arising from the undisturbed flow), $\mathbf{F}_{p}{ }^{(1)}$ (forces arising from the disturbed flow), and gravity. An important piece of information it conveyed is that only the forces due to the disturbed flow, i.e., the $\mathbf{F}_{p}^{(1)}$, will constitute the interphase momentum transfer. Albeit derived in the two-fluid setting, the concluded principle is equally applicable to the twoway coupled E-L approach. In light of this, the coupling forces for a dilute bubbly flow may be written down as 


$$
\mathbf{F}^{n} \equiv-\left(\mathbf{F}_{D}^{n}+\mathbf{F}_{L}^{n}+\mathbf{F}_{A}^{n}\right)
$$

with only the history effect being neglected.

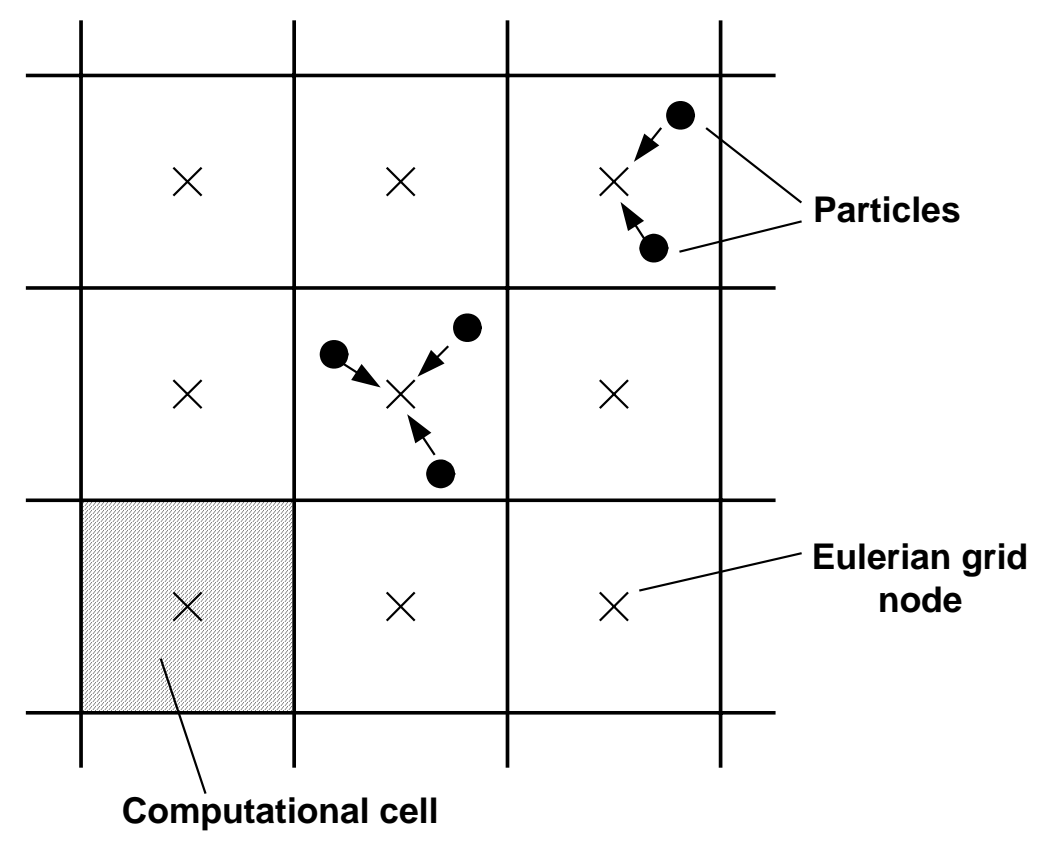

Figure 7-5 Schematic of Particle-Source-In Cell (PSI-Cell) method.

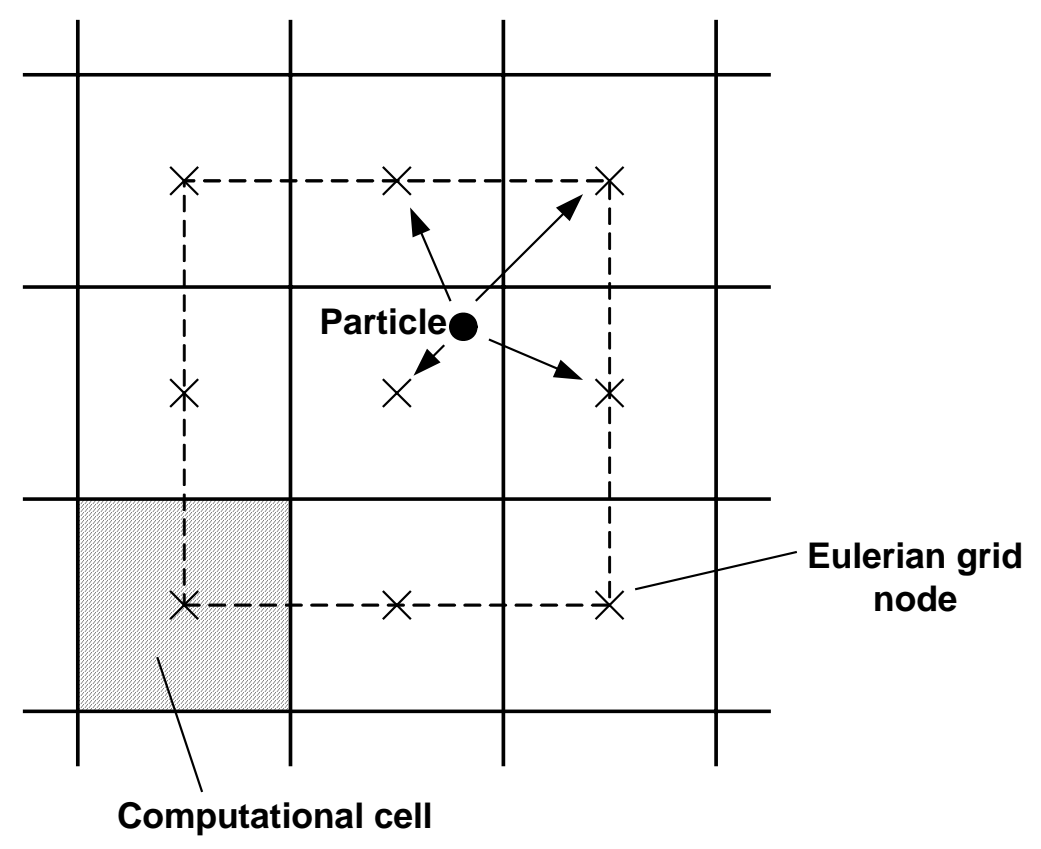

Figure 7-6 Schematic of PSI-Cell method with force distribution. 


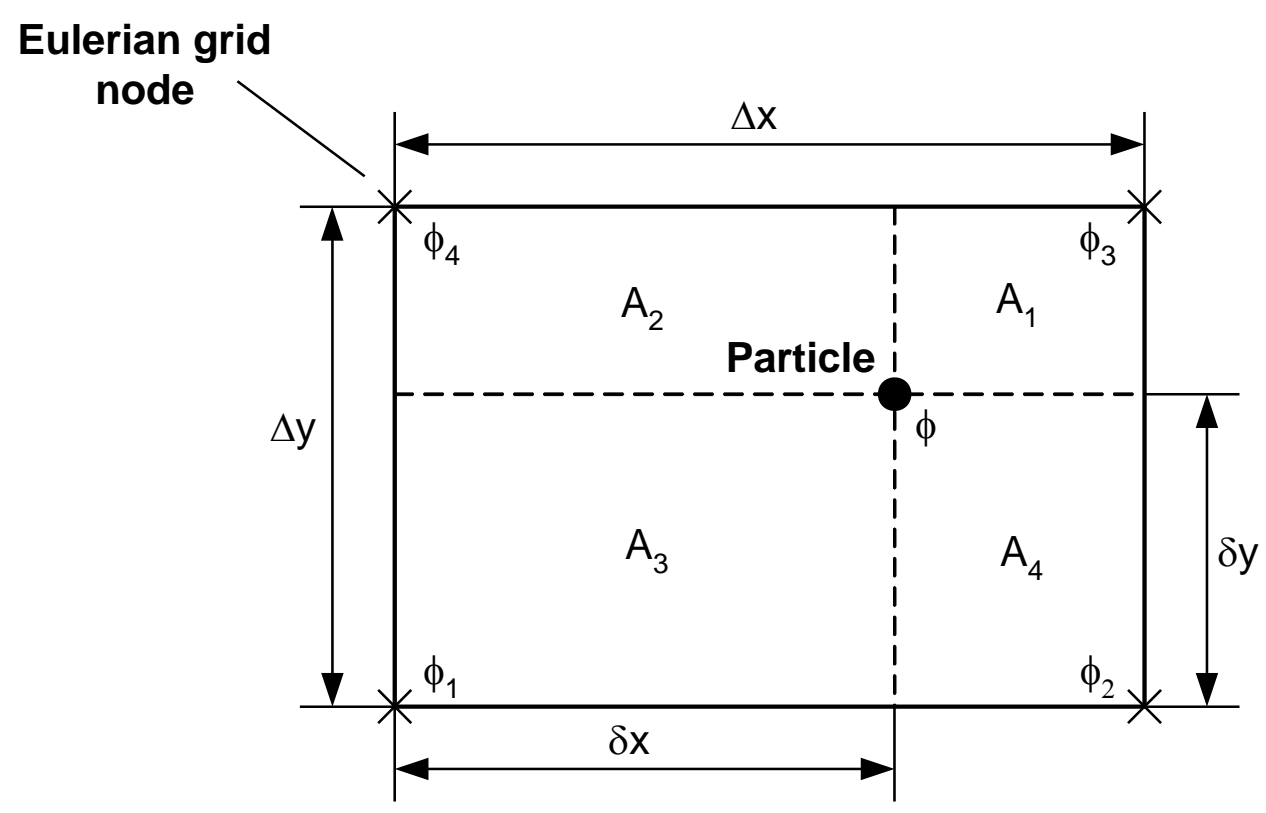

Figure 7-7 Schematic of area- or volume-weighted distribution of a quantity defined at the particle location.

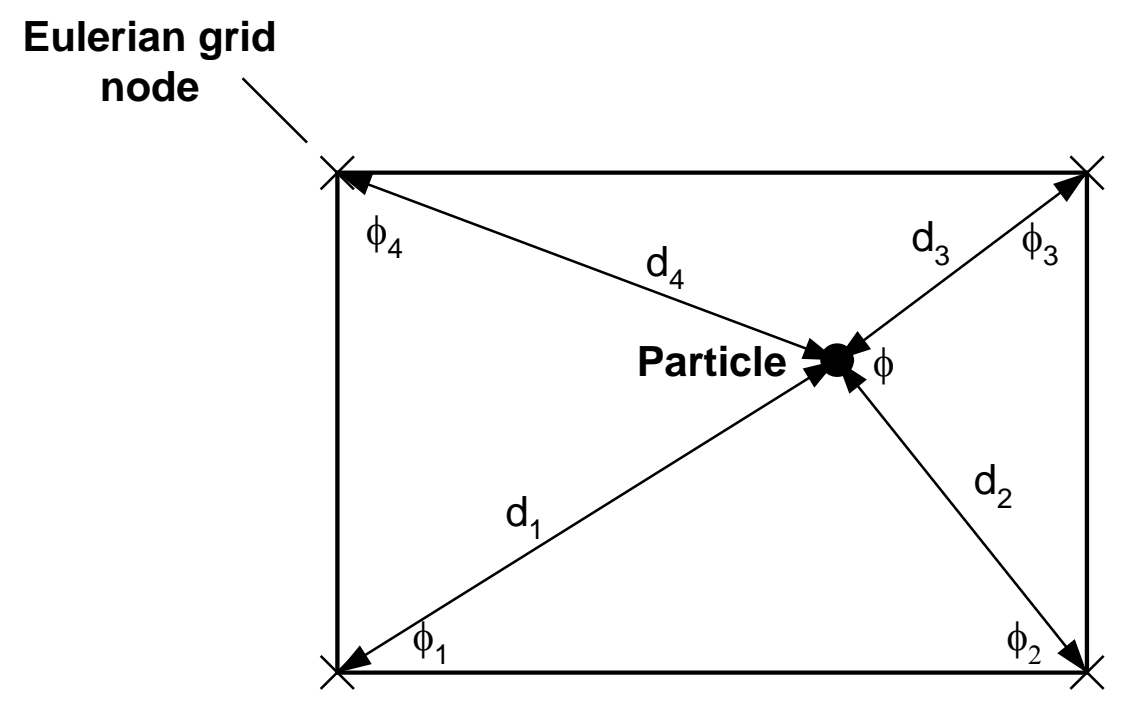

Figure 7-8 Schematic of inverse-distance-weighted distribution of a quantity defined at the particle location.

\subsubsection{Backward Coupling in E-L Approach: Velocity Coupling}

Notice that the backward-coupling formalism presented above rests on the point force assumption. The expression of the point force is interpreted as a reaction force to the force acting on the particle. This coupling mechanism is often termed force coupling, and is by far the most widely used method to account for the particle influence on the carrier phase. On the other hand, by noticing that the particle presence is essentially to generate the locally disturbed flow, (Pan and Banerjee 1996) proposed an alternative approach 
which uses a velocity coupling instead of the force coupling. In this approach, the Stokesian flow around the particle is assumed so that a local disturbance velocity field, $\tilde{\mathbf{u}}$, can be analytically obtained:

$$
\tilde{\mathbf{u}}=\frac{3}{8} d_{p} \mathbf{v}_{r e l} \cdot\left(1+\frac{d_{p}^{2}}{24} \nabla^{2}\right) \mathbf{O},
$$

In the above equation, $\mathbf{O}$ is the Oseen tensor defined as

$$
\mathbf{O}=\frac{1}{r} \delta_{i j}+\frac{1}{r^{3}}\left(x_{i}-y_{j}\right)\left(x_{j}-y_{j}\right),
$$

where $r$ is the distance between $\mathbf{x}$ and the particle location $\mathbf{y}$. Eq. (7.200) satisfies the following Stokes equations:

$$
\begin{gathered}
-\nabla \tilde{p}+\mu \nabla^{2} \tilde{\mathbf{u}}+\mathbf{F} \delta(\mathbf{x}-\mathbf{y})+\frac{d_{p}^{2}}{4} \mathbf{F} \nabla^{2} \delta(\mathbf{x}-\mathbf{y})=0, \\
\nabla \cdot \tilde{\mathbf{u}}=0,
\end{gathered}
$$

where $\tilde{p}$ is the disturbed pressure field, and $\mathbf{F}$ the point force acting on the fluid by the particle. Similar to $\tilde{\mathbf{u}}$, an analytical expression also exists for $\tilde{p}$ (Kim and Karrila 1991). The backward coupling effect is again based on the point force assumption. The point forces here are represented by the monopole and degenerated quadrupole, i.e., the last two terms on the LHS of Eq. (7.202). Hence, instead of putting the point forces in the coupling term, one may rephrase them using the disturbed flow field. This yields the following coupled equation for the case with a single particle:

$$
\rho \frac{D \mathbf{u}}{D t}=-\nabla p+\nabla \cdot(\mu \nabla \mathbf{u})+\rho \mathbf{g}-\left(-\nabla \tilde{p}+\mu \nabla^{2} \tilde{\mathbf{u}}\right) .
$$

When $N$ particles are present in the domain, the modified fluid momentum equation becomes

$$
\rho \frac{D \mathbf{u}}{D t}=-\nabla p+\nabla \cdot(\mu \nabla \mathbf{u})+\rho \mathbf{g}-\sum_{n=1}^{N}\left[-\nabla \tilde{p}^{n}\left(\mathbf{x}-\mathbf{y}^{n}\right)+\mu \nabla^{2} \tilde{\mathbf{u}}^{n}\left(\mathbf{x}-\mathbf{y}^{n}\right)\right],
$$

where the superscript $n$ denotes the particle index.

One sees that the above velocity coupling implementation is essentially another way of formulating the point-force coupling. Physically, this equivalence implies that a disturbance in the background velocity field $\mathbf{u}$, due to the existence of particle, can be generated by either imposing a point-force or imposing a stress tensor (Pan and Banerjee 1996). Numerically, however, these two approaches can be different. The velocity coupling approach can be advantageous when the carrier phase turbulence is mainly affected through disturbing the local velocity field, and the total kinetic energy the particles extract from the continuous phase is relatively small; for example, when the particle is slightly heavier than the fluid. One downside is that special treatment is necessary in the near-wall region, in order for $\tilde{\mathbf{u}}$ to satisfy the wall boundary condition. A way was proposed in the original paper, but at cost of increased computational effort. Also, the linear summation of the disturbance fields from all particles does not answer the question if there is any interaction between the disturbance fields. 


\subsubsection{Backward Coupling in E-L Approach: PHYSALIS}

PHYSALIS is a clever method aimed at a carrying out volume-resolving direct numerical simulation of dispersed two-phase flows in an efficient manner. This approach is devised and developed by Prosperetti, Takagi and their collaborators (Prosperetti and Oguz 2001; Huang and Takagi 2003; Takagi et al. 2003; Zhang and Prosperetti 2003, 2005a, b). In some sense it can be grouped into the velocity coupling model. But, rather than being called a coupling model, it may be more appropriately recognized as a numerical method. The basic idea originates from the fact that, due to the no-slip condition, in the reference frame of each particle, the fluid velocity in the immediate neighborhood of the particle is very small, so that the Stokes equations can be used as an excellent approximation to the full N-S problem. The key of the method is to utilize the analytical solution of the Stokes equations to "bridge" the no-slip condition from the particle surface to the adjacent grid nodes within a predefined "cage" around each particle. In this manner the Eulerian grid for the carrier phase can be constructed as if the particles were not present; as a result, the geometric complexity arising from the phase interfaces is avoided, yielding a big gain in the computational efficiency. Some key elements of this method are briefly described in the following. More details can be found in the aforementioned publications.

The Stokes equation around each particle can be written as

$$
-\nabla \tilde{p}+\mu \nabla^{2} \tilde{\mathbf{u}}=0
$$

where $\tilde{\mathbf{u}}$ and $\tilde{p}$ are the local velocity and pressure field respectively, expressed in the particle frame. The general solution of the Stokes equation near a spherical boundary is well-established, and can be written in the form

$$
\begin{gathered}
\tilde{\mathbf{u}}=\frac{v}{a^{2}} \sum_{n=1}^{\infty} \frac{1}{(n+1)(2 n+3)}\left[\frac{1}{2}(n+3) r^{2} \nabla p_{n}-n \mathbf{r} p_{n}\right]+\frac{v}{a} \sum_{n=1}^{\infty}\left[a \nabla \phi_{n}+\nabla \times\left(\mathbf{r} \chi_{n}\right)\right] \\
+\frac{v}{a^{2}} \sum_{n=1}^{\infty} \frac{1}{n(2 n-1)}\left[-\frac{1}{2}(n-2) r^{2} \nabla p_{-n-1}+(n+1) \mathbf{r} p_{-n-1}\right]+\frac{v}{a} \sum_{n=1}^{\infty}\left[a \nabla \phi_{-n-1}+\nabla \times\left(\mathbf{r} \chi_{-n-1}\right)\right], \\
\tilde{p}=\frac{\mu v}{a^{2}}\left[p_{0}+\sum_{n=1}^{\infty}\left(p_{n}+p_{-n-1}\right)\right]
\end{gathered}
$$

where $a$ is the sphere radius, $p_{n}, \phi_{n}, \chi_{n}$ are regular solid spherical harmonics of order $n$, while $p_{-n-1}, \phi_{-n-1}, \chi_{-n-1}$ are singular harmonics. Thus, e.g.,

$$
p_{n}=\left(\frac{r}{a}\right)^{n}\left[P_{n m} \cos m \varphi+\tilde{P} \sin m \varphi\right] P_{n}^{m} \cos \theta,
$$

where $P_{n m}$ and $\tilde{P}_{n m}$ are dimensionless coefficients, $P_{n}^{m}$ is an associated Legendre function, and $r, \theta$, and $\varphi$ are spherical coordinates centered at the particle center. See, e.g., (Zhang and Prosperetti 2005a) for more details of the expressions.

Eulerian grid is constructed covering the entire computational domain irrespective of the presence of the particles. Cages are subsequently defined for those grid nodes that enclose respective particles. With the help of the Stokes solution, velocity field are then imposed as boundary condition at those grid nodes falling inside the cages, while the N-S equations are solved on the entire domain as a whole. By using an iterative procedure, the 
calculated velocity field in the cage region will satisfy both the N-S and Stokes flows. The particle velocity and position are updated in the Lagrangian reference frame with the hydrodynamic forces obtained from the known Stokes flow field.

There are certain limitations of this method. First, it relies on the availability of an exact Stokes solution for a particular body shape. If the particle shape is not spherical, the Stokes solution given above is not valid, and the mapping to the cage grids will no longer be an easy task. This also implies that a sphere particle under consideration must be nondeformable. Second, the cage grid nodes should be well inside the boundary layer for the Stokes approximation to be applicable. Hence, the Eulerian grid, albeit ignoring the presence of the particles, must be constructed smaller than the particle size. Thus, if a dispersed system on the order of meters contains, say, microparticles, the required grid resolution will be prohibitive. Moreover, if the system is poly-dispersed, an adaptive grid becomes necessary in order to efficiently resolve particles with various sizes.

Overall, as compared to other regular DNS studies, PHYSALIS offers an exciting perspective in accurately and efficiently simulating dispersed two-phase systems. On the other hand, the volume resolving nature of this method may present a significant hurdle in its application to the practical engineering problems.

\subsection{Interpolation}

In the point-volume Lagrangian simulation for the dispersed phase, the particles are dispersed in the fluid field, and in general, their positions do not coincide with the grid points, on which the Eulerian fluid field is computed and updated. In order to integrate the particle equation of motion, fluid quantities computed on the Eulerian mesh, such as the velocity and vorticity, must be interpolated to the particle position (forward interpolation). When two-way coupling is implemented, such as the one formulated in Eq. (7.182), a reverse interpolation will also be needed. That is, the coupling force defined at each particle position is interpolated (or projected) back to the fluid grid nodes.

\subsubsection{Forward Interpolation}

Often times, one formulates an interpolation scheme of arbitrary order using the Lagrangian polynomial or the Hermite polynomial. The Hermitian interpolation is advantageous when the solution is represented in the spectral space, for example, from a spectral or pseudo-spectral method. Other schemes can also be used, such as the Chebyshev polynomial. For an interpolation scheme that uses weighted sum, such as the Lagrangian polynomial, one may write the interpolation function, for example for the fluid velocity at the particle location $\mathbf{y}^{n}$, in the following general form:

$$
\mathbf{u}\left(\mathbf{y}^{n}\right)=\sum_{v} S\left(\mathbf{y}^{n}, \mathbf{x}^{v}\right) \mathbf{u}\left(\mathbf{x}^{v}\right),
$$

where $\mathbf{x}^{v}$ represents a fluid grid point or vertex, the basis function (or coefficient polynomial, weighting function), $S\left(\mathbf{y}^{n}, \mathbf{x}^{v}\right)$, is constrained to satisfy 


$$
\sum_{v} S\left(\mathbf{y}^{n}, \mathbf{x}^{v}\right)=1
$$

and the summation goes through all the fluid vertices in the entire domain; however, the effectively participating nodes are only those with $S \neq 0$, usually located in the neighborhood of the particle position $\mathbf{y}^{n}$. The number of involved vertices determines the order of accuracy. In a structured grid system (where one can use $\mathrm{i}, \mathrm{j}, \mathrm{k}$ index to locate a vertex), the $N$-th order Lagrangian polynomial with $(N+1)$ participating points can be written down as

$$
\mathbf{u}\left(x_{p}, y_{p}, z_{p}\right)=\sum_{i=0}^{N} \sum_{j=0}^{N} \sum_{k=0}^{N} L_{i}\left(x_{p}\right) L_{j}\left(y_{p}\right) L_{k}\left(z_{p}\right) \mathbf{u}\left(x_{i}, y_{j}, z_{k}\right),
$$

where $\mathbf{y}^{\mathbf{n}}=\left(x_{p}, y_{p}, z_{p}\right), \mathbf{x}^{v}=(x, y, z)$, and the basis functions are given by

$$
L_{i}\left(x_{p}\right)=\prod_{\substack{l=0 \\ l \neq i}}^{N} \frac{x_{p}-x_{l}}{x_{i}-x_{l}}, L_{j}\left(y_{p}\right)=\prod_{\substack{l=0 \\ l \neq j}}^{N} \frac{y_{p}-y_{l}}{y_{j}-y_{l}}, L_{k}\left(z_{p}\right)=\prod_{\substack{l=0 \\ l \neq k}}^{N} \frac{z_{p}-z_{l}}{z_{k}-z_{l}} .
$$

The Lagrangian polynomial is exact at grid nodes; when $N=1$, it becomes a trilinear interpolation. One sees that Eq. (7.212) is an expensive operation if the order is high. For instance, a third order polynomial $(N=3)$ involves $4^{3}=64$ grid points and requires of the order of $3 \times 4^{3}$ operations. Additionally, $3 \times 4^{2}$ operations are required to evaluate each basis function.

Evaluation of accuracy of different forward interpolation schemes has been well documented by several researchers (Yeung and Pope 1988; Balachandar and Maxey 1989; Yeung and Pope 1989; Kontomaris et al. 1992; Boivin 1996; Wang and Squires 1996a; Fukagata et al. 1998). It is generally agreed that third-order accuracy is required at minimum to accurately track the particle trajectory, whereas the first-order linear interpolation can be inadequate, despite its computational simplicity and economy. Some more details are provided in the following review.

A way of testing the accuracy of an interpolation scheme is to distribute a large number of particles in the simulation domain, and compute fluid statistics based on the interpolated values at those particle locations. The fluid statistics collected in this manner should converge to those calculated using the fluid velocities directly available at the particle positions. By quantifying the difference of the two, one is able to evaluate the accuracy of the interpolation scheme. This approach was used, for example, by (Balachandar and Maxey 1989; Wang and Squires 1996a; Fukagata et al. 1998).

(Yeung and Pope 1988) studied the accuracy of interpolation schemes based on Taylor series and on cubic splines. The respective errors in computed trajectories are quantified for simple, frozen velocity fields. The third-order Taylor series with 13 points (TS13), and the piecewise cubic Lagrangian polynomial yield superior results.

(Balachandar and Maxey 1989) compared various interpolation schemes in a spectral simulation of homogeneous turbulence. They found that the fluid statistics were retained with a good accuracy when the sixth order Lagrangian interpolation, the Hermite interpolation, or the TS13 scheme proposed by (Yeung and Pope 1988) were used. They 
also pointed out that the linear interpolation generated large errors as compared to statistics of the original field.

(Wang and Squires 1996a) used LES field of fully developed turbulent channel for testing the accuracy of interpolation schemes. The schemes they tested were the linear interpolation, the fourth-order and the sixth-order Lagrangian polynomials. They demonstrated that, with respect to mean quantities, all the schemes yield accurate predictions. However, when comparing the second moment statistics (i.e., RMS values), or the Lagrangian statistics of the flow field, large errors (about $8 \%$ ) were found with the linear interpolation; the errors associated with the fourth-order and the sixth-order Lagrangian interpolations are rather small (within 1\%), consistent with previous findings.

(Fukagata et al. 1998) extended the study to particles with large inertia. They tested three different interpolation schemes, nearest grid point, linear and sixth order Lagrangian. Their results showed that both mean and RMS quantities are not largely influenced by the differences in interpolation scheme. It can be due to the fact that heavy particles are insensitive to small-scale turbulence.

It seems that the optimal interpolation method is the third-order piecewise cubic polynomial, a combination of accuracy and cost. E-L simulations using this order of interpolation are, for example, (Deutsch and Simonin 1991; Boivin 1996; Sundaram and Collins 1996; Boivin et al. 1998; Sundaram and Collins 1999; Boivin et al. 2000), among others. It should be noted that, a large body of point-volume Lagrangian simulation published in the literature has used the inexpensive linear, bi-linear, or tri-linear interpolation method due to their significant computational efficiency. It is conjectured by the author that, compared to the modeling error in LES, the accuracy loss due to the first order linear interpolation could be a minor issue. In the later bubble column LES it will be shown that with the tri-linear interpolation high-quality results can be attained.

With respect to the linear interpolation it is worthwhile to mention that the particle velocity may experience a jump when it crosses from one computational cell to the other in an unstructured mesh setting (Smirnov et al. 2005b). However, such a jump phenomena is neither observed in the present study where the grid is orthogonal, nor reported by other researchers that use unstructured grids. Therefore, a further study is necessary in order to clarify this jump issue.

\subsubsection{Reverse Interpolation}

The need for a reverse interpolation arises when the coupling term (e.g., Eq. (7.182)) in the carrier phase momentum equation is to be evaluated at the Eulerian fluid grid nodes based on the coupling force, which is defined at the particle location. The reverse interpolation of the coupling force $\mathbf{F}\left(\mathbf{x}^{v}\right)$ onto an arbitrary fluid grid node $\mathbf{x}^{v}$ can be generally expressed as

$$
\mathbf{F}\left(\mathbf{x}^{v}\right)=\sum_{n} S^{*}\left(\mathbf{y}^{n}, \mathbf{x}^{v}\right) \mathbf{F}\left(\mathbf{y}^{n}\right)
$$


where $S^{*}$ is the coefficient function of the reverse interpolation, the summation goes through all the particles in the domain; only an effectively participating particle has a non-zero $S^{*}$. Notice that one particle may participate in the $\mathbf{F}\left(\mathbf{x}^{v}\right)$ evaluation at several grid nodes. Thus, a different view of Eq. (7.214) is to see the backward interpolation as a "spreading" process, in which each $\mathbf{F}\left(\mathbf{y}^{n}\right)$, associated with a single particle, is redistributed over the several neighboring fluid nodes. Further, if $S^{*}$ is equal to $S$, the weight used in the forward interpolation and backward redistribution between each $\mathbf{y}^{n}-\mathbf{x}^{v}$ pair will be the same. In this case, the summation of the redistributed forces contributed by a single particle recovers the original point force, since

$$
\sum_{v} S^{*}\left(\mathbf{y}^{n}, \mathbf{x}^{v}\right) \mathbf{F}\left(\mathbf{y}^{n}\right)=\mathbf{F}\left(\mathbf{y}^{n}\right) \sum_{v} S^{*}\left(\mathbf{y}^{n}, \mathbf{x}^{v}\right)=\mathbf{F}\left(\mathbf{y}^{n}\right),
$$

the last equality is due to Eq. (7.211). However, it should be noted that $S^{*}=S$ is not the only way to conserve the force magnitude; for example, any statistical distribution can used to redistribute $\mathbf{F}\left(\mathbf{y}^{n}\right)$.

Commonly used redistribution approaches are volume-weighted (Squires and Eaton 1990; Boivin 1996; Delnoij et al. 1997a; Delnoij et al. 1997b; Boivin et al. 1998), and distance-weighted (Elghobashi and Truesdell 1993). (Boivin 1996) also reported that both approaches yield similar results. Note that the volume-weighted or distanceweighted redistribution corresponds to first-order reverse interpolation.

A remarkable result was obtained by (Sundaram and Collins 1996) who showed that in order to conserve the global kinetic energy (kinetic energy of flow field plus that of particles), the interpolation schemes used in the forward and backward coupling must be consistent, that is, $S=S^{*}$. However, this gives rise to an important dilemma: in general, one tends to use a high-order forward interpolation to improve the accuracy of the particle tracking. If the reverse interpolation is required to be on the same high order, the backward coupling contributed by a single particle will then spread its influence over a larger volume. On the other hand, the primary point force assumption requires that the local disturbance flow generated by a particle scales with the particle diameter, a length scale must be smaller than the resolved flow scales, typically represented by the smallest grid spacing. In this respect, the influence of a point force should be limited within the cell in which the corresponding particle resides. Therefore, a conceptual contradiction results.

(Sundaram and Collins 1996) also tested the effect of the "spreading" on the reverse coupling, and found it to be negligible. The third-order interpolation scheme suggested by the authors was applied in a later study of the same authors (Sundaram and Collins 1999) with success. In spite of that, hesitance still exists in accepting this methodology, mainly due to the open question addressed above. The author's intuition is that one should separate the physical aspect from the mathematical aspect. Deciding the influence region of a point force is a physical problem, since it depends on the flow feature, i.e., the smallest scales of turbulence. In fact, the spreading-effect test made by (Sundaram and Collins 1996) also suggests from another point of view that it is possible for the point force to have a larger influence region. Finally, one should not forget that although the overall kinetic energy is biased if the forward and reverse interpolation 
schemes are not symmetric, the momentum is still properly conserved independent of the interpolation method.

\subsection{Formulation of Two-way Coupled LES}

With the above preparation, it finally gets down to a point, where the issue of two-phase LES with two-way coupling can be addressed. Recall that the objective of a single-phase LES is to resolve details of energy-containing large scales. For a two-phase system, it is still possible to use single-phase LES under the one-way coupling assumption to predict particle dispersion, since the particle dispersion pattern is mainly controlled by the largescale motions (cf. discussions in Section 7.6.2). Yet, difficulties arise when LES needs to operate in a two-way coupled regime. The main obstacle here lies in the fact that the subgrid scales at which closure modeling is required are subject to possible interactions between the dispersed phase and turbulence. Consequently, the SGS models originally developed for a single phase LES may not be valid in a two-phase system; modifications or reconstruction of SGS models may be necessary. Thus, the benefits gained from only resolving the large scales would get lost by the approximations of the closure models (Elghobashi 1994).

At the same time, one also realizes two additional facts. First, the computational expense of a DNS is prohibitive. Even in the single-phase flow study, DNS is only used for the research purposes; application of DNS to high Reynolds number flows with complex geometry will be, at present and probably in a long while, still not possible. Second, in the two-fluid or mixed-fluid method, the mesoscale-averaging may sacrifice important turbulence scales, possibly including those at the energy containing levels. In spite of their wide use in practical engineering applications, it is not possible to ask this approach to recover sufficient details of turbulence. Between DNS and two-fluids stands the LES, which appears to be the optimal compromise between the cost and profit. It is generally believed that LES would show considerable promise, when better and fully verified closure models become available.

A side mark is that the mesoscale averaging in the two-fluid method should not be confused with the filtering used in LES. In the case when a box filter is used, both yield the same type of operation, namely, the volume averaging. However, the two operations are fundamentally different. The mesoscale is defined in a statistical sense, i.e., it should be large enough to yield an asymptotic statistical average, while the filter length in a filtering operation is defined according to the resolution need of the turbulence scales. It is possible that the two length scales overlap.

Resent research activities have shown an intense interest in using LES to predict turbulent two-phase flows. (Hewitt 1999; Loth 2000; Lakehal 2002; Simonin and Squires 2002; Sundaresan et al. 2003; Sirignano 2005) made some general comments about the formalism, difficulties and perspectives of the two-phase flow LES. In the theoretical aspect, (Yeh and Lei 1991; Yang and Lei 1998; Armenio et al. 1999) investigated the effect of LES field on the particle dispersion statistics. (Boivin et al. 2000; Miller and 
Bellan 2000; Okong'o and Bellan 2000, 2004; Leboissetier et al. 2005) performed $a$ priori and a posteriori SGS analysis for gas-liquid flows and transitional droplet-laden mixing layers. (Fukagata 2000; Lei et al. 2002; Milelli et al. 2002; Pandya and Mashayek 2002) proposed SGS models for LES of turbulent flows laden with particles or bubbles. In the application-related aspect, (Wang and Squires 1996a, b; Simonin et al. 1997; Tanaka et al. 1997; Fukagata et al. 1998; Fukagata 2000; Yamamoto et al. 2001) used LES to study the particle-laden turbulent channel flows. (Climent and Magnaudet 1998; Lakehal et al. 2002; Milelli 2002; Milelli et al. 2002) reported recent advances in the application of LES to turbulent bubbly mixing layers. (Yang et al. 2002a) and (Smirnov et al. 2005a) simulated bubbly jets and bubbly ship wakes, respectively. (Menon and Pannala 1998; Pannala and Menon 1998; Sankaran and Menon 2002; Apte et al. 2003a; Apte et al. 2003b) tackled problems using LES in the area of particle-laden combustions, such as the spray combustion.

With respect to the possible influence of the dispersed phase on the SGS closure models, there are three options: (i) discard SGS models developed in a single-phase LES completely, and build new SGS models from the ground up, (ii) accept those single-phase SGS models and do modifications on them, or (iii) neglect the influence of the dispersed phase on the SGS when condition allows. Probably no one would really like the first idea. Option (ii) was chosen in the study of (Fukagata 2000) and (Milelli 2002), where a Smagorinsky-type model is used to serve as the base model, and a particle-induced eddy viscosity, $\mu_{p}$, is incorporated into the expression of the effective viscosity of the carrier phase, $\mu_{t}$. Nevertheless, the semi-empirical expressions of $\mu_{p}$, such as that presented in (Zahrai et al. 1995; Tran 1997), are generally derived under a series of assumptions and approximations. An accurate assessment of the proposed two-phase SGS models may only be achieved by employing a volume-resolving DNS, which seems at present not quite possible.

In the present study, it is assumed that the backward coupling will not affect the well-established single-phase SGS models. This line of thinking follows the two-way coupling formalism presented in Section 7.6, where the N-S equations for the carrierphase are solved in their original form, and the point force assumption is made to account for the modulation effect of particles on the flow. Simulation based on that is typically a non-volume-resolving DNS coupled with point-volume Lagrangian tracking. As (Boivin et al. 2000) pointed out, this framework, namely, DNS with point force assumption, has in fact already involved a "subgrid" approximation: the local fluid flow perturbations induced by the particle presence are not fully resolved, and, the effects of the subgrid part of the disturbance on the resolved field are assumed to be negligible with respect to the influence of the molecular viscosity of the fluid.

It is necessary to recap some important assumptions that are commonly used in an E-L DNS with the point-volume treatment for the dispersed phase:

1. The dispersed two-phase system under consideration is dilute, loaded with small particles/bubbles/droplets whose length scales $\left(d_{p}\right)$ and time scales $\left(d_{p}{ }^{2} / v\right.$ or $\left.d_{p} / v_{\infty}\right)$ are smaller than, or on the order of the characteristic length and time scales of the 
flow, respectively. In a turbulent flow simulation, the characteristic flow scales refer to the Kolmogorov length scales or the smallest resolved scales.

2. Because of the dilute assumption, the governing Navier-Stokes equations for the carrier phase retain their original form (except for an additional interphase transfer term).

3. The particle-particle collision is neglected due to the dilute condition.

4. The effect of the particles on the carrier flow can be represented through point forces in the governing $\mathrm{N}-\mathrm{S}$ equations.

Note that condition (1) also implies (2) through (4). With the above assumptions, Eqs (7.180) through (7.182) consolidates their significance in serving as the governing equations for the point-volume E-L DNS. The logical extension to the filtered equations for a point-volume E-L LES is simply achieved by performing the filtering operation defined in Eq. (4.54) on the original equations. This yields:

$$
\begin{gathered}
\frac{\partial \bar{u}_{j}}{\partial x_{j}}=0 \\
\frac{\partial}{\partial t}\left(\rho \bar{u}_{i}\right)+\frac{\partial}{\partial x_{j}}\left(\rho \bar{u}_{j} \bar{u}_{i}\right)=-\frac{\partial \bar{p}}{\partial x_{i}}+\frac{\partial}{\partial x_{j}}\left(\mu \frac{\partial \bar{u}_{i}}{\partial x_{j}}\right)-\frac{\partial \tau_{i j}^{R}}{\partial x_{j}}+\overline{\mathcal{I}}_{i},
\end{gathered}
$$

where the fluid gravity is combined into the pressure term, overbar denotes a filtered quantity associated with a filter $G(\mathbf{x}), \tau_{i j}^{R}$ is the residual stress representing the SGS effect on the resolved scales, and needs to be modeled, $\tilde{\mathcal{I}}$ represents a "filtered" version of the coupling point-force $\mathcal{I}$ that is defined in Eq. (7.182). Also, due to the dilute condition, the subgrid scales generated by the local disturbance flow in the presence of the particles can be assumed to be negligible, with respect to their influence on the resolved field. Such an assumption justifies the use of SGS models developed from a single-phase LES setting.

The most critical part here is the coupling force $\mathcal{I}$ and its filtered counterpart $\tilde{\mathcal{I}}$. According to (Maxey et al. 1997) and (Boivin et al. 2000), a generalization of $\mathcal{I}$ and $\overline{\mathcal{I}}$ can be written in the form

$$
\begin{gathered}
\mathcal{I}(\mathbf{x}, t)=\sum_{n=1}^{N} \mathbf{F}\left(\mathbf{y}^{n}, t\right) g\left(\mathbf{x}-\mathbf{y}^{n}\right), \\
\overline{\mathcal{I}}(\mathbf{x}, t)=\sum_{n=1}^{N} \int_{R^{3}} \mathbf{F}\left(\mathbf{y}^{n}, t\right) g\left(\boldsymbol{\xi}-\mathbf{y}^{n}\right) H(\mathbf{x}-\boldsymbol{\xi}) d \boldsymbol{\xi},
\end{gathered}
$$

where $g$ is an envelope function controlling the shape of the point force $\mathbf{F}$ (cf. Section 7.6.3), $H(\mathbf{x})$ is a three-dimensional low-pass filter with a characteristic filter width of the order of mesh size. Other notations are same as those defined in Section 7.6.3. In the work of (Squires and Eaton 1990; Elghobashi and Truesdell 1993; Boivin et al. 2000) among others, $H$ is used to redistribute (or interpolate back) the point force over the computational nodes surrounding the particle. Since $H$ is an assumed function, it is not necessarily the same as the filter $G$. Selection of the $g$ and $H$ function is largely a matter of empirism. Typically, a Gaussian envelope function is chosen for the function $g$. For a bubbly flow, the interaction force $\mathbf{F}$ can be, for example, that given by Eq. (7.199). 
Traditional ways of the numerical implementation of the coupling force term have been discussed in Subsection 7.6.3.

\subsection{Two-layer Concept}

\subsubsection{Particle Size vs. Grid Size}

It is important to note that the assumptions used in deducing the governing equations for a two-way coupled LES (Eq. (7.216) and (7.217)) sets a considerable restriction on the size of the particles. Conventionally, in DNS, since the Kolmogorov scales are fully resolved, the particle size must be smaller than the Kolmogorov length scales. In LES the grid size represents the resolved length scales, thus the particle size must be much smaller than the computational cell size. This fact simply implies, for example, a wall-resolving DNS or LES for high-Re turbulent channel flow would very possibly have difficulties in accommodating even very small micro-particles, since in the wall layer very fine grid must be allocated (Figure 7-9). Moreover, regardless of the resolved flow scales, the particle size is also commonly required to be smaller than the grid size in an E-L simulation, mainly due to the computational needs and convenience (cf. Section 7.6.3).

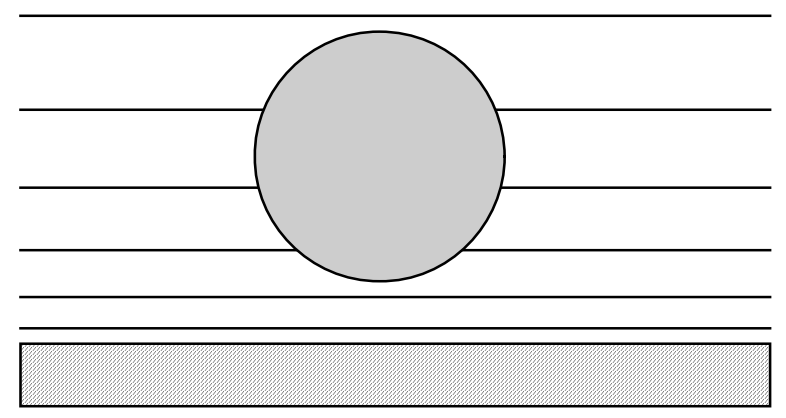

Figure 7-9 Schematic of a micro particle vs. grid distribution in a wall-resolving LES or DNS.

To help explain the potential problems with this particle size restriction, first consider a laminar flow with immersed particles. Suppose the particle size, $d_{p}$, is smaller than the grid size, $h$, which is sufficiently small to resolve the characteristic length scale of the laminar flow, say, $l$ (Figure 7-10a). Next, it is fully admissible if one wants to refine the original grid to achieve a better accuracy of the flow solution. Note that refining the grid does not affect the $d_{p}<l$ condition at all, because $l$ is determined by the flow nature not the grid. Now, at certain refinement factor, say ten times, the grid size $h$ eventually becomes smaller than the particle size, i.e., $d_{p}>h$ (Figure 7-10b). Then one would like to ask: do we really need to keep the particle size smaller than the grid size for such laminar flow case? The answer is apparently NOT, because in this scenario $d_{p}<l$ is not violated, and the flow length scales are not necessarily represented by the grid size! In other words, the particle dimension should have in theory no direct relation with the computational grid size just because the grid is allowed to be arbitrarily fine. 


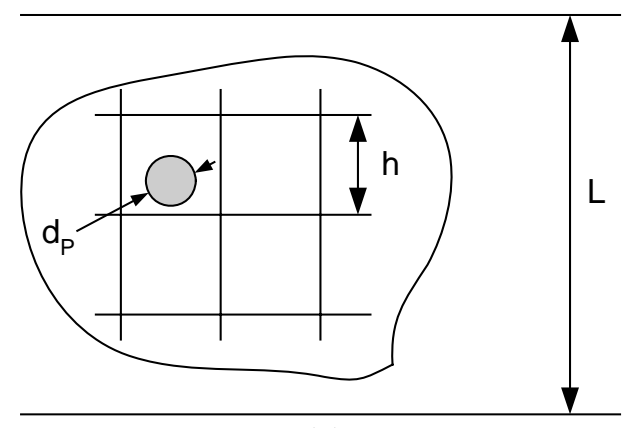

(a)

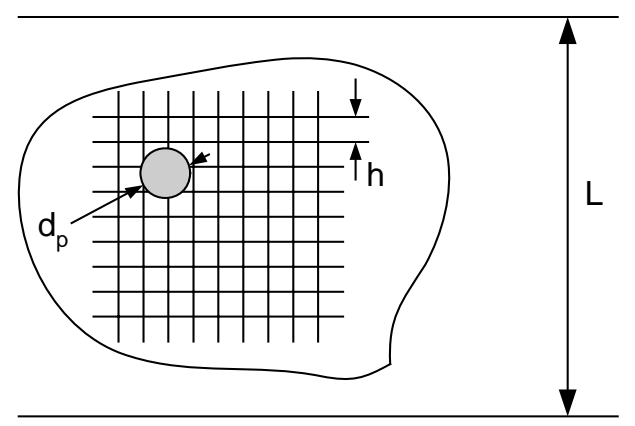

(b)

Figure 7-10 Schematic of particle size with respect to grid size. Eulerian grid is (a) coarse, (b) fine.

Now, let us return to the situation displayed in Figure 7-9, where the particle size is larger than the near wall grid spacing in a wall-resolving DNS or LES of a turbulent channel flow.

In the case with DNS, suppose the near-wall grid is excessively fine, i.e., it overresolves the Kolmogorov scales. As such, although $d_{p}>h$, the $d_{p}<l$ ( $l$ here represents the Kolmogorov length scale) condition is still met, and there should be no reason not to justify such a two-way coupled point-volume E-L simulation. But, since the particle overlaps several cells (not reside in a single one), and each of the overlapped cells (not only the one that encloses the center of the particle) should have the "equal right" to take a portion of the feeding back force, the conventional PSI-Cell based implementations for the reverse coupling (cf. Section 7.6.3) may become inappropriate and will need some modification to overcome this size conflict.

In the case with LES, one commonly asks the particle size, $d_{p}$, to be smaller than the smallest resolved scales, $l$, in order to justify the point-volume assumption. Note that, in LES $l$ is on the order of the grid spacing $h$; thus $d_{p}<l$ also implies $d_{p}<h$. However, this common practice needs to be elaborated. Suppose Figure 7-10a represents a typical LES grid containing particles. According to $d_{p}<h$, this grid qualifies for a point-volume LES. Now, if the grid is refined to Figure 7-10b, one finds $d_{p}>h$. Does that then mean the point-volume assumption is alright with a coarse resolution LES, but not OK with a fine resolution LES? Apparently, this presents a paradox. Further, consider the particle influence on the SGS model. In the situation of dilute flow with $d_{p}<h$, one may well argue that the small particles (relative to the grid size) have only minor effect on the SGS motion, so that a single-phase SGS model can be employed. However, if the same flow is solved on a fine grid as in Figure 7-10b, neglecting the particle influence on those locally overlapping SGS seems to be questionable. Thus, a second paradox arises: a coarse grid LES may use a single-phase SGS model, while a fine grid LES may not.

Given above discussion, the following viewpoints can be agreed. (i) In a laminar flow simulation or DNS of a turbulent flow, the applicability of the point-volume assumption depends on whether the particle size $d_{p}$ is smaller than the characteristic flow size $l$, i.e., $d_{p}<l$ ( or $d_{p}<<l$ in strict sense), and it is independent of the relation between $d_{p}$ and the grid size $h$. In other words, as long as the point-volume approach can be 
applied, the geometric feature of the particle should be decoupled from that of the grid. Hence, in the implementation of the reverse force coupling this size decoupling principle should also be reflected. (ii) In a point-volume LES, the conventional requirement of $d_{p}<$ $l$, where $l$ is the smallest resolved scales, lacks certain rigor. Further, whether or not a single-phase SGS model can be applied to a two-phase LES should be judged by possibly the diluteness condition, irrespective of how small the computational grid is. Thus, it is important to find a way that can justify the use of the point-volume assumption in a LES setting.

\subsubsection{Point Volume or PHYSALIS?}

One of the primary goals of this study is to boost the viability of the point-volume twoway coupled LES of dispersed two-phase turbulent flows. It is the author's belief that, in the interest of practical engineering and industrial applications, LES will represent the most promising approach in tackling two-phase turbulent flow problems with a highest

quality-price ratio. Sticking with the point-volume approach is due to the consideration of its capability to be extended to real-world flow problems, which typically involves a considerably large number of particles of various sizes.

A competitor to the point-volume LES is the so-called PHYSALIS, which has been reviewed in Section 7.6.5. In short, PHYSALIS is a clever volume-resolving DNS which is capable of handling many non-deformable particles, e.g., 1024 particles in the study of (Zhang and Prosperetti 2005b). Although this approach significantly eases the grid construction (by completely ignoring the presence of the spherical bodies), it does not ease the resolution of the computational grid, since the mesh size is required to be on the order of the particle size. Because of this, PHYSALIS would have difficulty when applied to, for example, a large-scale system containing even a small number of small particles. For practical engineering and industrial applications, the point-volume LES will still have its solid role.

\subsubsection{An Assumption: Point Volume and Relatively Dilute}

Now, coming back to the topic of point-volume LES. As elaborated in Section 7.9.1, to answer when the point-volume assumption can be valid in LES is a very difficult task. At this point, an engineering shortcut would like to be made: instead of letting the pointvolume assumption be built upon other primary assumptions, such as the dilute condition etc, a reformulated starting assumption is proposed.

Assumption: The point-volume treatment of relatively small particles is an applicable approach for the Lagrangian simulation of relatively dilute dispersed systems.

This assumption can be further interpreted with the following four aspects. (i) A system with relatively large void fraction and relatively large particles is fully permitted, so long as such E-L simulation is capable of delivering satisfactory results. For example, one should not rule out the possibility of simulating a dispersed two-phase system in a 1 
$\mathrm{m}^{3}$ box containing $10 \mathrm{~mm}$ particles with a $5 \%$ volume fraction for the dispersed phase. Although the particle size is not required to be smaller than the characteristic turbulence length scales, it should not be larger than a limit, above which the two-phase flow under consideration does not qualify for a dispersed system. Questions, such as what is the upper limit of the particle size and the system diluteness, are left for future studies. (ii) Since the dispersed system is not strictly dilute, but relatively dilute, whether or not to neglect the effect arising from the particle-particle interaction can be kept as an option, depending on a particular flow being considered. (iii) The point-volume treatment of the particles also implies that the filtered equations (7.216) through (7.219), without being volume-averaged, can still serve as the governing equations for the continuous phase in an E-L LES. (iv) The Lagrangian particle tracking is carried out by treating the finitesized particles as point volumes. Although particles can be relatively large, such pointvolume tracking approach is still viable in an engineering sense, because the RHS forces of the particle equation of motion are regarded as an engineering model that involves adjustable model parameters and capable of reproducing the particle motion captured in a laboratory measurement. Even when the point-volume assumption is not quite justified in a physical sense, such as the situation of relatively large particles in a thin wall boundary layer, one may formulate a proper model force, e.g., the wall force, to approximate the real hydrodynamic forces acting on the particles. In essence, the notion of a point-volume model is not very much different from that of the turbulence modeling. Although most of the hydrodynamic forces addressed in Section 7.5 are derived under a rigorous pointvolume assumption, in the sense of modeling however, they may still serve as a good approximation for relatively large particles.

\subsubsection{An Assertion: Geometric Decoupling}

Next, from the discussion provided in the Section 7.9.1, and the conclusions made there to the particle size condition in a point-volume E-L laminar or DNS simulation, it is seen that an extension of the uncorrelation between the particle size and mesh size to the LES seems plausible. This yields the following proposed assertion.

Assertion: The geometric feature of the particle should be decoupled from that of the computational grid.

As such, the particle size is no longer limited by an upper bound, the cell size, and the particles can now reside not only inside a computational cell, but also over multiple cells. The Eulerian computational grid can then be constructed to a desired fineness (e.g., in the wall layer) without concern for the particle size. This fact is particularly useful in the simulation practice as most of the time the characteristic flow length scales are not known a priori.

\subsubsection{An Approach: PSI-Ball (Particle-Source-in Ball)}

With respect to the reverse force coupling, the PSI-Cell (particle-source-in cell) based implementations have been discussed in detail in Section 7.6.3. With the PSI-Cell, a 
particle is required to be much smaller than the computational cell in which it resides, so that the distribution of the particle-to-fluid point force occurs only to either the associated cell center, or the cell corner nodes (see Figure 7-5 and Figure 7-6).

In the case when the particle size is relatively large with respect to the computational cell, such as the one displayed in Figure 7-10b (whose situation is fully allowed due to the assumption and assertion made in the previous two subsections), the PSI-Cell implementation for the reverse coupling yields an "unfair" distribution of the point force, because not all the Eulerian grid nodes that overlap the particle can obtain a share. As a result, the influence region due to the presence of a particle is only confined to a single cell that encloses this particle, and whether the particle in question is large or small will, except through the magnitude of the coupling force, have no other influence on the flow solution. Yet, the past experience has taught us that, the particle size can play a determinant role in both the local and global dynamics of a dispersed two-phase system, and this size factor must be properly taken care of in a simulation approach. Provided a volume-resolving approach is not the concern, such as in the current study, a way needs to be found to remedy the deficiency of the PSI-Cell method. Hence, an approach, named PSI-Ball (particle-source-in ball) is proposed.

Approach (PSI-Ball): The reverse coupling (dispersed phase on the carrier phase) is achieved by redistributing the interphase point force via a distribution function onto those Eulerian grid nodes falling inside a predefined local influence sphere (or cage) centered at the particle center.

The name PSI-Ball derives from PSI-Cell. However, PSI-Ball can be considered as a generalization and extension of those PSI-Cell based methods. For example, when the influence sphere is chosen to be the computational cells, it then becomes the PSI-Cell approach. When the defined sphere (or ball) contains multiple computational cells, the interphase exchange source will take effect in all those nodes that fall within the ball. Figure 7-11 shows this idea schematically in a 2D layout.

It should be pointed out that the PSI-Ball is physically justifiable. In a nonuniform grid, for example, with the PSI-Cell, small cells tend to have lower probability to "host" a particle and consequently have less chance to receive a share of the interphase force than a large cell does. On the other hand, such unfairness does not exist with the PSI-Ball method. Also, in a region of relatively large local volume fraction multiple balls centered at each particle location may overlap and produce an accumulative effect of the backward coupling. Thus, the PSI-Ball approach can properly reflect the local agglomeration of the particles.

One may ask: does the redistribution of the coupling point force contradict the point-volume assumption? The answer is yes and no. Strictly speaking, the point-volume assumption is not valid for a relatively large particle. However, in the present context, the point-volume should be understood as a modeling approach rather than the assumption itself. One has seen that, the point-volume treatment can be used to greatly facilitate the Lagrangian tracking technique. On the other hand, when the backward force coupling is 
concerned, the location of the point forces does not coincide with the Eulerian grid nodes in general, and a mapping from the particle location to the designated nodes becomes a must. The force redistribution in the backward coupling further conforms to the physical intuition: the local disturbances generated by a particle should occur in its immediate neighborhood, but not at a single point. It becomes further necessary in a LES approach because of the filtering operation imposed to the original N-S equations. Given above, the force redistribution in the LES has a numerical, physical and mathematical need, and it should not be confused with the point-volume assumption mainly applied as a model in the forward coupling.

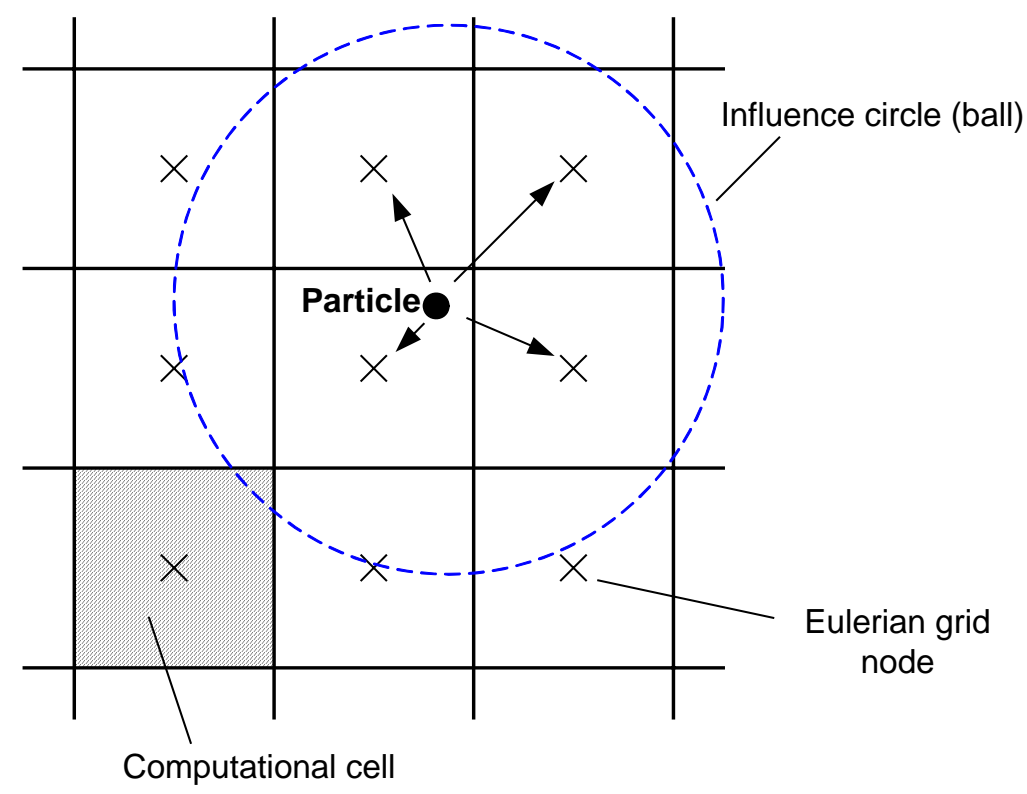

Figure 7-11 Schematic of force redistribution using a predefined influence circle or ball (PSI-Ball).

As to the selection of the influence ball diameter, $D$, several necessary conditions apply. Obviously, $D$ is larger or equal to the particle diameter, $d_{p}$. Second, $D$ should be larger than or equal to the maximum grid spacing, $h_{\max }$, because otherwise the ball can fall completely inside a cell and as such no coupling force can be transferred to the cell nodes. Third, $D$ should not be too large because the particle disturbance is local effect. Based on these, in the present study, $D$ is taken as:

$$
D=\max \left(2 d_{p}, 2 h_{\max }\right) .
$$

Effectively, Eq. (7.220) implies the following: when the particle is small as compared to the grid size, it takes the nearest neighbors (four nodes in Figure 7-11 or eight nodes in a corresponding $3 \mathrm{D}$ arrangement) for the coupling. When the particle is relatively large, the particle force will then be distributed to those nodes that fall within a sphere of $2 d_{p}$ in diameter.

A distribution function or envelope function (cf. Section 7.8), g, can be chosen to control the distribution weight lent to a nearby node or a faraway node. A typical choice 
of $g$ is the Gaussian function with zero mean. The variance should be selected according to the ball size. For example,

$$
\sigma=2 D
$$

\subsubsection{Two-layer View}

The two-layer view of a dispersed two-phase system is simply a convenient way to summarize and visualize the key contents presented in the previous subsections. In this concept, the carrier phase and the dispersed phase are viewed as two independent layers, whose interaction occurs at those discrete particle locations through modeled momentum exchange forces (Figure 7-12). Layer here means a computational entity or system, and it can be $1 \mathrm{D}, 2 \mathrm{D}$ or $3 \mathrm{D}$. In this study, the fluid layer is calculated in the Eulerian reference frame and the particle layer in the Lagrangian reference frame. As the picture suggests, the two layers are decoupled in terms of their geometry. Particles in the particle layer can be relatively large and their sizes are independent of the Eulerian grid size. The volume fraction of the particle layer can be relatively large so long as the dispersed condition is not violated. The particle-to-fluid interaction (backward coupling) is achieved with the proposed PSI-Ball method. In essence, the two-layer concept is a combination of the three A's, namely, an assumption, an assertion and an approach (PSI-Ball). With this concept, one expects a largely expanded class of two-phase flow problems that can be tackled using a point-volume E-L LES.

It should be stressed that one of the goal of this study is to break the size hurdle set for the particles as well as the computational cells. Also note that, before a wellestablished two-phase SGS model becomes available, single-phase SGS models will be the primary choice, in the present study, for the LES closure.

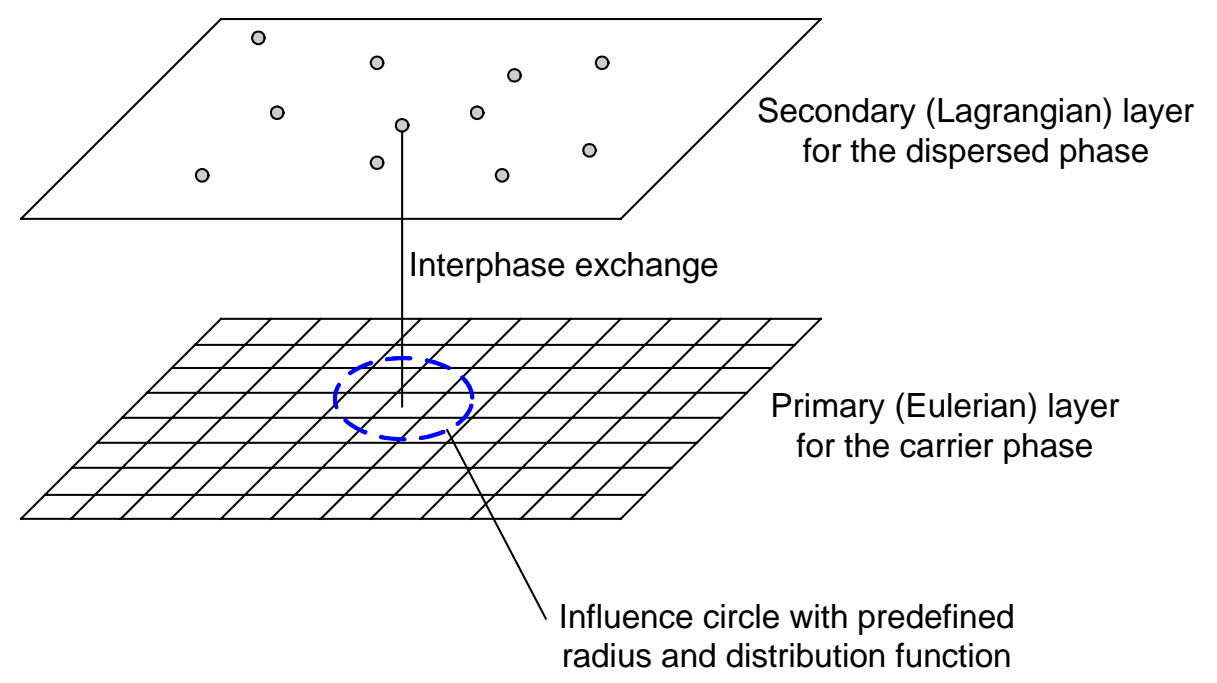

Figure 7-12 Schematic of two-layer concept. 


\section{Chapter 8 Large Eddy Simulation of Bubble Column}

Gas-liquid turbulent bubbly flow in a flat bubble column is studied by means of largeeddy simulation (LES), combined with Lagrangian particle tracking under the condition of two-way coupling. The incompressible spatially filtered Navier-Stokes equations are solved to compute the turbulent, bubble-driven liquid velocity field, while the motion of the dispersed phase is tracked in the Lagrangian reference frame with each individual bubbles being treated as a point source. The influence of the dispersed phase on the liquid phase is assumed to occur through the momentum-exchange terms. Here, the two-layer concept is adopted. Key elements of this new method to model dispersed two-phase flows have been described in Section 7.9. In short, it permits the existence of relatively large bubbles/particles, and the reverse coupling is implemented by way of PSI-Ball. With respect to the SGS closure, the classical Smagorinsky model is used. Bubble coalescence and break-up are neglected. Other theoretical aspects of the Eulerian-Lagrangian (E-L) LES of dispersed two-phase flows, such as hydrodynamic forces, two-way coupling, and interpolation, have been presented in detail in the preceding chapter.

Considered in this study is a locally aerated flat bubble column based on the experimental study of (Becker et al. 1994) and later (Sokolichin and Eigenberger 1999). The bubble-dispersion characteristics are examined in detail, the results are in very good agreement with the experimental data. The LES results also reveal that the bubble dispersion pattern and the vortical structure of the liquid phase differ significantly from that obtained from a 2D simulation reported by (Delnoij et al. 1997b). Influence of the grid resolution is also addressed.

\subsection{Introduction}

A flat bubble column is a liquid-containing apparatus with a rectangular cross-section and very small dimension in depth (see, for example, Figure 8-1). Gas spargers are mounted at the bottom of the column, from which air bubbles are continuously released. The initially stationary liquid is set into motion as a result of the driving effect of the buoyant bubbles. Owing to the small column depth, the flow developed can be regarded as twodimensional if only large-scale structures (whose size is greater than the column depth) are of interest. According to the bubble injection configurations, the bubble columns can be typically classified into partially (or locally) aerated and fully (or uniformly) aerated bubble columns. With the partially aerated ones, if the aeration is located at the center of the bottom, it is then called center-aerated (or symmetrically aerated) bubble column.

The flat bubble column has gained special interest in the gas-liquid dispersed twophase flow research because of its simple construction and complex flow phenomena 
associated with it. Knowledge gained from the study of laboratory bubble columns has served as the foundation, upon which related industrial applications, such as the bubble column reactors, are developed. The bubble column reactors have been widely employed in biological, chemical, petrochemical and other process-related industries.

In the multiphase CFD community the flat bubble columns have been widely used as a standard test to validate and evaluate two-phase flow models and simulation codes. First, reliable experimental data are available in the literature for various bubble column configurations. Second, most of the known hydrodynamic forces acting on an individual gas bubbles (cf. Section 7.5), including gravity, buoyancy, drag, lift, fluid stress and added mass forces, will play a role in the simulation. Neglecting any of these forces may lead to erroneous behavior of predicted bubble dispersion. Third, it is an excellent case to test a two-way coupling model. While in some other two-phase flow problems, such as the particulate channel flows, one-way coupling (i.e., without considering the reverse effect of particles on the flow) can be an acceptable alternative, the simulation of bubble columns however, must incorporate the two-way effect because otherwise the initially still fluid will not move. Moreover, it provides a crucial benchmark of evaluating E-E (Eulerian-Eulerian) models and E-L (Eulerian-Lagrangian) models.

In general, several factors can affect the fluid dynamics of a bubble column. The most prominent ones are location and uniformity of the bubble injection, gas flow rate, column aspect ratio (column height to width). Depending on a specific configuration, both the liquid phase and the dispersed phase will exhibit significantly different behaviors in terms of the flow regimes, vortical structures, characteristic time and length scales, and the bubble dispersion pattern. For example, (Chen et al. 1989) conducted an experiment on uniformly aerated bubble columns, and a cooling tower pattern is observed when the aspect ratio is small, whereas large aspect ratio produces staggered rows of vortices. (Becker et al. 1994) investigated a locally aerated bubble column and reported that at different gas flow rates the flow can be quasi-steady or oscillatory.

With respect to the fluid-dynamic modeling there are several scenarios that need to be considered with care. First, which approach is more suitable, the E-E or the E-L? Second, which governing equations should be used? Third, must a turbulence model be included, or will a laminar flow assumption suffice? The answers are presented in the following.

To question (i). A majority of the simulation work in the literature has adopted the E-E approach, for example, (Grienberger and Hofmann 1992; Ranade 1992; Sokolichin and Eigenberger 1994; Borchers et al. 1999; Sokolichin and Eigenberger 1999). Studies using the E-L approach are (Delnoij et al. 1997a; Delnoij et al. 1997b; Sommerfeld et al. 1997). In principle, both formulations should lead to close results, provided that similar terms are accounted for in both cases and the numerical discretization is sufficiently fine (Sokolichin et al. 1997). This suggests that the numerical diffusion inherited from the E-E approach can be critical, as there is the possibility of generating scheme-dependent falsedispersion of the particle (here bubble). On the other hand, although the E-L approach 
completely avoids the issue of numerical diffusion, the computational expense limits the number of particles that can be tracked, henceforth the quality of the statistical sample.

To question (ii). In principle, the governing equations for the liquid phase should be chosen according to the specific model formulation. With the E-E model, the volumeaveraged equation or the equation for a mixed fluid is typically formulated. With the E-L model, the volume-averaged equation or the Reynolds-averaged equation is often used for the liquid phase.

To question (iii). If the turbulence effect needs to be considered, the dominant turbulence models being applied are of RANS type, typically the $k-\varepsilon$ model developed from a single-phase turbulent flow, i.e. (also see Eq. (4.46) in Section 4.2),

$$
\mu_{e f f}=C_{\mu} \rho_{l} \frac{k^{2}}{\varepsilon} \text {. }
$$

In situations where the $k-\varepsilon$ model produces excessive dampings (corresponding to overpredicted effective viscosity), an empirical effective viscosity is often specified. For example, (Becker et al. 1994) increased the liquid viscosity by a constant factor of 100. In another work of (Yang et al. 2002b), albeit on a different bubbly flow case (bubble-laden mixing layer), the same magnifying factor was used. It is then further possible to simply neglect the turbulent viscosity and let the effective viscosity be approximated by the liquid viscosity, i.e.,

$$
\mu_{\text {eff }} \cong \mu_{l}
$$

where $\mu_{l}$ represents the liquid viscosity. Models using Eq. (8.2) is sometimes called the laminar model, and it was used for example in the study of (Sokolichin et al. 1997) and (Delnoij et al. 1997b). Overall, it appears that there is no unified answer to the question whether a turbulence model or a laminar model is more suitable, and the choice is judged on a case-by-case basis by the researchers. However, as the text develops, it will be seen that the flow field in the flat bubble columns under consideration does manifest itself with turbulence features, and the absence of a turbulence model lacks a theoretical justification.

It is interesting to note that the above survey is mainly taken from the multiphase research literature. In the turbulence community, direct numerical simulation (DNS) with two-way coupled Lagrangian particle tracking has served as a powerful tool in studying the particle dispersion and turbulence modulation effect. See (Squires and Eaton 1990, 1991b; Elghobashi and Truesdell 1992, 1993; Wang and Maxey 1993; Pan and Banerjee 1996; Maxey et al. 1997; Boivin et al. 1998; Sundaram and Collins 1999; Druzhinin and Elghobashi 2001; Ferrante and Elghobashi 2003). In this approach the original NavierStokes equations are solved directly. To reduce the computational cost, development of LES for particle-laden (or bubble-laden) turbulent flows have been made in recent years. See (Wang and Squires 1996b; Boivin et al. 2000; Fukagata 2000; Yamamoto et al. 2001; Milelli 2002; Apte et al. 2003b). Different from DNS, the LES solves the filtered N-S equations, and thus a subgrid-scale (SGS) model is required to close the resulting subgrid stresses. Although SGS models for single-phase phase flows have been well established, development of reliable SGS closures in the two-phase regime still presents the major 
challenge in applying LES to this category. Although several two-phase SGS models was attempted (Fukagata 2000; Milelli 2002), no generalization has been widely accepted. Before such a one becomes available, most of the up-to-date two-phase LES simulations still adopt the single-phase SGS models. This is very similar to the RANS-based twophase approaches where RANS turbulence models are directly applied. Two common points of the aforementioned DNS and LES two-phase research are that (i) the reverse coupling is formulated via an interphase force in the momentum equations, and (ii) the particle dimension is required to be much smaller than the smallest resolved length scales of turbulence, so that the point-mass of the particle can be assumed, and the equation of motion for a point mass can be used. Notably, the point (ii) sets a stringent restriction on the application of LES to a wide range of two-phase flow problems, such as many bubbly flows where bubble sizes are relatively large (e.g., on the order of millimeter). Also refer to Section 7.6 and 7.9 for more details.

In this study an engineering approach is sought to advance the LES capability in simulating dispersed two-phase flows with relatively large bubbles, such as the case in bubble columns. In Section 7.9 a two-layer concept has been proposed with the reverse coupling being implemented with the help of an influence circle (or ball, PSI-Ball). This approach attempts to break the size hurdle set for the particle as well as the computational cell. In the following sections, a classical bubble column is selected as an example to illustrate the vitality of such a LES calculation.

\subsection{Experimental Setup and Flow Feature}

Considered is a locally aerated flat bubble column according to the experiment conducted by (Becker et al. 1994). The experimental setup is depicted in Figure 8-1. The column has a dimension of $2 \mathrm{~m} \times 0.5 \mathrm{~m} \times 0.08 \mathrm{~m}$ in height, width and depth, respectively. The liquid level used in the experiment is $1.5 \mathrm{~m}$. The gas distributors, flush-mounted on the bottom of the column, are made up of $40 \mathrm{~mm}$ plastic discs with a mean pore size of $40 \mu \mathrm{m}$. In the experiment the gas was fed from the leftmost disk (shaded circle in Figure 8-1) located $0.15 \mathrm{~m}$ from the left wall.

With a given geometry, the two-phase flow pattern is mainly dependent of the gas flow rate, $Q$. It is found that at $Q$ below about $3.6 \mathrm{l} / \mathrm{min}$, the flow exhibits strong transient characteristics. The gas flow rate used in the experiment is $1.6 \mathrm{l} / \mathrm{min}$, which corresponds to a superficial gas velocity of $0.66 \mathrm{~mm} / \mathrm{s}$. At this gas throughput it is experimentally observed that, several staggered liquid recirculation zones are formed, which change their location, shape and size in time. The action of these unsteady, large structures leads to a swinging and meandering shape of the rising bubble clouds (Figure 8-2b). An interesting phenomena is that the direction of the lower part of the bubble swarm is relatively stable and it is always directed towards the near sidewall.

Time histories were recorded for the vertical velocity component at two selected locations (point A and B in Figure 8-2b). Point A (in bubbly flow zone) is located at $0.9 \mathrm{~m}$ above the distributor and $0.035 \mathrm{~m}$ from the left wall, while point B (in bubble-free zone) 
is 1.05 away from the bottom and 0.05 from the right wall. The two time series are shown in Figure 8-2a. Note that this figure is reproduced by (Sokolichin and Eigenberger 1999) based on the original work of (Becker et al. 1994). The turbulent nature of the flow field is thus evident: large-scale low-frequency structure is superimposed by small-scale highfrequency random fluctuations. From this figure one further sees a periodic change of the vertical velocity. The dominant period calculated from point $A$ is about $41 \mathrm{~s}$, which agrees with the visual observations of the oscillatory movement of the rising bubble swarm.

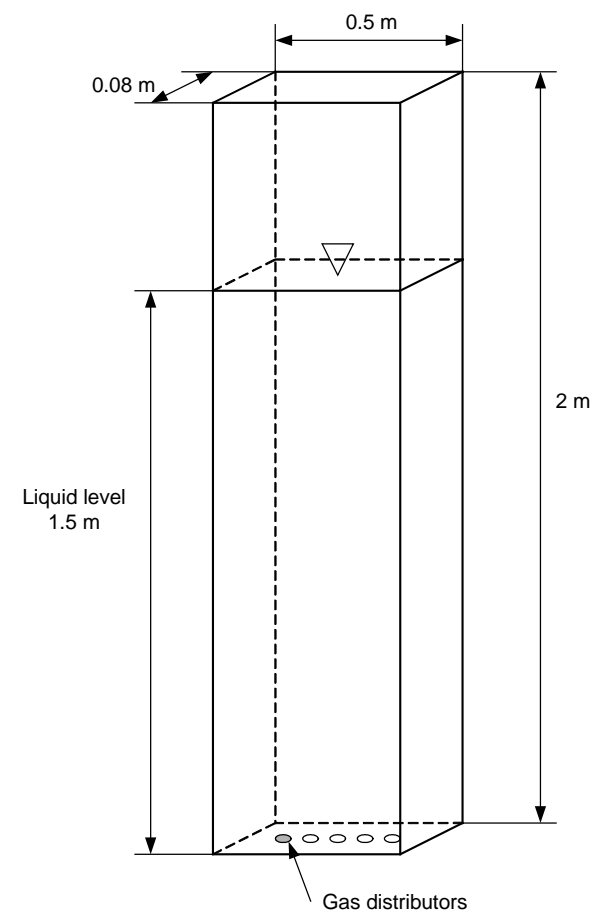

Figure 8-1 Sketch of flat bubble column used by (Becker et al. 1994).
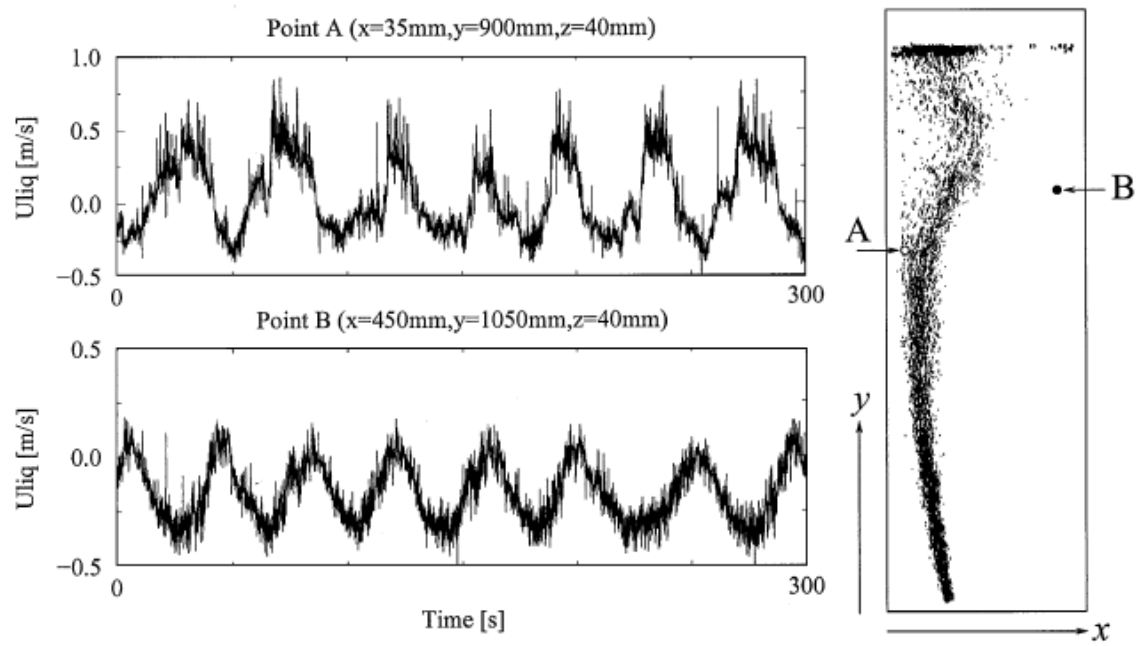

(a)

(b)

Figure 8-2 (a) LDA-measurements of vertical velocity at two selected locations A and B in a locally aerated bubble column. (b) A typical instantaneous snapshot of rising bubble swarm. (Reprinted from (Sokolichin and Eigenberger 1999) with permission). 


\subsection{Two-dimensional Test Simulation}

(Delnoij et al. 1997b) performed a two-dimensional simulation of this particular bubble column using the Eulerian-Lagrangian (E-L) approach on a $50 \times 100$ uniform grid. The volume-averaged equations were used in their study, with the phase coupling being modeled through a force term in the momentum equations:

$$
\begin{gathered}
\frac{\partial}{\partial t}\left(\alpha_{l} \rho_{l}\right)+\nabla \cdot\left(\alpha_{l} \rho_{l} \mathbf{u}_{l}\right)=0 \\
\frac{\partial}{\partial t}\left(\alpha_{l} \rho_{l} \mathbf{u}\right)+\nabla \cdot\left(\alpha_{l} \rho_{l} \mathbf{u u}\right)=-\alpha_{l} \nabla P+\nabla \cdot\left(\alpha_{l} \mu_{l} \nabla \mathbf{u}\right)+\alpha_{l} \rho_{l} \mathbf{g}+\mathcal{I}
\end{gathered}
$$

where

$$
\mathcal{I} V_{\text {cell }}=\sum_{n}\left(F_{D, n}+F_{L, n}+F_{A, n}\right)
$$

and the subscript $l$ denotes the liquid phase. The liquid void fraction $\alpha_{l}$ is calculated from

$$
\alpha_{l}=1-\frac{\sum_{n} V_{b, n}}{V_{\text {cell }}} \text {. }
$$

where $V_{b, n}$ is the volume of the $n$th bubble, and $V_{\text {cell }}$ is the volume of the computational cell. Due to the two-dimensional nature of their implementation, a virtual third dimension was introduced for the calculation of $V_{\text {cell }}$. This third dimension can be derived from the relation between the two-dimensional and three-dimensional global void fraction in the respective $2 \mathrm{D}$ and $3 \mathrm{D}$ space. A laminar viscosity (that of the liquid) was assumed in their calculation without a turbulence model. For the Lagrangian particle trajectory tracking, the following hydrodynamic forces are considered:

$$
\mathbf{F}_{p}=\mathbf{F}_{G}+\mathbf{F}_{S}+\mathbf{F}_{D}+\mathbf{F}_{L}+\mathbf{F}_{A} .
$$

This choice and significance of these forces for light particles, such as air bubbles under consideration, have been addressed in detail in Section 7.5. The bilinear interpolation was employed for both the forward and backward interpolation. Also, an elastic, irrotational collision model was exercised to account for the bubble-bubble interaction.

The work of (Delnoij et al. 1997b) is the closest found in the literature that has a direct relevance to both the two-way coupled E-L approach and the (Becker et al. 1994)'s flat bubble column case. A similar two-dimensional E-L simulation is then carried out in the present study based on the same formalism, numerical methods and computational grid as described in the preceding paragraph. Same as in the original calculation the bubble size issue is simply neglected as long as the computational cell size is larger (but not much larger) than the particle size. The only unmatched implementation is a collision model, which is applied in the original work, but neglected in the current study for the sake of simplicity. The goal of repeating such a $2 \mathrm{D}$ calculation is to verify the current EL implementation with two-way coupling. Some key computational parameters are listed in Table 8-1. Note that these parameters are same as those used in the Delnoij et al.'s simulation. 
Table 8-1 Parameters used in the 2D simulation of a locally aerated bubble column.

\begin{tabular}{|l|l|l|l|}
\hline Column height & $1.50(\mathrm{~m})$ & Gas flow rate & $1.6(1 / \mathrm{min})$ \\
\hline Column width & $0.50(\mathrm{~m})$ & Bubble diameter & $2.0(\mathrm{~mm})$ \\
\hline Orifice width & $0.04(\mathrm{~m})$ & Number of x-cells & 50 \\
\hline Liquid (water) density & $1.0 \times 10^{-3}\left(\mathrm{~kg} / \mathrm{m}^{3}\right)$ & Number of y-cells & 100 \\
\hline Liquid viscosity & $1.0 \times 10^{-3}\left(\mathrm{~N} \cdot \mathrm{s} / \mathrm{m}^{2}\right)$ & Time step & $5.0 \times 10^{-3}(\mathrm{~s})$ \\
\hline Gas (air) density & $1.2\left(\mathrm{~kg} / \mathrm{m}^{3}\right)$ & & \\
\hline
\end{tabular}

However, it is important to keep in mind that the results presented by (Delnoij et al. 1997b) should be interpreted with caution. (Sokolichin and Eigenberger 1999) studied the same bubble column using an E-E approach and with a systematic grid refinement. In the case of $50 \times 100$ grid nodes without using any turbulence model, they obtained very similar results to those of (Delnoij et al. 1997b). On the other hand, laminar simulation on other coarser or finer grids produced largely varying flow structures (more vorticities were resolved with the refined grid) and it did not lead to a convergent solution. In fact, this is typical of a laminar calculation for a turbulent flow. They concluded that under the condition of laminar model, the results of simulation is strongly grid-dependent; a good agreement with the experiments should be regarded as a pure coincidence, which could be partially attributed to the diffusive nature of the $1^{\text {st }}$ order upwind scheme.

One more comment should be made here. The solution of volume-averaged equations gives only volume-averaged velocity field, but not the instantaneous ones. Use of the volume-averaged quantities in computing the particle trajectories should be thus regarded as a model. As already mentioned in Section 7.6.2, the non-instantaneous field, with the exception of filtered field calculated from a LES, cannot address the turbulent random motion of the particle. Therefore, in a turbulent flow simulation with a RANS or laminar model, a random force or a random velocity is typically incorporated into the calculation of the particle trajectory.

Given above discussions, it should be emphasized that conducting such a 2D E-L simulation according to (Delnoij et al. 1997b) is merely for the verification purpose. No intention will be given to a physical interpretation of the results. In particular, a side-byside comparison is conducted with the simulation of (Delnoij et al. 1997b), (Sokolichin and Eigenberger 1999) as well as the experimental data. This is presented as follows.

For brevity, (Becker et al. 1994) shall be referred to as Becker, (Delnoij et al. 1997b) as Delnoij, and (Sokolichin and Eigenberger 1999) simply as S\&E.

Figure 8-3 presents a comparison of instantaneous bubble dispersion patterns at a 10s interval among S\&E's experimental observations, current 2D E-L simulation, Delnoij's 2D E-L simulation, and S\&E's 2D E-E simulation. It is noted that the bubble swarms in the three 2D simulations have in common in that (i) they have similar forms of undulation, (ii) they all exhibit a quasi-periodic motion with about the same period, (iii) 
the oscillatory motion of the bubble dispersion pattern does not synchronize with the one observed in the experiment, (iv) the lateral bubble spreading is underpredicted as compared to the experiment. An interesting experimental observation is that the lower part of the bubble swarm is firmly pushed towards the left wall, irrespective of the relatively large oscillation of its upper part. This feature is captured in the present $2 \mathrm{D}$ simulation, whereas from the 2D results of Delnoij and S\&E the lower part of the bubble swarm also oscillates.

Figure 8-4 depicts the liquid velocity fields at the same five instants as that in Figure 8-3. Compared with each other are the present and Delnoij's 2D E-L simulations, as well as the 3D E-E results of S\&E. The three independent calculations generally show more or less different predictions in terms of the number, size and arrangement of gross recirculation zones. The present $2 \mathrm{D}$ results are compared more favorably with the S\&E's ones, since both show the existence of either two large eddies or three. On the other hand, Delnoij's calculation only shows two gross recirculation zones most of the time (except the one in the third plot).

The long-time averaged mean flow field is an important indicator of the reliability of the predicted results. The is shown in Figure 8-5, along with Becker's (b) and S\&E's (d) measurements, as well as Becker's 2D E-E simulation (c). The mean field of this flow case was reported in Delnoij's publication. From these plots, to be identified are one primary gross recirculation zone, three secondary mid-sized recirculation zones (two in the upper and lower part respectively, and one in the middle near the left wall), and one small tertiary recirculation zone in the left upper corner. Results from all the simulations and experiments are all in qualitatively good agreement. The shape and position of the secondary near-wall eddy given by the current $2 \mathrm{D}$ simulation is, however, somewhat different from that of other results. Also note that the seemingly good agreement of the Becker's 2D result could be questionable, as discussed in S\&E's work.

Figure 8-6 shows the time histories of the vertical velocity recorded at a selected point $\mathrm{A}$ in the mid-depth plane ( $900 \mathrm{~mm}$ from the bottom, $35 \mathrm{~mm}$ from the left wall). Showed are, in order, the current 2D E-L simulation, Becker's experiment, Becker's 2D E-E simulation, S\&E's experiment, and S\&E's 3D E-E calculation. A Fourier transform of the sampled signal reveals a periodicity of $40.96 \mathrm{~s}$ in the present prediction, which is rather close to the observed 41s in Becker's and S\&E's measurements. In the Delnoij's 2D E-L study an approximate period of 30s is reported (signal not shown here). Notably, none of the numerical calculations (2D E-L, 2D E-E, 3D E-E) is able to capture the turbulent random fluctuations, as is evident in the experimental data. The calculated signals are considerably smoothened out, and this is normally to be expected in a volumeaveraging-based two-phase calculation.

Overall, the present 2D E-L simulation, as a testing step towards a full 3D LES, delivers results similar to other $2 \mathrm{D}$ and $3 \mathrm{D}$ calculations. 

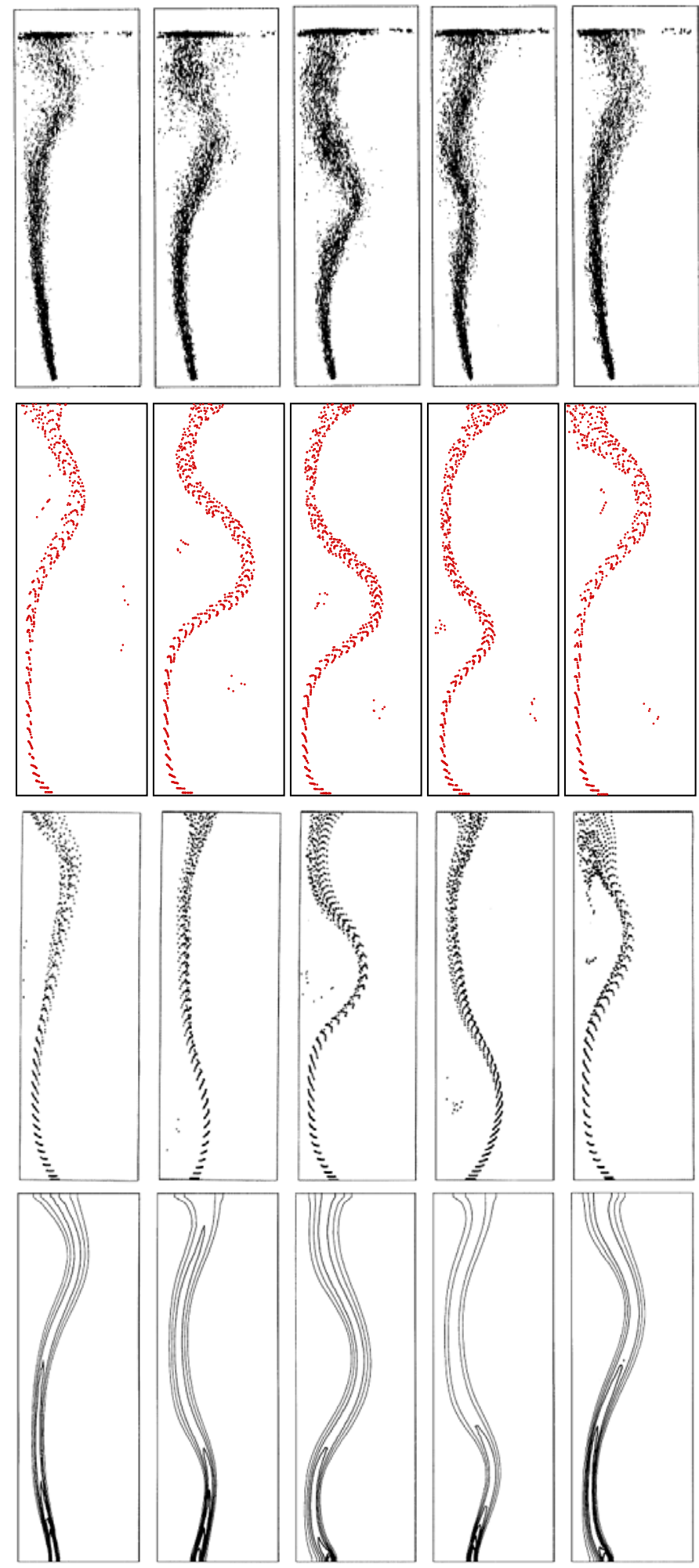

Figure 8-3 Instantaneous bubble dispersion in a locally aerated bubble column. From top to bottom: (a) Experiment (binary and inverted photograph) of (Sokolichin and Eigenberger 1999), (b) present 2D E-L simulation, (c) 2D E-L simulation of (Delnoij et al. 1997b), (d) 2D E-E simulation of (Sokolichin and Eigenberger 1999). Gas flow rate $=1.6 \mathrm{l} / \mathrm{min} . \Delta \mathrm{t}=10 \mathrm{~s}$. (Figures on $1^{\text {st }}, 3^{\text {rd }}$ and $4^{\text {th }}$ rows reprinted with permission). 

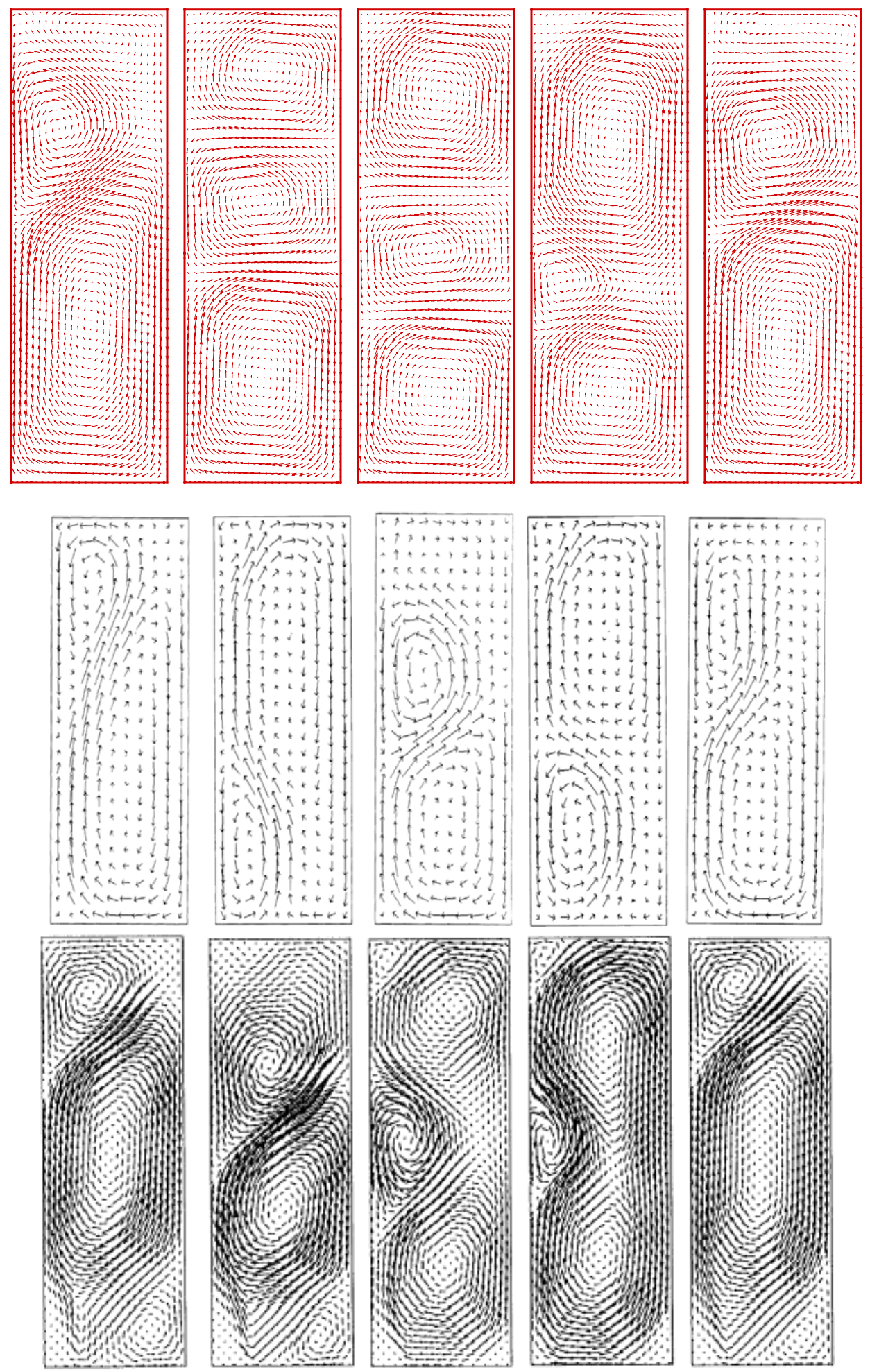

Figure 8-4 Instantaneous liquid vector field in a locally aerated bubble column. From top to bottom: (a) present 2D E-L simulation, (b) 2D E-L simulation of (Delnoij et al. 1997b), (c) 3D E-E simulation of (Sokolichin and Eigenberger 1999). Gas flow rate $=1.6 \mathrm{l} / \mathrm{min} . \Delta t=10 \mathrm{~s}$. (Figures on $2^{\text {nd }}$ and $3^{\text {rd }}$ rows reprinted with permission). 


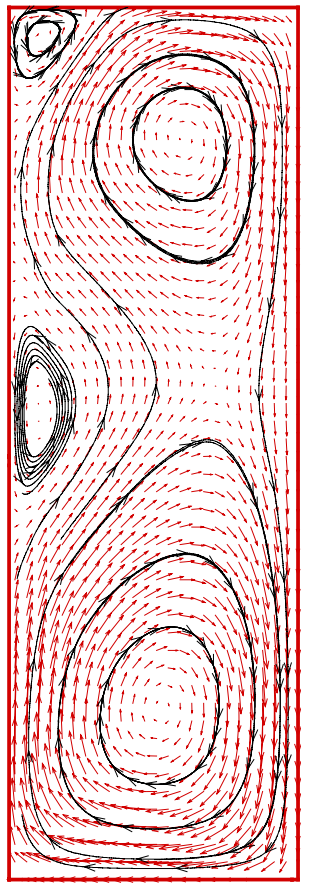

(a)

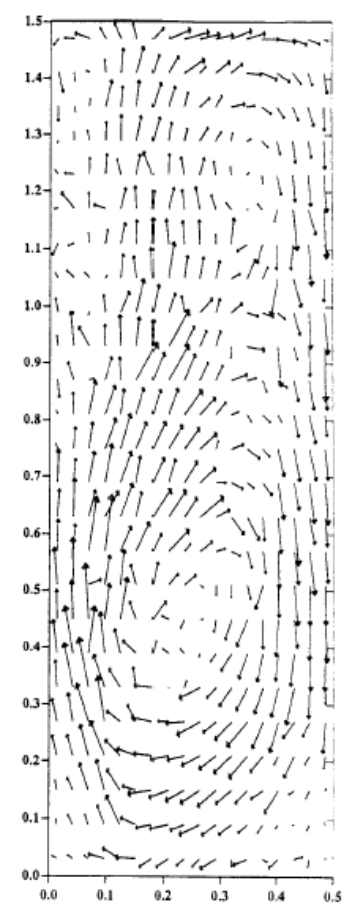

(b)

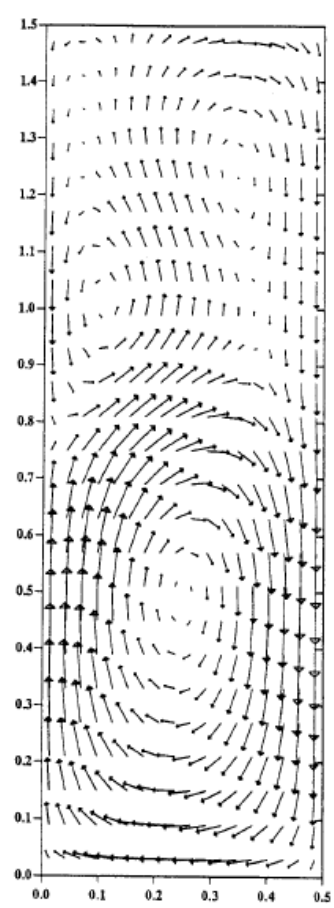

(c)

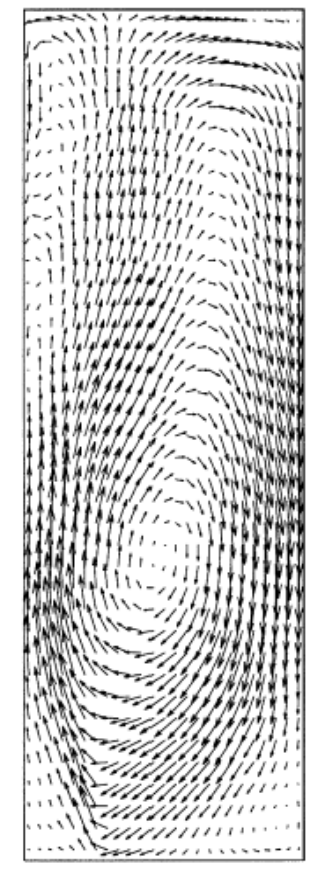

(d)

Figure 8-5 Long-time averaged liquid velocity field (a) present 2D E-L simulation (0 - 300s), (b) (Becker et al. 1994)'s experiment, (c) (Becker et al. 1994)'s 2D E-E simulation, (d) (Sokolichin and Eigenberger 1999)'s experiment. (Figures (b), (c) and (d) reprinted with permission). 

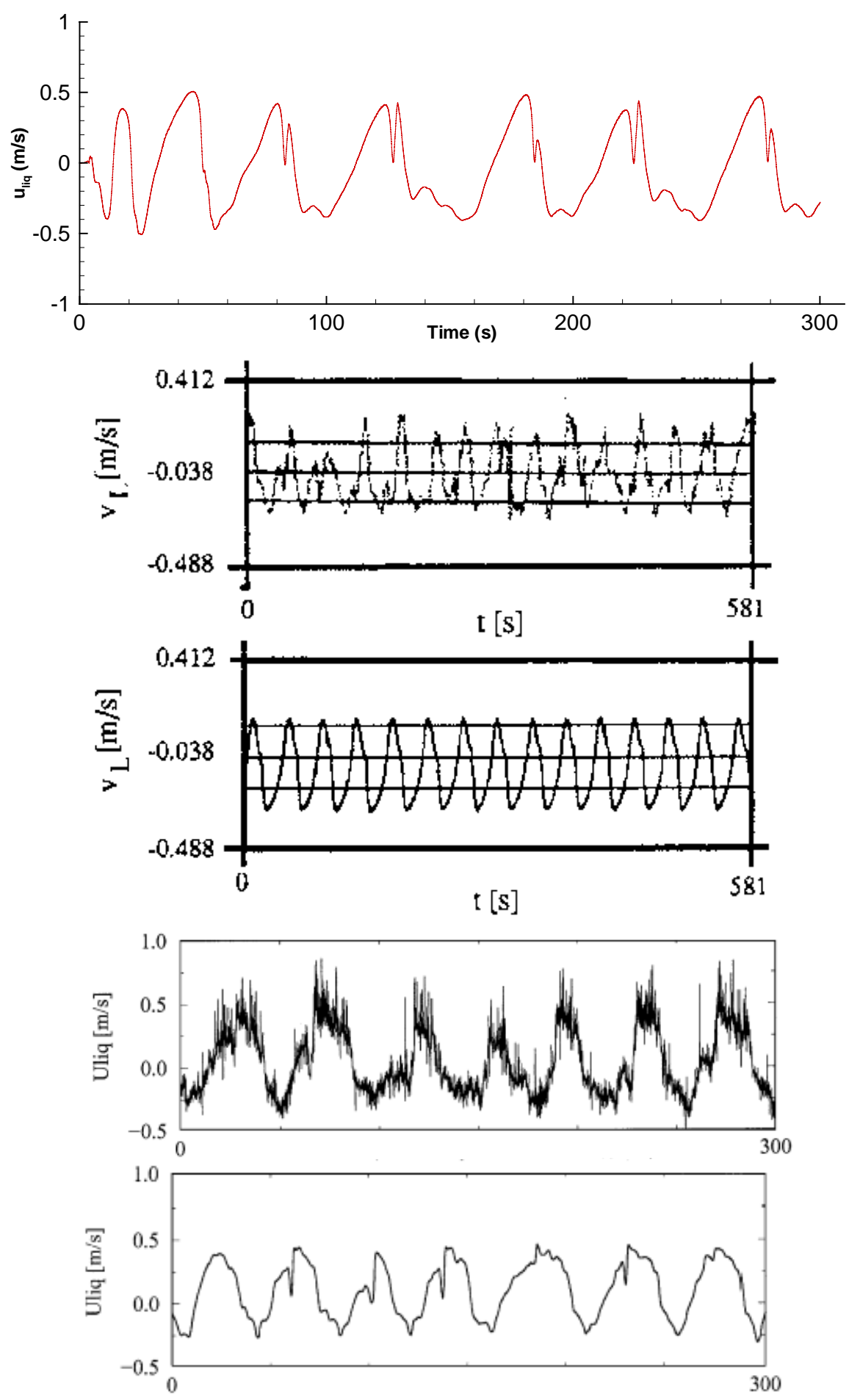

Figure 8-6 Time history of liquid vertical velocity sampled at point A ( $900 \mathrm{~mm}$ from bottom, $35 \mathrm{~mm}$ from left wall). From top to bottom: (a) present 2D simulation, (b) (Becker et al. 1994)'s experiment, (c) (Becker et al. 1994)'s 2D simulation, (d) (Sokolichin and Eigenberger 1999)'s experiment, (e) (Sokolichin and Eigenberger 1999)'s 3D simulation. (Last four figures reprinted with permission). 


\subsection{Large-eddy Simulation and Results}

The study of (Sokolichin and Eigenberger 1999) has demonstrated that, the solution of a 2D calculation without a turbulence model, i.e., only relying on the laminar assumption, is grid dependent and scheme dependent, irrespective of whether it is an E-L or E-E approach. The unsteady forms of undulation and periodicity predicted by such 2D simulations may be subject to serious error based upon comparison with the experimental measurements. They further pointed out that even when there exist a good agreement, e.g., the mean velocity field predicted by (Becker et al. 1994), it should be regarded as pure coincidence, in which the diffusive upwind scheme may play a role in dissipating turbulent energy.

Two-dimensional simulation using a turbulence model, typically the $k-\varepsilon$ model, has been performed in the past (Becker et al. 1994; Sokolichin et al. 1997; Sommerfeld et al. 1997; Sokolichin and Eigenberger 1999). But it is generally found that, the turbulent kinetic energy calculated in a 2D domain produces too excessive effective viscosity. For example, an $\mu_{\text {eff }}$ increase of up to a factor of 20,000 has been reported by (Becker et al. 1994). The failure lies in a complete neglect of the turbulence damping mechanism made by the front and back wall of the column. Moreover, since a $2 \mathrm{D}$ simulation with turbulence model rests on the assumption that the flow is statistically 2D, the use of a turbulence model usually leads to a steady-state solution (Sommerfeld et al. 1997; Sokolichin and Eigenberger 1999). The dynamic feature of the virtually 3D turbulent flow is simply lost.

A consequent effort is certainly a 3D simulation and with a turbulence model. Such an attempt has been made by (Sokolichin and Eigenberger 1999), who use an E-E formulation and pick the standard $k-\varepsilon$ to serve as the turbulence model. Close agreement with the experiments, with regard to the bubble dispersion pattern and the mean liquid velocity field, is obtained this time. The success is however, neither because of the E-E formulation, nor the turbulence model. The three-dimensionality helps the TKE to (have to) deliver strong damped values due to the existence of the front and back walls. Now, if only the mean quantity is concerned, then so far so good. However, when turbulence quantities are of primary interest, RANS-type E-E simulation can hardly survive, where the TKE or turbulence intensities are commonly underestimated to different degrees. This is evident by comparing a real-world signal (Figure 8-6d) with the calculated one (Figure $8-6 e)$.

The hope, if not DNS, must then be LES. In the previous chapters homework has been done regarding LES. Also a two-layer concept has been proposed that allows for an E-L LES with relatively large particles/bubbles, such as in the present bubble column case. Now, we would like to put all these together to carry out a quality E-L based LES for the same bubble column.

The geometry, physical properties and some key computational parameters are summarized in Table 8-2. The $2^{\text {nd }}$ order central differencing is applied to both convection 
and diffusion terms. Temporal integration is carried out using the $2^{\text {nd }}$ order AdamsBashforth. The standard Smagorinsky SGS model is used with the Smagorinsky constant $C_{S}$ set to 0.032 . It has been tested that larger values of $C_{S}$ may lead to excessive dampings of the liquid field, and eventually a steady-state solution. (So, this is the nice thing of the Smagorinsky model, where one can always adjust or fine-tune the results with only one constant, but not a bunch of them, as is the case with the RANS models!). It should be noted that this choice of $C_{S}$ is below the range $(0.05 \sim 0.25)$ which is commonly reported in the single-phase LES literature. A very possible cause of the low $C_{S}$ operating in the two-way coupled two-phase regime can be due to the interphase momentum exchange, which, besides the SGS model, may also partially dissipate TKE of the continuous phase. Considering possible insufficient wall-layer resolution (normal to the left and right walls), the van Driest wall-damping function is applied, as usual. Also, the two-layer concept with the PSI-Ball is implemented in this calculation. The influence sphere is set according to Eq. (7.220); the associated Gaussian distribution function has a variance of twice the sphere diameter. The calculation starts with the liquid at standstill and proceeds with a time step of 5.0E-3 for exactly 300 seconds (5 minutes). The instantaneous and post-processed results are presented as follows. Again, the shorthand S\&E is used in place of (Sokolichin and Eigenberger 1999), and Becker in place of (Becker et al. 1994).

Table 8-2 Parameters used in 3D E-L large-eddy simulation of a locally aerated bubble column.

\begin{tabular}{|l|l|l|l|}
\hline Column height & $1.50(\mathrm{~m})$ & Gas flow rate & $1.6(1 / \mathrm{min})$ \\
\hline Column width & $0.50(\mathrm{~m})$ & Bubble diameter & $1.6(\mathrm{~mm})$ \\
\hline Column depth & $0.08(\mathrm{~m})$ & Number of x-cells & 64 \\
\hline Orifice width & $0.04(\mathrm{~m})$ & Number of y-cells & 96 \\
\hline Liquid (water) density & $1.0 \times 10^{-3}\left(\mathrm{~kg} / \mathrm{m}^{3}\right)$ & Number of z-cells & 8 \\
\hline Liquid viscosity & $1.0 \times 10^{-3}\left(\mathrm{~N} \cdot \mathrm{s} / \mathrm{m}^{2}\right)$ & Time step & $5.0 \times 10^{-3}(\mathrm{~s})$ \\
\hline Gas (air) density & $1.2\left(\mathrm{~kg} / \mathrm{m}^{3}\right)$ & y-grid expansion ratio & 1.12 \\
\hline
\end{tabular}

Figure 8-7 shows bubble dispersion pattern at nine consecutive instants with a time interval of five seconds. The time between the first and last plot is 40 seconds and the shape of undulation between the two is similar, so the nine snapshots offer a complete picture of the oscillatory development of the bubble plume within one period. Comparing the simulated results (first row) with the experimental observations on row two and row four (post-processed binary and inverted photographs), a surprisingly good agreement is seen. Importantly, the lower part of the bubble plume is directed firmly towards the left wall, conforming to the experimental photographs. However, thinking of the lower part not participating in the swing motion can be wrong; it's safe to say it oscillates with very small amplitude, which is hardly appreciable. Note that the results from the S\&E's 3D EE simulation (third row) are also good.

Figure 8-8 presents a comparison of the continuous velocity vector field predicted by the current E-L simulation (middle row) and S\&E's E-E simulation (bottom row). The nine selected instants are same as those reported in Figure 8-7. It is seen that the primary gross recirculation zone and secondary smaller eddies from both simulations are similar 
in shape, size, position, orientation and their staggered arrangement. Also note, while the vector field calculated by the E-E approach is more organized, the instantaneous vortical structures captured by the E-L LES exhibit more random nature and more resemble a true turbulent flow field.

Figure 8-9 shows the time history of the vertical velocity at a selected point "A" ( $900 \mathrm{~mm}$ from the bottom, $35 \mathrm{~mm}$ from the left wall). There are two purposes of doing so. First, one can gain some preliminary picture of the turbulent fluctuations; and second, one may do an estimation of the natural period of the oscillating liquid field. It is evident that the signal produced by the present LES (a) resembles the measured (b) and (c) very well. A total of about seven periods within a 300s recording time are accurately captured. The peak magnitudes of the oscillation in the present LES tends to be smaller than S\&E's measurements but larger than Becker's ones. Also, the present LES signal (a) yields a mean amplitude of $-0.0276 \mathrm{~m} / \mathrm{s}$, while that reported by Becker et al. (c) is $-0.038 \mathrm{~m} / \mathrm{s}$. It is interesting to note that, although S\&E's E-E simulation (e) produces correct overall oscillatory behavior, the turbulent random fluctuations get lost due to the nature of their approach. This damped-out signal will be consequently linked to an underpredicted TKE level.

Note that, by only looking at the time series the signal can be regarded as quasiperiodic, i.e., not strictly periodic. By transferring the signal from the time domain into the frequency domain (e.g., using FFT), a dominant frequency surfaces. This is shown in Figure 8-11(a), which gives a period equal to 40.96s. Becker et al reported a measured period of $41 \mathrm{~s}$.

A similar time history comparison (Figure 8-10) is also made for a different point "B" located $1.05 \mathrm{~m}$ from the bottom, $50 \mathrm{~mm}$ from the right wall in the bubble-free zones. Similar comments as those in the preceding paragraph also apply here. The mean velocity calculated from signal (a) is $-0.118 \mathrm{~m} / \mathrm{s}$. The power spectrum of (a), given in Figure 8-11(b), shows a dominant frequency of $40.96 \mathrm{~s}$, which is same as that at point A.

The mean velocity vector field in the mid-depth plane is depicted in Figure 8-12(a), in a comparison with both experiments and S\&E's computation. It is noted that the gross recirculation zone is very well reproduced in the present LES (a). The shape, size and location of the upper-left-corner eddy are compared favorably with Becker's (b) and S\&E's (c) experimental measurements. With respect to the experiments, the S\&E's E-E simulation (d) underpredicts this left corner mean eddy to some degree. Also note that the strong clockwise mean recirculation in the lower part of the column explains why the bubble swarm in this lower region is pushed fest towards the left wall and thus is relatively insensitive to the overall oscillation.

A quantitative comparison of the mean vertical velocity at several vertical stations on the same mid-depth plane is presented in Figure 8-13. The overall agreement with the measurements is very good. At station $\mathrm{y} / \mathrm{H}=0.58$ and 0.7 , the measurements (dash-dot lines) may present some uncertainty near the left wall, as a typical sharp gradient in the near-wall region is not observed. 
Figure 8-14 show the turbulence intensity $\left(u_{\mathrm{rms}}\right.$ and $\left.v_{\mathrm{rms}}\right)$ as well as TKE obtained from the present LES. One sees that there is an increasing trend of the turbulence level from the bottom to the mid-height, reaching its maximum at about $\mathrm{y} / \mathrm{H}=0.58$, and then followed by a decreasing trend until turbulence is significantly suppressed in the near surface region. The locations of the peak TKE with an increasing liquid height undergo a slight shift towards the left wall up to $\mathrm{y} / \mathrm{H}=0.58$, then move back again towards the center. The largest TKE (valued at $0.036 \mathrm{~m}^{2} / \mathrm{s}^{2}$ ) turns out to be at about $\mathrm{y} / \mathrm{H}=0.58$ from the bottom and $0.07 \mathrm{~m}$ away from the left wall. This finding appears to be consistent with the bubble swarm motion shown in Figure 8-7, because at this height the curvature of the rising bubble cluster is subject to most frequent changes, and the oscillating amplitude is the largest. Contrary to this result, S\&E's 2D E-E simulation reported a maximum TKE at the mid-height of about $0.011 \mathrm{~m}^{2} / \mathrm{s}^{2}$ located in the center region (Figure $8-15$ ). This value is about three times smaller than the present prediction.

It is also necessary to take a look at the volume fraction of the dispersed phase. Since bubbles enter from the bottom and leave from the free surface at a constant or quasi constant rate, the global void fraction of the gas phase can be regarded as approximately constant. At time $t=300 \mathrm{~s}$, for example, the global void fraction obtained from the present simulation is about $0.24 \%$. Such a value is representative and suggests the system under consideration well qualifies for a dilute dispersed system (cf. Eqs. (7.19) and (7.20)). However, a small global volume fraction does not necessarily guarantee a small volume fraction locally. This is shown in Figure 8-16, where the nine instantaneous local volume fraction contours are computed from the same instants as in Figure 8-7. The comparison between the present (upper row) and S\&E's (lower row) results evinces a qualitatively similar distribution of the volume fraction from two independent simulations. From the upper row of Figure 8-16 it is seen that, a local void fraction as large as $1 \%$ to $2 \%$ is present near the injection region and along the center of the bubble swarm. This range is further confirmed in the contour plot of the mean (long-time averaged) local volume fraction (Figure 8-17). In strict sense, with the relatively high volume fraction at the center of the bubble plume, the particle-particle interaction may not be negligible and the two-phase flow may not be considered dilute locally. However, with the two-layer notion introduced in Section 7.9, the restrictive dilute condition (Eq. (7.19)) may be relaxed so that a relatively dilute flow, such as the current one with local void fraction of $1 \%$ or $2 \%$, is also admissible. In fact, this is one of the reasons that the bubble column is chosen for the validation study of the proposed idea. The excellent agreement with the experiments presented in this subsection conveys an important piece of information; that is, under the condition of relatively large particle size and relatively large volume fraction, the point volume approach can still serve as a viable model to accurately capture the dynamics of the both phases. 


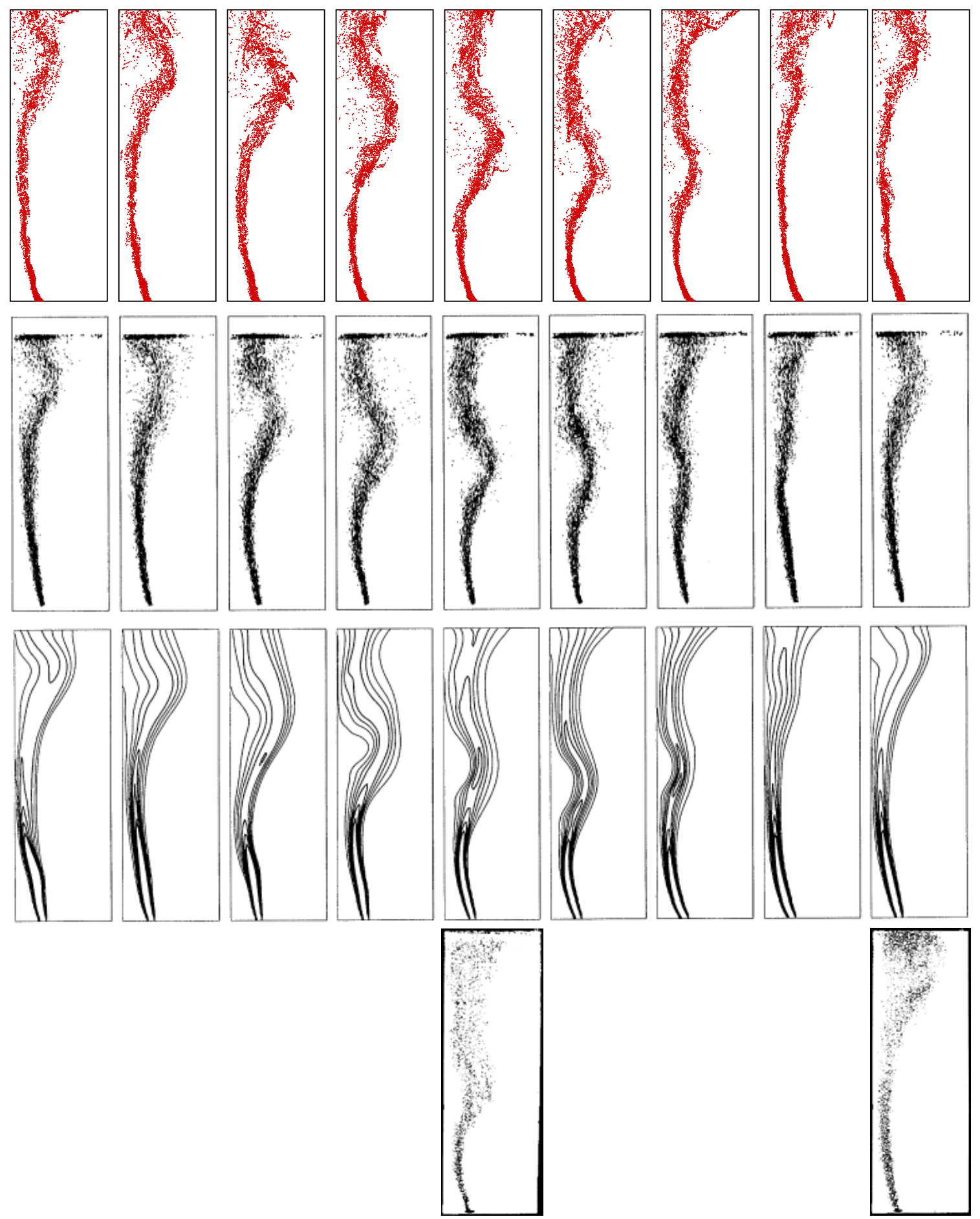

Figure 8-7 Instantaneous bubble dispersion in a locally aerated bubble column. From top to bottom:

(a) present LES, (b) experiment of (Sokolichin and Eigenberger 1999), (c) E-E 3D simulation of (Sokolichin and Eigenberger 1999), (d) experiment of (Becker et al. 1994). Gas flow rate $=1.6 \mathrm{l} / \mathrm{min}$. $\Delta t=5$ s. (Figures on last three rows reprinted with permission). 

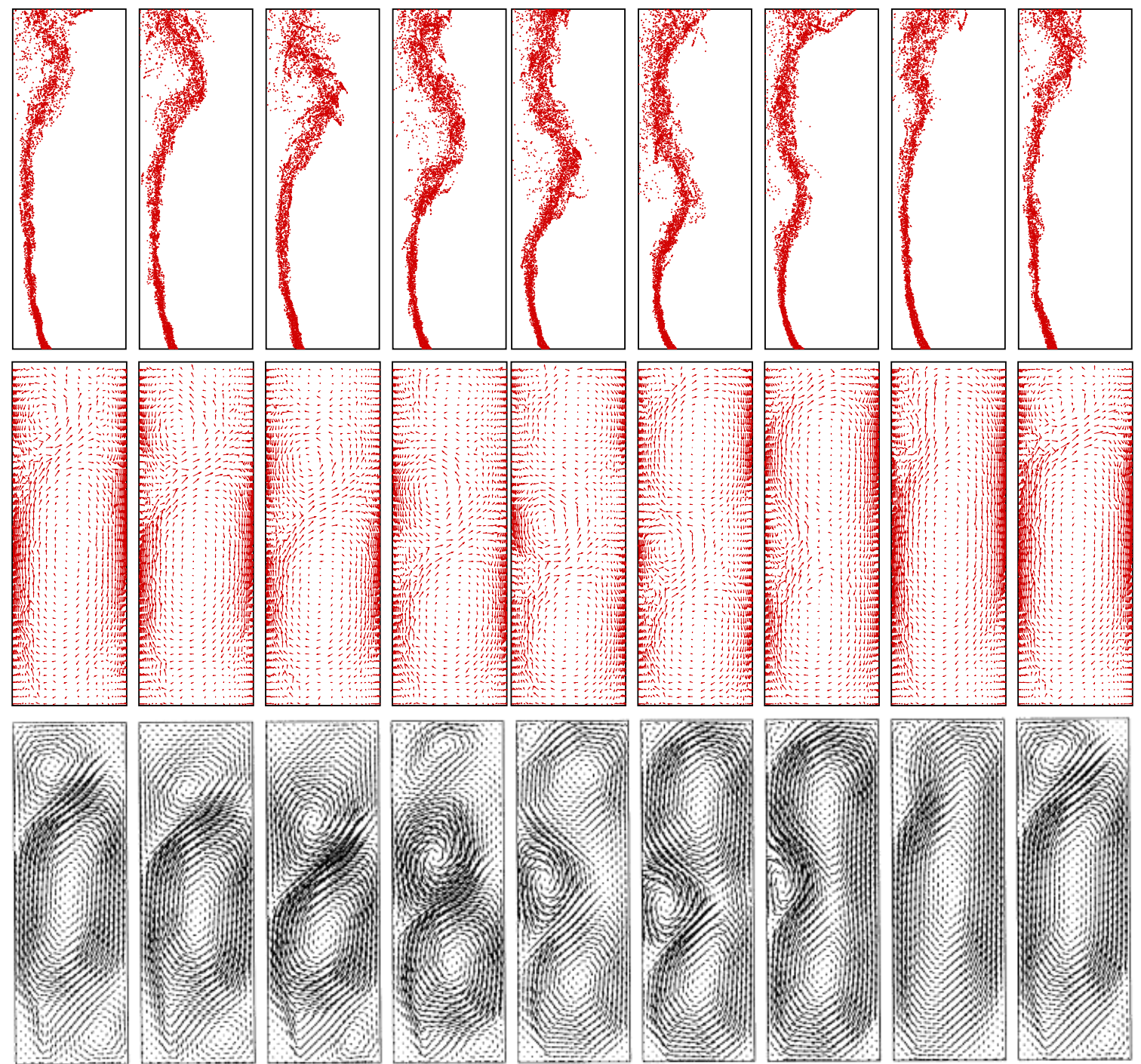

Figure 8-8 Instantaneous liquid vector field in a locally aerated bubble column. From top to bottom: (a) instantaneous bubble dispersion (from present LES), (b) present LES (grid index skip in both $x-$ and y-directions is 2), (c) E-E 3D simulation of (Sokolichin and Eigenberger 1999). Gas flow rate = $1.6 \mathrm{l} / \mathrm{min} . \Delta \mathrm{t}=5 \mathrm{~s}$. (Figure on $3^{\text {rd }}$ rows reprinted with permission). 

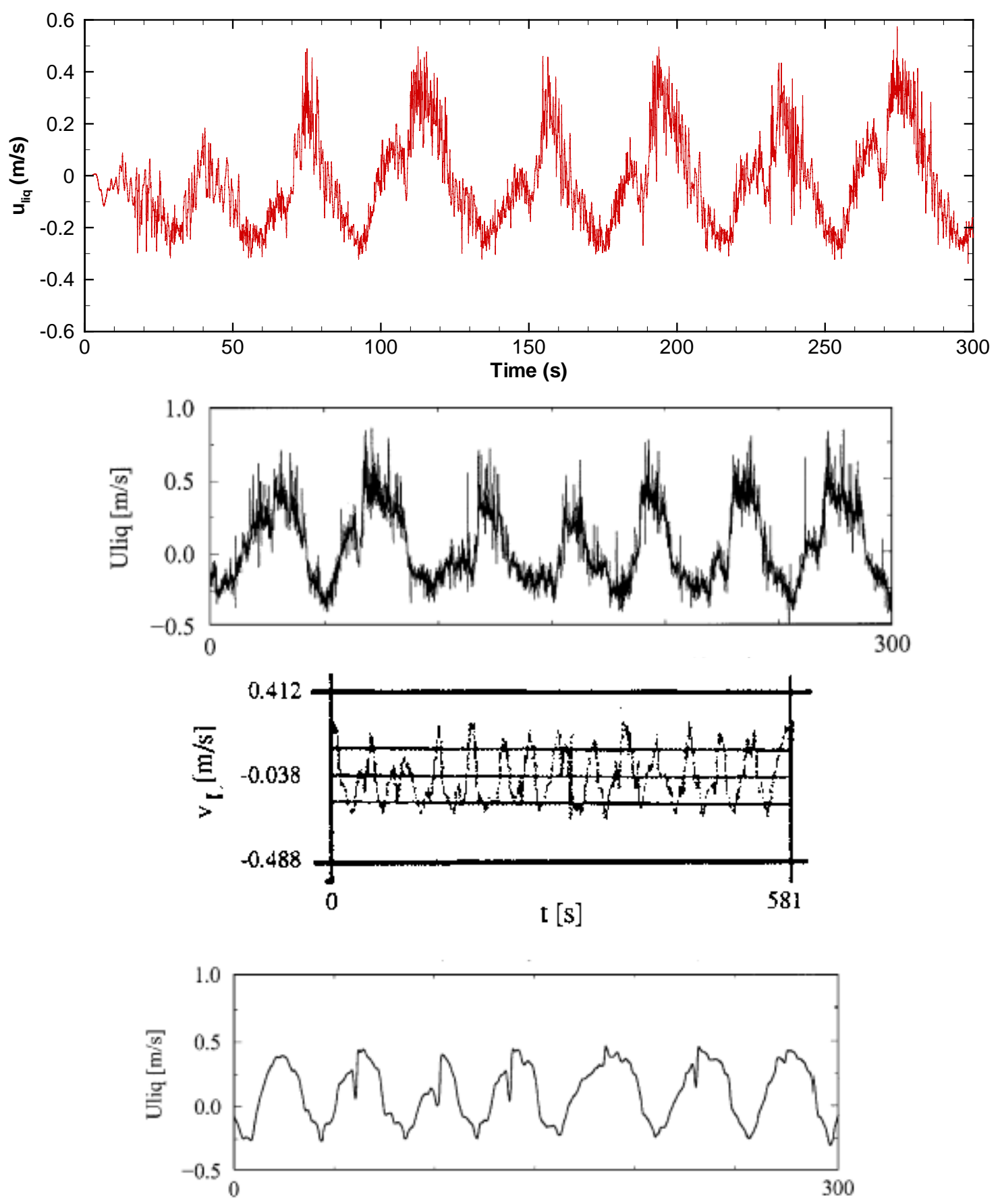

Figure 8-9 Time history of liquid vertical velocity sampled at point A ( $900 \mathrm{~mm}$ from bottom, $35 \mathrm{~mm}$ from left wall). From top to bottom: (a) present LES, (b) (Sokolichin and Eigenberger 1999)'s experiment, (c) (Becker et al. 1994)'s experiment ( 0 - 581s), (d) (Sokolichin and Eigenberger 1999)'s 3D simulation. (Last three figures reprinted with permission). 

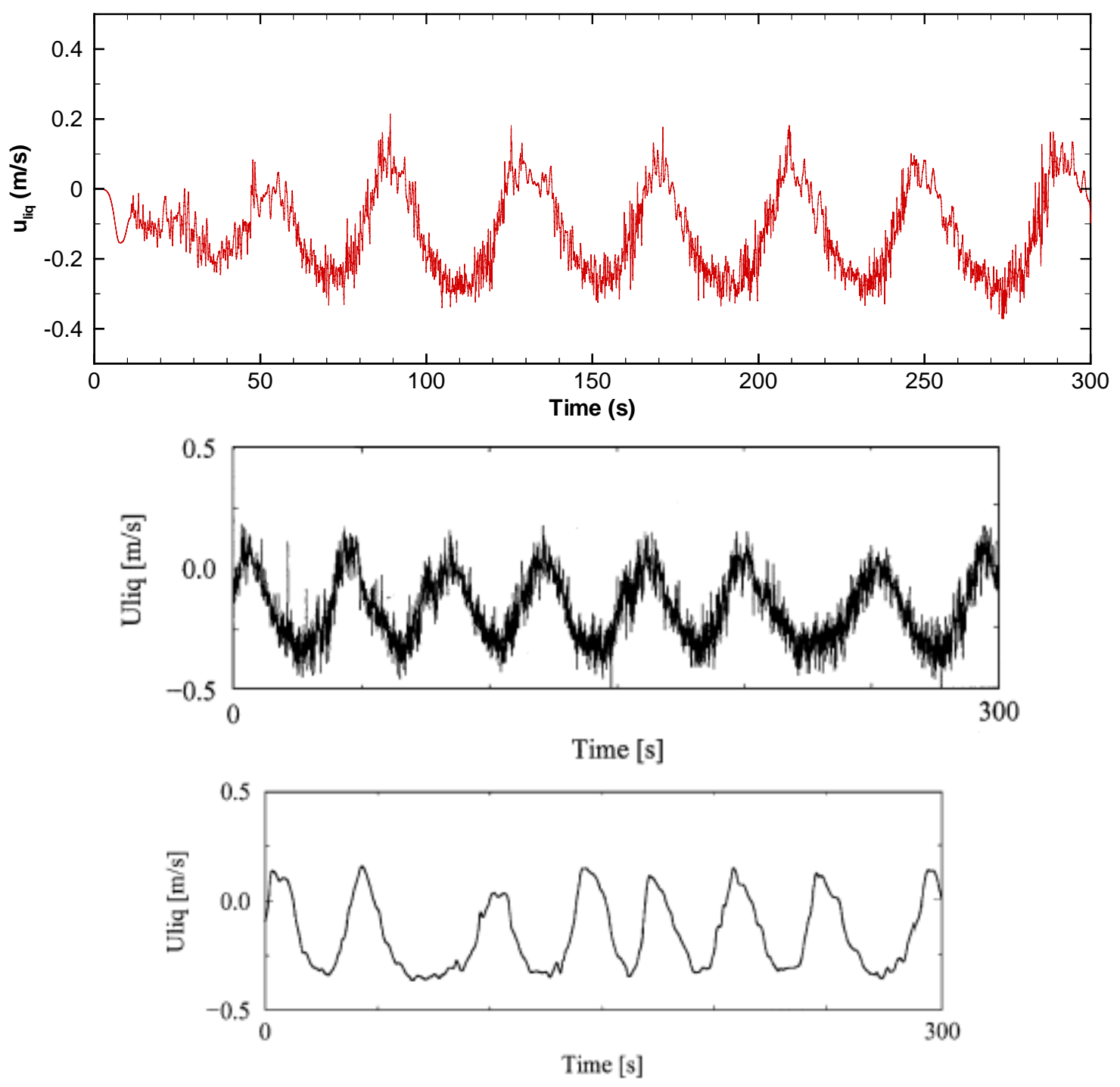

Figure 8-10 Time history of liquid vertical velocity sampled at point $B(1.05 \mathrm{~m}$ from bottom, $50 \mathrm{~mm}$ from right wall). From top to bottom: (a) present LES, (b) (Sokolichin and Eigenberger 1999)'s experiment, (c) (Sokolichin and Eigenberger 1999)'s 3D simulation. (Second and third figures reprinted with permission). 

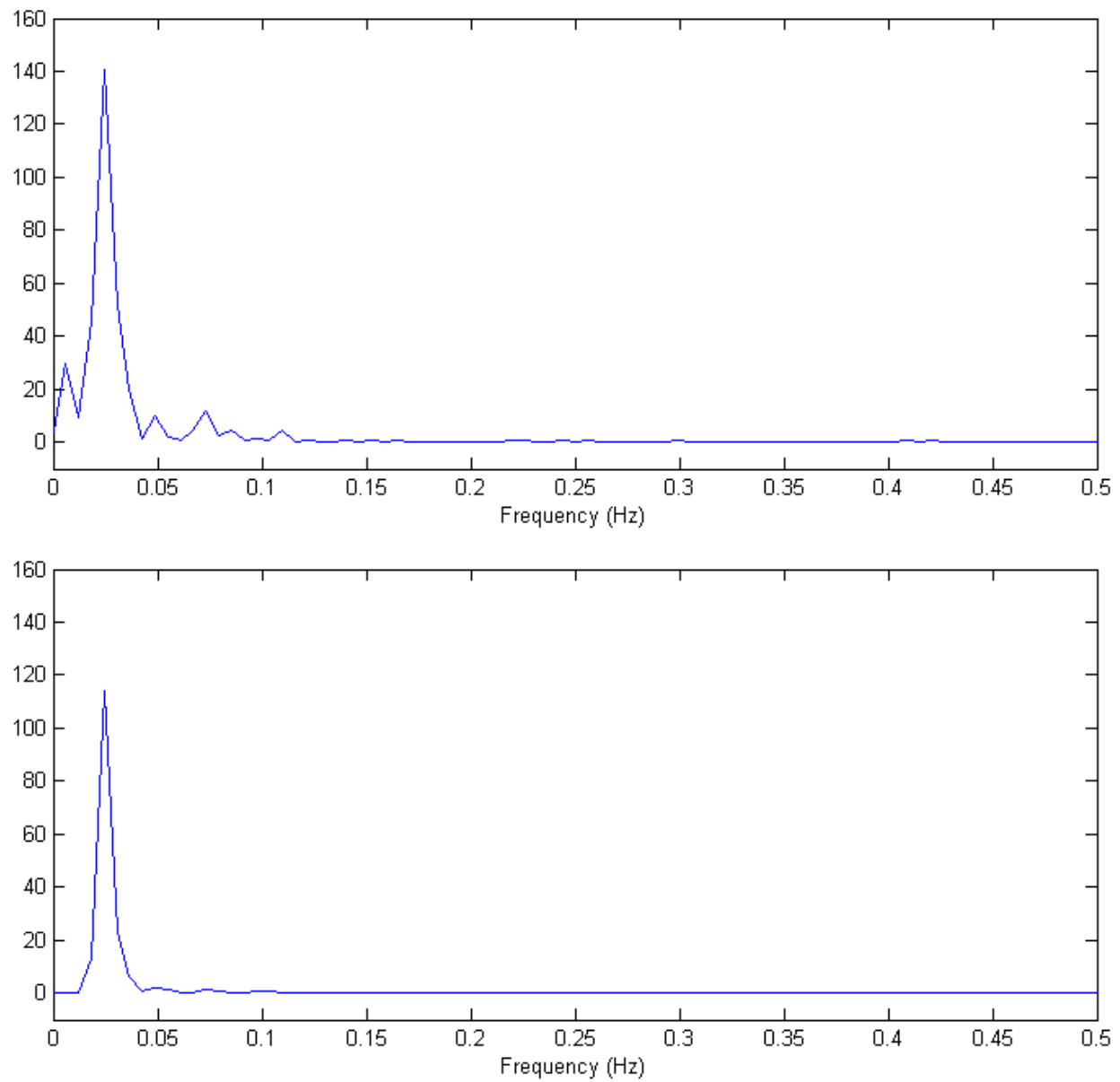

Figure 8-11 Power spectra of u-velocity fluctuations at Point A (upper) and B (lower). 


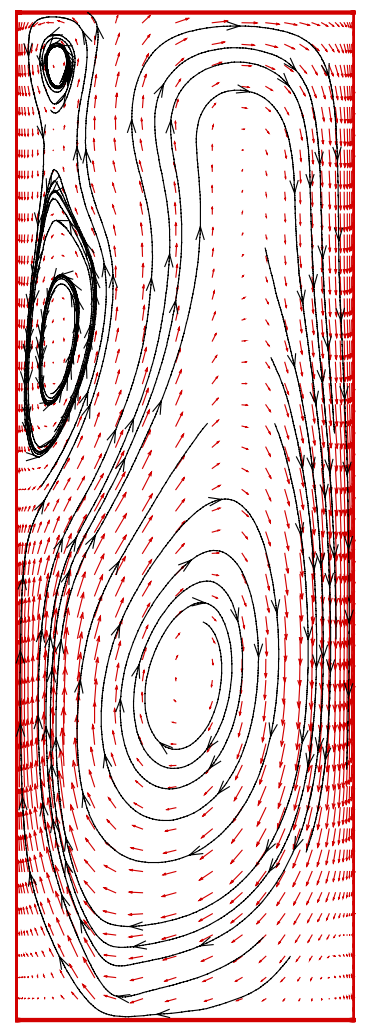

(a)

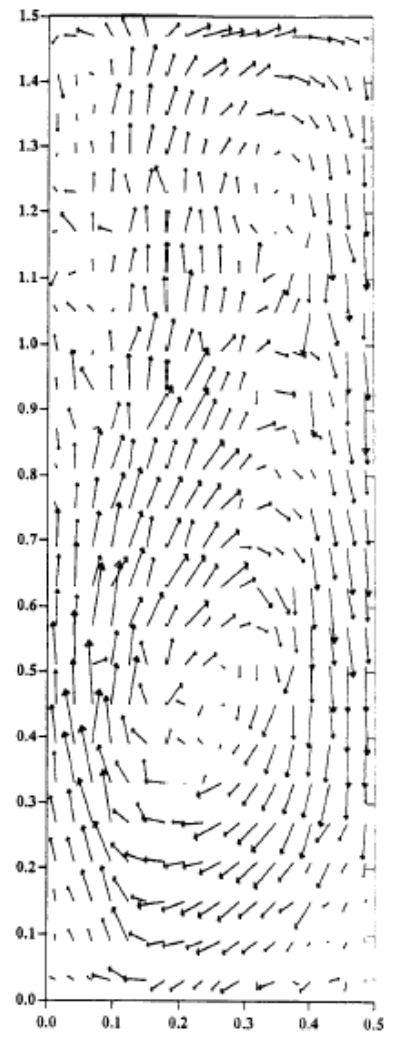

(b)

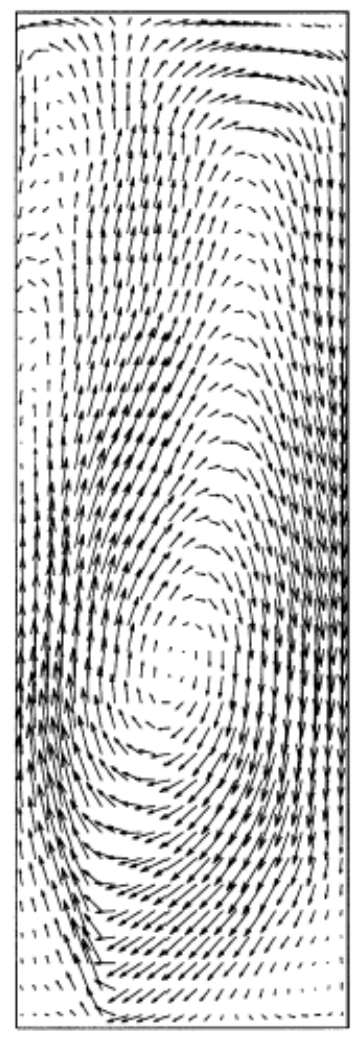

(c)

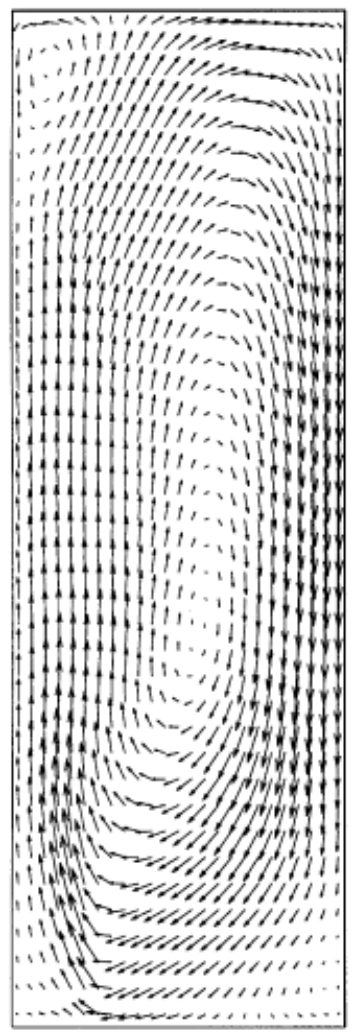

(d)

Figure 8-12 Long-time averaged liquid velocity field (a) present LES ( 0 - 300s, grid index skip in both $x$ - and $y$-directions is 2), (b) (Becker et al. 1994)'s experiment, (c) (Sokolichin and Eigenberger 1999)'s experiment, (d) (Sokolichin and Eigenberger 1999)'s 3D simulation. (Figures (b), (c), and (d) reprinted with permission) 


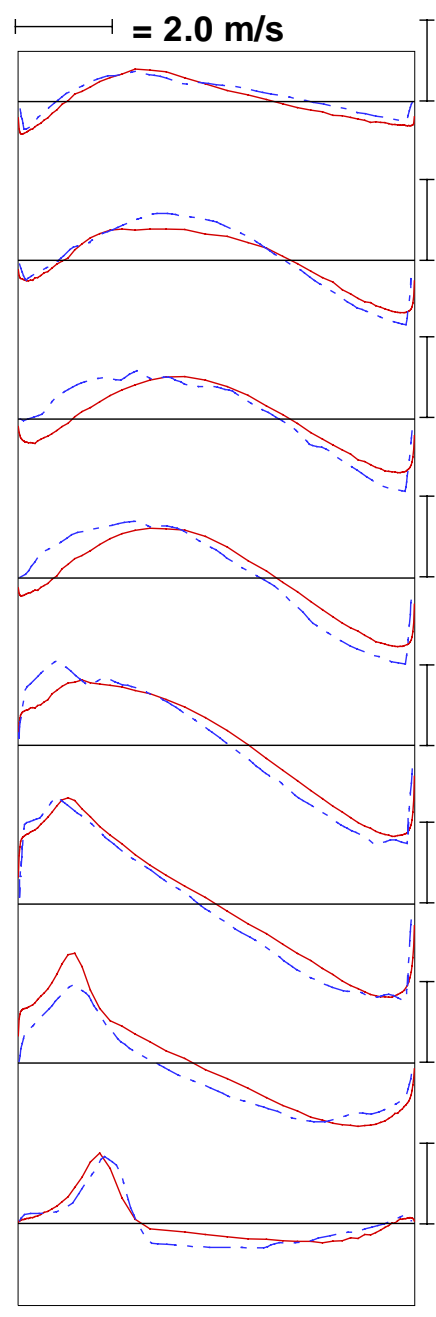

(a)

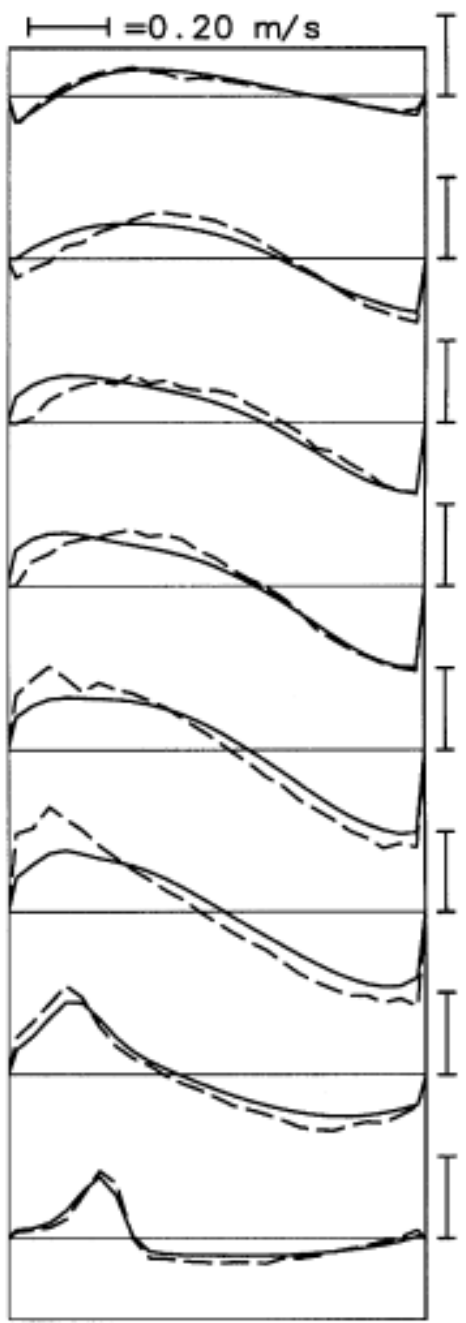

(b)

Figure 8-13 Long-time averaged vertical velocity profiles at different heights of the mid-depth plane.

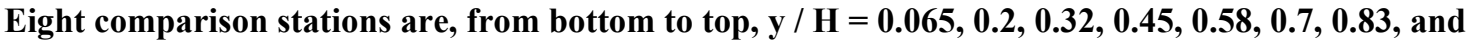
0.96. Solid lines are present LES (left) or (Sokolichin and Eigenberger 1999)'s 3D E-E simulation (right), dash-dot or dashed lines (Sokolichin and Eigenberger 1999)'s experiment. (Right figure reprinted with permission) 

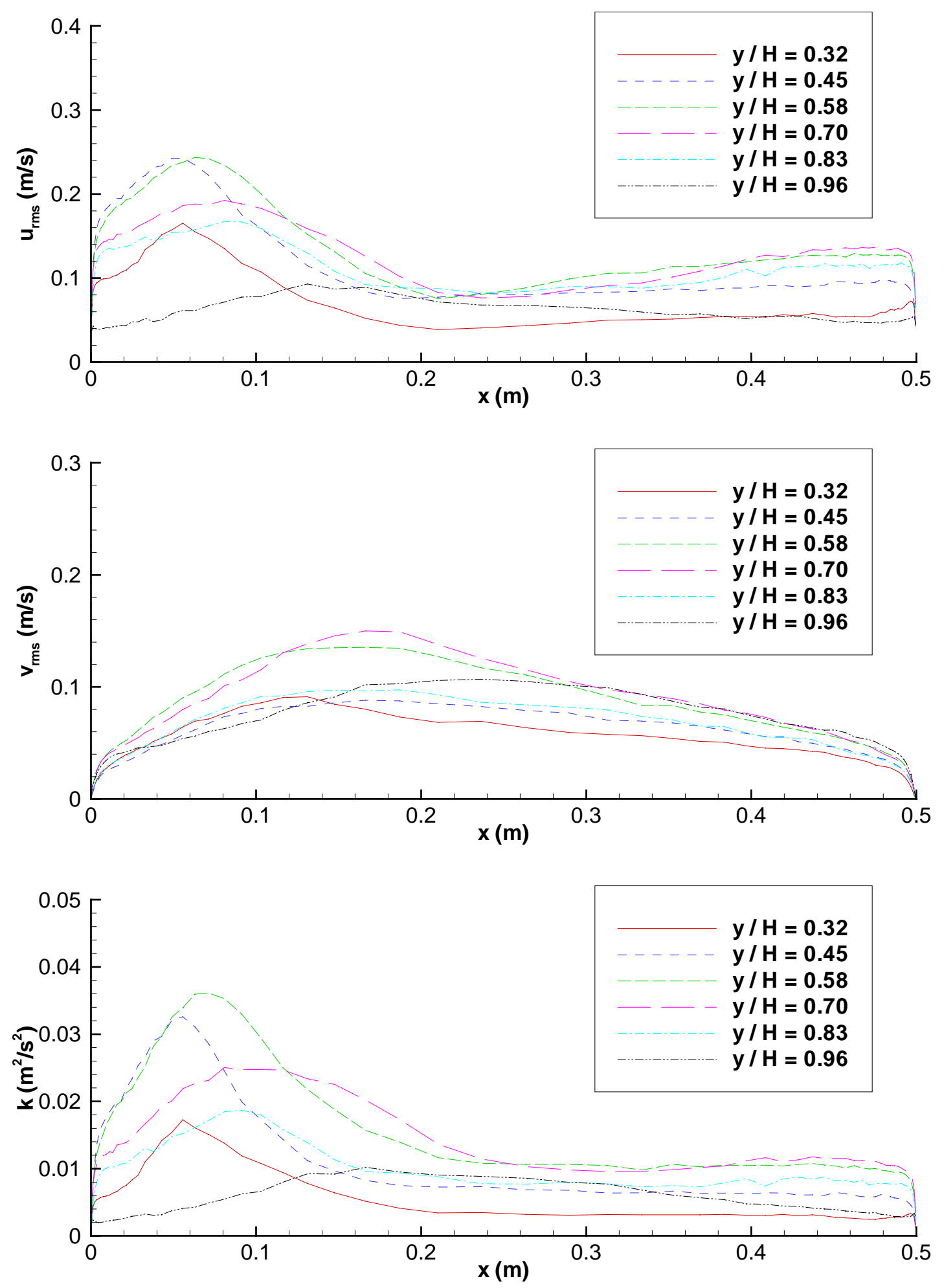

Figure 8-14 Turbulence intensities at different heights in the mid-depth plane, from top to bottom: (a) $u_{r m s}$, (b) $v_{r m s}$, (c) turbulent kinetic energy. 


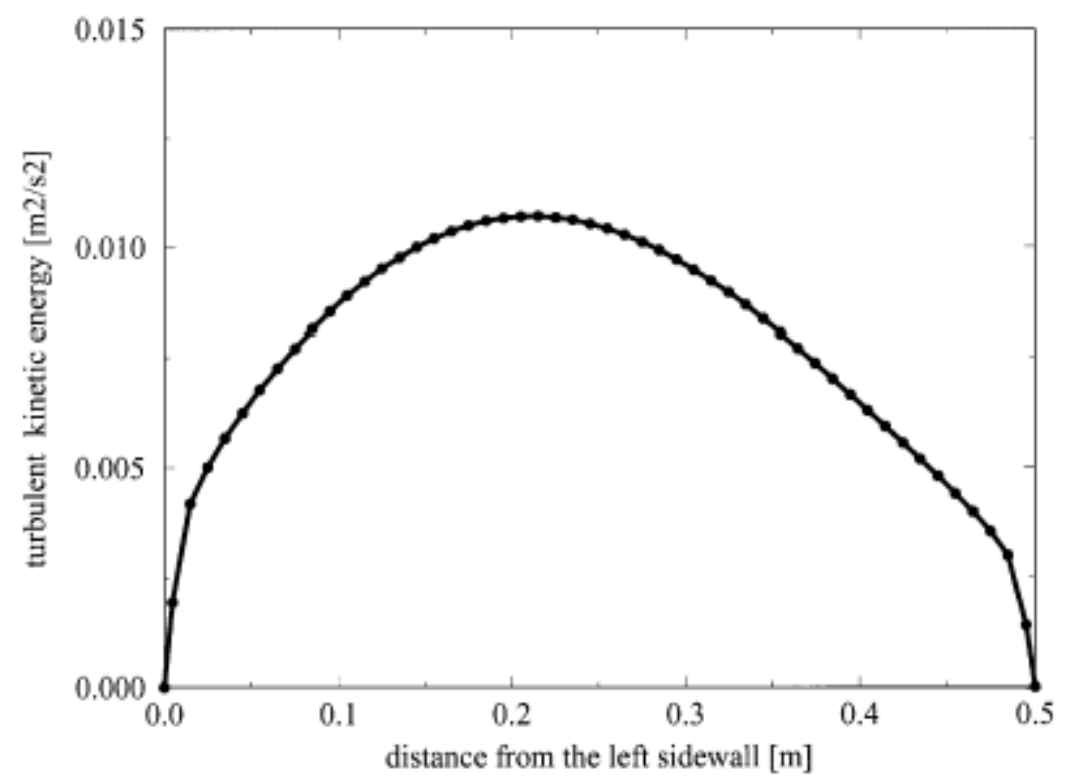

Figure 8-15 TKE profile at $\mathrm{y}$ / $\mathrm{H}=0.5$ calculated from (Sokolichin and Eigenberger 1999)'s 2D E-E simulation with $k-\varepsilon$ model. (Figure reprinted with permission).

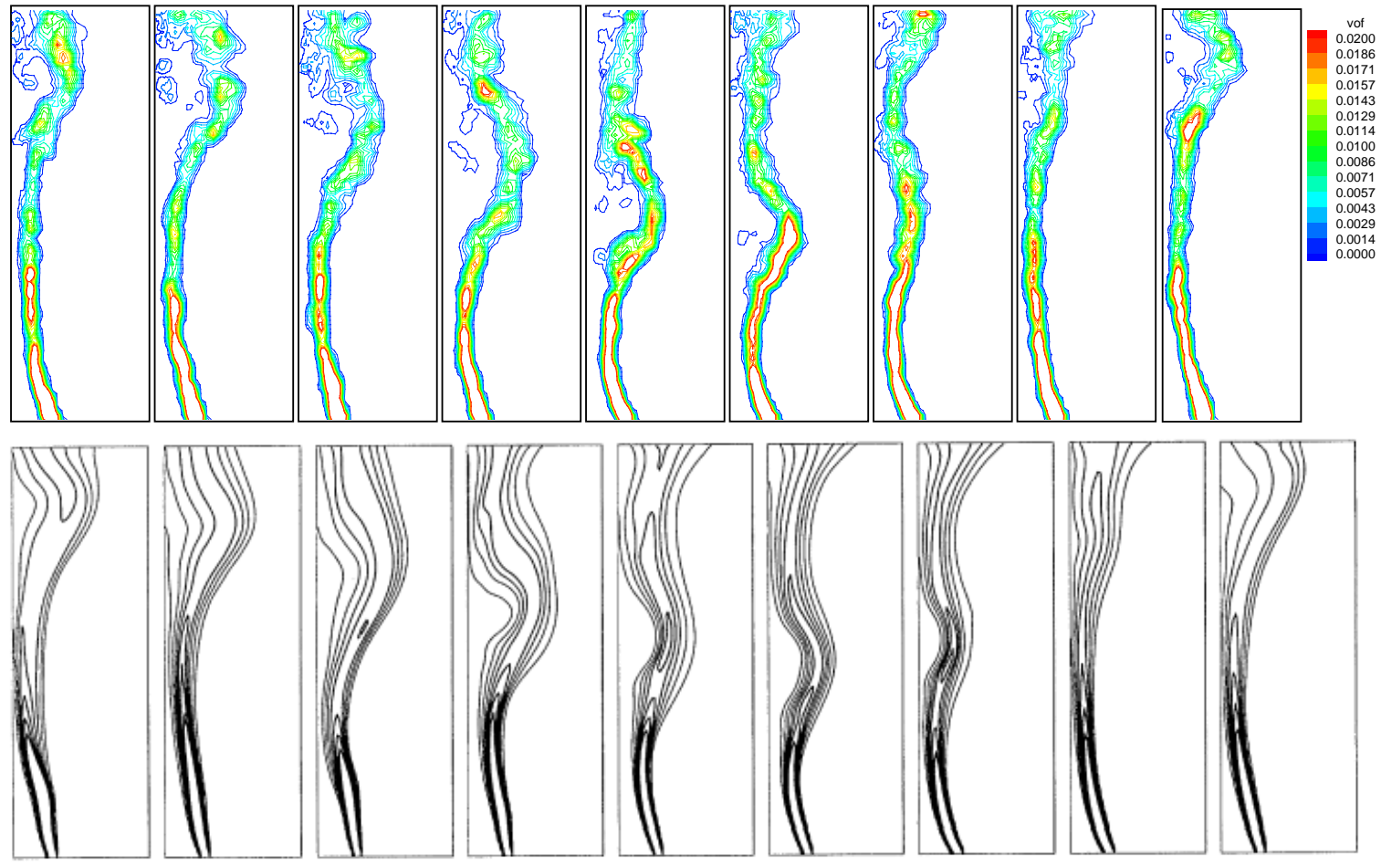

Figure 8-16 Instantaneous volume fraction of dispersed phase (bubble). Upper row: present LES; lower row: E-E 3D simulation of (Sokolichin and Eigenberger 1999). 


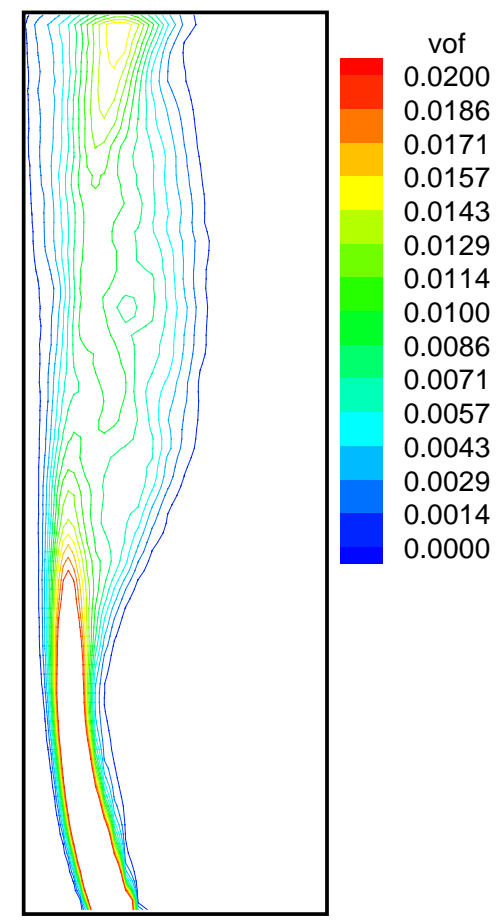

Figure 8-17 Mean volume fraction of dispersed phase (bubble).

\subsection{Grid Sensitivity Study}

First, let the LES presented in the previous section be referred to as the baseline case. A grid sensitivity study is then performed where the baseline grid is refined by a factor of $30 \%$ in all three directions. The two different grids under consideration are summarized in Table 8-3. All the computational parameters, except for the number of grid nodes, are kept the same, so that the difference between the two calculations will be only due to the spatial resolution. Plots of the same kinds are generated for the refined grid computation, and they are compared side-by-side with the baseline case in Figure 8-18 through Figure $8-23$.

A comparison of the coarser and finer-grid solution in Figure 8-18 through Figure 8-22 suggest that both calculations deliver very similar results, with respect to the bubble dispersion pattern and mean quantities. The period and mean vertical velocity at Point $\mathrm{A}$ and B calculated from the two grids (see Table 8-4) are also close.

In such a study it is also expected that with refined grid resolution, more TKE will be resolved. This is shown in the plot of Figure 8-23, where line profiles of the turbulence intensities and TKE are compared at two vertical stations. It is clearly seen, with the refined grid size, more turbulent fluctuations are resolved.

Based on these facts the present E-L LES can be well considered reliable. 
Table 8-3 Grid information in coarser and finer-grid computation.

\begin{tabular}{|l|l|l|l|}
\hline \multicolumn{2}{|c|}{ Coarser grid } & \multicolumn{2}{c|}{ Finer grid } \\
\hline Number of x-cells & 64 & Number of x-cells & 80 \\
\hline Number of y-cells & 96 & Number of y-cells & 120 \\
\hline Number of z-cells & 8 & Number of z-cells & 10 \\
\hline Time step & $5.0 \times 10^{-3}(\mathrm{~s})$ & Time step & $5.0 \times 10^{-3}(\mathrm{~s})$ \\
\hline y-grid expansion ratio & 1.12 & y-grid expansion ratio & 1.12 \\
\hline
\end{tabular}

Table 8-4 Comparison of period and mean vertical velocity at point $A$ and $B$.

\begin{tabular}{|c|c|c|c|c|}
\hline & \multicolumn{2}{|c|}{ Coarser grid } & \multicolumn{2}{c|}{ Finer grid } \\
\hline & Period (s) & $\begin{array}{c}\text { Mean vertical } \\
\text { velocity }(\mathrm{m} / \mathrm{s})\end{array}$ & Period (s) & $\begin{array}{c}\text { Mean vertical } \\
\text { velocity }(\mathrm{m} / \mathrm{s})\end{array}$ \\
\hline Point A & 40.96 & -0.0276 & 40.96 & -0.0257 \\
\hline Point B & 40.96 & -0.118 & 40.96 & -0.119 \\
\hline
\end{tabular}
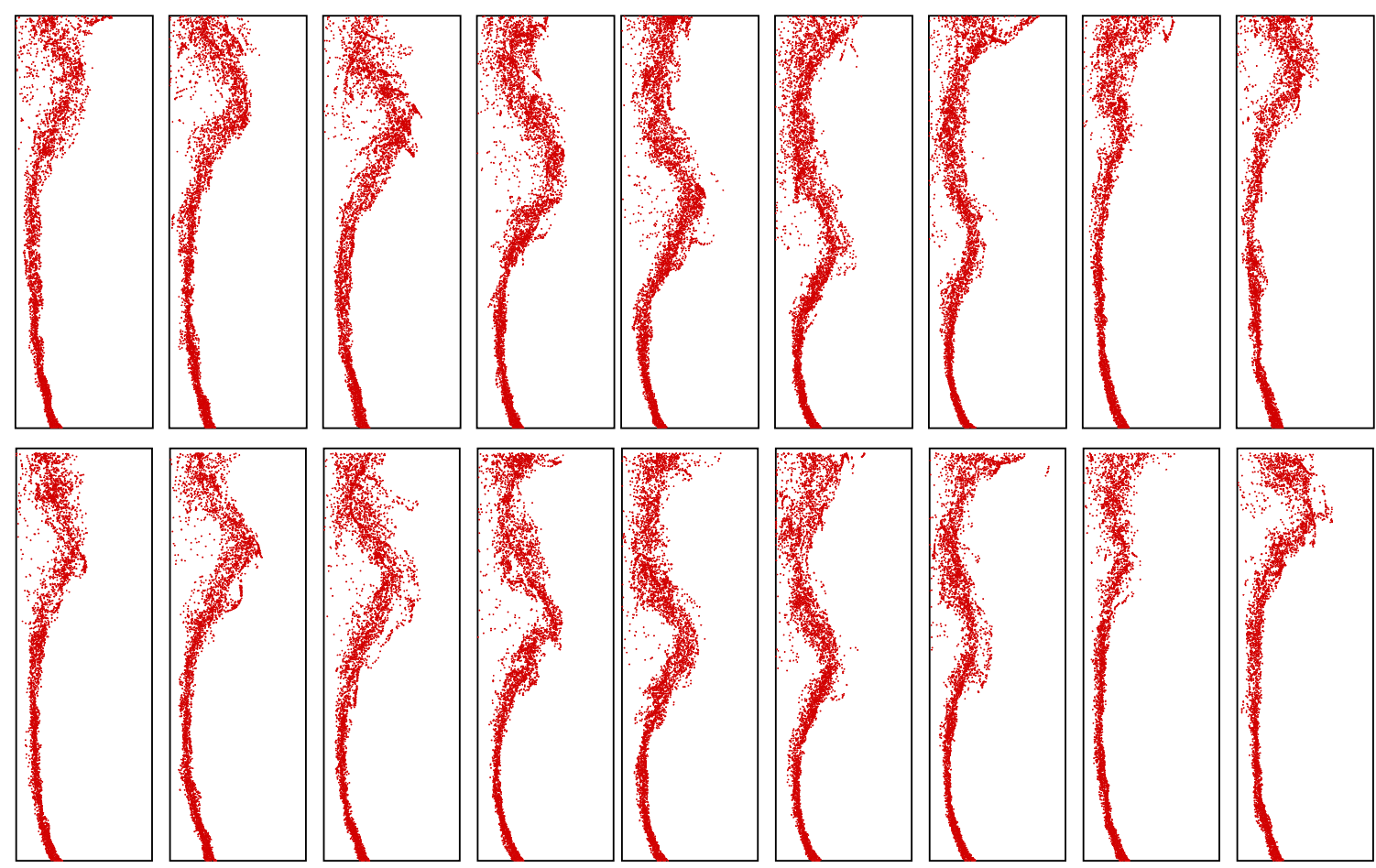

Figure 8-18 Comparison of instantaneous bubble dispersion calculated with two different grids. Upper row: 96x64x8; lower row: 120x80x10. 

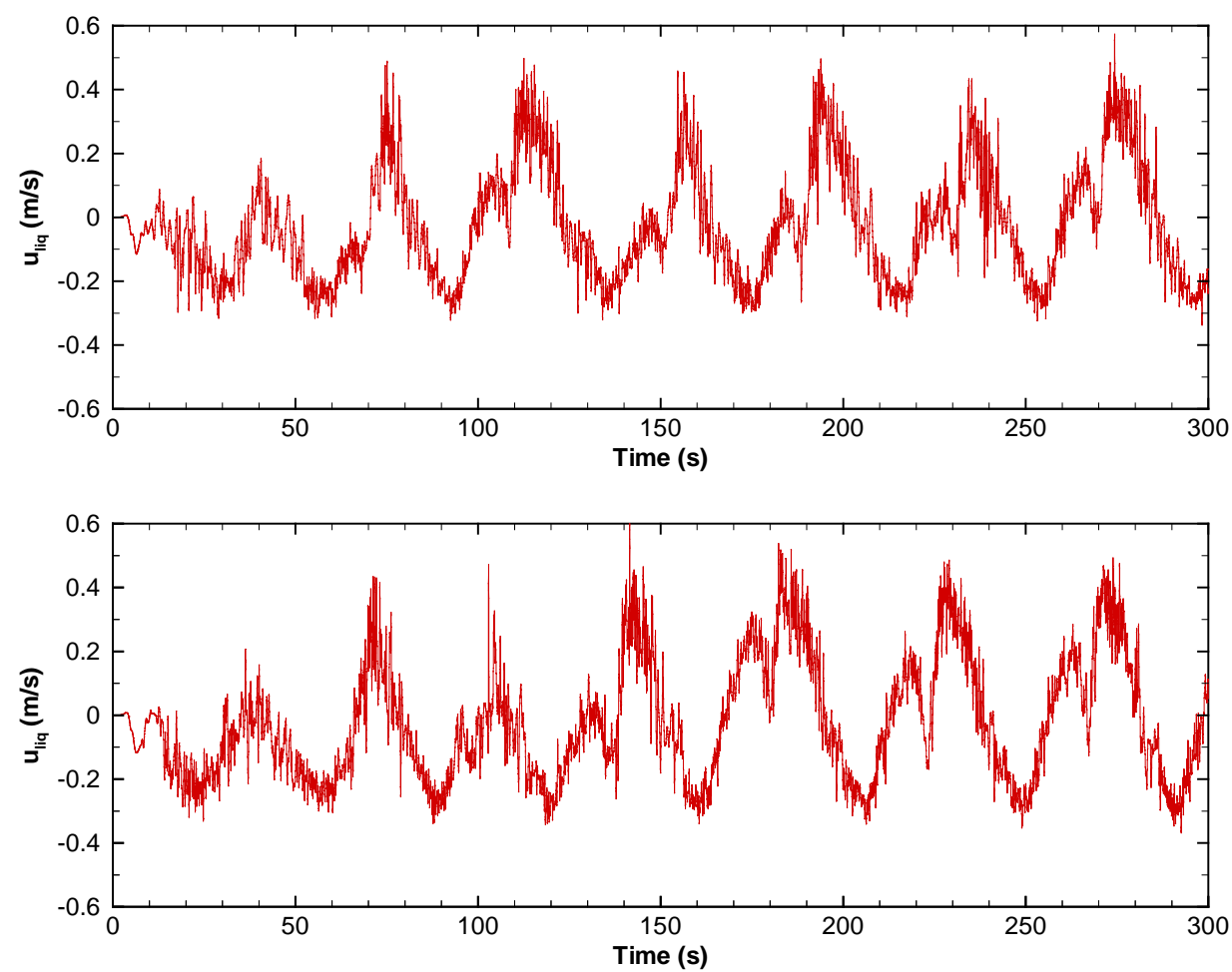

Figure 8-19 Comparison of time history of vertical velocity at Point A calculated with two different grids. Upper row: 96x64x8; lower row: 120x80x10.
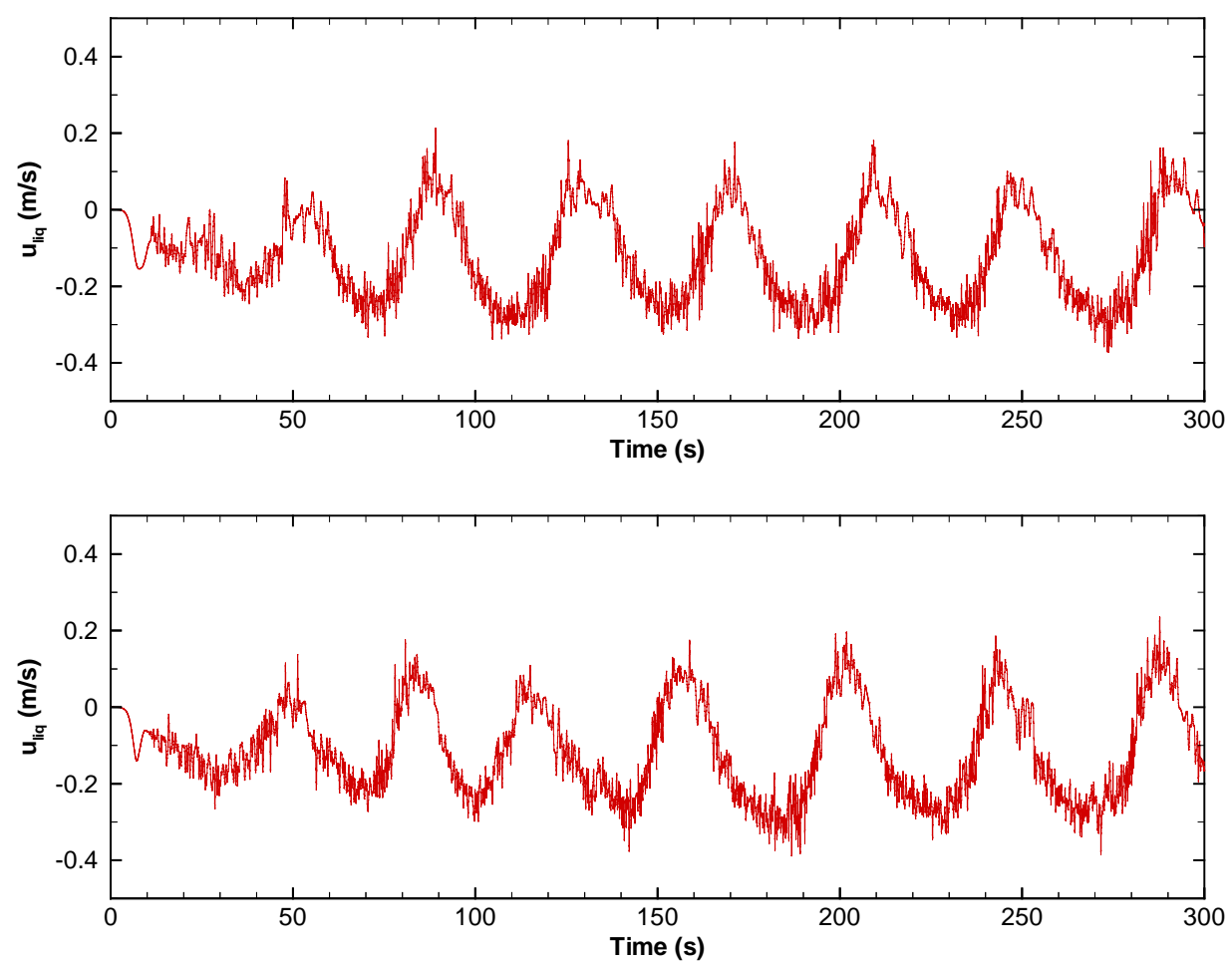

Figure 8-20 Comparison of time history of vertical velocity at Point B calculated with two different grids. Upper row: 96x64x8; lower row: 120x80x10. 


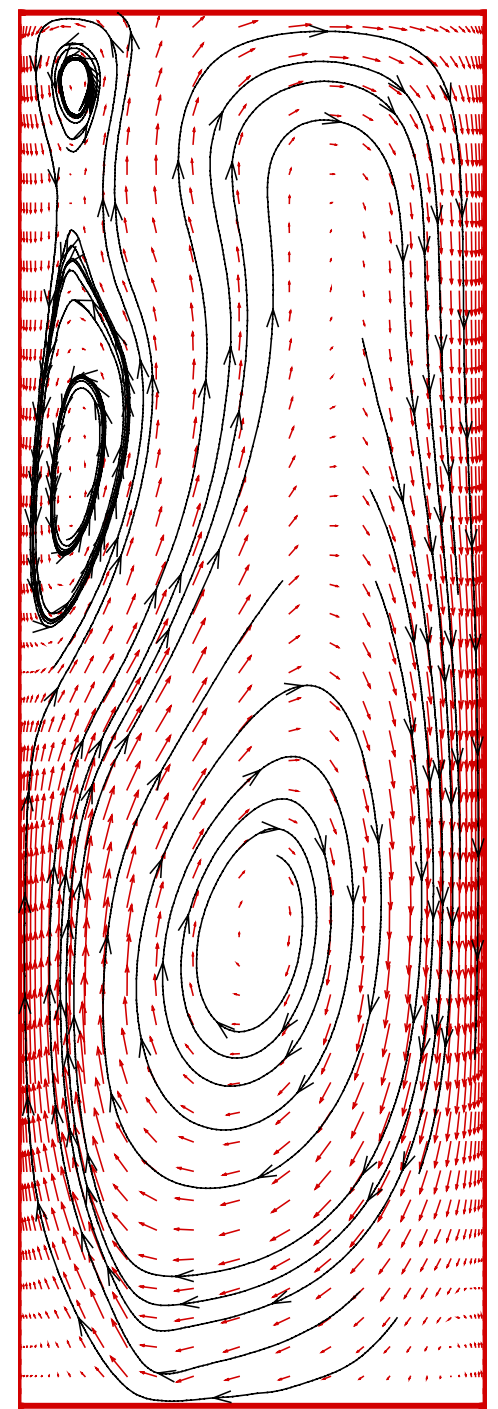

(a)

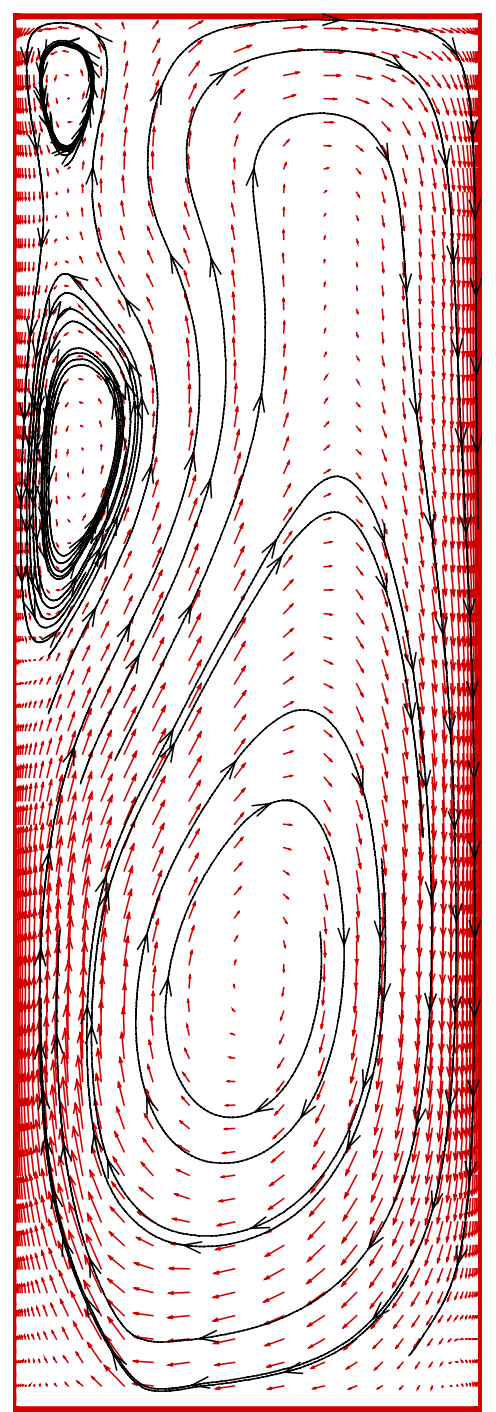

(b)

Figure 8-21 Comparison of long-time $(0$ - 300s) averaged liquid velocity field in the mid-depth plane calculated with two different grids: (a) 96x64x8, (b) 120x80x10. Grid index skip is 2 in both $x-$ and $y-$ directions. 


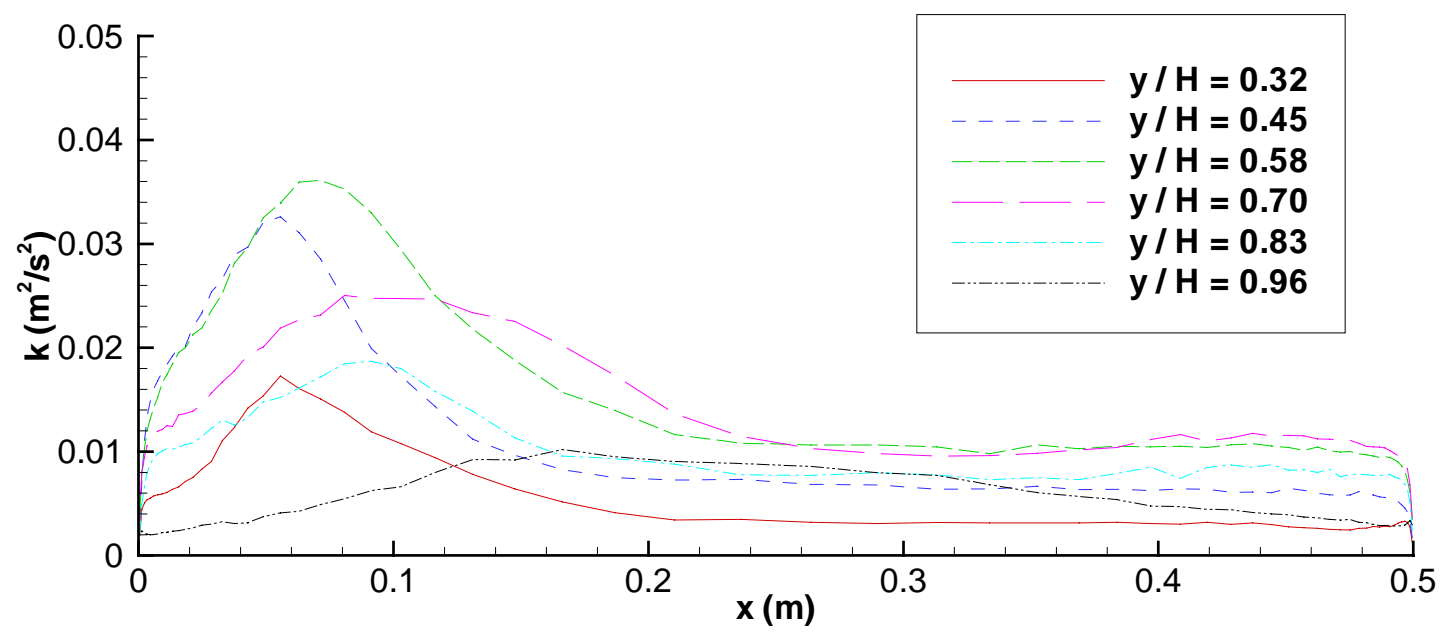

(a)

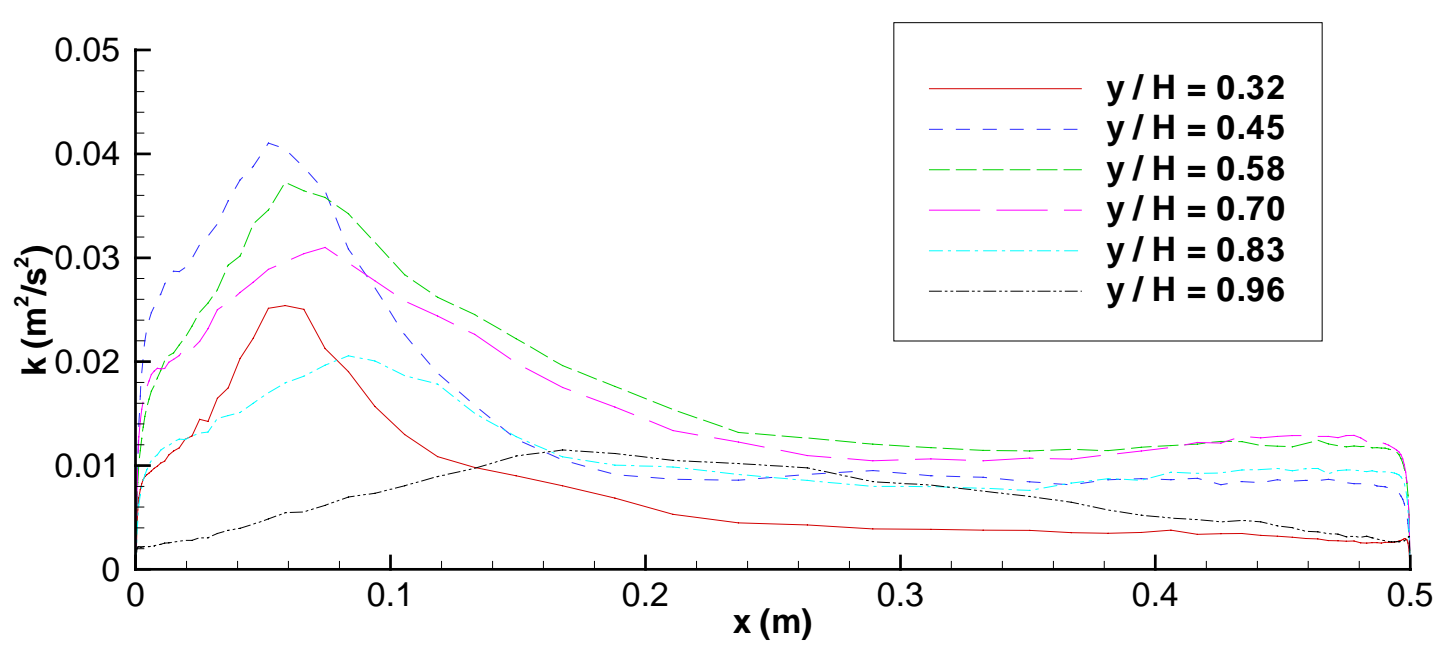

(b)

Figure 8-22 Comparison of turbulent kinetic energy profiles at different height in the mid-depth

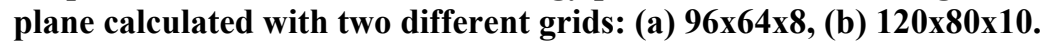



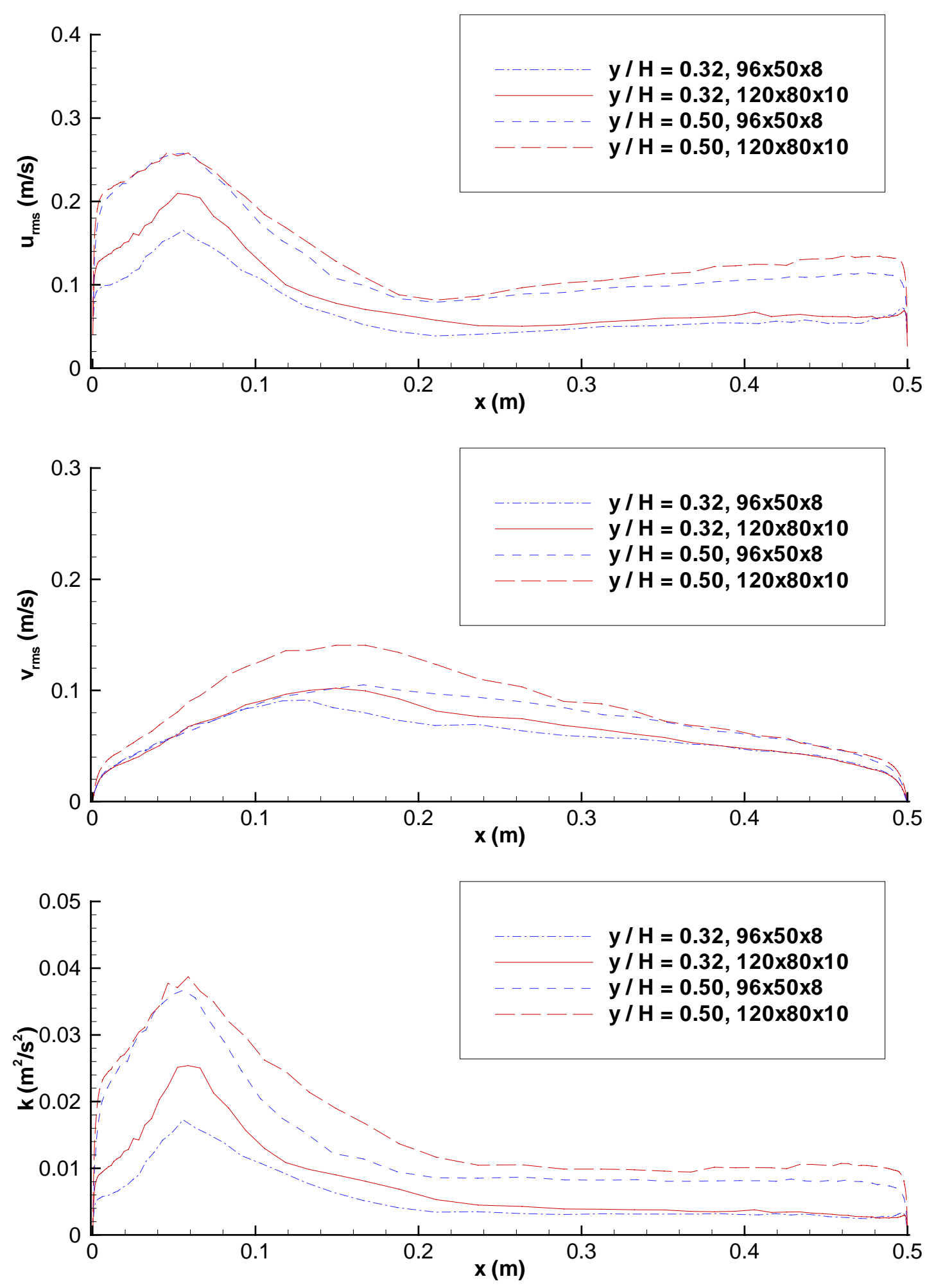

Figure 8-23 Comparison of turbulence intensity and turbulent kinetic energy profiles at two different height in the mid-depth plane calculated with two different grids. From top to bottom: (a) $\mathbf{u}_{\text {rmss }}$ (b) $\mathrm{v}_{\text {rms }}$, (c) TKE. 


\section{Chapter 9 Conclusions and Future Work}

\subsection{Summary}

In this study a transient, three-dimensional, finite-volume-method based Navier-Stokes (N-S) solver has been developed, which solves viscous, incompressible flow problems on an orthogonal and staggered grid. Discretization schemes have been derived in detail for both the momentum equations and the pressure Poisson equation. A fractional step method proposed by (Kim and Moin 1985) has been adopted for the solution of the coupled partial differential equations. The developed flow solver has been verified on a variety of benchmark flows including Taylor's vortex, free-shear layer, backward-facing step flow and square cavity, and it has been shown to be second-order overall accurate in both space and time.

Modeling strategies for and issues related to the large-eddy simulation (LES) of single-phase turbulent flows have been reviewed and summarized. Three subgrid scale (SGS) models, namely, Smagorinsky model, dynamic model and implicit model, have been implemented and investigated. Validation of the turbulent flow simulation has been carried out for three building-block turbulent flows: turbulent channel flow, developing plane mixing layer and flow past a square cylinder. The test results showed a superior quality of the present LES.

An extensive review has been performed for the characterization and description of a dispersed two-phase flow system. Summarized are the governing equations suitable for a DNS (direct numerical simulation), LES and RANS-type simulation based on the volume averaging. Both Eulerian and Lagrangian description of the dispersed phase has been addressed, with the emphasis on the latter. Various types of hydrodynamic forces acting on particles or bubbles, including drag, lift, fluid-stress, added mass, history and wall force, have been recapitulated. The assumptions and applicability of the EulerianLagrangian (E-L) based large-eddy simulation for a dispersed two-phase system have been elaborated. Various two-way coupling models as well as their numerical realization in the E-L LES have been reviewed. The importance of an accurate and efficient interpolation scheme is stressed.

The issue of the scale restrictions with respect to the particle/bubble sizes, characteristic flow length scales and computational grid size has been raised and carefully studied. A two-layer concept has been proposed, aimed at decoupling the geometric feature of the particle from that of the computational grid. In this approach, the primary carrier phase and the secondary dispersed phase are viewed as two independent, but interacting computational layers. The concept comprises three major elements (three A's): an assumption, an assertion and an approach. They are: 
- Assumption: The point-volume treatment of relatively small particles is an applicable approach for the Lagrangian simulation of relatively dilute dispersed systems.

- Assertion: The geometric feature of the particle should be decoupled from that of the computational grid.

- Approach (PSI-Ball): The reverse coupling (dispersed phase on the carrier phase) is achieved by redistributing the interphase point force via a distribution function onto those Eulerian grid nodes falling inside a predefined local influence sphere (or cage) centered at the particle center.

Finally, the proposed two-layer approach is applied to the large-eddy simulation of a partially aerated bubble column where bubbles are relatively large in size. Excellent agreement with the experiments has been obtained for both the bubble dispersion pattern and the statistics of the continuous carrier flow field.

\subsection{Achievements and Conclusions}

Three major accomplishments have been made in this study.

1. A framework of the two-way coupled Eulerian-Lagrangian LES covering a broad range of fundamental aspects, including governing equations, interphase coupling, particle force formulations, and interpolation has been formalized based upon previous research findings published in the open literature.

2. The commonly recognized size restriction on the particle or the computational cells has been challenged with a proposed two-layer concept. In this approach, the carrier phase and the dispersed phase are viewed as two standalone computational layers; the reverse coupling, i.e., from the particle to the carrier fluid, takes place at those discrete particle locations through modeled momentum exchange forces with the help of a predefined influence sphere (or circle in 2D). A significant advantage is that the Eulerian grid can be constructed to a desired fineness (e.g., in the wall layer) without concern for the particle size. Or equivalently, relatively large bubbles can be computed as long as the criteria of the dispersed flow regime (low volume fraction of the dispersed phase) can be still met. This convenience opens up the opportunity of applying E-L LES to a wider range of practical engineering flows such as bubble column reactors and bubbly channel flows.

3. In the literature the study of bubble columns has served as the foundation upon which industrial bubble column reactors are developed, because the flow pattern generated by the bubble motion, despite the simple geometry of the column, has been shown to be highly complex and to depend on many factors. The present study has successfully simulated a partially aerated bubble column using the E-L LES approach and the proposed two-layer concept. A high fidelity of the results has been attained. Second order statistics have been reported. 


\subsection{Future Work}

The following recommendations can be made for further investigations:

1. The proposed two-layer concept should be further evaluated on different types of dispersed flow systems, such as the bubbly mixing layer, bubbly channel flow and bubbly ship wakes.

2. Reliable two-phase SGS models are to be developed and rigorously verified.

3. The present study mainly investigated the force coupling between the two phases, where the wake effect of the particle/bubble is neglected. Another form of the two-way coupling, known as the velocity coupling, which may potentially take into account the influence of the particle wake, can be investigated.

4. Although it has been found in the literature that with LES a particle dispersion model can have minor influence on the particle dispersion patterns, the particle dispersion due to random turbulent fluctuation may still play a role in some notwell-resolving LES. Instead of fully relying on the filtered velocity in computing the particle trajectories, it can still be a good idea to develop a reliable particle dispersion model to supplement certain deficiency of the filtered velocity field.

5. There has been a vast discussion on the importance of the interpolation schemes in the literature. In the practical interest, the efficient tri-linear interpolation is still preferred over other expensive high-order interpolation. The present study has shown that even with the first-order tri-linear interpolation, fruitful results can be obtained in the bubble column simulation. It is certainly desirable to investigate the applicability and accuracy of the first-order interpolation as compared to higher order schemes, if only the mean or at most the second-order statistics of the carrier phase is of interest.

6. The applicable particle force in the near-wall region remains to be a big mystery. Although some investigations have been made in the literature, most available expressions are based on empirical correlations. A fundamental understanding on the mechanism of the wall-force is still lacking. Further research is needed in this seemingly small, but indeed very important area.

7. Despite the increasing power of the computational resources, a parallel algorithm and implementation for the E-L LES of the dispersed two-phase flows will still be a must to meet the challenges of industrial flow problems. On a single processor, based on the current computing capability, the number of computational particles or parcels is generally limited to an order of about $10^{5}$. A substantial removal of this bottleneck can be only achieved, in the author's opinion, with the help of massively parallelized implementation of the simulation program. However, difficulties still exist. For example, in the domain decomposition approach, the communication overhead and load imbalance hinders a linear speedup of the parallel program.

It is clear that, research in this area towards a well establishment still has a long way to go, and it calls for continuing and collaborative efforts among researchers, scientists and engineers. But, it is the author's belief that a good start has been made here. 


\section{Bibliography}

Abe, H., Kawamura, H., and Matsuo, Y. (2001). "Direct numerical simulation of a fully developed turbulent channel flow with respect to the Reynolds number dependence." Journal of Fluids Engineering, 123, 382-393.

Abramovich, G. N. (1963). The Theory of Turbulent Jets, MIT Press, Cambridge, MA. Alajbegovic, A. (2001) "Large eddy simulation formalism applied to multiphase flows." ASME 2001 Fluids Engineering Division Summer Meeting, New Orleans, LA, May 29 - June 1, FEDSM2001-18192.

Amsden, A. A., O'Rourke, P. J., and Butler, T. D. (1989). "KIVA-II: A computer program for chemically reactive flows with sprays." LA-11560-MS, Los Alamos National Lab, Los Alamos.

Apte, S. V., Gorokhovski, M., and Moin, P. (2003a). "LES of atomizing spray with stochastic modeling of secondary breakup." International Journal of Multiphase Flow, 29, 1503-1522.

Apte, S. V., Mahesh, K., Moin, P., and Oefelein, J. C. (2003b). "Large-eddy simulation of swirling particle-laden flows in a coaxial-jet combustor." International Journal of Multiphase Flow, 29, 1311-1331.

Aris, R. (1962). Vectors, Tensors and the Basic Equations of Fluid Mechanics, PrenticeHall, Englewood Cliffs, NJ.

Armaly, B. F., Durst, F., Pereira, J. C. F., and Schoenung, B. (1983). "Experimental and theoretical investigation of backward-facing step flow." Journal of Fluid Mechanics, 127, 473-496.

Armenio, V., and Fiorotto, V. (2001). "The importance of the forces acting on particles in turbulent flows." Physics of Fluids, 13(8), 2437-2440.

Armenio, V., Piomelli, U., and Fiorotto, V. (1999). "Effect of the subgrid scales on particle motion." Physics of Fluids, 11(10), 3030-3042.

Arunajatesan, S., and Shipman, J. (2004) "Numerical modeling of coupled VSTO-ship airwake flowfields." 2004 ASME Heat Transfer/Fluids Engineering Summer Conference, Charlotte, NC, July 25-29, HT-FED2004-56147.

Auton, T. R. (1981). "The dynamics of bubbles, drops and particles in motion in liquids," Ph.D. Thesis, Cambridge University, Cambridge, UK.

Auton, T. R. (1987). "The lift force on a spherical body in a rotational flow." Journal of Fluid Mechanics, 183, 199-218.

Auton, T. R., Hunt, J. C. R., and Prud'homme, M. (1988). "The force exerted on a body in inviscid unsteady non-uniform rotational flow." Journal of Fluid Mechanics, 197, 241-57.

Balachandar, S., and Maxey, M. R. (1989). "Methods for evaluating fluid velocities in spectral simulations of turbulence." Journal of Computational Physics, 83(1), 96125.

Baldwin, B., and Lomax, H. (1978). "Thin-layer approximation and algebraic model for separated turbulent flows." AIAA Journal, 78, 257. 
Bardina, J., Ferziger, J. H., and Reynolds, W. C. (1980) "Improved subgrid models for large eddy simulation." 13th AIAA Fluid \& Plasma Dynamics Conference, Snowmass, CO, July 14-16, AIAA Paper 80-1357, 80.

Barkla, H. M., and Auchterlonie, L. J. (1971). "The Magnus or Robins effect on rotating spheres." Journal of Fluid Mechanics, 47, 437.

Basset, A. B. (1888a). "On the motion of a sphere in a viscous liquid." Philos. Trans. R. Soc. Londer, Ser. A, 179, 43-63.

Basset, A. B. (1888b). Treatise on Hydrodynamics, Deighton Bell, London.

Bataille, J., Lance, M., and Marie, J. L. (1991). "Some aspects of the modeling of bubbly flows." Phase-interface Phenomena in Multiphase Flow, G. F. Hewitt, F. Mayinger, and J. R. Riznic, eds., Hemisphere Publishing Corporation.

Batchelor, G. K. (1967). An Introduction to Fluid Dynamics, Cambridge University Press, Cambridge.

Bear, J. (1972). Dynamics of Fluids in Porous Media, Amerian Elsevier.

Becker, S., Sokolichin, A., and Eigenberger, G. (1994). "Gas-liquid flow in bubble columns and loop reactors: part II. Comparison of detailed experiments and flow simulations." Chemical Engineering Science, 49(24B), 5747-5762.

Benjamin, A. S., and Denny, V. E. (1979). "On the convergence of numerical solutions for 2-D flows in a cavity at large Re." Journal of Computational Physics, 33(3), 340-358.

Bernal, L. P., and Roshko, A. (1986). "Streamwise vortex structure in plane mixing layers." Journal of Fluid Mechanics, 170, 499.

Bird, R. B., Stewart, W. E., and Lightfoot, E. N. (2002). Transport Phenomena, John Wiley \& Sons Inc., New York.

Boisson, N., and Malin, M. R. (1996). "Numerical prediction of two-phase flow in bubble columns." International Journal for Numerical Methods in Fluids, 23, 1289.

Boivin, M. (1996). "Etude de l'Influence des Particules sur la Turbulence a partir de Simulations Directes et de Simulations des Grandes Echelles d'ecoulementes Diphasiques Gaz-Solides Homogenes Isotropes Stationnaires," Universite de Rouen.

Boivin, M., Simonin, O., and Squires, K. D. (1998). "Direct numerical simulation of turbulence modulation by particles in isotropic turbulence." Journal of Fluid Mechanics, 375, 235-263.

Boivin, M., Simonin, O., and Squires, K. D. (2000). "On the prediction of gas-solid flows with two-way coupling using large eddy simulation." Physics of Fluids, 12(8), 2080-2090.

Borchers, O., Busch, C., Sokolichin, A., and Eigenberger, G. (1999). "Applicability of the standard k-e turbulence model to the dynamic simulation of bubble columns. Part II. comparison of detailed experiments and flow simulations." Chemical Engineering Science, 54, 5927-5935.

Boris, J. P., and Book, D. L. (1973). "Flux-corrected transport. I. SHASTA, a fluid transport algorithm that works." Journal of Computational Physics, 11(1), 38-69.

Boris, J. P., Grinstein, F. F., Oran, E. S., and Kolbe, R. L. (1992). "New insights into large eddy simulation." Fluid Dynamics Research, 10(4-6), 199-228. 
Bosch, G., and Rodi, W. (1998). "Simulation of vortex shedding past a square cylinder with different turbulence models." International Journal for Numerical Methods in Fluids, 28, 601-616.

Boussinesq, J. (1877). "Theorie de l'ecoulement tourbillant." Mem. Presentes par Divers Savants Acad. Sci. Inst. Fr., 23, 46-50.

Boussinesq, V. J. (1885). Applications L'etude des Potentiels, Blanchard, Paris.

Bracewell, R. N. (1978). The Fourier Transform and its Applications, McGraw-Hill, New York.

Brown, G. L., and Roshko, A. (1974). "On density effects and large structure in turbulent mixing layers." Journal of Fluid Mechanics, 64(pt.4), 775-816.

Bunner, B., and Tryggvason, G. (1997) "Simulation of large bubble systems." 1997 ASME Fluids Engineering Division Summer Meeting, June 22 - 26, FEDSM973409.

Burden, R. L., and Faires, J. D. (1989). Numerical Analysis, PWS-Kent Publishing Company, Boston.

Celik, I., and Badeau, A. (2003). "Verification and validation of Dream code with an introduction to LES." MAE-IC03-Tr\#3, Mechanical \& Aerospace Engineering Department, West Virginia University, Morgantown.

Celik, I., and Wang, Y.-Z. (1994). "Numerical simulation of circulation in gas-liquid column reactors: isothermal, bubbly, laminar flow." International Journal of Multiphase Flow, 20(6), 1053-1070.

Celik, I. B. (2005). Introductory Turbulence Modeling, Ararat Books \& Publishing, LLC., Morgantown, WV.

Chahine, G. L., Delepoule, E., and Hauwaert, P. (1993) "Study of the interaction between a bubble and a vortical structure." ASME Cavitation and Multiphase Flow Forum, FED Vol. 153, 39-46.

Chandrsuda, C., Mehta, R. D., Weir, A. D., and Bradshaw, P. (1978). "Effect of freestream turbulence on large structure in turbulent mixing layers." Journal of Fluid Mechanics, 85, 693.

Chang, E. J. (1992). "Accelerated motion of rigid spheres in unsteady flows at finite Reynolds numbers," Ph.D. thesis, Brown University.

Chapra, S. C., and Canale, R. P. (1998). Numerical Methods for Engineers, with Programming and Software Applications, WCB/McGraw-Hill, Boston.

Chen, J. J. J., Jamialahmadi, M., and Li, S. M. (1989). "Effect of liquid depth on circulation in bubble columns: a visual study." Chemical Engineering Research \& Design, 67(2), 203-7.

Chen, R. C., and Wu, T. L. (1999) "The flow characteristics between two interactive spheres." ASME Fluids Engineering Conference, San Francisco, June, FEDSM997776.

Cherukat, P., McLaughlin, J. B., and Graham, A. L. (1994). "The inertial lift on a rigid sphere translating in a linear shear flow field." International Journal of Multiphase Flow, 20(2), 339-353.

Choi, H., and Moin, P. (1994). "Effects of computational time step on numerical solutioins of turbulent flow." Journal of Computational Physics, 113(1), 1-4.

Chow, F. K., and Moin, P. (2003). "A further study of numerical errors in large-eddy simulations." Journal of Computational Physics, 184, 366-380. 
Chung, T. J. (2002). Computational Fluid Dynamics, Cambridge University Press, Cambridge.

Clark, J. A. (1968). "A study of incompressible turbulent boundary layers in channel flow." Transactions of the ASME, Series D, Journal of Basic Engineering, 90, 455.

Clark, J. A., and Markland, E. (1970). "Vortex structures in turbulent boundary layers." Aeronautical Journal, 74, 243-244.

Clift, R., Grace, J. R., and Weber, M. E. (1978). Bubbles, Drops and Particles, Academic Press, New York.

Climent, E., and Magnaudet, J. (1998). "Bubble-induced modifications of a vertical mixing layer." Comptes Rendus de l'Academie des Sciences, Serie II (Mecanique, Physique, Astronomie), 326(10), 627-34.

Colella, P., and Woodward, P. R. (1984). "The piecewise parabolic method (PPM) for gas-dynamical simulations." Journal of Computational Physics, 54, 174-201.

Collis, S., Lele, S. K., Moser, R. d., and Rogers, M. M. (1994). "The evolution of a plane mixing layer with spanwise nonuniform forcing." Physics of Fluids, 6(1), 381396.

Comte, P., Lesieur, M., and Lamballais, E. (1992). "Large- and small-scale stirring of vorticity and a passive scalar in a 3-D temporal mixing layer." Physics of Fluids A (Fluid Dynamics), 4(12), 2761.

Conte, S. D., and de Boor, C. (1980). Elementary Numerical Analysis, An Algorithmic Approach, McGraw-Hill Book Company, New York.

Corcos, G. M., and Lin, S. J. (1984). "The mixing layer: deterministic models of a turbulent flow. II. The origin of the three-dimensional motion." Journal of Fluid Mechanics, 139, 67-95.

Corrsin, S., and Lumley, J. (1956). "On equation of motion for particle in turbulent fluid." Applied Scientific Research, 6(2-3, Sec A), 114-116.

Cox, R. G., and Hsu, S. K. (1977). "The lateral migration of solid particles in a laminar flow near a plane." International Journal of Multiphase Flow, 3, 201-222.

Crowe, C., Sommerfeld, M., and Tsuji, Y. (1998). Multiphase Flows with Droplets and Particles, CRC Press, Boca Raton.

Crowe, C. T. (1982). "Review-numerical models for dilute gas-particle flows." Transactions of the ASME. Journal of Fluids Engineering, 104(3), 297-303.

Crowe, C. T. (2000). "On models for turbulence modulation in fluid-particle flows." International Journal of Multiphase Flow, 26, 719-727.

Crowe, C. T., Chung, J. N., and Troutt, T. R. (1988). "Particle mixing in free shear flows." Progress in Energy and Combustion Science, 14(3), 171-194.

Crowe, C. T., Gore, R. A., and Troutt, T. R. (1985). "Particle dispersion by coherent structures in free shear flows." Particulate Science and Technology, 3(3-4), 149158.

Crowe, C. T., Sharma, M. P., and Stock, D. E. (1977). "The particle-source-in cell (PSICELL) model for gas-droplet flows." Transactions of the ASME. Series I, Journal of Fluids Engineering, 99(2), 325-32.

Crowe, C. T., Troutt, T. R., and Chung, J. N. (1996). "Numerical models for two-phase turbulent flows." Annual Review of Fluid Mechanics, 28, 11-43. 
Csanady, G. T. (1963). "Turbulent diffusion of heavy particles in the atmosphere." Journal of Atmospheric Science, 20, 201-208.

Daily, J. W., and Harleman, D. R. F. (1973). Fluid Dynamics, Addison-Wesley Publishing Company, Reading, Massachusetts.

Daly, B. J., and Harlow, F. H. (1970). "Transport equations in turbulence." Physics of Fluids, 13, 2634-2649.

Davies, J. M. (1949). "The aerodynamics of golf balls." Journal of Applied Physics, 20(9), 821-828.

Deardorff, J. W. (1970). "A numerical study of three-dimensional turbulent channel flow at large Reynolds numbers." Journal of Fluid Mechanics, 41, 453-480.

Deardorff, J. W. (1974). "Differences between eddy coefficients for instantaneous and continuous vertical diffusion into the neutral surface layer." Boundary-Layer Meteorology, 5(4), 451-7.

Deardorff, J. W. (1980). "Stratocumulus-capped mixed layers derived from a threedimensional model." Boundary-Layer Meteorology, 18, 495-527.

Delhaye, J. M., Giot, M., and Riethmuller, M. L. (1981). "Thermohydraulics of TwoPhase Systems for Industrial Design and Nuclear Engineering." McGraw-Hill, New York.

Delnoij, E., Kuipers, J. A. M., and van Swaaij, W. P. M. (1997a). "Dynamic simulation of gas-liquid two-phase flow: effect of column aspect ratio on the flow structure." Chemical Engineering Science, 52(21/22), 3759-3772.

Delnoij, E., Lammers, F. A., Kuipers, J. A. M., and van Swaaij, W. P. M. (1997b). "Dynamic simulation of dispersed gas-liquid two-phase flow using a discrete bubble model." Chemical Engineering Science, 52(9), 1429-1458.

Denham, M. K., and Patrick, M. A. (1974). "Laminar flow over a downstream-facing step in a two-dimensional flow channel." Transactions of Institute of Chemical Engineers, 52, 361-367.

Deutsch, E., and Simonin, O. (1991) "Large eddy simulation applied to the motion of particles in stationary homogeneous fluid turbulence." ASME FED 110, 35.

Drew, D. A. (1983a). "Continuum modeling of two-phase flows." Theory of Dispersed Multiphase Flow, R. E. Meyer, ed., Academic Press, New York, 173-190.

Drew, D. A. (1983b). "Mathematical modeling of two-phase flow." Annual Review of Fluid Mechanics, 15, 261-291.

Drew, D. A., and Lahey, R. T. J. (1987). "Virtual mass and lift force on a sphere in rotating and straining inviscid flow." International Journal of Multiphase Flow, 13(1), 113-121.

Drew, D. A., and Lahey, R. T. J. (1990). "Some supplemental analysis concerning the virtual mass and lift force on a sphere in a rotating and straining flow." International Journal of Multiphase Flow, 16(6), 1127-1130.

Drew, D. A., and Lahey, R. T. J. (1993). "Analytical modeling of multiphase flow." Particulate Two-Phase Flow, M. C. Roco, ed., Butterworth-Heinemann, Boston, MA, 509-566.

Druzhinin, O. A., and Elghobashi, S. E. (2001). "Direct numerical simulation of a threedimensional spatially developig bubble-laden mixing layer with two-way coupling." Journal of Fluid Mechanics, 429, 22-61. 
Dukowicz, J. K. (1980). "A particle-fluid numerical model for liquid sprays." Journal of Computational Physics, 33, 229.

Durbin, P. A., and Pettersson Reif, B. A. (2001). Statistical Theory and Modeling for Turbulent Flows, John Wiley \& Sons.

Elghobashi, S. (1994). "On predicting particle-laden turbulent flows." Applied Scientific Research, 52, 309-329.

Elghobashi, S., and Truesdell, G. C. (1992). "Direct simulation of particles dispersion in a decaying isotropic turbulence." Journal of Fluid Mechanics, 242, 655-700.

Elghobashi, S., and Truesdell, G. C. (1993). "On the two-way interaction between homogeneous turbulence and dispersed solid particles. I: turbulence modification." Physics of Fluids A, 5(7), 1790-1801.

Esmaeeli, A., and Tryggvason, G. (1998). "Direction numerical simulations of bubbly flows. Part 1. low Reynolds number arrays." Journal of Fluid Mechanics, 337, 313-345.

Esmaeeli, A., and Tryggvason, G. (1999). "Direct numerical simulation of bubbly flows. Part 2. moderate Reynolds number arrays." Journal of Fluid Mechanics, 385, 325358.

Etheridge, D. W., and Kemp, P. H. (1978). "Measurements of turbulent flow downstream of a backward-facing step." Journal of Fluid Mechanics, 86, 545.

Faeth, G. M. (1987). "Mixing transport and combustion in sprays." Progress in Energy and Combustion Science, 13, 293-345.

Falco, R. E. (1978). "The role of outer flow coherent motions in the production of turbulence near a wall." Coherent Structures of Turbulent Boundary Layers, C. R. Smith and D. E. Abbott, eds., A.F.O.S.R., Lehigh.

Fan, L. S., and Tsuchiya, K. (1990). Bubble Wake Dynamics in Liquids and Liquid-Solid Suspensions, Butterworth-Heinemann, Boston.

Fan, L.-S., and Zhu, C. (1998). Principles of Gas-Solid Flows, Cambridge University Press, Cambridge.

Favre, A. (1983). "Turbulence: space-time statistical properties and behavior in supersonic flows." Physics of Fluids, 26(10), 2851-2863.

Faxen, H. (1922). "Der Widerstand gegen die Bewegung einer starren Kugel in einer zum den Flussigkeit, die zwischen zwei parallelen Ebenen Winden eingeschlossen ist." Ann. Phys. (Leipzig), 68, 89-119.

Felton, K., and Loth, E. (2001). "Spherical bubble motion in a turbulent boundary layer." Physics of Fluids, 13(9), 2564-2577.

Ferrante, A., and Elghobashi, S. (2003). "On the physical mechanism of two-way coupling in particle-laden isotropic turbulence." Physics of Fluids, 15, 315-329.

Ferziger, J. H. (1981). Numerical Methods for Engineering Application, John Wiley \& Sons, New York.

Ferziger, J. H., and Peric, M. (1996). Computational Methods for Fluid Dynamics, Springer-Verlag, New York.

Folland, G. B. (1992). Fourier Analysis and Its Applications, Brooks/Cole Publishing Company, Pacific Grove.

Fox, R. W., McDonald, A. T., and Pritchard, P. J. (2003). Introduction to Fluid Mechanics, John Wiley \& Sons, New York.

Frisch, U. (1995). Turbulence, Cambridge University Press, Cambridge. 
Froelich, J., and Rodi, W. (2002). "Introduction to large eddy simulation of turbulent flows." Closure Strategies for Turbulent and Transitional Flows, B. E. Launder, ed., Cambridge Press, 267-298.

Fukagata, K. (2000). "Numerical analysis of dispersed gas-particle two-phase turbulent flows," Ph.D. thesis, University of Tokyo.

Fukagata, K., Zahrai, S., and Bark, F. H. (1997) "Large eddy simulation of particle motion in a turbulent channel flow." 1997 ASME Fluids Engineering Division Summer Meeting, FEDSM97-3591.

Fukagata, K., Zahrai, S., and Bark, F. H. (1998). "Force balance in a turbulent particulate channel flow." International Journal of Multiphase Flow, 24, 867-887.

Fureby, C., and Grinstein, F. F. (1999). "Monotonically integrated large eddy simulation of free shear flows." AIAA Journal, 37(5), 544-556.

Fureby, C., and Grinstein, F. F. (2002). "Large eddy simulation of high Reynolds number free and wall bounded flows." Journal of Computational Physics, 181, 68.

Fureby, C., Tabor, G., Weller, H. G., and Gosman, A. D. (1997). "A comparative study of subgrid scale models in homogeneous isotropic turbulence." Physics of Fluids, 9(5), 1416-1429.

Galperin, B., and Orszag, S. A. (1993). "Large Eddy Simulation of Complex Engineering and Geophysical Flows." Cambridge University Press, Cambridge.

Germano, M. (1986). "A proposal for a redefinition of the turbulent stresses in the filtered Navier-Stokes equations." Physics of Fluids, 29, 2323-2324.

Germano, M. (1992). "Turbulence: the filtering approach." Journal of Fluid Mechanics, 238, 325-336.

Germano, M., Piomelli, U., Moin, P., and Cabot, W. H. (1991). "A dynamic subgridscale eddy viscosity model." Physics of Fluids A, 3(7), 1760-1765.

Ghia, U., Ghia, K. N., and Shin, C. T. (1982). "High-Re Solutions for Incompressible Flow Using the Navier-Stokes Equations and a Multigrid Method." Journal of Computational Physics, 48, 387-411.

Ghosal, S., Lund, T. S., and Akselvoll, K. (1995). "A dynamic localization model for large eddy simulation of turbulent flows." Journal of Fluid Mechanics, 286, 229255.

Ghosal, S., and Moin, P. (1995). "The basic equations for the large eddy simulation of turbulent flows in complex geometry." Journal of Computational Physics, 118, 24-37.

Goda, K. (1979). "A multistep technique with implicit difference schemes for calculating two- or three-dimensional cavity flows." Journal of Computational Physics, 30(1), 76-95.

Godunov, S. K. (1959). "A difference scheme for numerical computation of discontinuous solutions of hydrodynamic equations." Math. Sbornik, 47(271-306).

Goldstein, R. J., Eriksen, V. L., Olson, R. M., and Eckert, E. R. G. (1970). "Laminar separation reattachment, and transition of flow over a downstream-facing step." Transactions of ASME: Journal of Basic Engineering, 92(732).

Golub, G. H., and van Loan, C. (1990). Matrix Computations, Johns Hopkins University Press, Baltimore.

Gore, R. A., and Crowe, C. T. (1989). "Effect of particle size on modulating turbulence intensity." International Journal of Multiphase Flow, 15, 279-285. 
Gore, R. A., and Crowe, C. T. (1991). "Modulation of turbulence by a dispersed phase." Journal of Fluids Engineering, Transactions of the ASME, 113(2), 304-307.

Gosman, A. D., and Ioannides, E. (1981) "Aspects of computer simulations of liquidfueled combustors." AIAA Paper 81-0323.

Graham, D. I., and Moyeed, R. A. (2002). "How many particles for my Lagrangian simulations?" Powder Technology, 125(2-3), 179-186.

Gray, W. G., and Lee, P. C. Y. (1977). "On the theorems for local volume averaging of multiphase systems." International Journal of Multiphase Flow, 2(4), 333-40.

Green, G. (1833). "Researches on the vibration of pendulums in fluid media." Trans. $R$. Soc., 13, 54-68.

Gresho, P. (1991). "Incompressible fluid dynamics: some fundamental formulation issues." Annual Review of Fluid Mechanics, 23, 343-370.

Gresho, P., and Lee, R. (1981). "Don't suppress the wiggles-they're telling you something." Computers and Fluids, 9(2), 223-253.

Grienberger, J., and Hofmann, H. (1992). "Investigations and modelling of bubble columns." Chemical Engineering Science, 47, 2215-2220.

Grinstein, F. F., and Fureby, C. (2002). "Recent progress on MILES for high Reynolds number flows." Journal of Fluids Engineering, 124, 848-861.

Grinstein, F. F., and Guirguis, R. H. (1992). "Effective viscosity in the simulation of spatially evolving shear flows with monotonic FCT models." Journal of Computational Physics, 101, 165-175.

Harten, A. (1983). "High resolution schemes for hyperbolic conservation laws." Journal of Computational Physics, 49, 357-393.

Harten, A. (1984). "On a class of high resolution total-variation-stable finite-difference schemes." SIAM Journal on Numerical Analysis, 21(1), 1-23.

Harten, A., Hyman, J. M., and Lax, P. D. (1976). "On finite-difference approximations and entropy conditions for shocks." Communications on Pure and Applied Mathematics, 29, 297-322.

Hetsroni, G. (1989). "Particles-turbulence interaction." International Journal of Multiphase Flow, 15, 735-746.

Hewitt, G. F. (1999). "Multiphase flow: views of the future." Multiphase Science and Technology, 11(1), 59-77.

Hinze, J. O. (1972). "Turbulent fluid and particle interaction." Progress in Heat and Mass Transfer, G. Hestroni, S. Sideman, and J. P. Hartnett, eds., 433-452.

Hinze, J. O. (1975). Turbulence, McGraw-Hill, New York.

Hirsch, C. (1988). Numerical Computation of Internal and External Flows: Fundamentals of Numerical Discretization, John Wiley \& Sons, Chichester.

Hirsch, C. (1990). Numerical Computation of Internal and External Flows: Computational Methods for Inviscid and Viscous Flows, John Wiley \& Sons.

Hjelmfelt, J., A.T., and Mockros, L. F. (1966). "Motion of discrete particles in turbulent fluid." Applied Scientific Research, 16(2), 149-161.

Hjelmfelt, J., A.T., and Mockros, L. F. (1967). "Stokes flow behavior of accelerating sphere." American Society of Civil Engineers Proceedings, Journal of the Engineering Mechanics Division, 93(EM6), 87-102.

Ho, C.-M., and Huang, L.-S. (1982). "Subharmonics and vortex merging in mixing layers." Journal of Fluid Mechanics, 119, 443-473. 
Ho, C.-M., and Huerre, P. (1984). "Perturbed free shear layers." Annual Review of Fluid Mechanics, 16, 365.

Ho, C.-M., Zohar, Y., Foss, J. K., and Buell, J. (1991). "Phase decorrelation of coherent structures in a free shear layer." Journal of Fluid Mechanics, 230, 319-337.

Hoffmann, K. A., and Chiang, S. T. (1993). Computational Fluid Dynamics for Engineers, Engineering Education System.

Hoomans, B. P. B., Kuipers, J. A. M., Briels, W. J., and van Swaaij, W. P. M. (1995). "Discrete particle simulation of bubble and slug formation in a two-dimensional gas-fluidised bed: a hard-sphere approach." Chemical Engineering Science, 51(1), 99-118.

Howes, F. A., and Whitaker, S. (1985). "Spatial averaging theorem revisited." Chemical Engineering Science, 40(8), 1387-1392.

Hu, G. (2002). "Uncertainty assessment for CFD using error transport equation," Master Thesis, Mechanical and Aerospace Engineering Department, West Virginia University, Morgantown.

Huang, H., and Takagi, S. (2003). "PHYALIS: a new method for particle flow simulation. Part III: convergence analysis of two-dimensional flows." Journal of Computational Physics, 189, 493-511.

Hussain, A. K. M. F., and Reynolds, W. C. (1970). "The mechanics of an organized wave in turbulent shear flow." Journal of Fluid Mechanics, 41, 241-258.

Hussain, A. K. M. F., and Reynolds, W. C. (1975). "Measurements in fully developed turbulent channel flow." Transactions of the ASME, Series I, Journal of Fluids Engineering, 97, 568-578.

Hussain, A. K. M. F., and Zaman, K. M. B. Q. (1985). "An experimental study of organized motions in the turbulent plane mixing layer." Journal of Fluid Mechanics, 159, 85.

Hwang, R. R., and Peng, Y. F. (1995). "Computation of backward facing step flows by a second-order Reynolds stress closure model." International Journal for Numerical Methods in Fluids, 21(3), 223-235.

Isaacson, E., and Keller, H. B. (1967). Analysis of Numerical Methods, John Wiley, New York.

Ishii, M. (1975). Thermo Fluid Dynamics of Two-Phase Flows, Eyrolles, Paris.

Ishii, M. (1987) "Two-fluid model for two-phase flow." Proceeding of 2nd International Workshop on Two-Phase Flow Fundamentals 16-20.

Jimenez, J. (1983). "A spanwise structure in the plane shear layer." Journal of Fluid Mechanics, 13, 319.

Jones, W. P., and Launder, B. E. (1972). "The prediction of laminarization with a twoequation model of turbulence." International Journal of Heat and Mass Transfer, $15,301-314$.

Jordan, S. A., and Ragab, S. A. (1994). "On the unsteady and turbulent characteristics of the three-dimensional shear-driven cavity flow." Journal of Fluids Engineering, 116(3), 439-449.

Kaftori, D., Hetsroni, G., and Banerjee, S. (1995). "Particle behavior in the turbulent boundary layer. I. Motion, deposition, and entrainment." Physics of Fluids, 7(5), 1095. 
Katz, J., and Meneveau, C. (1996). "Wake-induced relative motion of bubbles rising in line." International Journal of Multiphase Flow, 22(2), 239-58.

Kelly, R. E. (1967). "On stability of inviscid shear layer which is periodic in space and time." Journal of Fluid Mechanics, 27(Part 4), 657-689.

Khosla, P. K., and Rubin, S. G. (1974). "A diagonally dominant second-order accurate implicit scheme." Computers and Fluids, 2, 207-209.

Kim, J., and Moin, P. (1985). "Application of a fractional-step method to incompressible Navier-Stokes equations." Journal of Computational Physics, 59, 308-323.

Kim, J., Moin, P., and Moser, R. (1987). "Turbulence statistics in fully developed channel flow at low Reynolds number." Journal of Fluid Mechanics, 177, 133166.

Kim, S., and Karrila, S. J. (1991). Microhydrodynamics: Principles and Selected Applications, Butterworth-Heinann, Boston.

Kline, S. J., Reynolds, W. C., Schraub, F. A., and Runstadler, P. W. (1967). "The structure of turbulent boundary layers." Journal of Fluid Mechanics, 30, 741-773.

Kojima, E., Akehata, T., and Shirai, T. (1975). "Behavior of single air bubbles held stationary in downward flows." Journal of Chemical Engineering of Japan, 8, 108-113.

Kolmogorov, A. N. (1942). "The equations of turbulent motion in an incompressible fluid." Izvestia Acad., USSR; Physics, 6(1-2), 56-58.

Kontomaris, K., Hanratty, T. J., and McLaughlin, J. B. (1992). "An algorithm for tracking fluid particles in a spectral simulation of turbulent channel flow." Journal of Computational Physics, 103(2), 231-42.

Koseff, J. R., Street, R. L., Gresho, P. M., Upson, C. D., Humphrey, J. A. C., and To, W. M. (1983) "Three-dimensional lid-driven cavity flow: experiment and simulation." Proceedings of Third International Conference on Numerical Methods in Laminar and Turbulent Flows, Seattle, Washington, August 8-11563581.

Kraichnan, R. (1970). "Diffusion by a random velocity field." Physics of Fluids, 11(1), 22-31.

Kreplin, H., and Eckelmann, M. (1979). "Behavior of the three fluctuating velocity components in the wall region of a turbulent channel flow." Physics of Fluids, 22, 1233-1239.

Kreyszig, E. (1993). Advanced Engineering Mathematics, John Wiley \& Sons, Inc., New York.

Kriebel, A. R. (1961). "Particle trajectories in a gas centrifuge." Journal of Basic Engineering, Transaction of ASME, 83(D), 333-340.

Kuo, T. C., Pan, C., and Chieng, C. C. (1997). "Eulerian-Lagrangian computations on phase distribution of two-phase bubbly flows." International Journal for Numerical Methods in Fluids, 24, 579-593.

Lahey, R. T., Jr., and Drew, D. A. (1988). "The three-dimensional time and volume averaged conservation equations of two-phase flow." Advances in nuclear science and technology. Vol.20, Plenum, 1-69.

Lakehal, D. (2002). "On the modelling of multiphase turbulent flows for environmental and hydrodynamic applications." International Journal of Multiphase Flow, 28, 823-863. 
Lakehal, D., Smith, B. L., and Milelli, M. (2002). "Large-eddy simulation of bubbly turbulent shear flows." Journal of Turbulence, 3, 025.

Lance, M., and Bataille, J. (1991). "Turbulence in the liquid phase of a uniform bubbly air-water flow." Journal of Fluid Mechanics, 222, 95-118.

Landau, L. D., and Lifschitz, E. M. (1971). Lehrbuch der Theoretischen Physik (Vol. VI): Hydrodynamk, Akademie, Berlin, Germany.

Laney, C. B. (1998). Computational Gasdynamics, Cambridge University Press, Cambridge.

Lapple, C. E., and Shepherd, C. B. (1940). "Calculation of particle trajectories." Industrial Engineering of Chemistry, 32, 605-617.

Lasheras, J. C., and Choi, H. (1988). "Three-dimensional instability of a plane free shear layer: an experimental study of the formation and evolution of streamwise vortices." Journal of Fluid Mechanics, 189, 53-86.

Lasheras, J. C., Choi, J. S., and Maxworthy, T. (1986). "On the origin and evolution of streamwise vortical structures in a plane, free shear layer." Journal of Fluid Mechanics, 172(231-258).

Launder, B. E., Reece, G. J., and Rodi, W. (1975). "Progress in the development of a Reynolds-stress turbulence closure." Journal of Fluid Mechanics, 68(3), 537-566.

Launder, B. E., and Sharma, B. I. (1974). "Application of the energy-dissipation model of turbulence to the calculation of flow near a spinning disc." Letters of Hear and Mass Transfer, 1, 131-138.

Launder, B. E., and Spalding, D. B. (1972). Mathematical Models of Turbulence, Academic Press, London.

Lazaro, B. J. (1989). "Particle dispersion in turbulent free-shear flows," PhD Thesis, University of Southern California.

Lazaro, B. J., and Lasheras, J. C. (1992a). "Particle dispersion in the developing free shear layer. Part 1. unforced flow." Journal of Fluid Mechanics, 235, 143-178.

Lazaro, B. J., and Lasheras, J. C. (1992b). "Particle dispersion in the developing free shear layer. part 2. forced flow." Journal of Fluid Mechanics, 235, 179-221.

Le, H., Moin, P., and Kim, J. (1997). "Direct numerical simulation of turbulent flow over a backward-facing step." Journal of Fluid Mechanics, 330, 349-374.

Leboissetier, A., Okong'o, N., and Bellan, J. (2005). "Consistent large-eddy simulation of a temporal mixing layer laden with evaporating drops. Part 2. A posteriori modelling." Journal of Fluid Mechanics, 523, 37-78.

Lei, K., Taniguchi, N., and Kobayashi, T. (2002). "Large eddy simulation of particleladen turbulent channel flow considering SGS coupling (A proposal of dynamic SGS model for two-way coupling)." JSME International Journal, Series B (Fluids and Thermal Engineering), 45(3), 164-73.

Leonard, A. (1974). "Energy cascade in large eddy simulations of turbulent fluid flows." Advances in Geophysics, 18A, 237-248.

Leonard, B. P. (1979). "A stable and accurate convection modelling procedure based on quadratic upstream interpolation." Computer Methods in Applied Mechanics and Engineering, 19, 59-98.

Leonard, B. P., and Drummond, J. (1995). "Why you should not use 'hybrid', 'power-law' or related exponential schemes for convective modelling - there are much better 
alternatives." International Journal for Numerical Methods in Fluids, 20(6), 421442.

Lesieur, M., and Metais, O. (1996). "New trends in large-eddy simulations of turbulence." Annual Review of Fluid Mechanics, 28, 45-82.

LeVeque, R. J. (1992). Numerical Methods for Conservation Laws, Basel.

Lighthill, M. J. (1970). Introduction to Fourier Analysis and Generalized Functions, Cambridge University Press, Cambridge.

Lilly, D. K. (1967) "The representation of small-scale turbulence in numerical simulation experiments." Proceedings of IBM Scientific Computing Symposium on Environmental Sciences, Yorktown Heights, NY, IBM form No. 320-1951, 195.

Lilly, D. K. (1992). "A proposed modification of the Germano subgrid-scale closure method." Physics of Fluids A, 4(3), 633-635.

Lin, S. J., and Corcos, G. M. (1984). "The mixing layer: deterministic models of a turbulent flow. III. The effect of plane strain on the dynamics of streamwise vortices." Journal of Fluid Mechanics, 141, 139-78.

Liou, T.-M., Chen, S.-H., and Hwang, P.-W. (2002). "Large eddy simulation of turbulent wake behind a square cylinder with a nearby wall." Journal of Fluids Engineering, 124, 81-90.

Liu, T. J., and Bankoff, S. G. (1993). "Structure of air-water bubbly flow in a vertical pipe - I. Liquid mean velocity and turbulence measurements." International Journal of Heat and Mass Transfer, 36(4), 1049-1060.

Lock, R. C. (1951). "The velocity distribution in the laminar boundary layer between parallel streams." Quarterly Journal of Mechanics and Applied Mathematics, 4, $42-63$.

Lopez, J. M., and Bulbeck, C. J. (1993). "Behavior of streamwise rib vortices in a threedimensional mixing layer." Physics of Fluids A, 5(7), 1694-1702.

Loth, E. (1997) "Dispersion of particles, droplets and bubbles in a turbulent free shear layer." ASME Fluids Engineering Summer Meeting, Vancouver, Canada, June, FEDSM97-3607.

Loth, E. (2000). "Numerical approaches for motion of dispersed particles, droplets and bubbles." Progress in Energy and Combustion Science, 26(3), 161-223.

Loth, E., Taeibi-Rahni, M., and Tryggvason, G. (1997). "Deformable bubbles in a free shear layer." International Journal of Multiphase Flow, 23(5), 977-1001.

Lumley, J. L. (1978). "Computational modeling of turbulent flows." Advances in Applied Mathematics, 18, 123-176.

Lyn, D. A., Einav, S., Rodi, W., and Park, J. H. (1995). "A laser-doppler velocimetry study of ensemble-averaged characteristics of the turbulent near wake of a square cylinder." Journal of Fluid Mechanics, 304, 285-319.

Maccoll, J. W. (1928). "Aerodynamics of a spinning sphere." Royal Aeronautical Society -- Journal, 32(213), 777-798.

Magaud, F., Najafi, A. F., Angilella, J. R., and Souhar, M. (2003). "Modeling and qualitative experiments on swirling bubbly flows: single bubble with Rossby number of order 1." Journal of Fluids Engineering, 125, 239-246.

Magnaudet, J., and Eames, I. (2000). "The motion of high-Reynolds-number bubbles in inhomogeneous flows." Annual Review of Fluid Mechanics, 32, 659-708. 
Manson, J., Pender, G., and Wallis, S. (1996). "Limitations of traditional finite volume discretizations for unsteady computational fluid dynamics." AIAA Journal, 34(5), 1074-1076.

Manson, J., and Wallis, S. (1997). "Accuracy characteristics of traditional finite volume discretization for unsteady computational fluid dynamics." Journal of Computational Physics, 132(1), 149-153.

Mansour, N. N., Kim, J., and Moin, P. (1988). "Reynolds-stress and dissipation-rate budgets in a turbulent channel flow." Journal of Fluid Mechanics, 194, 15-44.

Marble, F. E. (1970). "Dynamics of dusty gases." Annual Review of Fluid Mechanics, 2 , 397-446.

Marden, E., and Bakker, A. (2002). Computational Fluid Mixing, Fluent Inc., Lebanon, $\mathrm{NH}$.

Margolin, L. G., and Rider, W. J. (2002). "A rational for implicit turbulence modelling." International Journal for Numerical Methods in Fluids, 39, 821-841.

Margolin, L. G., Smolarkiewicz, P. K., and Wyszogrodzki, A. A. (2002). "Implicit turbulence modeling for high Reynolds number flows." Transactions of the ASME, 124, 862-867.

Marie, J. L., Moursali, E., and Tran-Cong, S. (1997). "Similarity law and turbulence intensity profiles in a bubbly boundary layer at low void fractions." International Journal of Multiphase Flow, 23(2), 227-247.

Mathieu, J., and Scott, J. (2000). An Introduction to Turbulent Flow, Cambridge University Press, Cambridge.

Maxey, M. R., Chang, E. J., and Wang, L.-P. (1994). "Simulation of interactions between microbubbles and turbulent flows." Applied Mechanics Review, 47(6), S70-S74.

Maxey, M. R., Chang, E. J., and Wang, L.-P. (1996). "Interactions of particles and microbubbles with turbulence." Experimental Thermal and Fluid Science, 12(4), 417-425.

Maxey, M. R., Patel, B. K., Chang, E. J., and Wang, L.-P. (1997). "Simulations of dispersed turbulent multiphase flow." Fluid Dynamics Research, 20(1-6), 143156.

Maxey, M. R., and Riley, J. J. (1983). "Equation of motion for a small rigid sphere in a nonuniform flow." Physics of Fluids, 26(4), 883-889.

McLaughlin, J. B. (1989). "Aerosol particle deposition in numerically simulated channel flow." Physics of Fluids A, 1, 1211.

McLaughlin, J. B. (1991). "Inertial migration of a small sphere in linear shear flows." Journal of Fluid Mechanics, 224, 261-274.

McLaughlin, J. B. (1993). "The lift on a small sphere in wall-bounded linear shear flows." Journal of Fluid Mechanics, 246, 249-265.

Mei, R., and Klausner, J. F. (1992). "Unsteady force on a spherical bubble with finite Reynolds number with small fluctuations in the free-stream velocity." Physics of Fluids A, 4(1), 63.

Mei, R., Lawrence, C. J., and Adrian, R. J. (1991). "Unsteady drag on a sphere at finite Reynolds number with small fluctuations in the free-stream velocity." Journal of Fluid Mechanics, 233, 613-631.

Meneveau, C., and Katz, J. (2000). "Scale-invariance and turbulence models for largeeddy simulation." Annual Review of Fluid Mechanics, 32, 1-32. 
Meneveau, C., and Lund, T. S. (1997). "The dynamic Smagorinsky model and scaledependent coefficients in the viscous range of turbulence." Physics of Fluids, 9(2), 3932-3934.

Meneveau, C., Lund, T. S., and Cabot, W. H. (1996). "A Lagrangian dynamic subgridscale model of turbulence." Journal of Fluid Mechanics, 224, 429-484.

Meneveau, C., and O'Neil, J. (1994). "Scaling laws of the dissipation rate of turbulent subgrid-scale kinetic energy." Physical Review E, 49(4), 2866-2874.

Menon, S., and Pannala, S. (1998) "Subgrid two-phase mixing and combustion modeling for large-eddy simulations." Proceedings of the 1998 International Gas Turbine \&amp; Aeroengine Congress \&amp; Exhibition, Jun 2-5 1998, Stockholm, Sweden8.

Menon, S., Yeung, P. K., and Kim, W. W. (1996). "Effects of subgrid models on the computed interscale energy transfer in isotropic turbulence." Computes and Fluids, 25(2), 165-180.

Michaelides, E. E. (1992). "A novel way of computing the Basset term in unsteady multiphase flow computations." Physics of Fluids A, 4(7), 1579-1582.

Michaelides, E. E. (1997). "Review: the transient equation of motion for particles, bubbles, and droplets." Journal of Fluids Engineering, 1997, 233-247.

Michaelides, E. E. (2003). "Hydrodynamic force and heat/mass transfer from particles, bubbles, and drops - the Freeman scholar lecture." Journal of Fluids Engineering, $125,1-30$.

Migdal, D., and Agosta, V. D. (1967). "A source flow model for continuum gas-particle flow." Journal of Applied Mechanics (ASME Transactions), 35(4), 860.

Milelli, M. (2002). "A numerical analysis of confined turbulent bubble plumes," Ph.D. thesis, University of Torino.

Milelli, M., Smith, B. L., and Lakehal, D. (2002). "Large-eddy simulation of turbulent shear flows laden with bubbles." Direct and Large-eddy Simulation, B. J. Geurts, O. Friedrich, and O. Metais, eds., Kluwer Academic Publishers, 461-470.

Miller, R. S., and Bellan, J. (2000). "Direct numerical simulation and subgrid analysis of a transitional droplet laden mixing layer." Physics of Fluids, 12(3), 650-71.

Mitchell, A. R., and Griffiths, D. F. (1980). The Finite Difference Method in Partial Differential Equations, John Wiley \& Sons Ltd., Chichester.

Moin, P., and Kim, J. (1982). "Numerical investigation of turbulent channel flow." Journal of Fluid Mechanics, 118, 341-377.

Moore, D. W., and Saffman, P. G. (1975). "The density of organized vortices in a turbulent mixing layer." Journal of Fluid Mechanics, 69(pt.3), 465-73.

Moraga, F. (1998). "Lateral forces on rigid spheres in turbulent uniform shear flow," $\mathrm{PhD}$ thesis, Rensselaer Polytechnic Institute, Troy.

Morsi, S. A., and Alexander, A. J. (1972). "An investigation of particle trajectories in two-phase flow systems." Journal of Fluid Mechanics, 55(2), 193-208.

Moser, R., Kim, J., and Mansour, N. (1999). "Direct numerical simulation of turbulent channel flow up to Re_tau=590." Physics of Fluids, 11(4), 943-945.

Moser, R. D., and Rogers, M. M. (1993). "The three-dimensional evolution of a plane mixing layer: pairing and transition to turbulence." Journal of Fluid Mechanics, 247, 275-320. 
Munson, B. R., Young, D. F., and Okiishi, T. H. (1994). Fundamentals of Fluid Mechanics, John Wiley \& Sons Inc., New York.

Murray, J. A., Piomelli, U., and Wallace, J. M. (1996). "Spatial and temporal filtering of experimental data for a priori studies of subgrid-scale stresses." Physics of Fluids, 8, 1978.

Naciri, A. (1992). "Motion of gas bubbles in a turbulent and rotating fluid," Ph.D. thesis, L'ecole Centrale De Lyon, Lyons.

Nakoryakov, V. E., Kashinsky, O. N., Randin, V. V., and Timkin, L. S. (1996). "Gasliquid bubbly flow in vertical pipes." Journal of Fluids Engineering, Transactions of the ASME, 118(2), 377-382.

Nallasamy, M., and Krishna Prasad, K. (1977). "On cavity flow at high Reynolds number." Journal of Fluid Mechanics, 79(2), 391-414.

Nygaard, K. J., and Glezer, A. (1991). "Evolution of streamwise vortices and generation of small-scale motion in a plane mixing layer." Journal of Fluid Mechanics, 231, 257-301.

Nygaard, K. J., and Glezer, A. (1994). "Effect of phase variations and cross-shear on vortical structures in a plane mixing layer." Journal of Fluid Mechanics, 276, 2159.

Odar, F. (1966). "Verification of proposed equation for calculation of forces on sphere accelerating in viscous fluid." Journal of Fluid Mechanics, 25(3), 591-592.

Odar, F., and Hamilton, W. S. (1964). "Forces on sphere accelerating in viscous fluid." Journal of Fluid Mechanics, 18(Part 2), 302-314.

Okong'o, N. A., and Bellan, J. (2000). "A priori subgrid analysis of temporal mixing layers with evaporating droplets." Physics of Fluids, 12(6), 1573-1591.

Okong'o, N. A., and Bellan, J. (2004). "Consistent large-eddy simulation of a temporal mixing layer laden with evaporating drops. Part 1. Direct numerical simulation, formulation and a priori analysis." Journal of Fluid Mechanics(499), 1-47.

Oran, E. S., and Boris, J. P. (1993). "Computing turbulent shear flows - a convenient conspiracy." Computers in Physics, 7, 523-533.

Oseen, C. W. (1910). "Ueber die Stokes'sche Formel und Ueber eine verwandte Aufgabe in der Hydrodynamik." Ark. Mat. Astron. Fys., 6(29).

Oseen, C. W. (1913). "Ueber den Goeltigkeitsbereich der Stokesschen Widerstandsformel." Ark. Mat. Astron. Fys., 9(19).

Pan, Y., and Banerjee, S. (1996). "Numerical simulation of particle interactions with wall turbulence." Physics of Fluids, 8(10), 2733-2755.

Pandya, R. V. R., and Mashayek, F. (2002). "Two-fluid large-eddy simulation approach for particle-laden turbulent flows." International Journal of Heat and Mass Transfer, 45(24), 4753-4759.

Pannala, S., and Menon, S. (1998) "Large eddy simulation of two-phase turbulent flows." 36th Aerospace Sciences Meeting and Exhibit, Reno, NV, January 12-15, AIAA 98-0163.

Parzynski, W. R., and Zipse, P. W. (1982). Introduction to Mathematical Analysis, McGraw-Hill, Inc., New York.

Patankar, S. (1980). Numerical Heat Transfer and Fluid Flow, Hemisphere Publishing Corporation, McGraw-Hill Book Company, New York. 
Pdeinotti, S., Mariotti, G., and Banerjee, S. (1993). "Direct numberical simulation of particle behavior in the wall region of turbulent flows in horizontal channels." International Journal of Multiphase Flow, 18, 927.

Peaceman, D. W., and Rachfod, H. H. (1955). "The numerical solution of parabolic and elliptic differential equations." Journal of Society of Industrial and Applied Mathematics, 3, 28.

Perng, C. Y., and Street, R. L. (1989). "Three-dimensional unsteady flow simulations: alternative strategies for a volume-averaged calculation." International Journal for Numerical Methods in Fluids, 9(3), 341-362.

Pierrehumbert, R. T., and Widnall, S. E. (1982). "Two- and three-dimensional instabilities of a spatially periodic shear layer." Journal of Fluid Mechanics, 114, 59-82.

Piomelli, U. (1993). "High Reynolds number calculations using the dynamic subgrid scale stress model." Physics of Fluids A, 5, 1484-1490.

Piomelli, U. (1999). "Large-eddy simulation: achievements and challenges." Progress in Aerospace Sciences, 35, 335-362.

Piomelli, U., Cabot, W. H., Moin, P., and Lee, S. (1991). "Subgrid-scale backscatter in turbulent and transitional flows." Physics of Fluids A, 3(7), 1766-1771.

Poisson, S. A. (1831). "Memoire sur les Mouvements Simultanes d'un Pendule et de L'air Environnemant." Mem. Acad. Sci., 9, 521-523.

Pope, S. (2000). Turbulent Flows, Cambridge University Press, Cambridge.

Pope, S. B. (2004). "Ten questions concerning the large-eddy simulation of turbulent flows." New Journal of Physics, 6(35).

Prandtl, L. (1925). "Bericht ueber die Entstehung der Turbulenz." Zeitschrift fuer Angewandte Mathematic und Mechanik, 5, 136-139.

Prandtl, L. (1945). "Ueber ein neues Formelsystem fuer die ausgebildete Turbulenz." Nachr. Akad. Wiss. Goettingen Math-Phys., K1, 6-19.

Press, W. H., Teukolsky, S. A., Vetterling, W. T., and Flannery, B. P. (1992). Numerical Recipes in Fortran, the Art of Scientific Computing, Cambridge University Press, Cambridge.

Prosperetti, A., and Oguz, H. N. (2001). "PHYSALIS: A new O(N) method for the numerical simulation of dispersed systems: potential flow of spheres." Journal of Computational Physics, 167, 196-216.

Rajagopalan, S., and Antonia, R. A. (1979). "Some properties of the large structure in a fully developed turbulent duct flow." Physics of Fluids, 22, 614-622.

Ranade, V. V. (1992). "Flow in bubble column: some numerical experiments." Chemical Engineering Science, 47, 1857.

Reeks, M. W. (1977). "On the dispersion of small particles suspended in an isotropic turbulent fluid." Journal of Fluid Mechanics, 83(3), 529-546.

Reutsch, G. R., and Meiburg, E. (1994). "Two-way coupling in shear layers with dilute bubble concentrations." Physics of Fluids, 6(8), 2656-2670.

Reynolds, O. (1895). "On the dynamical theory of incompressible viscous fluids and the determination of the criterion." Philosophical Transactions of the Royal Society of London, Series A, 186, 123.

Richardson, L. (1910). "The approximate arithmetical solution by finite differences of physical problems involving differential equations, with an application to the 
stresses in a masonary dam." Transactions of the Royal Society of London, 210(A), 307.

Richardson, L., and Gaunt, J. A. (1927). "The deferred approach to the limit." Transactions of the Royal Society of London, 226(A), 299.

Richtmyer, R. D., and Morton, K. W. (1967). Difference Methods for Initial Value Problems, John Wiley, New York.

Rightley, P. M. (1995). "Bubble dispersion and interphase coupling in a free shear flow," PhD Thesis, Department of Mechanical Engineering, University of California, San Diego, San Diego.

Rightley, P. M., and Lasheras, J. C. (2000). "Bubble dispersion and interphase coupling in a free-shear flow." Journal of Fluid Mechanics, 412, 21-59.

Riviere, N., Cartellier, A., Timkin, L., and Kashinsky, O. (1999). "Wall shear stress and void fraction in Poiseuille bubbly flows: Part II: Experiments and validity of analytical predictions." European Journal of Mechanics, B/Fluids, 18(5), 847867.

Rizk, M. A., and Elghobashi, S. E. (1985). "The motion of a spherical particle suspended in a turbulent flow near a plane wall." Physics of Fluids, 28(3), 806-817.

Roache, P. J. (1972). "On artificial viscosity." Journal of Computational Physics, 10(2), 351-366.

Rodi, W. (1980). Turbulence Models and Their Application in Hydraulics - a State-ofthe-art Review, IAHR (International Association of Hydraulic Research) Publication, Delft, The Netherlands.

Rodi, W. (1997). "Comparison of LES and RANS calculations of the flow around bluff bodies." Journal of Wind Engineering and Industral Aerodynamics, 69-71, 55-75.

Rodi, W. (1998). "Large-eddy simulations of the flow past bluff bodies: state of the art." JSME International Journal, Series B, 41(2), 361-374.

Rodi, W., Ferziger, J., Breuer, M., and Pourquie, M. (1995) "Proceedings of workshop on large-eddy simulation of flows past bluff bodies." Rottach-Egern, Germany, June.

Rodi, W., Ferziger, J. H., Breuer, M., and Pourquie, M. (1997). "Status of large eddy simulation: results of a workshop." Journal of Fluids Engineering, 119, 248-262.

Rogallo, R. S., and Moin, P. (1984). "Numerical simulation of turbulent flows." Annual review of fluid mechanics, 16, 99-137.

Rogers, M. M., and Moser, R. D. (1992). "The three-dimensional evolution of a plane mixing layer: the Kelvin-Helmholtz rollup." Journal of Fluid Mechanics, 243, 183-226.

Rogers, M. M., and Moser, R. D. (1994). "Direct simulation of a self-similar turbulent mixing layer." Physics of Fluids, 6(2), 903-923.

Roig, V., Suzanne, C., and Masbernat, L. (1998). "Experimental investigation of a turbulent bubbly mixing layer." International Journal of Multiphase Flow, 24(1), $35-54$.

Rubinow, S. I., and Keller, J. B. (1961). "The transverse force on spinning sphere moving in a viscous fluid." Journal of Fluid Mechanics, 11, 447.

Rudinger, G. (1980). Fundamentals of Gas-Particle Flow, Elsevier Scientific Publishing Company, Amsterdam. 
Sabin, C. M. (1965). "An analytical and experimental study of the plane, incompressible, turbulent free-shear layer with arbitrary velocity ratio and pressure gradient." Transactions of the ASME D, 87, 421.

Saffman, P. G. (1965). "The lift on a small sphere in a slow shear flow." Journal of Fluid Mechanics, 22, 385-400.

Saffman, P. G. (1968). "Corrigendum to "The lift on a small sphere in a slow shear flow"." Journal of Fluid Mechanics, 31, 624.

Saffman, P. G. (1970). "A Model for inhomogeneous turbulent flow." Proc. Roy. Soc. London, A317, 417-433.

Sagaut, P. (2002). Large Eddy Simulation for Incompressible Flows, Springer Verlag, Berlin.

Sandham, N. D., and Reynolds, W. C. (1989). "Some inlet plane effects on the numerically simulated spatially developing two-dimensional mixing layer." Turbulent Shear Flows, J. C. Andre, J. Cousteix, F. Durst, B. E. Launder, F. W. Schmidt, and Whitela, eds., Springer Verlag, 441-447.

Sankaran, V., and Menon, S. (2002). "LES of spray combustion in swirling flows." Journal of Turbulence, 2, 011.

Scarborough, J. B. (1958). Numerical Mathematical Analysis, Johns Hopkins University Press, Baltimore, MD.

Schlichting, H. (1979). Boundary-Layer Theory, J. Kestin, translator, McGraw-Hill Book Company, New York.

Schreiber, R., and Keller, H. B. (1983). "Driven cavity flows by efficient numerical techniques." Journal of Computational Physics, 49(2), 310-333.

Schuh, M. J., Schuler, C. A., and Humphrey, J. A. C. (1989). "Numerical calculation of particle-laden gas flows past tubes." AIChE Journal, 35(3), 466-480.

Schumann, U. (1975). "Subgrid scale model for finite difference simulations of turbulent flows in plane channels and annuli." Journal of Computational Physics, 18(4), 376-404.

Scotti, A. C., Meneveau, C., and Fatica, M. (1997). "Dynamic Smagorinsky model on anisotropic grids." Physics of Fluids, 9, 1856-1858.

Shepard, D. (1968) "A two-dimensional interpolation function for irregularly-spaced data." 23th National Conference ACM517-524.

Shih, T.-H., Liou, W. W., Shabbir, A., and Zhu, J. (1995). "A new k-e eddy viscosity model for high Reynolds number turbulent flows - model development and validation." Computes and Fluids, 24(3), 227-238.

Shiller, L., and Naumann, A. (1933). "Ueber die grundlegenden berechungen bei der schwerkraftaufbereitung." Verein Deutscher Ingenieure, 77, 318.

Shirolkar, J. S., Coimbra, C. F. M., and Queiroz McQuay, M. (1996). "Fundamental aspects of modeling turbulent particle dispersion in dilute flows." Progress in Energy and Combustion Science, 22, 363-399.

Simonin, O., and Squires, K. D. (2002) "What can be learned from LES of particle-laden turbulent flows." Proceedings of the 2002 ASME Joint U.S.-European Fluids Engineering Conference, Montreal, Que., United States, Jul 14-18, 257, 537-538.

Simonin, O., Wang, Q., and Squires, K. D. (1997) "Comparison between two-fluid model predictions and large eddy simulation results in a vertical gas-solid turbulent channel flow." Proceedings of the 1997 ASME Fluids Engineering Division 
Summer Meeting, FEDSM'97. Part 17 (of 24), Jun 22-26 1997, Vancouver, Can, $17,10$.

Sirignano, W. A. (1993). "Fluid dynamics of sprays - Freeman scholar lecture." ASME Journal of Fluids Engineering, 115, 345-378.

Sirignano, W. A. (1999). Fluid Dynamics and Transport of Droplets and Sprays, Cambridge University Press, Cambridge.

Sirignano, W. A. (2005). "Volume averaging for the analysis of turbulent spray flows." International Journal of Multiphase Flow, 31(6), 675-705.

Slattery, J. C. (1972). Momentum, Energy and Mass Transfer in Continua, McGraw Hill, New York.

Smagorinsky, J. (1963). "General circulation experiments with the primitive equations: I. the basic experiment." Monthly Weather Review, 91(3), 99-164.

Smirnov, A., Celik, I., and Shi, S. (2005a). "LES of bubble dynamics in wake flows." Computers and Fluids, 34(3), 351-373.

Smirnov, A., Hu, G., and Celik, I. (2005b) "Embarrassingly parallel computations of bubbly wakes." International Conference on Parallel CFD, College Park, MD, May.

Smirnov, A., Shi, S., and Celik, I. B. (2002). "Random flow generation technique for large eddy simulation and particle dynamics modeling." Journal of Fluids Engineering, 123, 359-371.

Smith, J. (1996). "An accurate Navier-Stokes solver with an application to unsteady flows," Dissertation, Mechanical and Aerospace Engineering Department, West Virginia University, Morgantown.

Smith, J., and Celik, I. (1999). "On time accuracy of numerical simulations of unsteady flows." International Journal of Applied Science \& Computations, 6(1), 18-32.

Smith, P. J., Fletcher, T. J., and Smoot, L. D. (1981) "Model for pulverized coal-fired reactors." 18th International Symposium on Combustion 1258.

Sod, G. A. (1985). Numerical Methods in Fluid Dynamics: Initial and Initial Boundaryvalue Problems, Cambridge University Press, Cambridge.

Sohankar, A., and Davidson, L. (2000). "Large eddy simulation of flow past a square cylinder of different subgrid scale models." Journal of Fluids Engineering, 122, $39-47$.

Sohankar, A., Davidson, L., and Norberg, C. (1999). "A dynamic one-equation subgrid scale model for simulation of flow around a square cylinder." Engineering Turbulence Modelling and Experiments, W. Rodi and D. Laurence, eds., Elsevier Science, 227-236.

Sohankar, A., Davidson, L., and Norberg, C. (2000). "Large eddy simulation of flow past a square cylinder: comparison of different subgrid scale models." Journal of Fluids Engineering, 122, 39-47.

Sokolichin, A., and Eigenberger, G. (1994). "Gas-liquid flow in bubble columns and loop reactors. Part I. Detailed modeling and numerical simulation." Chemical Engineering Science, 49, 5735.

Sokolichin, A., and Eigenberger, G. (1999). "Applicability of the standard k-e turbulence model to the dynamic simulation of bubble columns: Part I. Detailed numerical simulations." Chemical Engineering Science, 54, 2273-2284. 
Sokolichin, A., Eigenberger, G., and Lapin, A. (2004). "Simulation of buoyancy driven bubbly flow: established simplifications and open questions." AIChE Journal, 50(1), 24-45.

Sokolichin, A., Eigenberger, G., Lapin, A., and Luebbert, A. (1997). "Dynamic numerical simulation of gas-liquid two-phase flows. Euler/Euler versus Euler/Lagrange." Chemical Engineering Science, 52(4), 611-626.

Sommerfeld, M., Decker, S., and Kohnen, G. (1997) "Time-dependent calculation of bubble columns based on Reynolds-averaged Navier-Stokes equations with k-e turbulence model." 2nd Japanese-German Symposium on Multi-Phase Flow, Tokyo, Paper No. 3-1-4323-334.

Sonneveld, P. (1989). "CGS, a fast Lanczos type solver for non-symmetric linear systems." SIAM Journal on Scientific Computing, 10, 36-52.

Soo, S. L. (1967). Fluid Dynamics of Multiphase Systems, Blaisdell, Watham, MA.

Soo, S. L. (1989). Particulate and Continuum: Multiphase Fluid Dynamics, Hemisphere, New York.

Spalding, D. B. (1972). "A novel finite-difference formulation for differential expressions involving both first and second derivatives." International Journal for Numerical Methods in Engineering, 4, 551.

Speziale, C. G. (1985). "Galilean invariance of subgrid-scale stress models in large-eddy simulation of turbulence." Journal of Fluid Mechanics, 156, 55-62.

Speziale, C. G. (1987). "Second-order closure models for rotating turbulent flows." Quarterly of Applied Mathematics, 45, 721-733.

Speziale, C. G., Sarkar, S., and Gatski, T. B. (1991). "Modelling the pressure-strain correlation of turbulence. An invariant dynamical systems approach." Journal of Fluid Mechanics, 227, 245-272.

Squires, K. D., and Eaton, J. K. (1990). "Particle response and turbulence modification in isotropic turbulence." Physics of Fluids A, 2(7), 1191-1203.

Squires, K. D., and Eaton, J. K. (1991a). "Measurements of particle dispersion obtained from direct numerical simulations of isotropic turbulence." Journal of Fluid Mechanics, 226, 1.

Squires, K. D., and Eaton, J. K. (1991b). "Preferential concentration of particles by turbulence." Physics of Fluids A, 3(5), 1169-1178.

Sridhar, G., and Katz, J. (1995). "Drag and lift forces on microscopic bubbles entrained by a vortex." Physics of Fluids, 7(2), 389-399.

Stock, D. E. (1996). "Particle dispersion in flowing gases - Freeman scholar lecture." ASME Journal of Fluids Engineering, 118, 4-17.

Stokes, G. G. (1851). "On the effect of the internal friction of fluids on the motion of a pendulum." Trans. Cambridge Philos. Soc., 9, 8-27.

Stone, H. L. (1968). "Iterative solution of implicit approximations of multi-dimensional partial differential equations." SIAM Journal on Numerical Analysis, 5, 530-558.

Su, M., Chen, Q., and Chiang, C.-M. (2001). "Comparison of different subgrid-scale models of large eddy simulation for indoor airflow modeling." Journal of Fluids Engineering, 123, 628-639.

Sundaram, S., and Collins, L. R. (1996). "Numerical consideratins in simulating a turbulent suspension of finite-volume particles." Journal of Computational Physics, 124, 337-350. 
Sundaram, S., and Collins, L. R. (1999). "A numerical study of the modulation of isotropic turbulence by suspended particles." Journal of Fluid Mechanics, 379, 105-143.

Sundaresan, S., Eaton, J., Koch, D. L., and Ottino, J. M. (2003). "Appendix 2: Report of study group on disperse flow." International Journal of Multiphase Flow

Workshop on Scientific Issues in Multiphase Flow, 7-9 May 2002, 29(7), 1069-87.

Takagi, S., Oguz, H. N., Zhang, Z., and Prosperetti, A. (2003). "PHYSALIS: a new method for particle simulation. Part II: two-dimensional Navier-Stokes flow around cylinders." Journal of Computational Physics, 187, 371-390.

Tanaka, T., Yamagata, K., and Tsuji, Y. (1990) "Experiment on fluid forces on a rotating sphere and spheroid." Second KSME-JSME Engineering Conference, 1, 366.

Tanaka, T., Yamamoto, Y., Potthoff, M., and Tsuji, Y. (1997) "LES of gas-particle turbulent channel flow." ASME 1997 Fluids Engineering Division Summer Meeting, June 22-26, FEDSM97-3630.

Tang, L., Wen, f., Yang, Y., Crowe, C. T., Chung, J. N., and Troutt, T. R. (1992). "Selforganizing particle dispersion mechanism in free shear flows." Physics of Fluids $A, 4,2244$.

Taylor, G. I. (1923a). "On the decay of vortices in a viscous fluid." Philosophical Magazine, Series 6, 46, 671-674.

Taylor, G. I. (1923b). "Stability of a viscous liquid contained between two rotating cylinders." Philosophical Transactions of Royal Society London Series A, 223, 289-343.

Tennekes, H., and Lumley, J. L. (1972). A First Course in Turbulence, The MIT Press, Cambridge.

Thomas, P. J. (1992). "On the influence of the Basset history force on the motion of a particle through a fluid." Physics of Fluids A, 4(9), 2090-2093.

Tomiyama, A. (1998) "Struggles with computational bubble dynamics." Third International Conference on Multiphase Flows (ICMF98), Lyon, France, June.

Tomiyama, A., Kataoka, I., and Sakaguchi, T. (1995). "Drag coefficients of bubbles, 1st report: drag coefficient of a single bubble in a stagnant liquid." Transactions of JSME, Serial B., 61, 2357.

Tomiyama, A., Tampa, H., Zun, I., and Hosokawa, S. (2002). "Transverse migration of single bubbles in simple shear flows." Chemical Engineering Science, 57(11), 1849-1858.

Tran, M. L. (1997). "Modelisation instationnaire de la distribution spatiale des phases dans les ecoulementes diphasiques en regimes a bulles," $\mathrm{Ph} . \mathrm{D}$. thesis, University Claude Bernard Lyon.

Tri, B. D., Oesterle, B., and Deneu, F. (1990). "New results concerning the lift of a rotating sphere at intermediate Reynolds numbers." Comptes Rendus de l'Academie des Sciences, Serie II (Mecanique, Physique, Chimie Sciences de la Terre et de l'Univers), 311(1), 27-31.

Tryggvason, G., Bunner, B., Ebrat, O., and Tauber, W. (1998a). "Computations of multiphase flows by a finite difference/front tracking method. I multi-fluid flows." 29th Computational Fluid Dynamics. Lecture Series 1998-03, Von Karman Institute for Fluid Dynamics. 
Tryggvason, G., Bunner, B., Mortazavi, S., and Esmaeeli, A. (1997) "Direct numerical simulation of dispersed multiphase flows." JSME Conference, December.

Tryggvason, G., Esmaeeli, A., Mortazavi, S., Han, J., and Homma, S. (1998b).

"Computations of multiphase flows by a finite difference/front tracking method. II applications." 29th Computational Fluid Dynamics. Lecture Series 1998-03, Von Karman Institute for Fluid Dynamics.

Tryggvason, G., Juric, D., Che, J., Nobari, M. R. H., and Nas, S. (1998c). "Computations of multiphase flows by a finite difference/front tracking method. III variable surface tension and phase change." 29th Computational Fluid Dynamics. Lecture Series 1998-03, Von Karman Institute for Fluid Dynamics.

Tsao, H.-K., and Koch, D., L. (1997). "Observations of high Reynolds number bubbles interacting with a rigid wall." Physics of Fluids, 9(1), 44-56.

Tsuji, Y. (1991). "Review: turbulence modification in fluid-solid flows." Gas-Solid Flows, D. E. e. a. Stock, ed., ASME-FED, 1-6.

Tsuji, Y., Kato, N., and Tanaka, T. (1991). "Experiments on the unsteady drag and wake of a sphere at high Reynolds numbers." International Journal of Multiphase Flow, 17, 343-354.

Tsuji, Y., Morikawa, Y., and Mizuno, O. (1985). "Experimental measurement of the Magnus force on a rotating sphere at low Reynolds numbers." Journal of Fluids Engineering, 107, 484.

Turner, J. S. (1973). Buoyancy effects in fluids, Cambridge University Press, Cambridge.

Unverdi, S. O., and Tryggvason, G. (1992). "A front-tracking method for viscous incompressible, multi-fluid flows." Journal of Computational Physics, 100(1), 2537.

Van den Vorst, H. A. (1992). "BI-CGSTAB: a fast and smoothly converging variant of BI-CG for the solution of non-symmetric linear systems." SIAM Journal on Scientific Computing, 13, 631-644.

Van den Vorst, H. A., and Sonneveld, P. (1990). "CGSTAB, a more smoothly converging variant of CGS." Technical report 90-95, Delft University of Technology.

van Leer, B. (1974). "Towards the ultimate conservative difference scheme. II. Monotonicity and conservation combined in a second-order scheme." Journal of Computational Physics, 14(4), 361-370.

Vasilyev, O. V., Lund, T. S., and Moin, P. (1998). "A general class of commutative filters for LES in complex geometries." Journal of Computational Physics, 146, 82-104.

Vasseur, P., and Cox, R. G. (1977). "The lateral migration of spherical particles sedimenting in a stagnant bounded fluid." Journal of Fluid Mechanics, 80, 561591.

Versteeg, H. K., and Malalasekera, W. (1995). An Introduction to Computational Fluid Dynamics, Longman Scientific \& Technical.

Vojir, D. J., and Michaelides, E. E. (1994). "Effect of the history term on the motion of rigid spheres in a viscous fluid." International Journal of Multiphase Flow, 20(3), 547-556.

Voke, P. K. (1996). "Subgrid-scale modeling at low mesh Reynolds numbers." Theoretical and Computational Fluid Dynamics, 8, 131-143. 
Voke, P. K. (1997). "Flow past a square cylinder: test case LES2." Direct and LargeEddy Simulation II, J. P. Chollet, P. R. Voke, and L. Kouser, eds., Kluwer Academic, Dordrecht, 355-373.

von Karman, T. (1930) "Mechanische Aehnlichkeit und Turbulenz." Third International Congress of Applied Mechanics, Stockholm85-105.

Vreman, B., Geurts, B., and Kuerten, H. (1994). "Realizability conditions for the turbulent stress tensor in large eddy simulation." Journal of Fluid Mechanics, 278, 351-362.

Vreman, B., Geurts, B., and Kuerten, H. (1997). "Large-eddy simulation of the turbulent mixing layer." Journal of Fluid Mechanics, 339, 357-390.

Wang, L.-P., and Maxey, M. R. (1993). "Settling velocity and concentration distribution of heavy particles in homogeneous isotropic turbulence." Journal of Fluid Mechanics, 256, 27-68.

Wang, Q., and Squires, K. D. (1996a). "Large eddy simulation of particle deposition in a vertical turbulent channel flow." International Journal of Multiphase Flow, 22, 667.

Wang, Q., and Squires, K. D. (1996b). "Large eddy simulation of particle-laden turbulent channel flow." Physics of Fluids, 8(5), 1207-1223.

Wang, Q., Squires, K. D., Chen, M., and McLaughlin, J. B. (1997). "On the role of the lift force in turbulence simulation of particle deposition." International Journal of Multiphase Flow, 23(4), 749-763.

Wang, S. (1986). "Three-dimensional turbulence structure measurements in air/water two-phase flow," PhD thesis, Rensselaer Polytechnic Institute, Troy.

Wang, S., Lee, S., Jones, O. C., and Lahey, R. T. J. (1987). "Three-dimensional turbulence structure and phase distribution measurements in bubbly two-phase flow." International Journal of Multiphase Flow, 13, 327-343.

Wells, M. R., and Stock, D. E. (1983). "The effects of crossing trajectories on the dispersion of particles in a turbulent flow." Journal of Fluid Mechanics, 136, 3162.

Wendt, J. F., Anderson, J. D., Degrez, G., Dick, E., and Grundmann, R. (1996). Computational Fluid Dynamics: An Introduction, Springer-Verlag.

Weske, D. R., and Sturov, G. Y. (1974). "Experimental study of turbulent swirled flows in a cylindrical tube." Fluid Mechanics - Soviet Research, 3(1).

Whitaker, S. (1969). "Advances in the theory of fluid motion in porous media." Industrial and Engineering Chemistry, 61(12), 14-28.

Whitaker, S. (1999). The Method of Volume Averaging, Kluwer Academic Publishers, Dordrecht.

White, F. M. (1991). Viscous Fluid Flow, McGraw-Hill Inc., New York.

Wilcox, D. C. (1993). Turbulence Modeling for CFD, DCW Industries, La Canada, CA.

Wilson, R. V., and Demuren, A. O. (1996). "Two-dimensional spatially developing mixing layers." Numerical Heat Transfer, Part A, 29, 485-509.

Winant, C. D., and Browand, F. K. (1974). "Vortex pairing: the mechanism of turbulent mixing-layer growth at moderate Reynolds number." Journal of Fluid Mechanics, 63(2), 237-55.

Yakhot, V., and Orszag, S. A. (1986). "Renormalization group analysis of turbulence: I. basic theory." Journal of Scientific Computing, 1(1), 1-51. 
Yamamoto, Y., Potthoff, M., Tanaka, T., Kajishima, T., and Tsuji, Y. (2001). "Largeeddy simulation of turbulent gas-particle flow in a vertical channel: effect of considering inter-particle collisions." Journal of Fluid Mechanics, 442, 303-334.

Yang, C. Y., and Lei, U. (1998). "The role of the turbulence scales in the settling velocity of heavy particles in homogeneous isotropic turbulence." Journal of Fluid Mechanics, 371, 179.

Yang, M., Zhou, L. X., and Fan, L. S. (2002a) "A large-eddy simulation of bubble-liquid jets." ASME 2002 Fluids Engineering Division Summer Meeting, Montreal, Quebec, Canada, July 14-18, FEDSM2002-31214.

Yang, X., Thomas, N. H., Guo, L. J., and Hou, Y. (2002b). "Two-way coupled bubble laden mixing layer." Chemical Engineering Science, 57, 555-564.

Yee, H. C. (1987). "Construction of explicit and implicit symmetric TVD schemes and their applications." Journal of Computational Physics, 68(1), 151-79.

Yeh, F., and Lei, U. (1991). "On the motion of small particles in a homogeneous isotropic turbulent flow." Physics of Fluids A, 3, 2571.

Yeung, P. K., and Pope, S. B. (1988). "An algorithm for tracking fluid particles in numerical simulations of homogeneous turbulence." Journal of Computational Physics, 79(2), 373-416.

Yeung, P. K., and Pope, S. B. (1989). "Lagrangian statistics from direct numerical simulations of isotropic turbulence." Journal of Fluid Mechanics, 207, 531-586.

Yoshizawa, A., and Horiuti, K. (1985). "A statistically-derived subgrid-scale kinetic energy model for the large-eddy simulation of turbulent flows." Journal of the Physical Society of Japan, 54(8), 2834.

Young, J., and Leeming, A. (1997). "Theory of particle deposition in turbulent pipe flow." Journal of Fluid Mechanics, 340, 129-159.

Young, J. B., and Hanratty, T. J. (1991). "Trapping of solid particles at a wall in a turbulent flow." AIChE Journal, 37(10), 1529-1536.

Yuan, H., and Prosperetti, A. (1994). "On the in-line motion of two spherical bubbles in a viscous fluid." Journal of Fluid Mechanics, 278, 325-349.

Yuu, S., Yasukouchi, N., Hirosawa, Y., and Jotaki, T. (1978). "Particle diffusion in a dust laden round jet." AICHE J., 24, 509.

Zahrai, S., Bark, F. H., and Karlsson, R. I. (1995). "On anisotropic subgrid modeling." European Journal of Mechanics, B/Fluids, 14, 459-486.

Zang, Y., Street, R. L., and Koseff, J. R. (1993). "A dynamic mixed subgrid-scale model and its application to turbulent recirculating flows." Physics of Fluids A, 5, 31863196.

Zhang, D. Z., and Prosperetti, A. (1994). "Averaged equations for inviscid dispersed twophase flow." Journal of Fluid Mechanics, 267, 185-219.

Zhang, Z., and Prosperetti, A. (2003). "A method for particle simulation." Journal of Applied Mechanics, 70, 64-74.

Zhang, Z., and Prosperetti, A. (2005a). "A second-order method for three-dimensional particle simulation." Journal of Computational Physics, 210, 292-324.

Zhang, Z., and Prosperetti, A. (2005b) "Sedimentation of 1024 particles." ASME Fluids Engineering Division Summer Meeting and Exhibition, Houston, TX.

Zhu, J. (1994). "The second-order projection method for the backward facing step." Journal of Computational Physics, 117, 318-331. 
Zun, I., Kljenek, I., and Serizaw, A. (1992). "Bubble coalescence and transition from wall void peaking to core void peaking in turbulent bubbly flow." Dynamics of twophase flows, O. C. Jones and I. Michiyoshi, eds., 233-239. 


\section{Appendix A About the Navier-Stokes Solver "DREAM II"}

"DREAM II" is a transient, three-dimensional, finite-volume-method (FVM) NavierStokes solver used in this study. The solver is developed by the present author under the supervision of Dr. Ismail Celik, and it is a full reimplementation of the original DREAM code (written by Dr. Celik) with significant accuracy improvements, LES capability and many other new developments. Some key features include:

- Projection method according to (Kim and Moin 1985).

- Implicitness factor adjusting for both convection and diffusion terms.

- Third order QUICK (Quadratic Upwind Interpolation for Convective Kinematics)

- Fourth order central differencing.

- Fourth order deferred correction for pressure solution.

- $2^{\text {nd }}$ order Adam-Bashforth for time integration.

- LES with Implicit turbulence model.

- LES with Smagorinsky SGS (subgrid scale) model.

- LES with Dynamic SGS model.

- Efficient boundary condition setting.

- Capable of handing non-uniform grid.

- Reliable restart file.

\section{Description of Program Files}

Note that each file typically contains a collection of subroutines or other program units, which together performs a designated task. Also see Figure 2-4 for program flow chat.

DreamII.for: $\quad$ Main program, controls overall program flow, calls major solver subroutines, such as CALC_UVEL, CALC_VVEL, CALC_WVEL and CALC_PRES etc.

calc_pres.for: $\quad$ Calculates pressure or pseudo-pressure by solving the pressure Poisson equation.

calc_uvel.for: Calculates u-velocity component.

calc_vvel.for: Calculates v-velocity component.

calc_wvel.for: Calculates w-velocity component. 
config.for:

dream_cblock.for:

dream_param.for:

dump.for:

grid.for:

interp.for:

open_files.for:

projection.for:

properties.for:

psolv_cblock.for:

psolv_param.for:

psolvers.for:

restart.for:

set_bcons.for:

update_bcon.for: Updates boundary conditions.

utilities.for: Collection of utility subroutines.

Defines global variables.

Outputs results data. discretization schemes. Iteration) etc.

Output or read restart file. conditions of various types.
Takes input of flow and grid parameters, initializes field variables, performs other flow-related configurations.

Defines global program constants.

Generates orthogonal, staggered grid.

Collection of interpolation subroutines used by spatial

Opens input, output and restart files.

Projects predicted velocity field onto a divergence-free field.

Sets fluid properties and turbulence properties.

Defines shared variables used by matrix solvers.

Defines constants used by matrix solvers.

Collection of matrix solvers: SIP (Stone Implicit), CGSTAB (biConjugate Gradient Stabilized), ICCG (Incomplete Cholesky preconditioned Conjugate Gradient), ADI (Alternating Direction

Contains four subroutines for setting implicit and explicit boundary

Collection of utility subroutines. 


\section{Appendix B About the Lagrangian Particle Tracking Program "PART"}

"PART" is a Lagrangian particle tracking program developed by the present author. The program can be used in a combination with the N-S solver, DREAM II, to track particles velocity and trajectory in a three-dimensional space. The particles are advanced in time using the $2^{\text {nd }}$ or $4^{\text {th }}$ order Runge-Kutta method. Efficient particle population control is achieved by way of linked list.

\section{Description of Program Files}

part_cblock.for: Defines shared variables used by particle tracking routines.

part_param.for: $\quad$ Defines constants used by particle tracking routines.

part_start.for: $\quad$ Opens files and performs initialization tasks

part_typdef.for: Defines a derived type and its components for particle.

particles.for: $\quad$ Collection of particle tracking subroutines.

\section{Description of Major Subroutines in particles.for File}

Part_Initring: $\quad$ Sets up and initializes particle ring.

Part_Config: Configures particle properties and sets parameters used in the particle tracking routines.

Part_Iface_mesh: Takes grid information from the carrier flow solver.

Part_Iface_uvw: $\quad$ Takes velocity field from the carrier flow solver.

Part_Inject: Injects particles.

Part_Move: $\quad$ Moves particles.

Part_Move_onepart: Moves a single particle using RK2 or RK4.

Part_Append: Appends a particle to the particle ring. 
Part_Delete: Deletes a particle from the particle ring.

Part_Dump: $\quad$ Outputs particle results data.

Part_Write_restart: Writes particle restart file.

Part_Read_restart: Reads particle restart file.

Part_Get_icell: $\quad$ Locates the grid cell in which a particle resides.

Part_Interp_uc: $\quad$ Interpolates continuous fluid velocity onto the particle location.

Part_Interp_ucder: Interpolates fluid velocity gradients onto the particle location.

Part_Get_force: Computes hydrodynamic forces acting on a particle.

Part_Calc_vof: Calculates particle volume fraction.

Part_Calc_fcoup: Calculate interphase coupling force. 